Bernd Leiss, David Tanner, Axel Vollbrecht und Gernot Arp (Hg.)

\title{
Neue Untersuchungen zur Geologie der Leinetalgrabenstruktur
}

Bausteine zur Erkundung des geothermischen Potentials der Region Göttingen

GeothermieGruppeGöttingen (GGG)

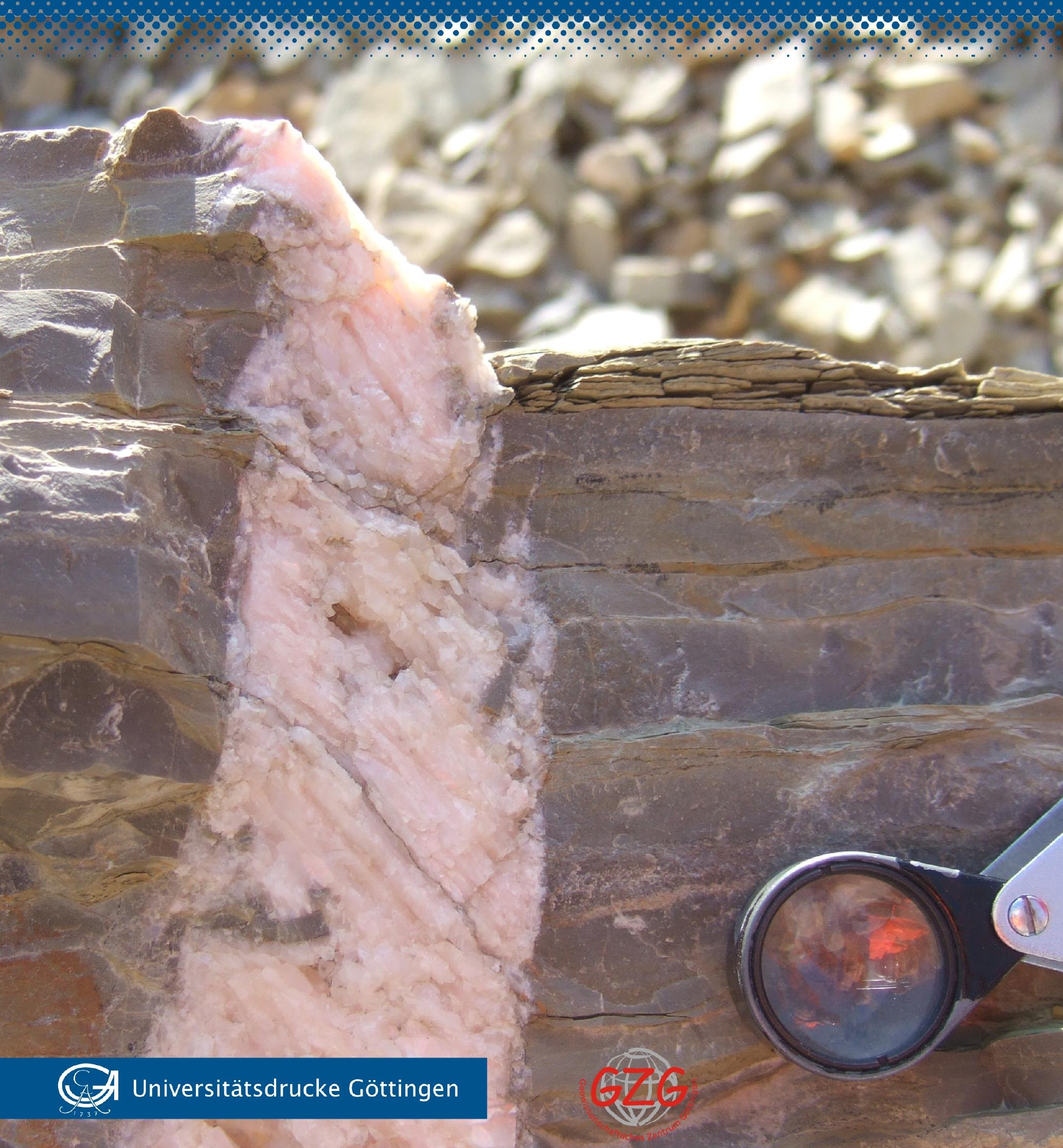


Bernd Leiss, David Tanner, Axel Vollbrecht, Gernot Arp (Hg.)

Neue Untersuchungen zur Geologie der Leinetalgrabenstruktur

This work is licensed under the Creative Commons License 3.0 "by-nd", allowing you to download, distribute and print the document in a few copies for private or educational use, given that the document stays unchanged and the creator is mentioned. You are not allowed to sell copies of the free version. 
erschienen in der Reihe der Universitätsdrucke

im Universitätsverlag Göttingen 2011 
Bernd Leiss, David Tanner,

Axel Vollbrecht, Gernot Arp (Hg.)

Neue Untersuchungen zur Geologie der

Leinetalgrabenstruktur

Bausteine zur Erkundung des geothermischen Potentials

der Region Göttingen

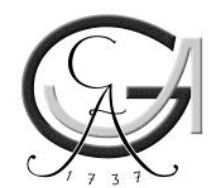

Universitätsverlag Göttingen 2011 


\section{Bibliographische Information der Deutschen Nationalbibliothek}

Die Deutsche Nationalbibliothek verzeichnet diese Publikation in der Deutschen Nationalbibliographie; detaillierte bibliographische Daten sind im Internet über < http://dnb.ddb.de> abrufbar

\section{Anschrift der Herausgeber}

Geowissenschaftliches Zentrum der Georg-August-Universität Göttingen Goldschmidtstr. 3

37077 Göttingen

Der Druck des Bandes wurde vom Geowissenschaftlichen Zentrum der Georg-August-Universität Göttingen und dem Verein der „Freunde der Geowissenschaften der Universität Göttingen e. V.“ finanziell unterstützt.
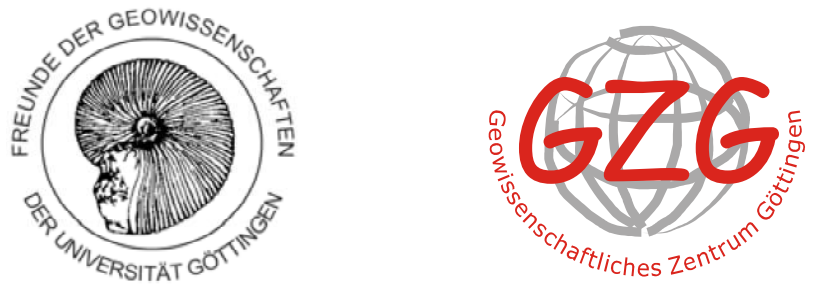

Dieses Buch ist auch als freie Onlineversion über die Homepage des Verlags sowie über den OPAC der Niedersächsischen Staats- und Universitätsbibliothek (http://www.sub.uni-goettingen.de) erreichbar und darf gelesen, heruntergeladen sowie als Privatkopie ausgedruckt werden Es gelten die Lizenzbestimmungen der Onlineversion. Es ist nicht gestattet, Kopien oder gedruckte Fassungen der freien Onlineversion zu veräußern.

Satz und Layout: David Tanner Umschlaggestaltung: Jutta Pabst Titelabbildung: Syntektonischer Calcitgang mit komplexer Entwicklung im Unteren Muschelkalk (Steinbruch Elvese)

Foto: Bernd Leiss 


\section{Inhaltsverzeichnis}

Vorwort

Bernd Leiss, David C. Tanner, Axel Vollbrecht und Gernot Arp vii

Zur Geologie des Leinetalgrabens - ein kurzer Überblick

Gernot Arp, Axel Vollbrecht, David C. Tanner und Bernd Leiss

Der Leinetalgraben als Teil einer regionalen Pull-Apart-Struktur

Axel Vollbrecht und David C. Tanner

Struktur der Leinetalgraben-Randstörung bei Reiffenhausen

(Autobahn 38 Heidkopftunnel-Westportal)

Gernot Arp, David C. Tanner und Bernd Leiss a

Ein Aufschluss im tieferen Steinmergelkeuper nahe der Leinetalgraben-

Randstörung (Kreuzberg Göttingen): Lithologische Abfolge und

Strukturgeologie

Gernot Arp, David C. Tanner und Bernd Leiss b

Exkursion nördliches Leinetal

David C. Tanner

Ebelhof-Abschiebungszone, Papenberg - extensionale Antikline und andere

Merkwürdigkeiten in den südlichen „Hausbergen“ des Göttinger Geozentrums

- der Luttertal Faltenzug

Till Heinrichs (mit einem Beitrag von Thomas Löffler)

3D-Störungsmorphologie bestimmt durch LIDAR-Messungen

David C. Tanner, Stefan Prüfer, Dirk Kuhn und Charlotte Krawczyk

Strukturgeologische 3D-Modellierung der Grabenrandstörung des Leinetals im östlichen Stadtgebiet von Göttingen

Jennifer Ziesch, David C. Tanner, Gernot Arp und Josef Paul

Störungskörper in einem Seitenverschiebungssystem bei Hardegsen

N. Wasja Bloch, David C. Tanner und Josef Paul

Struktur des Schwarzjura-Keuper-Vorkommens im Eichenberger

Grabenknoten bei Hottenrode

David C. Tanner, Gernot Arp, Frithjof A. Bense und Gabriele Ertl

Tiefer Mittlerer Muschelkalk im Liegenden der Hauptstörung temporäre Aufschlüsse am NE-Rand des Klausbergs, Göttingen NE

Till Heinrichs 
Sedimentgänge im Bausandstein der Solling-Folge NW' von Billingshausen (GK25, Blatt 4326 Lindau)

Malte Ritter, Axel Vollbrecht, Alfons M. van den Kerkhof und Klaus Wemmer

Lokalisierung von Störungen im Bereich der Leinetalgrabenstruktur

mittels VFL-Methode: Diskussion erster Ergebnisse

Michael Krumbholz, Axel Vollbrecht, Bernd Leiss, Johannes Großmann

und Hauke Hachmeister

Modellierung des Temperaturfeldes um eine tiefe Förderbohrung am östlichen Leinetalgrabenrand - Abschätzung des geothermischen Potentials France Albero und David Tanner

Zur Herkunft der Sole der Saline Luisenhall (Göttingen) mittels

Strontiumisotopie

Eike-Matthias Bultmann, Janina Ruprecht, Bent T. Hansen

und Ilka Kleinhanns

Gravitative Massenverlagerungen an der Röt/Muschelkalk-Schichtstufe

des Göttinger Waldes - GPS-gestützte Strukturkartierungen

Frithjof A. Bense, Gabriele Ertl, Axel Vollbrecht und Maurizio Battaglia

Mittlerer Keuper im direkten Hangenden des Göttinger Sprungs,

Göttingen, Georg-Dehio-Weg 5

Till Heinrichs und Bianca Wagner

Quartäre Akkumulation und Erosion am östlichen Rand der Leineaue

bei Göttingen — Detaillierte Quartärprofile aus den Baugruben

am Windausweg

Bianca Wagner, Christian Nieding, Christina Janssen und Maria Herold

Geoelektrische Profile entlang der Leineaue im nördlichen Göttingen

Bianca Wagner und Andreas Weller

Geotechnische Kartierung im Norden von Göttingen

Wiebke Jahnke, Alena Ohlmeyer, Marlene Rebens und Bianca Wagner

Tiefengeothermisches Potential in der Region Göttingen — geologische

Rahmenbedingungen

Bernd Leiss, David C. Tanner, Axel Vollbrecht und Klaus Wemmer 


\section{Vorwort}

\section{Bernd Leiss (GZG Göttingen), David C. Tanner (GZG Göttingen, LIAG Hannover), Axel Vollbrecht (GZG Göttingen) und Gernot Arp (GZG Göttingen)}

Der vorliegende Band enthält eine Sammlung von Arbeiten, die sich mit unterschiedlichen Aspekten der geologischen Entwicklung des Leinetalgrabens und seines strukturellen Rahmens befassen. Grundlage stellen neue und ältere, bislang nicht veröffentlichte Ergebnisse dar, die teilweise im Rahmen von geowissenschaftlichen Examensarbeiten erbracht wurden (i.W. Diplomarbeiten und -kartierungen, Bachelorarbeiten). Motivation für die Herausgabe dieses Bandes war es daher, diese häufig in Archiven ,versteckten' Daten interessierten Fachleuten, aber auch geologisch interessierten Laien zugänglich zu machen. In diesem Sinne dokumentiert der vorliegende Band auch neue Beobachtungen aus temporären Aufschlüssen und Bohrprofilen, die für strukturgeologische, aber auch stratigraphische Betrachtungen von Interesse sein können.

Herausgeber ist die Geothermie Gruppe Göttingen (GGG), in der GeowissenschaftlerInnen aus verschiedenen Abteilungen des Geowissenschaftlichen Zentrums der Georg-August-Universität Göttingen (GZG) tätig sind. Die GGG wurde im Jahre 2006 gegründet und hat sich zur Aufgabe gemacht, durch gezielte Untersuchungen und unter Einbindung älterer Daten zunächst ein dreidimensionales Strukturmodell für die Region Göttingen mit dem Leinetalgraben als zentraler Struktur zu erstellen. Dieses statische Modell

- Bernd Leiss - Geowissenschaftliches Zentrum der Universität Göttingen, Goldschmidtstr. 3, D-37077 Göttingen. bleiss1@gwdg.de

- David C. Tanner - GZG, jetzt Leibniz-Institut für angewandte Geophysik, Stilleweg 2, D-30655 Hannover. DavidColin.Tanner@liag-hannover.de

- Axel Vollbrecht - Geowissenschaftliches Zentrum der Universität Göttingen, Goldschmidtstr. 3, D-37077 Göttingen. avollbr@gwdg.de

- Gernot Arp - Geowissenschaftliches Zentrum der Universität Göttingen, Goldschmidtstr. 3, D-37077 Göttingen. garp@gwdg.de
(Ist-Zustand) stellt zunächst eine unabdingbare Grundlage für die anwendungsbezogene Bewertung geothermischer Potentiale dar, kann aber auch für die Entwicklung dynamischer Modelle im Rahmen der Tektonik Mitteleuropas in Betracht gezogen werden. Für diese Zielrichtung besteht eine wesentliche Aufgabe darin, aus den zahlreichen lokalen Beobachtungen zunächst jene elementaren Strukturmuster herauszufiltern, die sich in ein konsistentes Regionalmodell einbinden lassen. Ein weiterer wichtiger Themenkreis betrifft die Frage, inwieweit paläo-geothermische Anomalien noch die rezente Geothermie beeinflussen.

Da das Thema ,Geothermie unterschiedlichste Felder der Geowissenschaften erfasst, sind auch die Themen dieses Bandes entsprechend breit gefächert:

Der einleitende Artikel (Arp, Vollbrecht, Tanner und Leiss) gibt zunächst Überblick über die unterschiedlichen Vorstellungen zur Entwicklung des Leinetalgrabens. Dabei wird deutlich, dass die z.T. detaillierten Aufschlussbeobachtungen sehr unterschiedlich in die Tiefe projiziert wurden und zu entsprechend abweichenden Modellen führten. Eine wesentliche Frage war und ist weiterhin der Einfluss des Zechsteinsalinars auf die Strukturprägungen. Als Gemeinsamkeit stellt ein Großteil der früheren Arbeiten die strukturelle Asymmetrie des Leinetalgrabens heraus.

Die Arbeit von Vollbrecht und Tanner stellt den Leinetalgraben als ein Randelement des Mitteleuropäischen Dehnungsfeldes dar, das in der Oberkreide angelegt wurde und vor allem durch dextrale Pull-Apart-Strukturen charakterisiert ist. Ein Bezug zur Paläogeothermie wird in Verbindung mit Hochtemperaturdiagenese-Zonen im Deckgebirge und störungsgebundenen Mineralisationen diskutiert.

Die extremen rheologischen Kontraste innerhalb der mesozoischen Schichtenfolge führen offensichtlich zu komplexen Störungsgeometri- 
en und entsprechenden Verformungsstrukturen in den angrenzenden Schollen. Derartige Strukturen werden aus großflächigen Aufschlüssen am Heidkopftunnel-Westportal (Reiffenhausen) beschrieben, in denen die östliche Leinetalgraben-Randstörung angeschnitten wird (Arp, Tanner und Leissa). Die listrische Form der östlichen Randstörungen und eine damit verbundene Rotation der Schichten in ein E- bis ENE-Einfallen wurde durch die vorliegenden Arbeiten mehrfach bestätigt, u.a. für einen Abschnitt des Grabenrandes am Kreuzberg (Arp, Tanner und Leissb).

Die anschließenden Arbeiten dokumentieren anhand von detaillierten Strukturanalysen die Komplexität der tektonischen Entwicklung des Leinetalgrabens. Als wesentliche Erkenntnis daraus ist hervorzuheben, dass Seitenverschiebungen eine größere Bedeutung zukommt, als bislang angenommen wurde. Dies wird zunächst in einem Exkursionsführer von Tanner anhand von mehreren Aufschlüssen für den nördlichen Abschnitt des Leinetalgrabens dokumentiert. Entsprechendes zeigen Geländeaufnahmen im Bereich des Luttertals, wo sehr komplizierte Überlagerungen von NW-SE streichenden Falten und Störungen vorliegen, die von jüngeren $\mathrm{E}-\mathrm{W}$ streichenden Abschiebungen mit dextraler Komponente versetzt werden (Heinrichs mit einem Beitrag von Loeffler). Im Steinbruch Elvese war als Teil des Ahlsburg-Lineaments eine Störungsfläche von $120 \times 20 \mathrm{~m}$ aufgeschlossen. Tanner, Pruefer, Kuhn und Krawczyk haben die Störungsfläche mit Hilfe eines Laserscanners (LIDAR) aufgenommen und können auf Basis der dadurch darstellbaren Morphologie, die polyphase Kinematik ableiten.

Anhand von neuen Geothermiebohrungen und Verwendung archivierter Geländebefunde konstruierten Ziesch, Tanner, Arp und Paul ein 3D-Modell, das u.a. einen Versatz der östlichen Grabenrandstörung durch eine dextrale Seitenverschiebung beinhaltet. Am Westrand des Leinetalgrabens, im Steinbruch Hardegsen, finden sich innerhalb einer dextralen Störungszone spektakuläre Aufwölbungsstrukturen (Bloch, Tanner und Paul).

Besonders ausgeprägte Rotationen um vertikale Achsen, die an Schleppfalten über dextralen Störungen gebunden sind, werden aus Schichtfolgen des O' Keuper/U' Jura bei Hottenrode beschrieben (Tanner, Arp, Bense und Ertl).

Für die Entwicklung von Strukturmodellen sind ebenso Informationen über die Versatzbeträge an Störungen von Bedeutung. Für die Hauptstörungen am Ostrand des Leinetalgrabens werden Sprunghöhen im Bereich von mehreren hundert Metern angenommen. Einen Beleg hierfür liefert Heinrichs anhand von Beobachtungen in temporären Aufschlüssen am NE-Rand des Klausbergs.

Sandsteingänge im Bausandstein der SollingFolge, die vermutlich im Tertiär entstanden sind, werden als Beispiel für Kleinstrukturen beschrieben, die u.a. als Milieuanzeiger und Paläospannungs-Indikatoren dienen können (Ritter, Vollbrecht, v.d. Kerkhof und Wemmer). Die Öffnungsrichtung dieser Gänge könnte ebenfalls mit dextralen Bewegungen in Einklang gebracht werden.

Als eine Möglichkeit, auch verdeckte Bruchstrukturen genauer $\mathrm{zu}$ lokalisieren und Hinweise auf deren Verlauf in der Tiefe zu gewinnen, wird ein VLF-basiertes Verfahren in Form einer Pilotstudie vorgestellt (Krumbholz, Vollbrecht, Leiss, Grossmann und Hachmeister). Ein Einsatz dieses Verfahrens könnte vor allem die Kenntnisse über die offensichtlich komplex strukturierten Randstörungszonen des Leinetalgrabens erheblich verbessern.

Einen direkten Bezug zwischen einer konkreten Störungsstruktur in der östlichen Grabenrandzone bei Sudheim, dem damit verbundenen geothermischen Potential und die Auswirkung der langfristigen Nutzungsdauer einer $1000 \mathrm{~m}$ tiefen Förderbohrung zeigen die Modellierungen von Albero und Tanner.

Für die Modellierung konvektiver Wärmetransporte sind Kenntnisse über die Herkunft und den Charakter rezenter Fluidsysteme von Bedeutung. Isotopengeochemische Untersuchungen an Proben aus der Saline Luisenhall lassen darauf schließen, dass hier vor allem Salze der Roten Salztone aus der Aller-Folge (254 Ma) durch meteorische Wässer gelöst wurden (Bultmann, Ruprecht, Hansen und Kleinhanns).

Lokal wird die Geologie in bestimmten Teilregionen von jungen gravitativen Massenverlagerungen mitgeprägt, deren Ausmaß und genaue Ursachen (z.B. Einfluß von Subrosion) z.T. noch nicht vollständig geklärt sind. Die Arbeit von 
Bense, Ertl, Vollbrecht und Battaglia gibt ein Beispiel für hochauflösende GPS-gestützte Detailkartierungen entsprechender Strukturen, die auch die Möglichkeit eines Langzeitmonitorings eröffnen könnten. In temporären Aufschlüssen im Stadtgebiet von Göttingen wurden ebenfalls mächtige quartäre Rutschmassen nachgewiesen, die hier die östliche Hauptrandstörung des Leinetalgrabens überdecken (Heinrichs und Wagner).

Weitere Informationen zur jüngsten Entwicklungsgeschichte, in diesem Fall die weichselzeitliche bis jungholozäne Akkumulations- und Erosionsdynamik, liefern detaillierte Analysen quartärer Deckschichten, die temporär in Baugruben im östlichen Stadtgebiet von Göttingen aufgeschlossen waren (Wagner, Nieding, Janssen und Herold). Für zwei Profile entlang der Leineaue im nördlichen Göttingen wird demonstriert, wie durch geoelektrische Messungen die Mächtigkeit der quartären Deckschichten und die Morphologie der prä-quartären Geländeoberfläche genauer erfasst werden können (Wagner und Weller). Dadurch ergeben sich $u . U$. wichtige Informationen über verdeckte Störungen bzw. daran gebundene Hebungs- und Senkungszonen.

Für die konkrete Planung von Projekten zur Erschließung tiefer Geothermie sind für die ausgewählten Standorte auch geotechnische Kartierungen in Betracht zu ziehen. $\mathrm{Zu}$ einem Teilaspekt, im vorliegenden Fall die Beurteilung des Baugrunds, führten Jahnke, Ohlmeyer, Rebens und Wagner im Rahmen von Diplomarbeiten Untersuchungen im nördlichen Stadtgebiet von Göttingen durch.

Im abschließenden Beitrag wird der aktuelle geologische Kenntnisstand der Leinetalgrabenstruktur in Hinblick auf eine potentielle tiefengeothermische Nutzung zusammengefasst (Leiss, Vollbrecht, Tanner und Wemmer). Als Fazit werden eine Reihe offener Fragen formuliert, die die Notwendigkeit einer Fortführung intensiver geowissenschaftlicher Erkundung und deren Bedeutung herausstellen.

Es ist daher geplant, weitere Ergebnisse noch laufender, bzw. geplanter Untersuchungen in gleicher Weise zu publizieren.

B. Leiss, D.C. Tanner, A. Vollbrecht, G. Arp

(für die GeothermieGruppeGöttingen)

\section{Danksagung}

Die GeothermieGruppeGöttingen (GGG) dankt der Fakultät für Geowissenschaften und Geographie der Georg-August-Universität für die Unterstützung ihrer Arbeit.

Die GGG ist eine fachübergreifende, vom Mittelbau initiierte und getragene Arbeitsgruppe der Fakultät und hat die Förderung der geologischen Grundlagenforschung für die Nutzung geothermischer Energie in der Region Göttingen zum Ziel. 



\title{
Zur Geologie des Leintalgrabens — ein kurzer Überblick
}

\author{
Gernot Arp (GZG Göttingen), Axel Vollbrecht (GZG Göttingen), David C. Tanner (GZG \\ Göttingen, LIAG Hannover) und Bernd Leiss (GZG Göttingen)
}

\begin{abstract}
Zusammenfassung - Obwohl der Leinetalgraben schon seit dem späten 19. Jahrhundert als Senkungsgebiet bekannt ist, werden tektonische Entwicklungmodelle bis heute diskutiert. Gründe hierfür sind zum einen die Komplexität der Verformungsstrukturen, die aus der Mehrphasigkeit der tektonischen Entwicklung, dem Zusammenwirken von ,Ortho- und Halotektonik' und hohen rheologischen Kontrasten in den Deckgebirgsschichten resultiert. Zum anderen fand die Diskussion über die Entwicklung des Leinetalgrabens vor dem Hintergrund unterschiedlicher großregionaler Krustenmodelle statt.
\end{abstract}

\begin{abstract}
Although the Leinetal graben has been known as a zone of subsidence since the $19^{\text {th }}$ century, models of its tectonic development are still under discussion to date. On one hand the reasons for this are a result of the complexity of the structures, the polyphase tectonic development, the combination of ortho- and halotectonics and the strong rheological contrasts present in the overburden. On the other hand the development of the Leinetal graben has been discussed against a background of various large-scale crustal models.
\end{abstract}

Schlüsselworte—Leinetalgraben, südniedersächsisches Becken, Stockwerkstektonik.

Der Leinetalgraben (Abb. 1) ist eine der augenfälligsten tektonischen Strukturen im südlichen Niedersachsen. Seine zeitliche und strukturelle Entwicklung wird bis heute kontrovers diskutiert (z.B. Ritzkowski 1999). Dass hier eine generell N-S streichende, beidseitig von Störungen begrenzte Senkungszone vorliegt, wurde bereits von Lang (1880) erkannt. Folgende Bearbeitungen beschreiben den Leinetalgraben vor allem als Teil eines zentraleuropäischen Grabensystems, der sogenannten ,Mittelmeer-Mjösen-Zone': v. Koenen (1886, S. 198); Stille (1923/1925); Brinkmann (1933); Richter (1934); Wunderlich (1955, S. 79); Murawski (1960); Ortlam (1980). In neueren geodynamischen Modellen stellt der Leinetalgraben dagegen ein Teilelement des Niedersächsischen Beckens innerhalb des zentraleuropäischen

- Gernot Arp - Geowissenschaftliches Zentrum der Universität Göttingen, Goldschmidtstr. 3, D-37077 Göttingen. garp@gwdg.de

- Axel Vollbrecht - Geowissenschaftliches Zentrum der Universität Göttingen, Goldschmidtstr. 3, D-37077 Göttingen. avollbr@gwdg.de

- David C. Tanner - GZG, jetzt Leibniz-Institut für angewandte Geophysik, Stilleweg 2, D-30655 Hannover. DavidColin.Tanner@liag-hannover.de

- Bernd Leiss - Geowissenschaftliches Zentrum der Universität Göttingen, Goldschmidtstr. 3, D-37077 Göttingen. bleiss1@gwdg.de

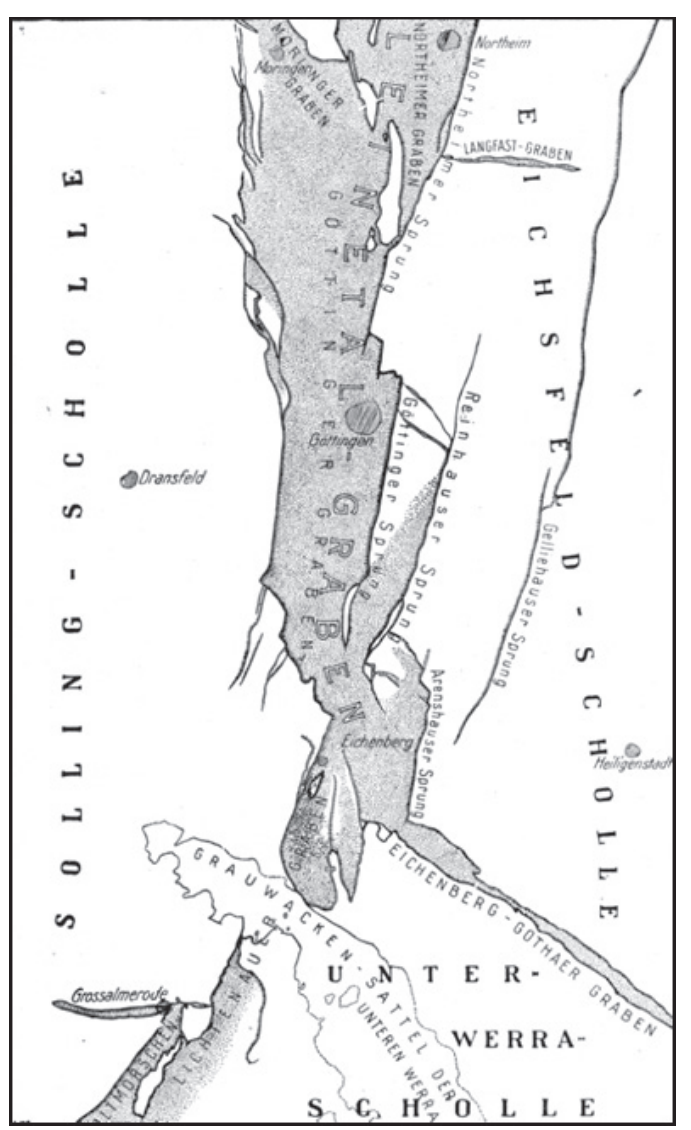

Abbildung 1. Strukturgeologische Kartenskizze des Leinetalgrabens (aus Stille \& Lotze 1933). 
Beckensystems dar (z.B. Littke et al. 2005), dass spätvariszisch angelegt und vorwiegend im jüngeren Mesozoikum bis Känozoikum durch großräumige Extensions- und Kompressionsphasen überprägt wurde (z.B. Betz et al. 1987, Ziegler 1990). Eine entsprechende mehrphasige Entwicklung kann auch für den Leinetalgraben angenommen werden. So wurde bereits früh eine mesozoische Anlage erkannt (z.B. Grupe 1909, Stille \& Lotze 1933, Brinkmann 1933 S. 8, Murawski 1951, 1960) und eine kretazisch/tertiäre Reaktivierung (heute als ,Inversion' bezeichnet) der mesozoisch geprägten Bruchstrukturen angenommen (z.B. Grupe 1909, Murawski 1951, 1957 S. 4, 1960, S. 301; Ziegler 1992, S. 92). Einige Autoren sehen sogar bis ins Quartär anhaltende Senkungen (Brinkmann 1933, Lüttig 1974, Wunderlich 1955, 1959).

Konkrete Beobachtungen, welche Zeitmarken der Grabenbildung setzen können, sind spärlich. Analogieausschlüsse können jedoch aus dem parallel streichenden Ohmgebirgsgraben ca. $30 \mathrm{~km}$ SSE' von Göttingen gezogen werden. Dieser zeigt über Störungen transgrediertes Cenoman, welches später weiter eingesunken ist (z.B. Frebold 1921, Wunderlich 1957, Nagel \& Wunderlich 1976). Aus dem Göttinger Raum wurden Relikte kreidezeitlicher Sedimente bisher nur auf der westlichen Grabenschulter bei Meensen in Form cenomaner Sandsteingerölle mit Inoceramen nachgewiesen (Murawski 1952).

Jüngere Sedimente sind erst wieder ab dem Eozän vertreten, und zwar fast ausschließlich auf der westlichen Grabenschulter (eozäne Tone, oligozäne marine Sande, miozäne limnischfluviatile Sande und Braunkohlen; Murawski 1960, Schüssler 1989, Ritzkowski 1999). Diese Sedimente greifen entlang einer paläogenen Rumpffläche diskordant über bereits verstellten Mittleren Buntsandstein bis Oberen Muschelkalk hinweg (Ritzkowski 1999). Auf der östlichen Grabenschulter sind einzig Bohnerz-Relikte mutmaßlich eozänen Alters zu finden, möglicherweise auch Relikte mariner Oligozän-Fossilien (Murawski 1955, S. 301; Ritzkowski 1999). Nach neueren Untersuchungen werden auch Sandsteingänge im Bausandstein der Solling-Folge als weitere Relikte eozäner Sedimentation in Betracht gezogen (Ritter et al., dieser Band).

Im Grabeninneren selbst findet sich dagegen kein Tertiär (Lang 1880), mit Ausnahme eines Oberoligozän-Vorkommens bei Moringen, das ver- mutlich in einer Subrosionssenke liegt (v. Koenen 1895; Frebold 1921, Jordan 1984). Die AlkaliOlivin-Basalte der Dransfelder Hochfläche, welche die oben genannten Tertiärsedimente vor vollständiger Abtragung im Pleistozän schützten, sind schließlich mittelmiozänen Alters (Kreuzer et al. 1973; Wedepohl 1982).

Die strukturgeologische Entwicklung des Leinetalgrabens ist äußerst komplex, vor allem bedingt durch die mehrphasige Tektonik in unterschiedlichen Spannungsfeldern und einer ausgeprägten Stockwerkstektonik. Letztere ergibt sich aus dem variszischen Grundgebirge, dem auflagernden Zechsteinsalinar und dem mesozoischen Deckgbirge, das wiederum aus Schichten sehr unterschiedlicher Kompetenz aufgebaut wird. So stellt sich bei vielen strukturgeologischen Beobachtungen die Frage nach einer möglichen Beteiligung von Salinar-beeinflussten Prozessen, entweder in Form von aktiven Fließbewegungen (Halotektonik) oder durch Subrosion, verbunden mit bruchhaften Kollapsstrukturen. Deutlichste Belege für halotektonische Einflüsse sind Intrusionen von Zechsteinsalzen in verschiedene Salinarhorizonte des hangenden Deckgebirges.

Das Resultat der Verknüpfung mehrphasiger Tektonik und unterschiedlicher Rheologien ist u.a. ein enges Nebeneinander von Dehnungsund Einengungsformen (z.B. Stille \& Lotze 1933, Wunderlich 1957) und eine häufig zu beobachtende Kombination von Bruch- und Faltungsstrukturen, welche auch in anderen Bereichen des südniedersächsischen Berglandes bzw. Weserberglandes verbreitet ist. Hierfür wurde von Stille (1910, 1925) der Begriff ,saxonische Faltung' geprägt.

Die wesentlichen, das Kartenbild prägende Strukturen, lassen sich vereinfacht wie folgt charakterisieren: der Grabenostrand wird im Wesentlichen durch bevorzugt NNE streichende Hauptabschiebungen gebildet, für die erhebliche Sprunghöhen nachgewiesen werden konnten (Stille 1932, Lotze 1932): Northeimer Sprung (700-900 m), Göttinger Sprung (700-800 m), Reinhäuser Sprung (ca. $400 \mathrm{~m}$ ) und Arenshäuser Sprung (ca. $400 \mathrm{~m}$ ).

Am Grabenwestrand sind dagegen stärker NS orientierte, z.T. stark aufgefiederte Störungen mit geringeren Versatzbeträgen entwickelt (Lotze 1932a, Patriciu 1930). Diese sind allerdings aufgrund der dort stärkeren Quartärbedeckung im Gelände schwieriger zu verfolgen. Bei Elliehausen ist ein Verwerfungsbetrag von insgesamt 

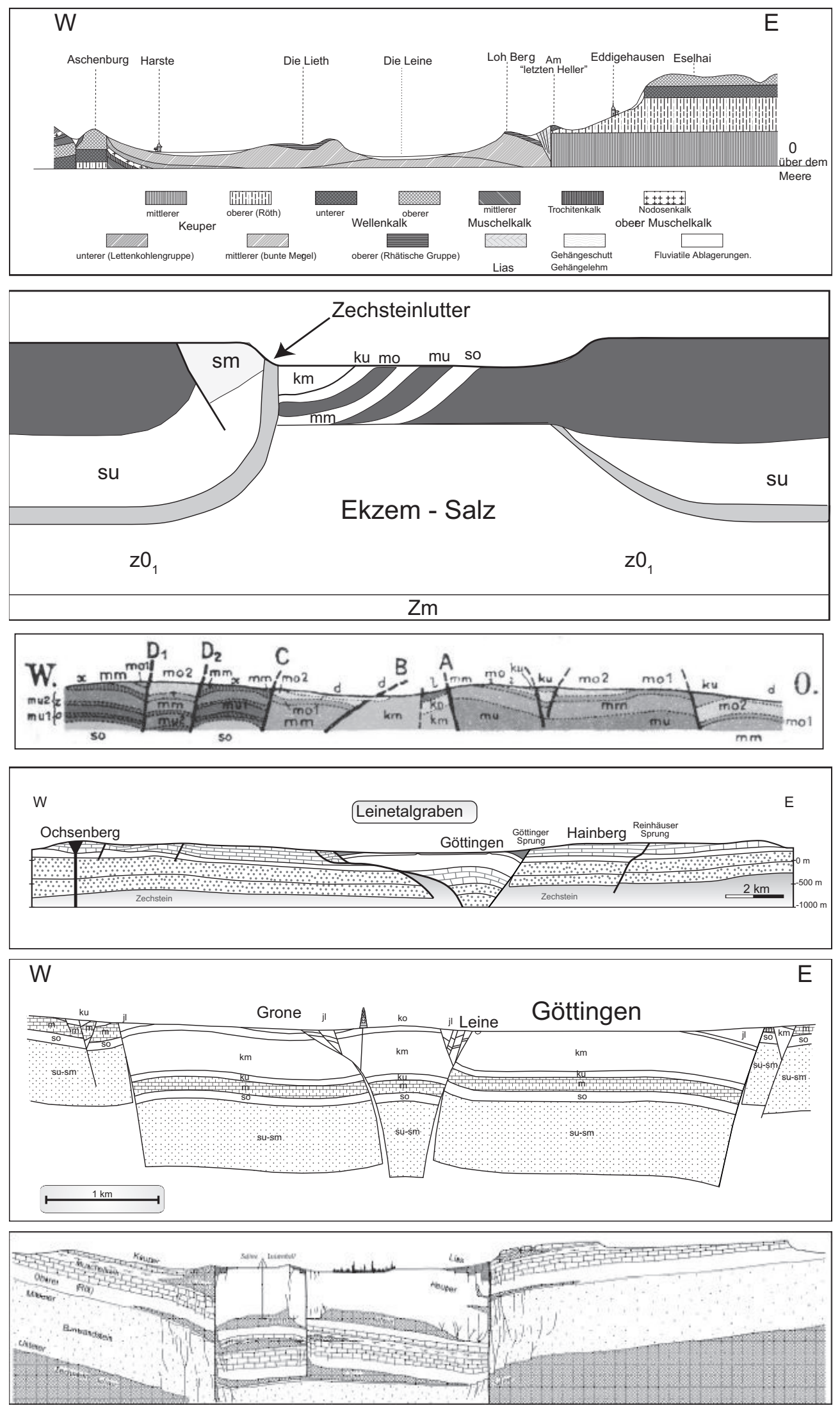

Abbildung 2. Geologische Querprofile des Leinetalgrabens als Dokumente einer langen Erforschungsgeschichte. Von oben nach unten: Lang (1880), Lachman (1917), Patriciu (1930), Stille und Lotze (1933), Wunderlich (1966) und Meischner (2002). 
ca. $650 \mathrm{~m}$, auf zwei Abschiebungen verteilt, zu beobachten. Beim Bau der Autobahn A 38 nahe dem Drammetal konnte ein Verwerfungsbetrag von etwa $400 \mathrm{~m}$ zwischen Rhätkeuper (Grabeninneres) und darauf aufgeschobenen höheren Lettenkohlenkeuper (Grabenschulter) beobachtet werden.

Inwieweit diese oberflächlich ausstreichenden Hauptstörungen sich durch das Zechsteinsalinar bis ins Grundgebirge fortsetzen, ist ungeklärt. Hochauflösende geophysikalische Untersuchungen hierzu sind aus dem Leintalgraben-Gebiet nicht bekannt. Auch liegen die wenigen Tiefbohrungen allesamt außerhalb des Grabens. In den Bohrungen Northeim 1 und Dransfeld 1 wurde das Grundgebirge bei etwa $1140 \mathrm{~m}$ unter NN angetroffen (Fabian 1957; Müller et al. 1961). Die weiter östlich gelegenen Bohrungen zeigen eine von $820 \mathrm{~m}$ unter NN (Northeim 3; Pöhlig 1986) bis auf $180 \mathrm{~m}$ unter NN (bei Duderstadt; Grupe 1909) ansteigende Grundgebirgsoberfläche, welche das Relief der Eichsfeldschwelle widerspiegeln. Ohnehin liegen die oben genannten Tiefbohrungen zwischen 20 und $40 \mathrm{~km}$ voneinander entfernt, so dass hieraus keine Aussagen über Verwerfungen und deren Beträge abgeleitet werden könnten. Ohne das Grundgebirge zu erreichen hat allerdings die Bohrung Sudheim 2 die östliche Grabenrandstörung durchteuft und ist auf die Zechstein-Gruppe im Bereich der Grabenschulter getroffen (Grupe 1923; Jordan 1984). Aus der Verbindung mit dem Oberflächenausbiss lässt sich ein durchschnittliches Einfallen der Grabenrandstörung von $40^{\circ}$ zum Grabenzentrum hin ableiten (Grupe 1923, Jordan 1984). Parallel zu den Randstörungen sind abschnittweise sog. „Streifenhorste' (z.B. Hardenberg-Horst; Gallbeutel S' Elliehausen; Sülzeberg bei Groß-Schneen: Lotze 1932b, Abb. 9) sowie ,Streifengräben' (z.B. Lias-Streifen zwischen Mariaspring und Weende) entwickelt. Insgesamt erscheint das Grabeninnere sattelartig gewölbt, so dass die Schichten oftmals beidseitig zu den Randstörungen hin einfallen (z.B. Lang 1880, S. 803).

Die in Abb. 2 dargestellten Strukturmodelle beziehen sich auf die Hauptstörungen der Grabenrandzonen sowie das Grabeninnere und dokumentieren unterschiedliche Interpretationsansätze bezüglich Tiefgang, Form und Verzweigung der Störungen, bzw. ihre Beziehungen zu halotektonischen Elementen. Im Grabeninneren wurde ein Salzlager durch die Salinenbohrung Luisenhall in 446,5 m Teufe erschlossen (296 m unter NN; Lang
1880, S. 802, v. Koenen 1894，1908, Tornquist 1892). Die eindeutige Zuordnung dieser Salze zu einem der bekannten Salinarhorizonte im Deckgebirge ist bis heute umstritten. Die Schwefelisotopenverhältnisse des Sulfats in der Salinenlauge lassen nach Wunderlich (1966, S. 683) auf eine Herkunft aus dem Keuper schließen. Nach neuen Untersuchungen von Bultmann et al. (dieser Band) kann jedoch angenommen werden, dass hier vor allem Salze des Roten Salztones aus der Aller-Folge (254 Ma) durch meteorische Wässer gelöst wurden. Die Kenntnis der Altersstellung derartiger Salzvorkommen ist für die Aufklärung des strukturgeologischen Baus des Leinetalgrabens von entscheidender Bedeutung, da eine stratigraphische Zuordnung zum Zechstein oder aber zum Keuper in diametral verschiedenen Profilschnitten und Erklärungsmodellen resultiert (vergl. Modelle in Abb. 2).

Neben den oben beschriebenen Hauptstörungszonen stellen NNW (herzynisch) streichende, schmale und im Auslaufbereich z.T. sigmoidal verbogene Gräben ein weiteres charakteristisches Strukturelement dar, welches bevorzugt auf der östlichen Grabenschulter vorkommt (s. Abb. 1). Hierzu zählen u.a. der Langfastgraben (Wunderlich 1957), der Herberhäuser Graben (v. Koenen 1894, 1908, Stille 1932, Stille \& Lotze 1933), die Kleperspalte (v.Koenen 1894, 1908, Stille 1932, Stille \& Lotze 1933) und die Friedländer Spalte (Lotze 1932b). Mit Ausnahme des Langfastgrabens sind diese 'Spezialgräben' offensichtlich auf das tektonische Stockwerk des Mittleren bis Oberen Muschelkalks beschränkt. Die o.g. tektonischen Strukturen wurden lokal noch durch gravitative Massenverlagerungen modifiziert bzw. von diesen überdeckt. Hierzu zählen u.a. ,wurzellose Schollen', bei denen es sich vor allem um isolierte Muschelkalkschollen auf Röt oder Mittlerem Keuper handelt. Größere Vorkommen wurden u.a. in einem Straßeneinschnitt zwischen Diemarden und Reinhausen (Blatt Reinhausen; v. Koenen 1894), vom Westportal des HeidkopfTunnels (Arp et al., dieser Band) und aus dem Bereich nördlich Marzhausen (Schott 1930) nachgewiesen. Auch der Grebenberg bei Angerstein ist ein wurzelloses Mosaik aus Muschelkalkschollen, die dem Mittleren Keuper auflagern (Schröder 1977, Jordan 1984).

Auch über mögliche Zusammenhänge zwischen Leintalgrabenbildung und tertiärem Basaltvulkanismus wurde lange diskutiert. So geht bereits 
v. Koenen (1886) von einem ursächlichen Zusammenhang aus, und Stille (1925, S. 170ff.) nimmt an, dass die Basalte an die N-S-verlaufenden Brüche des Leinetalgraben gebunden sind. Spekuliert wurde u.a. auch darüber, warum die Basalte auf die westliche Grabenschulter beschränkt sind. Nagel \& Wunderlich (1976) stellen in diesem Zusammenhang die Frage, ob eine geringe Zechsteinsalzmächtigkeit unter der Dransfelder Hochfläche das dortige Vorhandensein von Basaltdurchbrüchen begünstigt, während weiter östlich und nördlich mächtige Salzlager den Aufstieg der Magmen verhinderten.

Ein umfassendes strukturgeologisches Modell für den Leinetalgraben, das alle Beobachtungen miteinander verknüpft, besteht derzeit noch nicht. Neuere tektonische Konzepte beinhalten u.a. eine stärkere Beteiligung von transpressiven und transtensiven Deformationen, Rotationen an Störungen mit stark variierenden Sprunghöhen (Rampenbildung) sowie komplexe Verwerfungsmuster an unsteten Störungen. Beispiele hierfür liefern u.a. einige Beiträge dieses Bandes (dort weitere Zitate neuerer Literatur). Für Modelle mit größerem Tiefgang fehlen jedoch weitere geophysikalische Informationen, die eine fundierte Einbeziehung des variszischen Grundgebirges als Quelle der ,Orthotektonik` ermöglichen könnten.

\section{Literatur}

Arp, G., Tanner, D.C., Leiss, B. 2011. Struktur der Leinetalgraben-Randstörung bei Reiffenhausen (Autobahn 38 HeidkopftunnelWestportal), dieser Band.

Betz, D., Führer, F., Plein, E. 1987. Evolution of the Lower Saxony Basin. Tectonophysics 137, 127-170.

Brinkmann, R. 1933. Landschaftsformung und junge Krustenbewegungen im Leinegebiet. Die Naturwissenschaften 21, 7-11.

Bultmann E.-M., Ruprecht, J., Hansen, B.T., Kleinhanns, I. 2011. Zur Herkunft der Sole der Saline Luisenhall (Göttingen) mittels Strontiumisotopie, dieser Band.

Fabian, H.J. 1957. Die Bohrung, Northeim 1‘. Ergebnisse eines regionalgeologisch interessanten Aufschlusses am Leinetalgraben. Neues Jahrbuch Geologie Paläontologie Abhandlungen 105 (1), 113-122.

Frebold, G. 1921. Der Einfluss der Zechsteinsalze auf die Gestaltung des Göttinger Leinetales. Dissertation Universität Tübingen 1921, 35 S.
Grupe, O. 1909. Über die Zechsteinformation und ihr Salzlager im Untergrunde des hannoverschen Eichsfeldes und angrenzenden Leinegebietes nach den neueren Bohrergebnissen. Zeitschrift Praktische Geologie 17, 185-205.

Grupe, O. 1923. Zur Entstehung des Göttinger Leinetalgrabens. Jahrbuch der Preußischen Geologischen Landesanstalt, 42 (f. 1921), 595620.

Jordan, H. 1984. Erläuterungen zur geologische Karte von Niedersachsen 1:25000, Blatt Nr. 4325 Nörten-Hardenberg, 148 S.

Koenen, A.v. 1882. Über den geologischen Bau der Umgegend von Göttingen. Nachrichten der königlichen Gesellschaft der Wissenschaften zu Göttingen 1882, Nr. 11, 309-311.

Koenen, A.v. 1886. Ueber die Störungen, welche den Gebirgsbau im nordwestlichen und westlichen Deutschland bedingen. - Nachrichten der königlichen Gesellschaft der Wissenschaften zu Göttingen 1886, Nr. 6, 196-199.

Koenen, A.v. 1894. Erläuterungen zur Geologischen Spezialkarte von Preußen und den Thüringischen Staaten. - 62. Lieferung Blatt Göttingen [4425 Göttingen]. 58 S. [O. Hrsg. i.d. Königlich Preußische Geologische Landesanstalt und Bergakademie; Berlin] Berlin.

Koenen, A.v. 1895. Mitteilungen über Aufnahmen auf den Blättern Jühnde, Freden und Alfeld. Jahrbuch der Preußischen Geologischen Landesanstalt, 15 (f. 1894), XXXII-XXXIII.

Koenen, A.v. 1908. Erläuterungen zur Geologischen Spezialkarte von Preußen und den Thüringischen Staaten. 62. Lieferung Blatt Göttingen [4425 Göttingen]. 57 S. [Hrsg. Königlich Preußische Geologische Landesanstalt; Berlin] Berlin.

Kreuzer, H., Besang, C., Harre, W., Müller, P., Ulrich, H.-J., Vinken, R. 1973. K/ArDatierungen an jungtertiären Basalten aus dem Vogelsberg und aus dem Raum zwischen Kassel und Göttingen. Fortschritte der Mineralogie, 50, Beiheft 3, 10-11.

Lachmann, R. 1917. Ekzeme und Tektonik. [Posthum hrsgeg. v. F. Frech] Zentralblatt für Mineralogie, Geologie, Paläontologie, 1917, 414-426.

Lang, H.O. 1880. Über den Gebirgsbau des Leinetals bei Göttingen. Zeitschrift der Deutschen Geologischen Gesellschaft 32, 799-806.

Littke, R., Bayer, U., Gajewski, D. 2005. Dynamics of sedimentary basins: the example of the Central European Basin system, International 
Journal of Earth Sciences 94 (5-6), 779-781.

Lotze, F. 1930. Der Westrand des Leinetalgrabens zwischen Hardegsen und Moringen. Sonderdruck aus Göttinger Beiträge zur saxonischen Tektonik II. Abhandlungen der Preußischen Geologischen Landesanstalt. 9. Folge, Heft 116, $37 \mathrm{~S}$.

Lotze, F. 1932a. Überschiebungserscheinungen auf der Nordostseite der Ahlsburgachse bei Hillerse (südwestlich von Northeim). Abhandlungen der Preußischen Geologischen Landandesanstalt 139, Berlin.

Lotze, F. 1932b. Der Südteil des Göttinger Leinetalgrabens und der Eichenberger Grabenknoten. Abhandlungen der Preußischen Geologischen Landesanstalt, Neue Folge, 139; zgl. Göttinger Beiträge zur saxonischen Tektonik, IV, 5-48.

Lüttig, G. 1974. Geological history of the river Weser (Northwest Germany). In: Macar, P.[coord.]: Centenaire de la Societégéologique de Belgique - L'évolution quaternaire des bassins fluviaux de la mer du Nord merdionale, 318 S.; hier: S. 21-34, [ed. Soc. géol. d. Belgique] Liège 1974.

Meischner, D. 2002. Der Leinetalgraben - eine geologische Legende; populärwissenschaftlicher Vortrag am Geowissenschaftlichen Zentrum der Georg-August-Universität Göttingen, unveröffentlicht.

Müller, M., Boigk, H., Füchtbauer, H., Malzahn, E., Eckardt, F.J., Mattiat, B. 1961. Schichtenverzeichnis und geologischer Bericht über die Aufschlussbohrung Dransfeld 1, Niedersächsisches Landesamt für Bodenforschung, Bohrakte 33198, Hannover.

Murawski, H. 1951. Zusammenhänge zwischen Basaltvulkanismus und Tektonik in Niedersachsen. Geologische Rundschau 39, 114-119.

Murawski, H. 1952. Exkursion in die Ziegeleigrube der Fa. Levin in Göttingen und in die Basaltsteinbrüche der Bramburg bei Adelebsen. Zeitschrift der Deutschen Geologischen Gesellschaft $103,423-426$.

Murawski, H. 1955. Das Ausmaß der Vertikalleistung jungtertiärer Tektonik im Gebiet des Leinetalgrabens. Neues Jahrbuch Geologie Paläontologie Monatshefte 1955, 297-308.

Murawski, H. 1960. Das Zeitproblem bei der Tektogenese eines Großgrabensystems. Ein taphrogenetischer Vergleich zwischen Hessischer Senke und Oberrheintalgraben. Notizblätter
Hessisches Landesamt für Bodenforschung 88, 294-342.

Nagel, U., Wunderlich, H.G. 1976. Geologisches Blockbild der Umgebung von Göttingen. 2. Aufl. Veröffentlichungen des Niedersächsischen Instituts für Landeskunde und Landesentwicklung an der Universität Göttingen: Forschungen zur Landes- und Volkskunde; 91, Schriften der Wirtschaftswissenschaftlichen Gesellschaft zum Studium Niedersachsens; Neue Folge, 91, 50 S., Göttingen (Göttinger Tageblatt).

Ortlam, D. 1980. Die känozoische Entwicklung im Nordteil der Mittelmeer-Mjösen-Zone. Berliner Geowissenschaftliche Abhandlungen A 19, 160163.

Patriciu, V. 1930. Geologische und geophyiskalische (radioaktive) Untersuchungen am Westrande des Göttinger Leinetalgrabens. Abhandlungen der Preußischen Geologischen Landesanstalt Neue Folge 116, 163-194.

Pöhlig, C. 1986. Sedimentologie des Zechsteinkalks und des Werra-Anhydrits (Zechstein 1) in Südost-Niedersachsen. Göttinger Arbeiten zur Geologie und Paläontologie, 99 S.

Richter, G. 1934. Das Rheinische Element im Bilde Westeuropas. Nachrichten von der Gesellschaft der Wissenschaften zu Göttingen, Mathematisch-Physikalische Klasse, Fachgruppe IV Geologie und Paläontologie, Neue Folge, Band 1 (Nr. 3), 23-38.

Ritter, M., Vollbrecht, A., v. d. Kerkhof, A., Wemmer, K. 2011. Sedimentgänge im Bausandstein der Solling-Folge NW' von Billingshausen (GK25, Blatt 4326 Lindau). dieser Band.

Ritzkowski, S. 1999. Der Göttinger Leine-Graben im Paläogen (südliches Niedersachsen). Neues Jahrbuch für Geologie und Paläontologie, Abhandlungen 214, 237-256.

Schott, W. 1930. Überschiebungserscheinungen bei Marzhausen südlich Göttingen. Abhandlungen der Preußischen Geologischen Landesanstalt, Neue Folge 116, 233-237.

Schröder, H.G. 1977. Geologie und Tektonik am Leinetal-Graben-Rand zwischen Bovenden und Nörten-Hardenberg. Unveröffentlichte Diplomarbeit, Geologisch-Paläontologisches Institut, Georg-August-Universität Göttingen, 133 S.

Schüssler, P. 1989. Geologie des Ostteils der Dransfelder Hochfläche westlich Göttingen. Teil 1: Geologische Kartierung des Ostteils der Dransfelder Hochfläche zwischen Dransfeld, Varmissen, Jühnde und Meensen. Teil 2: 
Die tertiärzeitlichen Sedimente im Ostteil des Dransfelder Stadtwaldes. Sedimentologie, Stratigraphie, Fazies. Unveröffentlichte Diplomkartierung und Diplomarbeit, Universität Göttingen.

Stille, H. 1910. Die mitteldeutsche Rahmenfaltung. Jahresberichte der Niedersächsischen geologischen Vereinigung 3, 141-170.

Stille, H. 1923/1925. Die saxonischen Brüche. Göttinger Berichte zur Saxonischen Tektonik. Abhandlungen der preußischen geologischen Landes-Anstalt Neue Folge 95, 194-207.

Stille, H. 1932. Geologische Karte von Preußen und benachbarten deutschen Ländern - Lieferung 62 - Erläuterungen zu Blatt Göttingen Nr. 2520 [4425 Göttingen] Nr. 2520; 3., neu bearbeitete Auflage, [Hrsg.: Preußische Geologische Landesanstalt, Berlin]; Berlin 1932.

Stille, H., Lotze, F. 1933. Geologische Übersichtskarte der Umgebung von Göttingen 1: 100.000 mit Erläuterungsheft, Göttingen/Berlin.

Tornquist, A.J.H. 1892. Der Gypskeuper in der Umgebung von Göttingen. Unveröffentlichte Dissertation Universität Göttingen, 38 S.

Wedepohl, K.H. 1982. K/Ar-Altersbestimmungen an basaltischen Vulkaniten der nördlichen Hessichen Senke und ihr Beitrag zur Diskussion der Magmengenese. Neues Jahrbuch Mineralogie Abhandlungen 144, 172-196.

Wunderlich, H.G. 1955. Jüngste Tektonik im Gebiet des Leinetalgrabens. Geologische Rundschau 43, 78-93.

Wunderlich, H.G. 1957. Grenzfragen der saxonischen Tetonik Südniedersachsens -Fältelungserscheinungen und Bruchschollentektonik. Zeitschrift der Deutschen Geologischen Gesellschaft 109, 159-168.

Wunderlich, H.G. 1959. Zur Abfolge und Altersstellung quartärer Bildungen im Stadtgebiet von Göttingen. Eiszeitalter u. Gegenwart, 4155 .

Wunderlich, H.G. 1966. Ausweitung und Einengung an saxonischen Bauformen Südniedersachsens. Zeitschrift der Deutschen Geologischen Gesellschaft, 116 (f. 1964), 683-695.

Ziegler, P. A. 1990. Geological Atlas of Western and Central Europe [2nd ed.], 239 S., The Hague (Shell).

Ziegler, P.A., 1992. European Cenozoic rift system. In: Ziegler, P.A. (ed.), Geodynamics of rifting, Vol. I, Case History Studies on Rifts: Europe and Asia. Tectonophysics 208, 91-111. 



\title{
Der Leinetalgraben als Teil einer regionalen Pull-Apart-Struktur
}

\author{
Axel Vollbrecht (GZG Göttingen), David C. Tanner (GZG Göttingen, LIAG Hannover)
}

Zusammenfassung - Es wird ein Pull-Apart-Modell für das Südniedersächsische Deckgebirge vorgeschlagen, welches die Anlage bekannter Störungsmuster erklären kann und teilweise Verbindungen zu paläogeothermalen Strukturen herstellt. Der Leinetalgraben mit den Basaltvorkommen auf seiner westlichen Schulter stellt in diesem großräumigen Modell nur ein Teilelement des östlichen Randbereichs dar.

\begin{abstract}
We postulate a pull-apart model for the southern Lower Saxony cover rocks, which can explain the pattern of faults and is partially connected to paleo-geothermal structures. The Leinetal Graben, with its outcrops of basalt on its western shoulder, is only a small part of the eastern edge of this large-scale model.
\end{abstract}

Schlüsselworte—Südniedersachsen, Deckgebirge, Pull-Apart-Tektonik, Paläo-Geothermie

\section{Einführung}

Seit der ersten Erwähnung durch Lang (1880) werden bis heute verschiedene Modelle für die strukturgeologische Entwicklung des Leinetalgrabens diskutiert (s. zusammenfassende Darstellung in Arp et al. (2011); dieser Band). Dabei gingen die meisten Arbeiten von einer regionalgeologisch eher kleinräumigen Sichtweise aus, die sich weitgehend auf das Gebiet um Göttingen beschränkte. Eine übergreifende tektonische Einbindung erfolgte nur als Teil eines Europäischen Grabensystems, der sog. Mittelmeer-Mjösen-Zone (z.B. Ortlam 1980).

$\mathrm{Zu}$ den am häufigsten diskutierten Fragen zählen:

1) Die Asymmetrie des Leinetalgrabens, die vor allem durch unterschiedliche Störungsmuster in den östlichen und westlichen Randzonen sowie die nur auf der westlichen Grabenschulter vorkommenden tertiären Basalte zum Ausdruck kommt (z.B. Murawski 1951).

2) Die Beteiligung halotektonischer Prozesse und damit verbundener Subrosionsstrukturen (z.B. Wunderlich 1957).

- Axel Vollbrecht - Geowissenschaftliches Zentrum der Universität Göttingen, Goldschmidtstr. 3, D-37077 Göttingen. avollbr@gwdg.de

- David C. Tanner - GZG Göttingen, jetzt LeibnizInstitut für angewandte Geophysik, Stilleweg 2, D-30655 Hannover. DavidColin.Tanner@liag-hannover.de
3) Die enge Vergesellschaftung von Dehnungsund Einengungsstrukturen (z.B. Wunderlich 1966).

4) Die Bedeutung der in neueren Untersuchungen hervorgehobenen Seitenverschiebungen (z.B. Tanner et al. 2010).

5) Die Platznahme isolierter Schollen (z.B. Franke et al. 1977).

6) Die zeitliche Einordnung der strukturbildenden Ereignisse (z.B. Tanner et al. 2010).

Für die GGG sind genauere Kenntnisse über die Struktur des Leinetalgrabens vor allem im Hinblick auf geothermische Potentiale in Raum Südniedersachsen von Bedeutung. Das nachfolgende Modell soll hierzu einen vorläufigen Beitrag liefern.

\section{Das Modell}

Grundlage für die nachfolgende Modellskizze sind neuere Vorstellungen, nach denen das Zentraleuropäische Beckensystem zwischen der TeisseyreTornquist-/Sorgenfrei Tornquist-Zone im NE und dem Elbe-Störungssystem im SW weitgehend durch Blattverschiebungen bzw. transtensionale Störungssysteme angelegt wurde. Das Niedersächsische Becken stellt in diesem weitläufigen Dehnungsfeld ein Teilbecken dar, dessen südlicher Ast sich zwischen Harz und Rheinischem Massiv erstreckt (zusammenfassende Darstellungen z.B. in Betz et al. 1987; Mazur \& Scheck-Wenderoth 2005). In diesem Rahmen werden die am tiefsten abgesunkenen Teile des Niedersächsischen 


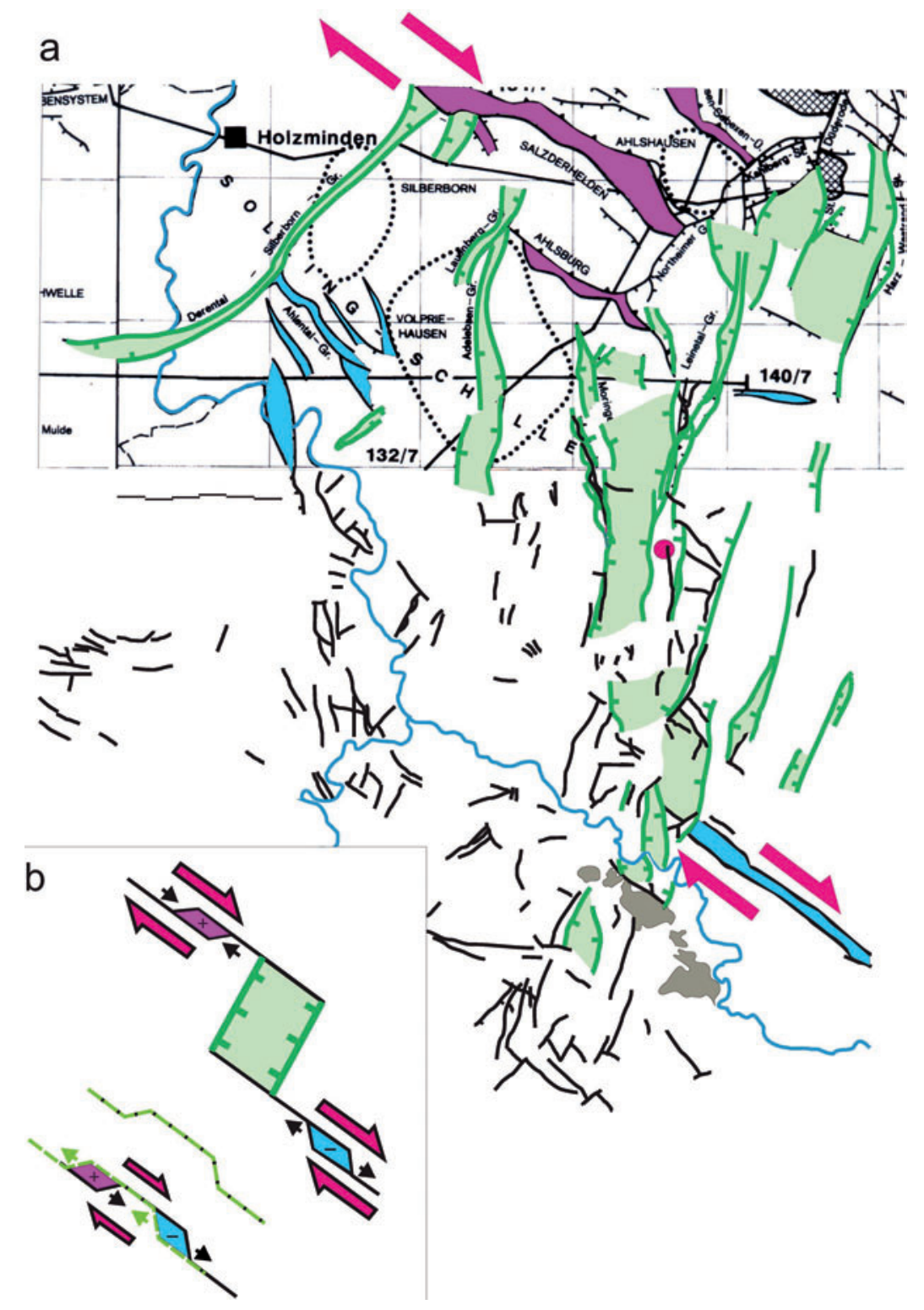

Abbildung 1. a) Modellskizze einer großregionalen Pull-Apart-Struktur im Bereich Südniedersachsen/Ostwestfalen; lokale Störungsmuster aus Tektonischer Atlas Niedersachsen, Geologische Karte Hessen. b) Prinzipskizze mit Dehnungsfeldern (-) und Einengungsfeldern (+) in gestaffelten und gekrümmten Blattverschiebungszonen.

Beckens als en-echelon angeordnete Pull-ApartBecken gedeutet, die in einem transtensionalem Regime während der Oberkreide angelegt wurden. Auch für Dehnungsstrukturen der Hessischen Senke werden entsprechende Modelle angenommen (zusammenfassende Darstellung u.a. in Möbus 2008).

Dementsprechend wird im Folgenden der Leinetalgraben als Teilelement einer größeren PullApart-Struktur betrachtet, die zwischen WNWESE streichenden dextralen Blattverschiebungen im südlichsten Teil des Niedersächsischen Beckens angelegt wurde (Abb. 1). Das Modell gilt somit zunächst für die frühe Dehnungsphase, die im Zeitraum Oberjura/Unterkreide beginnt. Im Rahmen der nachfolgenden Inversionstektonik ist von einer teilweisen kompressiven Überprägung der Pull-Apart bezogenen Strukturen auszugehen. Im Tertiär könnte es dann wieder zu einer Reaktivierung der vorgezeichneten Pull-Apart-Strukturen gekommen sein.

Zwischen den begrenzenden Haupt- 
Blattverschiebungszonen wird diese PullApart-Struktur vor allem durch N-S bis NNE-SSW streichende Abschiebungen mit entsprechenden Graben-/Horststrukturen abgebildet. Zum Teil weisen diese Abschiebungen auch Seitenverschiebungskomponenten auf (Tanner et al. 2010), die auf spätere Rotationen im dextralen Blattverschiebungssystem zurückgeführt werden könnten. Innerhalb der Blattverschiebungszonen wurden je nach Staffelung oder Krümmungsverlauf schmale Transpressions- bzw. Transtensionsstrukturen gebildet. So könnten in diesen Bereichen u.a. positive und negative Flower-Strukturen entstanden sein. Transtensionale Zonen wären auch als potentielle Salzaufstiegswege zu betrachten. Das Pull-Apart-Modell liefert somit eine Möglichkeit für die Erklärung zeitgleicher Dehnungs- und Einengungstektonik.

Die miozänen Basalte treten bevorzugt in einer N-S streichenden Zentralzone im südlichen Teil des Pull-Apart-Dehnungsfelds auf und sind wegen der östlichen Randlage des Leinetalgrabens nur auf dessen westlicher Schulter verbreitet. Weiter nach Norden wurde der Basalt-Aufstieg an die tertiäre Rumpffäche vermutlich durch mächtigere Zechsteinsalze verhindert.

Zahlreiche Untersuchungen haben nachgewiesen, dass in Teilen des Niedersächsischen Beckens die Sedimente im Deckgebirge eine Diagenese bei erhöhten Temperaturen erfahren haben, die nicht durch die stratigraphisch ableitbaren Versenkungstiefen bei normalen geothermischen Gradienten erklärt werden kann (z.B. Buntebarth \& Teichmüller 1979, Brauckmann 1984). Die dabei festgestellten Anomalien zeigen sowohl eine flächige Verbreitung (z.B. Inkohlung, Illitkristallinität) als auch eine lineare Anordnung (hydrothermale Mineralisationen). Für diese ab der Unterkreide nachweisbare ,Hochtemperatur- Diagenese‘ werden in zahlreichen Arbeiten zwei Ursachen diskutiert:

a) Positive Wärmeanomalien über hochkrustalen gabbroiden Plutoniten (hier: Bramsche- und Vlotho-Massiv), wobei entsprechende gravimetrische und magnetische Anomalien als Hauptargument angeführt wurden (z.B. Hahn \& Kind 1971). Neuere geophysikalische Modellierungen gehen jedoch von deutlich tiefer liegenden Intrusionen aus, weshalb ihre Bedeutung für thermische Anomalien im Deckgebirge zunehmend angezweifelt wird. b) Eine lokal starke Absenkung der Sedimente von bis zu ca. $4000 \mathrm{~m}$ in der Unterkreide (Berriasium bis Unter-Albium) und anschließende Heraushebung im Rahmen der in der Oberkreide (Mittel-Conacium bis Maastrichtium) stattfindenden Inversionstektonik (z.B. Hiss et al. 2005, Senglaub et al. 2006).

Ein entsprechendes kombiniertes Modell, mit dem auch erhöhte Inkohlungsgrade in Oberkarbon-Sedimenten erklärt werden, nimmt u.a. Teichmüller (1986) an. Unabhängig davon, welches dieser Modelle zutrifft, könnten die mit einem Pull-Apart-Szenario verbundenen lokal begrenzten Dehnungsstrukturen die wesentlichen Vorgänge erklären: Aufstieg und Platznahme von Magmen sowie starke Absenkung lokaler Sedimentbecken. Infolge der regionalen Krustendehnung käme eine Erhöhung des Wärmestroms als zusätzlicher, überlagernder Effekt hinzu. Neueren Untersuchungen von Adriasola Mun̂oz et al. (2007) bestätigen, dass dieser bereits im Obersten Jura einsetzte.

Für das vorgeschlagene Modell ergeben sich konkret folgende Korrelationen mit thermischen Anomalien (Abb. 2):

1) Das von Brauckmann (1984) dargestellte Muster der anomalen Illitkristallinitäten verläuft aus dem Bereich von Bramsche bis in das Gebiet der vorgeschlagenen PullApart-Struktur (Abb. 2a). Für diesen Teil der Anomalie-Zone zieht Brauckmann (1984) einen zusätzlichen Pluton im Bereich von Karlshafen als Ursache in Betracht.

2) Die lineare Anordnung der hydrothermalen Mineralisationen (Brauckmann 1984; Quarz, Mikroklin, Albit) deckt sich teilweise mit dem Verlauf der für das vorliegende Pull-ApartModell angenommenen nördlichen HauptBlattverschiebungszone und schwenkt am östlichen Ende in eine Richtung parallel zum Leintalgraben um (Abb. 2b bis d). Danach wären die Mineralisationen hier das Ergebnis eines konvektiven Wärmetransports in den Hauptbruchzonen. In gleicher Weise zeigt der Verlauf der Illitkristallinität-Isolinien eine Richtungsbeziehung zur nördlichen HauptBlattverschiebungszone.

3) Die Hauptverbreitung der tertiären Basalte zeigt eine symmetrische Beziehung zum $\mathrm{Mu}-$ ster der Illitkristallinitäten (Abb. 2e). Geht man von der o.g. Annahme eines Plutons 

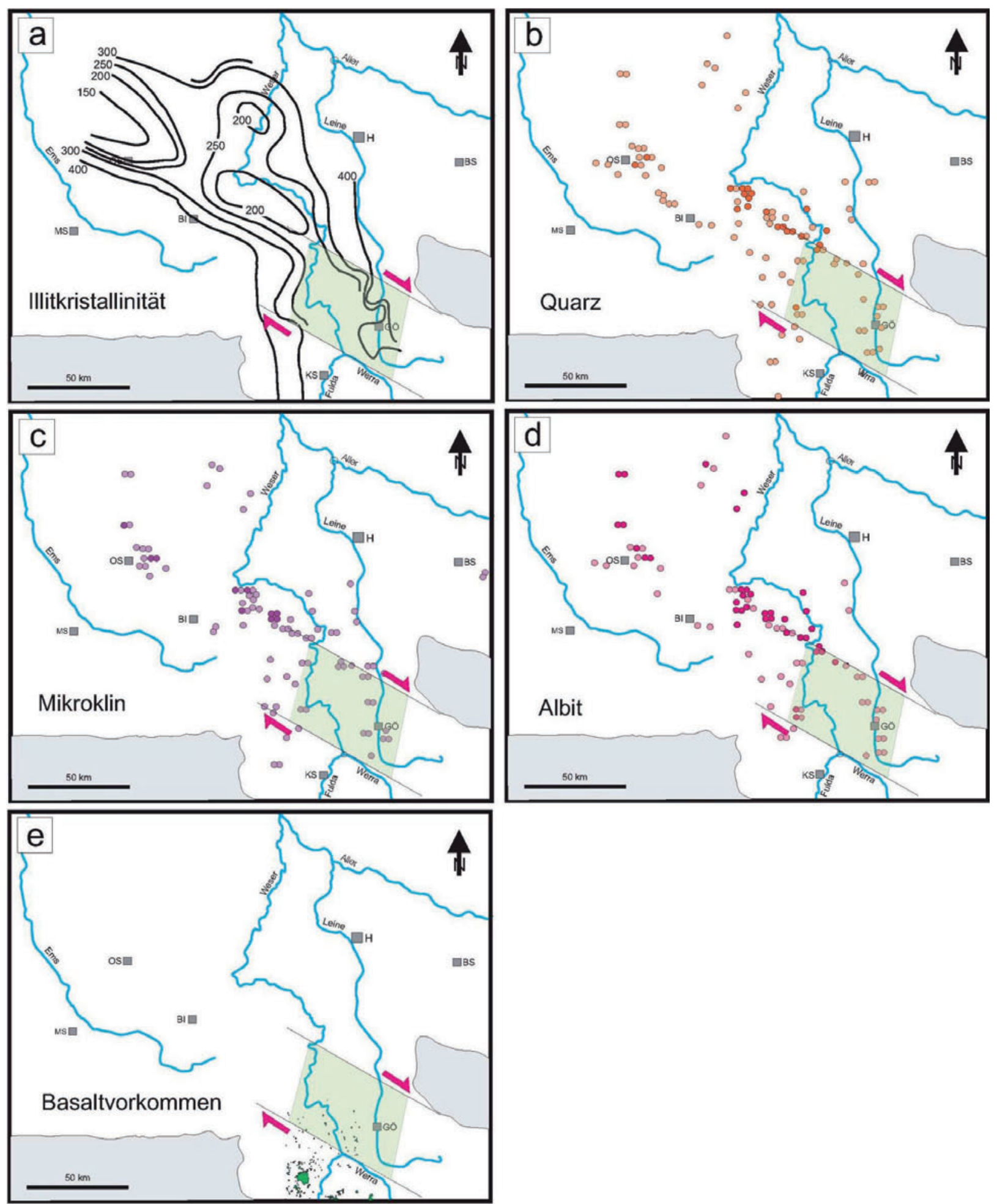

Abbildung 2. Paläo-geothermische Anomalien, abgebildet durch ,Hochtemperatur-Diagenese; vereinfacht nach Brauckmann (1984) mit Bezug zur Pull-Apart-Struktur (grüne Felder) und und zu tertiären Basalten. a) Illitkristallinitäten, b) Authigene Quarz-Mineralisationen, c) Authigene MikroklinMineralisationen, d) Authigene Albit-Mineralisationen, e) Verbreitung tertiärer Basalte (aus GK 300 Hessen). 


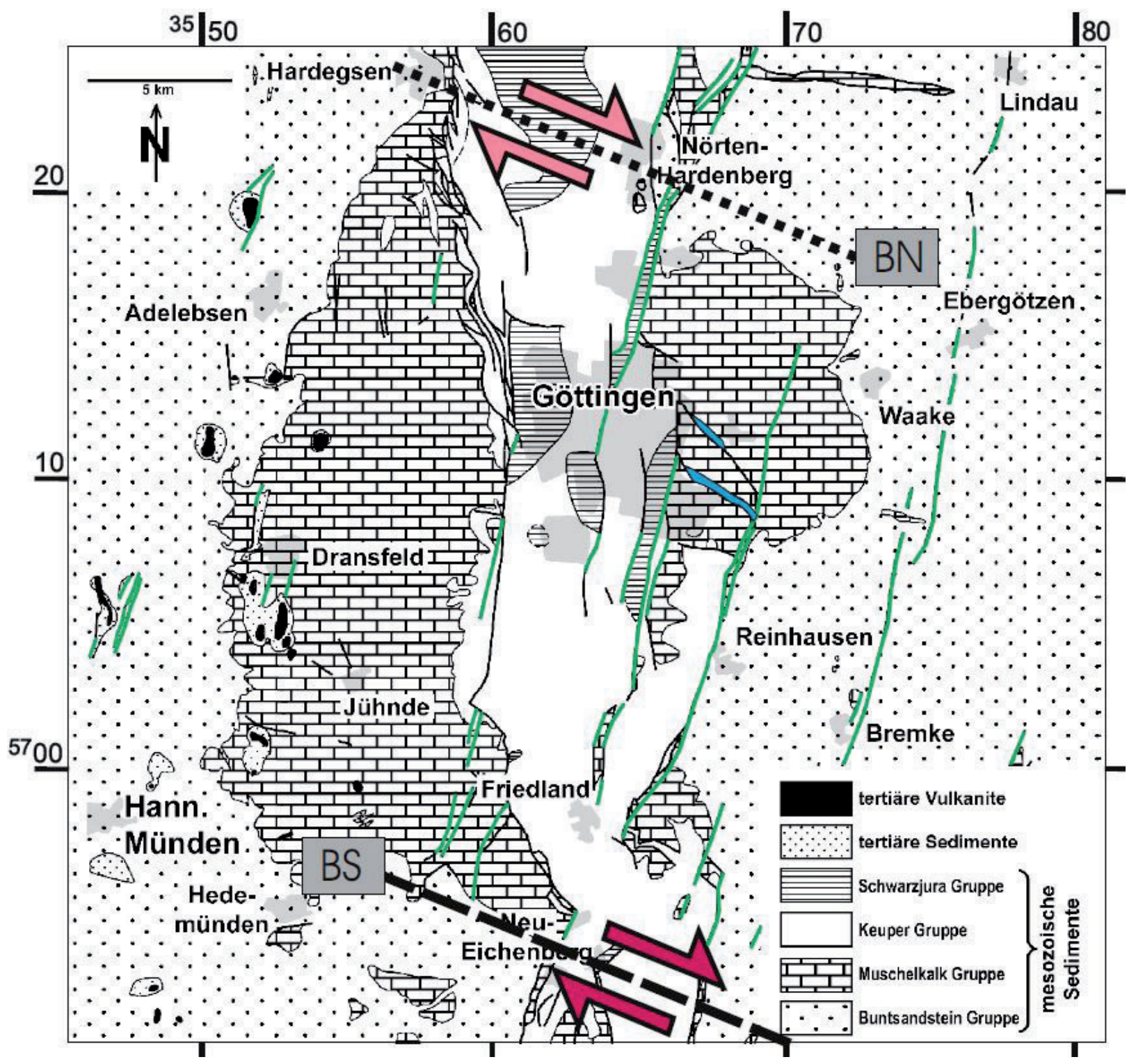

Abbildung 3. Kinematisches Modell für den Leinetalgraben. Kartengrundlage aus Arp et al. (2004); Farben wie in Abb. 1; weitere Erläuterungen im Text.

als Ursache für die kretazische Hochdiagenese aus, so könnte die Reaktivierung einer PullApart-Struktur im Tertiär Ursache für den erneuten Magmenaufstieg in diesem Bereich sein. Dadurch ergibt sich zumindest indirekt für diese Region ein Hinweis auf Magmatismus als Ursache für thermische Anomalien der Diagenese.

Generell ist davon auszugehen, dass in Blattverschiebungssystemen Pull-Apart-Strukturen in unterschiedlichen Dimensionen auftreten. Übertragen auf das vorliegende Modell für Südniedersachsen könnte spekuliert werden, ob z.B. im Bereich des Göttinger Leinetalgrabens das übergreifende ,Muschelkalk-Plateau' eine abgesunkene Scholle oder Mulde in einer kleiner dimensionierten Pull-Apart-Struktur darstellt (Abb. 3). Auf die Existenz entsprechender HauptBlattverschiebungszonen im Norden (BN) und Süden (BS) könnten folgende Strukturen hindeuten:

- BN: (a) Im eigentlichen Grabenbereich wären dies Keuper-Aufbrüche, die in der GÜK von Hessen in entsprechender Richtung eingetragen sind (s. Hessisches Geologisches Landesamt für Bodenforschung 1989). 
Nach eigenen Beobachtungen kann dies noch nicht bestätigt werden. (b) In der weiteren Verlängerung wird diese Störung morphologisch durch das Rodebachtal abgebildet. Auch gibt es Hinweise auf einen stratigraphischen Versatz über das Rodebachtal hinweg. (c) Sandsteingänge im Bereich des Rodebachtals (Ritter et al., dieser Band), deren Entstehung mit einer Reaktivierung der BN im Tertiär erklärt werden könnte. Ob und ggf. in welcher Weise die transpressiven Strukturen im Steinbruch Hardegsen II (Tanner et al., dieser Band), damit in Verbindung stehen, ist noch unklar.

- BS: (a) Im ESE' Abschnitt Verlauf einer deutlich entwickelten Grabenzone im Mittleren Buntsandstein, im wesentlichen mit Muschelkalk- und Keuperfüllung (s. Hessisches Geologisches Landesamt für Bodenforschung 1989), assoziiert mit Zechsteinvorkommen als möglicher Hinweis auf Salzintrusionen. (b) In der WNW' Verlängerung dieser Grabenzone Hinweise auf Störungszone im Bereich des Oberen Buntsandstein (reduzierte Ausstrichmächtigkeit und extrem geradliniger Grenzverlauf zwischen sm/so/mu in entsprechender Richtung; s. Hessisches Geologisches Landesamt für Bodenforschung 1989).

Vermutlich konnten sich im Bereich von Südniedersachsen diese Pull-Apart-Strukturen wegen nur geringmächtiger Zechsteinsalze deutlich in das Deckgebirge durchpausen. Das vorgeschlagene Modell kann hier die vorherrschenden Strukturelemente in der betrachteten Größenordnung ohne maßgebliche Beteiligung von Halotektonik erklären. Diese ist vermutlich eher für Strukturprägungen in kleinerer Dimension von Bedeutung. Dagegen sind Zechsteinsalze (auch als Intrusivkörper in höheren Deckschichten) vermutlich als Abscherhorizonte in Betracht zu ziehen.

\section{Schlussfolgerungen und Ausblick}

Das vorgeschlagene Modell ist als kinematisches Grundmuster für genauere bzw. weiterführende Betrachtungen zu verstehen. So wäre u.a. durch detaillierte Analysen zu prüfen, inwieweit bereits vorliegende Beobachtungen damit vereinbar oder evtl. neu zu interpretieren sind. Für vergleichende Betrachtungen bieten sich u.a. weitere Großstrukturen mit ähnlichen Störungsmustern in der Hes- sischen Senke an, für die auch Pull-Apart-Modelle diskutiert werden.

Die genauen Ursachen für die räumlichen Beziehungen zwischen tektonischen Strukturen und paläo-geothermischen Anomalien sind noch nicht geklärt. Die Prüfung möglicher Zusammenhänge mit der aktuellen Geothermie könnte ein interessanter Teilaspekt für die Modellierung geothermischer Potentiale in der betrachteten Region sein.

\section{Danksagung}

Wir danken allen Mitgliedern der GeothermieGruppe-Göttingen für viele anregende Diskussionen.

\section{Literatur}

Adriasola Mun̂oz, Y., Littke, R., Brix, M.R. 2007. Fluid systems and basin evolution of the western Lower Saxony Basin, Germany. Geofluids 2007/7, 335-355.

Arp, G., Hoffmann V.-E., Seppelt S., Riegel W. 2004. Trias und Jura von Göttingen und Umgebung, 74. Jahrestagung der Paläontologischen Gesellschaft, 2.-8.10.2004, Exkursion 6, 147192, Universitätsdrucke.

Arp, G., Vollbrecht, A., Tanner, D., Leiss, B., 2011. Zur Geologie des Leinetalgrabens - ein kurzer Überblick, dieser Band.

Betz, D., Führer, F., Plein, E. 1987. Evolution of the Lower Saxony Basin. Tectonophysics 137, 127-170.

Brauckmann, F.J. 1984. Hochdiagenese im Muschelkalk der Massive von Bramsche und Vlotho. Bochumer Geologische und Geotechnische Arbeiten 4, 195 S.

Buntebarth, G., Teichmüller, R., 1979. Zur Ermittlung der Paläotemperaturen im Dach des Bramscher Intrusivs aufgrund von Inkohlungsdaten. Fortschritte in der Geologie von Rheinland und Westfalen 27, 171-182.

Franke, W., Paul, J., Schröder, H.-G. 1977. Stratigraphie, Fazies und Tektonik im Gebiet des Leinetalgrabens (Trias, Tertiär). Exkursionsführer Geotagung '77, Bd. II, Exk. I: 41-62, Göttingen.

Hahn, A., Kind, E.G. 1971. Eine Interpretation der magnetischen Anomalie von Bramsche. Fortschritte in der Geologie von Rheinland und Westfalen 18, 387-394.

Hessisches Landesamt für Bodenforschung 1989. Geologische Übersichtskarte von Hessen. 4. 
Auflage, Hessisches Landesvermessungsamt, Wiesbaden.

Hiss, M., Mutterlose, J., Niebuhr, B., Schwerd, K. 2005. Die Kreide in der Stratigraphischen Tabelle von Deutschland 2002. Newsletter on Stratigraphy, 41, 287-306.

Lang, H.O. 1880. Über den Gebirgsbau des Leinetals bei Göttingen. Zeitschrift der Deutschen Geologischen Gesellschaft 32, 799-806.

Mazur, S., Scheck-Wenderoth, M. 2005. Constraints on the tectonic evolution of the Central European Basin System revealed by seismic reflection profiles from Northern Germany. Netherlands Journal of Geosciences Geologie en Mijnbouw 84/4, 389-401.

Möbus, H.M. 2008. Die Hessischen Gräben als mehrfach duktil entkoppelte ,pull apart"Strukturen. Geologisches Jahrbuch Hessen 135, 5-23, Wiesbaden.

Murawski, H. 1951. Zusammenhänge zwischen Basaltvulkanismus und Tektonik in Niedersachsen. Geologische Rundschau 39, 114 S.

Ortlam, D. 1980. Die känozoische Entwicklung im Nordteil der Mittelmeer-Mjösen-Zone. Berliner Geowissenschaftliche Abhandlungen, A 19, 160-163.

Scheck, M., Bayer, U., Otto, V., Lamarche, J., Banka, D., Pharaoh, T. 2002. The Elbe Fault System in North Central Europe - a basement controlled zone of crustal weakness. Tectonophysics 360, 281-299.

Senglaub, Y., Littke, R., Brix, M.R. 2006. Numerical modelling of burial and temperature history as an approach for an alternative interpretation of the Bramsche anomaly, Lower Saxony Basin. International Journal of Earth Sciences 95, 204-224.

Tanner, D.C., Leiss, B., Vollbrecht, A., the GGG 2010. The role of strike-slip tectonics in the Leinetalgraben, Lower Saxony. Zeitschrift der Deutschen Geologischen Gesellschaft 161/4, 369-377.

Teichmüller, M. 1986. Coalification and natural gas deposits in northwestern Germany. Geological Society, London, Special Publications, 1986/23, 101-112.

Wunderlich, H.-G., 1957. Tektogenese des Leinetalgrabens und seiner Randschollen. Geologische Rundschau, 46, 372-413.

Wunderlich, H.-G., 1966. Ausweitung und Einengung an saxonischen Bauformen Südniedersachsens. Zeitschrift der Deutschen Geologi- schen Gesesellschaft, 116, 683-695. 



\title{
Struktur der Leinetalgraben-Randstörung bei Reiffenhausen (Autobahn 38 Heidkopftunnel-Westportal)
}

\author{
Gernot Arp (GZG Göttingen), David C. Tanner (LIAG Hannover) und Bernd Leiss (GZG \\ Göttingen)
}

\begin{abstract}
Zusammenfassung - Während des Baus der Autobahn A38 etwa $15 \mathrm{~km}$ südsüdöstlich von Göttingen wurde erstmals großflächig der Bereich der östlichen Leinetalgraben-Randstörung angeschnitten. Der Störungsbereich zwischen Grabenschulter (Eichsfeld-Scholle mit höherer Buntsandstein-Gruppe anstehend) und Grabeninneren (Leinetal-Scholle mit Trochitenkalk bis basaler Gipskeuper anstehend) umfaßt eine von zwei Störungsbahnen umgrenzte Zone aus stark deformierten Gesteinen des Mittleren Muschelkalkes und der Röt-Formation. Die Störungsbahnen zeigen eine charakteristische Änderung des Einfallswinkels in Abhängigkeit der Lithologie, mit flachen Verläufen im Bereich der inkompetenten Röt-Formation und steilen Verläufen im Bereich der kompetenten Solling-Formation. Eine in Blöcke zerlegte Dolomitsteinscholle des Mittleren Muschelkalkes kann mit dem Abgleiten über diese stufenartige Geometrie erklärt werden. Die Störungsgeometrie eines variierenden Einfallswinkels verursacht zudem mehrfache Roll-Over-Strukturen im Bereich der Hangendscholle. Insgesamt können die beobachteten Strukturen allein mit extensiven Bewegungen erklärt werden. Einzig die sattelartige Wölbung der Hangendscholle im Aufschlußbereich könnte eine kompressive Inversionsbewegung dokumentieren.
\end{abstract}

\begin{abstract}
During the building of the A38 motorway tunnel, ca. $15 \mathrm{~km}$ south-southeast of Göttingen, the major eastern boundary fault of the Leine Valley graben was exposed. The fault zone between the footwall (Eichsfeld block; at the surface comprised of upper Buntsandstein strata) and the hanging-wall (Leine Valley block with Upper Muschelkalk (Trochitenkalk) up to basal Middle Keuper exposed) consists of a fault zones containing deformed rocks of the Middle Muschelkalk and Upper Buntsandstein (Röt Formation). The fault surfaces demonstrate a characteristic change in dip angle with respect to lithology, with shallow angles within the incompetent Röt-Formation and steep angles within the competent Solling Formation. Faulted blocks of dolomitic middle Muschelkalk were caused by movement over this step-like fault geometry. The fault shape also caused multiple roll-over structures in the hanging-wall block. In general, the observed structures can be explained by extensional tectonics. Some of the antiformal structures in the hanging-wall could also be attributed to compressive inversion.
\end{abstract}

Schlüsselworte-Buntsandstein, Muschelkalk, Keuper, Abschiebung, Hanging-wall roll-over.

\section{Einführung}

Der Bau des Heidkopftunnels der Autobahn A38 südsüdostlich von Göttingen durchschnitt im November 2003 den Bereich der östlichen Randstörung des Leinetalgrabens (Abb. 1). Dabei bot der etwa $400 \mathrm{~m}$ lange und bis zu $25 \mathrm{~m}$

- Gernot Arp - Geowissenschaftliches Zentrum der Universität Göttingen, Goldschmidtstr. 3, D-37077 Göttingen. garp@gwdg.de

- David C. Tanner - Leibniz-Institut für angewandte Geophysik, Stilleweg 2, D-30655 Hannover. DavidColin.Tanner@liag-hannover.de

- Bernd Leiss - Geowissenschaftliches Zentrum der Universität Göttingen, Goldschmidtstr. 3, D-37077 Göttingen. bleiss1@gwdg.de tiefe Einschnitt zum Westportal des Tunnels erstmal einen großdimensionierten Aufschluß der Randstörung (hier Arenshäuser Sprung) zwischen dem Grabeninneren (Hangendscholle) und der Eichsfeld-Scholle (Liegendscholle).

\section{Stratigraphie}

Die im Einschnitt des Westportals anstehenden Gesteinsformationen reichen von der SollingFormation (mittlere Buntsandstein-Gruppe) bis in die Grabfeld-Formation (mittlere KeuperGruppe).

Die Solling-Formation ist mit mittelkörnigen, mittel- bis dickbankigen Quarzsandsteinen 


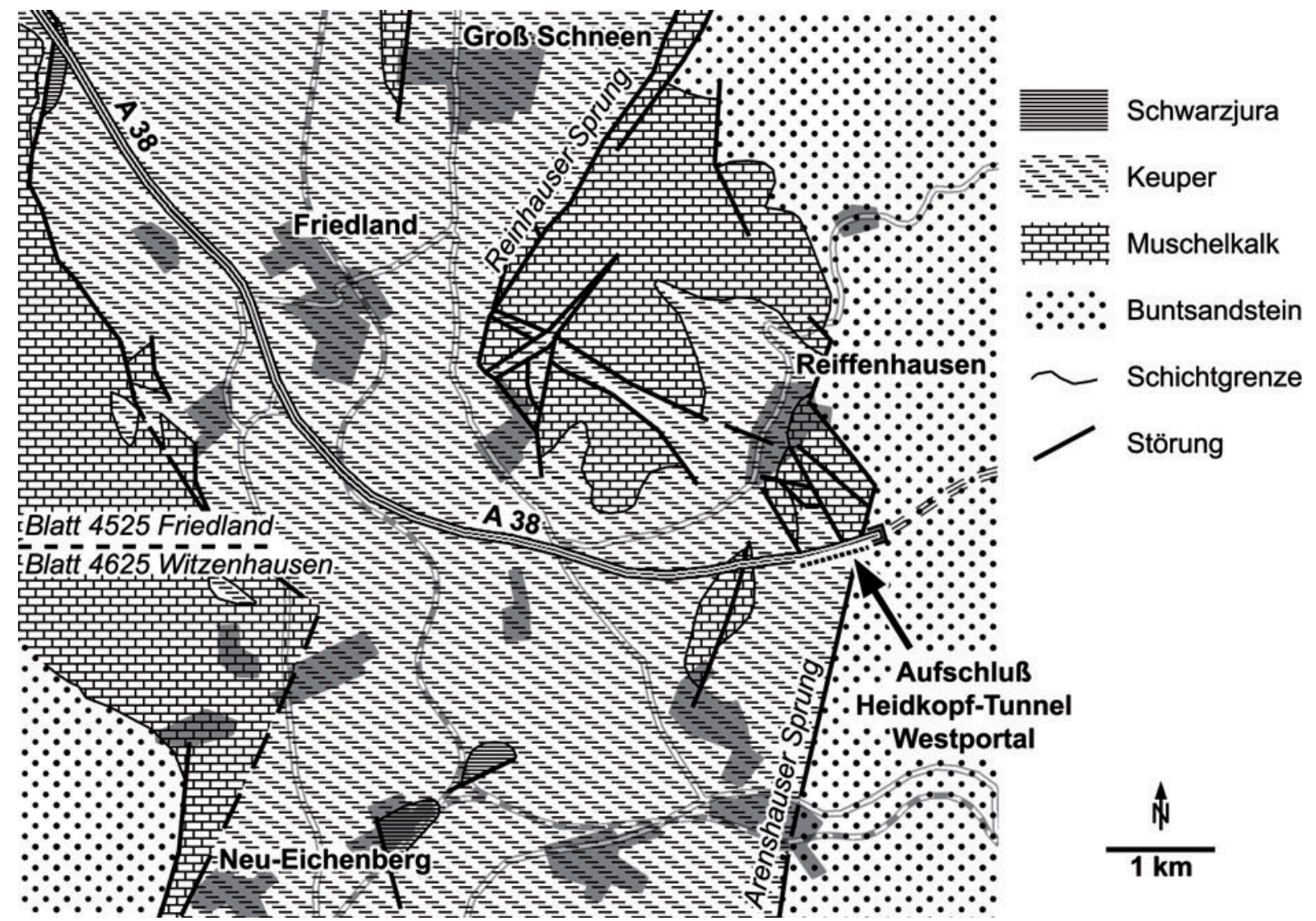

Abbildung 1. Geologische Übersicht des südlichsten Teils des Leinetalgrabens, auf Grundlage der Karten von Moesta (1884) und von Koenen (1893), mit Lage des Aufschlusses Heidkopf-Tunnel Westportal.

vertreten. Am Top der Formation sind über einem rotbraunen Karneol-Horizont (Paläoboden) noch knapp $1 \mathrm{~m}$ weißgraue Quarzsandsteine mit grünlichgrauen Tonschlieren (Thüringischer Chirotheriensandstein) entwickelt. Die nachfolgende Röt-Formation ist nur mit ihrem tiefsten Teil vertreten, in Form von grünlichgrauen und rotbraunen, vielfach brekziösen Tonsteinen (mehr als $10 \mathrm{~m}$ ). Gipssteine oder umfangreichere Residualbrekzien wurden nicht beobachtet. Gesteine des unteren MuschelkalkGruppe (Jena-Formation) waren nicht angeschnitten. Der mittlere Teil der MuschelkalkGruppe liegt in Form von mittelgrauen Dolomitmergelsteinen und mikrokristallinen, festen Dolomiten vor. Mächtigkeiten sind in diesem stark zerscherten Bereich nicht zu ermitteln. Der höhere Teil der Muschelkalk-Gruppe ist mit mittelgrauen, echinodermenschuttreichen Schillkalken der Trochitenkalk-Formation (oberste $3 \mathrm{~m}$ erschlossen), überlagert von der mittelgrauen Mikrit-
Schillkalk-Kalkmergel-Wechselfolge der Ceratitenschichten (Meißner- und Warburg-Formation; ca. $45 \mathrm{~m})$.

Die Keuper-Gruppe setzt mit scharfer Grenze über den Ceratitenschichten mit Feinsandsteinen, Siltsteinen, Kalkmergeln, dünnen Kohleflözchen und gelbgrauen Dedolomiten des Lettenkohlenkeupers (Erfurt-Formation; $38 \mathrm{~m}$ ) ein. Über dem letzten, etwa 1,5 m mächtigen Dedolomithorizont folgen mächtigere, rotbraune siltige Tonsteine mit einzelnen Residualknollen des tieferen Gipskeupers (Grabfeld-Formation).

\section{Methode}

Die Geländearbeiten wurden zwischen dem 2. und 16. November 2003 durchgeführt. Für Geländezeichnungen diente ein Fotomosaik als Grundlage. Lagerungswerte wurden mit einem Freiberger Geologenkompaß eingemessen. 


\section{Ergebnisse (Abb. 2)}

\subsection{Liegendscholle: Grabenschulter}

Die Grabenschulter wird oberflächennah aufgebaut von einer kompetenten ,Platte' aus Bausandstein (einschließlich aufliegendem ChirotherienSandstein) der Solling-Formation, welche flach mit bis $\mathrm{zu} 5^{\circ}$ nach Westen einfällt. Darüber folgen konkordant zunächst graue Tone der RötFormation. Im direkten Kontakt zur Störungszone ist der Chirotheriensandstein beulenartig nach oben aufgepreßt und von Dolomitmergel des Mittleren Muschelkalkes überlagert.

\subsection{Störungsbereich mit parautochthoner Muschelkalkscholle}

Der Störungsbereich umfaßt eine von zwei Störungsbahnen umgrenze Zone aus stark komprimierten und verfältelten Dolomitmergeln und Dolomitsteinen des Mittleren Muschelkalkes, mit mittig eingeschuppten rotbrauenen Tonsteinen der Röt-Formation. Festere Dolomitsteine des Mittleren Muschelkalkes liegen im höheren Aufschlußteil rotbraunen Röt-Tonsteinen auf und sind dabei in sich in eckige Blöcke zerschert. Im westlichen Teil der Störungszone zeigen die Gesteine des Mittleren Muschelkalkes noch eine Schichtlagerung $\left(260^{\circ} / 20^{\circ}, 260^{\circ} / 35^{\circ}\right)$ analog zu der der Ceratitenschichten und des Keupers des Grabeninneren am westlichen Aufschlußende $\left(270^{\circ} / 30^{\circ}\right.$; siehe unten). Im stärker zerblockten Bereich deutet der einzige gewonnene Lagerungswert $\left(20^{\circ} / 26^{\circ}\right)$ eine Schollenrotation an.

Abbildung 2. (Rechts) Strukturgeologisches Profil des Aufschlusses Heidkopf-Tunnel Westportal auf Grundlage eines Photomosaiks. Oben: Südböschung. Mitte: Temporäre Abbaustufe Autobahn-Mitte. Die eingefügten Geländeaufnahmen zeigen den Bereich der östlichen Störungsbahn mit beulenartige Aufpressungsstruktur an der Oberkante der Solling-Formation (links), die westliche Störungsbahn (Mitte) und das Abtauchen der Hangendscholle (hier Lettenkohlenkeuper und tieferer Gipskeuper) in westliche Richtung (rechts).

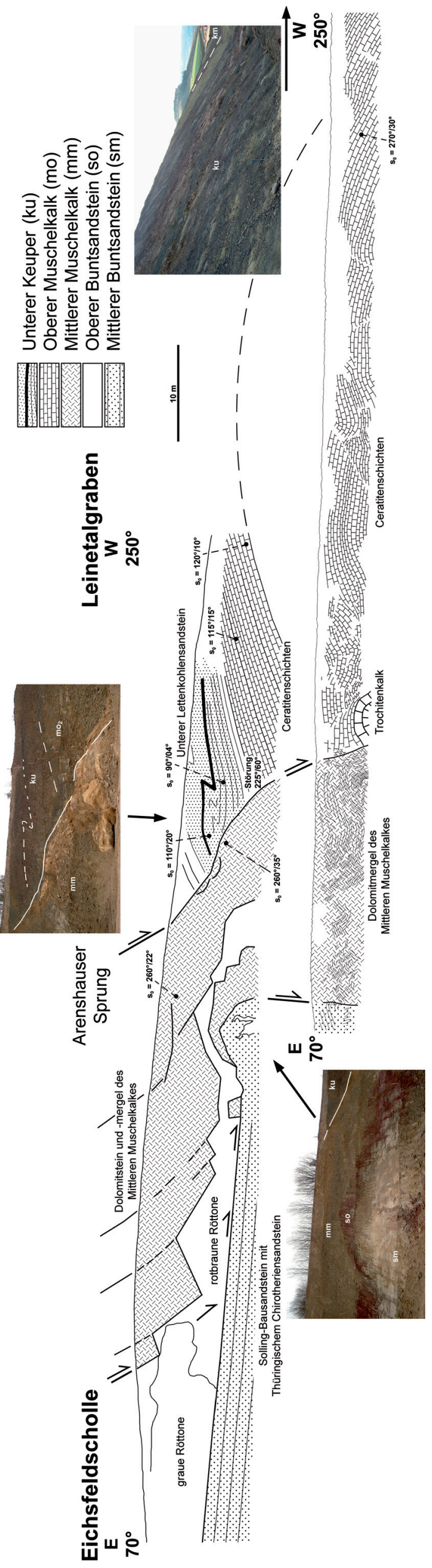


Die östliche Störungsbahn ist im tieferen Aufschlußteil steil und geht im höheren Aufschlußteil in eine flache Störungsbahn über. Die westliche Störungsbahn ist oberflächennah (am AufschlußTop) mit $45^{\circ}$ Einfallen vergleichsweise flach, versteilt aber in die Tiefe auf etwa $70^{\circ}$.

\subsection{Hangendscholle: Grabeninneres}

Der der Störungszone angrenzende Bereich der Hangendscholle fällt flach nach ESE, also zur Störungszone hin, ein $\left(110^{\circ} / 20^{\circ}, 115^{\circ} / 15^{\circ}\right)$. Die Aufschlußbasis zeigt an die Störung angrenzend eine lokale Aufbeulung der TrochitenkalkFormation. Auch die darüberliegenden Ceratitenschichten sind von mehreren Kleinstörungen und Flexuren durchsetzt. Eine Stauchung mit flacher, liegender Faltenstruktur ist schließlich im Bereich des Lettenkohlenkeupers (Erfurt-Formation) im höheren Aufschlußteil entwickelt. Nach Westen biegt das generelle Schichteinfallen der Formationen schließlich in Richtung Westen, also Einfallen zum Grabeninneren $\left(270^{\circ} / 30^{\circ}\right)$, um. Im westlichsten Aufschlußbereich konnten noch Werte von $240^{\circ} / 34^{\circ}$ bis $270^{\circ} / 20^{\circ}$ (Erfurt-Formation) gemessen werden.

\section{Diskussion}

Der großdimensionierte Autobahn-Aufschluß zeigt erstmal für den Leinetalgrabenrand direkt einsehbar eine Änderung des Einfallswinkels der Randstörung in Abhängigkeit der Lithologie: Die Abschiebungen verlaufen flach im Bereich der inkompetenten Röt-Formation, steiler im Bereich der kompetenten Solling-Formation (Abb. 2). Dieses Muster spiegelt letztlich die bevorzugte Abscherung entlang von „Schmierhorizonten' wie der Röt-Formation und des Mittleren Muschelkalkes wider (vgl. Möbus 2008). Schollenfragmente des Mittleren Muschelkalkes sind dabei teilweise an der Stufe der kompetenten Solling-Formation hängengeblieben. Die Störungsgeometrie eines variierenden Einfallswinkels verursacht zudem mehrfache Roll-Over-Strukturen (vgl. Seyferth \& Henk 2006), sichtbar als kleinteiliges Muster im Bereich der Ceratitenschichten, wie auch eine Rückfaltung (,accommodation structure' im Bereich des Lettenkohlenkeupers). Insgesamt kann diesen Beobachtungen folgend eine derartige stufenartige Geometrie für alle Hauptstörungen des Leinetalgrabens vermutet werden. Kompliziertere Erklärungsmodelle sind für die stufenartig zerlegte DolomitsteinScholle des Mittleren Muschelkalkes, die Mélange-artige Vermengung von Röt-Tonen mit Dolomitmergeln des Mittleren Muschelkalkes, sowie die beulenartige Aufpressungsstruktur an der Oberkante der Solling-Formation $\mathrm{zu}$ entwickeln (Abb. 2). Hierbei gilt es $\mathrm{zu}$ berücksichtigen, daß zur Zeit der tektonischen Bewegungen noch Evaporite (Gips, Anhydrit, Steinsalz) als zusätzlicher strukturmodifizierender Faktor wirkten. Diese Evaporite waren mit Sicherheit sowohl im tieferen Teil der RötFormation wie auch im Mittleren Muschelkalk vorhanden, sind aber heute oberflächennah vollständig abgelaugt. Entsprechend sind auch im vorliegenden Aufschluß kollabierte Strukturen zu erwarten.

Bezüglich der stufenartigen Zerlegung der Dolomitstein-Scholle des Mittleren Muschelkalkes kommt ein Dominostein-artiges Zerblocken während der Abschiebung auf der im betreffenden Abschnitt flachen Störungsfläche, mit anschließendem Einsinken in eine Ton/Evaporit-Mélange der Störungsbahn, als Erklärung in Frage. Ein Aufsteigen der Mélange aus Evaporiten (so, mm), Dolomitmergel (mm) und Tonen (so) könnte für die lokal auf die Oberkante der Solling-Formation begrenzte Kompressionsbeule verantwortlich sein.

\section{Schlussfolgerung}

In der Summe sind die beobachteten Strukturen entlang der hier aufgeschlossenen Grabenrandstörungszone allein durch Extension erklärbar. Einzig die sattelartige Wölbung des gesamten Schichtpacketes der Hangendscholle im Aufschlußbereich (im Osten mit Einfallen zur Störungszone hin, im Westen mit Einfallen zum Grabeninneren hin) kann als Hinweis auf eine großräumigere Kompression (Inversionsbewegung?) gedeutet werden.

\section{Literatur}

Möbus, H.M. 2008. Die Hessischen Gräben als mehrfach duktil entkoppelte ,pull apart'Strukturen. - Geologisches Jahrbuch Hessen 135, 5-23, Wiesbaden.

Moesta, Fr. 1884. Geologische Karte von Preussen und den Thüringischen Staaten 1:25000 GradAbtheilung 55, Blatt 40 [Neue Nr. 4625] Witzenhausen. 1 Kte., Berlin (Kraatz). 
von Koenen, A. 1893. Geologische Karte von Preussen und den Thüringischen Staaten 1:25000, Grad-Abtheilung 55, Blatt 34 [Neue Nr. 4525] Reinhausen. 1 Kte., Berlin (Kraatz). Seyferth, M., Henk, A. 2006. A numerical sandbox: high resolution distinct element models of halfgraben formation. International Journal of Earth Sciences 95, 189-203. 



\title{
Ein Aufschluss im tieferen Steinmergelkeuper nahe der Leinetalgraben-Randstörung (Kreuzberg Göttingen): Lithologische Abfolge und Strukturgeologie
}

\author{
Gernot Arp (GZG Göttingen), David C. Tanner (GZG, LIAG Hannover) \\ und Bernd Leiss (GZG Göttingen)
}

\begin{abstract}
Zusammenfassung - Eine Baugrube am Fuß des Kreuzberges im nordöstlichen Stadtgebiet von Göttingen erschloß eine überwiegend rotbraune Tonsteinabfolge der tieferen Arnstadt Formation (,Steinmergelkeuper'). Die Schichtung fällt hier, in Einklang mit bisherigen Erkenntnissen, mit etwa $25^{\circ}$ in Richtung ESE. Eine NNWSSE streichende Kleinabschiebung im Aufschluß deutet auf eine Fortsetzung eines schmalen Spezialgrabens der östlichen Grabenschulter bis in das Grabeninnere hin. Zwei Kluftrichtungen konnten festgestellt werden: Eine parallel zur beobachteten Kleinabschiebung sowie eine weitere senkrecht dazu.
\end{abstract}

\begin{abstract}
A construction excavation at the base of the Kreuzberg in the north-east region of Göttingen exposed a mainly red-brown clay sequence of the lower Arnstadt Formation (,Steinmergelkeuper'). The bedding dips here, in harmony with previous observations, ca. $25^{\circ}$ towards ESE. A small, NNW-SSE striking normal fault in the outcrop suggests an continuation of a thin extraordinary graben that occurs on the eastern graben shoulder into the hanging-wall of the graben. Two sets of joints could be distinguished; one parallel and one perpendicular to the observed normal fault.
\end{abstract}

Schlüsselworte-Keuper, Abschiebung, Klüftung

\section{Einführung}

Die Baugrube Reha-Zentrum Rainer Junge im nordöstlichen Stadtgebiet von Göttingen, Sprangerweg 3, bot im April 2005 einen temporären Aufschluß im Bereich des Steinmergelkeupers (Abb. 1). Der Aufschluß liegt am Fuß des Kreuzberges, direkt nördlich des Institutes für Leibesübungen (IfL) der Universität Göttingen auf Blatt 4425 Göttingen der topographische Karte 1: 25000 (R: 3566 260, H: 5713 290). Der ,Kreuzberges' ist aufgrund der Funde von PlateosaurierResten im hangend folgenden Rhätkeuper (von Koenen 1904, 1907) lokal bekannt. Der Aufschluß liegt ca. $575 \mathrm{~m}$ westlich der östlichen Randstörung des Leinetalgrabens (hier ,Göttinger Sprung'),

- Gernot Arp - Geowissenschaftliches Zentrum der Universität Göttingen, Goldschmidtstr. 3, D-37077 Göttingen. garp@gwdg.de

- David C. Tanner - GZG, jetzt Leibniz-Institut für angewandte Geophysik, Stilleweg 2, D-30655 Hannover. DavidColin.Tanner@liag-hannover.de

- Bernd Leiss - Geowissenschaftliches Zentrum der Universität Göttingen, Goldschmidtstr. 3, D-37077 Göttingen. bleiss1@gwdg.de und ca. $250 \mathrm{~m}$ südliche einer WNW-ESE verlaufenden Schollenzone bzw. Spezialgraben (,Herberhäuser Graben`; vgl. Stille 1932).

\section{Stratigraphie}

Die überwiegend rotbraune Abfolge fester siltiger Tonsteine (Abb. 2), gelegen zwischen überwiegend mittelgrauen dolomitischen Tonsteinen mit vereinzelten Estherien im Hangenden (Mittlere Graue Serie der Arnstadt-Formation; Deutsche Stratigraphische Kommission 2002) und rotbraunen siltigen Tonsteinen der Weser-Formation (,Rote Wand'; Deutsche Stratigraphische Kommission 2002) im Bereich des Universitätsklinikums weiter westlich, lassen eine lithostratigraphisch Einordnung in die ,Untere Bunte Serie ${ }^{6}$ der Arnstadt Formation (ehemals ,Steinmergelkeuper'), Keuper-Gruppe, zu. Chronostratigraphisch ist dieser tiefere Teil der Arnstadt-Formation dem mittleren Norium, Obertrias, zuzuordnen. Fossilien konnten im Bereich des Aufschlusses nicht gefunden werden. 

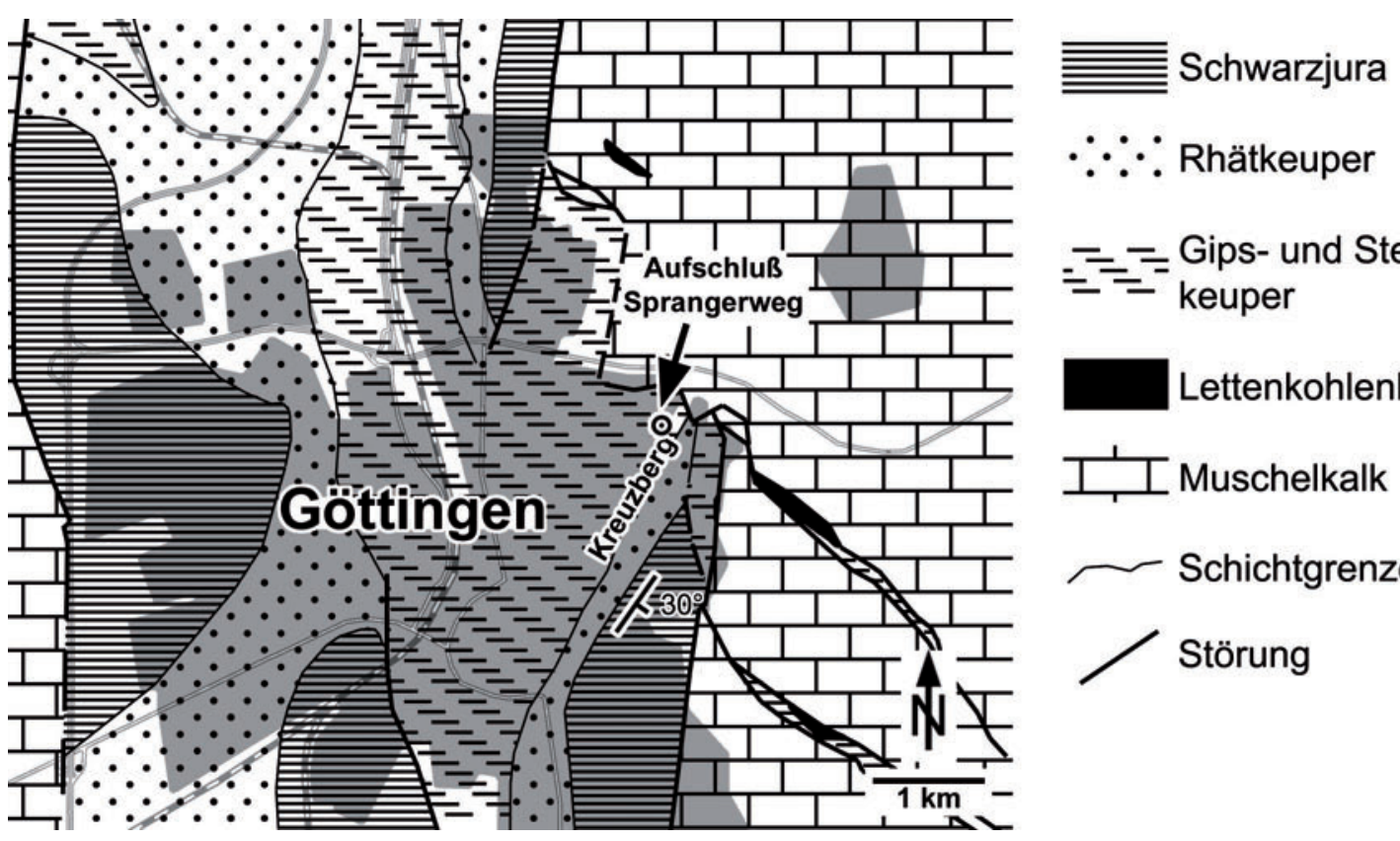

$\because \therefore$ Rhätkeuper

$\overline{=}=$ Gips- und Steinmergel-

$=$ keuper

Lettenkohlenkeuper

$\perp$ Muschelkalk

Schichtgrenze

Störung

Abbildung 1. Geologische Übersicht mit Lage des Aufschlusses am Sprangerweg 3, Göttingen.

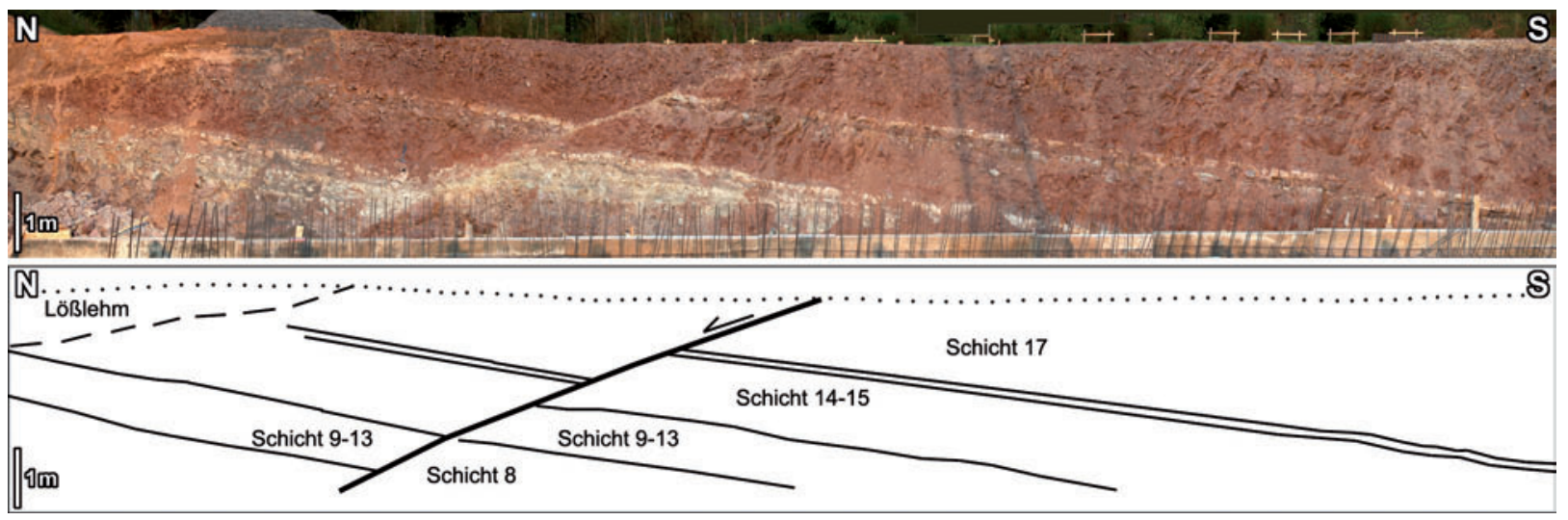

Abbildung 2. Ostwand der Baugrube Göttingen, Sprangerweg 3, mit Wechselfolge aus rotbraunen Tonsteinen und grünlichgrauen Dolomitmergelsteinbänken. Das scheinbar flache Einfallen der Abschiebung ist ein Anschnitteffekt.

\section{Methode}

Die Aufnahme und lithologische Beschreibung der aufgeschlossenen Sedimentgesteinsserie erfolgte am 15. und 17.04.2005. Eine Störungs- und 66 Kluftflächen wurden mit einem Freiberger Geologenkompaß eingemessen.

\section{Ergebnisse}

\subsection{Strukturgeologie}

Das Einfallen der Schichtflächen beträgt im Nordabschnitt der untersuchten Aufschlußwand $120^{\circ} / 25^{\circ}$, im Südabschnitt $110^{\circ} / 28^{\circ}$. Beide Abmit einem Versatzbetrag von etwa $1 \mathrm{~m}$ getrennt (Abb. 2). Diese Kleinabschiebung zeigt bei einer Fallrichtung von $60^{\circ}$ einen Einfallswinkel von $75^{\circ}$ (Abb. 3). Die ehemals überwiegend rotbraunen Tone sind im direkten Kontakt zur Störungsbahn grünlichgrau entfärbt. Die Lagerungswerte der gemessenen Klüfte ist in Tabelle 1 aufgeführt. Vielfach zeigen die Klüfte eine kalzitische Zementation. schnitte werden durch eine Kleinabschiebung 


\subsection{Lithologische Abfolge (Schichtenverzeichnis)}

\begin{tabular}{ll}
32 & $>30 \mathrm{~cm}$ \\
31 & $30 \mathrm{~cm}$ \\
30 & $30 \mathrm{~cm}$ \\
& \\
29 & $30 \mathrm{~cm}$ \\
& \\
28 & $10 \mathrm{~cm}$ \\
& \\
27 & $190 \mathrm{~cm}$ \\
& \\
26 & $20 \mathrm{~cm}$ \\
& \\
25 & $20 \mathrm{~cm}$ \\
& \\
24 & $160 \mathrm{~cm}$ \\
& \\
23 & $25 \mathrm{~cm}$ \\
& \\
22 & $100 \mathrm{~cm}$ \\
21 & $25 \mathrm{~cm}$ \\
20 & $120 \mathrm{~cm}$ \\
19 & $20 \mathrm{~cm}$ \\
\hline 21
\end{tabular}

$181 \mathrm{~cm}$

$17300 \mathrm{~cm}$

$16 \quad 12-14 \mathrm{~cm}$

$1513 \mathrm{~cm}$

$1490 \mathrm{~cm}$

$13 \quad 7-10 \mathrm{~cm}$

$12 \quad 12-14 \mathrm{~cm}$

$11 \quad 15 \mathrm{~cm}$

$10 \quad 6 \mathrm{~cm}$

$9 \quad 40 \mathrm{~cm}$

Hangendes: Bodenbildung; im Nordteil der Grube auch Lößlehmauflage

hellgrau-violettrotbraun, geflammter Tonstein, schichtungslos; splittrig-steinmergelartig; karbonatfrei; unscharfe Untergrenze

hellgrünlichgrauer Tonstein, schwach bis deutlich geschichtet; relativ scharfe Untergrenze; grünlichgrauer, schlierig geschichteter Tonstein; brekziös und mit Kalzit zementiert (Residuallage); mit dm-großen, feinsandig wirkenden Kalzitknollen in zwei Lagen (Residualknollen);

hellgrünlichgraue harte Tonsteinbank; ohne Schichtung, splittrig-steinmergelartig; karbonatfrei; scharfe Untergrenze;

hellgrünlichgrauer Tonstein, im Halbzentimeterbereich gut geschichtet infolge dünner Tonlamellen; scharfe Untergrenze;

schwach violettrotbrauner Tonstein, schichtungslos, grobstückig brechend; oberste 20-25 cm grünlichgrau entfärbt; fließende Untergrenze;

hellgrünlichgrau-rotbraun gescheckter Tonstein, schichtungslos, grobstückig brechend; fließende Untergrenze;

rotbrauner Tonstein, schichtungslos, grobstückig brechend; wellige Untergrenze mit in-situ Brekziierung und Entwässerungsstrukturen;

schwach violettrotbrauner Tonstein, schichtungslos, grobstückig brechend; untere Hälfte stellenweise mit schlieriger reliktischer Schichtung durch Siltlagen; fließende Untergrenze; grünlichgrauer Tonstein; untere Hälfte durch Siltlagen schwach bis gut geschichtet; sonst schichtungslos, grobstückig brechend; scharfe Untergrenze;

rotbrauner Tonstein; obere Hälfte schwach violettrotbraun; schichtungslos, grobstückig brechend; Top grünlichgrau entfärbt; fließende Untergrenze;

grünlichgrau-rotbraun gescheckter Tonstein, schichtungslos, grobstückig brechend; fließende Untergrenze;

rotbrauner Tonstein, schichtungslos, grobstückig brechend; scharfe Untergrenze;

hellgrünlichgraue harte Tonsteinbank; gut geschichtet durch Siltlagen, in der Mitte Schichtflächen mit schwachen polygonalen Trockenrissen; karbonatfrei; oberster $1 \mathrm{~cm}$ rauh brechender, brekziöser und kalzitisch zementierter, feinsandig wirkender Tonstein (Residuallage); scharfe Untergrenze;

grünlichgrauer, brekziöser und kalzitisch zementierter, feinsandig wirkender Tonstein (Residuallage); scharfe Untergrenze;

rotbrauner Tonstein, schichtungslos, grobstückig brechend; scharfe Untergrenze mit in-situ Brekziierung und Entwässerungsstrukturen;

grünlichgrau-hellviolettrotbraun gebänderter harter Tonstein; gut geschichtet infolge Siltlage; Topbereich feinschichtig-laminiert; karbonatfrei; scharfe Untergrenze;

rotbrauner Tonstein, schichtungslos, grobstückig brechend; scharfe Untergrenze;

rotbrauner Tonstein, schichtungslos, grobstückig brechend; oberste $5 \mathrm{~cm}$ grünlichgrau gescheckt (entfärbt); scharfe Untergrenze mit in-situ Brekziierung und Entwässerungsstrukturen;

feinstgeschichteter hellgrünlichgrauer bis rotbrauner Tonstein; fließende Untergrenze;

hellgrünlichgraue harte Tonsteinbank; unter schwach und schlierig geschichtet, mitte und oben gut geschichtet infolge Siltlagen; karbonatfrei; fließende Untergrenze;

hellgrünlichgrauer bis dunkelgrünlichgrauer, z.T. rotbraun geflammter, schlierig feingeschichteter Tonstein; von Entwässerungsstrukturen durchsetzt; 2-10 cm über Basis Lage aus brekziösen kalzitisch zementierter Tonsteinknollen (Residualknollen); scharfe Untergrenze; grünlichgrauer, kräftig dunkelgrasgrün gescheckter, schwach geschichteter Tonstein; oberster $1 \mathrm{~cm}$ brekziös, feinsandig wirkend und kalzitisch zementiert (Residuallage); scharfe Untergrenze;

hellgrünlichgraue harte Tonsteinbank; basale $5-8 \mathrm{~cm}$ hellgrünlichgrau und gut geschichtet; 8-14 cm über Basis dunkelgrau und feinschichtig infolge von Tonlamellen, Entwässerungsstrukturen; 14-40 cm über Basis hellgrünlichgrau und im 1/2 cm-Bereich durch Siltlagen gut geschichtet; leicht dolomitisch; scharfe Untergrenze;

rotbrauner Tonstein, schichtungslos, grobstückig brechend; oben blaßrotbraun, am Top dann grünlichgrau entfärbt (oberste $5 \mathrm{~cm}$ );

\section{— Störung - (Fortsetzung mit geringer Schichtverdopplung)}

rotbrauner Tonstein, schichtungslos, grobstückig brechend; mit Peloturbationsharnischen; 45-55 cm sowie 70-90 cm über Basis grünlichgrau gefleckt; scharfe Untergrenze; hellgrünlichgrau-blaßrotbraun gebänderte harte Tonsteinbank; gut geschichtet infolge Siltlagen; karbonatfrei; fließende Untergrenze; grau-hellviolettrotbraun geflammter Tonstein, schichtungslos; scharfe wellige Untergrenze; hellgrünlichgrauer Tonstein, grobstückig brechend; schwach bzw. schlierig geschichtet, pedogene Rißsysteme und Entwässerungsstrukturen; Topbereich leicht dolomitisch; fließende Untergrenze;

rotbrauner Tonstein, schichtungslos, grobstückig brechend; oberste $40 \mathrm{~cm}$ hellviolettrotbraun; fließende Untergrenze;

hellgrünlichgrauer Tonstein, schichtungslos; karbonatfrei; fließende Untergrenze;

Liegendes: rotbrauner Tonstein, schichtungslos, grobstückig brechend; karbonatfrei; 


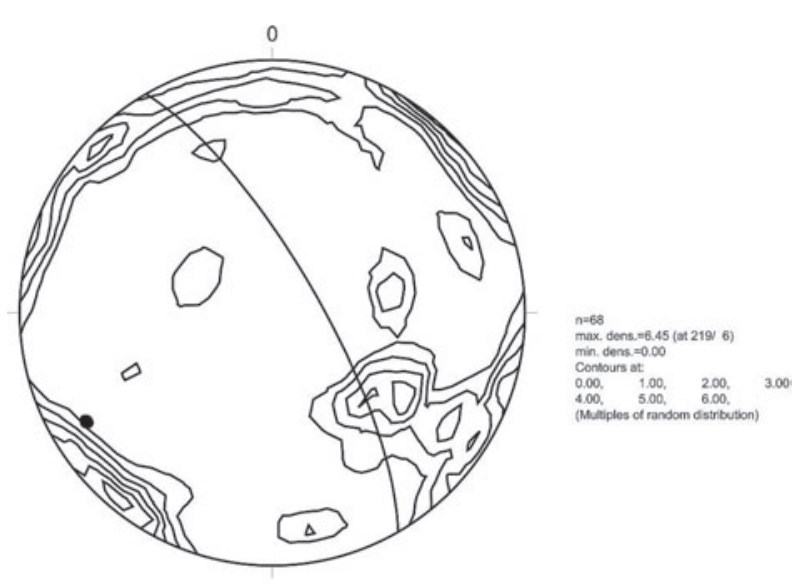

Abbildung 3. Flächentreue Projektion der Kluftpole und der Kleinabschiebung (Großkreis und zugehöriger Flächenpol)

\section{Diskussion und Interpretation}

Das gemessene Schichteinfallen mit $20-30^{\circ}$ in Richtung ESE hin zur östlichen Grabenrandstörung ist konform mit den bisherigen Erkenntnissen für den östlichen Randbereich der Grabenscholle (Stille 1932; vgl. Abb. 1).

Bezüglich der Klüftung können drei Richtungen unterschieden werden (Abb. 3). Die Hauptkluftrichtung läuft dabei parallel zur beobachteten Abschiebung im Aufschluß. Eine weitere Kluftschar ist senkrecht zu dieser Störung angelegt. Die beobachtete breite Streuung der Kluftwerte, insbesondere die der zweiten Kluftschar, spiegelt das unterschiedliche Bruchverhalten der verschiedenen Lithologien (massige gegenüber gut geschichteten Tonsteinen) wider.

Die NNW-SSE streichende Kleinabschiebung deutet auf eine Fortsetzung des auf der östlichen Grabenschulter entwickelten Herberhäuser Grabens bis in das Grabeninnere hin. Kalzitische Kluftfüllungen und Entfärbungen im Bereich der Störungsbahn belegen den Durchsatz von Grundwässern in dieser weitgehend pelitischen Formation der Keuper-Gruppe.

\section{Literatur}

Stille, H. 1932. Geologische Karte von Preußen und benachbarten deutschen Ländern. Erläuterungen zu Blatt Göttingen Nr. 2520. 3. Auflage, 40 S., 1 Kte., Berlin.

Deutsche Stratigraphische Kommission (Hg.) 2002. Stratigraphische Tabelle von Deutschland 2002. 16 S., 1 Tabelle, Potsdam.
Tabelle 1

Meßwerte der Klüftung in Tonsteinen der tiefern Arnstadt-Formation in der Baugrube Göttingen, Sprangerweg 3.

\begin{tabular}{|c|c|c|c|}
\hline Azimuth & Einfall & Azimuth & Einfall \\
\hline 048 & 84 & 312 & 76 \\
\hline 247 & 88 & 292 & 49 \\
\hline 194 & 78 & 059 & 89 \\
\hline 268 & 40 & 035 & 85 \\
\hline 330 & 60 & 214 & 62 \\
\hline 310 & 40 & 045 & 90 \\
\hline 206 & 89 & 324 & 48 \\
\hline 135 & 79 & 210 & 86 \\
\hline 110 & 90 & 300 & 70 \\
\hline 120 & 90 & 302 & 55 \\
\hline 034 & 84 & 043 & 90 \\
\hline 302 & 54 & 145 & 80 \\
\hline 060 & 84 & 299 & 42 \\
\hline 232 & 80 & 181 & 77 \\
\hline 310 & 40 & 350 & 76 \\
\hline 330 & 35 & 035 & 88 \\
\hline 111 & 82 & 092 & 90 \\
\hline 301 & 74 & 043 & 77 \\
\hline 287 & 86 & 038 & 85 \\
\hline 108 & 90 & 102 & 90 \\
\hline 033 & 79 & 030 & 68 \\
\hline 172 & 74 & 349 & 75 \\
\hline 248 & 69 & 312 & 60 \\
\hline 305 & 70 & 068 & 52 \\
\hline 252 & 70 & 312 & 48 \\
\hline 310 & 90 & 304 & 50 \\
\hline 252 & 42 & 134 & 78 \\
\hline 155 & 75 & 316 & 90 \\
\hline 238 & 90 & 232 & 88 \\
\hline 291 & 78 & 262 & 41 \\
\hline 132 & 90 & 161 & 84 \\
\hline 050 & 80 & 199 & 73 \\
\hline 159 & 58 & 178 & 78 \\
\hline
\end{tabular}

Koenen, A.v. 1894, 1907. Erläuterungen zur geologischen Specialkarte von Preussen und den Thüringischen Staaten. Blatt No. 28 Göttingen. 58 S., Berlin. 


\title{
Exkursion nördliches Leinetal
}

\author{
David C. Tanner (GZG Göttingen, LIAG Hannover)
}

\begin{abstract}
Zusammenfassung - Auf dieser Exkursion wird ein Ost-West Profil durch den nördlichen Leinetal Graben zwischen Sudheim und Hardegsen vorgestellt. Alle Aufschlüsse sind Steinbrüche im unteren Muschelkalk. Daher zielt der Inhalt dieser Exkursion nicht auf die Stratigraphie, sondern auf die Klein- und Meso-Strukturen, die hervorragend die Kinematik dieses Grabens zeigen. Insbesondere soll die Rolle von Blattverschiebungen hervorgehoben werden. Transpressive und -tensive Strukturen sind besonders im Steinbruch Elvese, innerhalb des Grabens, aufgeschlossen. Beispiele von steilen Blattverschiebungen mit reichlichem Durchfluß von Paläofluiden sind in den Steinbrüchen Hardegsen II und Papenberg zu sehen. Kompressive Strukturen sind in den Steinbrüchen Hardegsen I und Sudheim zu finden.
\end{abstract}

\begin{abstract}
This excursion demonstrates an east-west cross-section through the northern Leine Valley Graben between Sudheim and Hardegsen. All the outcrops are quarries in the Lower Muschelkalk. Therefore the aim of this excursion is to show not the stratigraphy, but the small and meso-structures that demonstrate excellently the kinematics of this graben. In particular, the role of strike-slip faults are emphasized. Examples of steep strike-slip faults with good evidence of fluid flow are to be seen in the quarries of Hardegesen II and Papenburg. Compressive structures can be found in the Hardegsen I and Sudheim quarries.
\end{abstract}

Schlüsselworte-Leinetalgraben, Muschelkalk, Exkursion, Seitenverschiebung

\section{Geologische Übersicht des Leinetal Grabens \\ Sedimentologie}

Die Sedimentologie im Bereich des Leinetals wird von Arp et al. (2004) sehr ausführlich beschrieben. Hier wird deshalb nur ein kurzer Abriss der Sedimentationsgeschichte gegeben.

Die ältesten Gesteine im weiteren Bereich des Leinetalgrabens (Abb. 1) sind variszisch geprägte unterkarbonische/devonische Grauwacken in der Bohrung Dransfeld 1 (Abb. 2; Müller et al. 1961) und unterkarbonische Grauwacken und Tonsteine des Visé und Namur in einer Bohrung bei Northeim (Abb. 2; Fabian, 1957). Diese Basementgesteine liegen jetzt in einer Tiefe von 1,5-1,8 km.

Als südlicher Rand des Zentralen Europäischen Beckens (Central European Basin - CEB) waren die Bereiche um Northeim von RotliegendFanglomeraten und -Arkosen bedeckt. Die Region von Göttingen dagegen blieb in dieser Zeit noch

- David C. Tanner - GZG Göttingen, jetzt LeibnizInstitut für angewandte Geophysik, Stilleweg 2, D-30655 Hannover. DavidColin.Tanner@liag-hannover.de

mit Hilfe und Daten von Bernd Leiss, Axel Vollbrecht, Wiebke Kallweit, Silke Meier, Asdis Oelrich \& Dorothea Reyer sedimentfrei. Eine weitere Senkung des CEB führte $\mathrm{zu}$ den ersten marinen Sedimenten der Zechstein Gruppe. In der Bohrung Dransfeld 1 wurden etwa $430 \mathrm{~m}$ Zechstein durchbohrt. Darauf folgte die Buntsandstein Gruppe (Abb. 3). Der Untere Buntsandstein begann mit Dolomit führenden Ton- und Siltsteinen (Arp et al. 2004). Dies entwickelte sich zu einer zyklischen Silt-, Tonund Sand-Ablagerung in ,Braided River Systems", die in einen zentralen Playasee mündeten (Paul 1982, 1999). Im Mittleren Buntsandstein wurde die Zyklizität der Sedimentation verstärkt, wobei viele Diskordanzen auftreten (Paul 1999).

Der obere Buntsandstein, die Röt Formation, ist eine Abfolge von grauen bis roten Tonsteinen mit Halit- und Gipseinlagen. Im obersten Röt findet zum ersten Mal eine starke marine Beeinflussung statt (Paul und Franke 1977).

In einer $1 \mathrm{~km}$ tiefen Bohrung bei Sudheim (s. Abb. 5) wurden die folgenden Mächigkeiten festgestellt: $100 \mathrm{~m}$ Keuper, $250 \mathrm{~m}$ Muschelkalk, $200 \mathrm{~m}$ Röt. Der Top des Zechstein-Salzes lag bei einer Tiefe von $700 \mathrm{~m}$ (Grupe 1909). Eine ebenfalls $1 \mathrm{~km}$ tiefe Bohrung bei Levershausen (s. Abb. 5), außerhalb des Grabens, stellte fest, dass das Röt $250 \mathrm{~m}$ mächtig, und der Mittlere und Untere Buntsandstein zusammen $280 \mathrm{~m}$ mächtig sind. Die 


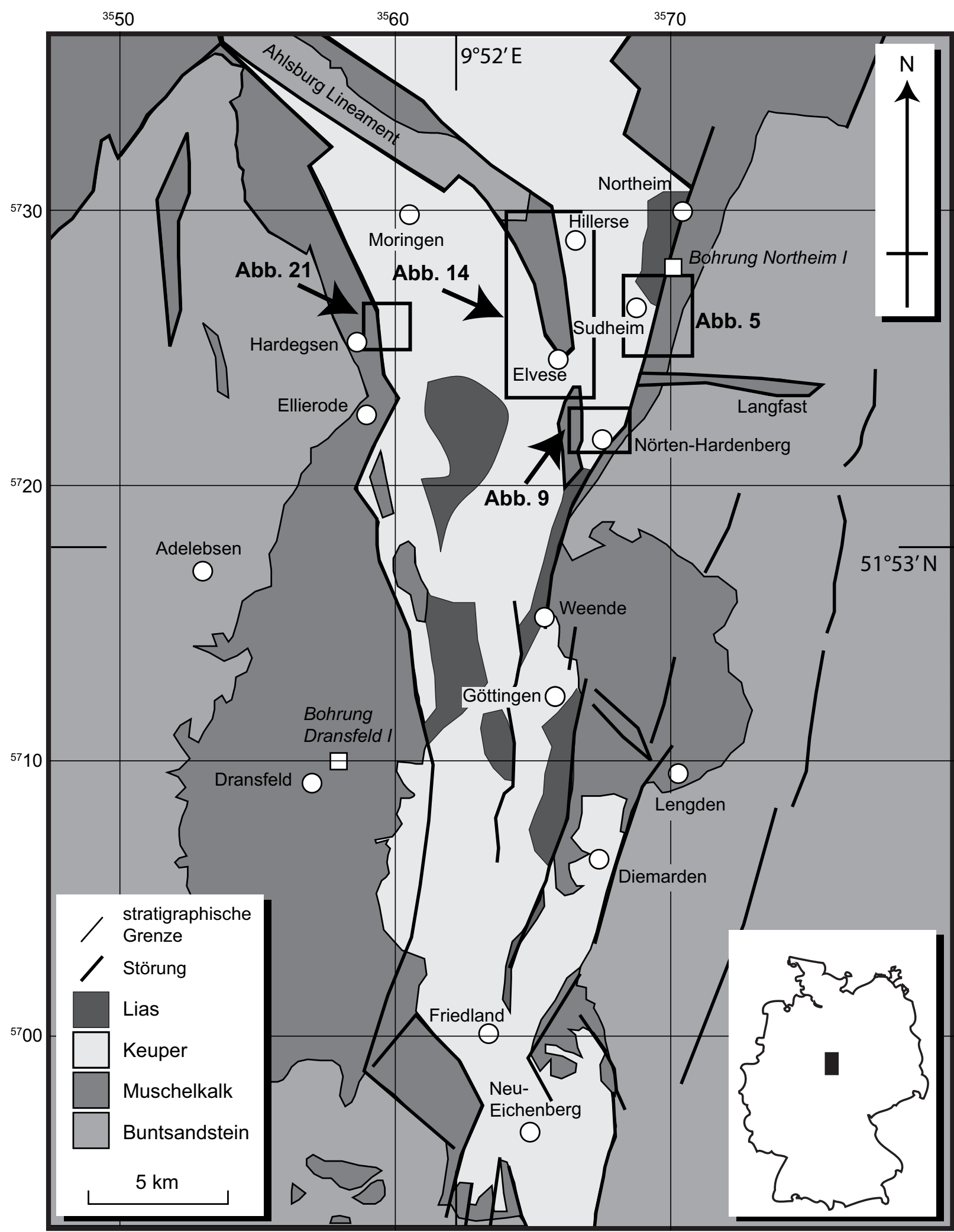

Abbildung 2. Vereinfachte geologische Karte des Leinetals und seiner Umgebung, nach Wunderlich (1955), Stille und Lotze (1933), Arp et al. (2004, und den geologischen Karten von Niedersachsen (GK25, 4325-6, 4224-6). Großmaßstäbliche Kartenausschnitte, die zusätzlich in diesem Führer zu finden sind, sind markiert. 


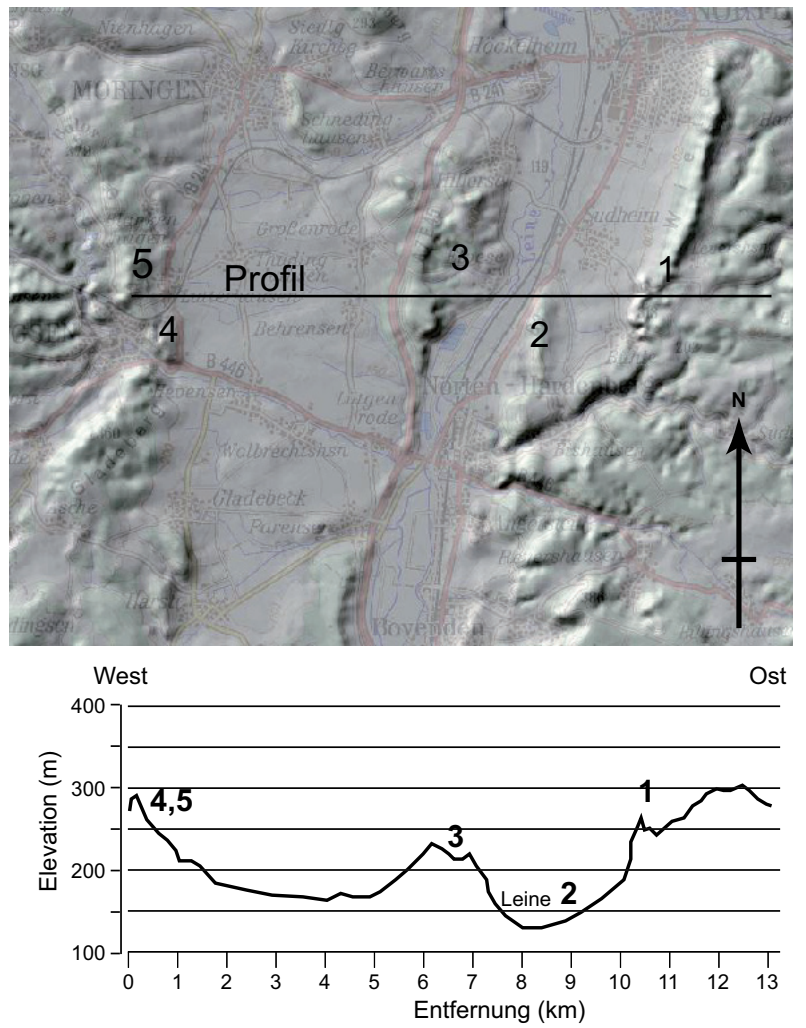

Abbildung 1. Oben: Topographische Karte, überlagert vom digitalen Höhenmodell (von der digitalen topographischen Karte Niedersachsen 1:50.000). Die Ziffern zeigen die Exkursionsaufschlüsse. Besonders gut zu sehen sind die Ostrandstörung bei Sudheim (1), das AhlsburgLineament (3) und die glatte Oberfläche innerhalb des Grabens. Unten: Ost-West Geländeschnitt im Bereich die Aufschlüsse.

Zechsteinsalze wurden bei einer Tiefe von $540 \mathrm{~m}$ erbohrt (Grupe 1909).

Zu Beginn der Muschelkalk Gruppe war das gesamte Gebiet marin überflutet (Arp et al. 2004). Die erste Sedimentation begann mit der Wellenkalk-Abfolge (Jena Formation) des Unteren Muschelkalkes. Dies ist das Gestein, das auf dieser Exkursion am häufigsten vorkommt. Auf die zunächst im offenen marinen Bereich abgelagerten Kalksteine, wurde der Gipse und Steinsalz führende Mittlere Muschelkalk in einem geschlossenen Milieu abgelagert. Offene marine Verhältnisse wurden im Oberen Muschelkalk wieder hergestellt (Arp et al. 2004).

Die Keuper Gruppe, obwohl im Göttinger Raum über $450 \mathrm{~m}$ mächtig, ist wegen der sehr schlechten Aufschlußverhältnisse kaum beschrie-

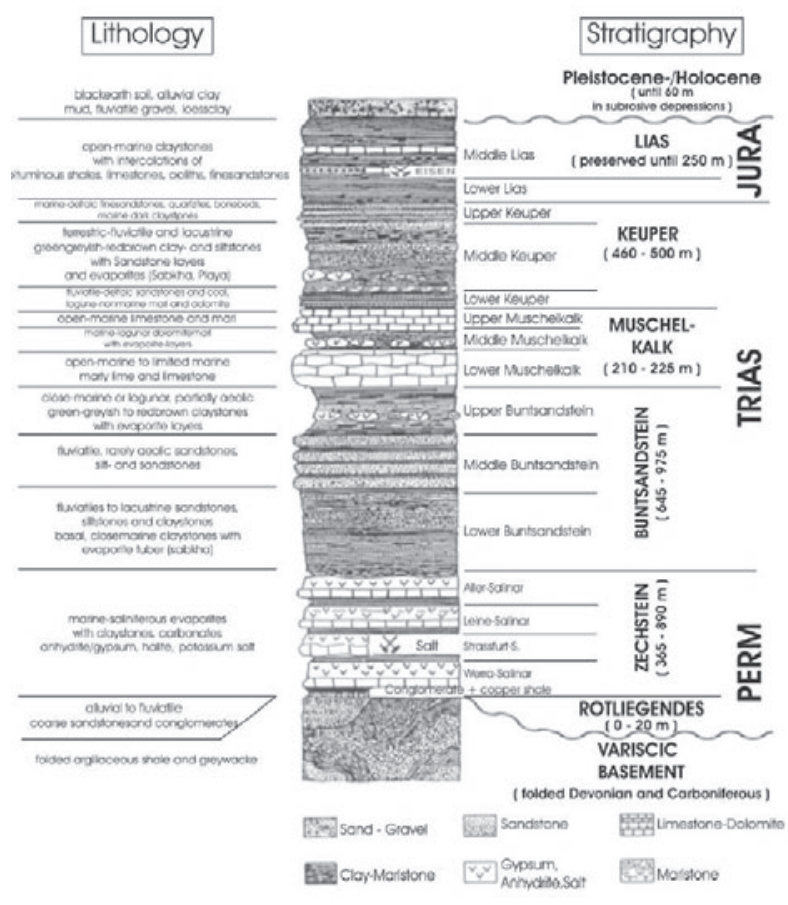

Abbildung 3. Ein stratigraphisches Profil des Niedersächsischen Beckens (nach Meyer 2000).

ben. Die besten Untersuchungen stammen aus Bohrungen. Dem Unteren Keuper (Erfurt Formation) werden brackisch-limnischer Kalkmergel mit Lettenkohlen und rotbunten Tonsteinen zugeordnet (Arp et al. 2004). Mit dem Unteren Gipskeuper wurden rotbunte Tonsteine mit Gipseinlagen und Steinsalzen abgelagert. Über einer Erosionsfläche wurde der kaum aufgeschlossene Schilfsandstein (Stuttgart Formation) sedimentiert (Arp et al. 2004).

Im Oberen Gipskeuper (Weser Formation) kam es wieder zu rotbunten Tonstein-Ablagerungen eines Playa-Systems. Im darauffolgenden Steinmergelkeuper (Amstadt-Formation) folgten rote bis graue Tonsteine in Zyklen von lacustrinen Systemen (Arp et al. 2004).

Die letzte mesozoische Sedimentation in der Leinetal-Gegend erfolgte mit der liassischen Schwarzjura Gruppe. Etwa $225 \mathrm{~m}$ dieser vollmarinen, schwarzen Tonsteine mit Kalkbänken und Konkretionen sind noch erhalten.

\section{Tektonik}

Obwohl die letzte mesozoische Sedimentation im Göttinger Raum im Mittleren Jura stattfand, entstand der Leinetalgraben wahrscheinlich während des gleichen Zeitraums (späte Oberkreide) 
wie z.B. die benachbarte Harznordrand-Überschiebung (Wunderlich 1957, Ritzkowski 1999). $\mathrm{Zu}$ dieser Zeit herrschte eine allgemeine Inversion des CEB, mit Reaktivierung von Abschiebungen als Aufschiebungen und Halokinese durch eine Nord-Süd gerichtete Verkürzung (Kockel 2003, Otto 2003, Mazur et al. 2005).

Der Leinetalgraben besteht aus zahlreichen Randstörungen, die auf der Ostseite en-echélon zueinander stehen (Abb. 2). Die Störungen versetzen Mittleren Buntsandstein bis Mittleren $\mathrm{Mu}$ schelkalk gegen Keuper bis Jura, so dass die maximale Sprunghöhe ca. $1000 \mathrm{~m}$ beträgt. Der Durchschnitt liegt bei etwa $500 \mathrm{~m}$ Sprunghöhe. Der Westrand hat deutlich weniger Versatz, so dass die Vermutung nahe liegt, dass der Graben eine Asymmetrie besitzt, wobei diese Asymmetrie von Nord nach Süd variiert.

Verschiedene Autoren haben Profile durch den Leinetalgraben konstruiert (Abb. 4). Deutlich ist zu sehen, dass die Meinungen über die Einfallwinkel der Randstörungen auseinandergehen, ebenfalls die Meinungen über die Rolle von ZechsteinSalz als Grabenbasis oder wie weit das Salz nach oben gedrungen ist. Was die meisten Autoren verbindet, ist ihre Vorstellung über die Wölbung des Grabeninneren und inbesondere dessen Einfallen in Richtung der Störungen.

Im Norden wird der Graben von einem markanten Streifen aus Buntsandstein und Muschelkalk durchquert; das Ahlsburg Lineament. Es wird postuliert, dass diese Struktur von Überschiebungen mit einer Seitenverschiebungskomponente flankiert wird (Lotze 1932b, Wunderlich 1959). Auf dieser Exkursion wird diese Hypothese untermauert, allerdings wird hier die Rolle der Seitenverschiebung hervorgehoben.

\section{Aufschluß 1: \\ Steinbruch Sudheim}

\section{Lage R: ${ }^{35} 69097 \mathrm{H:}{ }^{57} 25683$ \\ Karte TK25 4325 - Nörten-Hardenberg}

Der Steinbruch Sudheim liegt 1,2 km SW' des Dorfes Sudheim (Abb. 5). Der Steinbruch befindet sich in einem etwa $400 \mathrm{~m}$ schmalen Streifen von Unterem Muschelkalk, zwischen zwei Abschiebungen, die zusammen die Ostrandstörungszone des Leinetalgrabens bilden. Im Hangenden der westlichen Störung befindet sich Mittlerer Keuper, so dass der vertikale Versatz hier etwa $400 \mathrm{~m}$ beträgt. Die östlichen Abschiebungen haben jeweils weniger als $100 \mathrm{~m}$ vertikalen Versatz.
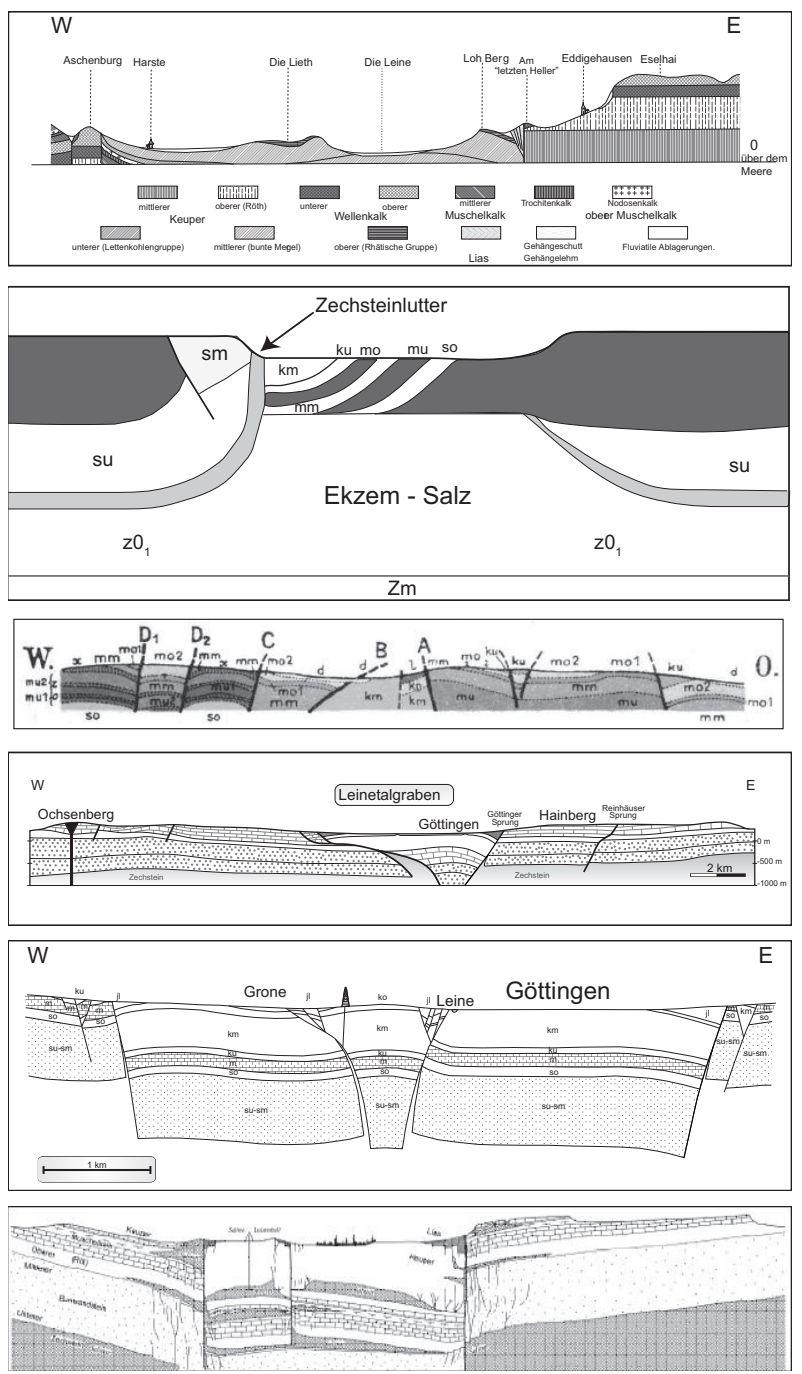

Abbildung 4. Verschiedene Ost-West Profile durch den Leinetalgraben, aus (von oben nach unten) Lang (1880), Lachman (1917), Patriciu (1930), Stille und Lotze (1933), Wunderlich (1966) und Meischner (2002).

Der Steinbuch besteht aus zwei Sohlen. Auf der unteren $4 \mathrm{~m}$ hohen, Ost-West streichenden Wand sind ein enger Sattel und eine Mulde mit nordwestlicher Vergenz aufgeschlossen. Deutlich ist zu sehen, dass die Klüftung auf dem steilen Schenkel viel stärker ausgeprägt ist als die auf dem flachen Schenkel. Abbildung 6 zeigt die Raumlage der Schichtung im Bereich der Falte. Die konstruierte Faltenachse hat die Raumlage 199/14.

Auf dem Weg zur oberen Sohle ist eine Schichtfläche mit hervorragender Mineralharnischbil- 


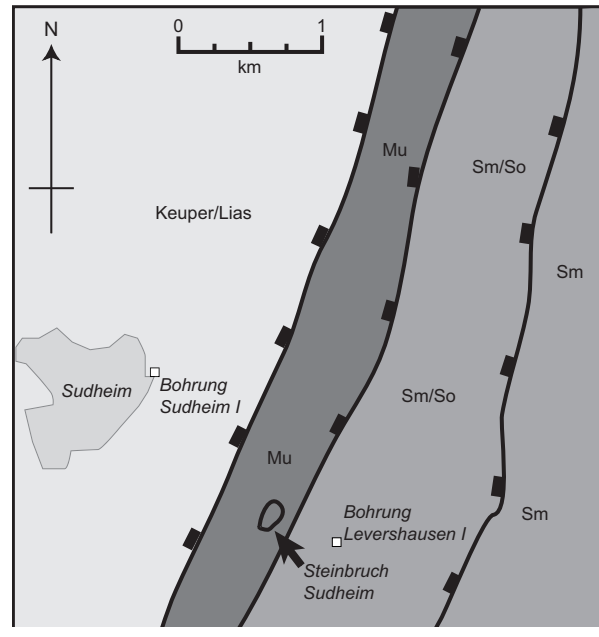

Abbildung 5. Vereinfachte geologische Karte des Gebietes um den Steinbruch Sudheim (nach den GK25, 4325 Nörten-Hardenberg und 4526 Katlenburg).

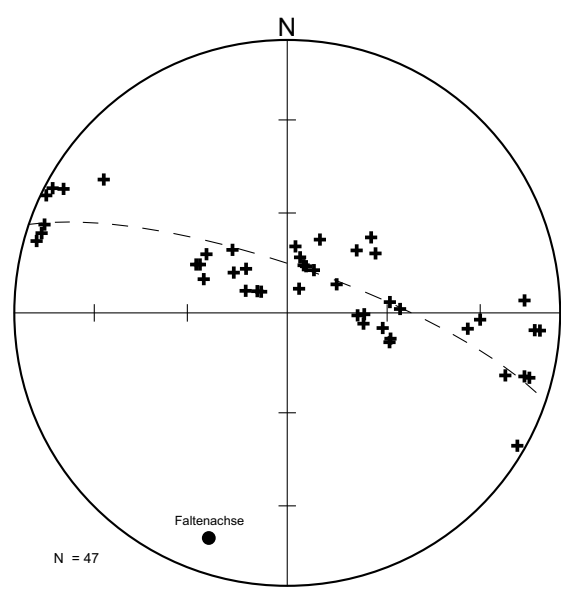

Abbildung 6. Schichtflächenpole im Bereich der auf der unteren südlichen Wand aufgeschlossenen Falte in Steinbruch Sudheim (nach Reyer 2008). Schmidt'sches Netz, untere Halbkugel.

dung aufgeschlossen (Abb. 7). Die meisten Harnischlineare zeigen einen Top-NW- oder Top-NSchersinn.

Die obere Sohle schließt auch Falten auf, die allerdings eine kleinere Amplitude haben als die in der unteren Wand. Abbildung 8 zeigt die entsprechende Raumlage der Schichtung und Lage der konstruierten Faltenachse (012/03). Besonders gut zu sehen ist eine kleine Aufschiebung, die sich aus dem östlichen Schenkel einer Falte in den Bereich der Umbiegungszone fortsetzt, und damit eine zusätzliche Verkürzung während und nach der Faltung anzeigt.

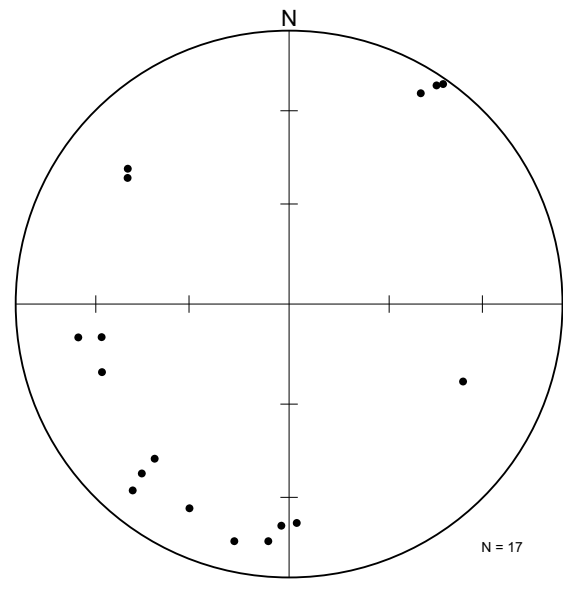

Abbildung 7. Harnischlineare im Steinbruch Sudheim (nach Reyer 2008). Schmidt'sches Netz, untere Halbkugel.

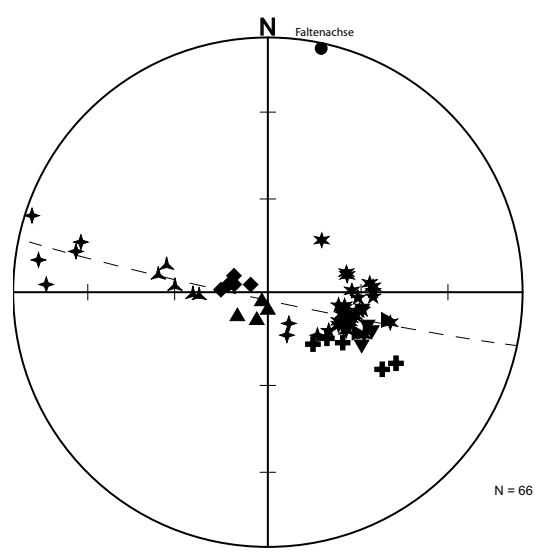

Abbildung 8. Schichtflächenpole im oberen Bereich der aufgeschlossenen Falte. (nach Reyer 2008). Schmidt'sches Netz, untere Halbkugel.

\section{Aufschluß 2: \\ Steinbruch Papenberg}

Lage: $R:{ }^{35} 66632 \mathrm{H:}{ }^{57} 23533$

\section{Karte TK25 4325 - Nörten-Hardenberg}

Der Steinbruch Papenberg liegt etwa $1 \mathrm{~km}$ NW' von Nörten-Hardenberg. Der Steinbruch erfasst den Unteren Muschelkalk (Wellenkalk) an der Ostseite des Ahlsburg Lineaments (Abb. 9). Dieses Lineament hier streicht etwa N-S und trifft bei Nörten-Hardenberg auf die Leinetal-Ostrandstörung.

Der erste Aufschluß im Steinbruch zeigt eine steile, etwa $4 \mathrm{~m}$ breite Störungszone (Abb. 10), in der ein keilförmiges Vorkommen von rot gefärbtem Material steckt. Dieser Keil besteht vermutlich aus Material des Röts $\left(\mathrm{So}_{2-4}\right)$, das hier 


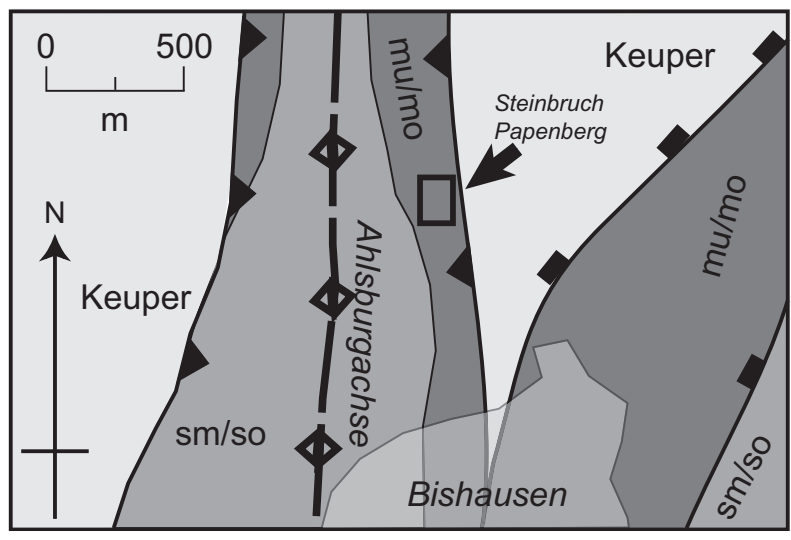

Abbildung 9. Vereinfachte geologische Karte des Gebietes um den Steinbruch Papenberg (nach der GK25 4325 Nörten-Hardenberg).

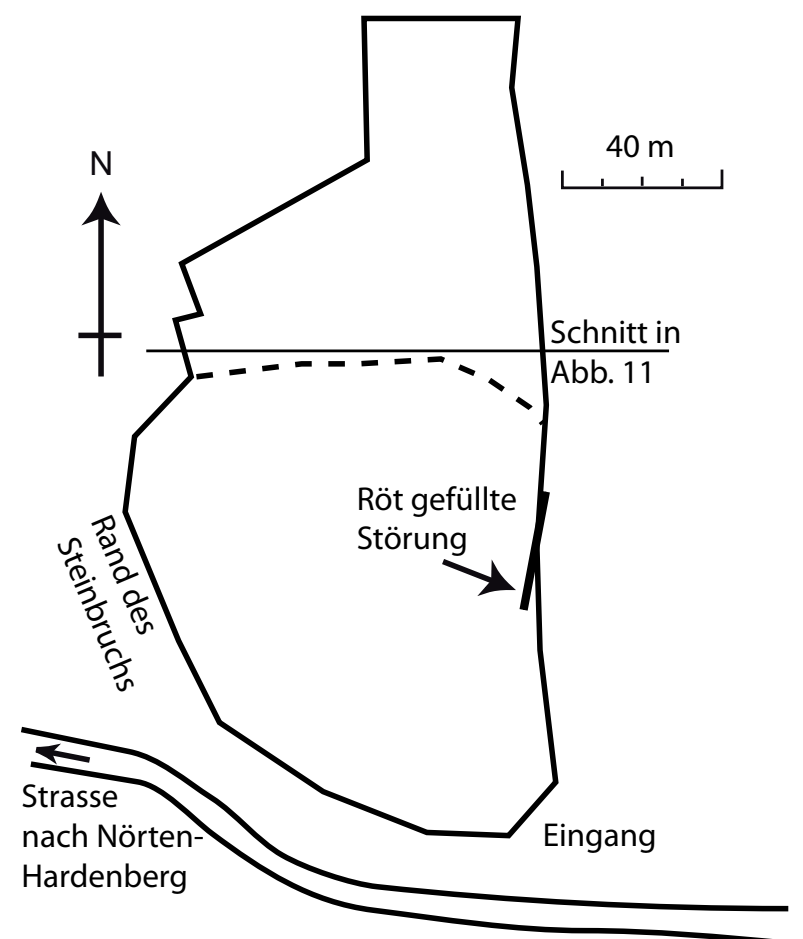

Abbildung 10. Lageplan von Steinbruch Papenberg.

allerdings stark sekundär zementiert und dadurch kaum noch erkennbar ist. Zu bemerken ist auch das sehr steile Einfallen des Muschelkalks nach NE in diesem Bereich.

Die nördliche Wand des Steinbruchs zeigt eine Reihe von steilen Abschiebungen (Abb. 11), mit jeweils 1-2m Versatz. Die Schichtung definiert eine sehr offene Sattelstruktur, mit $30^{\circ}$ nach NE fallenden Schichten an der Ostseite und fast horizontalen Schichten an der Westseite.

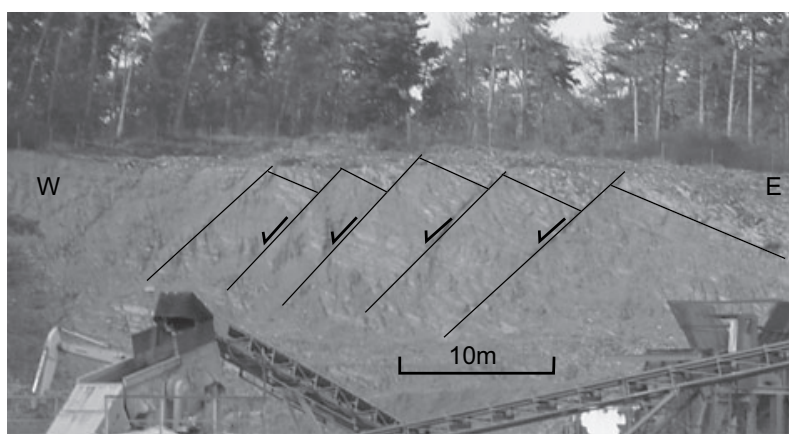

Abbildung 11. Blick auf die Nordwand des Steinbruchs Papenberg. Die Störungen fallen mit $60^{\circ}$ ein, hier sieht man nur den scheinbaren Einfallswinkel.

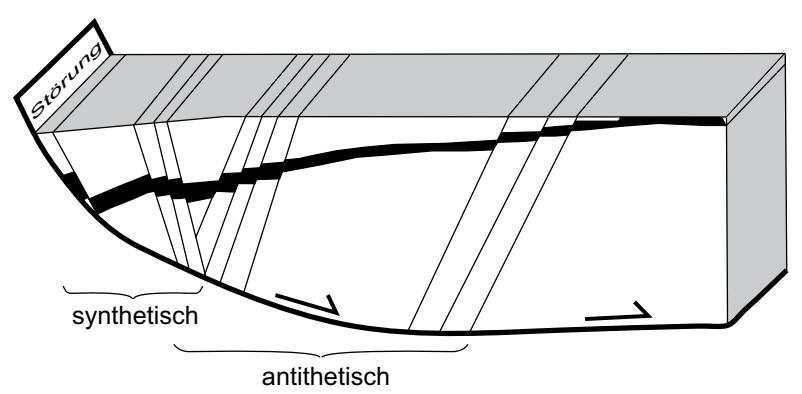

Abbildung 12. Rollover Struktur im Hangenden einer listrischen Abschiebung mit untergeordneten synthetischen und antithetischen Abschiebungen. Abgebildet von einem SandkastenExperiment, pers. Mitt. Jo Nagel, GFZ-Potsdam.

Es entsteht der Eindruck, dass die Abschiebungen zu einer ,Roll-over Struktur' als synthetische Abschiebungen gehören könnten (Abb. 12). Mißt man jedoch die Harnischlineare ein, die auf fast allen Schichtflächen zu sehen sind, sieht man, dass die Lineare und damit die Verschiebungen der Struktur, etwa parallel zu der Streichrichtung der Abschiebungen verlaufen. Wenn man ferner beachtet, dass das Ahlsburg-Lineament eine Blattverschiebung ist, dann paßt diese N-S orientierte, schichtparallele Kinematik besser, und man kann sich vorstellen, dass die Abschiebungen eigentlich ,transtensive' Strukturen sind, und die schwache Faltenstruktur zu der positiven ,Blumen' -Struktur gehört (Abb. 13, links), die den größten Teil des Ahlsburg-Lineaments ausmacht (z.B. besser zu sehen in Aufschluß 3). 

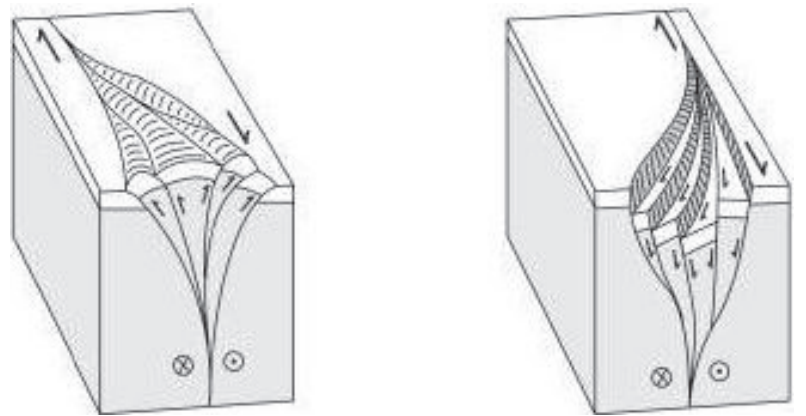

Abbildung 13. Positive (links) und negative (rechts) ,Flower'-Strukturen (aus Ramsay und Huber, 1987)

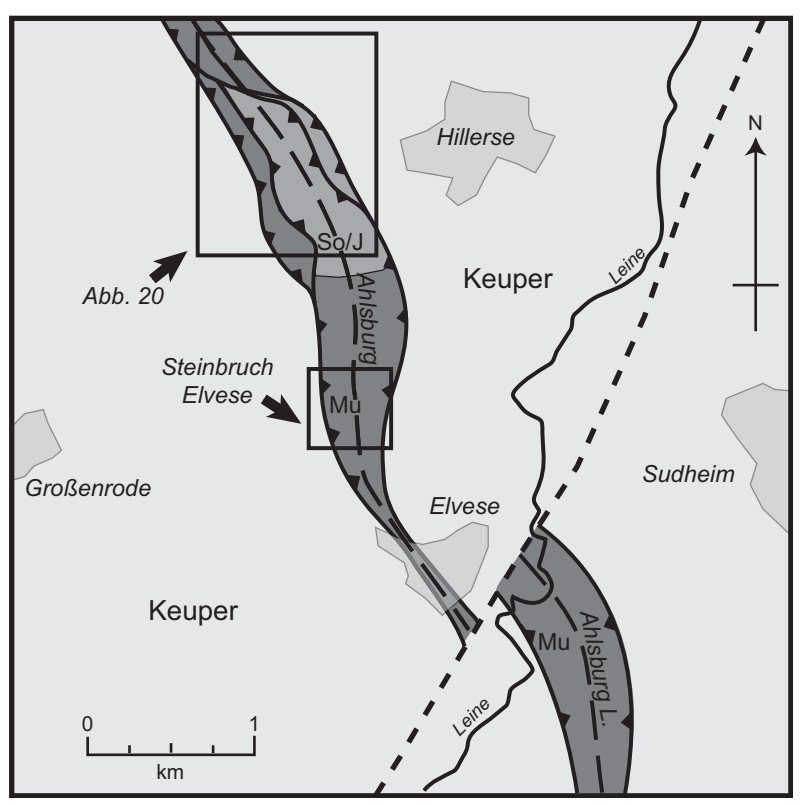

Abbildung 14. Vereinfachte geologische Karte des weiteren Gebietes um den Steinbruch Elvese (nach GK 254325 Nörten-Hardenberg). so$\mathrm{J}$ - Obere Buntsandstein-Jura, mu - Untere Muschelkalk. Gestrichelte Linie — vermutete sinistrale Blattverschiebung.

\section{Aufschluß 3: Steinbruch Elvese}

Lage: R: ${ }^{35} 64917$ H: ${ }^{57} 26928$

Karte TK25, 4325 - Nörten-Hardenberg

Der Steinbruch Elvese liegt etwa $1 \mathrm{~km}$ NW' von der gleichnamigen Ortschaft entfernt (Abb. 14). Dieser sehr große Steinbruch schließt ein gesamtes Ost-West-Querprofil durch die Ahlsburgachse auf. Die obere nördliche Wand des Steinbruches schneidet durch einen offenen, aufrechten Sattel (Abb. 16). Diese Falte, typisch für Muschelkalk-

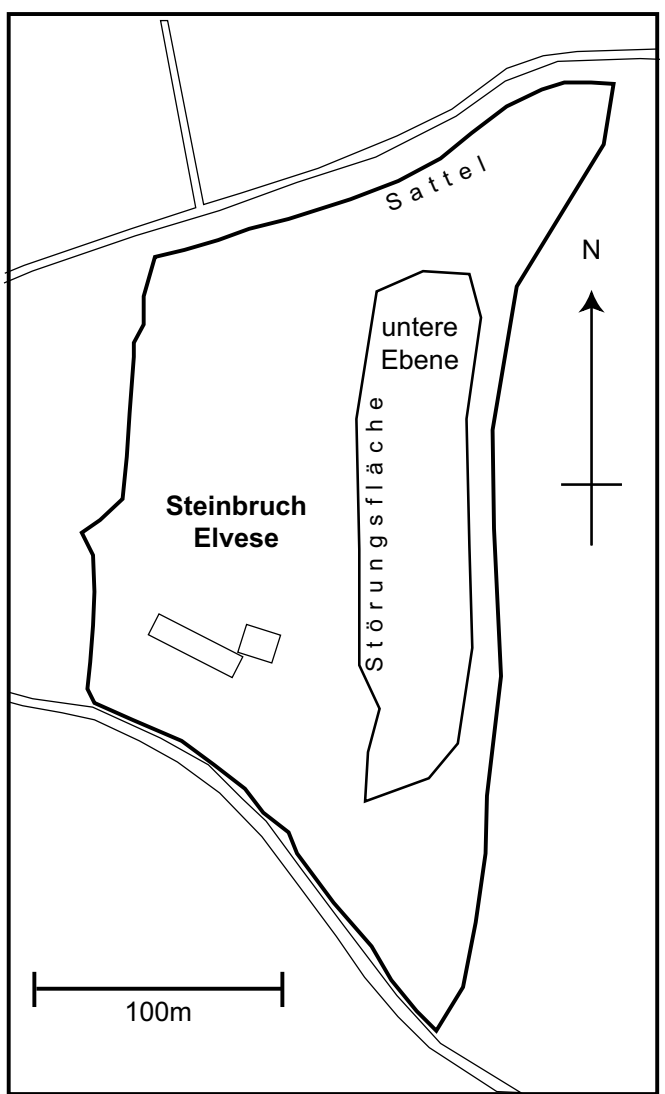

Abbildung 15. Skizze vom Steinbruch Elvese.

falten in dieser Region, ist verbunden mit bruchhaften Strukturen, insbesondere einem Fächer von ost und west-gerichteten Aufschiebungen mit minimalem Versatz. An den beiden Flanken der Falte sind diese Aufschiebungen mit engen Knickfalten verbunden.

In der unteren Ebene des Steinbruchs Elvese wird die westliche Wand von einer nach Osten einfallenden Störungsfläche, mit einer Länge von über $120 \mathrm{~m}$ und einer Höhe von etwa $20 \mathrm{~m}$ gebildet (siehe Tanner et al., 3D-Störungsmorphologie, dieser Band für weitere Informationen).

Mineral- und Reibungsharnische sind sehr häufig auf dieser Störung zu sehen mit gegenseitiger Überprägungen durch verschieden orientierte und gebogene Harnische, die eine Drehung der lokalen Störungsbewegung um bis zu $60^{\circ}$ anzeigen. Auf der gesamten Fläche ist eine $180^{\circ}$ Richtungsstreuung von abschiebenden, aufschiebenden und blattverschiebenden Harnischlinearen zu sehen, wobei steiles Einfallen nach NE überwiegt (Abb. 17).

Die Störungsfläche ist gewölbt um eine EWstreichende Achse (Abb. 18). Dass die individu- 


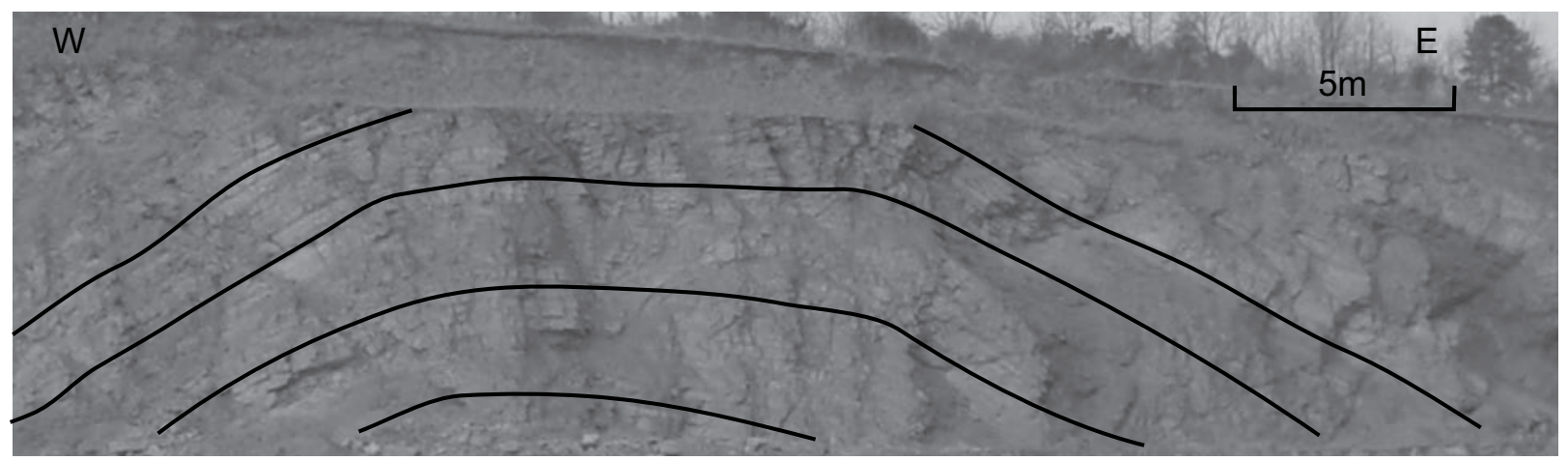

Abbildung 16. Sattel an der oberen, nördlichen Wand des Steinbruchs Elvese.

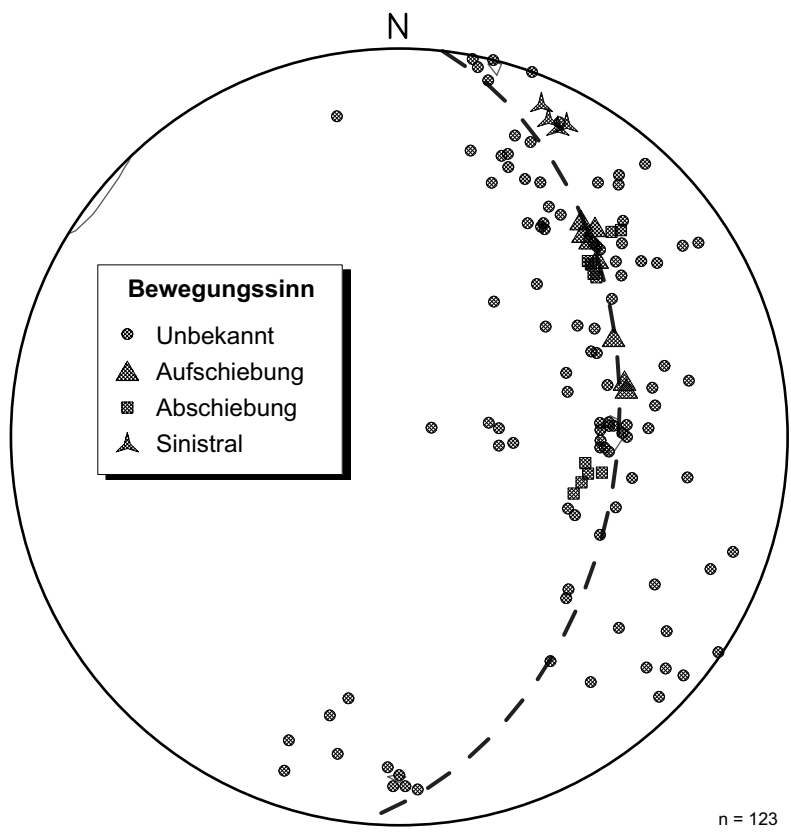

Abbildung 17. Harnischlineare. Der Großkreis repräsentiert die durchschnittliche Lage der Störungsfläche auf der die Harnischlineare liegen. Daten von Meier (2008). Schmidt'sches Netz, untere Halbkugel.

ellen Störungsflächen zum Teil eine Klein- und Großkreisverteilung besitzen, bedeutet, dass die Störungsfläche helicoidal ist. Das ist die typische Form einer Blattverschiebung, die in der Tiefe in kompetentem Gestein eine ebene Fläche besitzt und nach Aufsteigen in weniger kompetente Gesteine eine gebogene Form annimmt (Sagan und Hart 2004; s. Abb. 19). Weitere Informationen lieferte ein Laser-Scan dieser Störungsfläche (Tanner et al., dieser Band).

Meine Interpretation dieser Struktur ist, dass es sich um eine positive ,Flower'-Struktur über

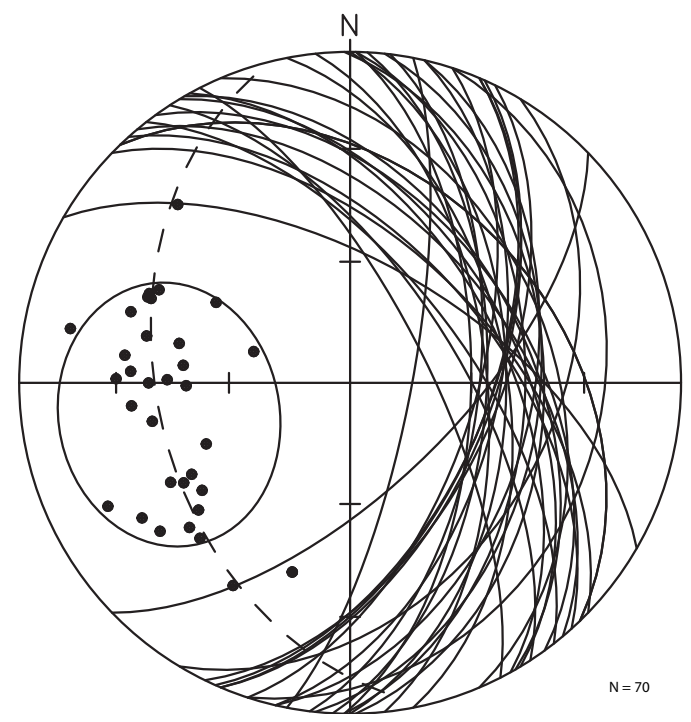

Abbildung 18. Die Störungsfläche als Großkreise und Flächenpole. Verteilungsmuster deutet auf eine helikoidale Form der Störungsfläche hin. Daten von Meier (2008). Schmidt'sches Netz, untere Halbkugel.

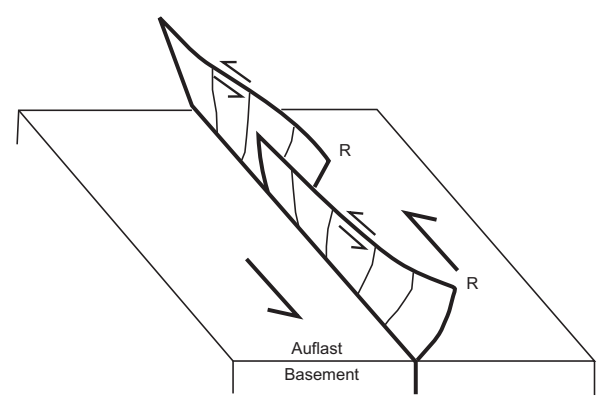

Abbildung 19. Helicoidale Störungsflächen (aus Sagan und Hart 2004); R - synthetische sinistrale Riedel-Scherflächen über einer sinistralen Hauptstörung. 


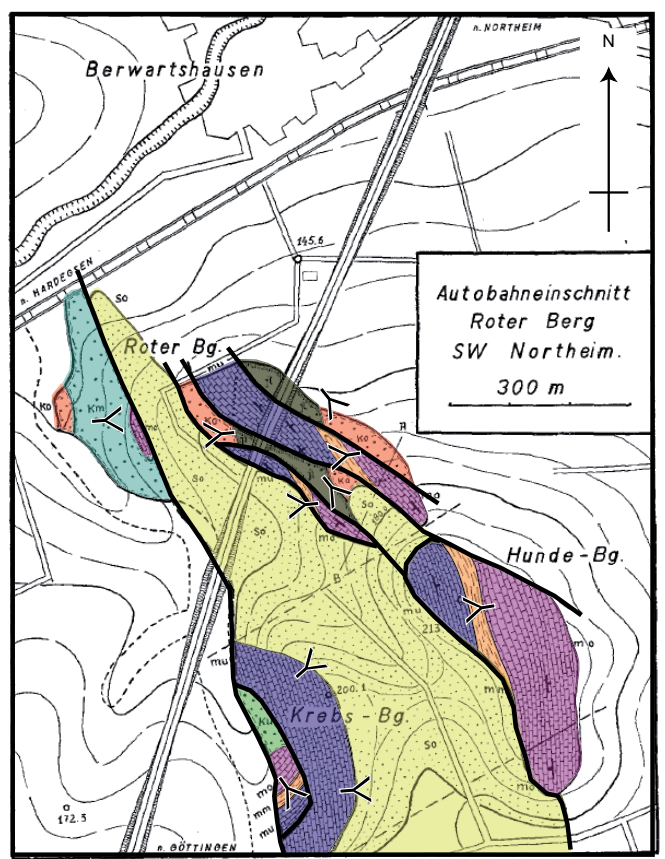

Abbildung 20. Geologische Karte des Ahlsburg Lineaments, SW' von Hillerse (geändert, ergänzt, und koloriert nach Abb. 5, Wunderlich 1957). Dicke Linien sind Blattverschiebungen, die Spitzen der , $Y$ ' zeigen in Richtung der jüngeren Schichten; so - gelb, mu - dunkelblau, mm — orange, mo - lila, ku — grün, km — türkis, ko - Hellrot, j - grau.

einem sinistralen Blattverschiebungssystem handelt. Das erklärt, warum die Falte bivergente Aufschiebungen enthält und die aufgeschlossene Störungsfläche ein solches Spektrum von Harnischen zeigt. Als auf den gewölbten Störungen Bewegungen stattfanden, bewirkten manche Krümmungen ,dehnende' (engl. releasing bends) und andere ,einengende' (engl. confining bends) Verformungen. Im ersten Fall wurde das Hangende nicht nur seitlich bewegt, sondern auch abgeschoben. Im zweiten Fall wurde das Hangende aufgeschoben. Deshalb bewegte sich das Hangende nacheinander in fast alle möglichen Richtungen, was auch durch die starke Änderung der Richtung von manchen Harnischen zu erkennen ist.

Diese Interpretation als Blattverschiebung wird unterstützt durch die Kartierung des Ahlsburg Lineaments (Wunderlich 1957; Abb. 20), etwa $1 \mathrm{~km}$ nördlich vom Steinbruch Elvese (Abb. 14). Obwohl dieses Gebiet stark gestört ist, zeigen die Störungsblöcke ein einheitliches Bild. Die jeweils jüngeren Schichten liegen weiter entfernt von der
Mitte der Achse (also Verjüngung nach SW oder NE) oder weiter im Nordwesten. Dies bedeutet, dass es sich ursprünglich um eine Sattel-Struktur handelt, deren Faltenachse nach NW abtaucht. In Abbildung 20 kann man auch sehen, dass sich manche Störungsblöcke um ca. $90^{\circ}$ gedreht haben, was nur durch Seitenverschiebungen erklärbar ist.

\section{Aufschluß 4: Steinbruch Hardegsen I}

\section{Lage R: ${ }^{35} 58302 \mathrm{H:}{ }^{57} 24603$}

Karte TK25, 4325 - Nörten-Hardenberg

Der Steinbruch Hardegsen I liegt etwa $200 \mathrm{~m} \mathrm{E'}$ von der Stadt Hardegsen, und etwa $700 \mathrm{~m} \mathrm{SE}$ ' vom Steinbruch Hardegsen II (Abb. 21).

Auf einer N-S Wand sind eine Reihe von aufrechten Falten aufgeschlossen (Abb. 22). Die Faltenachsen haben ihre höchste Belegungsdichte bei 090/35, aber viele weichen mit bis zu $90^{\circ}$ von dieser Richtung ab. Diese Variation zeigt sich schon innerhalb von 2-3 m Abstand zwischen übereinander liegenden Faltenachsen. Diese breite Variation in der Faltenachsenorientierung spiegelt sich ebenfalls in der Raumlage der Faltenachsenflächen wider.

Auffällig ist auch, dass die Falten disharmonisch sind und eine sehr kleine laterale Ausbreitung besitzen. Schon in der N-S Wand stirbt die Faltung in beiden Richtungen aus (Abb. 22).

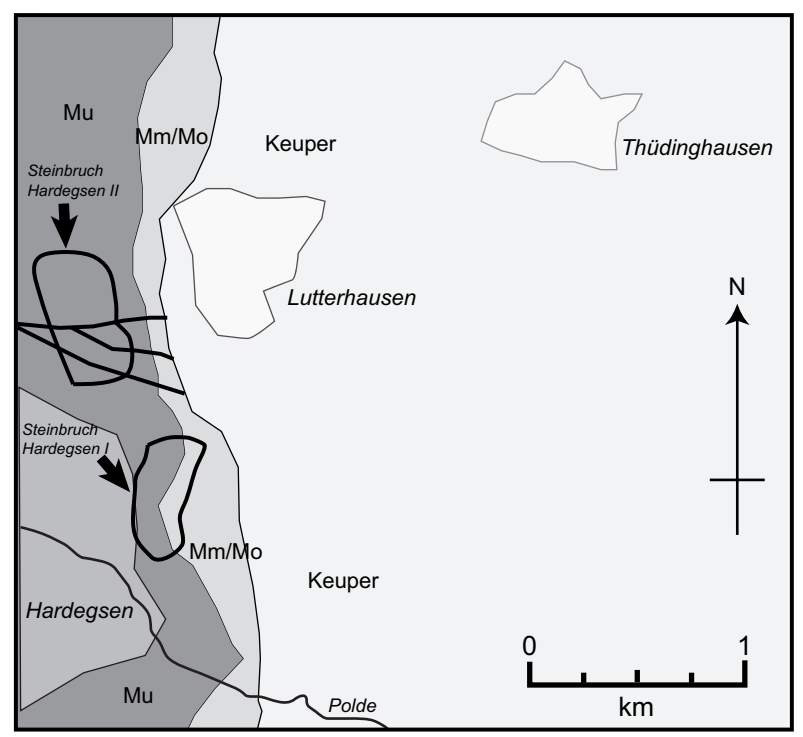

Abbildung 21. Vereinfachte geologische Karte des Gebietes um die Steinbrüche Hardegsen I und II (nach GK25 4325 Nörten-Hardenberg). 


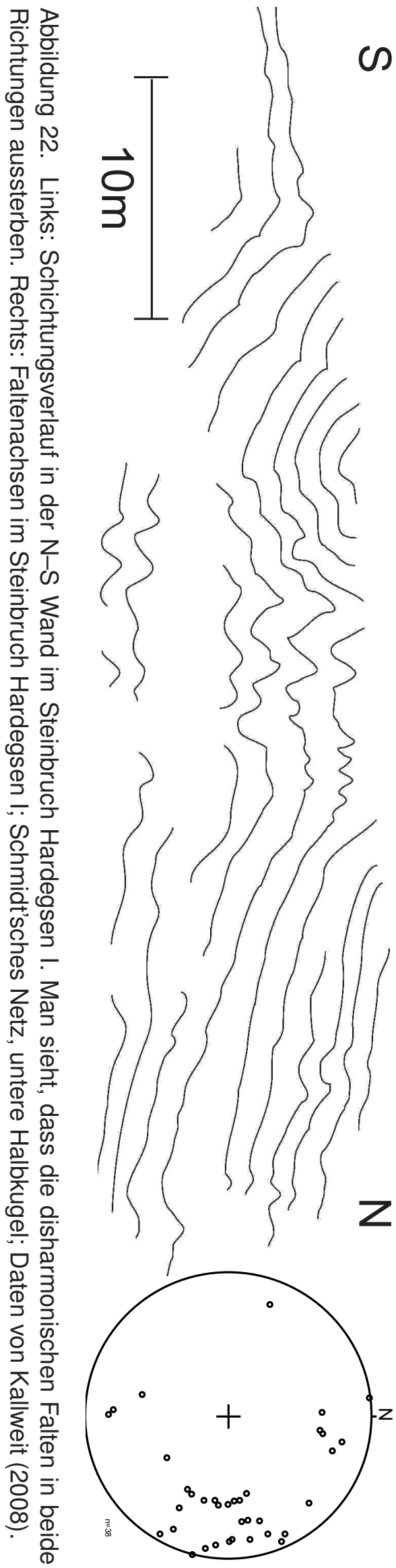

Man sieht auch, dass an der südlichen Wand des Steinbruchs keine Faltung vorhanden ist. Wunderlich (1957) beschreibt, dass hier auch im Jahre 1950, bevor weitere 5-10 m abgetragen wurden, ebenfalls keine Hinweise auf Falten vorhanden waren. Deshalb sind diese Falten offensichtlich sehr lokal begrenzt, mit einer etwa runden oder elliptischen Ausbreitungszone.

Ich vermute, dass es sich hier um eine Push-Up-Struktur an einem einengenden Übergang zwischen zwei Blattverschiebungen handelt (Abb. 23). Dies könnte das breite Spektrum der Faltenachsenraumlage und die begrenzte Ausbreitung der Falten erklären. Dieser Mechanismus ist mittlerweile gut bekannt (z.B. Segall \& Pollard 1980, 1983; Brankman \& Aydin 2004). Leider gibt

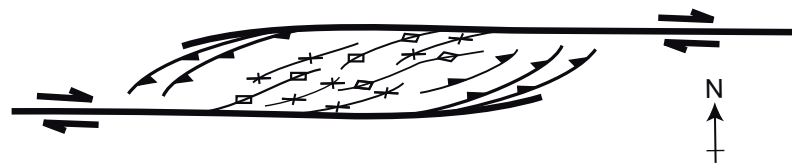

Abbildung 23. Gegen den Uhrzeigersinn versetzte dextrale Blattverschiebungen. Im Interaktionsbereich der Störungen wird eine ,Push-Up'Struktur produziert. Die Spuren von Faltenachsen oder Überschiebungen sind eingezeichnet. Die Kennzeichen einer solchen Struktur sind eine sehr begrenzte Ausbreitung und kurvilineare Faltenachsen und Aufschiebungen, die ein Richtungsspektrum von über $90^{\circ}$ besitzen können (nach Twiss und Moores 1992).

es in diesem Steinbruch keinen direkten Hinweis auf entsprechende Störungen, was die Vermutung nahe legt, dass die Störungen in tieferen Niveaus oder außerhalb des Steinbruchs verlaufen.

\section{Aufschluß 5: Steinbruch Hardegsen II}

Lage: R: ${ }^{35} 57982 ~ H:{ }^{57} 25283$ Karte TK25, 4325 - Nörten-Hardenberg

Der Steinbruch Hardegsen II liegt etwa $1 \mathrm{~km}$ SW' von Lutterhausen (Abb. 21). Er schliesst hauptsächlich den unteren Muschelkalk auf, der flach nach E einfällt. Durch die Mitte des Steinbruchs verläuft eine Blattverschiebungszone in E-W-Richtung, innerhalb derer sich Röt $\left(\mathrm{sO}_{2-4}\right)$ befindet (Abb. 24). Im Gegensatz zu der Blattverschiebung im Steinbruch Papenberg, ist in diesem Röt-Material noch eine deutliche Schichtung zu 


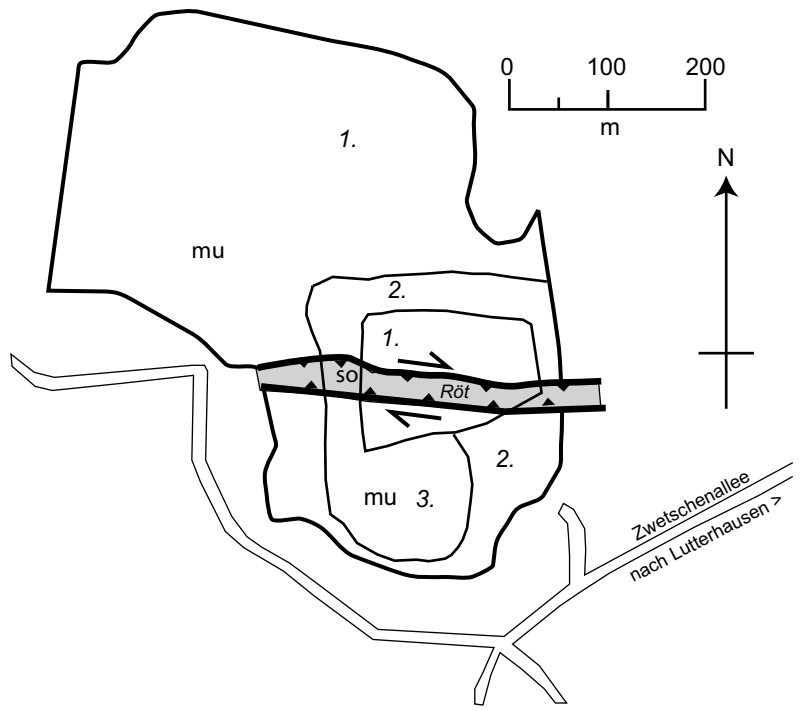

Abbildung 24. Skizze des Steinbruchs Hardegsen II. Die Ziffern bezeichnen die Stufen im Steinbruch.

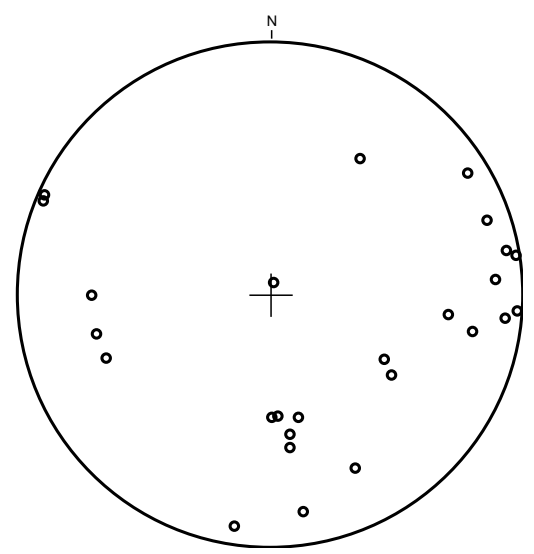

Abbildung 25. Faltenachsen innerhalb der RötMaterial in der Blattverschiebung, Steinbruch Hardegsen II.

erkennen. Sie ist um sub-horizontale Achsen gefaltet, die eine breite Richtungsstreuung besitzen (Abb. 25). Vergleicht man diese Achsen mit denen in Abb. 22, so gibt es sehr gute Übereinstimmung. In diesem Fall bin ich der Meinung, dass Falten zunächst dextral-transpressiv in den Muschelkalk eingepresst waren (positive Flower-Struktur) und subsequent dextral geschert wurden.

Die Frage ist, wie solche Blöcke (in diesem Fall Röt 2-4) entlang einer Störungsfläche aufsteigen können, ohne sichtbaren vertikalen Versatz des angrenzenden Muschelkalks. Vermutlich ist der Aufstieg durch die Steinsalz und GipsEinlagerungen im Röt erleichtert worden. Wenn das Material gelöst wird, nach oben transportiert und wieder abgelagert wird, wie dies für den Steinbruch Papenberg vermutet wird, ist dieser Prozeß ohne weiteres vorstellbar. Hier jedoch ist der Rötblock über $10 \mathrm{~m}$ breit, noch deutlich geschichtet, und kaum deformiert (offene Faltung). Im Eingangsbereich dieses Aufschlusses befinden sich sekundäre Dolomitlagen am Kontakt zum Röt, was auf Fluidmigrationen hindeutet (siehe auch Bloch et al., dieser Band, für weitere Diskussionen).

\section{Danksagung}

Ich möchte mich bei den Betreibern der Steinbrüche Hardegsen (Biomasse-VerwertungsGesellschaft) und Elvese (Südniedersächsische Kalksteinwerke $\mathrm{GmbH}$ \& Co. KG) für die Erlaubnis die Steinbrüche zu betreten, bedanken.

\section{Literatur}

Arp G., Hoffmann V.-E., Seppelt S., Riegel W. 2004. Trias und Jura von Göttingen und Umgebung, 74. Jahrestagung der Paläontologischen Gesellschaft, 2.-8.10.2004, Exkursion 6: 147192, Universitätsdrucke.

Bloch, N.W, Tanner, D.C., Paul, J. (dieser Band). Störungskörper in einem Seitenverschiebungssystem bei Hardegsen.

Brankman, C.M., Aydin, A. 2004. Uplift and contractional deformation along a segmented strike-slip fault system: the Gargano Promontory, southern Italy. Journal of Structural Geology, 26, 807-824.

Fabian H.-J. 1957. Die Bohrung, ,Northeim 1' - Ergebnisse eines regionalgeologisch interessanten Aufschlusses am Leinetalgraben, Neues Jahrbuch für Geologie und Paläontologie, Abhandlungen 105, 113-122.

Grupe O. 1909. Über die Zechsteinformation und ihr Salzlager im Untergrunde des hannoverschen Eichsfeldes und angrenzenden Leinegebietes nach den neueren Bohrergebnissen, Zeitschrift für praktische Geologie 17, 185-205.

Jordan, H. 1984. Erläuterungen zur geologischen Karte von Niedersachsen 1:25000, Blatt Nr. 4325 Nörten-Hardenberg. 148pp, 1 Map.

Kallweit, W. 2008. Geologische Kartierung am Westrand des Leinetalgrabens zwischen Har- 
degsen und Moringen. Diplomkartierung, Universität Göttingen (unpubl.).

Kockel, F. 2003. Inversion structures in Central Europe - expressions and reasons, an open discussion, Geologie en Mijnbouw, 82/4, 367382.

Lachmann, R., 1917. Ekzeme und Tektonik. Centralbl. f. Min., 414-426.

Lang, H.O. 1880. Über den Gebirgsbau des Leinetals bei Göttingen. Z. dt. geol. Ges. 32(4), 799-806.

Lotze, F. 1932a. Der Südteil des Göttinger Leinetalgrabens und der Eichenberger Grabenknoten. Abhandlung der Preußischen Geologischen Landesanstalt, Neue Folgen, Heft 139, 6-47.

Lotze, F. 1932b. Überschiebungserscheinungen auf der Nordostseite der Ahlsburgachse bei Hillerse (südwestlich von Northeim). Abhandlung der Preußischen Geologischen Landesanstalt, Neue Folgen 139, 87-99.

Mazur, S., Scheck-Wenderoth, M., Krzywiec, P. 2005. Different modes of the Late Cretaceous Early Tertiary inversion in the North German and Polish basins., International Journal of Earth Sciences, 94 (5-6), 782-798.

Meier, S. 2008. Geologische Kartierung im Leinetalgraben südlich der Ortschaft Hillerse. Unpubl. Diplomkartierung, Univ. Göttingen, Göttingen.

Meischner, D. 2002. Der Leinetalgraben - eine geologische Legende; populärwissenschaftlicher Vortrag am Geowissenschaftlichen Zentrum der Georg-Au-gust-Universität Göttingen. Unveröffentlichtes Skript (unpubl.).

Meyer, R.H. 2000. Geologische Untersuchungen auf der Burg Plesse 1983-92, in: Moritz T.: Eine feste Burg - die Plesse, Interdisziplinäre Burgenforschung 1, 245-262.

Müller M., Boigk H., Füchtbauer H., Malzahn E., Eckardt F.J., Mattiat B., 1961. Schichtenverzeichnis und geologischer Bericht über die Aufschlussbohrung Dransfeld 1. Niedersächsisches Landesamt für Bodenforschung, Bohrakte 33198.

Otto, V. 2003. Inversion-related features along the southeastern margin of the North German Basin (Elbe Fault System). Tectonophysics, 373, 107-123.

Patriciu, V. 1930. Geologische und geophyiskalische (radioaktive) Untersuchungen am Westrande des Göttinger Leinetalgrabens. Abhandlungen der Preußischen Geologischen Lan- desanstalt Neue Folge 116, 163-194.

Paul, J. 1982. Der Untere Buntsandstein im Germanischen Becken. Geologische Rundschau 71, 795-811.

Paul, J. 1999. Fazies und Sedimentstrukturen des Buntsandsteins. In: Hauschke, N. \& Wilde, V. (Hrsg.) Trias - Eine ganz andere Welt. 105114; München (Pfeil-Verlag).

Paul, J., Franke, W. 1977. Sedimentologie einer Transgression: Die Röt/Muschelkalk-Grenze bei Göttingen. Neues Jahrbuch für Geologie und Paläontologie, Monatshefte 3, 148-177.

Ramsay, J.G., Huber, M.I. 1997. The Techniques of Modern Structural Geology: Folds and Fractures. Wiley.

Reyer, D. 2008. Geologische Kartierung im Bereich der östlichen Grabenrandstörung des Leinetalgrabens südlich Sudheim. Unpubl. Diplomkartierung, Universität Göttingen.

Ritzkowski, S. 1999. Der Göttinger Leine-Graben im Paläogen (südliches Niedersachsen). Neues Jahrbuch für Geologie und Paläontologie, Abhandlungen 214 (1), 237-256.

Sagan, J., Hart, B. 2004. Seismic and Structural Analysis of a Trenton-Black River Hydrothermal Dolomite Reservoir. AAPG Search and Discovery Article \# 40129.

Segall, P., Pollard, D.D. 1980. Mechanics of discontinuous faults. Journal of Geophysical Research 85, 4337-4350.

Segall, P., Pollard, D.D. 1983. Nucleation and growth of strike-slip faults in granite. Journal of Geophysical Research 88, 555-568.

Stille H., Lotze F. 1933. Erläuterungen zur Geologischen Übersichtkarte der Umgebung von Göttingen, Preus. Geol. Landesamt, 1-67.

Tanner, D.C., Prüfer, S., Kuhn, D., Krawczyk, C. (dieser Band) 3D-Störungsmorphologie bestimmt durch LIDAR-Messungen.

Twiss, R.J., Moores, E.M. 1992. Structural Geology, 532pp. Palgrave Macmillan.

Wunderlich, H.-G. 1955. Jüngste Tektonik im Gebiet des Leinetalgrabens. Geologische Rundschau 43, 78-93.

Wunderlich, H.-G. 1957. Tektogenese des Leinetalgrabens und seiner Randschollen. Geologische Rundschau, 46, 372-413.

Wunderlich, H.-G. 1966. Ausweitungen und Einengungen an saxonischen Bauformen Südniedersachsens. Zeitschrift der deutschen Geologischen Gesellschaft 116, 683-695. 


\title{
Ebelhof-Abschiebungszone, Papenberg-extensionale Antikline und andere Merkwürdigkeiten in den südlichen ,Hausbergen' des Göttinger Geozentrums - der Luttertal Faltenzug
}

\author{
Till Heinrichs (GZG Göttingen), mit einen Beitrag von Thomas Löffler (Göttingen)
}

Zusammenfassung-Vom Südhang des Luttertals zwischen Klausberg und Papenberg wird ein Zug NW-SE streichender Falten und Störungen beschrieben. Er liegt in der NW-Fortsetzung des Herberhäuser Grabens und mit diesem zusammen wird er als herzynische Achse Papenberg-Herberhausen aufgefaßt. Diese Achse setzt sich am Papenberg über die Relay-Abschiebung zwischen Northeimer und Göttinger Sprung in den Leinegraben fort und reicht vermutlich bis zur Stumpfen Eiche. Am Papenberg werden die herzynischen Strukturen durch SW- und WSW- gerichtete Abschiebungen durchsetzt. Teilweise übernehmen sie vermutlich die Funktion der Relay-Abschiebung.

Das jüngste Störungsmuster besteht aus etwa E-W streichenden Störungen, die nicht nur die Papenberg Antikline zerlegen, sondern an anderen Orten auch die Relay-Störung versetzen. Am Papenberg-Südhang wird aus Falten und bruchhaftem Versatz in der dortigen breiten E-W-laufenden Rote-Erde-Störungsgszone ein Abschiebungssinn nach SSW abgeleitet, was auf eine geringe dextrale Komponente hinweisen könnte.

Das Strukturensemble der herzynischen Achse Papenberg-Herberhausen aus Antiklinen und Gräben zeigt wahrscheinlich eine spätkretazisch reaktivierte Permische Störungszone an, die für eine tiefengeothermische Erkundung im prä-Zechstein von Interesse sein könnte.

\begin{abstract}
The Luttertal Fold Train, situated between Papenberg and Klausberg, is shown to consist of an assemblage of folds and faults with NW-SE, eg. Hercynian, grain. It forms the NW extension of the Herberhausen Graben and anticline, and so both are suggested to jointly form the Papenberg-Herberhausen Hercynian Axis. Near the Papenberg the normal fault that relays between two master faults on the eastern rim of the Leinegraben appears to be crossed by the hercynian axis, which may extend even further into the graben, up to Stumpfe Eiche. It is probably due to this interference that late normal faults with SW to WSW slip intersect the older Papenberg anticline of a strictly Hercynian trend. These faults partly take on the function of relay normal faults.

The youngest fault family consists of roughly E-W trending normal faults. They locally offset the relay fault. On Papenberg S-slope they form a wide normal fault zone associated with small WNW trending grabens suggesting a small dextral slip component.

The structural assemblage of the Hercynian axis Papenberg-Herberhausen with anticlines, small thrusts and grabens indicates a Permian fault in the pre-Zechstein basement that was transpressively reactivated in the lateCretaceous to early Paleogene. It may be of interest for deep geothermal exploration in pre-Zechstein strata.
\end{abstract}

Schlüsselworte-herzynische Richtung, reaktivierte Störung, Untere Muschelkalk Gruppe, Untere Keuper Gruppe

\section{Problemstellung}

- Till Heinrichs - Abt. Angewandte Geologie, Geowissenschaftliches Zentrum der Universität Göttingen, Goldschmidtstr. 3, D-37077 Göttingen. theinri@gwdg.de

- Thomas Löffler - Leonard-Nelson-Str.31, 37073 Göttingen

Der Ostrand des Leinetalgrabens wird dominiert von NNE verlaufenden Abschiebungen, die z.B. in Göttingen oberen Keuper oder sogar Schwarzschiefer des Lias neben Mittleren Muschelkalk brachten, also die westliche Hangendscholle um mehr als $0,5 \mathrm{~km}$ absenkten. Diese Hauptstörungen sind in einer N-S-Zone en-echelon angeordnet, 


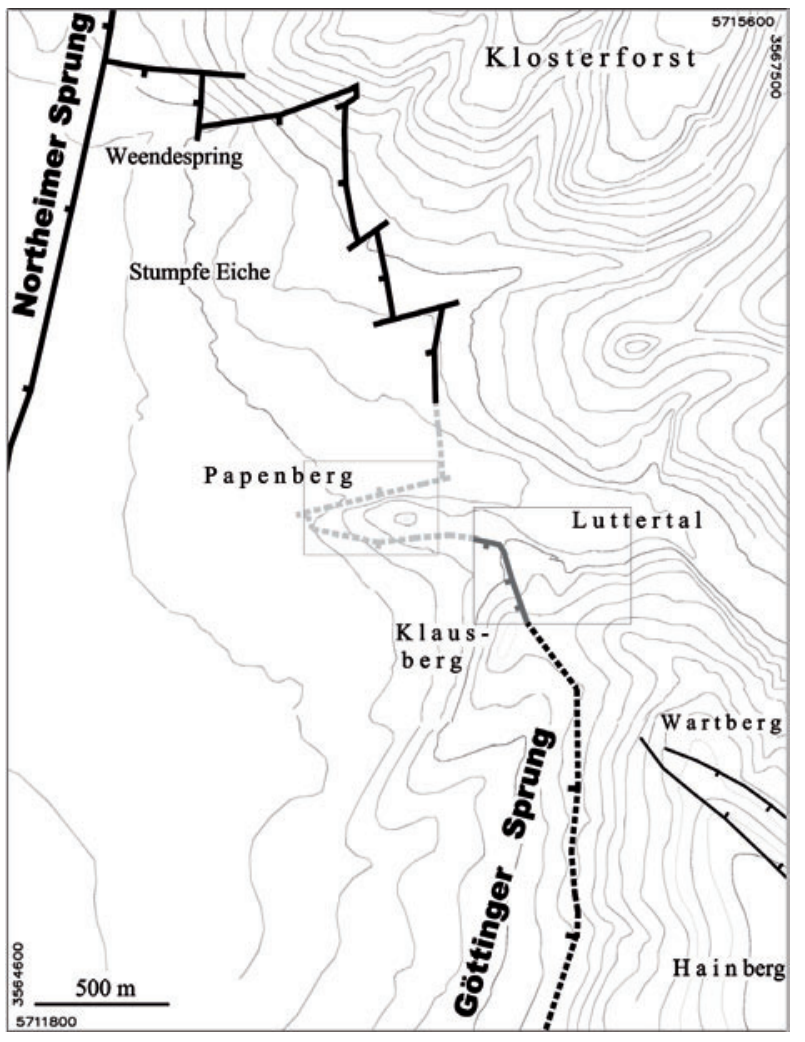

Abbildung 1. Übersicht Relay zwischen Northeimer- und Göttinger Sprung: RelayStörung trennt in der Regel $\mathrm{mm}$ im NE von $\mathrm{km}$ im SW; der Sporn des Papenberges ist mu2 bis ku umgeben von km; Störungsverlauf nördlich des Luttertals weitgehend belegt durch dichte Flachbohrungen nach Meischner (1980); Verlauf am Klausberg nach Stille (1932) und eigenen Aufnahmen temporärer Aufschlüsse; südlich davon unsicher; Göttinger Sprung nach Stille, am Hainberg durch quartäre Rutschungen verdeckt; S des Wartberges: NW-Ende des Herberhäuser Graben; Kästen : Lage der Detailaufnahmen. Isohypsen $10 \mathrm{~m}$-Intervall: 150 m ü. NN im SW, 320 m im NE.

d.h. sie verspringen linksseitig, wie im Norden Göttingens zwischen Weende und Klausberg um etwa $1,5 \mathrm{~km}$, der von Norden hereinziehende Northeimer Sprung gegen den östlicheren Göttinger Sprung (Stille 1928,1932; Stille \& Lotze 1933 und spätere).

Solche verspringenden Abschiebungen sind von vielen Grabensystemen bekannt. In Analogie zu diesen würde man als Transferstruktur im einfachsten Fall eine Rampe als Relay zwischen zwei ausklingenden Hauptstörungen erwarten (weiche Ver- bindung; z.B. Delicate Arch Relay, Utah; Fossen et al. 2005; Kenya-Rift: Sambu-Kirikiti System im Nguruman Escarpment, Heinrichs 2001:77). In der nächsten Entwicklungsstufe wird eine Rampe durch eine oder mehrere fest verbundene Störungen überprägt, die von einer zur anderen Hauptstörung gerade verlaufen oder auch konkav oder konvex zum Graben gekrümmt sind (z.B. Kenya Rift: Saimo-Kito Pass Fault System, Chapman et al. 1978; Hold with Hope Relay, Grönland, Peacock et al. 2000).

Eine Rampe, überprägt durch eine bogenförmige Relay-Abschiebung nach $\mathrm{S}$ und $\mathrm{W}$, mit fester Verbindung zum Northeimer Sprung W des Weendespring kann man ansatzweise nördlich des Luttertals realisiert sehen (Abb. 1), wo die Muschelkalkplatte vom Klosterforst mit 30 bis 40 $\mathrm{m} \mathrm{km}^{-1}$ nach SW absinkt, um dann mit der durch kleine, jüngere $\mathrm{E}-\mathrm{W}$-Sprünge gegliederten Relay-Abschiebung an den mittleren Keuper der Hangendscholle zu grenzen (Meischner 1980). Aber die weitere südostwärtige Verbindung des Relays mit dem Göttinger Sprung wird unterbrochen, morphologisch und, wie im Folgenden gezeigt wird auch strukturell, durch das hier E-W verlaufende Luttertal unbekannter Struktur, und durch einen WNW-ESE-Riegel von Hügeln aus unterem Muschelkalk, der sich südlich der Lutter vom Sattel nördlich des Klausberges bis zum Papenberg zieht (Stille 1932) und -entlang nicht ganz gesicherter Störungen- in die Hangendscholle hineinragt. Ziel des Beitrags ist daher, erstmals die Detail-Struktur dieses Riegels, soweit durch Tages-Aufschlüsse zugänglich, zu untersuchen und den möglichen Zusammenhang mit der RelayStruktur im Kontext der saxonischen Tektonik darzustellen.

\section{Methodische Vorbemerkung}

Alle Angaben zur Orientierung der Strukturelemente sind in Clar-Notation, die Deklination korrigiert nach NGDC magnetischem Modell. Diagramme der Orientierungsdaten wurden mit dem Programm Stereo-32 von Klaus Roeller und Claudia Trepmann, Ruhr-Universität erstellt, wobei die Gürtel und Achsen als ein Eigenvektor berechnet werden. Einige Daten wurden in einer eigenen Variante des SCAT-Plots (Bengtson 1981) dargestellt, um die starke Streuung der Orientierungsmessungen in ihrer räumlichen Variation entlang von Profilen zu erfassen. In Verbindung 
mit einem Stereogramm lässt sich dann der strukturbedingte Teil von dem durch Unzulänglichkeiten der sedimentären Vorzeichnung, oberflächliche gravitative Verstellung oder Überprägung erzeugten Teil der Streuung besser unterscheiden.

\section{Daten}

\subsection{Profil Klausberg-Hohlweg Nikolausber- ger Weg-Hoffmanshof}

Im Profil HOL stehen nach Stille (1932) Schaumkalk,zone' und höherer Unterer Muschelkalk (Top des mu, Jena Fm.) an. Im fast kontinuierlich aufgeschlossenen Westteil des Profils (Hohlweg; HOL-A) sind es vermutlich mittlere Teile der Schaumkalke. Den basalen Gelbkalken entsprechende Gesteine wurden nur östlich des Hohlweges und oberhalb der Aufschlüsse HOL-14 und -15 als gehäufte Lesesteine gefunden (GKk, Karte Abb. 4), so daß diese Aufschlüsse selbst wohl in Wellenkalk-3 (Wk3) stehen.

Im W, d.h. in Nähe der Hauptabschiebung, erstreckt sich über ca. 70 Profilmeter eine Domäne mit E- und NE-Fallen. Sie gruppiert mit den folgenden SW fallenden Schichten in einer flachen Mulde um eine NNW-SSE-streichende Achse. Der SW-fallende Flügel endet an der ersten der folgenden Flexuren. Weiter nach E folgt die Domäne mit dominantem SW-Fallen, in der weitere Flexuren mit Amplituden von einigen Metern beobachtet oder angenommen werden können (Abb.2 und 4). Die Achsenflächen der Flexuren I und II fallen gegeneinander ein, sodaß man beide zusammen mit dem schwach aufgewölbten Zwischenbereich als unsymmetrische Antikline mit mehreren Scheitellinien auffassen kann. Die Struktur belegt ein komplexes Deformations-Feld mit moderater Einengung in W-E- bis WNW-ESE-Richtung.

\subsection{Aufgelassener Steinbruch Geobotani- scher Garten Süd}

Der Steinbruch zeigt an seinem SE-Ende ein etwa $16 \mathrm{~m}$ langes Querprofil, vom SE-Flügel eines Sattels, über dessen verstürzten First, in eine vollständige offene Mulde. Nahe dem Sattelkern, am Fuß des Südflügels, sind in diesem Profil etwa $1 \mathrm{~m}$ mächtige Gelbkalke aufgeschlossen, die als Basis der Schaumkalk,zone' anzusehen sind. Im weiteren Verlauf des Stoßes nach NW stehen über ca. $60 \mathrm{~m}$ zunehmend höhere Teile des Schaumkalkbereiches an, die die streichende Fortsetzung des Sattel-Südflügels bilden. Am NW-Ende des
Steinbruchs zeigt sich dieser Sattelfügel in zwei etwa 6-8 m langen querschlägigen Stößen, d.h. Klippen mit einheitlichem SW-Fallen.

Alle Orientierungsdaten, sowohl die der Mulde als auch die der Wellung des Sattelflügels ordnen sich in zylindrischen Gürteln um nahezu gleiche NW-SE-Achsen an (Abb. 3). Eine Ausnahme bilden nur die Gelbkalke nahe dem nicht aufgeschlossenen Kern des Sattels. Sie sind wahrscheinlich gravitativ verstellt (Abb. 3; dolostones). Die Orientierungsdaten wurden daher in einem SCAT-Plot zusammengefasst, in dem die Daten vom südwestlichen Sattelflügel entsprechend ihrer Position zu einer extrapolierten mittleren Faltenachse in das NE-SW-Profil projiziert sind.

Da die Gelbkalke vom Sattelkern sich in der nordöstlich anschließenden Mulde, soweit aufgeschlossen, nicht fortsetzen und da charakteristische Bänke zwischen den SW-Flügeln des Sattels und der Mulde versetzt scheinen, wird eine steile Aufschiebung im Sattelkern mit Versatz im Meterbereich vermutet. Aber auch ohne diese Annahme zeigen die Falten eine SW-NE-Einengung an.

Der Aufschluss HOL-B (Karte Abb. 4), ein Subcrop unter flachgründigem Ranker, zeigt eine seichte Mulde allerdings mit unsicherer Raumlage der Achse, weil evtl. verdrifted, in vermutlich höherem Schaumkalkbereich. Die Grenze mu/mm wird nach ähnlichen oberflächennah stark verdrifteten Ausbissen von mu und mm etwa $50 \mathrm{~m}$ SW' der GBS-Sattelachse vermutet.

\subsection{Strukturkarte der HOL- und GBS- Aufschlüsse}

Aus den vereinzelten Aufschlüssen wurde unter Berücksichtigung der mittleren Orientierungsdaten die Grenzen des Schaumkalkbereichs konstruktiv ermittelt. Dies stellt sicher nur eine erste Näherung dar, da die stratigraphische Kontrolle hier gering und der tektonische Einfluss auf die Mächtigkeit (ca. $11 \mathrm{~m}$ ) unbekannt ist. Das Kartenbild der Schaumkalke zeigt, dass die RelayStörung ausgehend vom Göttinger Sprung sich nicht direkt nach $\mathrm{N}$ unter dem Luttertal fortsetzen kann, sondern an seinem N-Ende nach Westen umbiegt, etwa wie schon von Stille (1932) angegeben. Dieser Befund wird auch durch Kartierung der Leitbänke im Nordhang des Luttertales gestützt (Heinrichs, unpubl.), für die sich zwischen Faßberg, Bärenberg und Umgebung kein nennenswerter Versatz ergab. 


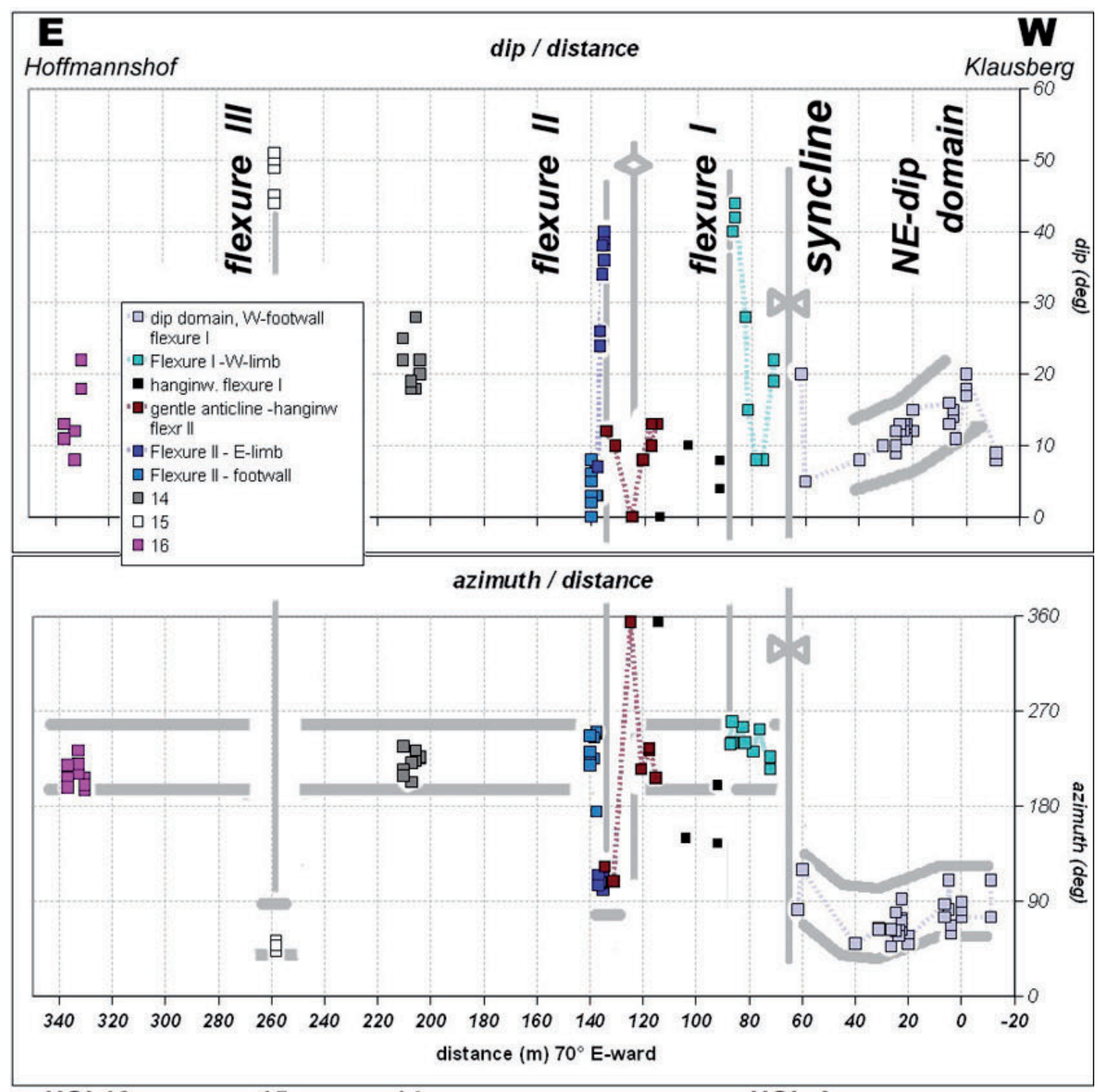

HOL16 $15 \quad 14 \quad$ - 15 ths 1010

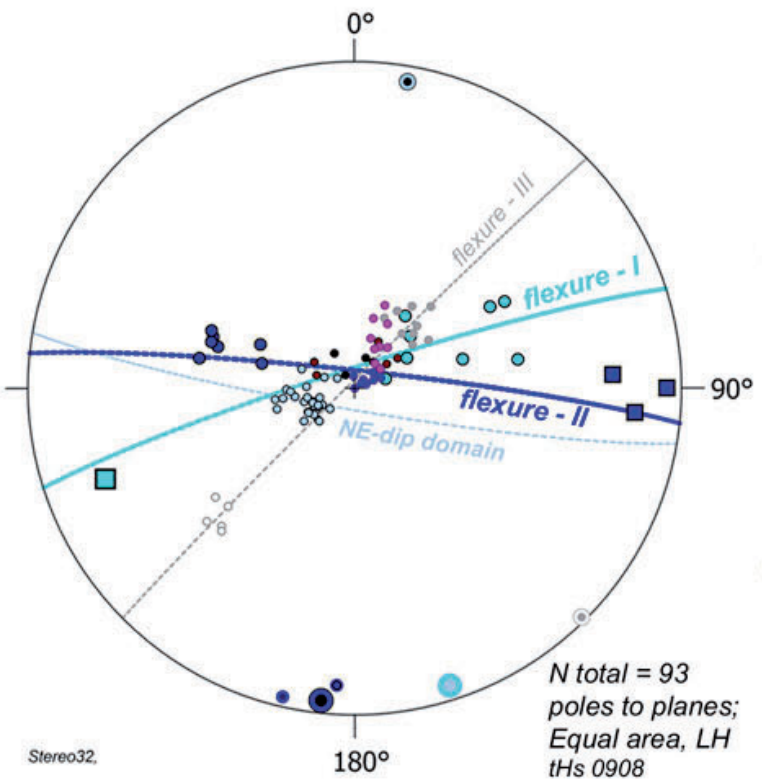

HOL

- ss (28), NE-dip domain

- girdle + axis [010/05]

- ss (9), W-limb

- ss (7), top E

- girdle + axis [162/05]

$\square$ axial plane (070/70)

- ss (4) gentle anticline, top

- ss (7), E-limb

- joints (3), (bc) = axial plane

- ss (11), footwall

- axis ss E-limb [193/03]

- axis E-limb + (bc) [184/10]

- girdle + axis, global [186/04]

- ss (9) HOL-14

ss (5) E-limb, HOL-15

girdle + axis ? [135/02]

Stereo32 tHs 0908

- ss (9) HOL-16

Abbildung 2. Profil Klausberg-Hohlweg Nikolausberger Weg- Hoffmannshof : SCAT-Plot und Stereogramm; zur Lage vergleiche Struktur-Karte (Abb.4). 


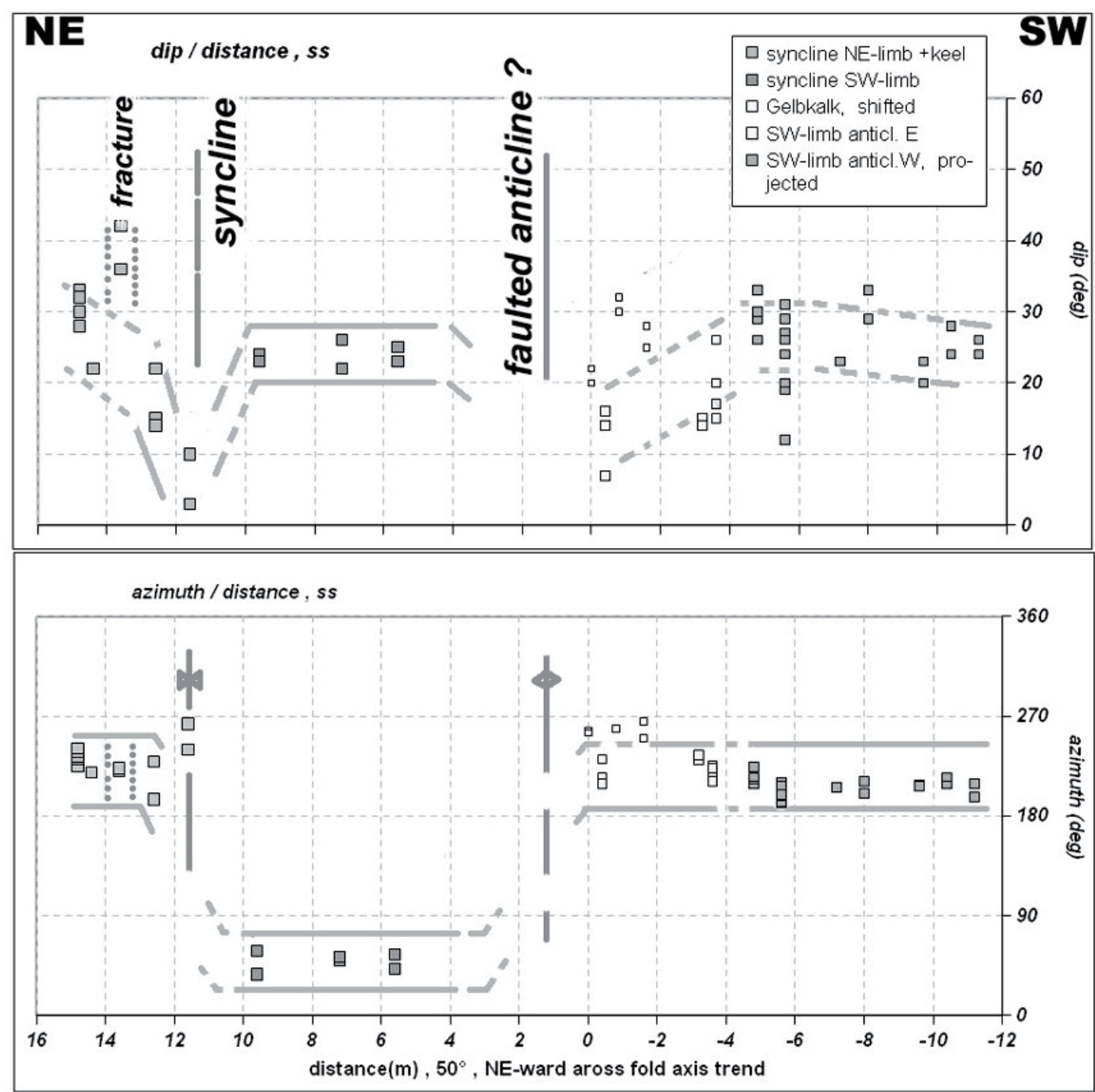

GBS-A Old quarry - Geobotanical Garden South tHs GZG 0910

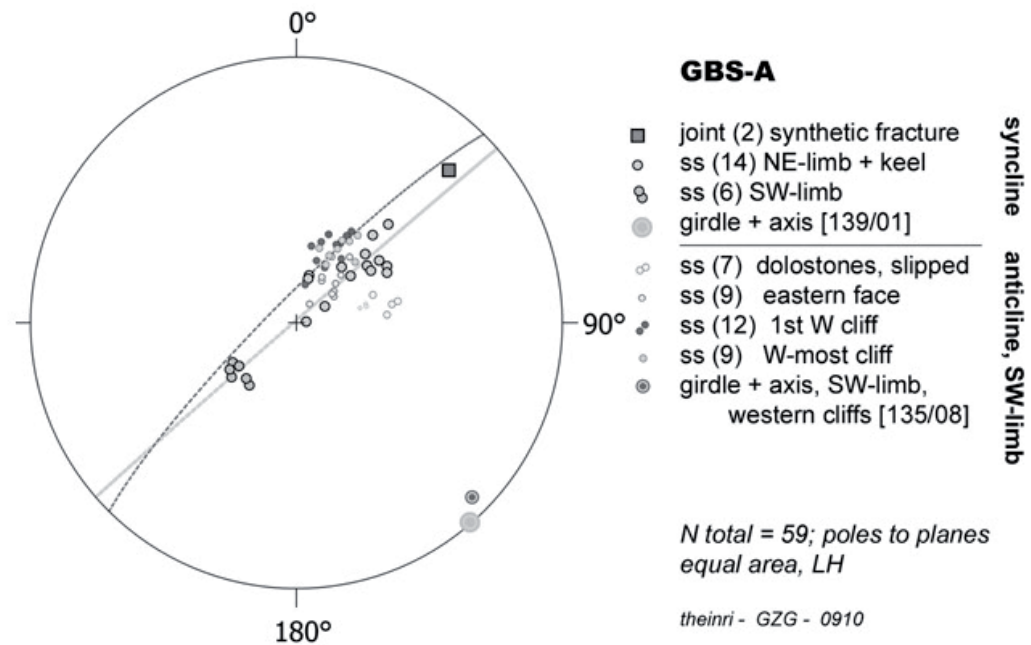

Abbildung 3. Aufgelassener Steinbruch Geobotanischer Garten-Süd; SCAT-Plot und Stereogramm 


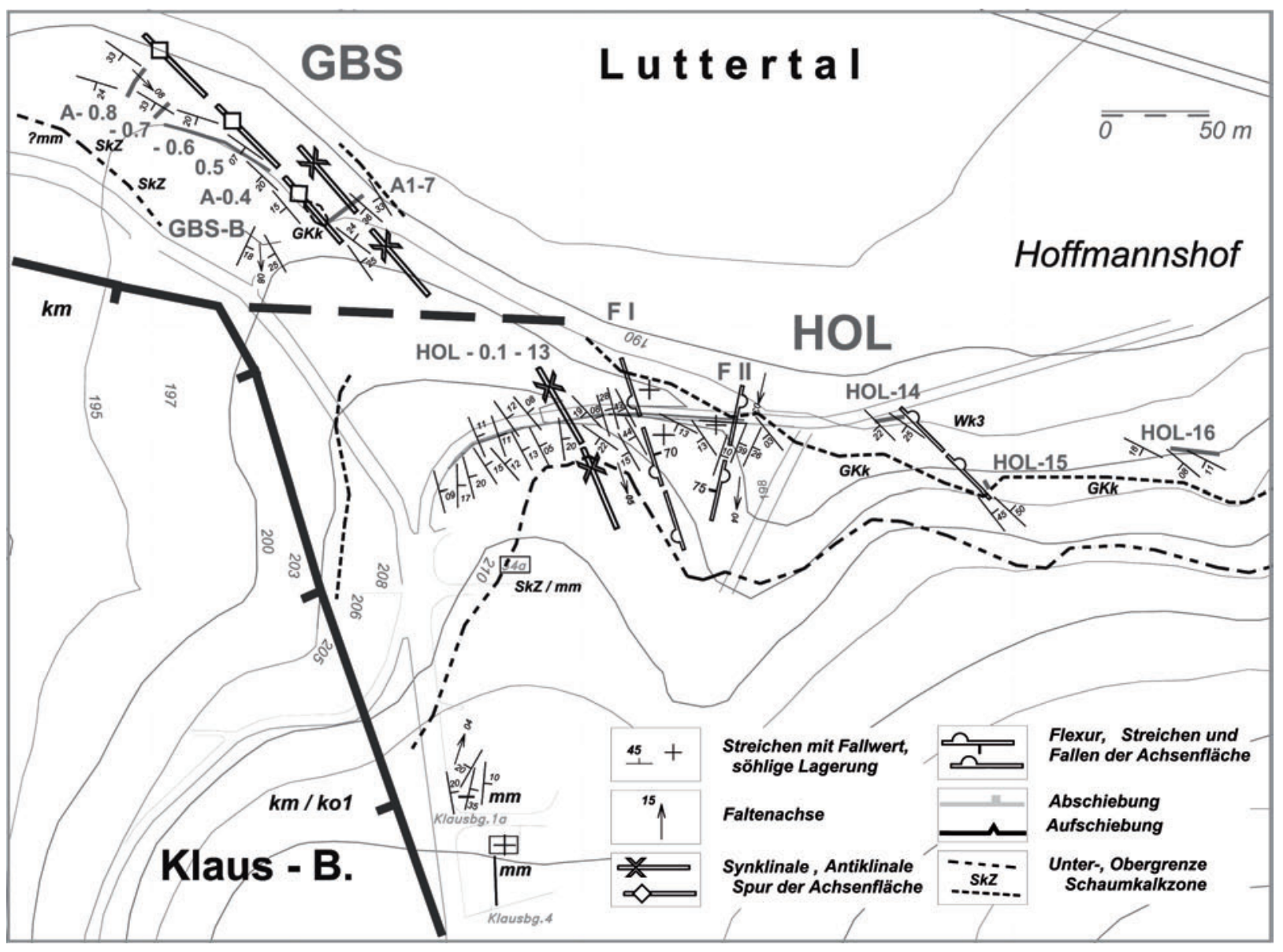

Abbildung 4. Strukturkarte Hoffmannshof-Geobotanischer Garten Süd; Schaumkalk-Bereich, konstruierte Basis = strichliert, konstruierter Top = strichpunktiert, s. Text; graue Majuskeln und Zahlen = Aufschlusscode ; kursiv = stratigraphische Beobachtung; hellgraue Linien=Lage der aufgenommenen Profile; Verlauf der Relay-Störung im N nach Stille (1932), im S gestützt durch eigene Aufnahme temporärer Aufschlüsse.

Das Umbiegen der Hauptstörung im Kartenbild könnte ein Effekt von flachem Einfallen sein. Wahrscheinlicher ist eine echte Umbiegung, die sich als feste Verbindung (hard-linkage) zweier Störungen entwickelte. Denn der stratigraphische Versatz entlang der Umbiegung verringert sich nicht entscheidend von Klausberg-NE mit $500 \pm 10 \mathrm{~m}$ (km-ko1-Grenze/tiefster $\mathrm{mm}$ in Baugrube Klausberg 4) zu ca. $400 \mathrm{~m}$ am SW-Rand des Geobotanischen Gartens (mu-mm-Grenze/ km? Gipskeuper-Steinmergel? = Kartierung Stille 1932). Dies könnte darauf hinweisen, dass der EW-Störungsast eine laterale Rampe zur Hauptabschiebung bildet, in die ja der Klausberg-Keuper als Rollover mit $15^{\circ}$ bis $25^{\circ}$ nach ENE einfällt (Heinrichs, unpubl.).

Die Falten und Flexurachsen scheinen sich um das N-Ende des Göttinger Sprungs zu schmiegen, aber ohne Zusatzannahmen lassen sich die einzelnen Elemente nicht über den nicht aufgeschlossenen Bereich zwischen HOL-A und GBS hinweg verknüpfen:

1) Angenommen die Antiklinale GBS-A taucht nach $\mathrm{S}$ ab oder lenkt nach $\mathrm{S}$ ein wie die unsichere Muldenachse in GBS-B, dann könnten die Synklinalen in GBS-A und HOL-A ineinander übergehen.

2) Ohne eine Verbindung der Strukturen ließe sich aus dem Strukturmuster auch eine rechtslaterale E-W Seitenverschiebung lesen, die sich aus dem E-W-Ast der Hauptstörung nach E fortsetzt und im Luttertal ausklingt. Insbesondere das Einengungsmuster der HOL-Aufschlüsse kann damit erklärte werden, daß sich die Nordschol- 


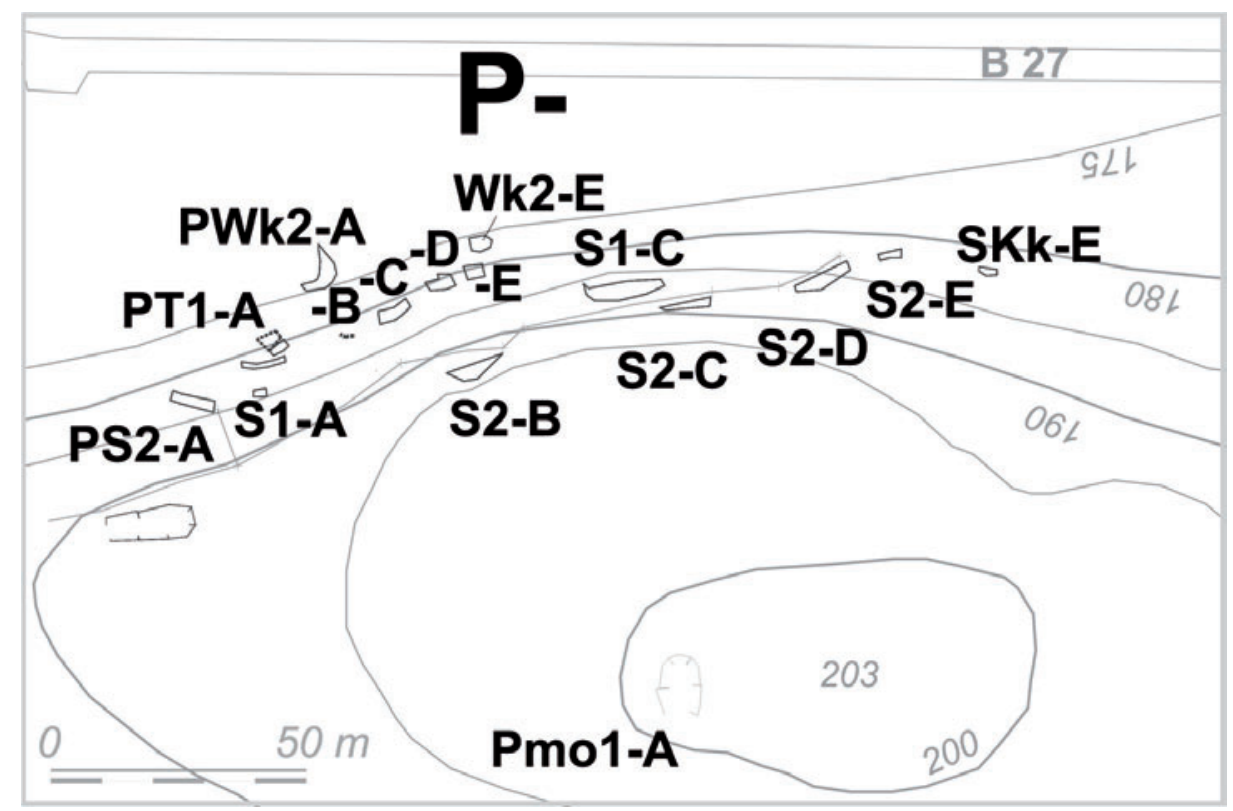

Abbildung 5. Situation der aufgenommenen Aufschlüsse, Papenberg N-Hang.

le (GBS) nach E verschob. Die GBS-Falten sind allerdings einem anderen Einengungsplan zuzuordnen. Bisher gibt es jedoch keine mesoskaligen Beobachtungen, die Seitenverschiebung hier direkt belegen. Jedenfalls erlauben die stratigraphischen Beobachtungen keinen Vertikalversatz zwischen HOL und GBS nur annähernd vergleichbar dem am direkt südlich anschließenden Ast der RelayStörung.

\subsection{Papenberg}

\subsubsection{Stratigraphie und Strukturen im Nord- hang: die Antikline}

Stille's Kartierung des Papenberges zeigt eine flache Antikline ohne interne Struktur aus Terebratelzone und Schaumkalkzone (Stille 1932:32, Karte), Unterer Muschelkalk (Jena Fm., Anis). Die eigene Aufnahme konzentriert sich auf ausgewählte Klippen und Pingen im steilen Nordhang, der nach Wissen des Autors bisher noch nicht kleintektonisch untersucht wurde. Die stark überwachsenen Aufschlüsse sind zwar nicht kontinuierlich, doch war über kurze Interpolation ein hinreichend repräsentatives Bild zu erwarten (Karte Abb. 5). Im Hangfuß bis etwa $4 \mathrm{~m}$ über der Talsohle zeigen sich in den Aufschlüssen PWk2A, -C, -E feingeschichtete Kalk- und Kalkmergelsteine, die auf Grund ihrer Position unter den

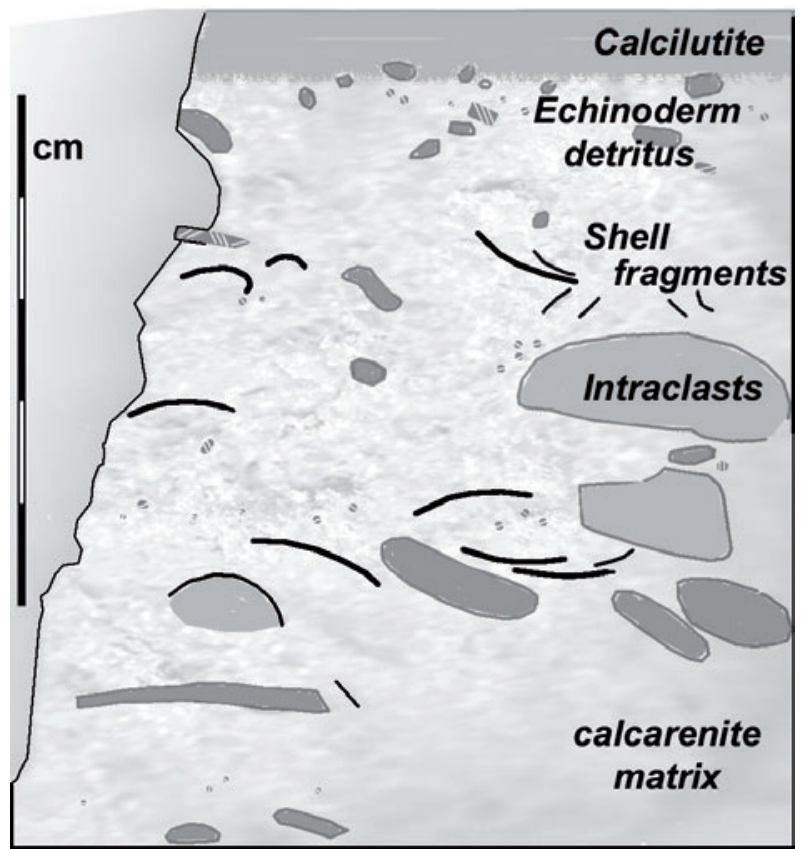

Abbildung 6. Internstruktur der T2-Bank; Papenberg, über Aufschluss PT1-A, Hangendscholle; Zeichnung nach Handstück von Oberkante der Bank.

Aufschlüssen der unteren Terebratelbank (T1) als Wellenkalk 2 angesehen werden.

Im Aufschluss PT1-A wird eine massige Folge von 2,7 m Mächtigkeit, aus kaum geschichteten, harten, intern nahezu strukturlosen, dichten 


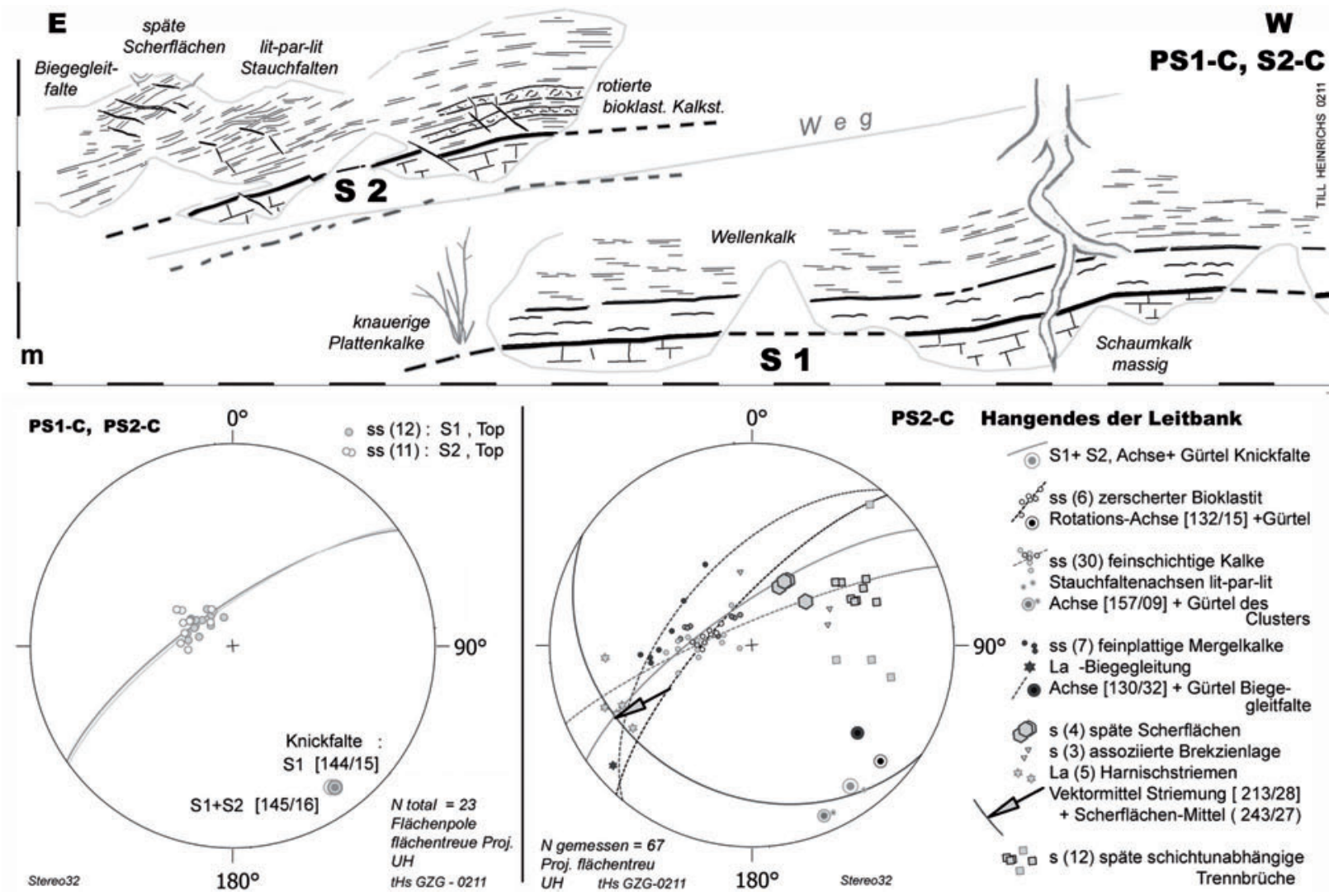

Abbildung 7. oben: Aufschlussskizze PS1-C / PS2-C; bestmögliche Übersicht Stratigraphie der Schaumkalke des Papenberg; links u.: Stereogramm Raumlage der Leitbänke, Aufschluss PS1-C und S2-C : stabile Achsenlage der offenen Knickfalten; vergleiche fast identische globale Faltenachse der Antikline (Abb.8); rechts u.: Stereogramm Detailstrukturen der feinschichtigen Kalke, Hangendes der Leitbank in PS2-C; komplexe Struktur durch Überlagerung verschieden alter Verformungen; d.h. Leitbänke überliefern herzynische Faltungsgeometrie während die feingeschichteten Kalke nach anderen, z.T. jüngeren Deformationsplänen überprägt werden.

feinkörnigen Kalkbänken angetroffen. Sehr wenige Mergelfilme konzentrieren sich auf Schichtflächen nahe Basis und Top der dort eher knauerigen, dickplattigen Lagen. An der Basis der Folge sind spärliche Intraklasten zu finden; 2 bis $2,4 \mathrm{~m}$ über der Basis tritt ein bioklastischer Kalkarenit auf mit einzelnen Crinoiden-Stielgliedern und einem geringen Gehalt an feinkörnigem Pseudoolith. In den Aufschlüssen PT1-D und -E sind nur etwa 2 bis $2,3 \mathrm{~m}$ Mächtigkeit erhalten. Mächtigkeit und Internstrukturen entsprechen im regionalen Vergleich der unteren Terebratelbank, T1, Leitbank 16 (Stein 1966; Dünkel \& Vath 1990). Darauf folgen in den teils deformierten Bereichen der Aufschlüsse PT1-A und -D ca. 1,6 bis $1,8 \mathrm{~m}$ feingeschichteter Wellenkalk. Über diesem Zwischenmittel wurde eine $0,7 \mathrm{~m}$ mächtige, lokal brekziös erscheinende Bank gefunden. Es handelt sich aber eindeutig nicht um eine tektonische, sondern um eine primär sedimentäre Einheit mit basalen $50 \mathrm{~cm}$ feinklastischer Kalke mit wenig Bioklasten. Der höhere Teil der Bank ist ein ruditischer Kalkstein mit zT. dachziegel-gelagerten Intraklasten neben Schill von Gastropoden, Muscheln, Crinoidenstielgliedern und Seeigelstacheln in Kalkarenit-Matrix (Abb. 6). Diese Einheit wird als Entsprechung der oberen Terebratelbank interpretiert, T2, Leitbank 17.

Im Vergleich zu den Standardstratigraphien sind die beobachteten Mächtigkeiten reduziert, insbesondere das Wellenkalkmittel, für das im Göttinger Raum am W-Hang der Eichsfeldschwelle relativ gleichförmig 3,4 bis $3,7 \mathrm{~m}$ angeben werden. Dagegen schwanken die Mächtigkeiten für T1 ( 2,1 bis $3,5 \mathrm{~m})$ und $\mathrm{T} 2(0,6$ bis $2,1 \mathrm{~m}$ ) stärker (Dünkel \& Vath 1990), so daß 
die am Papenberg beobachteten Mächtigkeiten primär sein können. Die signifikante Reduktion des Wellenkalk-Zwischenmittels spricht vielleicht für tektonische Extension (s.u.).

Über T2 folgen in diskontinuierlichen Aufschlüssen ca 5-9m Wellenkalk (Wk3) bis zu den isolierten Ausbissen (z.B. PS1-A) einer wohl $>0,8 \mathrm{~m}$ mächtigen, vorwiegend feinkörnig dichten, harten Kalkbank mit etwas zuckerkörnigem Top. Diese entspricht wohl der in PS1-C besser aufgeschlossenen Leitbank S1 (Abb. 7). Sie lässt dort an hinreichend verwitterten Anbrüchen regelmäßig große Bereiche mit der für die Schaumkalke charakteristischen, feinporigen pseudoolitischen Struktur erkennen. Im oberen Teil ist auch dm-skalige Schrägschichtung zu erkennen. Andere Bereiche bestehen aus massigen lutitischen Kalken, in denen jedoch EchinodermenDetritus, einzelne disartikulierte Stielglieder und cm-große mikritische Intraklasten auf klastische Sedimentation hinweisen. Es wird daher vermutet, dass es sich bei S1 um einen Vertreter der Unteren Schaumkalkbank (X1, Dünkel \& Vath 1990) handeln könnte.

Ihre stratigraphische Zuordnung ist unsicher, denn ihre Basis ist nicht aufgeschlossen, anstehende Gelbkalke waren nicht zu finden. Allerdings wurden oberhalb Aufschluss PS2-C (Top mu?) und in der Halde (Werksteinabbau) unterhalb PS1-C Gelbkalk-Lesesteine beobachtet. Für die Einordnung als tiefste Schaumkalkbank spricht auch die in Abbildung 7 dargestellte Folge mit einer zweiten Bank vom Schaumkalk-Typus, S2, über etwa $3 \mathrm{~m}$ Wellenkalk Zwischenmittel, und womöglich einer rudimentär erhaltenen dritten Bank nur wenige $m$ über Aufschluss PS2-C (nicht dargestellt).

Für die weitere strukturgeologische Analyse ist entscheidend, daß die Bank S2 durch Werksteinabbau so häufig freigelegt wurde (PS2-A bis -E), daß sie als eigenständiger Horizont die Struktur des Papenberges nachzeichnet. Auch weil die feingeschichteten Kalke, wie für S2-C gezeigt (Abb. 7), in großen Teilen des Sattelfirstes polyphas deformiert sind, wurde die globale Falten-Orientierung durch selektive Analyse der Messungen an den beiden mächtigen Hartkalkbänken T1 und S2 ermittelt. Trotz lokal starker Streuung (unparallele, wulstige ss; Verkippung durch jüngere Abschiebung nach S; lokal auch oberflächliche Verstellung) ergab sich eine sehr stabile, näherungsweise zylindrische Struktur

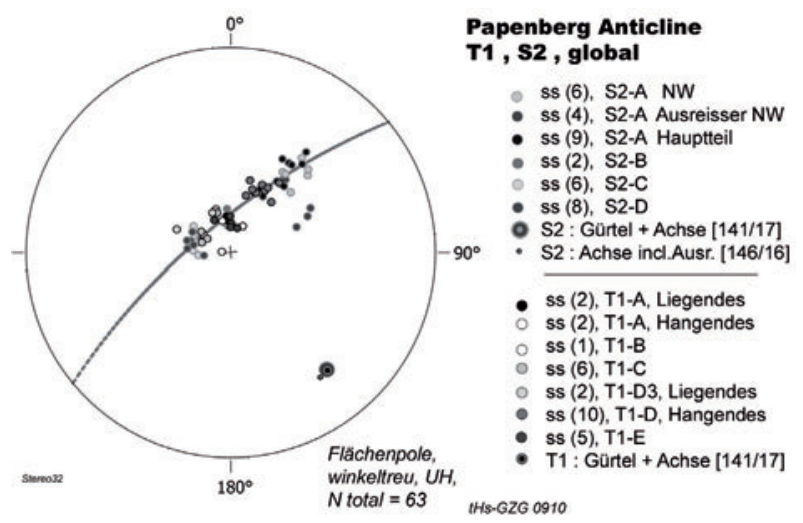

Abbildung 8. Sattel des Papenbergs: Faltung der Terebratelbank T1 und der Schaumkalkbank S2 um statistisch identische Achsen: eine genähert zylindrische Struktur mit leichter SWVergenz; die S2-A-Ausreisser wohl am steilen Hang (ca. $30^{\circ}$ ) atektonisch verstellt. Liegendes bzw. Hangendes bezieht sich auf die Abschiebungen im jeweiligen Aufschluss; Lage der Aufschlüsse PT1-A bis -E und PS2-A bis-D siehe Abb. 5.

(Abb. 8). Dies wird auch aus dem Vergleich der Teilpopulationen deutlich, wonach sich der Gürtel der T1-Antiklinalstruktur nicht von dem der S2Struktur und sogar kaum von dem der lokalen offenen Knickfaltung im NE-Flügel unterscheidet (Abb. 7; PS1-C/2-C). Die Statistik zeigt auch, daß die räumlich etwas getrennte Kalkbank des Aufschlusses PS2-A mit steilerem Einfallen zur Antikline gehört. Der Papenberg-Sattel ist also als eine herzynisch streichende Struktur angelegt.

\subsubsection{Hercynische Einengung und Extension im Kern des Sattels: eine Flat-ramp-Störung}

Im nordöstlichen First des Sattels ist die untere Terebratelbank T1 in den Aufschlüssen PT1-C, -D, -E dokumentiert. Während in T1-C mit flach gelagerter T1 eine Abschiebung nach SW, die östlichste der Ebelhof Störungsschar auftritt, ist kaum $10 \mathrm{~m}$ östlich davon, in PT1-D, eine nach NE- abschiebende Flat-Ramp-Störung zu beobachten (Abb. 9). In ihrem Hangenden bilden Wk2 und T1 mit TWk einen kleinen Sattel. Das Wellenkalkzwischenmittel TWk darüber deformiert in der direkten Fortsetzung des Flats nicht-coaxial entsprechend seiner Anisotropie der Scherfestigkeit und bildet dort einen Stapel von lit-par-lit Duplexen, d.h. es zerschert Schicht-für-Schicht. Kinematisch erklärt sich die Struktur dadurch, 


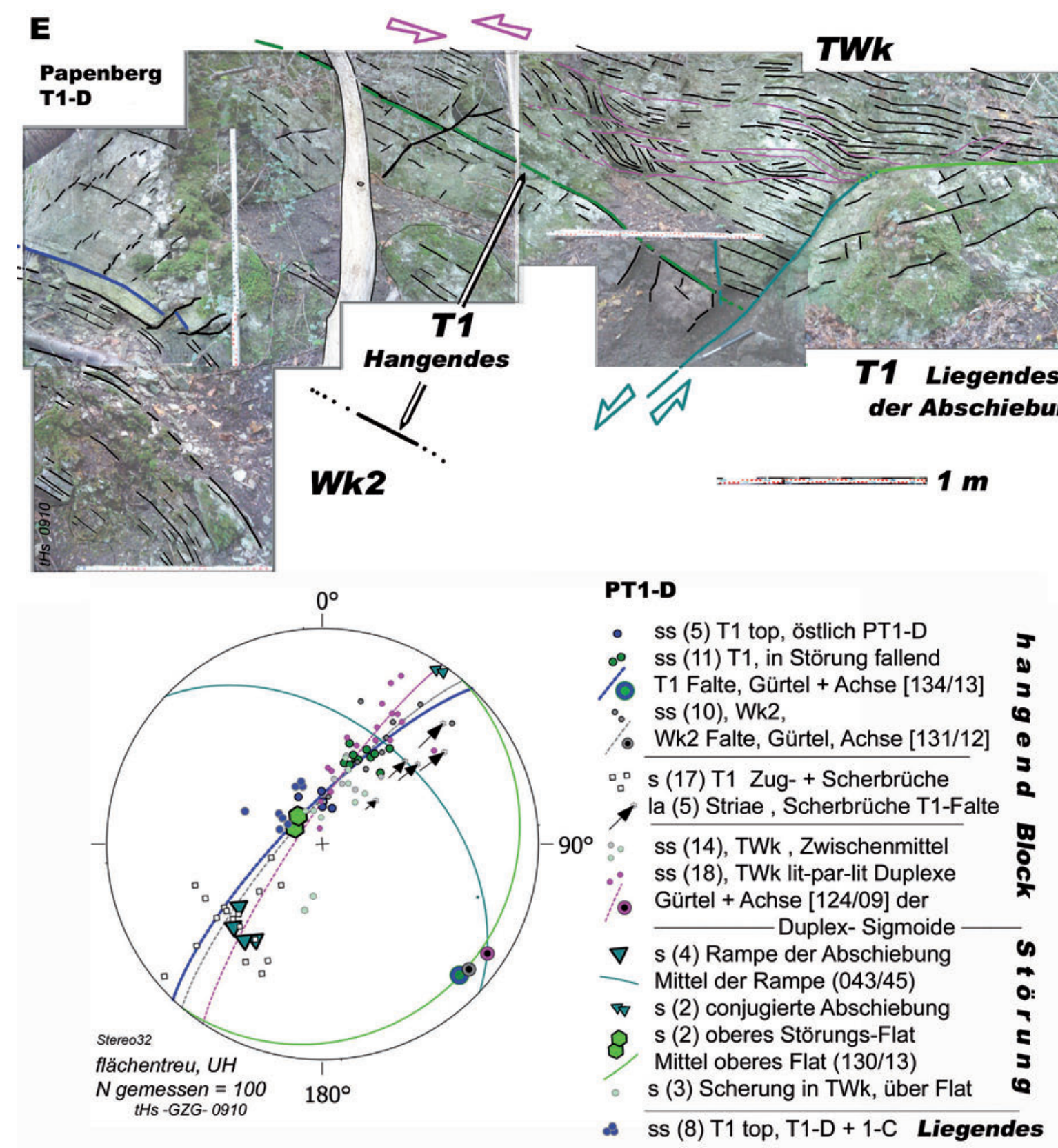

Abbildung 9. Aufschlussbild PT1-D (verkürzter Blick hangauf); Untere Terebratelbank, Wellenkalk-2 im Liegenden und diskordant auflagerndes Wellenkalk-Zwischenmittel (TWk) im Hangenden von T1. Ca. 1,8 m über T1 der Hangendscholle folgt $70 \mathrm{~cm}$ mächtige T2-Bank; farbcodiertes Stereogramm; (vergl. Abb. 10, Zentrum). 


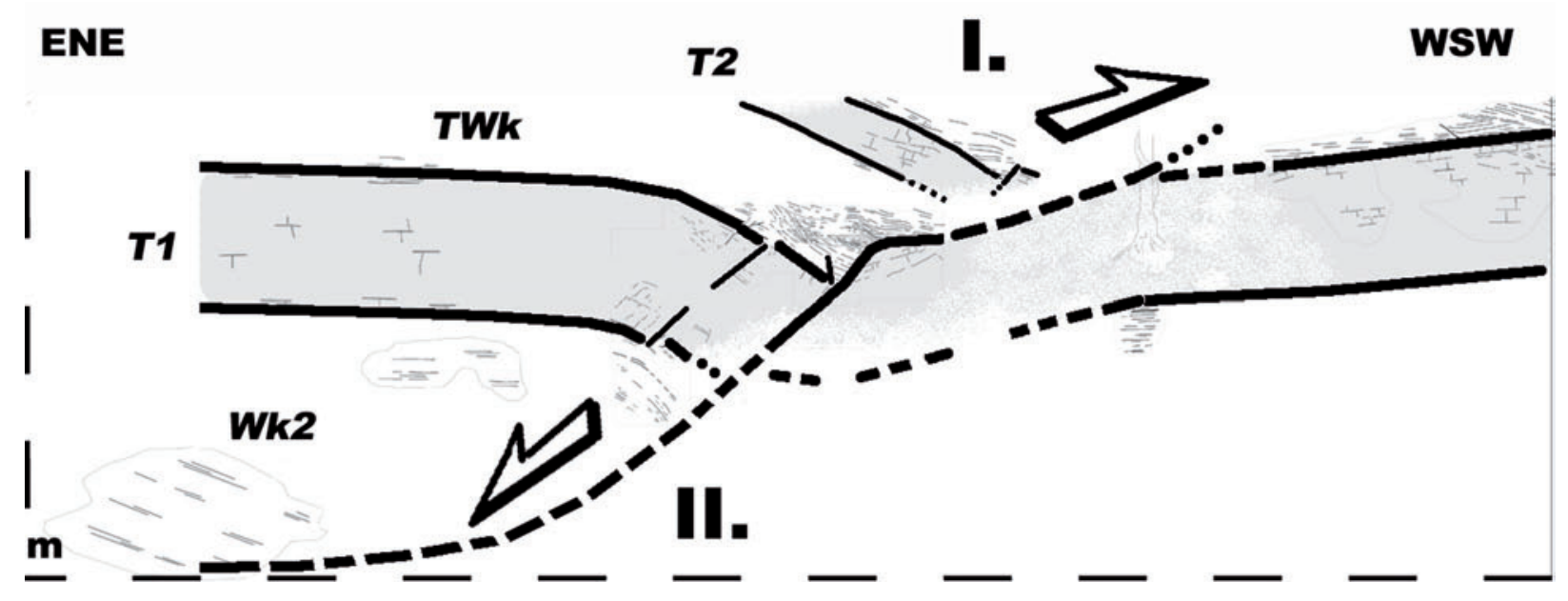

Abbildung 10. Rollover-Antikline oder reaktivierte Aufschiebung?; Profil Aufschluss PT1-E bis PT1-C mit PT1-D (vgl.Abb.9) im Zentrum; sowie PWk2-E und -C im Liegenden und PT2-D im Hangenden; die gestrichelten Kontakte sind nicht aufgeschlossen; Phasen: vermutete Überschiebung (I.), beobachtete Abschiebung (II.); Erläuterung im Text.

daß ältere, höherliegende Scherbahnen durch Aufrichtung in den darunterliegenden, jüngeren Scherlingen gefaltet und blockiert werden. Aus der Richtung der sigmoidalen Verbiegung der Scherlinge und der mittleren Krümmungsachse ergibt sich ein subhorizontaler Transport des Hangenden nach NE.

Die Orientierungsdaten (Abb. 9, Stereogramm) belegen nahezu homoaxiale Faltung von Wk2 und T1 zusammen mit der sigmoidalen Biegung des TWk in den Lit-par-lit Duplexen. Außerdem ist die Interndeformation der T1-Bank im Sattel, d.h. Scherflächenmaximum und Lage der Harnischstriemen parallel zur Abschiebungs-Rampe. Dies bedeutet, daß die einengenden wie die ausweitenden Transporte des herzynischen Deformationsplans in einer fast identischen Hauptebene senkrecht zu den ermittelten Achsen liegen, wobei für Einengung und Ausweitung lediglich die Richtungen der größten und kleinsten Hauptnormalspannung tauschen.

Das Verständnis des Störungsablaufes ist hier kompliziert durch die in $\mathrm{T} 1$ und $\mathrm{Wk} 2$ der Hangend-Scholle entwickelte antiklinale Falte, da deren Kontakt mit dem Fuß der Abschiebung nicht aufgeschlossen ist. Vermutlich setzt sich die Abschiebung unter dem aufgeschlossenen Wk2 in einem flachen Störungsteil fort (,unteres Flat'), entsprechend der geringeren Scherfestigkeit der Wellenkalke, wie es das das Aufschlussbild für ,das hangende Flat' zeigt. Dann wäre eine Deutung der Antikline als Rollover (reverse drag) zu bedenken.
Eine der Abschiebung entsprechende Schleppung von T1 könnte auch fehlen, wenn die Falte nach der Abschiebung entstanden wäre. Zur besseren Einschätzung der kinematischen Entwicklung trägt das nachfolgende zusammengesetzte Profil bei, das alle direkt benachbarten kleinen Aufschlüsse einbezieht (Abb. 10). Dabei sind die nicht aufgeschlossenen Teile der Hauptterebratelbank mit einer als minimal anzusehenden Mächtigkeit von $2 \mathrm{~m}$ ergänzt. Minimal deshalb, weil die Oberkante von T1 im Liegenden des Flat (Abb. 9, rechts) eindeutig tektonisch erodiert ist, angezeigt durch gekappte T1-Schichtung sowie ausgerissene Partien, in die dann Wellenkalk-Material eingepresst wurde.

Daß die T2-Bank auf das Flat hin ebenso steil einfällt wie die $\mathrm{T} 1$ in die Rampe, spricht eher gegen einen Rollover. Vor allem bauen die Rampen-parallel abschiebenden Scherungen in der hangenden T1-Bank die Antiklinale eher ab als auf, die Abschiebung folgt also auf die Faltung. In Umkehrung wird daraus geschlossen, dass die Antikline eine Auf- bis Überschiebung anzeigt. Diese ging offensichtlich der Abschiebung resp. Extension voraus, in deren Gang dann Teile des TWk mittels Schicht-interner einfachen Scherung auf die bereits vorliegende T1-Antikline transportiert wurden. Im Verlauf wurden daraus die litpar-lit Duplex-Stapel. Das Profil (Abb. 10) ist jedoch in 2-D bisher nicht eindeutig restorierbar, bedingt durch die Aufschlusslücke tiefere Rampe 


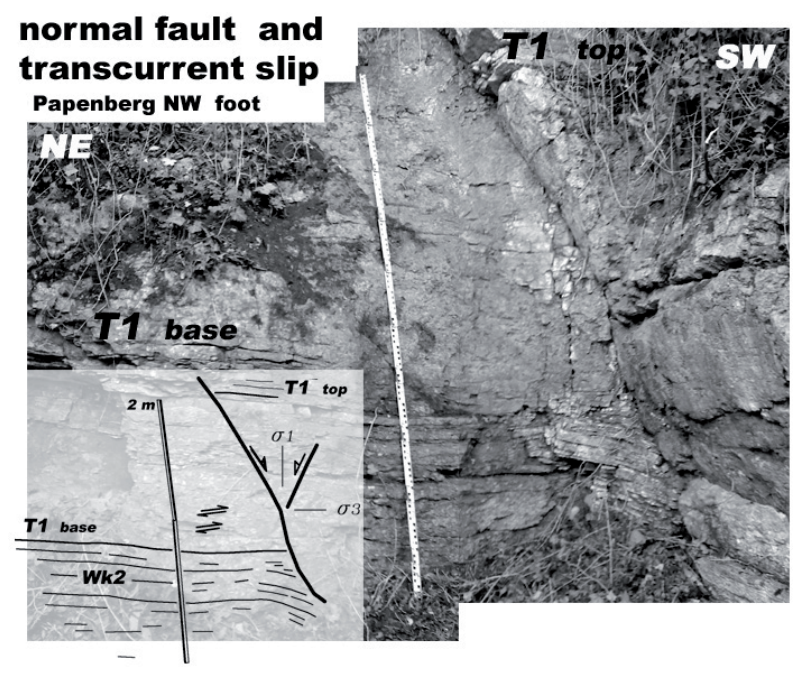

Abbildung 11. PT1-A (,Efeu-Zimmer'), NW-Fuß des Papenberges, Zollstock $=2 \mathrm{~m}$; rechts: 2 . Störungsfläche der Ebelhof-Abschiebungsschar mit Schleppung des Liegenden, und abzweigender konjugierter Fläche in der Hangendscholle, beide mit Störungsharnisch; in Front: NE-SWstreichende Großkluft in T1, mit subhorizontalen Striae; Inset Interpretation: Hauptspannungsgeometrie $\longrightarrow$ horizontale Extension in NE-SW; stratigraphischer Versatz ca. 1,5 m.

und die nicht abgesicherte Geometrie von T2 in der Liegendscholle. Die Frage Rollover oder Überschiebung wird also in weiteren Studien zu untersuchen sein, sowohl in derartigen FaltenAbschiebungs-Ensembles, die in kleineren Aufschlüssen auch weiter östlich beobachtet wurden, als auch im Zusammenhang der von Stille beschriebenen Überschiebungen an den herzynischen Kleper- und Herberhausen-Gräben.

\subsubsection{Die Ebelhof-Abschiebungszone}

In den Aufschlüssen PT1-A, -B, -C wird die untere Terebratelbank $\mathrm{T} 1$ an nachweisbar 4 parallelen Störungen jeweils um Meterbeträge nach SW abgeschoben. Diese Störungsschar, hier EbelhofAbschiebungszone genannt, durchsetzt nahezu symmetriekonform den höheren SW-Flügel der Antiklinale. Rotation beschränkt sich auf Schleppung in die Störung mit unter $1 \mathrm{~m}$ Wellenlänge, die Versteilung des SW-Flügels der Antiklinale folgt erst weiter südwestlich und ist daher wohl unabhängig von den und älter als die Abschiebungen.

Die etwa horizontale kleinste Hauptnormalspannung des Aufschlusses PT1-A (Abb. 11) und

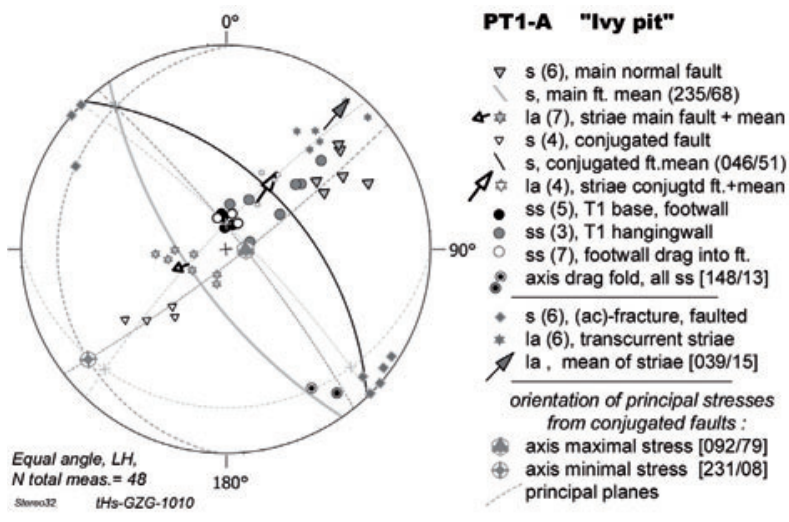

Abbildung 12. Aufschluss Papenberg T1A ,Efeu-Grube; Orientierungsdaten; StressAnalyse für die Ebelhof-Abschiebungsschar.

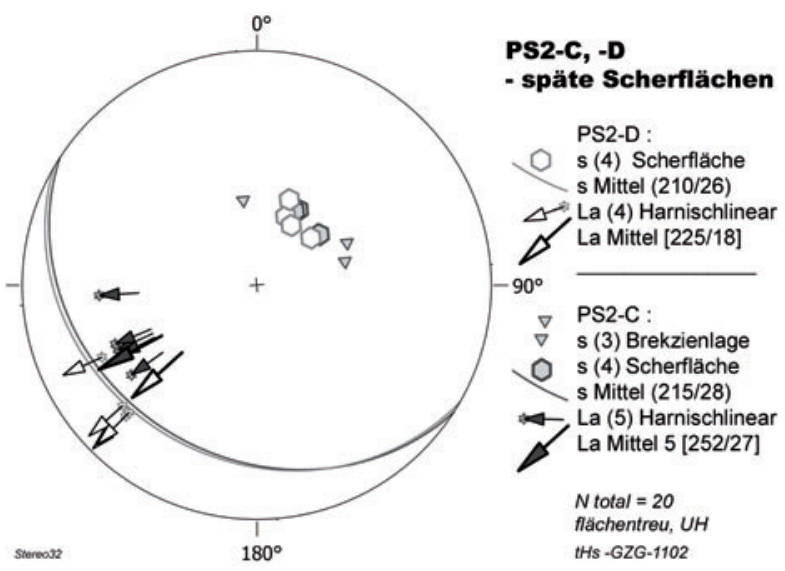

Abbildung 13. Stereogramm, PS2-C, -D, späte flache Abschiebungen im NE-Flügel PapenbergSattel.

anderer der Ebelhof-Abschiebungszone (T1-B und -C) ist stabil in SW-NE-Richtung. Kleine Varianten ergeben sich aus Konstruktion mit den gemittelten Harnischstriemen (hellgraue Hauptflächen Abb. 12). Die gefundenen Hauptspannungsrichtungen werden für zumindest lokal relevant angesehen, denn die T1-Bank stellt ein nahezu isotropes Material dar, so daß die Deformation sicher koaxial ist, und durch die Präsenz der konjugierten zur Hauptstörung sind auch keine Zusatzannahmen zum Winkel der inneren Reibung nötig. In den Wellenkalken oberhalb fällt die Störung flacher ein, was der nicht-koaxialen Verformung dieser Gesteine geschuldet ist.

Die Harnische der Hauptstörung dieses Aufschlusses enthalten eine Teilpopulation mit nach W gerichteter Schräg-Abschiebung. Dies kann eine mehrzeitige Nutzung der Abschiebung be- 
deuten, aber der Probenumfang ist für eine Bewertung hier zu klein. Siehe jedoch den wahrscheinlichen Zusammenhang mit den im Folgenden gezeigten flachen abschiebenden Scherflächen im höheren Schaumkalk-Bereich des NE-Flügels des Sattels.

Die Harnischlineare des (ac)-Querbruches in PT1A sind die einzigen nennenswerten mesoskopischen Anzeichen für Seitenverschiebung, die am Papenberg gefunden wurden. Ihre Bedeutung bleibt daher unklar. Evtl. lassen sie sich durch Raumprobleme bei der segmentweisen Abschiebung erklären, bei der durch die Versteilung der Störung im Kern der T1-Bank eine subhorizontale Ausweichbewegung erzwungen wird, die zu beiden Seiten des wohl älteren (ac)-Bruches leicht unterschiedliche Beträge erreicht.

\subsubsection{SW- bis WSW-wärtiges Zergleiten des Wellenkalks im Sattel-First und NE-Flügel (Auf- schlüsse $P S 2-B,-C,-D$ )}

In 3.4.1., Aufschluss PS2-C, wurde bereits aus dem Hangenden der S2-Bank eine Scherflächenschar mit SW- bis WSW-fallenden Harnischlinearen beschrieben. Die Scherflächen gehen dort zT. in Brekzien über, durchsetzen zudem eine ältere Biegegleitfalte (Abb. 7). In Aufschluss PS2$\mathrm{D}$ (ohne Abb.) wurde eine weitere derartige, einzelne große Scherfläche gefunden, ebenfalls nach SW abschiebend, die die NE-fallende S2-Bank zusammen mit den hangenden feinschichtige Mergelkalken SW-fallend diskordant durchsetzt. Die feinschichtigen Kalke in Aufschluss PS2-B (ohne Abb.) sind ebenfalls geprägt von Diskordanzflächen, die ein Zergleiten der Gesteine nahelegen. Diese relativ jüngere Überprägung im NE-Flügel der herzynischen Struktur könnte insbesondere mit den mehr WSW-gerichteten Abschiebungen den Bewegungen an der Graben-kontrollierten Relay-Störung entsprechen. Im SW-Flügel ist eine Entsprechung evtl. in der WSW-fallenden Teilpopulation der Harnische der Ebelhof-Störungsschar zu sehen.

\subsubsection{Internstruktur der Papenberg-Süd Ab- schiebungen - ,Störungszone Rote Erde' (Tho- mas Löffler \& Till Heinrichs)}

Die Datengrundlage bildet eine umfangreiche geologische Aufnahme von temporären Aufschlüssen im Zuge der Erschließung und Bebauung des Papenberg-Südwesthanges in den Jahren 1991-95 durch Th. Löffler. Dabei wurde eine Feinstratigraphie erarbeitet, die zugleich die entscheidende Grundlage der strukturellen Kartierung bildet (Abb. 14; 16). Sie beruht auf dem lithostratigraphischen Konzept mit formaler Untergliederung bis zum Member (Subformation), eingefügt in die Standard-Formationsgliederung der Trias (Grupe 1907; Beutler et al. 2005; Vath 2005). Im obersten Muschelkalk unterstützen biostratigraphische Befunde die Einstufung in die Ceratiten-Zonen.

Die strukturgeologisch wesentlichen Beobachtungen sind WNW-ESE bis W-E-verlaufende mo2- und ku- Einheiten, die mit ähnlichen Streichrichtungen intern gefaltet und gestört sind. Die Detailprofile (Abb. 15) dokumentieren, daß sowohl Einengung durch Falten als auch Ausweitung durch Abschiebungen vorliegt. Die Falten des mo2 zeigen häufig Asymmetrie, in Antiklinen mit einem steileren Süd- und flachem Nordschenkel. Dies kann man als Stauchung in einem Südabwärts- Transport deuten, der damit die dominierend Süd-abschiebende Bewegung in der Rote Erde-Störungszone unterstützt. Die bruchhaften Strukturen zeigen überwiegend Dehnung an. Selten sind kleine flache Überschiebungen mit etwa Top-Süd-Transport.

In Profil PS-A bietet sich im untersten Keuper (kuSU) das Bild einer antithetischen Schollenrotation, die numerisch eine Dehnung von $>10 \%$ anzeigt. Keuper ist um ca. $5-10 \mathrm{~m}$ auf dem Top des nördlich angrenzenden mo2 abgeschoben. Dieser mo2 seinerseits fällt mit ca $30^{\circ}$ nach $\mathrm{SW}$ und versteilt nach oben, wo er in eine enge, zT. südwärts überschobene, S-vergente Falte übergeht (Abb. 15). Wenig jenseits des Südrandes dieses Profils ist eine steil N-fallende Abschiebung des Keupers auf dem südlich angrenzende mo2-Block beobachtet. Der höhere Teil des aufgeschlossenen Keupers unterhalb der Solifluktionsschuttdecke zeigt Schleppung und nahezu liegende Falten mit Südvergenz. Es ist wahrscheinlich, dass sich hier eine tiefgreifende gravitative Bewegung abbildet. Ob diese südwärtigen Bewegungen dem Hangkriechen im Permafrost-Milieu zuzuschreiben ist, oder ob es sich um der Grabenabsenkung geschuldetes Abgleiten der heute erodierten Hangendscholle und damit einhergehende Schleppung des Liegenden handelt, ist nur zu vermuten.

Im Beispiel des Profil PS-B besteht die Nördliche Scholle aus mittelsteil S-fallendem mo2 mit Ceratites nodosus in anstehendem Mergelstein (Obere Ceratitenschichten, Meißner Fm.), bis zu 


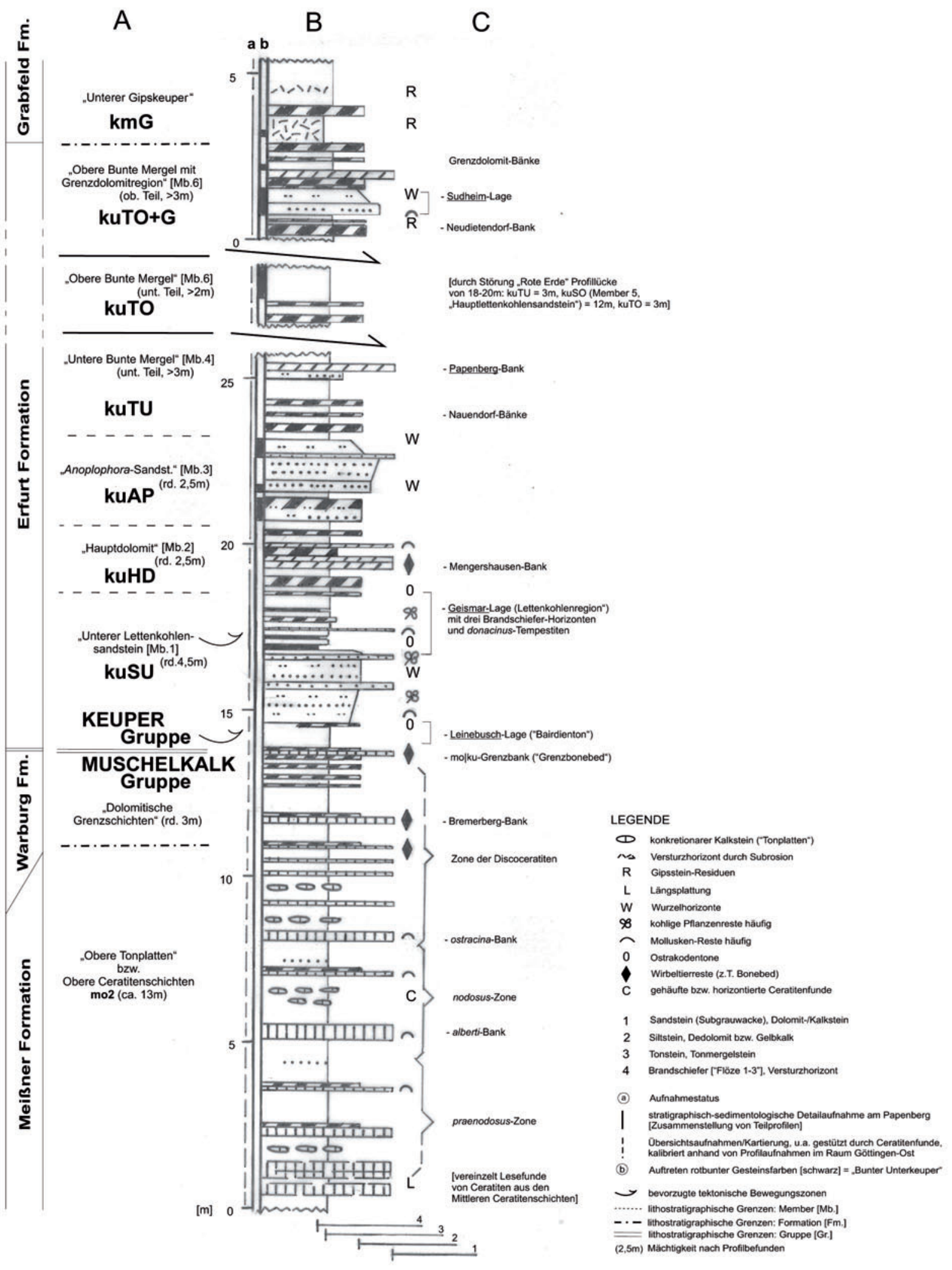

Abbildung 14. Stratigraphisches Übersichtsprofil Grenzbereich Muschelkalk/Keuper am Ostrand des Leinetalgrabens in Göttingen; Zusammenstellung von Aufschlußbefunden u.a. von der PapenbergSüdwestflanke im Zuge der Bebauung ,In der Roten Erde' ; Autor: Th. Löffler. A — Formationsgliederung des Unterkeupers weitgehend nach Grupe (1907) mit modifizierter Grenzziehung; Symbolgebung der geologischen Landesaufnahme aus Preuss (2010), vgl. auch Vath (2005). B - vereinfachte Lithologie. C - Leithorizonte und Ceratiten-Zonen, z.T. nur informell für Südniedersachsen. 

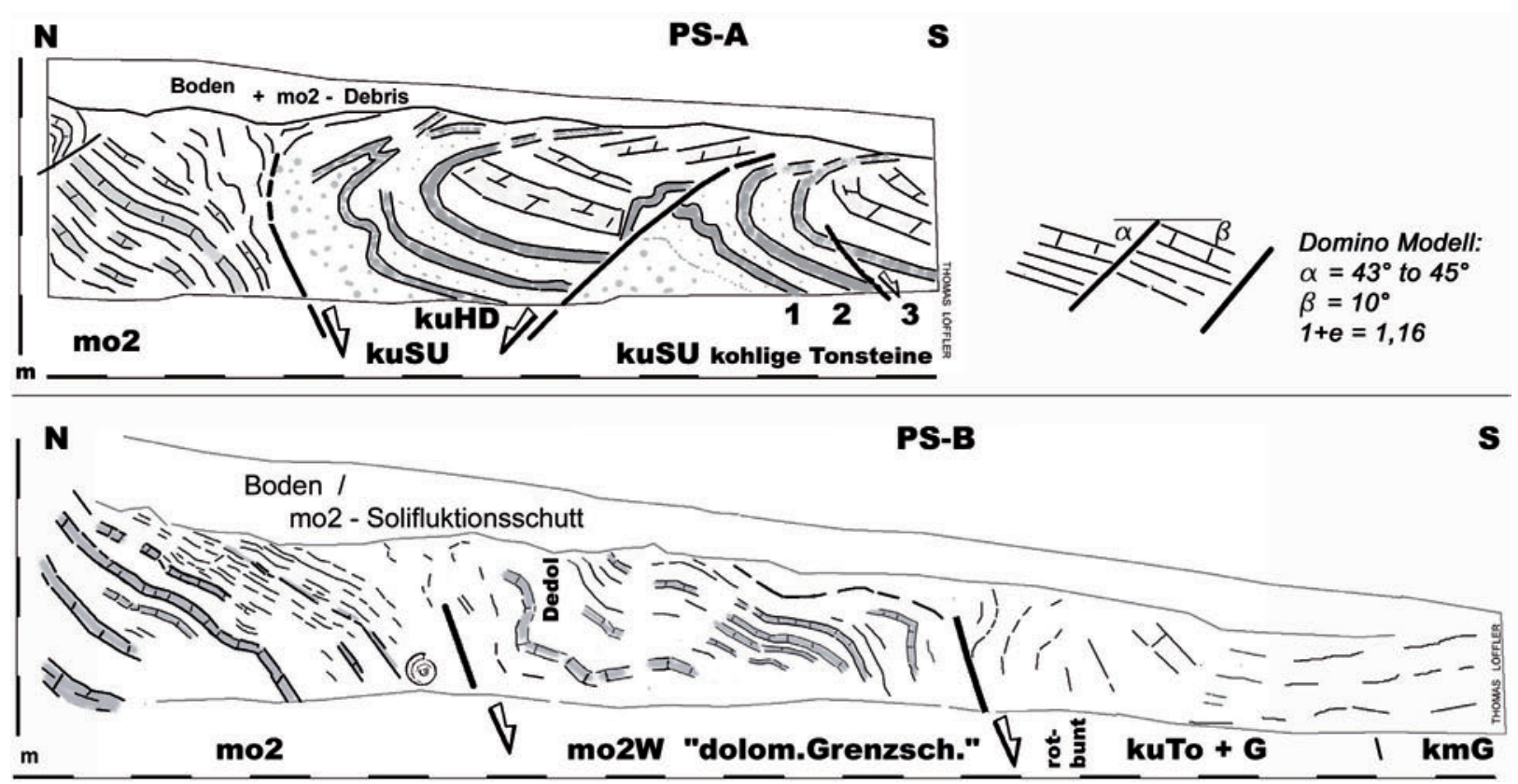

Abbildung 15. Detail-Profile Papenberg-Südwesthang; wenig südlich von PS-A liegt eine weitere $\mathrm{N}$-fallende Abschiebung; zur Lage vgl. Strukturkarte Abb. 16.

einer ersten steil S-fallenden Abschiebung. Die mittlere Scholle besteht aus S-vergenten Falten aus Mergelstein und Dedolomit-Bänken, wahrscheinlich dolomitische Grenzschichten (Warburg Fm.) überlagert von unterstem Keuper. Jenseits einer weitern steil S-fallenden rotbunten Störungszone mit z.T. calcitisch verheilter DedolomitTonstein-Brekzie folgt die südliche Scholle mit fast söhligem kuTO endend in kuG/ kmG Grenzschichten im Übergang von Unterem zu Mittlerem Keuper mit verstärkt einsetzenden Residualhorizonten.

\subsubsection{Strukturkarte des Papenberges}

Nach Stille's Kartierung wird die flache Antikline des Nordhangs nach S ungestört gefolgt von mm und mo1. Diese gesamte Einheit ist nach seiner Karte südwärts auf mo2 und ku durch eine flach N-wärts einfallende Störung überschoben, der ku im S-Hang des Papenbergs dann selbst wieder an einer steil N-fallenden Störung auf mittleren Keuper aufgeschoben (Stille 1932:32, Karte, Erl.). Die eigene Aufnahme bestätigt die Antikline, deckt aber ihre Überprägung durch im wesentlichen symmetriekonforme Abschiebungen auf. Der Südhang inclusive des mo1-Rückens wird als breite Abschiebungszone aufgefasst.

Das flache bewaldete Plateau des Berges ist ohne Aufschlüsse, eine Pinge im Westhang an der
Grenze zu den ersten Häusern erreichte das Anstehende nicht. Der Aushub besteht aus mikritischen Kalkplatten vom mo2-Typ, bis dm-starken Schillkalkbänkchen (Packstones) und oft bemerkenswert brekziierten grobklastischen Kalkareniten vom mo1-Typ mit bis cm-starkem CalcitMineralisat auf Klüften. Ob es sich dabei allein um eine Solifluktionschuttdecke handelt, bleibt unklar. Lesesteine belegen mm nur sporadisch auf dem höchsten unbebauten Teil des Südwesthanges. Der markante E-W streichende Kamm mit Höhe 203 wird von mo1 gebildet, der in einer Pinge über etwa 6 Profilmeter mit einheitlich $45^{\circ}$ bis $50^{\circ} \mathrm{S}$-Fallen, aufgeschlossen ist (Pmo1-A, ohne Abb.).

Auf der Strukturkarte (Abb. 16) ist die theoretische Obergrenze des Unteren Muschelkalks im Papenberg Sattel eingetragen, die aus dem mittleren kartierten Fallen kombiniert mit dem gemessenen Streichen der S2-Bank $5 \mathrm{~m}$ über letzterer rechnerisch in 3-D ermittelt wurde. Rein konstruktiv liegt auf dem NE-Flügel der Antikline bei mittlerem kartierten Einfallen von 10 bis $15^{\circ}$ in dem dort anschließenden sanften NE-Hang ausschließlich mm. Dagegen ergibt die Konstruktion auf dem steiler, um $45^{\circ}$ einfallenden SW-Flügel einen hypothetischen, etwa NWstreichenden mo1-Ausbiss auf halber Höhe des NNW-Hanges. 


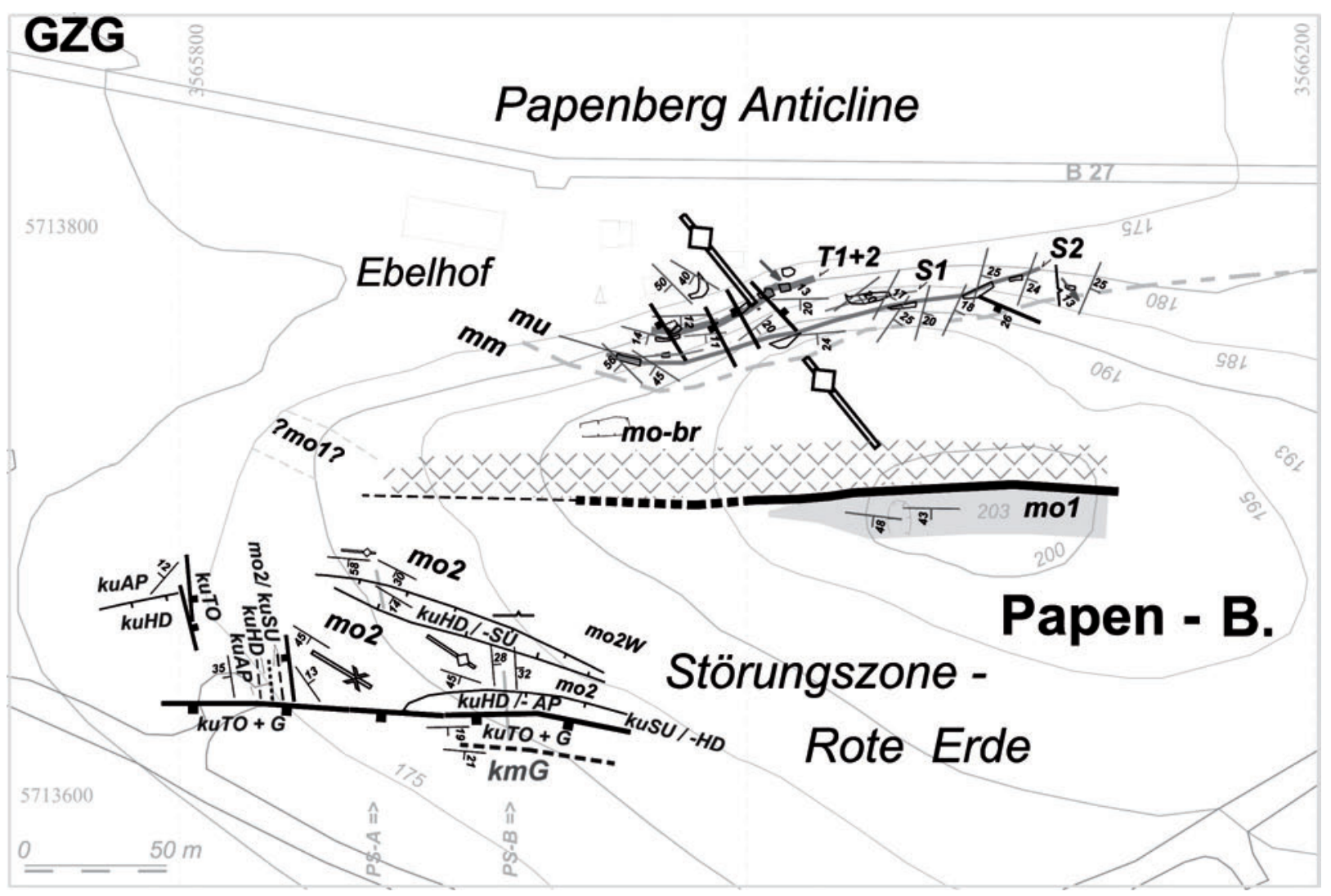

Abbildung 16. Strukturkarte des Papenbergs; Struktursymbole wie in Abb. 4; graue starke Linien über PS-A bzw. -B = Profile Abb. 15, Stz. Rote Erde; umrandete Aufschlüsse Nordhang, siehe Codes in Abb. 5; durchgezogene Linien = kartiert, gestrichelte = konstruiert/extrapoliert; $T 1+2=$ Untere und obere Terebratelbank, S1 und $-2=$ Leitbänke Schaumkalkzone, mo-br= brekziierter Oberer Muschelkalk; für stratigraphische Codes in Störungszone Rote Erde vgl. Abb. 14; gemustert = vermutete, im wesentlichen abschiebende Störungszone unter Trochitenkalk; vgl. Erl. im Text.

Aus der ermittelten mu-mm-Grenze folgert, daß bei einem Fortstreichen der Sattelachse nach SE diese im Achsenfallen von $15^{\circ}$ abrupt auf $>30^{\circ}$ versteilen müsste, wenn im Sattelfirst $55 \mathrm{~m} \mathrm{~mm}$ Standardmächtigkeit zwischen die Oberkante mu und den anstehenden mo1 zu liegen kommen sollen. Dies und das diskordante Streichen verdeutlichen, dass unter der E-W streichenden mo1Platte eine etwa E-W verlaufende Störungszone liegen muß, in die womöglich größere Teile des mm , mo1 und evtl. mo2 einbezogen sind. Der unterdrückte Ausstrich eines Teils des SWSattelflügels könnte eine $\mathrm{N}$-fallende Aufschiebung, ähnlich wie von Stille angenommen, nahelegen. In der Detailaufnahme des Nordhanges gibt es aber keine Hinweise auf eine solche späte, d.h. die Falte überprägende aufschiebende Deformation, im Gegenteil späte Bewegungen sind abschiebend. Wegen der anschließenden Abschiebungszo- ne im Papenberg Südhang (vergl. Kap.3.4.5) gilt für wahrscheinlicher eine S-fallende Abschiebung, steil unter die $>45^{\circ}$ geneigte Hangendscholle einfallend, womöglich mit einer Seitenverschiebungskomponente. Die Stille'schen Funde von mo2 auf NNW- und NE -Hang, allerdings nicht durch Anstehendes bestätigt, wären mit einer solchen komplexeren, evtl. repetitiven Störungszone zwischen N- und S-Hang Papenberg verträglich.

Die Befunde auf dem SW-Hang des Papenberges belegen eine breite Abschiebungszone in den Schichtfolgen, die der am Gipfel aufgeschlossenen mo1-Platte stratigraphisch auflagern. In die SEbis ESE-streichenden, gefalteten mo2-Einheiten sind jedoch mit 5-10 m Sprunghöhe kleine Gräben von tiefstem Keuper eingesunken, die einer Extension in N-S bis NNE-SSW-Richtung entsprechen. Diese Rote Erde-Störungszone endet im $\mathrm{S}$ an einer E-W streichenden markanten Abschiebung mit 20 
bis $30 \mathrm{~m}$ stratigraphischer Sprunghöhe innerhalb des Unteren Keupers. Daran legen sich nach S in weitgehend primärem Verband S-fallender oberster Unterkeuper unter tiefstem Mittleren Keuper an.

Die Extensions-Richtung der Rote ErdeGräben schräg zu den beobachteten oder vermuteten Hauptstörungen ist verträglich mit einer rechtshändigen Komponente der Abschiebungszone, das Faltenstreichen der mo2-Einheiten allerdings nicht. Es handelt sich daher wohl um ältere Faltung der herzynischen Richtung, die partiell in der Abschiebungszone überprägt wurde.

Am SW-Rand des Papenberges wird in Zusammenfassung mehrerer Aufschlüsse ein kleiner N-S streichender Graben ausgeschieden, in dem eine W-fallende Schichtfolge von höchstem Muschelkalk (mo2 mit Discoceratiten) bis AnoplophoraSandstein und, nach einer Aufschlusslücke, oberstem Unterkeuper (Obere Bunte Mergel) belegt ist. Dieser Graben wird als älter als die RoteErde-Störungszone angesehen, denn er wird von deren südlicher Randstörung überprägt.

\section{Diskussion}

Die saxonische (alpidische) Deformation nach Stille \& Lotze (1933, u.w.) beinhaltete bereits ein Konzept von richtungsabhängiger Dehnung und Einengung: NNE-streichende, ,rheinische', Brüche kontrollieren Extension, NW-SE streichende, ,herzynische', sind Einengungsstrukturen. Weitspannige Antiklinen, begleitet von Überschiebungen werden für den Göttinger Hainberg beschrieben in deren Scheiteln der Keuper der Kleperspalte und des Herberhäuser-WartbergGrabens eingesunken sind (Stille 1932:32; Stille \& Lotze 1933:44). Die vorgelegte Studie zeigt, dass der Luttertal-Faltenzug in diesen Zusammenhang $\mathrm{zu}$ stellen ist.

Die am Papenberg aufgeschlossenen Strukturen des unteren Muschelkalkes kann man als einen Einblick in ein etwas tieferes strukturelles Niveau unter den Hainberg-Gräben verstehen, wo oberer Muschelkalk und tiefer Keuper im Aufschlussniveau liegen. Allerdings kann die Stille'sche Vorstellung des Papenbergs als einer nach S überschobenen Struktur nicht bestätigt werden. Im Gegenteil wird in dieser Arbeit eine Verbindung aus Faltung und fast homoaxialer Extension belegt.

Die herzynische Achse HerberhausenWartberg-Papenberg erstreckt sich mögicher- weise NW' des Papenbergs bis Stumpfe Eiche und Weendespring. Denn dort ist im Hangenden der Relay-Abschiebung unter mächtiger Quartärbedeckung ein ebenfalls nach NE einfallendes Feld von Unterem und tieferem Mittlerem Keuper durch bisher unpublizierte Bohrungen nachgewiesen (Meischner 1980; Heinrichs \& Leiss (2011 in Vorber.)), das den NEFlügel eines herzynischen Sattels mit einem First im Bereich des Weender Schützenhauses bilden könnte. Die herzynische Achse HerberhausenWartberg-Papenberg modifiziert offensichtlich den Verlauf der Relay-Störung (Abb. 1), wobei das zeitliche Verhältnis beider nicht eindeutig ist, möglicherweise weil beide sich in mehreren Schritten gegenseitig beeinflussen.

Regionale Analogien von herzynisch streichenden Strukturen finden sich im gesamten saxonisch geprägten Bereich (u.a. Binot 2001, s.a. Tanner et al. 2010), so insbesondere auch im nicht durch Salinar beeinflussten Südrand des Niedersächsischen Tektogens mit den auf- und überschiebenden Strukturen des Osning und dem Ibbenbührener Aufbruch (pop-up) mit seinen bergbaulichen Aufschlüssen (Drozdzewski 1988). Dort ist abgeleitet, daß die herzynisch streichenden Falten und Überschiebungen des Deckgebirges durch schiefe, rechtslaterale Einengung über reaktivierten steilstehenden Tiefenbrüchen zu erklären sind. Die Übertragung von Schlussfolgerungen aus den Tiefenaufschlüssen von Ibbenbühren und ihren Zusammenhang mit der Deckgebirgsdeformation auf Bereiche mit einem teilweise salinaren Oberbau wie im Göttinger Raum ist sicherlich mit einzelnen Modifikationen verbunden, die der Spannungsumverteilung und -ablenkung zwischen duktilen und spröden Gesteinseinheiten und dem Auftrieb der Halit-reichen Einheiten geschuldet sind. Doch zeigen alle Analog-Experimente von unterschiedlich aufgebauten spröd-duktilen Multilayer, daß die Falten- und Bruchgeometrie auch im partiell entkoppelten suprasalinaren spröden Deckgebirge weitgehend von den Hauptspannungsrichtungen und Geometrien der reaktivierten Störungen des Sockels kontrolliert wird (z.B. Richard et al. 1991, etc.).

Zeitlich lässt sich sich die einengende Reaktivierung, durch die Verbindung mit der Sedimentationsgeschichte im Niedersächsischen Becken einordnen, wo mesozoische divergente Strukturen invertiert wurden (Betz et al. 1987). Danach wird die Inversion in späte Kreide und frühes $\mathrm{Pa}$ - 
laeogen gelegt, d.h. die sub-herzyne Inversion im frühen Campan und die laramische Inversion im mittleren Palaeozän. Das regionale Spannungsfeld zeigt in der Oberkreide eine etwa $\mathrm{N}-\mathrm{S}$ gerichtete horizontale Hauptspannung, die zu divergenter Reaktivierung von N-S-Tiefenbrüchen und vor allem transpressiv zu konvergenter Reaktivierung an alten herzynischen Störungen führt (Betz et al. 1987). Die jüngere Intraplatten-Deformation belegt dann eine Gegenuhrzeiger -Rotation dieser horizontalen Hauptspannung $\mathrm{s}_{H}$ (Bergerat 1987). Dies entspricht auch etwa den Beobachtungen in Süddeutschland, wo die Heldburger Gangschar und die zuerst divergenten Bewegungen an den Rheintalgraben-Randstörungen eine ältere $\mathrm{s}_{H^{-}}$ Richtung dokumentieren, auf die sinistrale Bewegungen folgen und damit eine Drehung von $\mathrm{s}_{H}$ in die heutige etwa NW-SE-Richtung belegen (Meier \& Eisbacher, 1991). Die rezenten Spannungsrichtungen variieren aber regional beträchtlich und systematisch, woraus man schlußfolgern muß, daß der lokalen Paläo-Spannungsanalyse weiterhin Bedeutung zukommt.

Die geometrische Analyse am Papenberg zeigt die Ähnlichkeit von konvergentem und divergentem Deformationsplan. Mehrere kinematische Szenarien sind denkbar. Eine Deutung durch Umkehr der kleinsten und größten Hauptspannungsrichtungen im aufgewölbten Sattel bei abklingender Kompression/ Transpression ist sehr gut verträglich mit der in Aufschluss PT1-D gezeigten konstanten Symmetrie der Hauptebene des tektonischen Transportes bei einengender Überschiebung/Faltung und nachfolgender ausweitender Deformation. Dieses Modell erscheint momentan am attraktivsten.

Ein weiteres Szenario besteht aus wiederum an einer transpressiv reaktivierten herzynischen Störung entwickelten älteren Falten. Die jüngeren Abschiebungen könnten sich jedoch bei nur leicht nach $\mathrm{N}-\mathrm{S}$ gedrehtem $\mathrm{s}_{H}$ zwischen nun linkslateral reaktivierten NNE-SSW-Tiefenstörungen bilden, wie durch Northeimer und Göttinger, Reinhäuser, etc. Sprung angezeigt. Dann würde z.B. die herzynische Achse von Klausberg über Papenberg bis Stumpfe Eiche zwischen den linksseitig verspringenden linkslateralen Störungen in ein NESW-Dehnungsregime geraten. Die symmetrischen Abschiebungen am Papenberg und damit auch die Grabenbildung des Herberhäuser Graben wäre dann wie im ersten Szenario ebenfalls noch jungkretazisch oder Palaeogen.
Im Licht der referierten Drehung der Rotation des äußeren großräumigen Spannungsfeldes ist als dritte Variante auch eine erste Einengung bei etwa $\mathrm{N}$-S-gerichteter $\mathrm{s}_{H}$ und, in größerem zeitlichen Abstand, nachfolgende Ausweitung der Papenberg Antikline bei dann nach NW-SE gedrehter $\mathrm{s}_{H}$ vorstellbar. Die Situation in der RoteErde-Abschiebungszone mit NW-SE und WNWESE verlaufenden kleinen Gräben scheint diese gedrehte Spannungsorientierung ebenfalls widerzuspiegeln. Die südliche Abschiebung dort und damit wohl auch die Rote-Erde-Gräben sind auch jünger als die E-W gerichtete, dem Leinegraben entsprechende Extension. Allerdings fehlen im Bereich des Leinegrabens Belege für Neogene Sedimentation in derartigen NW-SE laufenden Gräben. Ob bei dieser Situation das Spannungsfeld modifizierende Intraplatten-Deformation in Form von großräumigen Schollenrotationen eine Rolle spielen, ist kontrovers (Schreiber \& Rotsch 1998). Gerade hier ist die lokale Situation zu bedenken, denn eine divergente rechtshändige Verschiebung in der Rote Erde-Zone kann auch darauf zurückzuführen sein, daß die Zone Teil einer nördlichen lateralen Rampe zur W-gerichteten Abschiebung auf dem Göttinger Sprung ist (vergl. $3.3)$.

Für die Prognose von Strukturen im subsalinaren Sockel ist es zwar nicht unwichtig, dass die Existenz von großskaligen varistischen Strukturmustern, z.B. Überschiebungsstapeln, im Basement der hessischen Senke und des Göttinger Raumes seismisch nachgewiesen wurde (DEKORP-Profil 3N, $20 \mathrm{~km} \mathrm{~W} \mathrm{Göttin-}$ gen; Heinrichs et al. 1994). Allerdings dürften jüngere Störungssysteme, insbesondere solche die reaktiviert wurden, wegen der zu erwartenden Permeabilitäten ebenfalls ein interessantes Target für geothermische Exploration darstellen. Deswegen sei hier erinnert, daß das varistisch konsolidierte Grundgebirge Europas einen Permokarbonen strukturellen Umbruch erlebte (,Ural-Agadir Scherzone', Arthaud \& Matte 1977), in dem in unserem Raum — reflexionsseismisch im PräZechstein-Sockel des Niedersächsischen Beckens bis etwa Northeim kartiert (Betz et al. 1987) viele meridionale und herzynische Störungen angelegt wurden, die dann in einem günstigen Spannungsfeld in Oberkreide/Paläozän erneut aktiv wurden. 


\section{Dank}

Dank geht an Josef Paul und Frank Langenstrassen, Göttingen, für stratigraphischen, sedimentpetrographischen und paläontologischen Rat, an Dave Tanner, Hannover, für einen anregenden Exkursionsführer (siehe Tanner 2011, dieser Band), und an Martin Sauter, Göttingen, für konstante Rückendeckung.

\section{Literatur}

Arthaud F., Matte P. 1977. Late Paleozoic strikeslip faulting in southern Europe and northern Africa: Result of a right-lateral shear zone between the Appalachians and the Urals. Bulletin of the Geologicial Society of America 88, 13051320, Boulder.

Bengtson C.A. 1981. Statistical curvature analysis techniques for structural interpretation of dipmeter data. American Association of Petroleum Geologists Bulletin 65, 312-332.

Bergerat, F. 1987. Stress fields in the European platform at the time of Africa-Eurasia collision. Tectonics 6 (2), 99-132.

Betz D., Führer F.X., Greiner G., Plein E., 1987. Evolution of the Lower Saxony Basin. Tectonophysics 137: 127-170.

Binot, F., Baldschuhn, R., Fleig, St., Kockel, F. 2001. Geotektonischer Atlas von NordwestDeutschland und dem deutschen NordseeSektor. Geologische Jahrbuch A153, 88 S., 3 CDROM.

Chapman, G.R., Lippard S.J., Martyn J.E. 1978. The stratigraphy and structure of the Kamasia Range, Kenya Rift Valley. Journal of the Geological Society of London 135, 265-281.

Deutsche Stratigraphische Kommission / Koordinator G. Beutler, 2005. Stratigraphie von Deutschland IV: Keuper. Courier ForschungsInstitut Seckenberg 253, 296 S., Frankfurt a.M..

Drozdzewski, G. 1988. Die Wurzel der OsningÜberschiebung und der Mechanismus hercynischer Inversionsstörungen in Mitteleuropa. Geologischer Rundschau 77/1, 127-141.

Dünkel, H., Vath U. 1990. Ein vollständiges Profil des Muschelkalks von der Dransfelder Hochfläche. Geologische Jahrbuch Hessen 118, 87126.

Fossen, H., Johansen, T.E.S., Hesthammer, J., Rotevatn, A. 2005. Fault interaction in porous sandstone and implications for reservoir managment; examples from southern Utah. American
Association of Petroleum Geologists Bulletin 89, 1593-1606.

Grupe, O. 1907. Der Untere Keuper im südlichen Hannover. - In: Festschr. A. v. Koenen, 65-134, 1 Tab., 1 Taf.; Stuttgart, E. Schweitzerbartsche Verl.-Buchhandlg.

Heinrichs T., Giese P., Bankwitz P., Bankwitz E., DEKORP Research Group C 1994. DEKORP 3 / MVE 90 West - preliminary geological interpretation of a deep near-vertical reflection profile between the Rhenish and Bohemian Massifs, Germany. Zeitschrift für Geologische Wissenschaften 22, 771-801.

Heinrichs T. 2001. Geologie - Ostafrika $\left(2^{\circ} \mathrm{N}-2^{\circ} \mathrm{S}\right.$, $\left.32^{\circ}-38^{\circ} \mathrm{E}\right)$. Afrika-Kartenwerk, Beiheft E3, 231 SS., 10 Abb., Bornträger, Berlin - Stuttgart.

Koenen, A. von 1894. Erläuterungen zur geologischen Specialkarte von Preussen und den Thüringischen Staaten. Blatt No. 28 Göttingen: 58 S.; Berlin (Schropp) [2. Aufl. 1907]

Meier, L., Eisbacher, G.H. 1991. Crustal kinematics and deep structure of the northern Rhine Graben, Germany. Tectonics 10, 621-630.

Meischner, D. 1980. Geologisches Gutachten zur Gefährdung des Wasserwerkes Weende-Spring durch geplante Baumaßnahmen, insbesondere Bebauungsplan Nr.29 ,Stumpfe Eiche‘. 28 S., 9 Anl., maschinenschriftlich.

Peacock, D.C.P., Price, S.P., Pickles, C.S., 2000. The world's biggest relay ramp: Hold with Hope, NE Greenland. Journal of Structural Geology 22, 843-850.

Preuß, 2010. Symbolgebung, 3. digitale Fassg.

Richard, P., Mocquet, B., Cobbold, P.R. 1991. Experiments on simultaneous faulting and folding above a basement wrench fault. Tectonophysics 188, 133-141.

Ritzkowski, S. 1999. Der Göttinger Leine-Graben im Paläogen (südliches Niedersachsen). Neues Jahrbuch für Geologie und Paläontologie, Abhandlungen 214 (1): 237-256; Stuttgart.

Roeller, K., Trepmann, C. 2003-2007, Stereo-32 Ver.094, Ruhr-Universität, Bochum.

Schreiber, U., Rotsch S. 1998. Cenozoic block rotation according to a conjugate shear system in central Europe — indications from palaeomagnetic measurements Tectonophysics 299, 111-142.

Stein V. 1968. Stratigraphische Untersuchungen im Unteren Muschelkalk Südniedersachsens. Zeitschrift der Deutschen Gesellschaft für Geowissenschaften 117, 819-828. 
Stille, H., 1932. Geol. Karte von Preussen, Blatt Göttingen Nr.2520 , mit Erläuterungen,40 S., Preuß. Geol. Landesanst., Berlin.

Stille, H., Lotze, F., 1933. Erläuterungen zur geologischen Übersichtskarte der Umgebung von Göttingen (Hochschulexk. Kte Nr.3) , 67 S., Preuß. Geol. Landesanst., Berlin.

Tanner, D.C., 2011. Exkursion nördliches Leinetal, dieser Band.

Tanner, D.C., Leiss B., Vollbrecht A. \& The Göttingen Geothermal Group, 2010. The role of strike-slip tectonics in the Leinetal Graben, Lower Saxony. Zeitschrift für Geologische Wissenschaften 161/4, 369-377.

Vath, U., 2005. 5.4. Der Keuper im südlichen Niedersachsen. Courier Forschungs-Institut Seckenberg 253, 163-178.

Wunderlich, H.G. 1957. Tektogenese des Leinetalgrabens. Geologische Rundschau 46, 372-413.

Wunderlich, H.G, 1966. Ausweitung und Einengung an saxonischen Bauformen Südniedersachsens. Zeitschrift der Deutschen Gesellschaft für Geowissenschaften 116(1964), 683-695.

http://www.ngdc.noaa.gov/geomagmodels/

Declination.jsp 


\title{
3D-Störungsmorphologie bestimmt durch LIDAR-Messungen
}

\author{
David Tanner (GZG, LIAG Hannover), Stefan Prüfer (BGR Hannover), \\ Dirk Kuhn (BGR Hannover) und Charlotte Krawczyk (LIAG Hannover)
}

\begin{abstract}
Zusammenfassung - Mit Hilfe eines Laser Scanners (LIDAR) wurde eine $120 \mathrm{~m}$ lange und $20 \mathrm{~m}$ hohe, dreidimensionale Störungsfläche in Kalksteinen der Trias, mit einer räumlichen Auflösung von $4 \mathrm{~cm}$, aufgenommen. Mittels eines Softwareverfahrens wurde die Störungsfläche analysiert und beschrieben. Fünf unterschiedliche Strukturen wurden erkannt. Sie sind, in absteigender Rangordnung, 1) lange und kurze Segmente der Störungsfläche, wobei der Winkel zwischen den Elementen ca. $75^{\circ}$ beträgt, 2) jedes lange Segment ist in sich schraubenförmig, 3) die Störungsfläche fällt zwischen $20-80^{\circ}$ ein, 4) Unebenheiten (Amplitude 10-20 cm), die parallel zur Einfallsrichtung der Störungsfläche sind, 5) Eine Gaußsche Kurvaturanalyse stellt die Präsenz von Zonen unterschiedlicher Kurvatur dar, die eine Dimension von $4 \mathrm{~m}$ besitzen. Die fünfte Struktur ist mit dem ersten Wachstum der Störung verbunden. Strukturen 1 und 2 sind eine Konsequenz von $\mathrm{P}$ und $\mathrm{R}^{\prime}$ Riedelscherflächen, die sich unter sinistraler Seitenverschiebung entwickelt haben. Die vierte Struktur wurden durch Versatz auf der Störung in der Einfallsrichtung produziert.
\end{abstract}

\begin{abstract}
We used a laser-ranging (LIDAR) device to measure a $120 \mathrm{~m}$ long und $20 \mathrm{~m}$ high, three-dimensional fault surface within Triassic carbonates, with a spatial resolution of $4 \mathrm{~cm}$. Using software techniques we analysed the surface und determined five different fabrics. They are, in order of decreasing scale, 1) long und short segments of the fault surface, where the angle between the different segments is ca. $75^{\circ}, 2$ ) each long segment is helicoidal within itself, 3) the fault surface dips between $20-80^{\circ}, 4$ ) asperities (amplitude $10-20 \mathrm{~cm}$ ) that are parallel to the dip direction of the fault, 5) Gaussian curvature analysis reveals the presence of zones of different curvature within the fault surface with dimensions of ca. $4 \mathrm{~m}$. Fabric 3 is due to the stiffness of the bedding. Fabric 5 reflects the initial growth of the fault at spaced initiation points. Fabrics 1 und 2 are due to Riedel $P$ und $R^{\prime}$ planes which developed under sinistral strike-slip movement. Fabric 4 was caused by subsequent dip-slip movement.
\end{abstract}

Schlüsselworte—Laser-Scanner, Störung, Kinematik

\section{Einleitung}

LIDAR (auf englisch: Light Detection und Ranging, zu Deutsch: Lichterkennung und Entfernungsmessung) ist eine Methode der Fernerkundung, die ein Laserimpulsverfahren verwendet, um Informationen über ein entferntes Ob-

- David Tanner - GZG, jetzt Leibniz-Institut für Angewandte Geophysik, Stilleweg 2, D-30655 Hannover. DavidColin.Tanner@liag-hannover.de

- Stefan Prüfer - Bundesanstalt für Geowissenschaften und Rohstoffe, Stilleweg 2, D-30655 Hannover.s.pruefer@bgr.de

- Dirk Kuhn - Bundesanstalt für Geowissenschaften und Rohstoffe, Stilleweg 2, D-30655 Hannover. d.kuhn@bgr.de

- Charlotte Krawczyk - Leibniz-Institut für Angewandte Geophysik, Stilleweg 2, D-30655 Hannover. lotte@liag-hannover.de jekt zu bekommen (Cracknell und Hayes, 2006; Kemeny et al., 2007). Typische Verwendungen sind: Luftvermessung zur Erzeugung digitaler Geländemodelle (DGM) und Kartierung von Architektur. Für geologische Zwecke ist der Hauptverwendungszweck die Erstellung digitaler Aufschlussmodelle (DOMs), um virtuelle geologische Aufschlüsse zu produzieren (Ahlgren und Holmlund, 2002; Buckley et al., 2008; Clegg et al., 2005; Hennings et al., 2000; McCaffrey et al., 2005; Pringle et al., 2004). Eine bestimmte Anzahl von Autoren haben dreidimensionale Störungsmorphologien untersucht, wo die Aufschlussverhältnisse dies zulassen; meist in aktiven Steinbrüchen (Jones et al., 2009b; Wilson et al., 2005). Sinnvolle Verfahren brauchen eine hohe Auflösung, was einen großen Datensatz und spezialisierte Software für die Bearbeitung, erfordert (Kemeny 


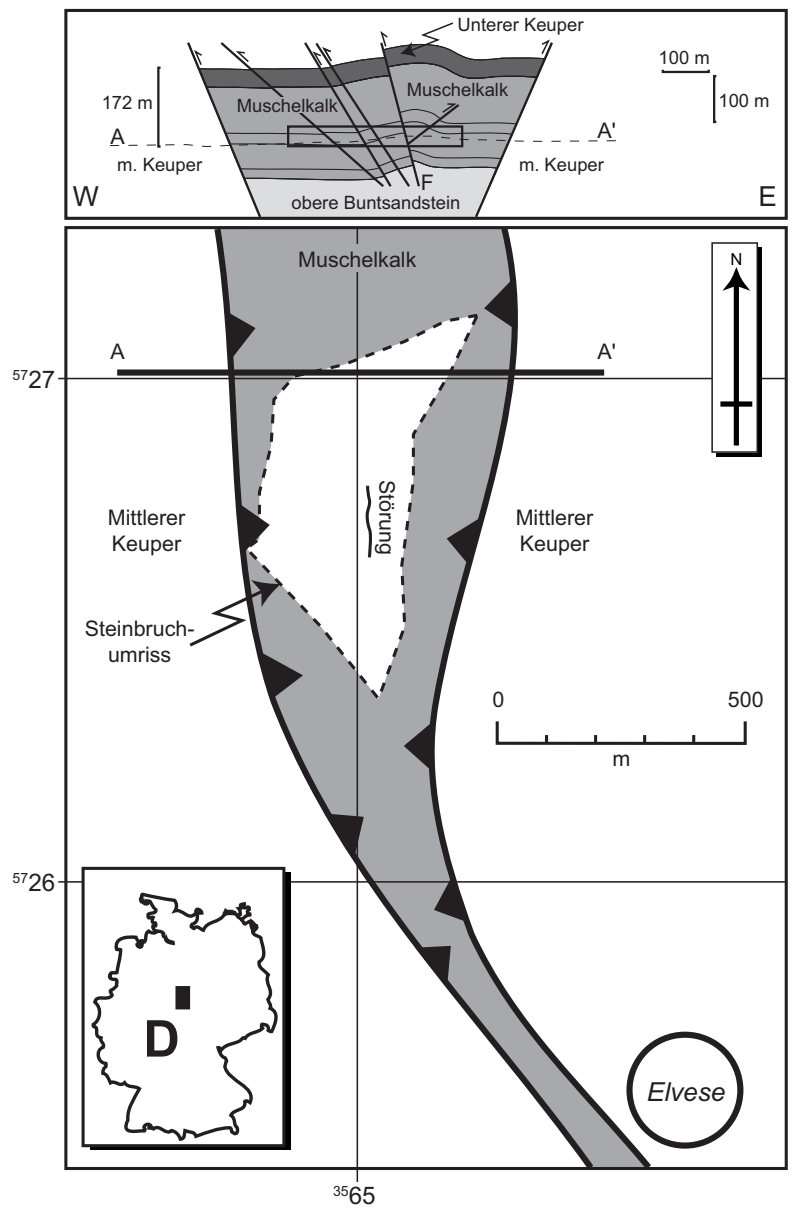

Abbildung 1. Unten: Vereinfachte geologische Karte des Gebietes um den Elveser Steinbruch, die zeigt, dass hier Muschelkalk auf mittlerem Keuper nach Ost und West überschoben wurde. Oben: Profil entlang der Linie A-A', basierend auf der in der Nordwund des Steinbruchs beobachteten Struktur. Der Versatz der äußersten Überschiebungen wird auf $172 \mathrm{~m}$ konservativ geschätzt. Die gestrichelte Linie stellt die Topographie dar. Koordinaten im deutschen GaussKrüger System.

et al., 2007).

Störungsflächen sind exzellente Ziele für LIDAR Analyse, da sie so selten in drei Dimensionen aufgeschlossen sind; im natürlichen Aufschluss sind sie meist nur in zwei Dimensionen zu sehen (typischerweise in sub-vertikalen Aufschlüssen). Störungen werden häufig im Rahmen zwei- und dreidimensionaler Seismik erkannt und interpretiert, jedoch mit einer sehr niedrigen Auflösung (meist schlechter als etwa $25 \mathrm{~m}$; Berkhout 1985). Kleinmaßstäbliche Strukturen, unterhalb seismi- scher Auflösungsmöglichkeiten, werden daher als ,sub-seismisch' bezeichnet (Pickering et al., 1996; Lohr et al., 2008). LIDAR-Fernerkundung kann diese Lücke schließen. Durch die geometrische Analyse am Rechner, werden im Gegensatz zur geologischen Betrachtung im Aufschluß vermehrt Strukturen, deren Wellenlänge größer als die Aufschlussgröße ist, erkannt.

Diese Arbeit basiert auf der Erfassung einer $120 \mathrm{~m}$ langen und $20 \mathrm{~m}$ hohen Störungsfläche mit LIDAR, die innerhalb triasischer Muschelkalk Karbonate in einem Steinbruch bei Elvese im Leinetal aufgeschlossen war ${ }^{1}$ (Abb. 1). Der Datensatz hat eine räumliche Auflösung von $4 \mathrm{~cm}$. Wir stellen eine geometrische Analyse der Störungsfläche vor, und zeigen fünf verschiedene Strukturen auf der Störungsfläche, mit Skalen zwischen $2 \mathrm{~cm}$ bis $20 \mathrm{~m}$. Danach interpretieren wir die Strukturen bezüglich Störungskinematik und -wachstum.

\section{Geologischer Rahmen}

Die analysierte Störungsfläche stammt aus dem Elveser Steinbruch (lat/long. 51 $40^{\prime} 19^{\prime \prime} \mathrm{N}$, $9^{\circ} 56^{\prime} 19^{\prime \prime} \mathrm{E}$ ), nahe Göttingen (Abb. 1). Der Steinbruch schließt ausschließlich triassischen $\mathrm{Mu}-$ schelkalk (Anis; Unterer Wellenkalk) auf, der aus fast reinen mikritischen Karbonatschichten mit mergeligen Zwischenschichten besteht. Die Schichtmächtigkeit variiert von 2 bis $25 \mathrm{~cm}$.

Der Steinbruch befindet sich innerhalb eines N-S/NW-SE-streichenden Lineaments (des sogenannten ,Ahlsburg Lineaments'; Stille und Lotze, 1931; Jordan, 1984; Tanner et al., 2010). Entlang dieses Lineaments ist der Muschelkalk über den mittleren Keuper nach Ost und West überschoben (Abb. 1). Basierend auf den stratigraphischen Mächtigkeiten nach Jordan (1984), wurde berechnet, dass der vertikale Versatz auf diesen Störungen mindestens $172 \mathrm{~m}$ beträgt (Abb. 1). Innerhalb des Ahlsburg Lineaments ist der $\mathrm{Mu}-$ schelkalk offen gefaltet und gestört (siehe E-W streichendes Profil, Abb. 1). Die Störung, die in dieser Arbeit analysiert wurde, ist wahrscheinlich die Verlängerung von Störung F in dem E-W Profil (Abb. 1). Weil der Aufschluß $20 \mathrm{~m}$ hoch ist und der untere Muschelkalk in diesen Gebiet eine Mächtigkeit von ca. $100 \mathrm{~m}$ aufweist (Dünkel und Vath, 1990), läßt sich auf einen vertikalen Versatz dieser Störung von weniger als $80 \mathrm{~m}$ schließen.

1. war, weil seit März 2010, durch fortschreitenden Bergbau die Hälfte der Störungsfläche vernichtet worden ist 

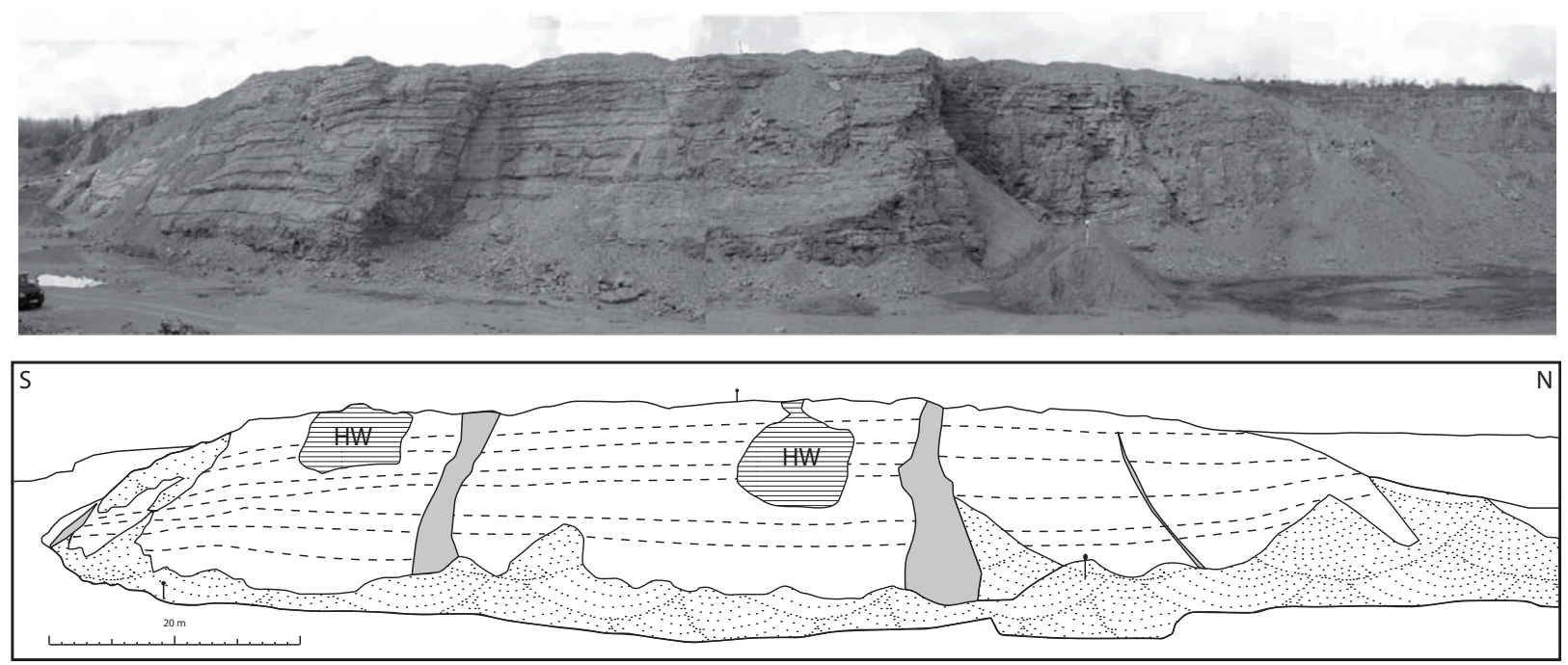

Abbildung 2. Oben; Panorama-Abbildung der Störung. Unten; die Hauptmerkmale der Störungsfläche. Graue Bereiche sind Segmente der Störungsfläche mit einem stumpfen Winkel zur Hauptfläche. Gestrichelte Bereiche sind Fragmente des Hangenden (HW), die noch an der Störung kleben. Schutt und loses Gestein sind gepunktet.

\section{Methoden}

\subsection{Im Gelände}

Die Störungsfläche ist etwa $120 \mathrm{~m}$ lang und $20 \mathrm{~m}$ hoch. Hauptsächlich ist nur der Liegendblock aufgeschlossen (Abb. 2). Wir verwendeten einen Optech Ilris 3D Laserscanner, der mit einer Entfernung zur Störungsfläche von $30 \mathrm{~m}$ aufgebaut wurde. Die geographischen Koordinaten des Scanners wurden mittels GPS Gerät festgestellt. Ein räumliche Aufösung von $4 \mathrm{~cm}$ wurde gewählt, woraus sich für den ursprünglichen Datensatz etwa 1.5 Million Punkte ergeben. Das verwendete LIDAR Gerät hat eine Entfernungsmessgenauigkeit von $7 \mathrm{~mm}$ bei einer Entfernung zum Objekt von $100 \mathrm{~m}$ (Optech, 2010).

\subsection{Geometrische Analyse der Störungs- fläche}

Die folgende Analyse wurde mit der Software 3Dmove ausgeführt (Midlund Valley Exploration Ltd, 2010). Teile der Punktwolke des Laserscans, die nicht zu Störung gehörten, wie z.B. Lose Steine, Pflanzen, und die Teile des Hangenden, die noch an der Störungsfläche klebten, wurden manuell entfernt. Die daraus resultierende Punktwolke wurde für die Erzeugung einer Fläche mittels Tessellation verwendet.

Die Software-Werkzeuge in 3Dmove wurden verwendet, um die Raumlage der Flächendreiecke

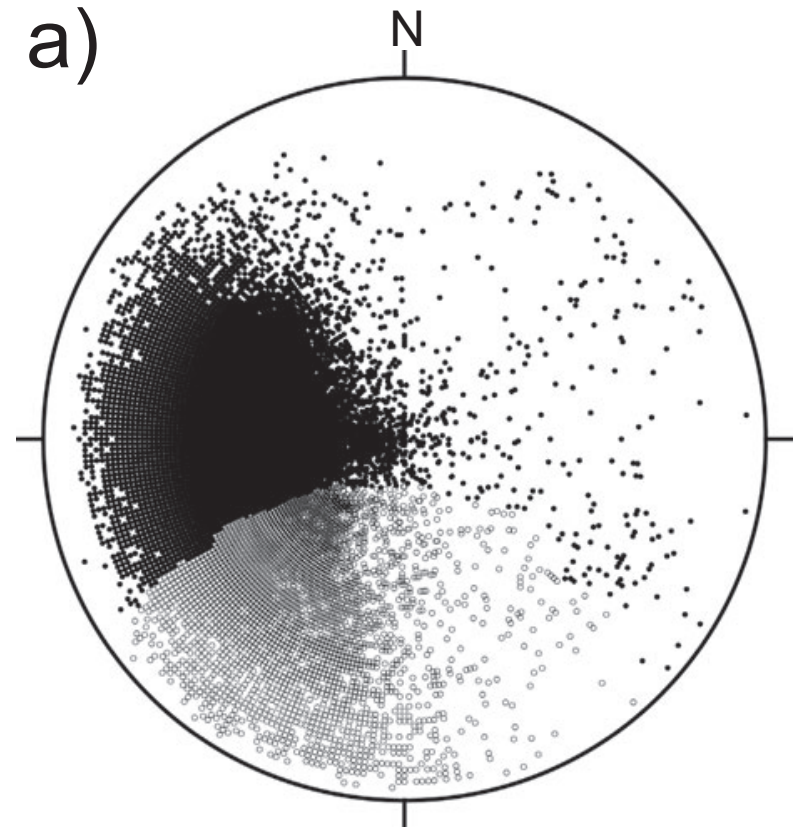

Abbildung 3. a) Schmidt-Netz Projektion der Dreieckspole der konstruierten Störungsfläche $(n=137000)$. Volle und offene Kreise stammen von den langen und kurzen Segmenten der Störungsfläche. 

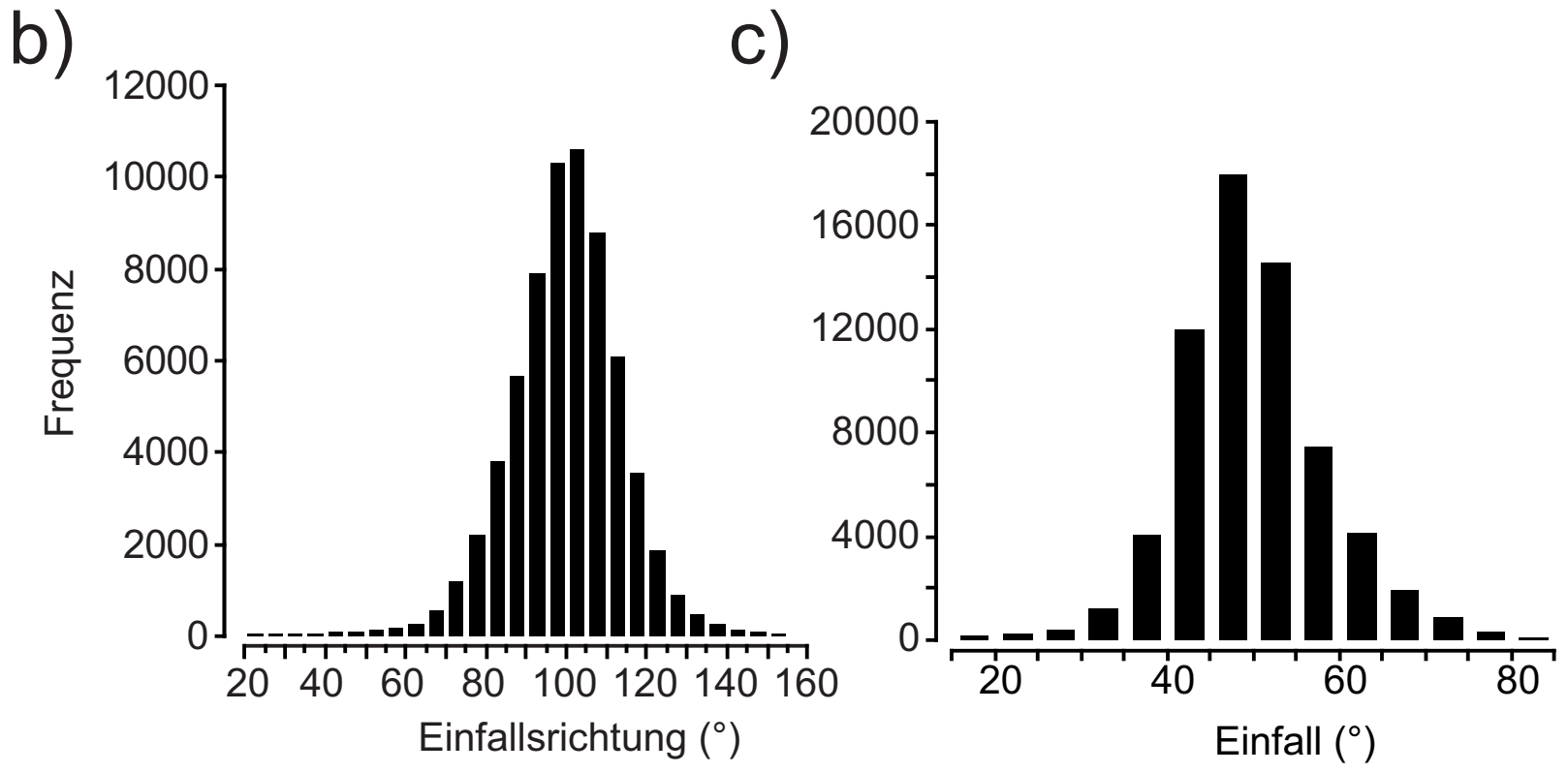

Abbildung 3. b) Histogram der Einfallsrichtung aller Dreiecke, c) Histogram der Einfallswinkel aller Dreiecke.

zu analysieren, und diese Attribute wieder auf die Fläche zu importieren. Die Werkzeuge, die hier verwenden wurden, sind: Filtern zum Erfassen und Abfärben von Einfallsrichtung, Einfall und Gaußscher Krümmung, die alle auf die Störungsfläche zurück projiziert wurden, Isolinien und Schnittdarstellungen, Raumlagebestimmung von Dreiecken, und verschiedene Licht- und Projektionstechniken.

Messung von Gaußscher Krümmung ist abhängig vom Lange (Lisle, 1994). In dieser Arbeit werden die besten Ergebnisse in einem Lange von $12 \mathrm{~cm}$, also der dreifachen räumlichen Auflösung, erzeugt.

\section{Ergebnisse}

Die Analyse der Störungsfläche zeigt, dass die Einfallsrichtung über $140^{\circ}$ und der Einfallwinkel über $60^{\circ}$ variiert (Abb. 3). Die grosse Varianz liegt zum Teil in der Tatsache begründet, dass die Störungsfläche aus langen und kurzen Segmenten besteht, die zu einander mit einen Winkel von $75 \pm 5^{\circ}$ stehen. Dies wird Gefüge 1 genannt.

Innerhalb der langen Segmente der Störung fällt die Störungsfläche immer steiler nach Süden ein, bis eine Verbindung mit den kurzen Segmenten, wie z.B. die Konvergenz der Streich- linien zeigt, hergestellt wird (Abb. 4b \& c). Deshalb können die langen Segmente als ,schraubenförmig' bezeichnet werden (Gefüge 2).

Der Einfallen der Störung (gleicht dem Abschnittswinkel des Liegenden, weil die Schichten des Liegenden horizontal einfallen) variiert stark senkrecht zur Schichtung (d.h. in der Einfallsrichtung, Abb. 5; Gefüge 3). Profile der Störung zeigen, dass für die Bänke (die aus mehreren Schichten bestehen), die etwa $10-50 \mathrm{~cm}$ hoch sind, die Störungseinfallswinkel zwischen 30 bis über $70^{\circ}$ variieren $(\mathrm{Abb} .5 \mathrm{~b})$. Oft liegen steile und flache Einfallswinkel nebeneinander.

Wenn die Einfallsrichtung der Störungsdreiecke auf der Störung dargestellt wird, ist ein starkes einfallsrichtungsparalleles Gefüge (Gefüge 4) zu sehen, wobei die Störungseinfallsrichtung um bis $\mathrm{zu} 40^{\circ}$ variiert. Abbildung 6 zeigt, dass das Gefüge aus durchgehenden, über $20 \mathrm{~m}$ langen, in Einfallsrichtung der Störung liegenden Oberflächenunebenheiten auf der Störungsfläche gebildet wird. Diese besitzen Amplituden von 10-20 cm.

Die Analyse der Gaußschen Krümmung (GC; Abb. 7) zeigt deutlich, dass es ein Muster von negativ und positiv GC gibt (Gefüge 5). Jede GCAnomalität hat die Dimensionen von etwa $4 \mathrm{~m}$ in Höhe und Breite. 

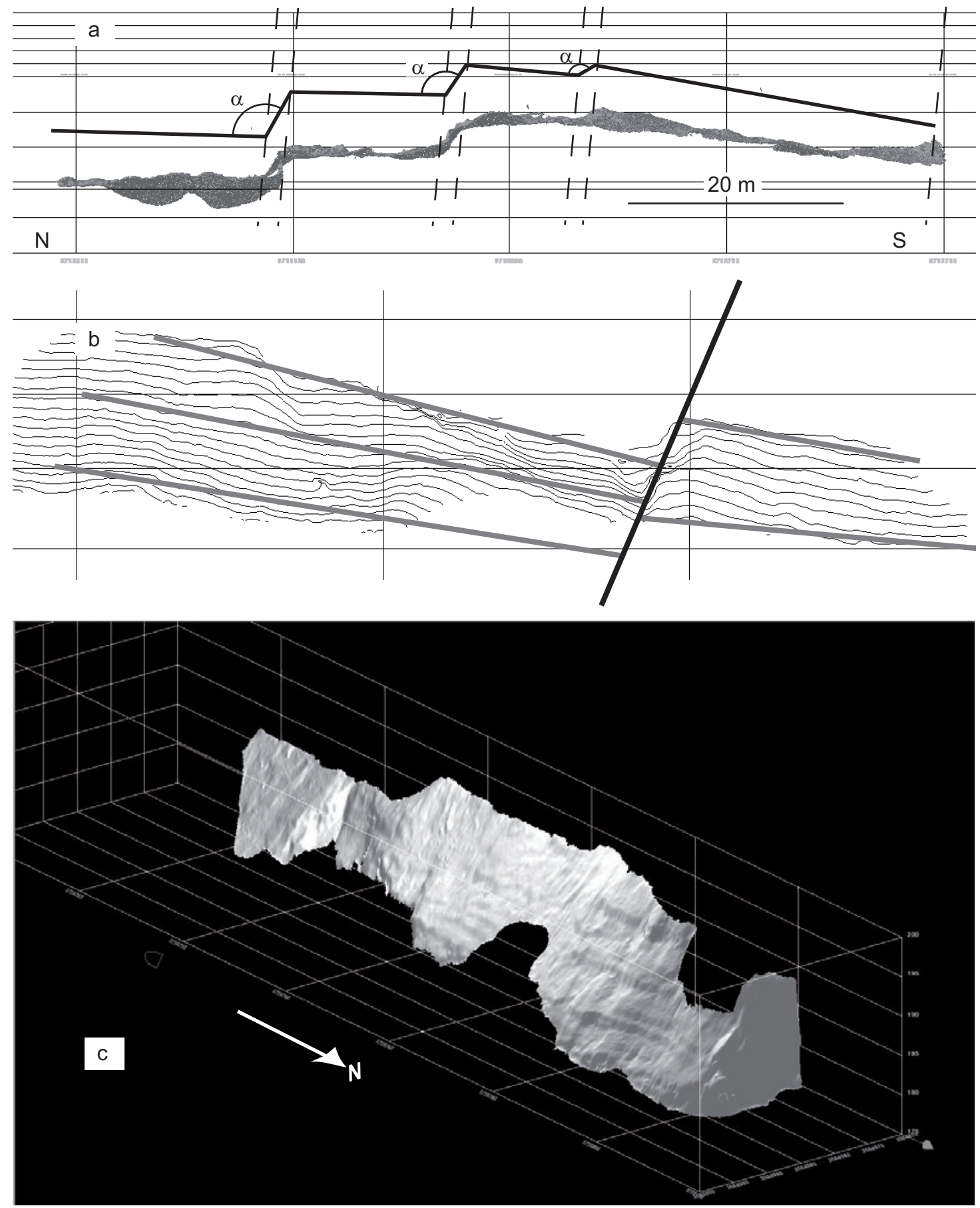

Abbildung 4. a) Blick von oben, in Richtung der Einfallsrichtung. Die Störungsfläche besteht aus vier langen, N-S streichenden Segmenten, getrennt von drei ESE-WSW streichenden Segmenten. Der Winkel zwischen langem und kurzem Segment beträgt $75 \pm 5^{\circ}$. Das Gebiet, das in b) gezeigt ist, ist als Rechteck dargestellt. b) Nahaufnahme des zentralen Teils der Störungsfläche. Die Störungsfläche wird mit Isolinien der Elevation im Abstund von $2 \mathrm{~m}$ gezeigt. Dicke graue Linien zeigen den generellen Trend der Isolinien, Schwarze Linien repräsentieren die kurzen Störungssegmente. Streichlinien auf dem langen Segment konvergieren (d.h. der durchschnittliche Einfallswinkel der Störungsfläche wird grösser), in Richtung der kurzen Segmente (nach Süden). Das zeigt, dass die langen Segmente schraubenförmig sind. c) Schwarzweiß-Bild der Störung in drei Dimensionen. Licht von Nordost. Hier sieht man, dass das lange Störungssegment nach Süden steiler wird. 

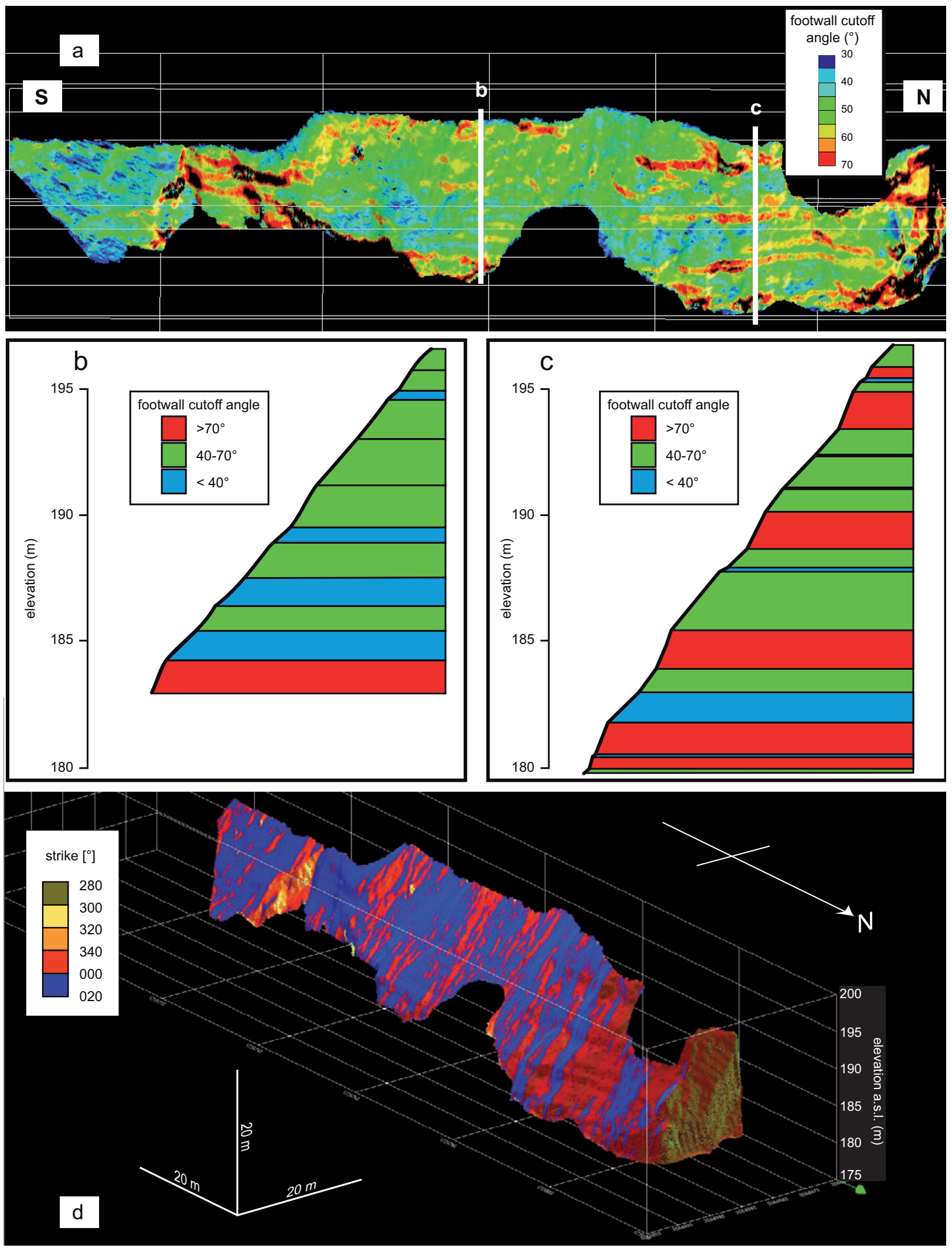

Abbildung 5. a) Blick auf die Störung, parallel der Störungsfläche. Die Dreiecksflächen sind nach Abschnittswinkel gegen das Liegende gefärbt. Die Winkel variieren von $30-70^{\circ}$, wobei Bereiche gleicher Winkel horizontal, parallel der Schichtung liegen. b) und c) Profile der Störung (Lokation siehe a.). Unterschiedlich liegende Abschnittswinkel werden von Bänken unterschiedlicher Steifen verursacht. d) Schräger, paralleler, dreidimensionaler Blick auf die Störung, wobei die bestehenden Dreiecke abhängig von der Einfallsrichtung gefärbt sind. Ein deutliches, einfallsrichtungsparalleles Gefüge ist zu sehen. 

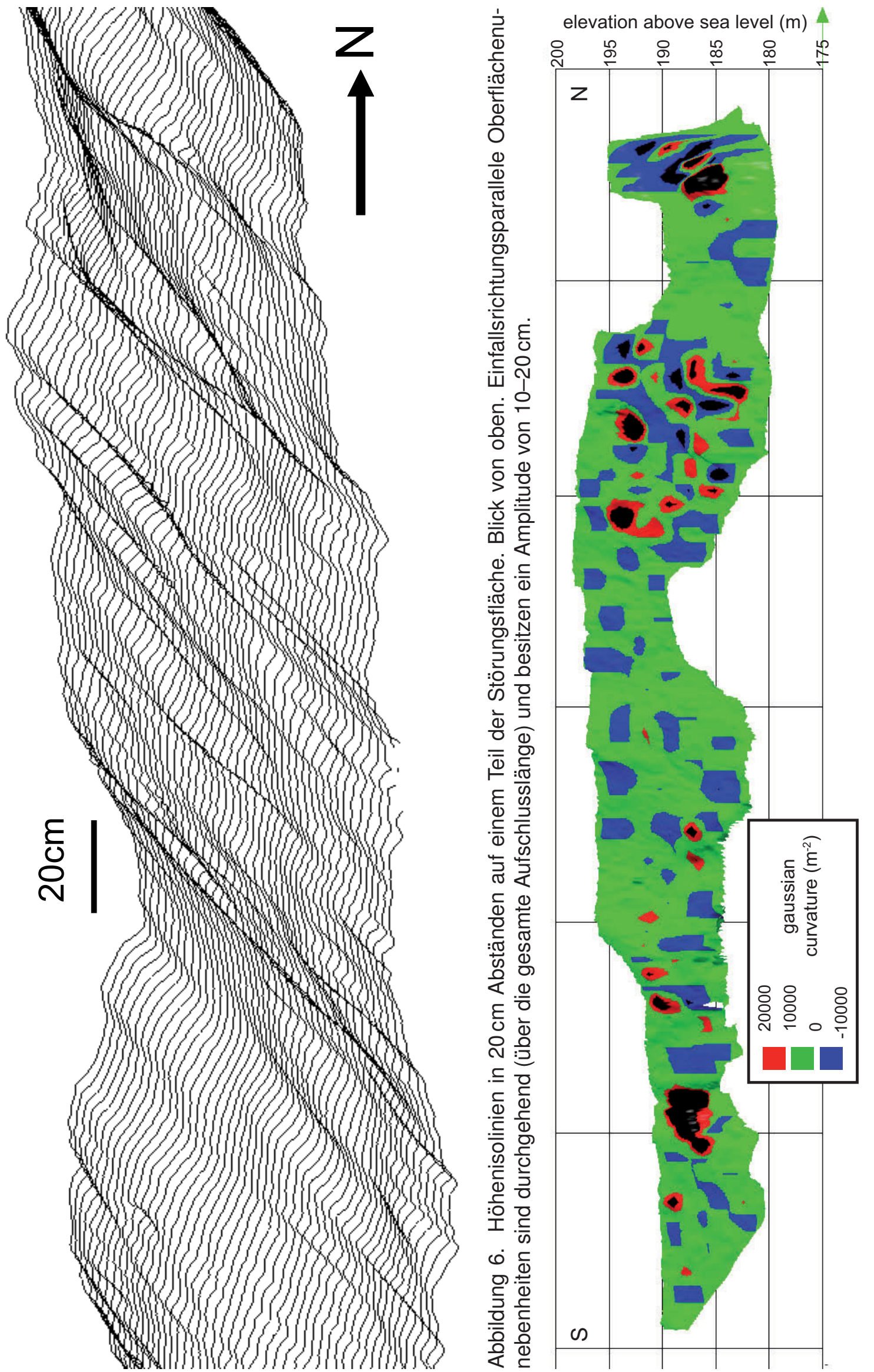

তิ

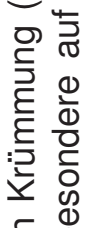
든 灾 ()

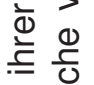
을 : 엉 응 :인 क्ष (1) ป ํำ : 은 品 ঠั ঠे ปั 电 ঠे $\frac{\overline{0}}{\overline{0}}$ .ᄃ (1) 穴 : ত 임 ฏ 㐫 ஸे ฮั 응 Ф

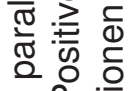
음. Ј टं ฮ :음 응 을 홍 ডัড บํㅓㅇ

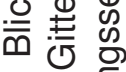
N

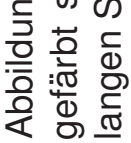




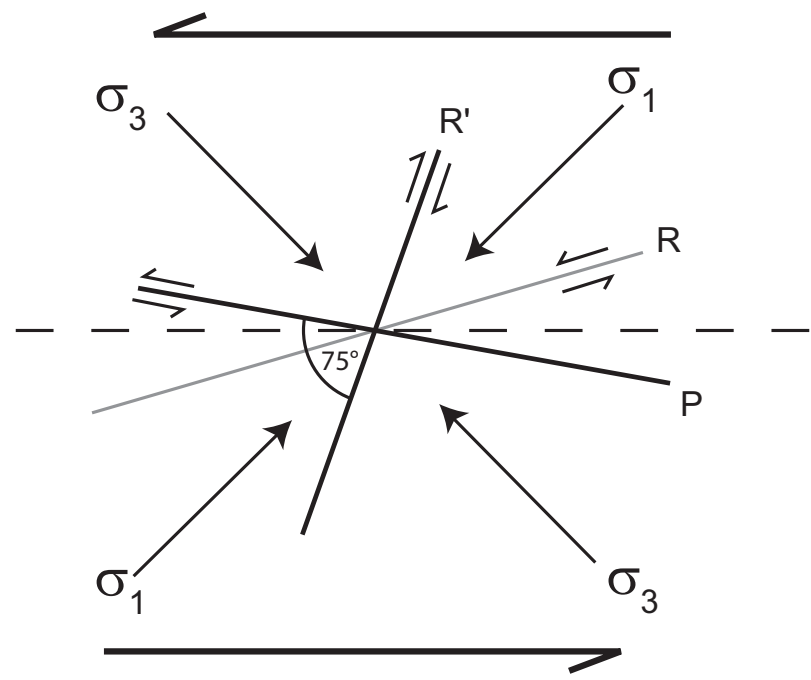

Abbildung 8. Riedel Schersystem für linkslaterale Scherung (Riedel 1929). In dieser Arbeit wird angenommen, dass lange bzw. kurze Segmente der Störungsfläche mit den P- bzw. R'-Flächen zusammenhängen. Der Winkel zwischen $\mathrm{P}$ - und $\mathrm{R}^{\prime}$-Fläche beträgt jeweils $75^{\circ}$.

\section{Interpretation und Diskussion}

Wir interpretieren Gefüge 1, d.h. die langen und kurzen Segmente der Störung, als $\mathrm{P}$ und $\mathrm{R}^{\prime}$ Riedelscherflächen (Abb. 8; Riedel, 1929). Das heißt, dass die Störung als sinistrale Seitenverschiebung zu sehen ist. Man erkennt, dass die Winkel zwischen den Störungssegmenten (Fig. 2; $75 \pm 5^{\circ}$ ) und zwischen $\mathrm{P}$ und $\mathrm{R}^{\prime}$ Riedelscherflächen (Abb. 8) sich gleichen. Gefüge 2, die geschraubte Form der langen Segmente, wird oft in Riedelscherflächen und Störungen erkannt (Barlett et al. 1981, Wilcox et al. 1973). Sie wird durch mit der Tiefe zunehmende Scherspannung erklärt. Dies ist typisch für eine Störung die durch Basementversatz bedingt ist (Barlett et al., 1981).

Es wurde dargestellt, dass Gefüge 3 (mit 30 bis $70^{\circ}$ Störungseinfallen) sich mit der Bankung ändertändert, entsprechend der Lithologie des unteren Muschelkalkes in diesem Aufschluss. Die Störungseinfallen hängt von der Steifheit der Schichtung ab.

Gefüge 4, die Unebenheiten parallel zur Einfallrichtung müssen durch Versatz auf der Störung in diese Richtung entstanden sein. Die Entstehung eines neuen Gefüges durch Störungsversatz ist nicht unbekannt; z.B. die Arkitsa-Störung in Mittel Griechenland; dort gibt es ebenfalls einfallsrichtungsparallele Unebenheiten (Jones et al. 2009a). Die Besonderheit hier ist, wie stark dieses Gefüge durchgeprägt ist und auch andere Gefüge überlagert.

GC-Analyse zeigt die Existenz von Panelen einheitlicher (negativ oder positiv) GC auf der Störungsfläche (Gefüge 5), wobei jedes Panel Dimensionen von etwa $4 \mathrm{~m}$ Höhe und Breite besitzt. Es wird postuliert, dass sie ursprüngliche Nukleierungspunkte der Störung repräsentieren. Diese wuchsen, wie im Störungswachstumsmodell von Walsh et al. (2003) beschrieben, bis zum Zusammentreffen und anschließender Vereinigung mit Nachbarpunkten, bis zur Entstehung der Störungsfläche. Es ist wichtig zu zeigen, dass diese Gefüge noch erkennbar sind, auch wenn sie von späteren Gefügen überdeckt sind.

Sämtliche Gefüge, mit Ausnahme von Gefüge 1, können als sub-seismisch klassifiziert werden. Deshalb sind sie im Rahmen einer seismichen Untersuchung nicht erkennbar, insbesondere wenn die Störung nicht horizontal liegt. Obwohl alle Gefüge mit der Kinematik der Störung zusammenhängen und nicht aus der umliegenden Geologie ableitbar sind, kann Gefüge 3 indirekt an Bohrungslogs beobachtet werden.

Wir postulieren, dass alle Gefüge dieser Störung durch sinistrale Seitenverschiebung, gefolgt von einfallsrichtungsparallelem Versatz (ob Ab- oder Aufschiebung ist unklar) verursacht wurden. Die Störung ist Teil der in Abb. 1 dargestellten geologischen Struktur (positive Flower-Struktur).

\section{Zusammenfassung}

Mit Hilfe eines LIDAR-Geräts wurde eine $120 \times$ $20 \mathrm{~m}$ große Störungsfläche analysiert, die in triasischem Kalkstein in einem aktiven Steinbruch aufgeschlossen war. Folgende fünf unterschiedliche Gefüge wurden auf der Störungsfläche bestimmt:

1) Kurze und lange Segmente der Störung; Segmente beschreiben einen Winkel von etwa $75^{\circ}$. Wir interpretieren sie als $\mathrm{R}^{\prime}$ und $\mathrm{P}$ Riedelscherflächen in einem sinistralen Seitenverschiebungssystem.

2) Schraubenförmige Form der langen Störungssegemente.

3) Änderung der Einfallwinkel zwischen 30 und $70^{\circ}$ bei Schichtwechseln, abhängig von der Steifheit des Materials.

4) Dip-slip Eigenschaften, die auffielen, nachdem der Azimuth des Einfallens der Störung analysiert worden war. Höchstwahrscheinlich 
sind diese auf eine dip-slip Bewegung zurückzuführen.

5) Auf der Störungsoberfläche liegende Panele mit einem Durchmesser von ca. $4 \mathrm{~m}$, die eine positive oder negative Gaußsche Kurvatur aufweisen. Diese sind vermutlich das Resultat von initialen sekundären Scherflächen, die an verschiedenen Stellen der Hauptstörung angelegt wurden und bei fortschreitender Bewegung zusammenliefen.

Die Untersuchung läßt die Komplexität einer dreidimensionalen Störungsfläche, speziell im subseismischen Maßstab, erkennen. Es wird außerdem deutlich, dass, bei Betrachtung einer Störung in weniger als drei Dimensionen oder bei schlechter Auflösung, eine Anzahl von Merkmalen übersehen wird. Um solche Informationen aus unbearbeiteten LIDAR scans zu extrahieren, ist der Einsatz spezieller Software notwendig. Abhängig vom Zustand ihrer Erhaltung können solche Gefüge Hinweise auf die Art der Störungsentstehung und -kinematik geben.

\section{Danksagung}

Wir danken Nadine Friese und Frithjof Bense für ihre Hilfe im Gelände. Wir danken Axel Vollbrecht für viele anregende Diskussionen. Dank auch an die Südniedersächische Kalksteinwerke GmbH \& Co. KG für die Erlaubnis, ihren Steinbruch zu betreten.

\section{Literatur}

Ahlgren, S., Holmlund, J. 2002. Outcrop scans give new view. American Association of Petroleum Geologists Explorer July 2002.

Barlett, W., Friedman, M., Logan, J. 1981. Experimental folding und faulting of rocks under confining pressure. Part IX: Wrench faults in limestone layers. Tectonophysics 79, 255-277.

Berkhout, A.J. 1985. Seismic resolution: a key to detailed geologic information. World Oil 201, 47-51.

Buckley, S., Howell, J., Enge, H., Kurz, T. 2008. Terrestrial laser scanning in geology: data acquisition processing und accuracy considerations. Journal of the Geological Society, London $165,625-638$.

Clegg, P., Trinks, I., McCaffrey, K.J., Holdsworth, R.E., Jones, R.R., Hobbs, R., Waggott, S. 2005. Towards the virtual outcrop. Geoscientist 15, 8-9.
Cracknell, A.P., Hayes, L. 2006. Introduction to Remote Sensing. Taylor und Francis, London. (2nd ed.) edition. OCLC 70765252.

Dünkel, H., Vath, U. 1990. Ein vollstandiges Profil des Muschelkalks (Mitteltrias) der Dransfelder Hochflache, SW Göttingen (Südniedersachsen). Geologisches Jahrbuch Hessen 118, 87-126.

Hennings, P., Olson, J., Thompson, L. 2000. Combining outcrop data und three-dimensional structural models to characterise fractured reservoirs: an example fromWyoming. American Association of Petroleum Geologists Bulletin 84, 830-849.

Jones, R.R., Kokkalas, S., McCaffrey, K.J. 2009a. Quantitative analysis und visualization of nonplanar fault surfaces using terrestrial laser scanning (LIDAR) - the Arkitsa Fault, central Greece, as a case study. Geosphere 5, 465-482.

Jones, R.R., McCaffrey, K.J., Clegg, P., Wilson, R., Holliman, N.S., Holdsworth, R.E., Imber, J., Waggott, S. 2009b. Integration of regional to outcrop digital data: 3D visualisation of multi-scale geological models. Computers \& Geosciences 35, 4-18.

Jordan, H. 1984. Geologische Karte von Niedersachsen, 1:25000. Niedersächsisches Landesamt für Bodenforschung, Hannover.

Kemeny, J., Turner, K., Norton, B. 2007. LIDAR for rock mass characterization: Hardware, software, accuracy und best-practices, in: Tonon, F., Kottenstette, J.T. (Eds.), Laser und Photogrammetric Methods for Rock Face Characterization. American Rock Mechanics Association, Alexandria, VA, pp. 49-61.

Lisle, R.J. 1994. Detection of zones of abnormal strains in structures using gaussian curvature analysis. American Association Petroleum Geologists, Bulletin 78, 1811-1819.

Lohr, T., Krawczyk, C.M., Tanner, D.C., Samiee, R., Endres, H., Thierer, P.O., Oncken, O., Trappe, H., Bachmann, R., Kukla, P.A. 2008. Prediction of sub-seismic faults und fracture deformation, und well data on an example of deformation around an inverted fault. American Association Petroleum Geologists, Bulletin 92, 473-385.

McCaffrey, K.J., Jones, R.R., Holdsworth, R.E., Wilson, R., Clegg, P., Imber, J., Holliman, N., Trinks, I. 2005. Unlocking the spatial dimension: digital technologies und the future of geoscience fieldwork. Journal of the Geological Society London 162, 1-12. 
Midlund Valley Exploration Ltd 2010. 3DMove 2010.1.

Optech 2010. Technical overview of the ILRIS system.

Pickering, G., Bull, J.M., Sanderson, D.J. (Eds.) 1996. Scaling of fault displacements und implications for the estimation of sub-seismic strain. Volume 99 of Modern developments in structural interpretation, validation und modelling. Geological Society of London, London.

Pringle, J., Westerman, A., Clark, J., Drinkwater, N., Gardiner, A. 2004. 3D high-resolution digital models of outcrop analogue study sites to constrain reservoir model uncertainty: an example from Alport Castles, Derbyshire, UK. Petroleum Geoscience 10, 343-352.

Riedel, W. 1929. Zur Mechanik geologischer Brucherscheinungen. Zentralblatt für Mineralogie, Geologie und Paläontologie Abhandlungen B, 354-368.

Stille, H., Lotze, F. 1931. Zur Frage der Überschiebungserscheinungen im Leinetal. Nachrichten von der Gesellschaft der Wissenschaften zu Göttingen, Mathematisch-Physikalische Klasse, 227-235.

Tanner, D.C, Leiss, B., Vollbrecht, A., the GGG 2010. The Role of Strike-Slip Tectonics in the Leinetal Graben, Lower Saxony. Zeitschrift der Deutschen Gesellschaft für Geowissenschaften 161/4, 369-377.

Walsh, J.J., Bailey, W.R., Childs, C., Nicol, A., Bonson, C. 2003. Formation of segmented normal faults: a 3-D perspective. Journal of Structural Geology 25, 1251-1262.

Wilcox, R., Harding, T., Seely, D. 1973. Basic wrench tectonics. American Association of Petroleum Geologists Bulletin 57, 74-96.

Wilson, R., McCaffrey, K.J., Jones, R.R., Imber, J., Clegg, P., Holdsworth, R.E. 2005. Lofoten has its faults! detailed fault analysis und $3 \mathrm{~d}$ digital mapping in the Norway's Lofoten Islands. Geoscientist 15, 4-9. 


\title{
Strukturgeologische 3D-Modellierung der Grabenrandstörung des Leinetals im östlichen Stadtgebiet von Göttingen
}

\author{
Jennifer Ziesch (GZG Göttingen, LBEG Hannover), David C. Tanner (GZG Göttingen, \\ LIAG Hannover), Gernot Arp (GZG Göttingen) und Josef Paul (GZG Göttingen)
}

\begin{abstract}
Zusammenfassung - Im Rahmen von verschiedenen Geothermie-Projekten wurden im östlichen Stadtgebiet von Göttingen Bohrungen beprobt, analysiert und beschrieben. Die Bohrung 2 (Tannenweg 20) durchteufte dabei die hier unter der Lias-Gruppe liegende, östliche Grabenrandstörung und traf auf Gipse des Mittleren Muschelkalkes. Für einen Aufstieg von Zechstein-Evaporiten entlang der Grabenrandstörung, so wie vormals vermutet, finden sich damit keine Belege. Unter Einbindung von weiteren Bohrungen und Fundstücken aus dem Museum des GZG sowie Fundangaben aus geologischen Kartierungen des frühen 19. Jahrhunderts hat sich der Verlauf der östlichen Grabenrandstörung gegenüber der publizierten Karte von Stille (1932) um etwa $100 \mathrm{~m}$ in Richtung Osten verschoben. Weiterhin zeigte sich in der erstellten, geologischen Karte, dass eine dextrale Seitenverschiebung eine Umbiegung der Lias-Schichten im Norden und einen dextralen Versatz der östlichen Grabenrandstörung von etwa $70 \mathrm{~m}$ verursacht. Das generierte, strukturgeologische 3D-Modell zeigt, dass die Basis der als Leithorizont genutzten Lias- $\gamma$-Kalksteinbank im nördlichen Bereich einen ausgeprägten Knick aufweist und mit $30^{\circ}$ steiler als der südliche Bereich einfällt. Ferner konnte anhand der 3D-Modellierung die Orientierung der östlichen Grabenrandstörung mit 289/55 ermittelt werden.

Abstract - Within the context of various geothermal projects, wells were sampled, analysed and described from the eastern side of the city of Göttingen. The well No. 2 (Tannenweg 20) encountered, in addition to the Lower Jurassic Lias Formation, the eastern Leinetal border fault and then gypsum layers of the Middle Muschelkalk. The previous assumption that Zechstein had risen along the border faults, could not be confirmed in this work. With the information from wells and finds from the museum of the GZG, and geological maps and their memoirs from the early $19^{\text {th }}$ Century, the trace of the main eastern border fault was moved about 100 metres toward the east. From the resulting geological map it becomes apparent that a dextral strike-slip fault caused a bend in the Liassic strata to the north and a dextral displacement of the eastern border fault by ca. 70 metres. The generated 3D structural geological model indicates that the base of Lias $\gamma$ horizon in the northern area has a pronounced bend and dips $30^{\circ}$ steeper than the southern area. By the use of 3D modelling, it was possible to determine that the orientation of the eastern border fault in this area is $289 / 55$.
\end{abstract}

Schlüsselworte-Lias $\gamma$, Jura, Göttingen, 3D-Modellierung

\section{Einleitung}

Das in Abbildung 1 aufgezeigte Arbeitsgebiet ist etwa $21 \mathrm{~km}^{2}$ groß und befindet sich im südlichen

- Jennifer Ziesch - GZG, jetzt Landesamt für Bergbau, Energie und Geologie, Stilleweg 2, D-30655 Hannover. Jennifer.Ziesch@lbeg.niedersachsen.de

- David Tanner - GZG, jetzt Leibniz-Institut für angewandte Geophysik, Stilleweg 2, D-30655 Hannover. DavidColin.Tanner@liag-hannover.de

- Gernot Arp - Geowissenschaftliches Zentrum der Universität Göttingen, Goldschmidtstr. 3, D-37077 Göttingen. garp@gwdg.de

- Josef Paul - Geowissenschaftliches Zentrum der Universität Göttingen, Goldschmidtstr. 3, D-37077 Göttingen.jpaul@gwdg.de
Niedersachsen im Landkreis Göttingen (TK 25, Blatt 4425 Göttingen). Der östliche Teil zeigt die Grabenschulter des Leinetalgrabens, mit weitflächig anstehenden Gesteinen der MuschelkalkGruppe. Der westliche Teil, also die Mittelscholle des Leinetalgrabens, wird von Gesteinen der Keuper- und Lias-Gruppe eingenommen. Diese sind allerdings überwiegend von quartären Fließerden, fluviatilen Schottern sowie Lösslehmen bedeckt, sodass sowohl Lagerungsverhältnisse, Ausstrich der einzelnen Formationen wie auch der präzise Verlauf und das Einfallen der Grabenrandstörung bis dato mit großen Unsicherheiten behaftet waren (vgl. Stille 1932, Wunderlich 1957).

Im Rahmen einer Diplomarbeit (Ziesch 2010) 


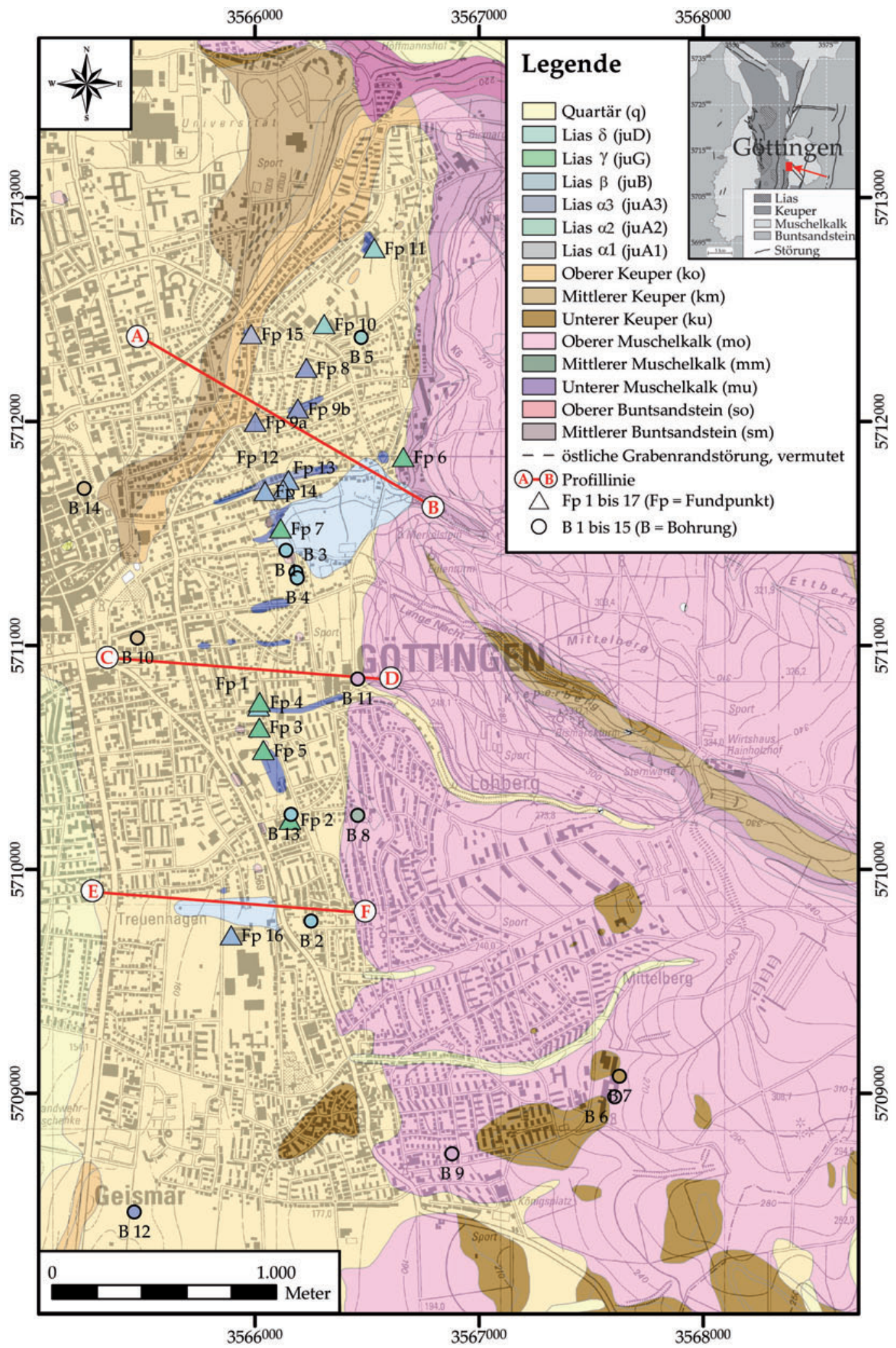

Abbildung 1. Aussschnitt aus der Geologischen Karte von Niedersachsen 1:25000, Blatt 4325 Göttingen (Stille 1932; digitalisierte Version des LBEG-Kartenservers), mit Lage der Bohrungen, Lesestein-Fundpunkte und Profilschnitte (Auflistung in Ziesch 2010); Projektion Koordinatensystem: Gauss Krüger Zone 3, Datum: Deutsches Hauptdreiecksnetz Sphäroid: Bessel 1841. Oben rechts: Lage des Arbeitsgebietes auf der vereinfachten, geologischen Übersichtskarte des Leinetalgrabens (auf Grundlage von Stille \& Lotze 1933, Quartär abgedeckt). 
wurde Bohrklein von mehreren flachen Geothermiebohrungen mit Teufen bis zu $139 \mathrm{~m}$ lithostratigraphisch ausgewertet. Von ausgewählten Gipsstein-Proben und Vergleichsproben wurden neben Haupt- und Spurenelementen (RFA) auch ${ }^{87} \mathrm{Sr} /{ }^{86} \mathrm{Sr}$-Isotopenverhältnisse zwecks stratigraphischer Zuordnung gemessen. Ziel der vorliegenden Publikation ist es auf Grundlage dieser neuen Bohrungen, unter Hinzuziehung von publizierten Bohrprofildaten, Literaturangaben sowie Sammlungsmaterial (Handstücke, Fossilien), ein räumliches strukturgeologisches 3D-Modell sowie eine abgedeckte, geologische Karte für das östliche Stadtgebiet von Göttingen vorzustellen.

\section{Material und Methoden}

Das Bohrklein von Bohrung 1, 2, 6 und 7 (Abb. 1) wurde alle zwei bzw. drei Meter mit einem Sieb abgefangen, im Labor gespült, geschlämmt und mit einem Binokularmikroskop analysiert. Als weitere Datengrundlage für das 3D-Modell dienten die vom Landesamt für Bergbau, Energie und Geologie (LBEG Niedersachsen) archivierten Bohrprofile sowie Fundstücke aus dem Lias, die im Laufe der letzten 100 Jahre aufgenommen wurden.

Die Analyse der Haupt- und Spurenelemente von neun ausgewählten Proben erfolgte mit dem Gerät PANalytical AXIOS advanced sequential XRay spectrometer (Software Super Q 4) der Abteilung Geochemie des Geowissenschaftlichen Zentrums Göttingen. Für die Strontium-Isotopie wurden die neun Proben nasschemisch aufbereitet und mit dem Thermoionen-Massenspektrometer Triton(C) der Firma ThermoFinnigen der Abteilung Isotopengeologie des Geowissenschaftlichen Zentrum Göttingens analysiert (s. Tab. 4). Zur Überprüfung der Messgenauigkeit wurde der internationale Standard NBS 987 verwendet. Der gemessene Langzeitwert für das ${ }^{87} \mathrm{Sr} /{ }^{86} \mathrm{Sr}-$ Verhältnis liegt bei $0,710260 \pm 79 \quad(n=191)$ und für das ${ }^{84} \mathrm{Sr} /{ }^{86} \mathrm{Sr}-$ Verhältnis bei $\quad 0,056482 \pm 79$ $(\mathrm{n}=191)$.

Die geometrische, strukturgeologische 3DModellierung erfolgte mit der Software 4DMove und 3DMove von Midland Valley Exploration Ltd. Für die Erstellung der stratigraphischen Fläche wurde der Algorithmus Tesselation verwendet, bei dem die erzeugte Fläche durch alle Orts- und Tiefenangaben der Bohrungen und Fundpunkte verlaufen muss.
Tabelle 1

a: Kurzprofil B1 Hainholzweg, b: Kurzprofil B2 Tannenweg, c \& d: Kurzprofile B6 \& B7 Charlottenburgerstraße 19, aus Ziesch (2010). a:

\begin{tabular}{cl} 
Bohrtiefe $[\mathrm{m}]$ & Stratigraphie \\
\hline 4,0 & Quartär \\
11,4 & Lias $\delta$ \\
11,7 & Lias $\gamma$ \\
139,0 & Lias $\beta$ \\
\hline
\end{tabular}

$$
\text { b: }
$$

\begin{tabular}{ll} 
Bohrtiefe $[\mathrm{m}]$ & Stratigraphie \\
\hline 6 & Quartär \\
10 & Lias $\delta$ \\
11 & Lias $\gamma$ \\
48 & Lias $\beta$ \\
& - S t ö r u n g - \\
69 & Mittlerer Muschelkalk \\
\hline
\end{tabular}

\begin{tabular}{ll} 
Bohrtiefe $[\mathrm{m}]$ & Stratigraphie \\
\hline 4 & Anthropogener Schutt \\
10 & Quartär \\
56 & Ceratitenschichten \\
69 & Trochitenkalk \\
\hline
\end{tabular}

$\mathrm{d}:$

\begin{tabular}{ll} 
Bohrtiefe $[\mathrm{m}]$ & Stratigraphie \\
\hline 2 & Quartär \\
14 & Unterer Keuper mit Bruchstücken \\
& von Mittleren Muschelkalk \\
54 & Ceratitenschichten \\
60 & $\mathrm{mo}_{1}$ Trochitenkalk \\
\hline
\end{tabular}

\section{Ergebnisse}

Die stratigraphischen Kurzprofile der untersuchten Bohrungen sind in Tabelle 1 aufgelistet. Die Bohrungen 6 und 7 (Charlottenburger Straße 19), auf der Grabenschulter gelegen, durchteuften nahezu den gesamten Oberen Muschelkalk, mit Endteufe in der Trochitenkalk-Formation. Die untersuchten Bohrungen 1, 3 und 4 im Grabeninneren (Hainholzweg 54, Merkelstraße 16 und Merkelstraße 18) setzen im Lias- $\delta$ (AmaltheentonFormation) an, durchteufen die markante Kalkbank des Lias- $\gamma$ (Belemnitenschichten) und finden ihre Endteufe innerhalb des mächtigen Lias- $\beta$ (Obtususton-Formation). Von besonderem Interesse ist die Bohrung 2 (Tannenweg 20). Sie durchteuft zunächst Tonsteine des Lias- $\delta$, geringmächtige Kalke und Eisenoolithe des Lias- $\gamma$ sowie Tonsteine des Lias- $\beta$, bevor sie in $48 \mathrm{~m}$ Teufe die östliche Grabenrandstörung antraf. Darunter wurden mittelgraue, feinschichtige Gipssteine mit dünnen, 
grünlichgrauen Tonhäutchen und Karbonatlagen beobachtet. Die drei gewonnenen ${ }^{87} \mathrm{Sr} /{ }^{86} \mathrm{Sr}$-Werte von Proben aus 54, 63 und $69 \mathrm{~m}$ Teufe liegen im Bereich der Vergleichsproben der MuschelkalkGruppe aber auch der Röt-Formation. Sie unterscheiden sich jedoch erheblich von den niedrigen Werten der Zechstein-Gruppe (Tab. 3). Da die rötlichen Gesteinsfarben komplett fehlen und die östlich angrenzende Grabenschulter zuoberst von Muschelkalk-Formationen aufgebaut wird, werden die in der Bohrung 2 (Tannenweg 20) angetroffenen Gipssteine dem Mittleren Muschelkalk zugeordnet. Die etwas niedrigeren ${ }^{87} \mathrm{Sr} /{ }^{86} \mathrm{Sr}$-Verhältnisse der faserigen Gipse könnten auf einen Isotopenaustausch mit Fluiden aus der Trochitenkalk-Formation hinweisen, wohingegen der Wert des feinschichtigen Mikrites aus Teufe $69 \mathrm{~m}$ den Vergleichswerten der Mittleren Muschelkalk-Formation entspricht.

Anhand dieser neuen Bohrung kann nun zusammen mit weiteren Bohrungen (Reinkebrunnen, Ackermann unpubliziert; Bohrungen GeismarSüd, Feldmann 1983) der Verlauf der östlichen Grabenrandstörung revidiert werden. Südlich von Geismar, nahe des Reiterhofes, sind in der Bohrung 549 dunkelgraue Tonsteine der Lias-Gruppe angetroffen worden, bereits $200 \mathrm{~m}$ nordöstlich davon in der Bohrung 561 grüngraue, schluffige Tonsteine des Unteren Keupers (Feldmann 1983). Entsprechend ist der Oberflächenausstrich der Störung im Vergleich zu Stille (1932) bzw. Stille (1933) um ca. $300 \mathrm{~m}$ bzw. $100 \mathrm{~m}$ nach Osten zu verlegen.

Weiter nördlich, im Bereich des HainbundDenkmales bzw. an der Ecke Herzberger Landstraße/Hainbundstraße ist die Lokation von Fundpunkt 6 (,Tongrube unterhalb des Credo'schen Hauses $^{6}$ ) vermutlich nicht richtig überliefert. Hier zeichnete von Koenen (1893) ein fleckenhaftes Lias-Vorkommen noch an der Biegung des BrunoJung-Weges ein und beschrieb die Lokation auch in seinen Erläuterungen (von Koenen 1907; vgl. Abb. 2a). Jedoch ist in der geologischen Karte von Stille (1932), welche die Grundlage für die digitalisierte Karte auf dem Server des LBEG bildet, dieser Punkt um etwa $100 \mathrm{~m}$ nach Westen verschoben (Abb. 2b). Da keine anderen Informationen bekannt sind, ist davon auszugehen, dass diese Fläche dennoch die Tongrube darstellen soll. Aufgrund dessen verschiebt sich der Störungsverlauf um etwa $100 \mathrm{~m}$ in Richtung Osten.

Im Zusammenhang mit dem neuen Verlauf der
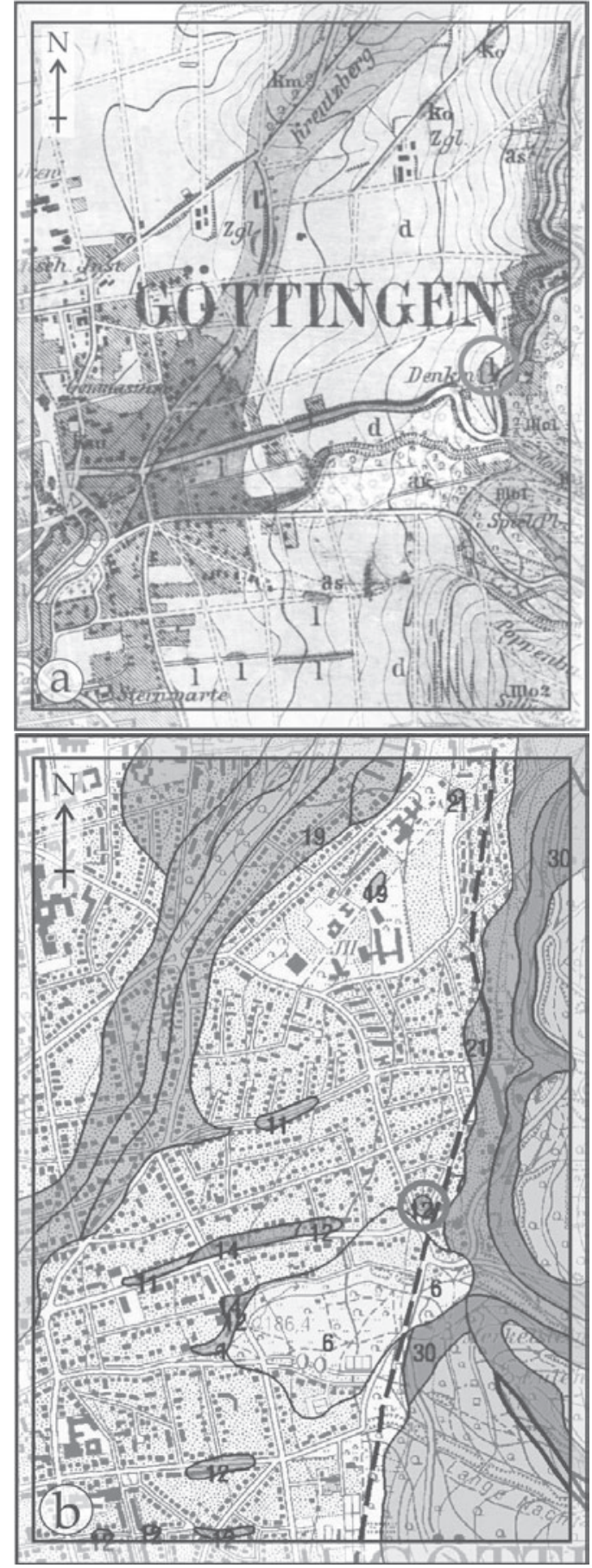

Abbildung 2. Vergleich der geologischen Karten von v. Koenen (1893) und Stille (1932). Man beachte, dass die Lokation der Tongrube ursprünglich an der Biegung des Bruno-Jung-Weges liegt, der vom Hainbund-Denkmal in Richtung RohnsTerrassen führt; der Kreis kennzeichnet die Lokation der Tongrube a) Ausschnitt aus der Geologischen Karte von Koenen (1893) b) Ausschnitt aus der digitalisierten, geologischen Karte des LBEG (Ausgabe 2005) nach Stille (1932). 

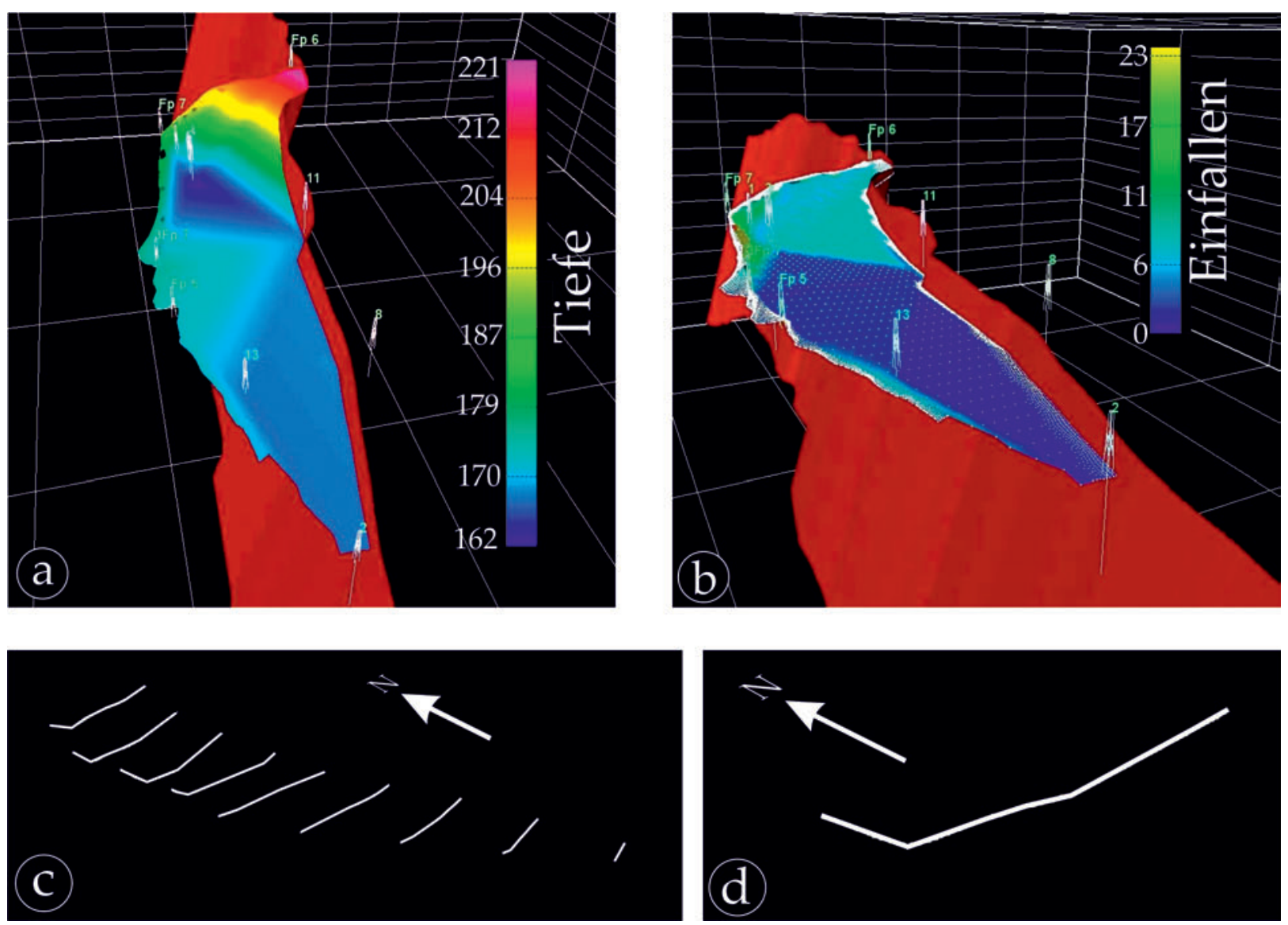

Abbildung 3. Fläche des Lias- $\gamma$ in der 3D-Ansicht, die Störungsfläche ist rot gefärbt a) Fläche des Lias- $\gamma$ gefärbt nach der Höhe ü. NN (Blickrichtung nach Nord, 5.5 fach überhöht) b) Basis von Lias$\gamma$ gefärbt nach dem Einfallen (Blickrichtung nach Nordost, 5.5 fach überhöht) c) zehn Querschnitte durch Lias- $\gamma$ zeigen eine Verflachung von Norden nach Süden (4fach überhöht) d) ein Einzelschnitt verdeutlicht, dass Lias- $\gamma$ nach dem Knick das Einfallen ändert (4fach überhöht).

Störung, der bekannten Tiefe in den Bohrungen und der Morphologie, wurde die Störungsfläche, die bis in eine Tiefe von $200 \mathrm{~m}$ reicht, dreidimensional erzeugt (Abb. 3a und b). Es konnte der durchschnittlicher Orientierungswert 289/55 für die gesamte Störungsfläche ermittelt werden.

Bezüglich Lagerung und potentiellen Schleppungen im Randbereich der Grabenmittelscholle im östlichen Stadtgebiet von Göttingen eignet sich die markante Kalkbank des Lias- $\gamma$ als hervorragender Leithorizont, um die Raumlage der Lias-Schichten zu modellieren. In verschiedenen Bohrungen (Hainholzweg 54, Merkelstraße 16 und 18, Tannenweg 20 und Breslauer Straße 10; Ziesch 2010) konnte die Tiefe dieser nur $30 \mathrm{~cm}$ bis $1 \mathrm{~m}$ mächtigen Schicht bis auf $1 \mathrm{~m}$ genau aufgenommen werden. Im 3D-Modell wurde die Basis des Lias- $\gamma$ mit Hilfe der Bohrungen und Fundpunkte erstellt (Abb. 3a und b). Das 3D-Modell zeigt, dass die Lagerung des Lias- $\gamma$ in Richtung Süden flacher wird und abtaucht (Abb. 3a). Der südliche Bereich fällt sehr flach mit einem Winkel bis zu $6^{\circ}$ ein, während der nordwestliche Randbereich bis zu $30^{\circ}$ Einfallen aufweist (Abb. 3b). Im nördlichen Teilbereich ändert sich die Morphologie in der Streichrichtung E-W stärker, als im Süden. Es zeigt sich, dass im Norden ein Knick deutlich ausgeprägt ist, während im Süden die Schicht nahezu söhlig lagert (Abb. 3c). Der Einzelschnitt in Abbildung 3d verdeutlicht, dass der Lias- $\gamma$ bis zum Knick in Richtung Osten einfällt und nach dem Knickpunkt das Einfallen ändert.

Auf Basis der 3D-Modellierung wurden drei Querprofile über das östliche Stadtgebiet von Göttingen gelegt, um den Ausstrich der einzelnen Lias-Formationen näher einzugrenzen (Abb. 1). 

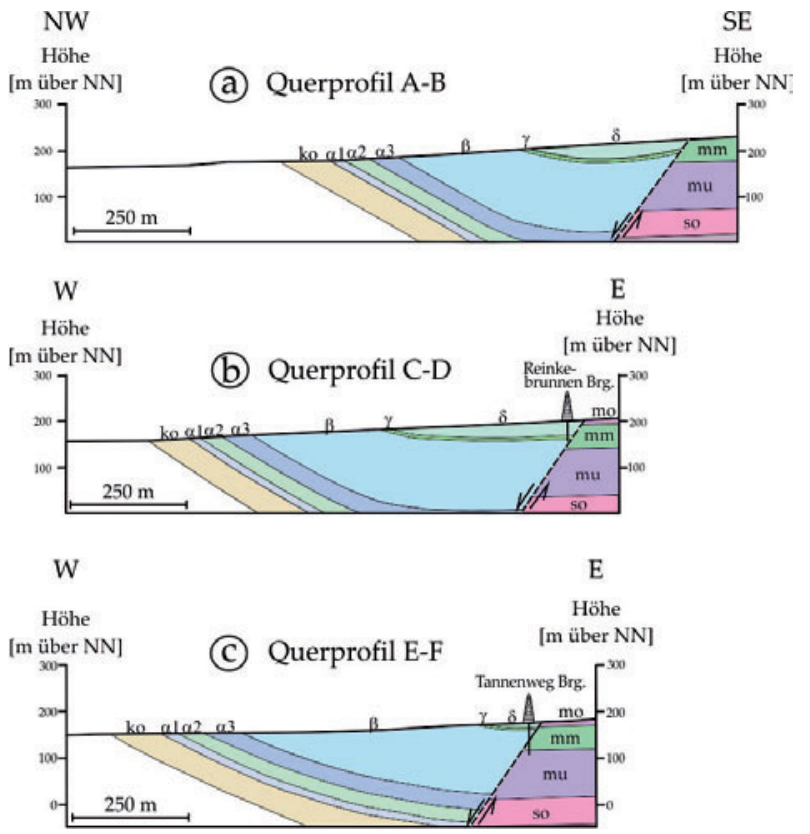

Abbildung 4. Maßstabsgetreue Querprofile: a) NW-SE orientiertes Querprofil zwischen den Punkten A (R: $\left.{ }^{35} 65510 / \mathrm{H}:{ }^{57} 12371\right)$ und B (R: $\left.{ }^{35} 65766 / \mathrm{H}:{ }^{57} 11635\right)$ b) W-E orientiertes Querprofil zwischen den Punkten C (R: ${ }^{35} 65378 / \mathrm{H}$ : $\left.{ }^{57} 10946\right)$ und $\left.\mathrm{D}\left(\mathrm{R}:{ }^{35} 66563 / \mathrm{H}:{ }^{57} 10854\right) \mathrm{C}\right) \mathrm{W}-\mathrm{E}$ orientiertes Querprofil zwischen den Punkten E (R: $\left.{ }^{35} 65313 / \mathrm{H}:{ }^{57} 09900\right)$ und $\mathrm{F}\left(\mathrm{R}:{ }^{35} 66456 / \mathrm{H}\right.$ : $\left.{ }^{57} 09811\right)$.

Das Querprofil A-B (Abb. 4) zeigt einen Schnitt durch den nördlichen Teil des Göttinger Ostviertels, Querprofil C-D (Abb. 4) und E-F (Abb. 4) verlaufen von $\mathrm{W}$ nach $\mathrm{E}$ durch das Ostviertel. In allen drei Querprofilen wurde der modellierte Knick der Schichtung abgerundet dargestellt. Die Geometrie der Lias- $\gamma$-Kalkbank ist auf die Formationen im Liegenden übertragen und in die Tiefe fortgesetzt worden. Die Schichten im Grabeninneren fallen mit etwa $30^{\circ}$ in Richtung Osten ein, so wie es u.a. von v. Koenen (1907) und Stille (1932) gemessen wurde. Die Schichten auf der Grabenschulter fallen nur sehr flach mit bis zu $3^{\circ}$ ein. Die Entwicklung von Norden (Querprofil A-B) nach Süden (Querprofil E-F) zeigt folgende Veränderungen:

1) Die Verringerung der Ausstrichsbreite des Lias- $\delta$,

2) eine Erhöhung der Ausstrichsbreiten des Lias- $\beta$ und des Lias- $\alpha_{1-3}$ durch die flachere Lagerung und

3) die Sprunghöhe der Ostrandstörung nimmt
Tabelle 2

Minimale und maximale Sprunghöhe entlang der östlichen Grabenrandstörung für die Querprofile A-B, C-D und E-F von Norden nach Süden (Für Lage siehe Abb. 1).

\begin{tabular}{|c|c|c|}
\hline Querprofil & \multicolumn{2}{c|}{ Minimum ${ }_{\mathrm{N}}$ Maximum } \\
\hline A-B & $765 \mathrm{~m}$ & $830 \mathrm{~m}$ \\
\hline C-D & $758 \mathrm{~m}$ & $798 \mathrm{~m}$ \\
\hline E-F & $736 \mathrm{~m}$ & $776 \mathrm{~m}$ \\
\hline \multicolumn{2}{|c}{$\mathrm{S}$} \\
\hline
\end{tabular}

entlang seines Streichens von N nach $\mathrm{S}$ von etwa $800 \pm 30 \mathrm{~m}$ auf etwa $755 \pm 20 \mathrm{~m}$ ab (Tab. 2).

\section{Diskussion und Interpretation}

Im nördlichen Bereich des Göttinger Ostviertels kann eine angedeutete Umbiegung von LiasSchichten beobachtet werden (Abb. 5a). In diesem Bereich befindet sich östlich der Grabenrandstörung ein schmales, mit Keupersedimenten gefülltes Störungssystem (Kleperspalte), das NW-SE streicht.

Die Kleperspalte wird im Süden von einer flachen Überschiebung und im Norden von einer Abschiebung begrenzt (Stille 1932, Abb. 5a). Die leicht sigmoidale Verformung dieses Störungssystems weist auf eine, zum Grabenrand parallele Horizontalbewegung hin (Wunderlich 1957). Weiterhin kann in Abbildung 5a ein seitlicher dextraler Versatz der östlichen Grabenrandstörung um etwa $70 \mathrm{~m}$ beobachtet werden. Auch im nördlichen Leinetalgraben sind späte, dextrale Seitenverschiebungen von Tanner et al. (2010b) nachgewiesen worden. Eine schematische Skizze zur Entstehung solcher Strukturen ist in Abbildung 5b und c dargestellt. Dadurch könnte die dextrale Seitenverschiebung den Störungsversatz und die Umbiegung von Lias- $\gamma$ und $-\delta$ verursacht haben.

Anhand der drei Querprofile und der 3DModellierung wird nachgewiesen, dass die Sprunghöhe entlang des Streichens der östlichen Grabenrandstörung von ca. $765 \mathrm{~m}$ (maximal $830 \mathrm{~m}$ ) im Norden auf ca. $736 \mathrm{~m}$ (maximal $776 \mathrm{~m}$ ) im Süden verringert (Tab. 2). Der Versatz entlang des Streichens einer Störung ändert sich von einem Maximum nahe des Initiationspunktes 


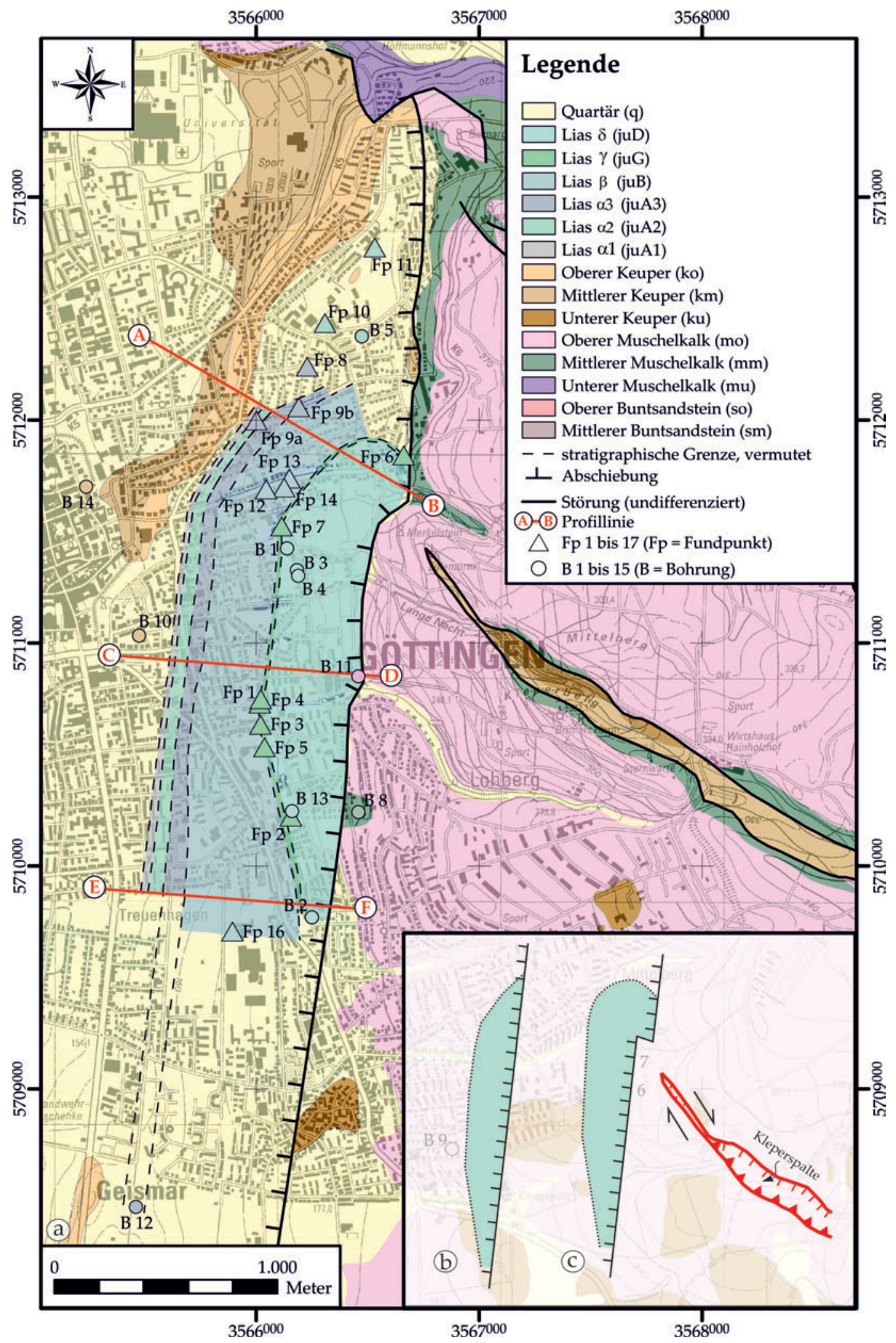

Abbildung 5. a) die Geologische Karte des Stadtgebietes von Göttingen zeigt die neue Kartierung der Lias-Schichten und der östlichen Grabenrandstörung, Auflistung aller Fundpunkte und Bohrungen in Ziesch (2010), Kartengrundlage: Geologische Karte Niedersachsen TK 25 vom LBEG; Projektion Koordinatensystem: Gauss Krüger Zone 3, Datum: Deutsches Hauptdreiecksnetz Sphäroid: Bessel 1841, b+c) Die Umbiegung der Schichten kann durch eine dextrale Seitenverschiebung hervorgerufen werden, die roten Linien markieren die Störungen in der Kleperspalte, die mögliche Ursache für die Umbiegung der Schichten: b) vermuteter Ausstrich der Lias-Schichten vor der dextralen Blattverschiebung c) Umbiegung der Lias-Schichten durch die dextrale Blattverschiebung. 
(ältester Versatzanfang) zu einem Minimum an den Rändern (jüngster Versatzanfang). Im Fall der östlichen Grabenrandstörung ist es möglich, dass sich die Störung von Norden nach Süden entwickelt hat und somit der Versatz im Norden etwas höher als im Süden ist.

Neue Erkenntnisse über die Geometrie der LiasSchichten und der östlichen Grabenrandstörung wurden gewonnen. Mittels der 3D-Modellierung mit 3DMove wurde verdeutlicht, dass die Fläche des Lias- $\gamma$ im nördlichen Bereich einen ausgeprägten Knick aufweist (Abb. 3c und d). Dieser ist vermutlich eine Schleppfalte, die durch eine ungleichmäßige Abschiebung entstanden ist. Ferner konnte der Einfallwinkel der Grabenrandstörung auf $55^{\circ}$ bestimmt werden. Insofern ist das Einfallen etwas niedriger, als bei einer ,normalen' Abschiebung (Anderson 1951). Eine Erklärungsmöglichkeit wäre, dass die Gipse aus dem Mittleren Muschelkalk, die entlang der Störungsfläche migrieren und in Bohrung 2 (Tannenweg 20) nachgewiesen worden sind, die interne Reibung herabgesetzt haben (McKenzie 1969, Byerlee 1978 und Sibson 1985). Eine andere Erklärung für den etwas flacheren Einfallwinkel könnte in einer möglichen listrischen Abschiebung liegen. Zechsteinsalze im Untergrund könnten für eine listrische Störung den Abscherhorizont darstellen (Tanner et al. 2010a). Der Zechstein liegt in einer Tiefe von etwa $900 \mathrm{~m}$. Auch Grupe (1909) nimmt aufgrund seiner Beobachtungen in der Sudheimer und Levershäuser Bohrung für die östliche Grabenrandverwerfung an, dass sie in der Tiefe ein erheblich flacheres Einfallen zeigt. Es wäre demzufolge vorstellbar, dass die östliche Grabenrandstörung eine listrische Abschiebung mit einem Abscherhorizont im Zechstein ist. Unter der Annahme, dass der steilere Abschnitt der Störung gemeinsam mit den umliegenden Sedimenten schon erodiert ist, hat die Störungsfläche an dem jetzigen Schnittniveau im Bereich des Arbeitsgebietes einen Einfallwinkel von etwa $55^{\circ}$.

\section{Schlussfolgerung}

Unter Einbindung von Bohrungen, Fundstücken sowie geologischen Karten und Literaturangaben konnte der Ausstrich der Lias-Formationen und der Verlauf der östlichen Grabenrandstörung näher eingegrenzt werden (Abb. 5a). Die Umbiegung der Lias-Formationen und der Störungsversatz sind durch eine dextrale Seitenverschiebung verursacht worden. Die 3D-Modellierung verdeutlicht, dass die Fläche der Kalkbank des Lias- $\gamma$ im nördlichen Bereich einen ausgeprägten Knick aufweist und mit $30^{\circ}$ steiler als weiter südlich einfällt (Abb. 3b). Ferner wurden in dieser Ausarbeitung neue Erkenntnisse über die Geometrie der östlichen Grabenrandstörung gewonnen. So konnte anhand der 3D-Modellierung die Orientierung der östlichen Grabenrandstörung mit einem Wert von $289^{\circ} / 55^{\circ}$ ermittelt werden.

\section{Danksagung}

Ein herzliches Dankeschön gilt Dr. Gerald Hartmann für die RFA-Messungen und die Besprechung der Daten. Ebenso bedanken wir uns bei den Mitarbeitern der Isotopengeologie: Prof. Dr. Bent Hansen, Nicole Nolte, Brigitte Dietrich und Franziska Wilsky für die isotopengeochemische Analyse der Proben und die Hilfe bei der Auswertung.

\section{Literatur}

Anderson, E. M. 1951. The dynamics of faulting and dyke formation with applications to Britain. 2nd ed., 206 S., Edinburgh.

Byerlee, J.D 1978. Friction of rocks. Pure appl. Geophys. 116, 615-626.

Feldmann, R. 1983. Ingeneurgeologische Kartierung der Stadt Göttingen, Teilblatt GeismarSüd, unveröffentlichte Diplomarbeit Universität Göttingen, $68 \mathrm{~S}$.

Grupe, O. 1909. Über die Zechsteinformation und ihr Salzlager im Untergrunde des hannoverschen Eichsfeldes und angrenzenden Leinegebietes nach den neueren Bohrergebnissen. Zeitschrift für praktische Geologie 17, 185-205, Berlin.

Koenen, A.v. 1907. Erläuterungen zur geologischen Specialkarte von Preußen und den Thüringischen Staaten. Lieferung 91. Gradabtheilung 55, No. 28, Blatt Göttingen.

Koenen, A.v. 1893. Geologische Specialkarte von Preußen und den Thüringischen Staaten 1:25 000, Königliche Preussische geologische Landesanstalt, Blatt Göttingen 4425.

Küster, F.W., Thiel A., Fischbeck K. 1985. Rechentafeln für die Chemische Analytik 103, bearb. Auflage, 310 S. Berlin, New York (de Gruyter).

McKenzie, D.P. 1969. The relation between fault plane solutions for earthquakes and the directions of the principal stresses. Bulletin of the 
Seismological Society of America 59/2, 591601.

Sibson, R.H. 1985. A note on fault reactivation. Journal of Structural Geology 7/6, 751-754.

Stille, H. 1932. Geologische Karte von Preußen und benachbarten deutschen Ländern. Erläuterungen zu Blatt Göttingen Nr. 2520. [3. Aufl.] Preußische Geologische Landesanstalt, 40 S., Berlin.

Stille, H., Lotze, F. 1933. Erläuterungen zur Geologischen Übersichtskarte der Umgebung von Göttingen (Hochschul-Exkursionskarte Nr. 3). Preußische Geologische Landesanstalt, 67 S., Berlin.

Tanner, D.C., Albero F., Leiss, B., the GGG 2010a. Modelling the Geothermal Potential of the Eastern Border of the Leinetal Graben, Lower Saxony. Zeitschrift für geologische Wissenschaften 38/1, 61-68, Berlin.

Tanner, D.C., Leiss, B., Vollbrecht, A., the GGG 2010b. The Role of Strike-Slip Tectonics in the Leinetalgraben, Lower Saxony, Zeitschrift der Deutschen Gesellschaft für Geowissenschaften, 161/4, 369-377.

Wunderlich, H.G. 1957. Tektogenese des Leinetalgrabens und seiner Randschollen. Die postvariszischen Sedimentbecken Mittel- und Westeuropas. Geologische Rundschau 46, 372-413, Stuttgart.

Ziesch, J. 2010. Strukturgeologische Modellierung der Grabenrandstörung des Leinetals im östlichen Stadtgebiet von Göttingen auf der Basis flacher Geothermiebohrungen, unveröffentlichte Diplomarbeit Universität Göttingen, 92 S.

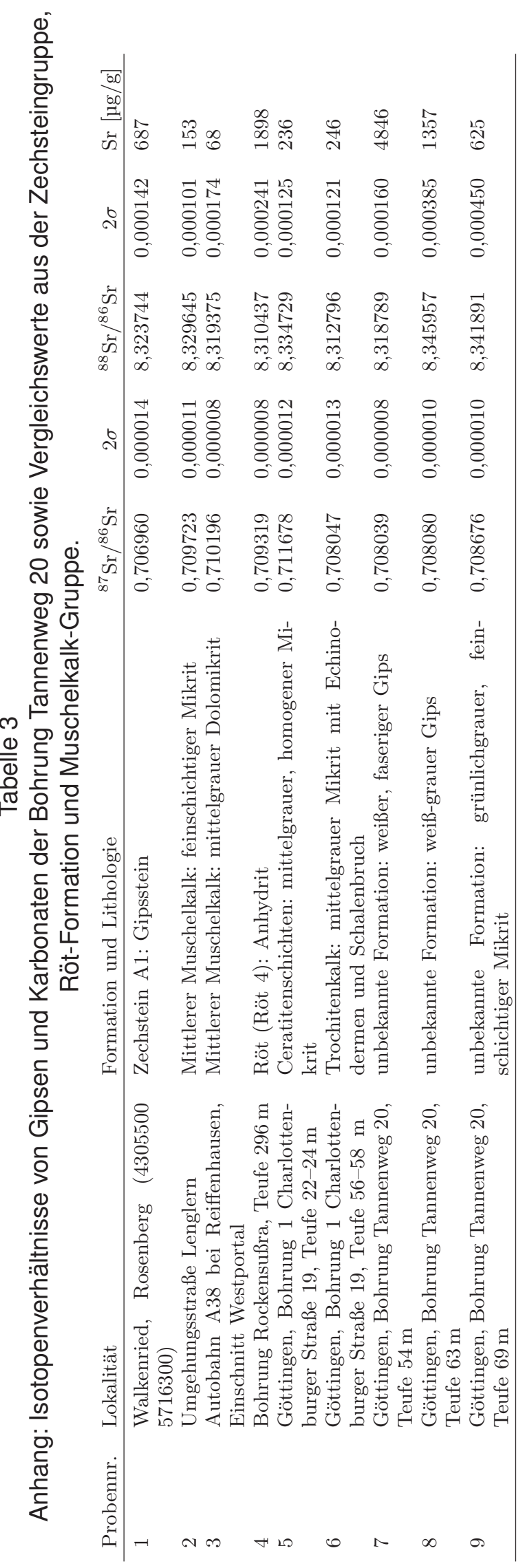


Nㅗㅇ

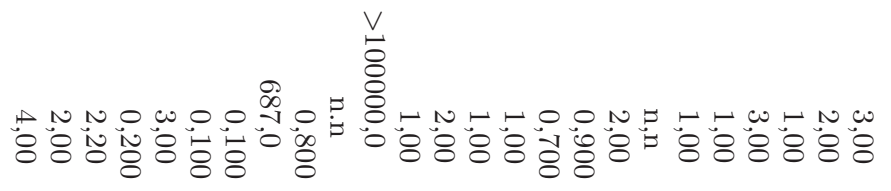

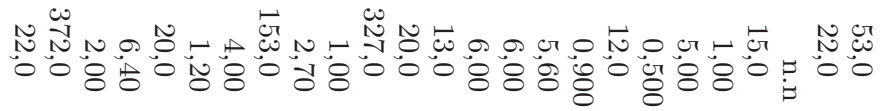

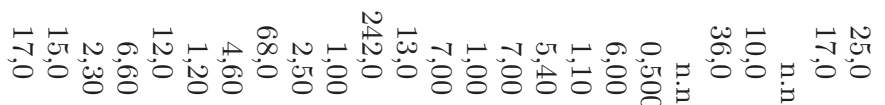

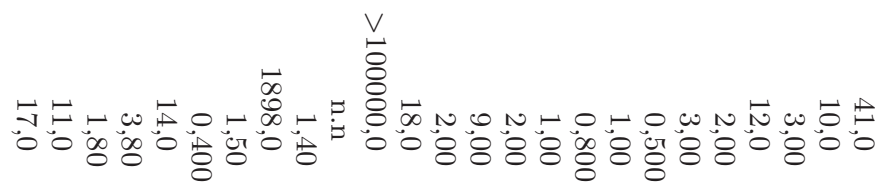

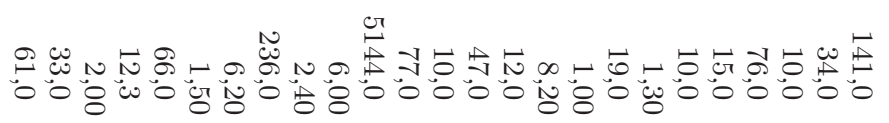

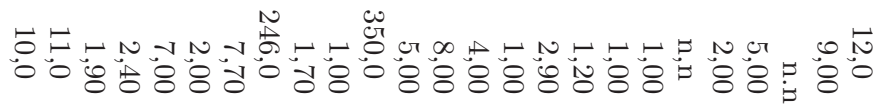

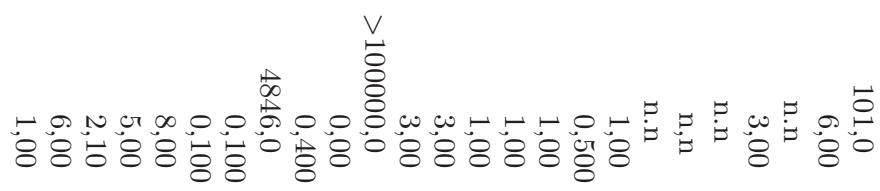

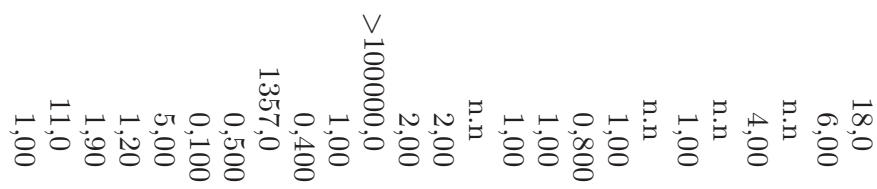

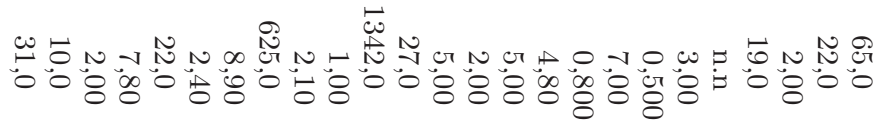

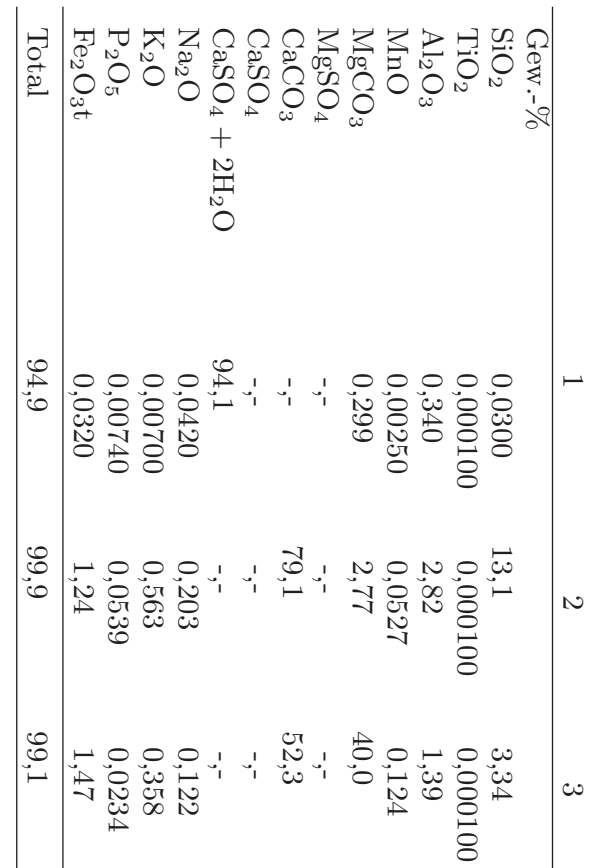

离:

(1)

$\cong$ गुग

仓市

응

․ $\frac{0}{2}$.

उิ

○ㅇำ

กิ

○ิ $\frac{5}{1}$

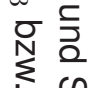

$>0$

ऽ.

है

a

륜 $\frac{\mathrm{D}}{3}$

क

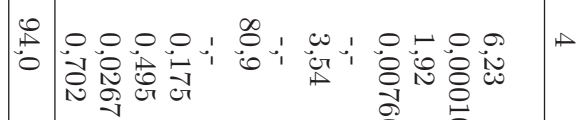

吾

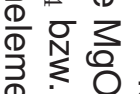
का

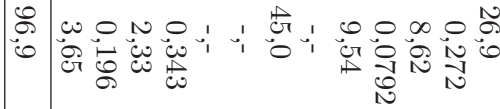

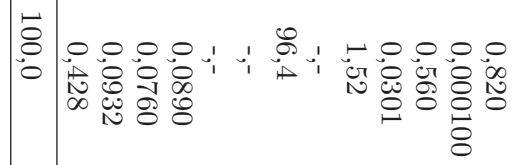

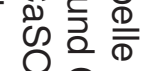

○ـ

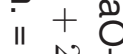

근. ำ

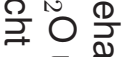

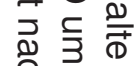

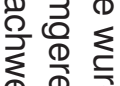

D. 通

음 운 을

里

$\Phi$

壱

ठำ

유 요

$\overline{\bar{c}} \overline{\mathrm{o}}$

용

0

¿ $\frac{3}{3}$

류:

工 옥

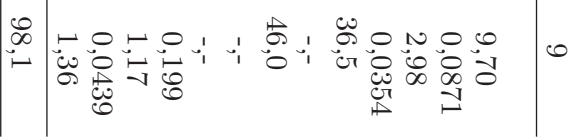




\title{
Ein Störungskörper in einem Seitenverschiebungssystem bei Hardegsen
}

\author{
N. Wasja Bloch (GZG Göttingen, FU Berlin), David C. Tanner (GZG Göttingen, LIAG \\ Hannover) und Josef Paul (GZG Göttingen)
}

\begin{abstract}
Zusammenfassung - Der stillgelegte Kalksteintagebau bei Hardegsen am westlichen Rand des Leinetalgrabens schließt Röt-Material zwischen Muschelkalk-Schollen, das durch saigere, E-W streichende, dextrale Seitenverschiebungen getrennt ist, auf. Mit Hilfe von Laserentfernungs- und Kompassmessungen wird der genaue Kontakt der Rötenklave kartiert und ein 3D-Modell produziert. Die Form dieser Enklave variiert stark; an der Basis der Steinbruchs ist sie über $20 \mathrm{~m}$ breit, am Top des Steinbruchs aber nur $5 \mathrm{~m}$. Wir stellen eine Hypothese vor, nach der das Röt-Material durch Seitenverschiebung tektonisch getrennt wurde und anschließend durch hohen inneren Druck in das Hangende eindrang.
\end{abstract}

\begin{abstract}
The disused open-pit limestone quarry at Hardegsen on the western border of the Leinetal Graben exposes Röt material sandwiched between Muschelkalk, separated by vertical, E-W striking, dextral strike-slip faults. We used laser range-finding and compass measurements to directly map the contact of the Röt enclave and produce a three-dimensional model. The form of the enclave varies greatly; it is over $20 \mathrm{~m}$ wide at the base of the quarry but only $5 \mathrm{~m}$ wide at the top. We suggest the Röt material was detached tectonically by the strike-slip faults. It has penetrated the Muschelkalk in the hanging-walls due to high internal pressure.
\end{abstract}

Schlüsselworte-Muschelkalk, Röt, Seitenverschiebung

\section{Einführung}

Störungen stellen oft verschiedene Gesteine nebeneinander, seltener ist allerdings, dass Fremdmaterial zwischen zwei nahezu parallele Störungen eingebracht wird. Ein solcher Fall liegt in einem Steinbruch bei Hardegsen, an der westlichen Seite der Leinetals, vor, wo Oberer Buntsandstein (Röt 3) zwischen Schichten des Unteren Muschelkalks liegt, die in einer seigeren, E-W streichenden, dextralen Seitenverschiebungzone vorkommt (Abb. 1, Vollbrecht (1985)). Die versetzte Grenze der Basis des Muschelkalks (Punkt X in Abb. 1) und die Tatsache, dass Unterer Muschelkalk auf beiden Seiten der Störung vorkommt, kann verwendet werden, um den gesamten (horizontalen)

- N. Wasja Bloch - GZG, jetzt Freie Universität Berlin, Fachrichtung Geophysik, Malteserstr. 74-100, D-12249 Berlin.wasja.bloch@fu-berlin.de

- David C. Tanner - GZG, jetzt Leibniz-Institut für angewandte Geophysik, Stilleweg 2, D-30655 Hannover. DavidColin.Tanner@liag-hannover.de

- Josef Paul - Geowissenschaftliches Zentrum der Universität Göttingen, Goldschmidtstr. 3, D-37077 Göttingen.jpaul@gwdg.de
Versatz auf der Störung zu bestimmen; in diesem Fall $326 \mathrm{~m}$ (Tanner et al. 2010). Ferner sieht man in Abb. 1, dass die dextralen Störungen die NS streichende Leinetal-Westrandstörung versetzen (Vollbrecht 1985), folglich sind sie hier die jüngsten Störungen im Leinetalgraben (Tanner et al. 2010).

Bloch (2009) hat im Rahmen einer BachelorArbeit die Form der Röt-Störungsenklave im dreidimensional modelliert und die Kontakte der Enklave mit dem Muschelkalk untersucht, um Kinematik und Art der Entstehung der Enklave genauer zu verstehen. Diese Arbeit ist ein Abriss von Bloch (2009).

\section{Stratigraphie}

Der Steinbruch schließt an seiner Nordwand ein längeres Profil der Röt 4-Schichten, Oberer Buntsandstein, (Abb. 1, Punkt Y) bis hin zu den Terebratelbänken des Unteren Muschelkalks auf. Südlich der Störung ist nur der Untere Wellenkalk 1 aufgeschlossen. Der stratigraphische Kontakt von Röt und Muschelkalk ist sowohl westlich des Steinbruchs aufgeschlossen (Arp et al. 2004, Paul 


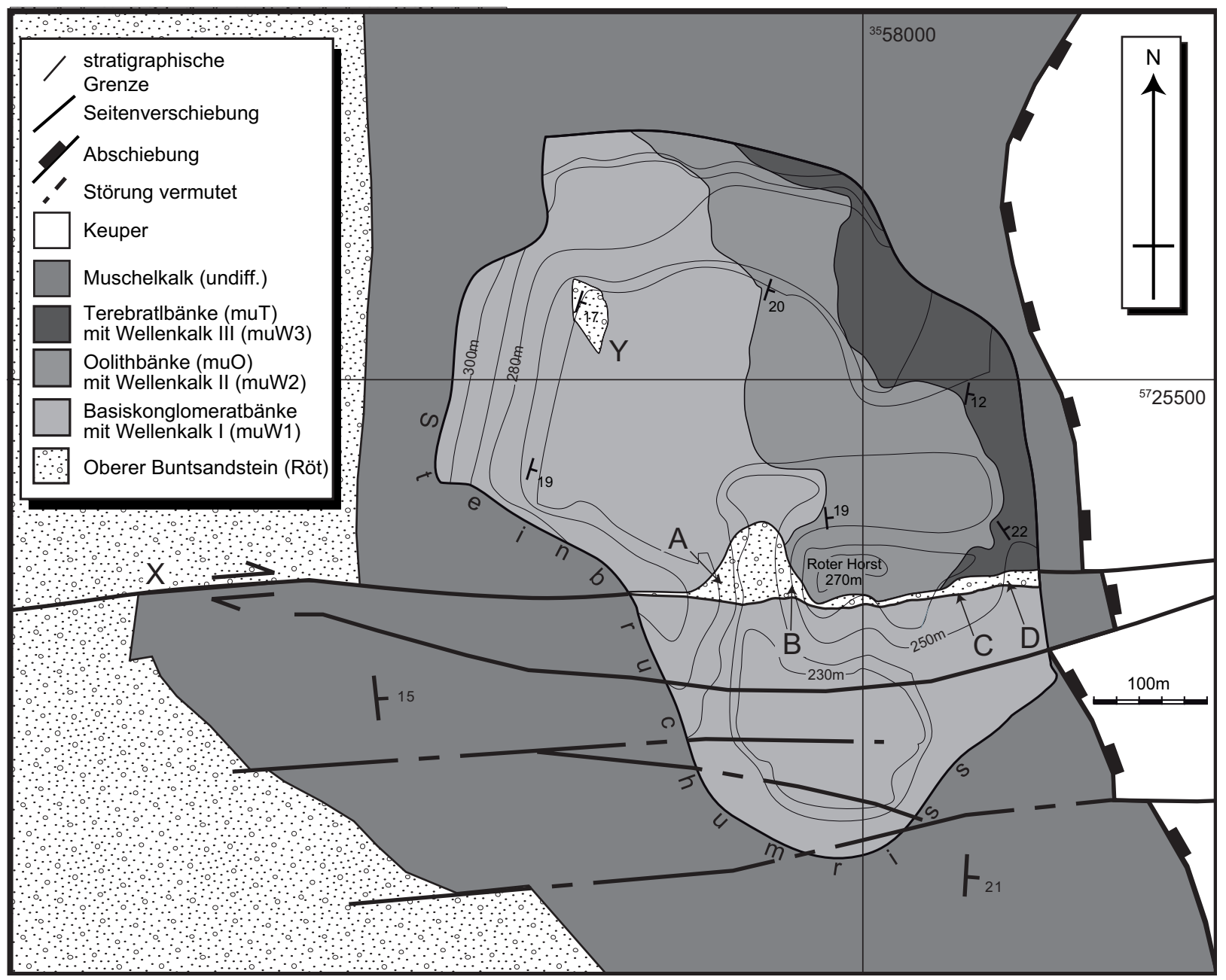

Abbildung 1. Geologische Karte vom Steinbruch Hardegsen, die die Position des eingeschuppten Röts (Oberer Bundsandstein) innerhalb der Störungszone zeigt (nach Vollbrecht (1985) und Bloch (2009)). Unterer Muschelkalk ist nur innerhalb des Steinbruchs differenziert. Punkte $X$ und $Y$ werden im Text erklärt. A-D kennzeichnen Aufschlußwände. Höhenlinien je $10 \mathrm{~m}$, Gauss-Krüger Koordinaten.

\& Franke 1977) als auch im Steinbruch (Abb. 1, Punkt Y). Der Kontakt zwischen Muschelkalk und Keuper ist immer durch Störungen getrennt.

Die Störung-Enklave enthält Buntsandstein Röt 3 Material (so3), das aus dünnen roten und grünen Feinsilt- und Tonschichten besteht (Abb. 2).

\section{Methode}

Wir verwendeten einen elektronischen Theodoliten der Marke Leica (TPS1100), um Messpunkte am Kontakt der Störungsenklave zu bestimmen. Da der Theodolit mit einem GPS-Gerät genau geortet war, konnte die Kontaktspur direkt mit dreidimensionalen Koordinaten versehen werden.

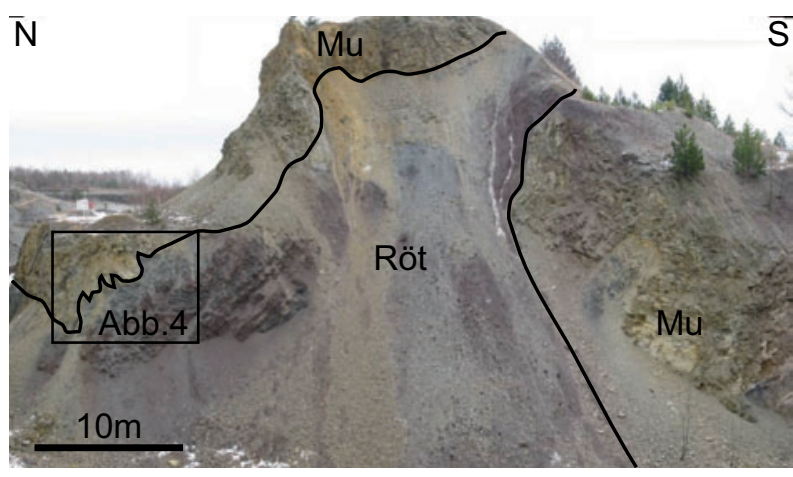

Abbildung 2. Foto der Aufschlußwand B (Lage siehe Abb. 1). Durch perspektivische Effekte z.T. deutliche Unterschiede zwischen realer und scheinbarer Raumlage der markierten Grenzfläche. 


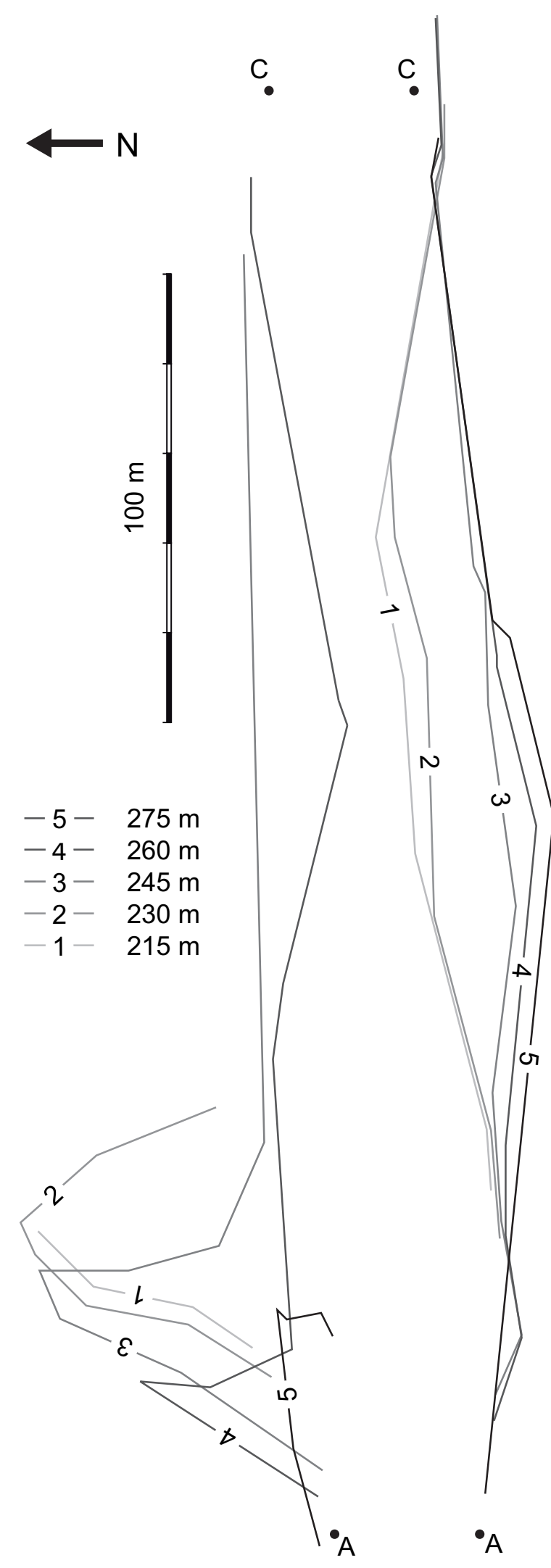

Abbildung 3. Höhenlinien (je $15 \mathrm{~m}$ ) der nördlichen und südlichen Kontakte der Enklave zwischen den Wänden A-C. 1 - tiefste, 5 - höchste.

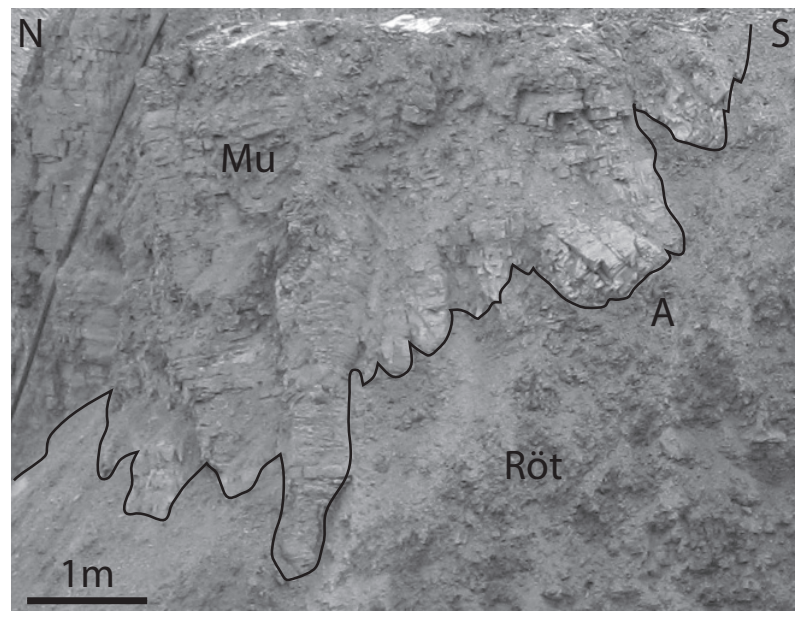

Abbildung 4. Detailfoto eines Teils des Enklavenkontaktes auf Aufschlußwand B (Lage siehe Abb. 2). Bei Punkt $A$ ist der Muschelkalkblock leicht rotiert.

Zusätzlich wurde mit einem Geländekompass die Raumlage des Kontaktes an der Stelle, an der die Position mit Hilfe des Theodoliten bestimmt worden war, vermessen.

In dieser Art wurden alle sichtbaren freien Kontakte der Enklave an vier Wänden (A-D, Abb. 1) gemessen. Mit Hilfe von PYTHON-Skripts wurden die Raumdaten der Kontaktflächen in ihren Streich- und Einfallrichtungen verlängert. Die Datenpunkte wurden dann in das dreidimensionale Software-Programm GoCAD eingelesen und zusammen in Dreiecksflächen umgewandelt. Daraus wurden Isolinienbilder erzeugt, da dies die beste 2D-Darstellung ergibt.

\section{Ergebnisse}

Die Enklave hat eine steil einfallende südliche Kontaktfläche und eine flach bis steil einfallende nördliche Kontaktfläche. Der gesamte Enklavenkontakt ist mittels $15 \mathrm{~m}$-Isolinien in Abb. 3 dargestellt. Meist ist der Kontakt scharf, aber vor allem im flacher einfallenden Teil ist die Fläche sehr gewölbt (Abb. 4). In Abb. 3 kann man erkennen, dass der südliche Kontakt sogar zum größten Teil ,überkippt‘ ist.

\section{Diskussion}

Der vertikale stratigraphische Abstand zwischen Röt 3 und Unterem Muschelkalk beträgt mindestens $17 \mathrm{~m}$. Wir stellen uns eine Situation vor, bei der ein Block von Röt 3 Material zwischen zwei 
oder mehreren Seitenverschiebungen tektonisch getrennt wird. Durch die leicht anastomosierte Störungenfläche ist die Enklave während des mehrere hundert Meter horizontalen Versatzes etwa $20 \mathrm{~m}$ nach oben gewandert. Jedoch war die tektonische Bewegung nicht der einzige Prozess: Die Tonenklave drang, durch internen Druck, in umgebende Schichten ein, was die gewölbten Stellen am flacher einfallenden Nordkontakt beweisen (Abb. 4). Tondiapire sind häufige Erscheinungen, insbesondere wenn die Tone unter starkem Druck stehen und höheren internen Porendruck besitzen (Brown 1990, van Rensbergen et al. 1999). Das weicht von der ursprünglichen Idee ab, dass Diapire durch Dichteunterschiede entstehen, stattdessen ist hier gemeint, dass sich Tonenklave und Störungen gegenseitig unterstützen, d.h. die Tone erniedrigen die Reibung in der Störungszone, und die Störungszone gibt den Tonen den nötigen internen Druck, um sich zu bewegen. Ein solches Modell ist im Golf von Mexiko für die Präsenz von Salz entlang der Störungzone (Rowan et al. 1999) denkbar. Durch diesen (internen) Druck, gelingt es dem Ton entlang von Rissen und Bruchflächen in den Muschelkalk einzudringen (Abb. 4).

\section{Schlussfolgerung}

Im Steinbruch Hardegsen kommen jüngere (als die Hauptstörungen des Leinetalgrabens) dextrale E-W-streichende, vertikale Seitenverschiebungen im Unteren Muschelkalk vor. Zwischen zwei der Störungen liegt eine Enklave von Röt-Sedimenten. Durch genaue Messung mit einen Theodolit und Projektion der Daten in GoCAD stellen wir fest, dass der südliche Kontakt steil ist, der nördliche Kontakt jedoch steile und flache Komponenten besitzt. Wir stellen die Hypothese auf, dass das Röt-Material tektonisch von der Störung gelöst wurde, sich anschließend jedoch zum Teil diapirisch nach oben bewegte.

\section{Danksagung}

Wir bedanken uns bei dem Besitzer des Steinbruchs, August Oppermann Kiesgewinnungs- und Vertriebs-GmbH für die Zutrittsgenehmigung.

\section{Literatur}

Arp, G., Hoffmann, V.-E., Seppelt, S., Riegel, W. 2004. Trias und Jura von Göttingen und Umgebung, 74. Jahrestagung der Paläontologischen Gesellschaft, 2.-8. 10. 2004, Göttingen (Universitätsdrucke).

Bloch, N.W. 2009. Dreidimensionale strukturgeologische Aufnahme einer Störungszone am westlichen Rand des Leinetalgrabens bei Hardegsen, Süd-Niedersachsen. Unveröffentlichte B.Sc.-Arbeit, Universität Göttingen.

Brown, K.M. 1990. The nature and hydrologic significance of mud diapirs and diatremes for accretionary prisims. Journal of Geophysical Research 95/B6, 8969-8982.

Paul, J., Franke, W. 1977. Sedimentologie einer Transgression: Die Röt/Muschelkalk- Grenze bei Göttingen. Neues Jahrbuch für Geologie und Paläontologie, Monatshefte 1977/3, 148177.

Rowan, M.G., Jackson, M.P.A., Trudgill, B.D. 1999. Salt-Related Fault Families and Fault Welds in the Northern Gulf of Mexico. AAPG Bulletin 83/9, 1454-1484.

van Rensbergen, P., Morley, C. K., Ang, D. W, Hoan, T. Q., Lam N. T. 1999. Structural evolution of shale diapirs from reactive rise to mud volcanism: 3D seismic data from the Baram Delta, offshore Brunei Darussalam. Journal of the Geological Society of London 156, 633-650.

Vollbrecht, R. 1985. Der Westrand des Leinetalgrabens zwischen Lutterhausen und Hardegsen. Unveröffentlichte Diplomarbeit, Universität Göttingen. 


\title{
Struktur des Schwarzjura-Keuper-Vorkommens im Eichenberger Grabenknoten bei Hottenrode
}

\author{
David C. Tanner (GZG Göttingen, LIAG Hannover), Gernot Arp (GZG Göttingen), \\ Frithjof A. Bense (GZG Göttingen) und Gabriele Ertl (GZG Göttingen, LBEG Hannover)
}

\begin{abstract}
Zusammenfassung - Die Tongrube Hottenrode südlich Friedland erschließt Teile der Sedimentabfolgen der Schwarzjura-Gruppe (Arietenton-/Obtususton-Formation) und der höheren Keuper-Gruppe (Arnstadt Formation), welche voneinander durch eine vertikale Störung getrennt sind. Mit Hilfe einer GPS unterstützten Untersuchung der Schichtflächenlagerung wurde eine genaue Strukturkarte produziert. Auffällig ist die Raumlage der Schichtflächen des Jura direkt an der Störung: Manche Schichtflächen sind um $180^{\circ}$ aus ihrer normalen Lage gedreht. Ein Model wird vorgestellt, in dem durch eine dextrale Seitenverschiebungsbewegung auf der Störung Schleppfalten erzeugt werden, die sich weiter durch Externrotation um eine vertikale Achse drehen.
\end{abstract}

\begin{abstract}
The clay pit at Hottenrode, south of Friedland, exposes part of a sedimentary sequence of Jurassic Lias (Arieten Clay/Obtusus Clay Formation) and an upper Keuper group (Arnstadt Formation), which are separated by a vertical fault. We used a GPS device to directly map bedding planes and produce an exact structural map. The orientation of Jurassic beds directly against the fault surface is striking: some beds are rotated by up to $180^{\circ}$ from their normal orientation. A model is proposed in which dextral strike-slip movement on the fault caused drag fold to be generated, which were then further rotated by body rotation around a vertical axis.
\end{abstract}

Schlüsselworte-Schwarzjura, Keuper, Seitenverschiebung.

\section{Einführung}

Die Hottenroder Tongrube liegt auf halbem Wege zwischen Hottenrode und NeuEichenberg, südlich von Friedland und nahe der Thüringischen-Hessischen-Niedersächsischen Ländertripelpunktgrenze (TK 25 Blatt 4625 Witzenhausen R $\left.{ }^{35} 64950, \mathrm{H}^{56} 94300\right)$. In der kleinen Grube $(170 \times 70 \mathrm{~m})$ stehen Gesteine des Steinmergelkeuper-Rhätkeupers angrenzende an Gesteine des Schwarzjuras an, beide voneinander durch eine Störung getrennt (Abb. 1). Strukturgeologisch befindet sich der Aufschluß im

- David C. Tanner — GZG, jetzt Leibniz-Institut für angewandte Geophysik, Stilleweg 2, D-30655 Hannover. DavidColin.Tanner@liag-hannover.de

- Gernot Arp - Geowissenschaftliches Zentrum der Universität Göttingen, Goldschmidtstr. 3, D-37077 Göttingen. garp@gwdg.de

- Frithjof A. Bense - Geowissenschaftliches Zentrum der Universität Göttingen, Goldschmidtstr. 3, D-37077 Göttingen. fbense@gwdg.de

- Gabriele Ertl - GZG, jetzt Landesamt für Bergbau, Energie und Geologie, Stilleweg 2, D-30655 Hannover. Gabriele.Ertl@lbeg.niedersachsen.de
Kreuzungsbereich des Leinetal-Grabens und des Eichenberg-Gothaer Grabens, dem sogenannten ,Eichenberger Grabenknoten' (Lotze 1932).

Die Tongrube dient der gelegentlichen Entnahme von pyritführenden Tonmergeln zur Beimischung in der Ziegel-Herstellung der Ziegelei Friedland. Künstliche Aufschlüsse wie der vorliegende sind für regionalgeologische Fragestellungen bedeutsam, da sie Einblicke in geologische Strukturen in verwitterungsanfälligen Gesteinsabfolgen gewähren, welche auf natürlichem Wege nie erschlossen sind. Im vorliegenden Fall sind dies insbesondere störungsnahe Strukturen in weichen Tongesteinen.

Ziel dieser Arbeit war es, mit Hilfe einer GPS unterstützten Untersuchung der Schichtflächenlagerung eine genaue Strukturkarte zu erzeugen. Die Daten wurden als Grundlage zur Ermittlung der Störungskinematik benutzt. 


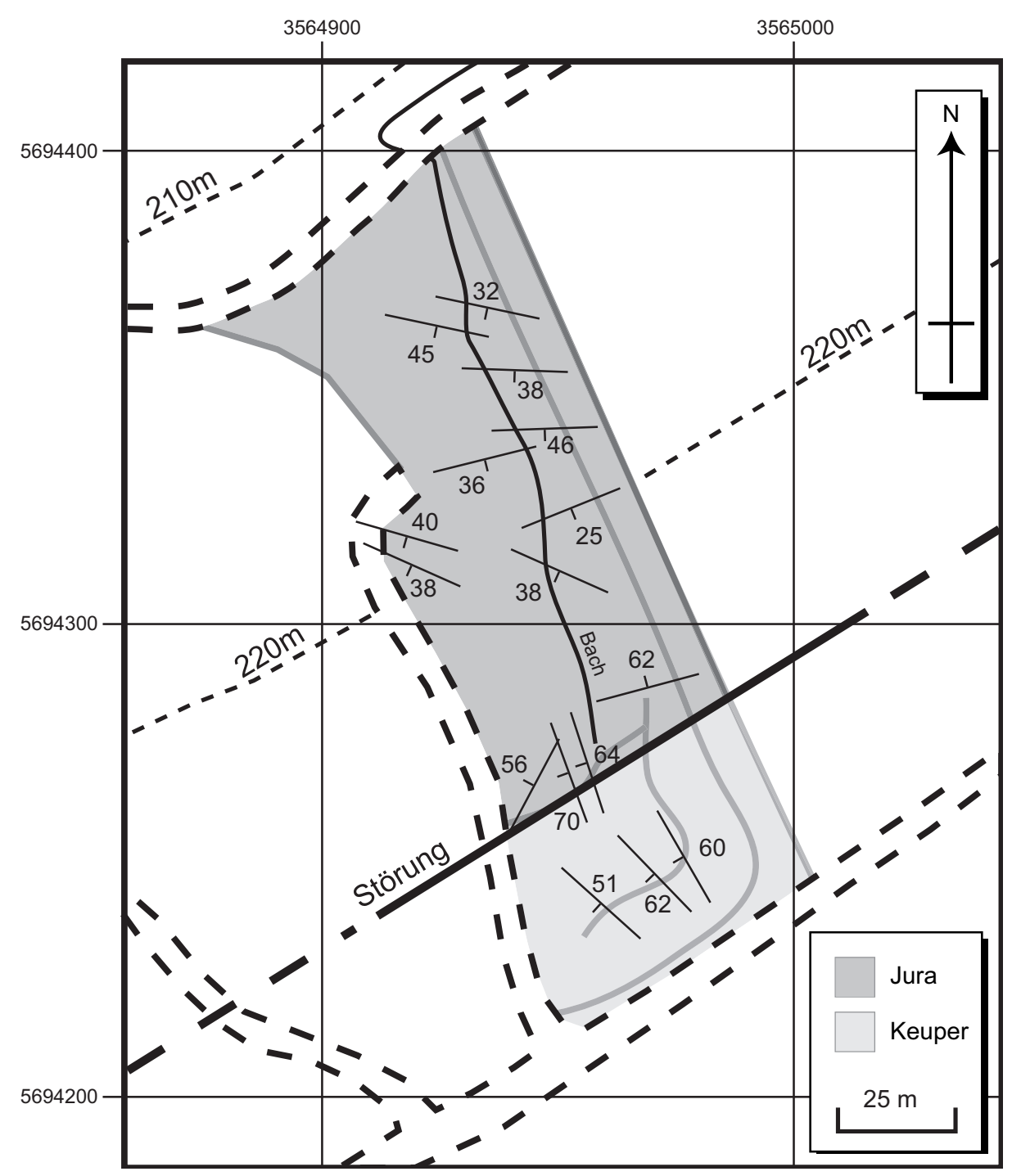

Abbildung 1. Geologische Karte der Tongrube Hottenrode mit Raumlage der Schichten. Dicke graue Linien sind Geländekanten.

\section{Stratigraphie}

Arp et al. (2004) ordnen das ca. $21 \mathrm{~m}$ aufgeschlossene Keuperprofil dem Übergangsbereich Steinmergelkeuper-Rhätkeuper zu, einem Bereich der nach neuer lithostratigraphischer Nomenklatur der Arnstadt-Formation zuzurechnen ist (Deutsche Stratigraphische Kommission 2002, Vath 2005). Fossilfunde, welche eine chronostratigraphisch Zuordnung zum Nor oder Rhät zulassen würden, konnten allerdings nicht gemacht werden.

Der in der Grube aufgeschlossene Teilabschnitt der Schwarzjura-Gruppe gehört zur höheren Arietenton- und tiefsten Obtususton-Formation (Sinemurium) (Arp et al. 2000). Er besteht aus einer Mergelschiefer/Ölschiefer-Chondrites-
Mergel/Tonmergel-Folge (Arp et al. 2006).

\section{Methode}

Die vorliegende strukturgeologische Studie der Tongrube wurde im April 2007 durchgeführt. Beide Formationsgruppen waren frisch angeschnitten, und sogar die Störung war sichtbar. Mit Hilfe eines 12-Kanal, Doppelfrequenz GPS Receivers, wurde die genaue Position der im Steinbruch gemessenen strukturellen Raumlage der Schichtflächen erfasst. Alle Messwerte wurden zusammen mit den Positionsdaten mit Hilfe von Geländeakquisitionssoftware verbunden und abgespeichert. Die Genauigkeit der horizontalen Positionsinformation liegt unterhalb $1 \mathrm{~m}$. 


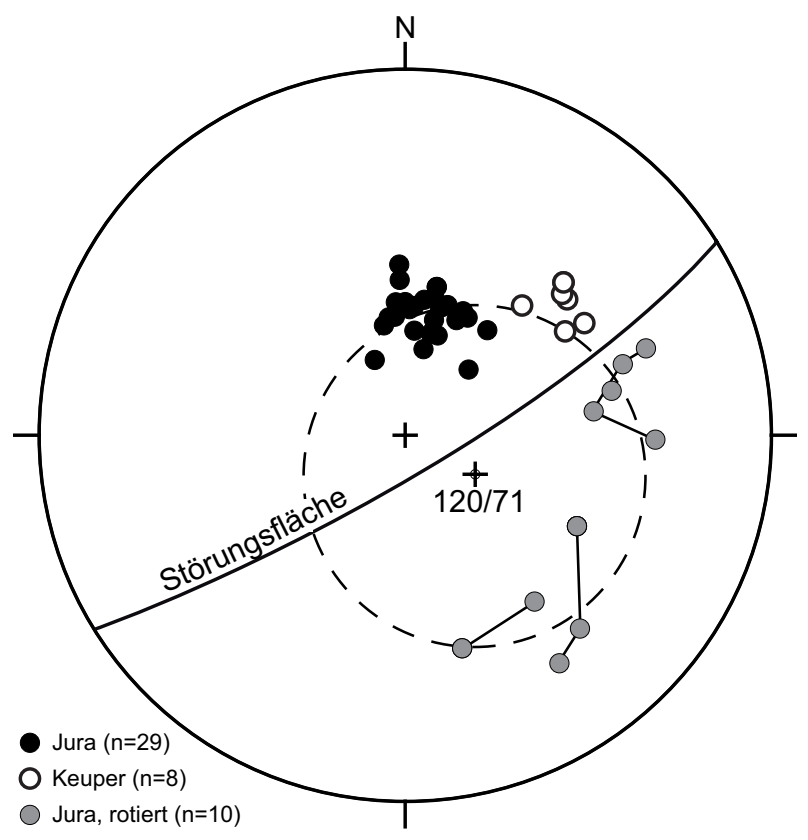

Abbildung 2. Winkeltreue stereographische Projektion der Raumlage der Pole zu den Schichtflächen. Die Störungsfläche ist als Großkreis dargestellt. Linien verbinden Daten von der gleichen Schichtfläche.

\section{Ergebnisse}

Abbildung 1 zeigt die Lage der Schichtflächen und der Störung. Es ist auffällig, dass der Schwarzjura in der Nähe der Störung ein unterschiedliches Einfallen zeigt, während $30 \mathrm{~m}$ und weiter von den Störungen entfernt sowohl Schwarzjura- wie auch Keuper-Schichten jeweils sehr einheitlich einfallen, jedoch ca. $20^{\circ}$ gegeneinander gedreht. Weiterhin fallen die Pole der Schwarzjura-Schichtflächen, die nahe an der Störungsfläche liegen, auf einen Kleinkreis, der einen Öffnungswinkel von $45^{\circ}$ um 120/71 besitzt (Abb. 2).

Die Störung erscheint als eine $5-10 \mathrm{~cm}$ breite, mit Kalzit gefüllte Zone und fällt steil nach Südost ein (Abb. 2).

\section{Diskussion}

Die Schwarzjura-Schichten nahe der Störung sind deutlich vom Versatz auf der Störung betroffen. Im Gelände bilden die Schichtflächen Teile von offenen, steil abtauchenden Falten. In der stereographischen Projektion sieht man jedoch, dass, obwohl die Schichtflächen Teilgroßkreise wegen dieser Faltung bilden, sie auf einem gemeinsamen Kleinkreis (Abb. 2) liegen. Zum Teil sind die

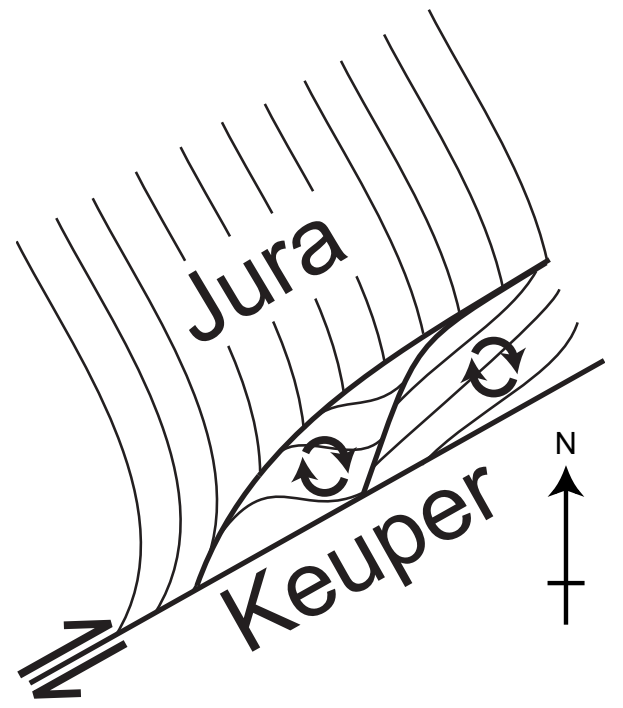

Abbildung 3. Durch Versatz auf der Störung entstehen Schleppfalten, die durch weiteren Versatz als Blöcke weitergedreht wurden.

Schwarzjura-Schichten um mehr als $180^{\circ}$ gedreht. Der Mittelpunkt dieses Kleinkreises (120/71) liegt nahe der Störungsfläche.

Die beobachtete Kleinkreisrotation von Schichtflächen könnte durch konische Faltung (Ramsay \& Huber 1987) oder durch Block-Rotation erzeugt worden sein. Wir halten das letztere für wahrscheinlicher, da einzelne Schichten zylindrische, offene Falten zeigen. Reibung an der Störung könnte demnach offene (Schlepp-) Falten erzeugt haben, die danach als abgescherte Blöcke durch die gleiche Bewegung weitergedreht wurden. Diese Hypothese ist in Abbildung 3 dargestellt.

Wenn diese Hypothese stimmt, war der Versatzvektor an der Störung nahezu horizontal, weil die Rotationsachse an der Störung in der Einfallsrichtung liegt. Aus der Verteilung der SchwarzjuraSchichtflächen im Uhrzeigersinn (Abb. 2), lässt sich ein dextraler Versatz folgern.

Vermutlich gehört diese Störung zu einer Schar von E-W bzw. ENE-WSW streichenden, dextralen Seitenverschiebungen.

\section{Schlussfolgerung}

Die Hottenroder Tongrube erschließt Gesteine der tieferen Schwarzjura-Gruppe (Arietenton/Obtususton-Formation) und der höheren Keuper-Gruppe (,Steinmergelkeuper ${ }^{6}$ und ,Rhätkeuper', Arnstadt-Formation), die durch eine Störung getrennt sind. Bei einer genauen Untersuchung der Struktur stellen 
sich die Schwarzjura-Schichtflächen innerhalb $30 \mathrm{~m}$ von der Störung entfernt als aus ihrer normalen Lage gedreht dar. Die Analyse der Schichtflächen ergibt, dass ihre Pole entlang eines Kleinkreises verteilt sind. Es wird postuliert, dass durch dextralen Versatz auf der Störung Schleppfaltungen erzeugt wurden, die danach in Form von abgescherten Blöcken weitergedreht wurden.

\section{Danksagung}

Wir danken dem Eigentümer der Tongrube, Freiherr Melchior von Bodenhausen (Niedergandern), für die Erlaubnis, in der Grube zu arbeiten.

\section{Literatur}

Arp, G., Peršoh, D., Reimer, A., Reitner, J., Sosnitza, M. 2000. Lias-Fossilien aus der Tongrube Eichenberg, Nordhessen. Fossilien 17 (2), 108113.

Arp, G., Hoffmann, V.-E., Seppelt, S. \& Riegel, W. 2004. Exkursion 6: Trias und Jura von Göttingen und Umgebung. 74. Jahrestagung der Paläontologischen Gesellschaft, 02.-08. Oktober 2004.

Deutsche Stratigraphische Kommission (Hrsg.) 2002. Stratigraphische Tabelle von Deutschland 2002 .

Lotze, F. 1932. Der Südteil des Göttinger Leinetalgrabens und der Eichenberger Grabenknoten. Abhandlungen der Preußischen Geologischen Landesanstalt, Neue Folge 139, 5-48.

Ramsay, G.J. \& Huber, M.I. 1987. The techniques of modern structural geology. Volume 2: Folds and Fractures. Academic Press.

Vath, U. 2005. Der Keuper im südlichen Niedersachsen bei Göttingen. In: Stratigraphie von Deutschland. Keuper (Kap. 5.4). Courier Forschungs-Institut Senckenberg 253, 163-178.
Tabelle 1:

Die in Abb. 1 und 2 verwendeten Meßwerte. $\mathrm{J}$ - Jura, J(R) - Jura, rotiert, $\mathrm{K}$ - Keuper

\begin{tabular}{|lcc|lcc|}
\hline & Azimuth & Einfall & & Azimuth & Einfall \\
\hline $\mathrm{J}$ & 204 & 39 & $\mathrm{~J}$ & 204 & 38 \\
$\mathrm{~J}$ & 224 & 28 & $\mathrm{~J}$ & 218 & 40 \\
$\mathrm{~J}$ & 196 & 40 & $\mathrm{~J}$ & 198 & 41 \\
$\mathrm{~J}$ & 198 & 32 & $\mathrm{~J}$ & 169 & 34 \\
$\mathrm{~J}$ & 172 & 36 & $\mathrm{~J}$ & 184 & 39 \\
$\mathrm{~J}$ & 192 & 45 & $\mathrm{~J}$ & 180 & 40 \\
$\mathrm{~J}$ & 208 & 40 & $\mathrm{~J}$ & 175 & 36 \\
$\mathrm{~J}$ & 192 & 45 & $\mathrm{~J}$ & 185 & 32 \\
$\mathrm{~J}$ & 192 & 27 & $\mathrm{~J}$ & 194 & 32 \\
$\mathrm{~J}$ & 194 & 36 & $\mathrm{~J}$ & 176 & 40 \\
$\mathrm{~J}$ & 182 & 38 & $\mathrm{~J}$ & 192 & 42 \\
$\mathrm{~J}$ & 188 & 41 & $\mathrm{~J}$ & 182 & 39 \\
$\mathrm{~J}$ & 195 & 40 & $\mathrm{~J}$ & 178 & 46 \\
$\mathrm{~J}$ & 178 & 50 & $\mathrm{~J}$ & 158 & 25 \\
$\mathrm{~J}$ & 205 & 41 & $\mathrm{~J}(\mathrm{R})$ & 263 & 55 \\
$\mathrm{~J}(\mathrm{R})$ & 271 & 69 & $\mathrm{~J}(\mathrm{R})$ & 252 & 64 \\
$\mathrm{~J}(\mathrm{R})$ & 250 & 70 & $\mathrm{~J}(\mathrm{R})$ & 258 & 60 \\
$\mathrm{~J}(\mathrm{R})$ & 298 & 56 & $\mathrm{~J}(\mathrm{R})$ & 318 & 71 \\
$\mathrm{~J}(\mathrm{R})$ & 326 & 74 & $\mathrm{~J}(\mathrm{R})$ & 322 & 60 \\
$\mathrm{~J}(\mathrm{R})$ & 345 & 62 & $\mathrm{~K}$ & 237 & 55 \\
$\mathrm{~K}$ & 230 & 60 & $\mathrm{~K}$ & 238 & 60 \\
$\mathrm{~K}$ & 226 & 62 & $\mathrm{~K}$ & 228 & 60 \\
$\mathrm{~K}$ & 226 & 62 & $\mathrm{~K}$ & 222 & 51 \\
\hline
\end{tabular}




\title{
Tiefer Mittlerer Muschelkalk im Liegenden der Hauptstörung — temporäre Aufschlüsse am NE-Rand des Klausbergs, Göttingen NE
}

\author{
Till Heinrichs (GZG Göttingen)
}

\begin{abstract}
Zusammenfassung-Mittlerer Muschelkalk der Karlstadt-Formation im unmittelbar Liegenden der Ostrandstörung zeigt geringe Deformation oder liegt söhlig. In Präzisierung der Stille'schen Karte wird der Verlauf der Hauptstörung eingegrenzt und der stratigraphische Versatz zu $500 \pm 10 \mathrm{~m}$ bestimmt.
\end{abstract}

Abstract-Middle Keuper found below Middle Muschelkalk in Quaternary landslides documents the northern extension of the main normal fault that was predicted by Stille's map. The stratigraphic separation is determined as $500 \pm 10 \mathrm{~m}$.

Schlüsselworte—Keuper, Abschiebung, Klüftung

\section{Einführung}

Das Übersichtsbild Baugrube Klausberg 4 (Abb. 1, vgl. Karte Abb. 3) zeigt den SW-Teil der fertigen Ausschachtung, Klausberg 4. Alle Details der Abfolge lassen sich über den gesamten Stoß verfolgen. Zwei verfärbte Bruchzonen werden nur in dem offensichtlich spröderen oberen Teil der Gesteinsfolge beobachtet. Der östliche dieser Brüche wird teilweise von einer kleinen Flexur, cm-Versatz W-abwärts, begleitet, die in

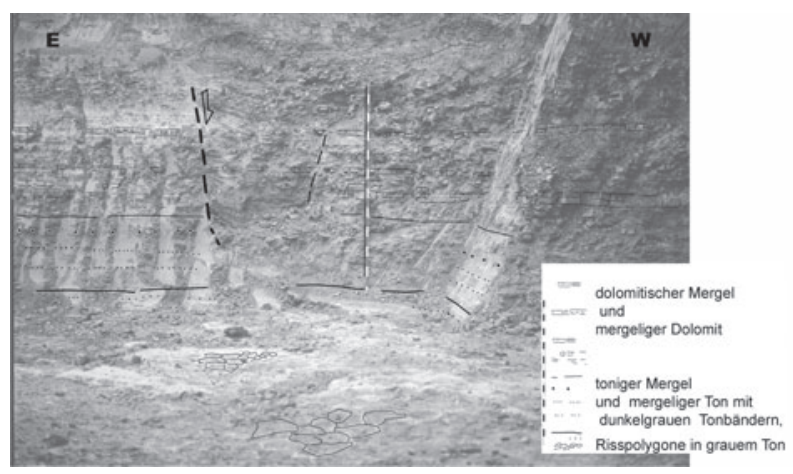

Abbildung 1. Am Klausberg 4, Baugrube 27.11.2007; Blick nach S; Maßstab mit dmTeilung.

- Till Heinrichs - Abt. Angewandte Geologie, Geowissenschaftliches Zentrum der Universität Göttingen, Goldschmidtstr. 3, D-37077 Göttingen. theinri@gwdg.de

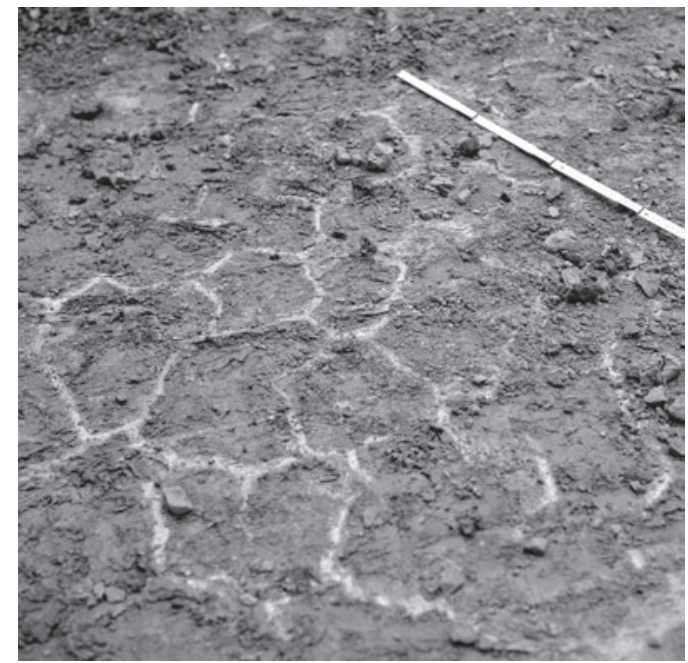

Abbildung 2. Polygonmuster in Tonlage mit hellen Karbonatstegen, Sohle Baugrube am Klausberg 4.

der unteren tonigeren Abfolge verschwindet. Die gesamte Sohle besteht aus der gleichen, einige cmmächtigen dunkelgrauen Tonschicht, die in ein Polygonmuster (Abb. 2) zerlegt ist - Trockenrisse, Synaerese? Sie zeigt keinen der Flexur entsprechenden Versatz.

Stratigraphisch fügen die Gesteine sich gut in die Karlstadt Formation, den tiefsten Mittleren Muschelkalk, ein, der als ,Untere Mergel-DolomitFolge' oder ,Unterer Dolomit‘ beschrieben wurde, 

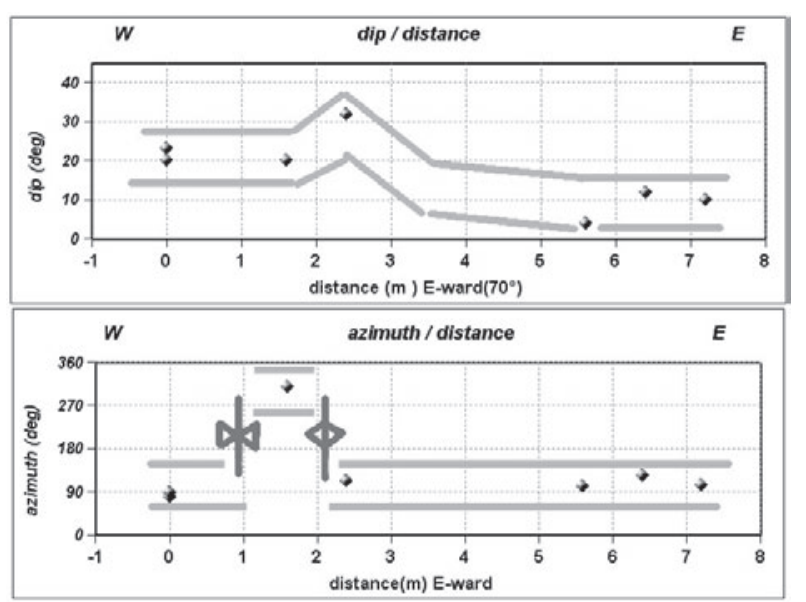

Abbildung 3. Am Klausberg 1a; Orientierung von ss im Graben entlang südlicher Kellerwand, ca. 2,5 m tief, 31.10.2007

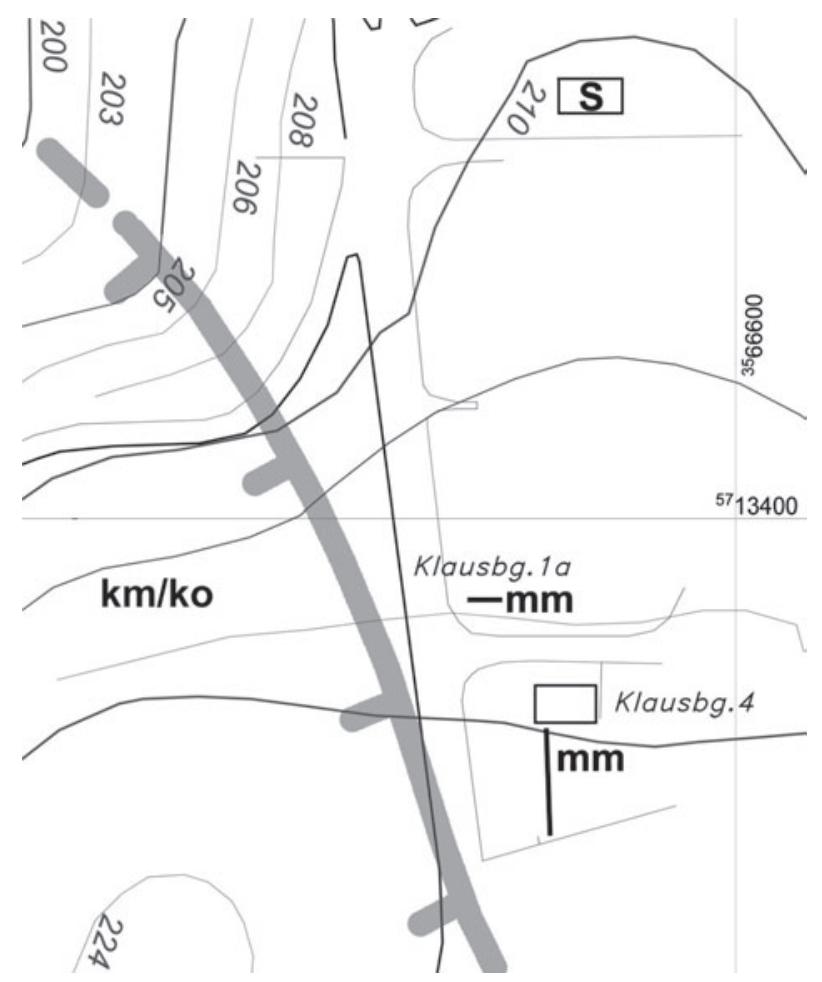

Abbildung 4. Karte der temporären mmAufschlüsse, Nordostrand Klausberg; in Baugrube Haus Nr. 34 wurden sehr harte Bankkalke angetroffen, wohl Schaumkalkbereich oberster mu (,S'); wahrscheinlicher Verlauf der Hauptabschiebung. oft mit Trockenrissen (vgl. Dünkel \& Vath 1990). Diese stratigraphische Position wird unterstützt durch Auftreten harter Bankkalke, wohl oberster mu, in Baugrube Haus Nr 34 (vgl. Abb. 4).

Entlang der südlichen Kellerwand des Hauses 1a sind feingeschichte dolomitische Mergel mit vorwiegend sanftem Einfallen nach $\mathrm{E}$ anstehend. Nach W ist eine kleine offene Knickfalte ausgebildet. Gleichzeitig vergrößert sich der Fallwinkel leicht. Nullpunkt der SCAT Plots (Abb. 3) ist die SW Hausecke. Die Faltenachsen verlaufen etwa 020/04, also schief zur Hauptstörung.

Ein ca $1 \mathrm{~m}$ tiefer $\mathrm{N}-\mathrm{S}$-verlaufender Leitungsgraben südlich des Hauses Nr.4 (Abb. 4) zeigt ebenfalls anstehenden mm. Etwa $50 \mathrm{~m}$ westwärts oberhalb des Nikolausberger Weges finden sich als Lesesteine rotbraun und grau marmorierte Feinsand- und Siltsteine, wohl höchster Mittlerer Keuper oder Übergang zu tiefstem Oberem Keuper. Zufolge aller dargestellten Aufschlüsse wird die Hauptstörung, Teil des Relay zwischen Northeimer und Göttinger Sprung, in der Rinne zwischen Klausberg und Klausberg-Siedlung zu lokalisieren sein.

Der stratigraphische Versatz zwischen einem Niveau etwa 5-8m über der Basis des $\mathrm{mm}$ und höchstem km ist hier $500 \pm 10 \mathrm{~m}$ (Mächtigkeiten nach Dünkel \& Vath 1990, Stille 1932, Vath 2005).

\section{Literatur}

Dünkel, H., Vath, U. 1990. Ein vollständiges Profil des Muschelkalks von der Dransfelder Hochfläche. Geologisches Jahrbuch Hessen 118, 87-126.

Stille, H. 1932. Geologische Karte von Preußen und benachbarten deutschen Ländern. Erläuterungen zu Blatt Göttingen Nr. 2520. - 3. Auflage, 40 S., 1 Kte., Berlin.

Vath, U. 2005. 5.4. Der Keuper im südlichen Niedersachsen. Courier Forschungsinstitut Senckenberg 253, 163-178. 


\title{
Sedimentgänge im Bausandstein der Solling-Folge NW' von Billingshausen
}

\author{
Malte Ritter, Axel Vollbrecht, Alfons van den Kerkhof, Klaus Wemmer (GZG Göttingen)
}

\begin{abstract}
Zusammenfassung - In zwei ehemaligen Steinbrüchen NW' von Billingshausen ca. 10 km NE' von Göttingen sind im Bausandstein der Solling-Folge isolierte Reste von steilen N-S streichenden Sedimentgängen erhalten. Makroskopisch heben sich die Karbonat-zementierten Gänge durch eine nach S einfallende Internschichtung, gröbere siliziklastische Komponenten und einen hohen, lokal jedoch stark wechselnden Gehalt an Eisenerzen von den flach gelagerten Rahmengesteinen ab. Die scharfe Grenzfläche zwischen Gängen und Rahmengesteinen ist z.T. mit Calcit-Kristallrasen belegt. Die Eisenerze bestehen fast ausschließlich aus opaken und transparenten Ooiden, die größtenteils unregelmäßige Wachstumszonierungen und Übergänge zu verzweigten Krusten zeigen und deshalb als in situ-Mineralisationen interpretiert werden. Nur wenige isolierte, streng konzentrisch aufgebaute Eisenooide werden als eingespülte Komponenten gedeutet. Anhand von KathodolumineszenzFarben und charakteristischen Zonierungen lassen sich drei Karbonatzement-Generationen unterscheiden. Eine daraus zu folgernde Mehrphasigkeit der Gangbildung wird zusätzlich durch Gangfragmente mit früh gebildetem Zement dokumentiert, die als Klasten in jüngeren Gangfüllungen vorkommen sowie durch komplexe Altersbeziehungen zwischen verschiedenen Zement- und Eisenerzgenerationen.

Viele Geröllkomponenten im Gang können weder aus den Rahmengesteinen noch aus den unmittelbar hangenden Einheiten stammen. Es wird daher angenommen, dass die Sedimentgänge mit der tertiären Rumpffläche in Verbindung standen und über diese mit Detritus gefüllt wurden. Die Eisenerze könnten demnach als Äquivalente der an anderen Stellen oberflächennah, konkretionär gebildeten eozänen Bohnerze betrachtet werden. Auch waren die Paläospannungsrichtungen zu dieser Zeit günstig für die initiale Öffnung der N-S streichenden Gänge, die durch eine ältere Hauptkluftschar vorgezeichnet waren.
\end{abstract}

\begin{abstract}
In two abandoned quarries, NW of the village Billingshausen, about $10 \mathrm{~km} \mathrm{NE}$ of Göttingen, isolated remains of sedimentary dykes are exposed within Triassic sandstones of the Solling-Formation (Middle Buntsandstein). Macroscopically, the carbonate-cemented dykes are distinguished from the flat-lying country rocks by S-dipping bedding, larger grain size of siliciclastic components, and a high, but varying amount of iron ore. Locally, the sharp boundary between dykes and country rock is coated with patches of calcite crystals. The iron ore components mostly consist of opaque or transparent ooids with irregular growth zones, which often merge into branched incrustations and are thus interpreted as in-situ mineralisation. Only a few isolated iron ooids with regular concentric growth zones are regarded as detrital components. On the basis of different cathodoluminescence colours and specific growth zoning, three generations of carbonate cement can be distinguished. This points to multiple dyke formation, which is confirmed by clasts of early-formed cement incorporated in younger dyke infill and complex relationships between different generations of cement and iron ores.

Many clasts in the dykes do not come from the country rock nor from the surrounding strata. Therefore we suggest that the sedimentary dykes were connected to the Tertiary peneplain and were filled by detritus. We propose that the iron ores are equivalent to iron concretions of Eocene age. In addition, the paleostress field was so oriented that opening of $\mathrm{N}-\mathrm{S}$ striking dykes was favoured along an older joint system.
\end{abstract}

Schlüsselworte-Sedimentgang, Tertiär, Eisenooide, Mikrogefüge, Kathodolumineszenz

\section{Einführung}

- Malte Ritter — m.ritter@stud.uni-goettingen.de

- Axel Vollbrecht - avollbr@gwdg.de

- Alfons van der Kerkhof - akerkho@gwdg.de

- Klaus Wemmer — kwemmer@gwdg.de

Geowissenschaftliches Zentrum der Universität Göttingen, Goldschmidtstr. 3, D-37077 Göttingen
Bei den untersuchten Aufschlüssen handelt es sich um zwei ehemalige Steinbrüche ca. $500 \mathrm{~m}$ NW' von Billingshausen am Nordosthang des Rodebachtals (Abb. 1). Anstehend sind mittlere Abschnitte des Bausandsteins der ca. $90 \mathrm{~m}$ mächtigen 
Solling-Folge (z.B. Müller et al. 1961), der hier eine 5 bis $10 \mathrm{~m}$ hohe Steilkante bildet. Im darüber liegenden Hang gehen die höheren Abschnitte der Solling-Folge in die vorwiegend tonigen Basisschichten des Oberen Buntsandsteins (Röt 1) über (Abb. 1b), in die, nach Osten zunehmend, größere Gipskörper eingeschaltet sind. In beiden Steinbrüchen sind jeweils Relikte eines Sandsteingangs in Form von isolierten, unregelmäßig verteilten Belägen auf einer steilen $\mathrm{N}-\mathrm{S}$ streichenden Hauptkluftfläche erhalten (Abb. 2).

Generell sind für die Entstehung von Sedimentgängen unterschiedliche Prozesse und Szenarien in Betracht zu ziehen (z.B. Hayashi 1966). Bei sog. Injektionsgängen werden unverfestigte (,flüssige') Sedimente durch hydrostatischen Überdruck von unten oder durch lithostatischen Druck von oben in Gesteinsspalten eingepresst. In ähnlicher Weise können auch halbverfestigte Sedimente durch Überdrucke oder tektonische Beanspruchung in Spalten des unmittelbar angrenzenden Nebengesteins eingepresst werden. Eine andere Gruppe stellen Sedimentgänge dar, die von der freien Oberfläche aus mit Detritus gefüllt werden und oft auch Fragmente aus dem Nebengestein (Brekziierung bei Öffnung der Spalten) sowie in situ-Mineralisationen enthalten (z.B. Wright et al. 2009). Dabei können komplexe Gangfüllungsmuster u.a. dadurch entstehen, dass die Gang- bzw. Spaltenöffnung mehrphasig erfolgt (z.B. Friese et al. 2010), oder dass über Lösungsprozesse sekundäre Hohlräume für jüngere Füllungen erzeugt werden.

Ziel der vorliegenden Untersuchung war, anhand von Makro- und Mikrogefügeanalysen erste Informationen über die Entstehung und Altersstellung der Sedimentgänge zu gewinnen und sie in die regionalgeologische Entwicklungsgeschichte einzubinden.

Bei den ersten Untersuchungen standen daher folgende Fragestellungen im Vordergrund:

1) Typisierung und mögliche Herkunft der siliziklastischen Gangkomponenten,

2) Mineralisationen im Gang und deren mögliche Altersabfolge.

3) Aus (1) und (2) ableitbares Modell für die Gangentwicklung.

Wesentliche Grundlage für die nachfolgenden Ausführungen stellt eine 12-wöchige BachelorArbeit dar, die am Geowissenschaftlichen Zentrum der Georg-August-Universität in Göttingen angefertigt wurde (Ritter 2010). Weiterführen- de Untersuchungen unter Einbeziehung stofflicher Analytik sind geplant. Dies betrifft vor allem die Zusammensetzung von Zementen und Erzmineralen.

\section{Methodik \\ 2.1 Probennahme}

Die Proben wurden bis auf wenige Handstücke als Bohrkerne gewonnen. Als Bohrgerät kam hierbei ein Akkubohrer (Metabo BS 18 LI) zum Einsatz, der mit einer handelsüblichen Lochsäge für Bohrungen in Zement und Bausteinen ausgestattet war (Hartmetallsplitterbesatz, Innendurchmesser etwa $2 \mathrm{~cm}$ ). Zur Kühlung wurde Wasser mit einer Labor-Spritzflasche von außen auf die Lochsäge und in die Bohrung gespritzt. Mit diesem Verfahren konnten bis zu ca. $5 \mathrm{~cm}$ lange Bohrkerne gewonnen werden. Um die Kerne nach dem Bohren aus dem Gestein zu brechen, wurde ein der Länge nach halbiertes Rohr mit entsprechendem Durchmesser als Hebel verwendet. Alle ProbenahmeStellen wurden fotographisch dokumentiert. Vor Anfertigung der Dünnschliffe wurden alle Proben sowohl mit einer herkömmlichen Digitalkamera als auch mit stärkerer Vergrößerung mit einem Auflicht-Mikroskop mit integrierter Kamera fotografiert.

Zur Schonung der wenigen erhaltenen Gangrelikte wurde die Anzahl der Proben beschränkt. Dadurch konnten nicht alle in situ beobachteten Makrogefüge auch mikroskopisch untersucht werden.

\subsection{Probenpräparation}

Wegen der hohen Porosität und geringen Festigkeit des Gesteins wurden die Proben zunächst mehrere Tage in Kunstharz (Araldit 2020) getränkt, bevor sie gesägt und zu polierten, nicht abgedeckten Dünnschliffen für die Polarisations- und die Kathodolumineszens-Mikroskopie (KLM) verarbeitet werden konnten. Als Bedampfungsmittel wurde Kohlenstoff verwendet, der mittels Widerstandsbedampfung im Hochvakuum auf die Dünnschliffe aufgetragen wurde. Die KristallrasenProben wurden vor Anfertigung der Dünnschliffe durch Tränkung in verdünnter Ameisensäure $\left(\mathrm{CH}_{2} \mathrm{O}_{2}\right)$ von Flechtenbewuchs befreit.

\subsection{Mikroskopische Verfahren}

Die Dünnschliffe wurden zunächst mit einem handelsüblichen Polarisationsmikroskop (Leica) untersucht. Zur Dokumentation wurde eine an das 


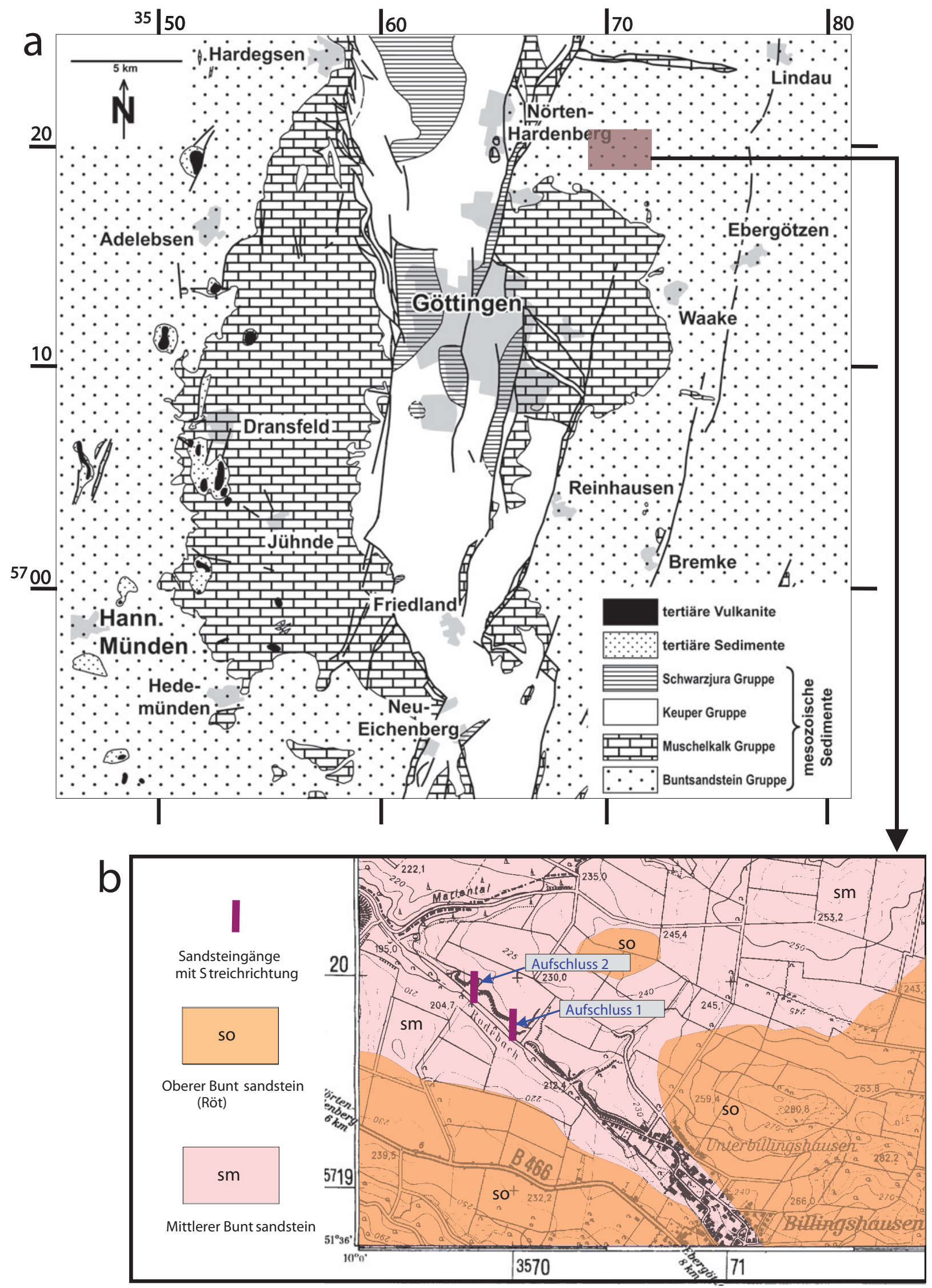

Abbildung 1. Geologischer Rahmen. a) Geologische Übersichtskarte von Göttingen und Umgebung (aus Arp et al. 2004; Quartär abgedeckt); farbiges Rechteck zeigt Lage von Karte in b). b) Aufschlüsse NW' Billingshausen mit Sandsteingängen im Mittleren Buntsandstein (sm); Kartengrundlage TK 25, 4326 Lindau; geologische Grenzen sm/so nach Heinrich (1977). 

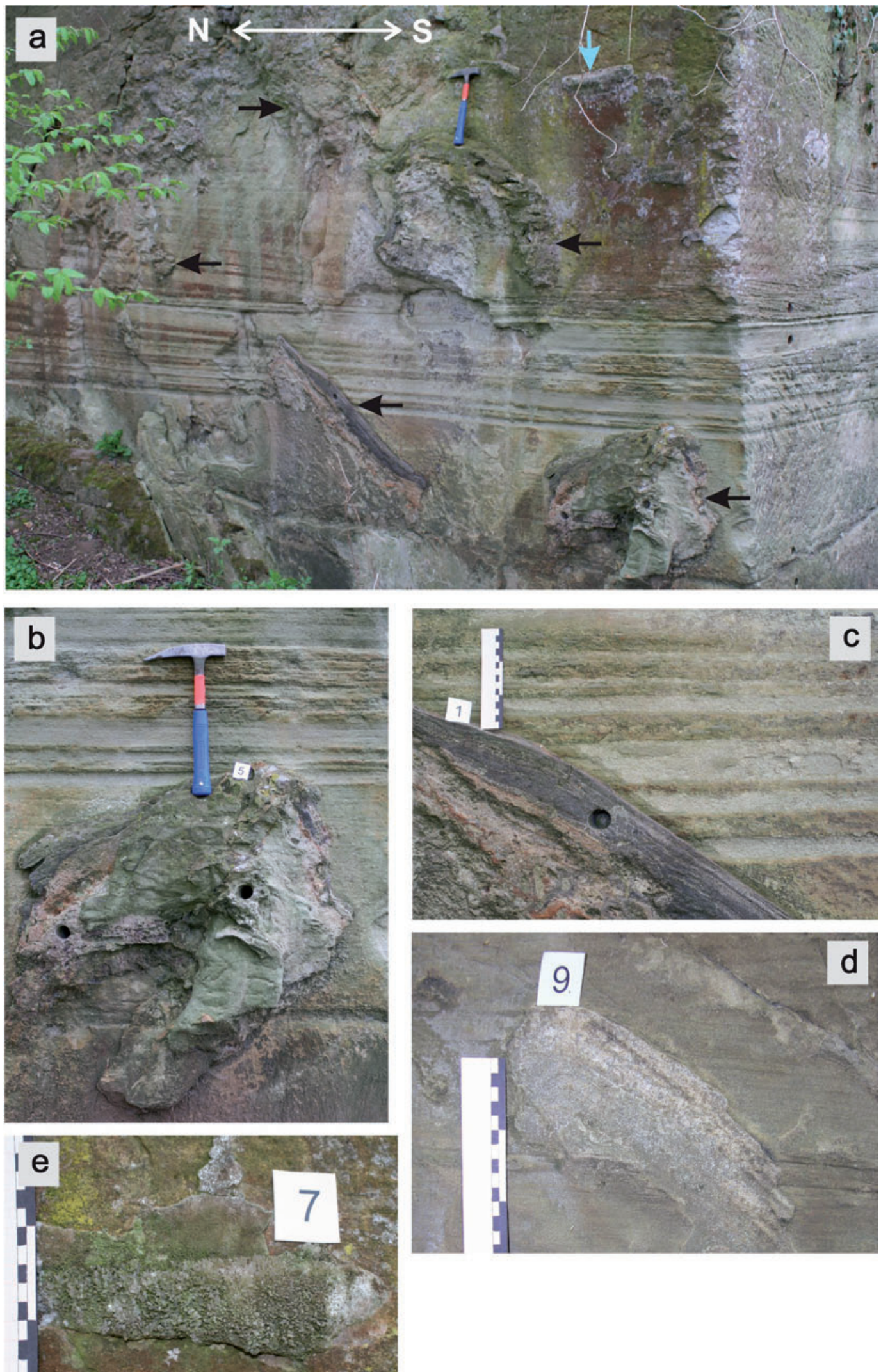

Abbildung 2. Sandsteingänge in Aufschluss 1) Isolierte Reste des Sandsteingangs in Aufschluß 1 auf Grenzfläche zum Rahmengestein (Solling-Bausandstein mit weitgehend horizontaler Schichtung); N-S streichende Grenzfläche stellt eine der beiden Hauptkluftscharen dar (vgl. Abb. 4); größte Gangrelikte mit schwarzen Pfeilen markiert, b) Gangrelikt aus zellig verwitterndem Sandstein, c) Gangrelikt mit braun gebändertem Sandstein; Schichtung mit ca. $40^{\circ}$ nach S einfallend, d) Gangrelikt aus hellem undeutlich geschichtetem Sandstein, e) Calcitkristall-Rasen auf Grenzfläche zum Rahmengestein (blauer Pfeil in a). (Weitere Erläuterungen im Text) 

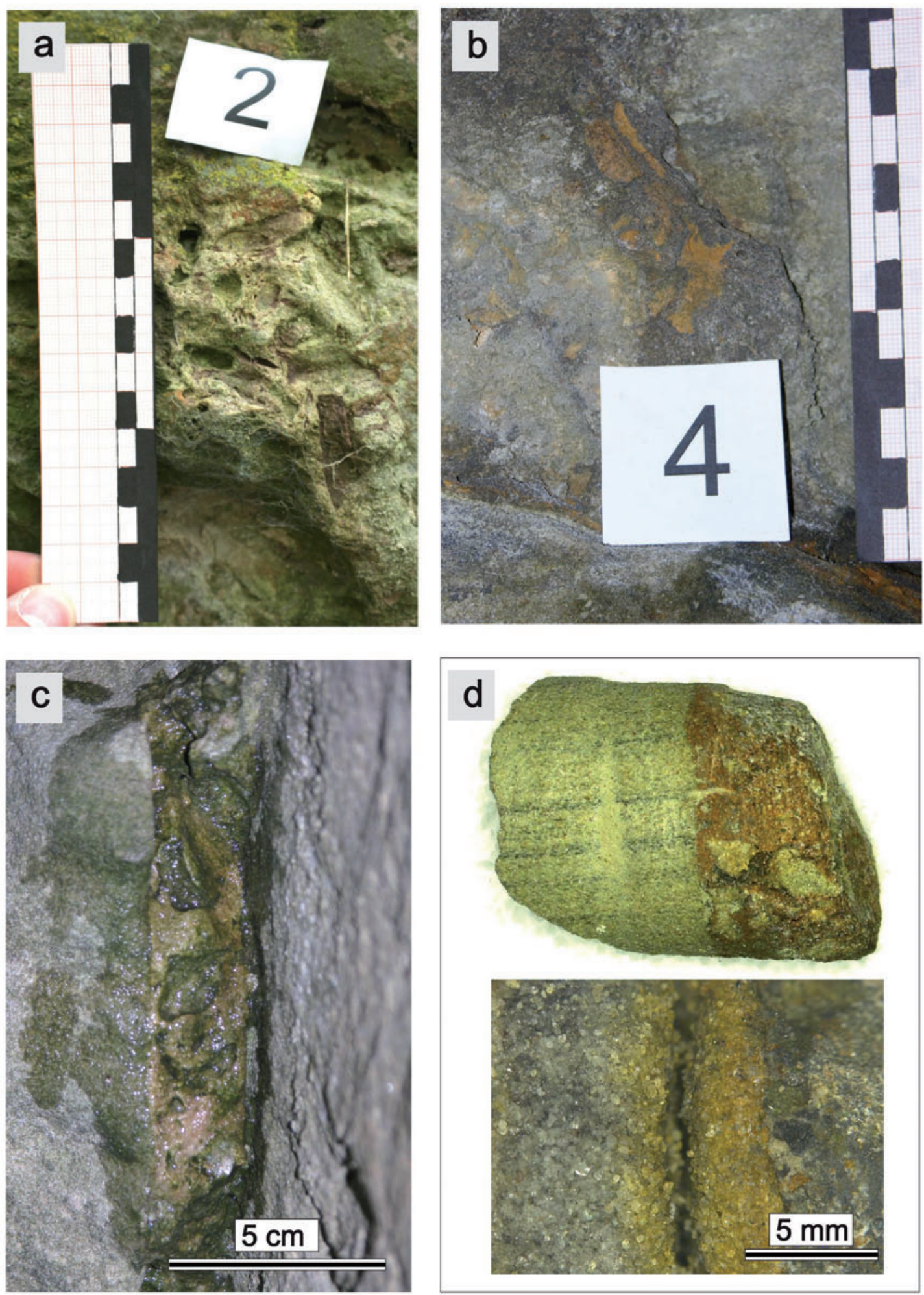

Abbildung 3. Details der in Abb. 2 gezeigten Gangrelikte (Makroaufnahmen). a) Gehäuftes Vorkommen von Geröllen im zellig verwitternden Gangfüllungstyp, b) Anreicherung von Eisenerz-Geröllen, c) Beidseitig vom Rahmengestein begrenztes Gangrelikt mit Geröllen und Eisenerzkruste an rechter Grenzfläche, d) Bohrkern mit scharfer Grenzfläche zwischen fein geschichtetem Rahmengestein (links) und Geröll führendem Gang (rechts); Bohrkerndurchmesser ca. 1,5 cm, e) Eisenoxid reiche Grenzzone zwischen Rahmengestein (links) und Gang (rechts). (Weitere Erläuterungen im Text) 
Mikroskop angeschlossene Digitalkamera (Canon EOS 350D) verwendet, die direkt mit einem Computer verbunden ist und über eine spezielle Software (DSLR Remote Pro von Breeze Systems Limited) von diesem aus gesteuert wird.

Übersichtsbilder der Dünnschliffe wurden mit einem Dia-Scanner (Nikon SUPER COOLSCAN 5000 ED mit zugehöriger Software) angefertigt. Dabei wurden zwei eigens zu diesem Zweck konstruierte Diarahmen verwendet, die jeweils einen Dünnschliff aufnehmen können. In einen der Diarahmen sind gekreuzte Polarisatorfolien eingesetzt, so dass Scan-Aufnahmen sowohl in normalem als auch polarisiertem Durchlicht möglich sind. Die Schliffscans dienten vor allem zur Lokalisierung von Beobachtungspunkten bei der KLM.

Die Kathodolumineszenzaufnahmen wurden mit einem sog. ,Heissfilament'-KL-Mikroskop durchgeführt (Modell HC3-LM Lumic-SimonNeuser Special Microscopes; Neuser 1995). Hierbei handelt es sich um ein Polarisationsmikroskop, das an Stelle eines Probentisches über eine evakuierbare Probenkammer mit eingebauter Elektronenstrahlröhre verfügt. In der Probenkammer wird die Dünnschliffoberfläche bei einem Vakuum von 10-5 mbar mit Elektronen beschossen (Beschleunigungsspannung $14 \mathrm{kV}$, Stromstärke ca. $0,18 \mathrm{~mA}$ ). Die bestrahlte Fläche hat einen Durchmesser von $3 \mathrm{~mm}$, die entsprechende Strahlstromdichte beträgt etwa $35 \mathrm{~W} \mathrm{~cm}^{-2}$. Das Mikroskop ist mit einer Peltier-gekühlten Digitalkamerasystem (Kappa DX 40C) ausgestattet, von der aus die Bilder direkt auf einen Computer übertragen werden. Für detaillierte Erläuterungen der KLM und Anwendungsmöglichkeiten in den Geowissenschaften siehe z.B. Neuser et al. (1995).

\section{Ergebnisse}

\subsection{Geländebeobachtungen}

Die Rahmengesteine der Sedimentgänge bilden hellgraue bis rotbraune, feinkörnige Sandsteine der Solling-Folge (Bausandstein). Der Sandstein bildet meist homogene Bänke von bis zu mehreren Metern Mächtigkeit, die etwa horizontal gelagert sind (Abb. 2). Oft weisen die Bänke eine interne Schrägschichtung auf, wobei die einzelnen Lagen bis zu etwa $5 \mathrm{~cm}$ mächtig sind. An der Kontaktfläche zu den Sedimentgängen sind einige Lagen rotbraun gefärbt und verwitterungsresistenter als die hellgrauen Lagen. Diese rotbraune Färbung reicht jedoch nur wenige Millimeter in das Rahmengestein hinein. Mit der Lupe lässt sich eine mäßige bis gute Kornrundung und Sortierung erkennen. Stellenweise findet man kreisrunde, zentimetergroße Löcher, welche auf die Herauswitterung karbonatisch zementierter Linsen zurückgeführt werden (z.B. Heinrich 1977). Den Sandsteinbänken sind mehrere tonige Lagen zwischengeschaltet, von denen eine besonders auffällig ist und sich in beiden Aufschlüssen verfolgen lässt. Sie ist zwischen zehn und zwanzig Zentimeter mächtig und führt Tongerölle von bis zu mehreren Zentimetern Durchmesser.

Die Sedimentgänge sind in beiden Aufschlüssen nur noch in Form von isolierten, reliktischen Belägen auf steilen, NS streichenden Kluftflächen im Rahmengestein erhalten (Abb. 2a). Vereinzelt findet man jedoch auch Bereiche, in denen das Rahmengestein noch auf beiden Seiten eines Gangs erhalten geblieben ist (Abb. 3c). Die sich aus den Gangrelikten ergebenen minimalen Mächtigkeiten liegen zwischen 2 und $20 \mathrm{~cm}$.

Nach makroskopischen Merkmalen lassen sich bei den Gangfüllungen prinzipiell drei Formen unterscheiden (Abb. 2 und 3):

1. Helle, zellig verwitternde Sandsteine, die etwa $20 \mathrm{~cm}$ dicke Beläge auf der Gangwand bilden (Abb. 2b). Einige dieser zentimetergroßen, eckigen Zellen sind mit eckigen Fragmenten eines roten, sehr fein laminierten Sandsteins ausgefüllt. Diese Fragmente lassen sich jedoch leicht herauslösen und zerbröckeln, ebenso leicht wie das angrenzende Rahmengestein. Vor allem in dieser Art Gangfüllung finden sich auch unregelmäßig begrenzte Zonen mit Anreicherungen unterschiedlicher Gerölle, darunter auch Sandsteingerölle, die aus dem Buntsandstein stammen könnten (Abb. 3a).

2. Lange, schmale nur etwa 2 bis $5 \mathrm{~cm}$ dicke Sandsteinlinsen, deren Langachsen und interne Schichtflächen in der Regel mit 30 bis $40^{\circ}$ nach Süden einfallen (Abb. 2c). Sie sind deutlich härter als das Rahmengestein und entweder dunkel oder wechselnd braunrot und hellbraun gefärbt. Gelegentlich findet man beide Färbungen auch in ein und demselben Sandsteinstück, wobei die dunkel gefärbte Partie immer die Oberseite bildet und scharf von der helleren abgegrenzt ist. In Teilen der dunklen Partien findet man, vor allem im Aufschluss 2, gelb-braune rundliche Vererzungen (Abb. 3b und c), die in Größe und Form den 
tertiären (eozänen) ,Bohnerzen` ähneln (z.B. Ritzkowski 1999).

3. Ebenfalls relativ harte Sandsteine, die jedoch grau gefärbt sind und keine oder nur eine undeutliche Schichtung aufweisen (Abb. 2d). Sie bilden meistens rundliche Anhaftungen auf der Kontaktfläche zum Rahmengestein, zum Teil aber auch gestreckte Körper, deren Langachsen ebenfalls mit etwa $40^{\circ}$ nach Süden einfallen.

Bei allen genannten Formen ist der Kontakt zum Rahmengestein scharf. Ebenso reicht dessen Feinschichtung stets ungestört bis direkt an die Gangfüllungen heran (Abb. 3d). Oft ist entlang der Kontaktfläche eine erzreiche Zone bzw. Kruste zu beobachten (Abb. 3c und d).

Die Gänge zeigen keine spezifischen Interngefüge, die auf Einströmbewegungen und damit auf hydraulische Bildungsprozesse (Injektion von fließfähigen Sedimenten; s.o.) hindeuten, wie z.B. Lagenbau oder Körngrößengradienten parallel zur Gangwand. Dagegen wurde an einer Stelle eine taschenförmige Internschichtung beobachtet, die auf ein Einsacken während eine jüngeren Öffnungsphase des Gangs hindeuten könnte.

Der Sedimentgang in Aufschluss I enthält in Teilbereichen zusätzlich Calcitkristallrasen, die von der Grenzfläche zum Rahmengestein ausgehend in den Gang hineingewachsen sind (Abb. 2e). An vielen Stellen durchgeführte $\mathrm{HCl}-$ Karbonattests lassen bereits darauf schließen, dass auch in der Gangmatrix Karbonat enthalten ist.

Das Haupt-Kluftsystem wird von zwei zuein-

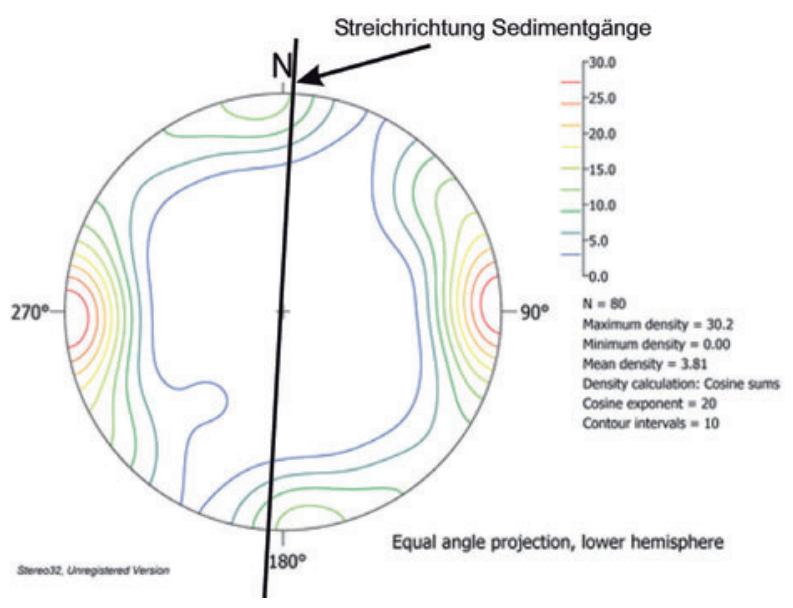

Abbildung 4. Kluftflächenpole im SollingBausandstein; Schmidt-Netz, untere Halbkugel; mit Streichrichtung der Gänge. ander ungefähr orthogonal stehenden senkrechten Scharen gebildet (Abb. 4), wobei die Hauptschar N-S streicht. Entsprechende Richtungen, jedoch mit stärkerer Ausprägung der E-W-streichenden Kluftschar, wurden auch in der näheren Umgebung festgestellt (z.B. Beer 1977)

\subsection{Mikrogefüge}

\subsubsection{Rahmengesteine}

Die mikroskopische Beschreibung der Rahmengesteine beschränkt sich auf einen kurzen Überblick und konzentriert sich auf Aspekte, welche für die Diskussion der Sedimentgang-Genese von Interesse sind.

Das häufigste Rahmengestein ist eine mittelbis feinkörnige Arkose mit deutlicher Schichtung (Abb. 5a bis c). Die Sortierung ist generell gut. Die Quarzkörner sind eckig bis mäßig gerundet und zeigen im KL-Bild drei verschiedene Farben (mit abnehmender Häufigkeit): braun, violett und dunkel blau. Monokristalline Quarzkörner überwiegen, polykristalline Quarze mit stark verzahnten Kristallgrenzen treten dagegen nur vereinzelt auf. Des Weiteren gibt es Lagen, die fast nur aus Quarzkörnern bestehen.

Die Kalifeldspäte sind ebenfalls gut sortiert und besitzen unregelmäßig eckige bis schwach gerundete, z.T. auch längliche Formen. Im KLBild treten sie durch intensive, hellblaue Farben hervor und überstrahlen dadurch oft angrenzende Quarzkörner. In geringen Mengen tritt auch Plagioklas auf. Die Feldspäte besitzen stellenweise dünne Karbonat-Anwachsungen.

Opake Erze sind im Vergleich zu den Ganggesteinen (s.u.) recht selten. Sie treten vor allem als feinkristalline Zwickelfüllungen zwischen den Quarz- und Feldspatkörnern auf und sind demnach als in-situ-Mineralisationen zu betrachten.

In einigen Lagen des Gesteins sind größere Hellglimmerschuppen angereichert, die durch mechanische Kompaktion wellig verbogen sind und eine deutliche Feinschichtung abbilden (Abb. 5c; s.a. Abb. 3d). In diesen Bereichen findet man stellenweise auch kleinere Domänen mit karbonatischem Zement. In Proben aus dem Nahbereich zu den Sedimentgängen treten feinkristalline CalzitMikrogänge auf, die vereinzelt unterschiedlich geformte Quarzkörner enthalten (Abb. 5d). Die Entstehung dieser Mikrogänge steht wahrscheinlich mit den Sedimentgängen in Verbindung, da sie in deren Nähe gehäuft auftreten. 

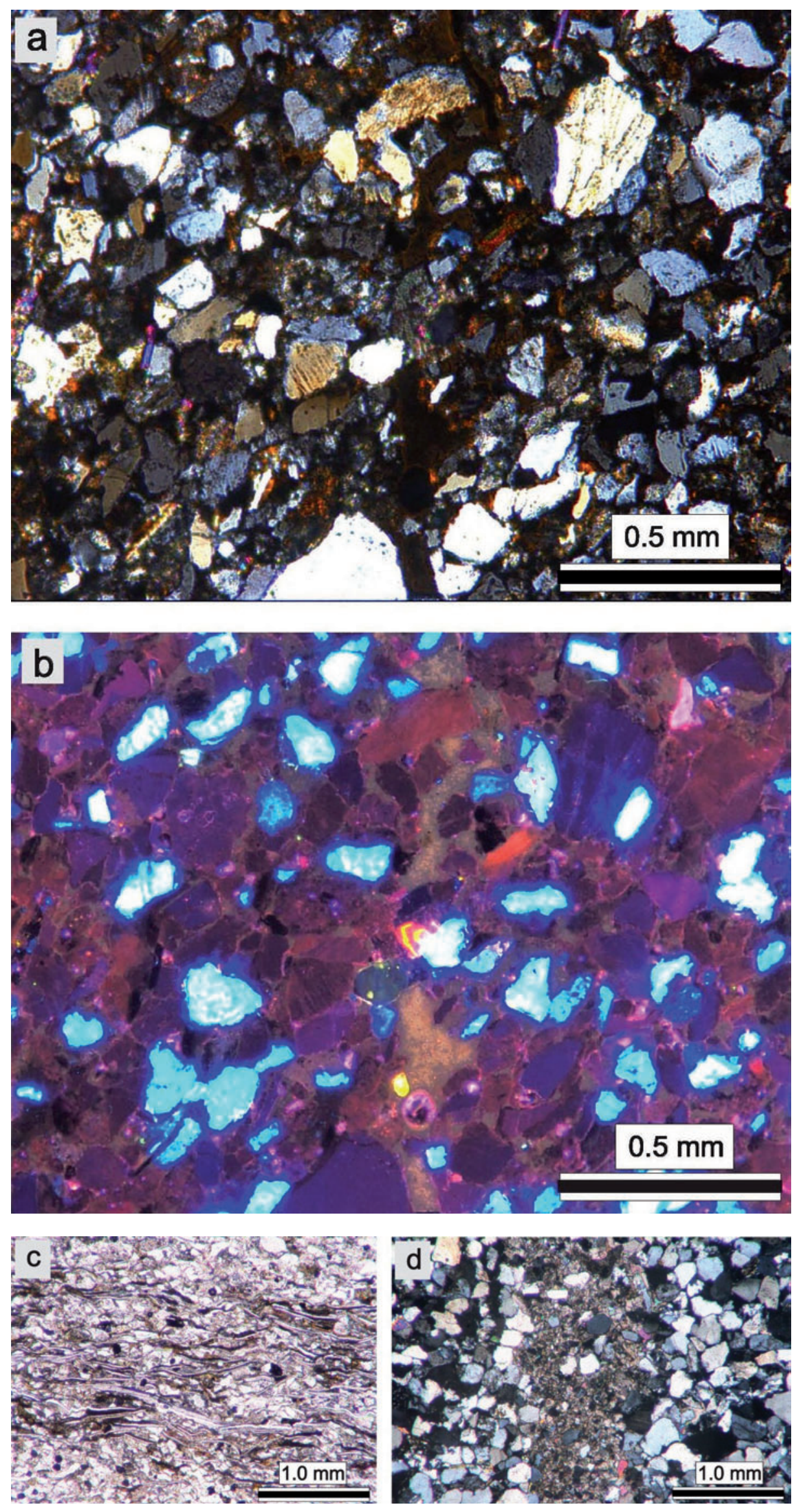

Abbildung 5. Mikrogefüge des Rahmengesteins: a) Übersichtsbild; X Pol., b) KL-Bild desselben Ausschnitts, c) Durch glimmerreiche Lagen abgebildete Feinschichtung; II Pol., d) Calcit-Mikrogang (Bildmitte) mit vereinzelten Quarzkörnern; in der Entstehung vermutlich dem Hauptgang zuzuordnen. (Genaue Erläuterungen im Text). 

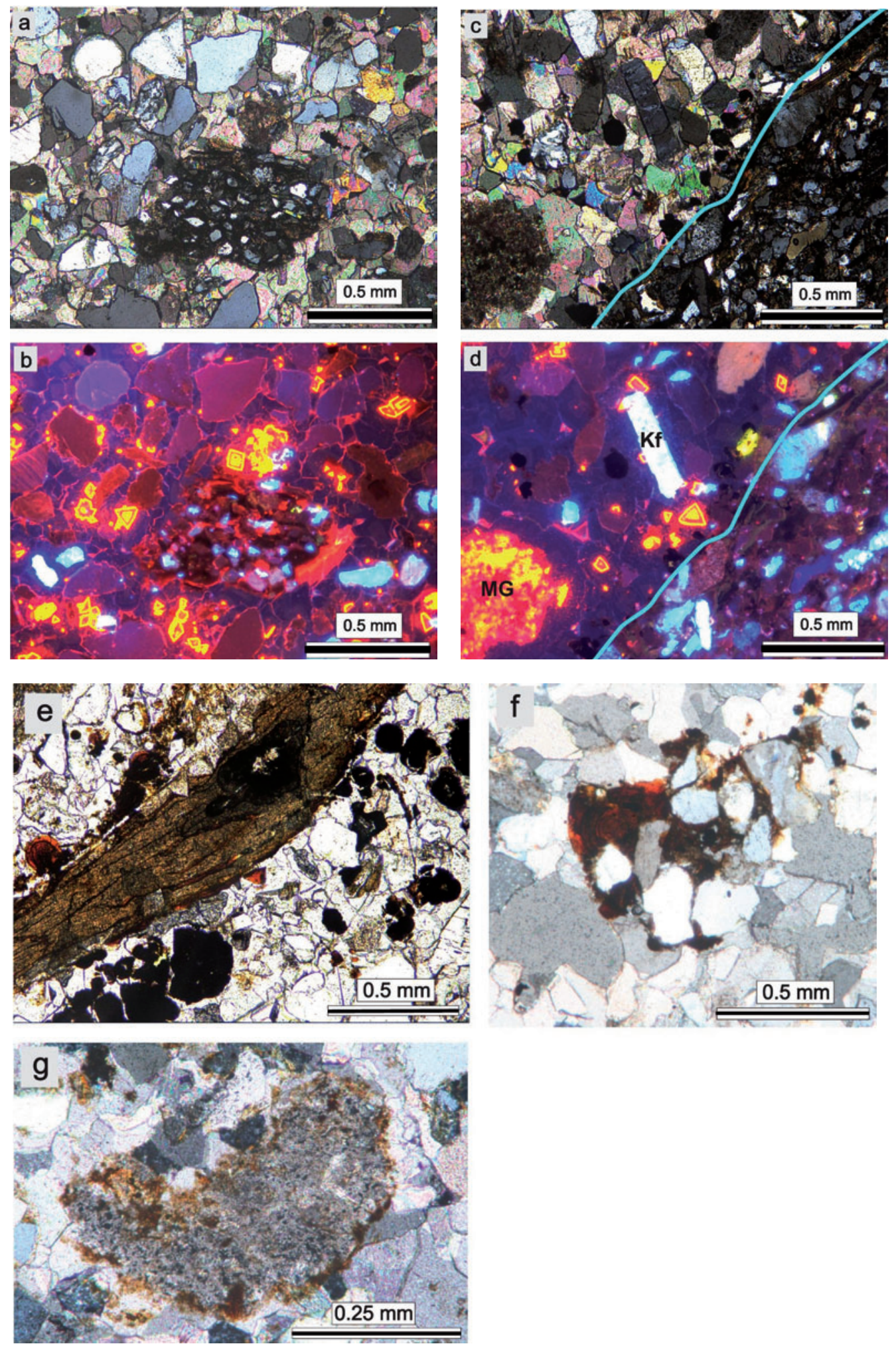

Abbildung 6. Detritische Komponenten im Sedimentgang: a) Übersichtsbild; X Pol.; im Zentrum ein Gesteinsfragment, das wegen seiner eckigen Forn und Zusammensetzung aus dem unmittelbaren Rahmengestein stammen könnte (Bausandstein), b) KL-Bild desselben Ausschnitts; Quarzkörner mit polymiktem KL-Farbspektrum; hellblau: Kalifeldspat; Erläuterungen zu Zementfarben (rot, gelb, dunkelblau) s. Abb. 7, c) Kontaktzone zwischen Gang (links) und Rahmengestein (rechts), Grenze markiert durch blaue Linie; Gang im Vergleich zum Rahmengestein mit deutlich gröberen detritischen Komponenten und ohne Kornformregelung, d) KL-Bild desselben Ausschnitts; im Gang neben Quarzkörnern als weitere Komponenten u.a. ein Geröll eines mikritischen Kalksteins (MG) mit undeutlichem Zement-Anwachssaum und ein hypidiomorpher Kalifeldspat ( Kf, ? authigen), e) Feinkristallines deutlich foliiertes Metapelit-Geröll, f) Mit Eisenerz (Ooide, Krusten) zementiertes polykristallines Quarzit-Korn, g) Mikritisches Kalkstein-Korn. (Weitere Erläuterungen im Text) 


\subsubsection{Sedimentgänge}

\subsubsection{Detritische Komponenten:}

Die detritischen Komponenten im Ganggestein bestehen im Wesentlichen aus mittel- bis grobkörnigen Quarzkörnern, wobei monokristalline Körner gegenüber polykristallinen überwiegen (Abb. 6a und b). Die Quarzkörner zeigen sehr unterschiedliche Rundungsgrade, sind tendenziell aber besser gerundet als die in den Rahmengesteinen. Sie zeigen im KL-Bild dasselbe polymikte Farbspektrum wie die Quarze im Rahmengestein (braun, violett und dunkelblau; Abb. 6b). Polykristalline Körner aus Kristallen sehr unterschiedlicher Größe mit stark verzahnten Grenzflächen, wie sie im Rahmengestein zu finden sind, kommen ebenfalls vor.

Vereinzelt treten auch in den Sedimentgängen Kalifeldspäte auf. Diese sind meist schlecht gerundet oder zeigen z.T. hypidiomorphe Umrisse (Abb. 6c und d), was auf eine authigene Bildung hindeuten könnte. Des Weiteren findet man auch Gesteinsbruchstücke, von denen einige aus den unmittelbaren Rahmengesteinen stammen könnten (Abb. 6a und b). Die meisten Gesteinbruchstücke sind jedoch als exotisch einzustufen. Hierzu zählen in erster Linie feinkristalline phyllosilikatreiche Körner (bis Geröllgröße). Diese Metapelite können jedoch aufgrund ihrer starken Alteration nicht mehr eindeutig klassifiziert werden (Abb. 6e). Einige dieser MetapelitGerölle enthalten mikritische Karbonatlinsen, bei denen es sich vermutlich um Alterationsprodukte handelt (Pseudomorphosen). Selten beobachtet wurden Körner, die kleinere Quarzkörner enthalten und durch Eisenerze (s.u.) zementiert sind (Abb. 6f).

In den Gängen treten vereinzelt größere, fast undeformierte Hellglimmer-Schuppen auf. Diese stammen vermutlich auch nicht aus den Rahmengesteinen, in denen die Glimmer i.d.R. deutlich deformiert sind (s.o., Abb. 5c).

Neben den o.g. siliziklastischen Komponenten kommen weniger häufig auch unregelmäßig geformte, mikritische Karbonatkörner vor (Abb. 6c, d und g). Bei polarisiertem Licht werden fleckenhafte Auslöschungsmuster sichtbar, die vermutlich ein ursprünglich grobkristallines Gefüge abbilden. Ihre hellgelben bis roten KL-Farben lassen indirekt auf eine Mg-reiche calcitische bzw. dolomitische Zusammensetzung schließen (Abb. 6d). Die Körner besitzen z.T. unscharfe Begrenzungen mit Übergängen in den Zement, weshalb eine eindeutige Ansprache nicht immer möglich ist. So kann auch eine in situ-Bildung nicht ausgeschlossen werden (evtl. mikritisierter Zement). Teilweise besitzen sie auch undeutliche Zement-Anwachssäume (Abb. 6c und d).

\subsubsection{Karbonatzemente:}

Der karbonatische Zement ist weitgehend blockig ausgebildet (Abb. 7), wobei polariationsmikroskopisch grobkörnige und eine feinkörnige Domänen erkennbar sind. Im KL-Bild lässt sich eine genauere Abgrenzung durchführen, so dass insgesamt drei Zementgenerationen unterschieden werden können (Z I bis Z III). Deren Altersabfolge lässt sich eindeutig im Bereich eines zementierten/verheilten Mikrogangs nachweisen (Abb. 7a und $b$ ):

Z I ist ein feinkörniger Zement, der im KLBild einen rot-gelben sehr regelmäßigen Zonarbau mit scharf begrenzten, ebenen Umrissen zeigt. Dazwischen befinden sich schwach dunkelblau lumineszierende Zonen mit feiner Interstruktur, die nur bei niedrigen Lichtintensitäten sichtbar sind (Abb. 7e), ansonsten aber in den Bildern durch die angrenzenden rot-gelb Farben überstrahlt werden (Abb. 7b und d). Der Z I bildet den Rahmen des Mikrogangs und stellt damit die älteste Generation dar. Im Zentrum einiger Z I-Karbonatkristalle sind konzentrisch zonierte rundliche Strukturen zu erkennen, die Ooiden ähneln (Abb. 7e). Möglicherweise bildeten sie die Kristallisationskeime für den frühen Karbonatzement, wobei die zuerst gebildeten Kristalle dann im Laufe der Zeit zu größeren Aggregaten zusammenwuchsen.

Z II ist ein grobkörniger, blockiger Zement, der im KL-Bild dunkelblau bis violett erscheint und den Mikrogang in Z I verheilt hat (Abb. 7b). Meist zeigt Z II keinen oder nur undeutlichen Zonarbau (Abb. 7c) oder nur eine sehr schwache KL. Stellenweise ist jedoch (wie in Abb. 7b) ein sehr deutlicher, regelmäßiger Zonarbau entwickelt. Nach den bisherigen Beobachtungen kann keine Aussage über die genetischen Beziehungen dieser beiden Z II-Varietäten gemacht werden.

Z III ist nur an wenigen Stellen sichtbar. Er ist ähnlich grobkörnig wie der Z II und wächst auf dessen Kristallen auf (Abb. 7b). Er leuchtet im KL-Bild rot. Ansonsten tritt Z III lokal be- 

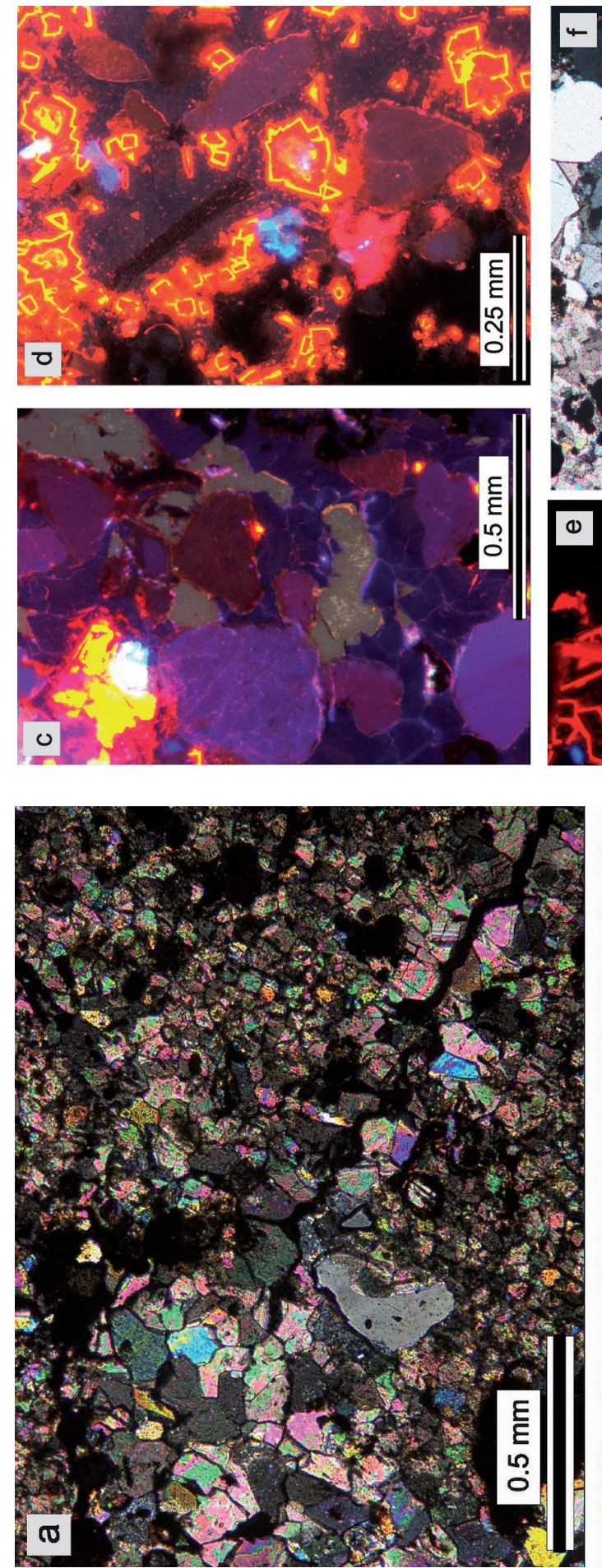
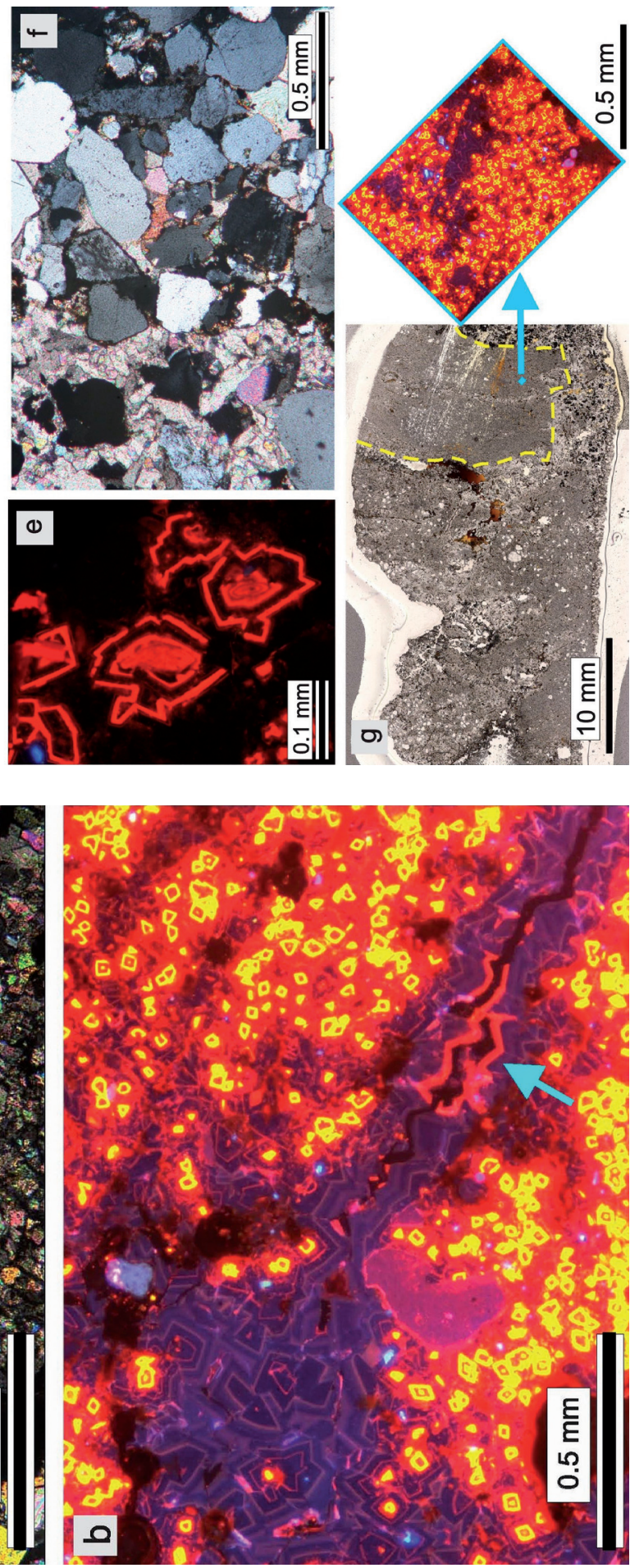

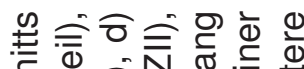

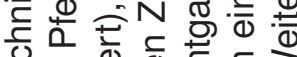

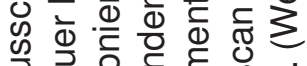

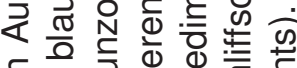

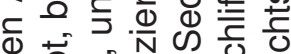

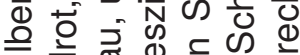

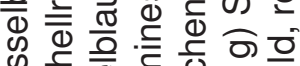

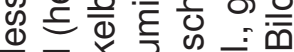

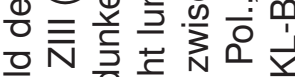

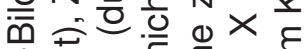
光产䒕 ว O N 芒茪 ¿ 은

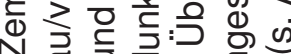
N 넘흐웡

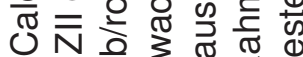
$\varepsilon$ 잉 잉 윤 ब. बे

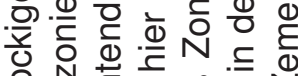
응 긍

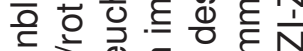
헤응 产 ळ

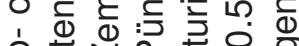
은돈

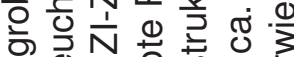

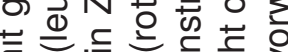

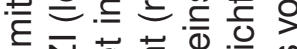

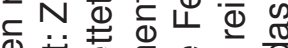

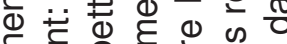
: ह ह ठ N

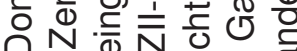

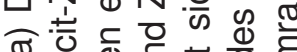
정 कृष $\frac{1}{\bar{\sigma}}$ ฮั ธ

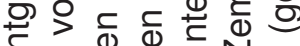

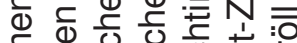
E는 8 음 फ $\frac{\pi}{0} 0$ Е Ф ब( 0 듣

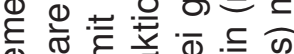

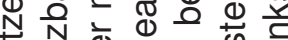

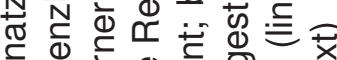
ธัँ :

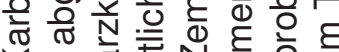

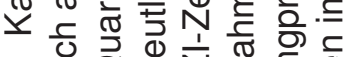

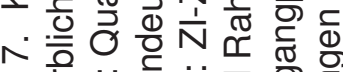

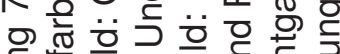

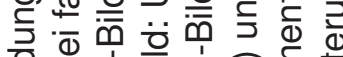

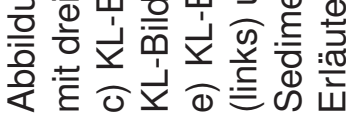


grenzt als dünner Saum zwischen Z II-Kristallen und Calcitkristallen (Kristallrasen, s.u.) sowie in Mikrorissen auf.

Die verschiedenen KL-Farben von Karbonaten werden durch Fremdatome erklärt, wobei insbesondere unterschiedliche $\mathrm{Fe}^{2+} / \mathrm{Mn}^{2+}$-Verhältnisse und deren absolute Konzentration von Bedeutung sind (z.B. Bruhn et al. 1995). Ein Überwiegen von $\mathrm{Mn}^{2+}$ erzeugt helle orange bis gelbe $\mathrm{KL}$, während Dominanz von $\mathrm{Fe}^{2+}$ schwache orange bis rote KL hervorruft. Tiefblaue bis schwach violette oder fehlende KL lässt auf fremdatom- und fehlstellenarme Kristalle (intrinsische KL) bzw. nur sehr geringe $\mathrm{Mn}^{2+}$-Gehalte schließen (Details zu Ursachen der KL in Karbonaten u.a. in Braithwaite (1993) sowie Richter und Zinkernagel (1981)). Unter Berücksichtigung weiterer Spurenelemente erlauben die KL-Farben auch Rückschlüsse auf die am Zementationsablauf beteiligten Wässer (z.B. Bruhn et al. 1995). Danach könnten die dunklen KL-Farben/geringe $\mathrm{Mn}^{2+}$-Gehalte des Z II auf ein meteorisch beeinflusstes, oxidisches Milieu hindeuten. Dem Z III entsprechende Rotfarben werden i.d.R. Mg-reichen Karbonaten zugeordnet, insbesondere wenn $\mathrm{Mn}^{2+}$-Ionen die $\mathrm{Mg}$ Gitterpositionen besetzen (z.B. Richter et al. 2003). Karbonate mit den hier für Z I festgestellten rot/gelben KL-Farben wurden von Friese et al. (2010) als Rissfüllungen in Sedimentgängen beobachtet, die zweifelsfrei mit marinen Wässern in Verbindung standen.

Die KL-Farbmuster bilden stellenweise eine Kontaktaureole zwischen Z I und Z II ab, in der die blauen Z II-Körner rote Flecken enthalten, die mit zunehmender Entfernung vom Kontakt immer kleiner und weniger werden (Abb. 7d). Vermutlich handelt es sich dabei um Reaktionszonen zwischen beiden Zementtypen, in denen sekundärer diffusiver Stoffaustausch stattfand (Fe, Mn).

Der Z I dringt von der Kontaktfläche aus z.T. in einer wenige Körner breiten Zone in das Nebengestein ein (Abb. 7f). Weiter entfernt vom Kontakt sind gelegentlich auch einzelne Z I-Kristalle zu finden (Abb. 5b). Danach waren die Kontaktflächen offensichtlich versiegelt; denn Z II Zement wurde im Rahmengestein nicht beobachtet.

Außerdem wurden im Gang auch Fragmente gefunden, die vorwiegend aus Z I bestehen (Abb. $7 \mathrm{~g}$ ). Hierbei handelt es sich um früh zementierte Gangbereiche, die zerbrochen sind und als Klasten in jüngere Gangsedimente gelangten.

\subsubsection{Calcitkristall-Rasen:}

Die Calcitkristall-Rasen bestehen aus klaren, durchscheinenden Kristallen (Abb. 8a), deren Größe von der Anwachsfläche zur freien Oberfläche hin deutlich zunimmt (Abb. 8b). Die Kristalle zeigen dunkelblaue KL-Farben, die in Schnitten senkrecht zur Langachse eine undeutliche, sehr engständige, regelmäßige und leicht gewellte Wachstumszonierung abbilden (Abb. 8c). Diese könnte durch zyklische Konzentrationsänderungen während des Ausscheidungsprozesses hervorgerufen sein. In Schnitten parallel zur Langachse wurde zusätzlich eine auf wenige Abschnitte begrenzte KL-Wachstumszonierung mit unregelmäßigen Abständen und deutlicheren Blau-Kontrasten beobachtet (Abb. 8d), die auf episodische Einschübe von Lösungen mit unterschiedlich (hohen) $\mathrm{Fe}^{2+} / \mathrm{Mn}^{2+}$ Verhältnissen hinweist.

Die Zwickel zwischen den Kristallen sind z.T. mit feinlamellierten, bräunlichen Eisenerzkrusten gefüllt, die dem unten beschriebenen Typ II ähneln (Abb. 8e). Gelegentlich enthalten diese Zwickel auch ein Gemenge von Eisenerzen und Calcitfragmenten (Abb. 8f).

Die Alterstellung der Calcitkristallrasen im Zuge der Gangbildungen ist ungeklärt, da mögliche strukturelle Beziehungen zu den Karbonatzementen wegen ihres isolierten Vorkommens nicht untersucht werden konnten. Wegen der ähnlichen KL-Farben wäre eine Beziehung zu Z II denkbar. Wegen der schwachen KL wäre auch hier eine Bildung aus meteorisch beeinflussten Wässern anzunehmen. Entsprechend könnten die feinen karbonatischen Säume und Rissfüllungen mit roter KL (Abb. $8 \mathrm{c}$ und d) dem vermutlich Mg-reichen Z III zugeordnet werden.

\subsubsection{Eisenerze:}

Ein weiterer Bestandteil der Sedimentgänge sind Eisenerze. Neben Eisenerz-Krusten in Poren, Mikrorissen und an Korngrenzen kommen vor allem verschiedene Arten ooidischer Eisenerze vor. Die Klassifikation als ,Ooide' wird hier rein deskriptiv angewendet für sphäroidale bis ellipsoidale Körner von maximal $2 \mathrm{~mm}$ Durchmesser, die aus konzentrisch um einen Kern angeordneten, glatten Schichten bestehen, wobei die Schichtdicke variieren kann (Tucker und Wright 1990). Entsprechen- 

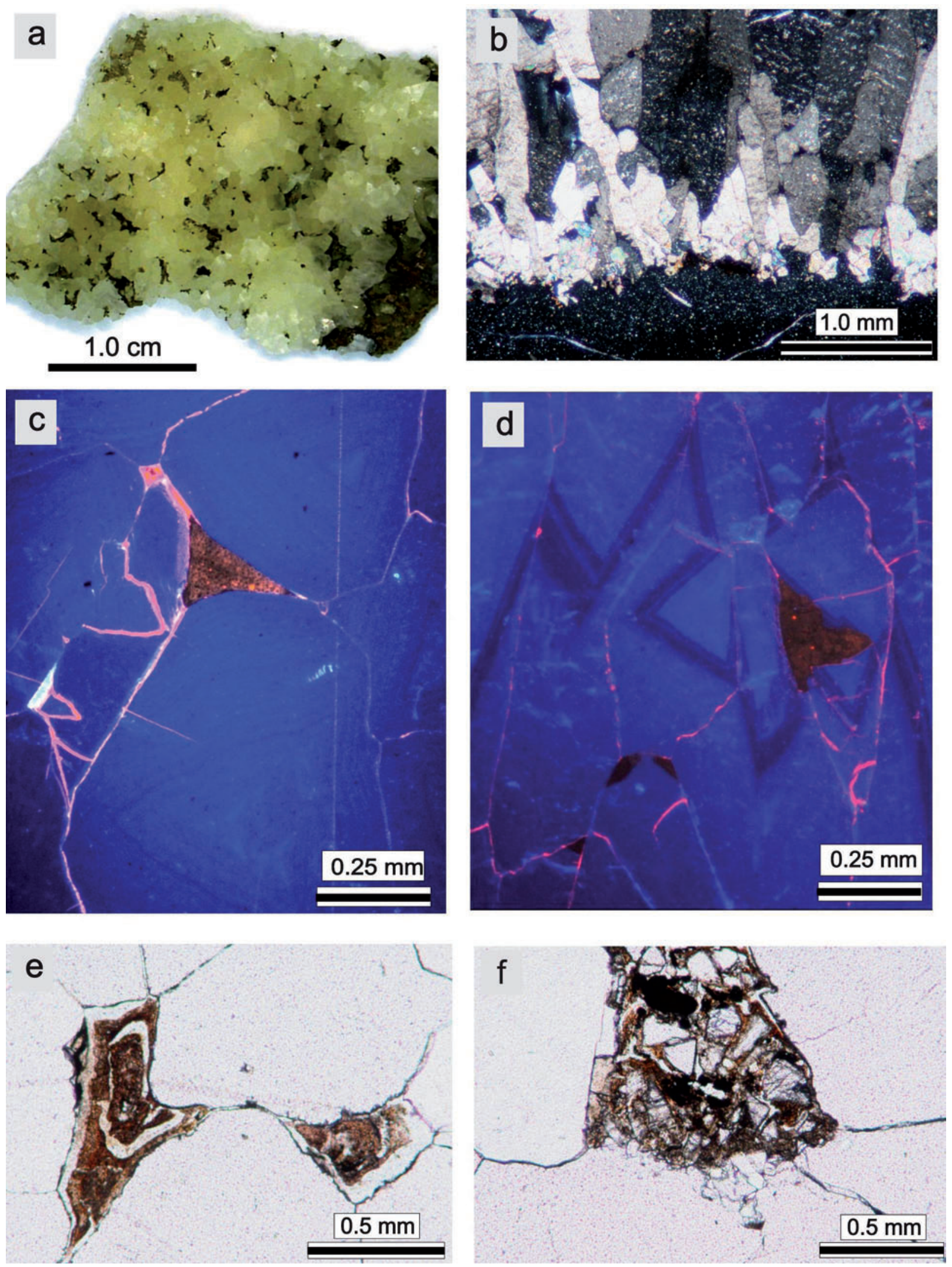

Abbildung 8. Calcit-Kristallrasen: a) Handstück, Blick auf die Kopfflächen, b) Dünnschliffbild von Kristallrasen im Querschnitt; X Pol.; Kristallgrößen in Wachstumsrichtung zunehmend, c) KL-Bild: Schnitt senkrecht zu Kristall-Langachsen, d) KL-Bild: Schnitt parallel zu Kristall-Langachsen, e) Eisenerzkrusten in Zwickel zwischen Calcitkristallen; II Pol., f) Aggregate aus Calcitbruchstücken und Eisenerzkrusten als Zwickelfüllung; II Pol. 
de Körner, deren Durchmesser größer als $2 \mathrm{~mm}$ ist, bezeichnet man nach dieser Klassifikation als Pisoid.

Für nachfolgend beschriebenen Eisenerz-Typen konnte noch keine generell gültige Altersbeziehung nachgewiesen werden (s.u). Die Auflistung erfolgt daher nach der relativen Häufigkeit; Abb 9):

Typ I: Opake, unregelmäßig geformte Eisenooide mit Durchmessern bis zu $100 \mu \mathrm{m}$ (Abb. 9a), Sie gehen z.T. in Krusten über, die stellenweise auch einzelne opake Ooide oder Ooid-Aggregate ummanteln. Typ I tritt gehäuft in den Randzonen der Gänge (Abb. 9b) oder im Kontakt zu siliziklastischen Geröllen auf (Abb. 9c). Internstrukturen sind im Durchlicht aufgrund der Opazität, die vermutlich durch hohe Mangangehalte bedingt ist, nicht zu erkennen. Möglicherweise stellen ringförmige Calcit-Zonen (Abb. 9a und d) gefüllte Dehnungsfugen dar, die konzentrische Wachstumszonen innerhalb der Ooide nachzeichnen. Eindeutige Belege für Calcitzement gefüllte Dehnungsstrukturen sind in Form von Ooid durchschlagenden Mikrogängen vorhanden (Abb. 9d).

Typ II: Rundliche z.T. auch unregelmäßig geformte, braun-rote transparente Eisenooide mit Durchmessern bis zu $100 \mu \mathrm{m}$ (Abb. 9e). Sie treten i.d.R. in Form von Aggregaten auf, in denen die einzelnen Ooide meistens unvollständige Rundkörper bilden, die direkt oder durch Krusten miteinander verwachsen sind (Abb. 9f). Die Ooide zeigen interne Wachstumszonen, deren Dicke z.T. recht konstant ist (Kernbereiche), meistens aber stark schwankt mit den größten „Mächtigkeiten im mittleren Sektor. Teilweise stoßen die Wachstumszonen auch diskordant aneinander (Abb. 9g), die Kerne sind häufig opak mit undeutlicher Abgrenzung. Der äußerste Anwachssaum ist gelegentlich auch ebenflächig begrenzt (Abb. 9f). Auch Typ II bildet z.T. Krusten um siliziklastische Gerölle (Abb. 9f). Ein Teil dieser Ooide kommt auch in Form von flächenförmig angeordneten, feinkörnigen Aggregaten im Karbonatzement vor (Abb. 9h), wobei in den einzelnen Ooiden nur selten eine Wachstumszonierung erkennbar ist (Abb. 9i und k). Hierbei handelt es sich höchstwahrscheinlich um in situ-Mineralisationen in Mikrorissen. An wenigen Stellen treten auch größere Ooide im Bereich dieser z.T. komplex verzweigten Risse auf (Abb 9h und k).

Die meisten der oben beschriebenen Struk- turmerkmale lassen darauf schließen, dass beide Ooid-Typen in situ gebildet wurden. Vereinzelt treten aber auch isolierte, gut gerundete Ooide auf (opak und transparent), die auch als eingespülte, d.h. detritische Komponenten interpretiert werden könnten (Abb. 91). Sie unterscheiden sich von den vermeintlichen in situ-Ooiden zusätzlich durch regelmäßigere Wachstumszonen bis an die Ränder und tendenziell geringere Korngrößen. Möglicherweise werden sie als ,klassische Ooide in bewegtem Wasser an der Oberfläche gebildet.

Aufgrund der häufigen Verwachsungen ist jedoch eine eindeutige Zuordnung der Ooide oft problematisch. Komplexe Strukturen könnten z.B. dadurch entstanden sein, dass Aggregate oder Bruchstücke von eingespülten Ooide in situ noch von Eisenerzkrusten ummantelt wurden. An wenigen Stellen wurde beobachtet, dass opake Krusten transparente Ooide ummanteln (Abb. 9e und f), womit lokal eine eindeutige Altersbeziehung gegeben wäre, deren Allgemeingültigkeit allerdings noch anhand weiterer Beobachtungen bestätigt werden müsste.

Möglicherweise handelt es sich bei diesen Vererzungen um Entsprechungen der tertiären Bohnerze (s.o.) im Mikromaßstab. Die Vererzungen vom Typ II wurden auch in umgelagerter Form als Bestandteil von detritischen Geröllen im Sedimentgang beobachtet (s.o.; Abb 6f).

\section{Interpretation}

Insgesamt betrachtet kann aufgrund der geologischen Rahmenbedingungen und der beschriebenen Makro- und Mikrogefüge folgende Entwicklung der Sedimentgänge angenommen werden:

Der scharfe Kontakt zwischen Gängen und Rahmengesteinen, der sich bis in den Mikrobereich abbildet, belegt, dass die Rahmengesteine zum Zeitpunkt der initialen Gangbildung bereits vollständig verfestigt waren, und eine synsedimentäre bis frühdiagenetische Bildung während des Mittleren Buntsandsteins unwahrscheinlich ist. Auch die im Gang auftretenden eckigen Fragmente der Rahmengesteine belegen ihren hohen Verfestigungsgrad zum Zeitpunkt der Gangbildungen. Gegen eine frühe Gangbildung im Mittleren Buntsandstein sprechen auch viele detritische Komponenten, die wegen ihrer Korngröße und Lithologie nicht direkt aus den Rahmengesteinen bezogen werden. Auch die im Gang vorhandene Anhäufung von Eisenerzen wurde im Rahmengestein nicht beobachtet. 

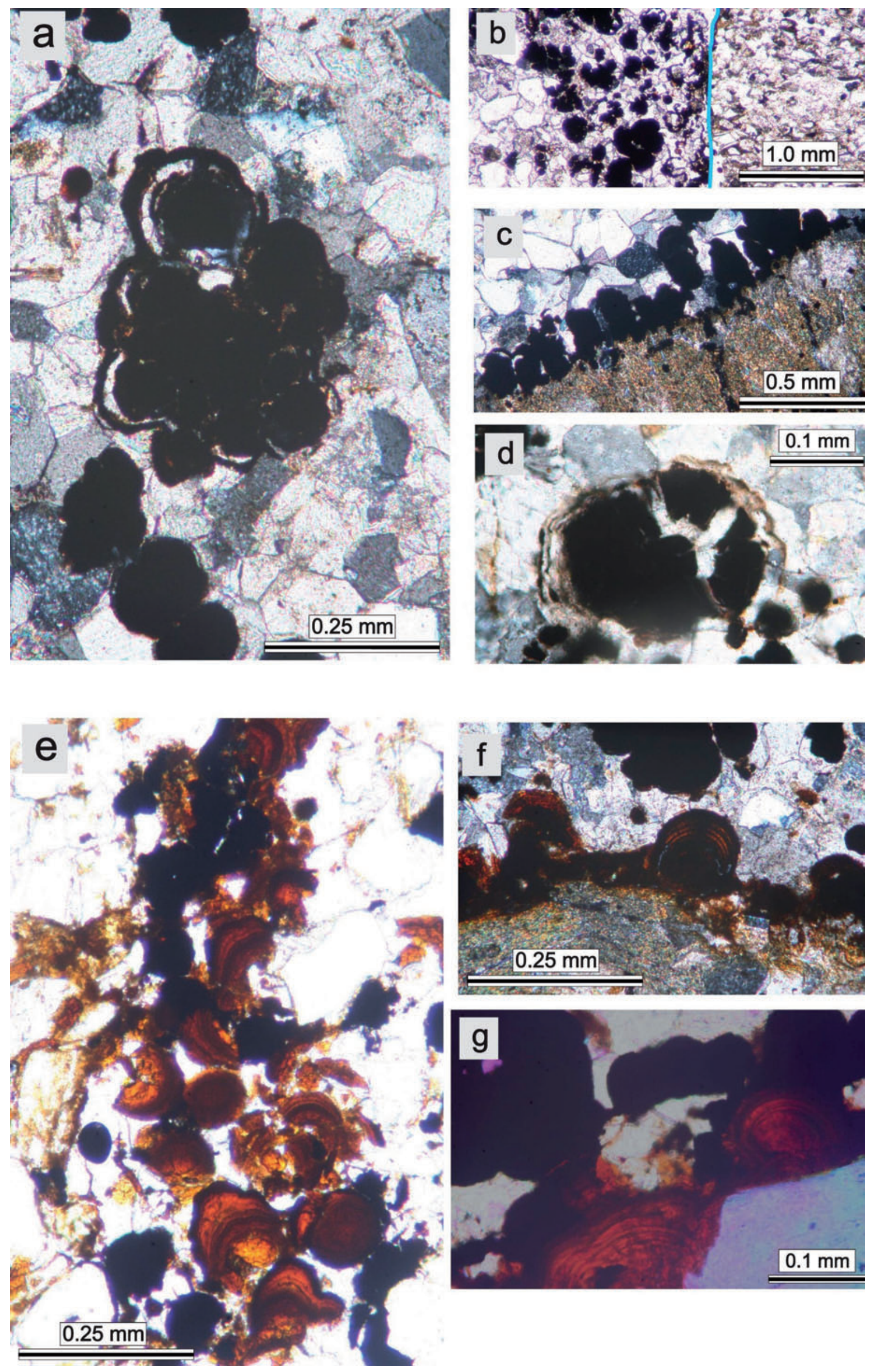

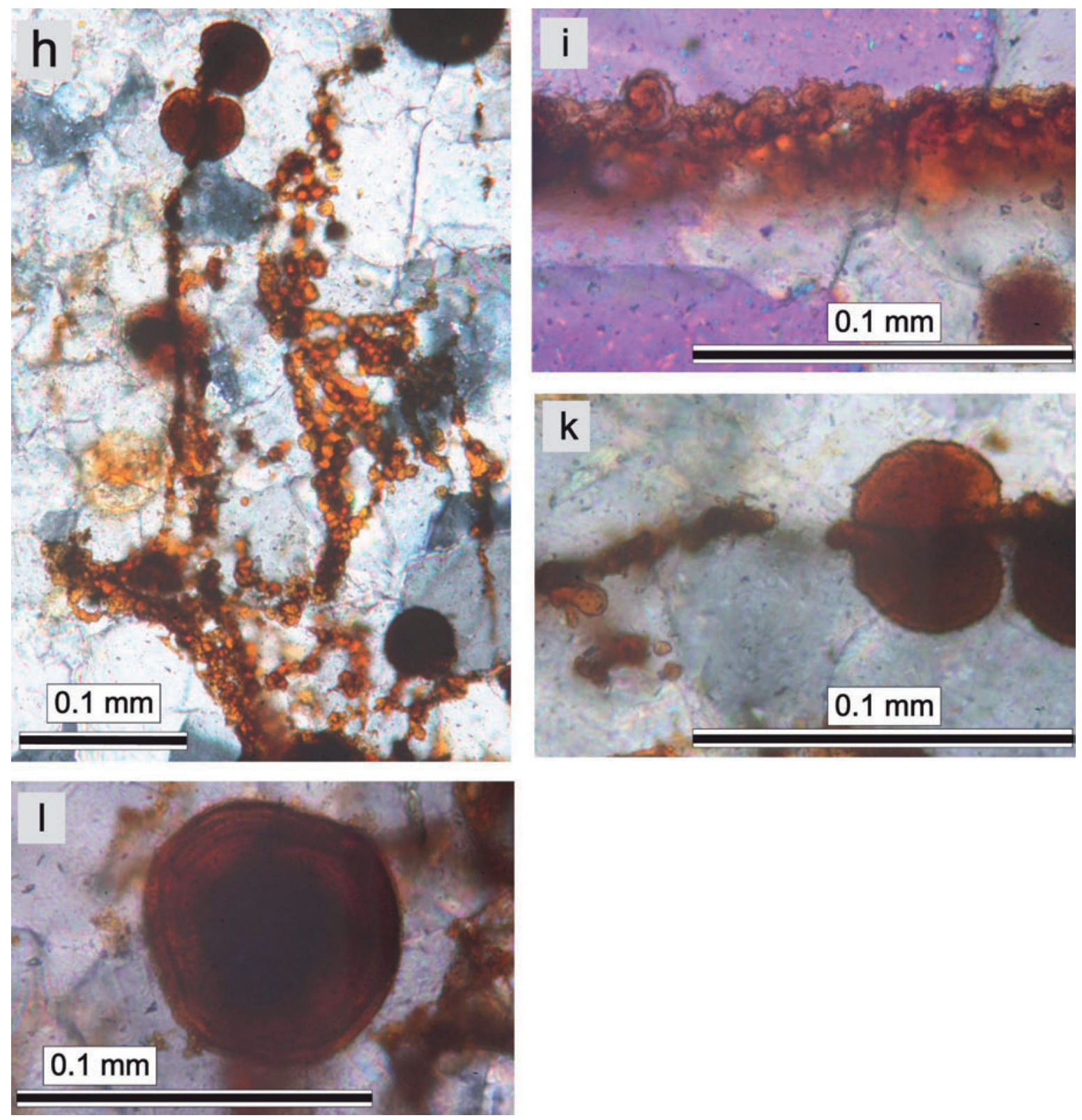

Abbildung 9. Eisenerze im Sedimentgang: a) Opake Ooide (Typ I), b) Gehäuftes Vorkommen von Typ I- Ooiden in Nähe zum Gangrand, c) Saum von Typ I-Ooiden am Rand eines Phyllosilikat reichen Gerölls, d) Einzelnes opakes Ooid mit von Calcitsäumen abgegrenzten Wachstumszonen und transgranularem Calcit-Mikrogang, e) Aggregat aus transparenten Typ II-Ooiden, f) Eisenerzkruste mit Typ II-Ooiden am Rand eines Phyllosilikat reichen Gerölls; vereinzelt sind eckige Umrisse entwickelt, g) Transparente Typ II-Ooide mit z.T. diskordanten Wachstumszonen, teilweise von opaken Typ I-Erzen umkrustet, h) Verzweigte Aggregate aus unterschiedlich großen Typ II-Ooiden, die von Mikrorissen ausgehend gebildet wurden, i) Flächiges Aggregat aus feinkörnigen Typ IIOoiden (vergl. Abb.9 h), k) Einzelnes Typ II-Ooid als Teil der verzweigten Aggregate (Ausschnitt aus Abb. 9h); doppelte Halbkugelform deutet auf zweiseitig gerichtetes Wachstum vom Riss ausgehend hin, I) Singluläres transparentes Ooid mit regelmäßigen konzentrischen Wachstumszonen. 
Ebenso scheiden wegen des Ganginhalts die Abschnitte Röt und Muschelkalk als Bildungszeitraum aus. So fehlen z.B. tonige Gangfüllungen, die auf Einspülungen während der Rötsedimentation hindeuten könnten. Umgekehrt wäre bei einer Bildung während des Muschelkalks kein siliziklastischer Eintrag zu erwarten. Als nächst höhere stratigraphische Einheit als Quelle für siliziklastische Komponenten käme der Untere Keuper in Frage. In diesem Fall müsste allerdings eine Tiefenstreckung der Gänge von min. ca. $200 \mathrm{~m}$ (durch Muschelkalk und Röt) angenommen werden, was bei Gangbreiten im cm-Bereich höchst unwahrscheinlich ist. Auch wurden entsprechende Gangsegmente in den zwischen liegenden Einheiten in der Region nicht beobachtet.

Es ist daher wahrscheinlicher, dass es sich bei den Gangsedimenten vorwiegend um Abtragungsprodukte der triassischen Schichtenfolge handelt, die auf der tertiären Rumpffläche aufgearbeitet, z.T. oberflächennah zementiert und dann als detritische Gerölle in den Gängen resedimentiert wurden. Ein Beleg hierfür könnten u.a. die durch Eisenerze zementierten siliziklastischen Gerölle sein. Dementsprechend könnten (fragliche) Karbonatgerölle aus Gebieten stammen, wo Muschelkalk zu dieser Zeit im Abtragungsbereich lag. Für die Metapelit-Gerölle müssten fernere Liefergebiete (? Harz) angenommen werden. Demnach wären die Gänge Zeugen einer wieder erodierten Auflage eozäner oder evtl. auch jüngerer tertiärer Sedimente auf der Rumpffäche der östlichen Schulter des Leintalgrabens, wohingegen sie auf der westlichen Grabenschulter, durch auflagernde miozäne Basalte vor Erosion geschützt, teilweise erhalten geblieben sind (z.B. Murawski 1953) Betrachtet man die in situ gebildeten Eisenerze in den Sedimentgängen als Äquivalente der ,Bohnerze', so wäre ein eozänes Alter der Gänge anzunehmen. Die Ausrichtung des für diese Zeit anzunehmenden Spannungsfelds (Sippel et al. 2009) war günstig für die initiale Öffnung der N-S-streichenden Gänge, die durch eine ältere Hauptkluftschar vorgezeichnet waren. Eine direkter struktureller Zusammenhang könnte mit einer von Vollbrecht \& Tanner (dieser Band) postulierten dextralen Blattverscheibung im Verlauf des Rodebachtals bestehen.

Die Bildung der Gänge erfolgte mehrphasig was durch deutlich unterscheidbare Zementgenerationen und durch Gangfragmente mit früh gebildetem Zement dokumentiert wird, die als Klasten in jüngeren Gangfüllungen vorkommen. Der Wechsel vom hell lumineszierenden Z I- zum dunkelblau bis nicht lumineszierenden Z II-Zement könnte auf einen Wechsel von evtl. marinen zu meteorisch beinflussten Aquiferen hindeuten.

\section{Danksagung}

Die Autoren danken N. Hildebrand und H. Tonn für die Herstellung der Dünnschliffe und B. Dietrich für die chemische Reinigung einiger Proben.

\section{Literatur}

Arp, G., Hoffmann, V.-E., Seppelt, S., Riegel, W. 2004. Exkursion 6: Trias und Jura von Göttingen und Umgebung. In: Exkursionsführer zur 74. Jahrestagung der Paläontologischen Gesellschaft, 147-192.

Beer, W. 1977. Röt und Unterer Muschelkalk am Ostrand des Göttinger Waldes zwischen Södderich und Billingshausen (TK 25: 4425 Göttingen, 4426 Waake, 4326 Lindau). Unveröff. Diplomarbeit und Diplomkartierung, Geologisch-Paläontologisches Institut, GeorgAugust-Universität Göttingen, 176 S.

Braithwaite, C.J.R. 1993. Cement stratigraphy in carbonates. Journal Sedimentology. Petrology 63, 295-303.

Bruhn, F., Bruckschen, P., Richter, D.K. Jan Meijer, J., Andreas Stephan, A., Ján Veizer, J. 1995. Diagenetic history of sedimentary carbonates: Constraints from combined cathodoluminescence and trace element analyses by microPIXE. Nuclear Instruments and Methods in Physics Research B104, 409-414.

Friese, N., Vollbrecht, A., Leiss, B., Jacke, O. 2010. Cambrian sedimentary dykes in the Proterozoic basement of the Västervik area (Southeast Sweden): cyclic formation inferred from macro- and microfabrics. International Journal of Earth Sciences 100, 741-752.

Hayashi, T. 1966. Clastic dikes in Japan. Transactions Japanese Journal of Geology and Geography $37,1-20$.

Heinrich, H. 1977. Oberer Buntsandstein und Unterer Muschelkalk am Nordrand des Göttinger Waldes zwischen Leinetal-Graben und Billingshausen. Unveröff. Diplom-Arbeit, GeorgAugust-Universität Göttingen, 143 S.

Müller, M., Boigk, H., Füchtbauer, H., Malzahn, E., Eckardt, F. J., Mattiat, B. 1961. Schichtenverzeichnis und geologischer Bericht über die 
Aufschlußbohrung Dransfeld 1. Niedersächsisches Landesamt für Bodenforschung, Bohrakte 33198.

Murawski, H. 1953. Die Entwicklungsgeschichte des Jüngeren Tertiär westlich des Leinetalgrabens. Geologisches Jahrbuch 67, 495-528.

Neuser R.D. 1995. A new high-intensity cathodoluminescence microscope and its application to weakly luminescing minerals. Bochumer Geologische und Geotechnische Arbeiten 44, 116-118.

Neuser R.D., Bruhn F., Götze J., Habermann D., Richter D.K. 1995. Cathodoluminescence: method and application. Zentralblatt für Geologie und Paläontologie, Teil I H. 1/2, 287-306.

Richter, D. K., Zinkernagel, U. 1981. Zur Anwendung der Kathodolumineszenz in der Karbonatpetrographie. Geologische Rundschau 70, 1276-1302.

Richter, D.K., Götte, T., Götze, J., Neuser, R.D. 2003. Progress in application of cathodoluminescence (CL) in sedimentary petrology. Mineralogy and Petrology 79, 127-166.

Ritter, M. C. 2010. Sedimentgänge im Bausandstein (Solling-Folge) bei Billingshausen (GK 25, Blatt 4326). Unveröff. Bachelor-Arbeit, GeorgAugust-Universität Göttingen, $59 \mathrm{~S}$.

Ritzkowski, S. 1999. Der Göttinger Leine-Graben im Paläogen (südliches Niedersachsen). Neues Jahrbuch für Geologie und Paläontologie, Abhandlungen 214, 237-256.

Sippel, J., Scheck-Wenderoth, M., Reicherter, K., Mazur, S. 2009. Paleostress states at the southwestern margin of the Central European Basin System - Application of fault-slip analysis to unravel a polyphase deformation pattern. Tectonophysics 470, 129-146.

Tucker, M. E., Wright, V.P. 1990. Carbonate Sedimentology. Blackwell Science, 1-3.

Vollbrecht A., Tanner, D.C. 2011. Der Leinetalgraben als Teil einer regionalen Pull-ApartStruktur, dieser Band.

Wright, V., Woodcock, N.H., Dickson, J.A.D. 2009. Fissure fills along faults: Variscan examples from Gower, South Wales. Geological Magazine 146, 890-902. 


\title{
Lokalisierung von Störungen im Bereich der Leinetalgrabenstruktur mittels VFL-Methode: Diskussion erster Ergebnisse
}

\author{
Michael Krumbholz (GZG Göttingen, UU), Axel Vollbrecht (GZG Göttingen), \\ Bernd Leiss (GZG Göttingen), Johannes Großmann (GZG Göttingen) \\ und Hauke Hachmeister (GZG Göttingen)
}

\begin{abstract}
Zusammenfassung - Durch den Einsatz einer modifizierten VFL-Methode wurde versucht, bekannte und vermutete Störungen des Leinetalgrabens genauer zu lokalisieren und Hinweise auf deren Raumlage zu gewinnen. Insbesondere sollte geprüft werden, inwieweit auch Störungen unter quartärer Bedeckung detektierbar sind. Erste Messungen entlang von vier Profilen zeigen, dass die Methode sehr gute Ergebnisse liefern kann. So lassen sich deutliche Maxima der VFL-Signale gut mit dem Verlauf bekannter Störungen korrelieren. Zusätzlich variiert die Asymmetrie dieser Maxima in charakteristischer Weise mit dem Einfallwinkel der Störungen. Entsprechende Signalcharakteristika werden auch in Bereichen mit quartärer Bedeckung beobachtet und lassen sich dort als Anzeiger für verdeckte Störungen verwenden. Für die bislang untersuchten Störungen ergibt sich danach ein einheitliches Einfallen zur Mitte des Leinetalgrabens hin, mit steilen Einfallwinkeln zumindest in den oberen 100 bis $200 \mathrm{~m}$. Listrische Krümmungen wären demnach erst in größeren Tiefen zu erwarten.

In stark besiedelten Gebieten ist diese Methode aufgrund von Störeinflüssen offensichtlich nur begrenzt einsetzbar, was u.a. durch Störsignale über Versorgungsleitungen belegt wird. Hier kann evtl. durch einen sorgfältig geplanten Profilverlauf der Einfluss von Störkörpern minimiert werden.
\end{abstract}

\begin{abstract}
Using a modified version of the VLF method, we attempt to determine the approximate attitude of known and probable faults in the Leinetal Graben. We particularly focus on the applicability of this approach to detect faults below Quaternary cover. First measurements along four profiles demonstrate that the method produces very good results: Distinct peaks of the VLF signals show a good correlation with the location of known faults. The asymmetry of these peaks can be related to the dip direction of the faults. Corresponding signals also occur in areas covered by Quaternary sediments and can therefore be used as indicators of hidden faults. In general, the studied faults show a steep (at least in the uppermost 100 to $200 \mathrm{~m}$ ) dip towards the centre of the Leinetal Graben. Listric geometry of the faults is thus only expected at greater depths. In densely populated areas, application of the method is limited by disturbances due to e.g. supply lines. In this case careful planning of the location of profiles may help to minimize their influence.
\end{abstract}

Schlüsselworte—VLF, Störungslokalisierung, Leinetalgraben, Randstörungen

- Michael Krumbholz - GZG, jetzt Institut für Geowissenschaften, Uppsala Universität, Villavägen 16, 75236 Uppsala, Schweden. mkrumbh@gwdg.de

- Axel Vollbrecht - Geowissenschaftliches Zentrum der Universität Göttingen, Goldschmidtstr. 3, D-37077 Göttingen. avollbr@gwdg.de

- Bernd Leiss - GZG Göttingen. bleiss1@gwdg.de

- Johannes Großmann - GZG Göttingen. johannes.grossmann@stud.uni-goettingen.de

- Hauke Hachmeister - GZG Göttingen. Hauke.Hachmeister@gmx.de

\section{Einleitung}

Wegen der oft schlechten Aufschlussverhältnisse sind die Kenntnisse über den genauen Verlauf, die Breite und die Raumlage der Störungen bzw. Störungszonen in den Randbereichen und zentralen Bereichen des Leinetalgrabens sehr begrenzt. Deswegen werden im Rahmen der aktuellen Untersuchungen des Leinetalgrabens hinsichtlich seiner geothermischen Potentiale auch geophysikalische Methoden wie Seismik und im vorliegenden Fall die VLF-Methode (s.u.) eingesetzt. Die ersten Messungen hierzu wurden auf Profillinien durchgeführt, die in etwa senkrecht 


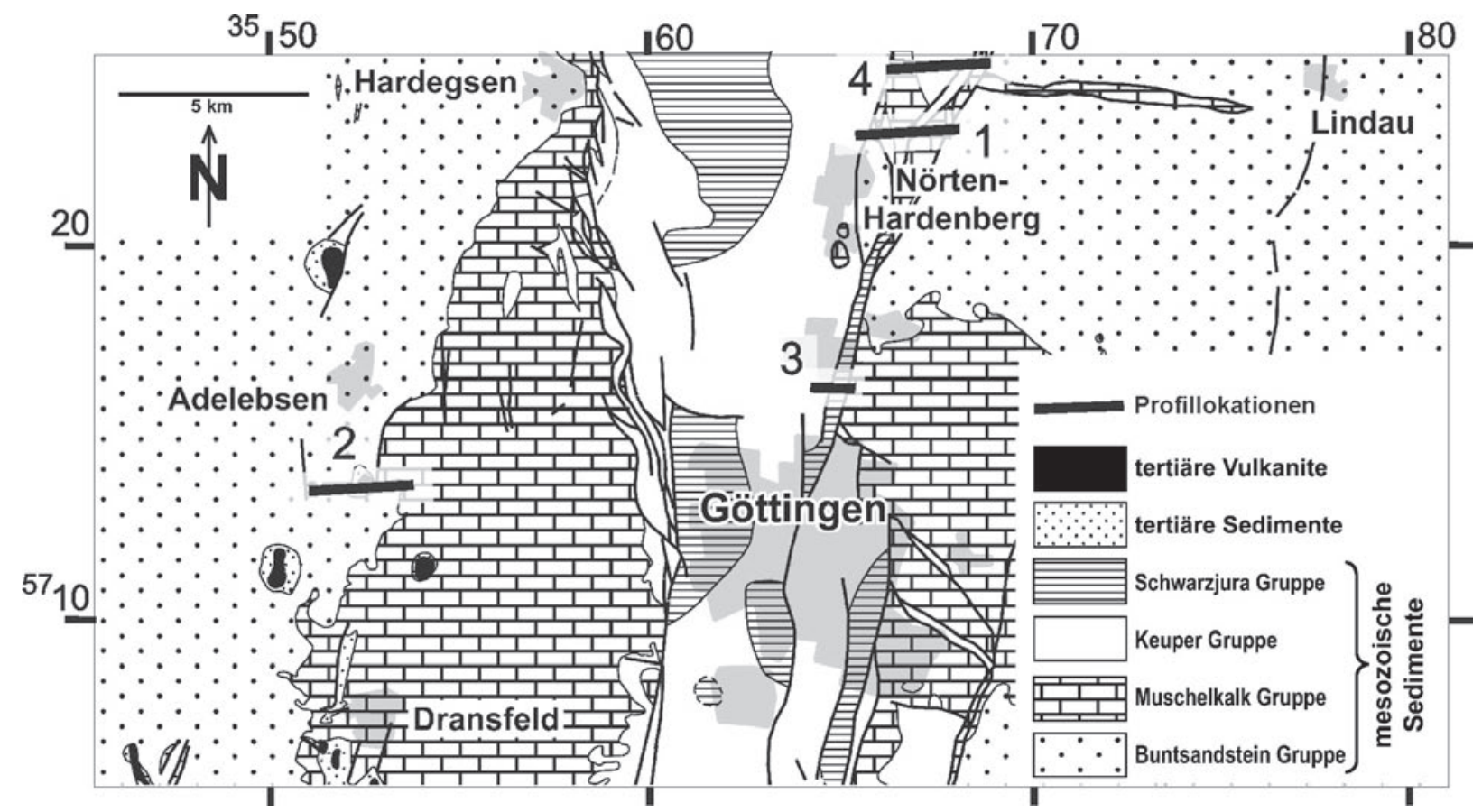

Abbildung 1. Lokationen der vorgestellten Messprofile. Karte modifiziert nach Arp et al., 2004.

zum vermuteten Störungsverlauf ausgerichtet sind (Abb. 1). Grundlage für vorliegende Arbeit sind Teile der Dissertation von Krumbholz (2010) und darauf aufbauende Bachelorarbeiten (Grossmann 2010, Hachmeister 2010).

\section{Methodik}

Für die vorliegenden Untersuchungen wurde eine abgewandelte Form der Very Low FrequencyMethode (VLF) eingesetzt. Während bei der herkömmlichen VLF-Methode die Phasenverschiebung und Amplitudenverhältnisse zwischen dem primären elektromagnetischen Feld starker VLF-Sender und dem sekundären elektromagnetischen Feld bestimmt werden, die an Strukturen höherer Leitfähigkeit wie z.B. Metallkörpern, Rohrleitungen aber auch geologischen Strukturen wie Störungen auftreten, wurde bei dieser Methode die Orientierung und die Intensität des sekundären elektromagnetischen Feldes bestimmt (Abb. 2). Die Messungen wurden mit dem sog. Cereskop, das von der Firma GE \& O (Dr. H. Obermeyer, Karlsruhe) produziert wird (Obermeyer, 2001), durchgeführt. Dieses Gerät wurde ursprünglich dazu konzipiert, natürliche elektromagnetische Emissionen (EMR) in einem Bereich zwischen 5 und $50 \mathrm{kHz}$ zu messen, die durch Mikrorissbildung und -ausbreitung entstehen (z.B.
Rabinovitch et al., 2007). Laut Herstellerangabe könnten Störungen demnach über erhöhte natürliche, mikrorissinduzierte EMR detektiert werden. Allerdings wurde von Krumbholz (2010) gezeigt, dass entsprechende Messungen mit diesem Gerät von künstlichen Störquellen dominiert werden und letztlich der VLF-Methode zuzuordnen sind. Die verwendeten Messgrößen sind nach der Neubetrachtung der Methode als Einzelpulse [Anzahl/0,1 s] und als Signalstärke [dimensionslos] zu betrachten und hängen primär von der Sendefrequenz der VLF-Sender, ihrer Sendeleistung und Entfernung, der Tageszeit, sowie von den elektrischen Eigenschaften der untersuchten Strukturen ab.

Die gemessene Signalstärke hängt weiterhin von der Orientierung des Sensors von der Tiefe der detektierten Anomalie (hier: Störung) ab, da das überdeckende Gesteins- und Bodenvolumen dämpfend wirkt. Der Sensor besteht aus einer ferritkernbasierten Stabantenne mit einer omnidirektionalen Richtwirkung (maximale Empfindlichkeit senkrecht zu Langachse bei Ausrichtung parallel zu den magnetischen Feldlinien). Ferritkernantennen gehören $\mathrm{zu}$ den magnetischen Antennen und sind nur für die magnetische Komponente $(B)$ eines elektromagnetischen Feldes sensitiv.

Dabei kann die Eindringtiefe $\delta[\mathrm{m}]$ annähe- 

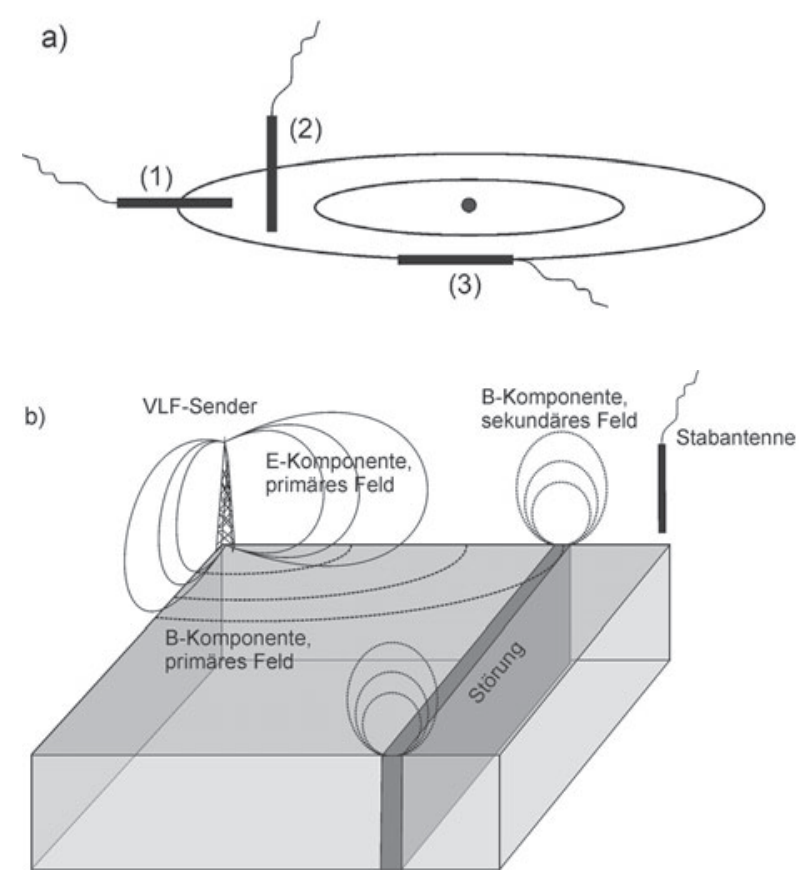

Abbildung 2. a) Abhängigkeit der Empfangseigenschaften einer Ferritantenne von ihrer Ausrichtung zu den magnetischen Feldlinien; ( 1 und 2) unempfindlich, (3) maximale Empfindlichkeit bei Ausrichtung parallel zu den Feldlinien; b) Vereinfachte Darstellung der Methodik: An elektrisch leitfähigeren Strukturen (hier: Störungen) im Untergrund induziert das primäre VLF-Feld einen elektrischen Strom, der wiederum ein sekundäres elektromagnetisches Feld hervorruft, das senkrecht zur Erdoberfläche orientiert ist; Stabantenne in Position mit maximaler Empfindlichkeit für das sekundäre elektromagnetische Feld.

rungsweise nach (1), einer Vereinfachung der Maxwell-Gleichungen, unter der vereinfachenden Annahme, dass die Materialien als elektrische Leiter darstellen, berechnet werden:

$$
\delta=\frac{1}{\sqrt{\frac{\mu_{0} \omega}{2 R}}} \quad[\mathrm{~m}]
$$

wobei $R$ der spezifische Widerstand $[\Omega \mathrm{m}], \mu_{0}$ die magnetischen Permeabilität $\left[\mathrm{V} \mathrm{s} \mathrm{A}^{-1} \mathrm{~m}^{-1}\right]$ und $\omega$ die Winkelfrequenz $2 \Pi f$ ( $f$ entspricht der Frequenz in $[\mathrm{Hz}])$, ist.

Somit wäre unter den geologischen Verhältnissen im Leinetalgraben Eindringtiefen von bis zu ca. $300 \mathrm{~m}$ zu erwarten (Abb. 3).

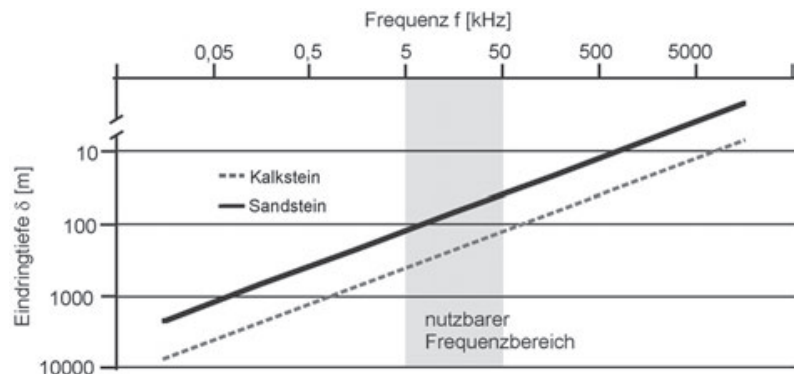

Abbildung 3. Zu erwartende Eindringtiefen für Kalkstein und Sandstein berechnet nach Formel (1). Im nutzbaren Frequenzbereich zwischen 5 und $50 \mathrm{kHz}$ kann demzufolge mit Eindringtiefen des Signals zwischen etwa 100 und $300 \mathrm{~m}$ gerechnet werden.

\section{Vorgehen}

An den ausgewählten Profilen wurden die Intensitäten des sekundären VLF-Feldes gemessen. Die Profile wurden vornehmlich an der östlichen Grabenschulter mit einer Orientierung in etwa senkrecht zur Hauptstreichrichtung der Grabenrandstörungen aufgenommen. Wegen ihrer omnidirektionalen Richtcharakteristik wurde die Antenne dabei senkrecht ausgerichtet, sodass sie sich zur horizontal propagierenden magnetischen Komponente des primären elektromagnetischen Felds der VLF-Transmitter in der sogenannten Nullposition (unempfindlich) befand (Abb. 2). Auf den Profilen wurde vornehmlich mit Messzeitintervallen von $1 \mathrm{~s}$ gearbeitet, was bei normaler Laufgeschwindigkeit Messpunktabstände von etwa $1,5 \mathrm{~m}$ ergibt. Zusätzlich wurde bei einigen Profilen das Signal mit gleichzeitig aufgenommen GPS-Signalen verknüpft, sodaß in diesen Fällen eine exakte geographische Lokalisierung der Messprofile möglich war.

\section{Resultate}

Das Profil 1 in Abbildung 4 (Lage siehe Abb. 1) wurde ca. $500 \mathrm{~m}$ nördlich von Nörten Hardenberg auf der östlichen Grabenschulter des Leinetalgrabens aufgenommen. Das Profil ist ca. $2 \mathrm{~km}$ lang und wurde mit einem Messzeitintervall von $4 \mathrm{~s}$ aufgenommen. Erkennbar sind drei Anstiege der Intensitäten, deren Bezug zu Störungen sowohl durch vorliegende Kartierungen, direkte Geländebeobachtungen als auch durch Fernerkundungsdaten (Geländekanten und Bewuchsgrenzen) bestätigt werden kann. Störung (1), die zwischen Profilmeter 400 und 430 ausbeißt, zeigt 

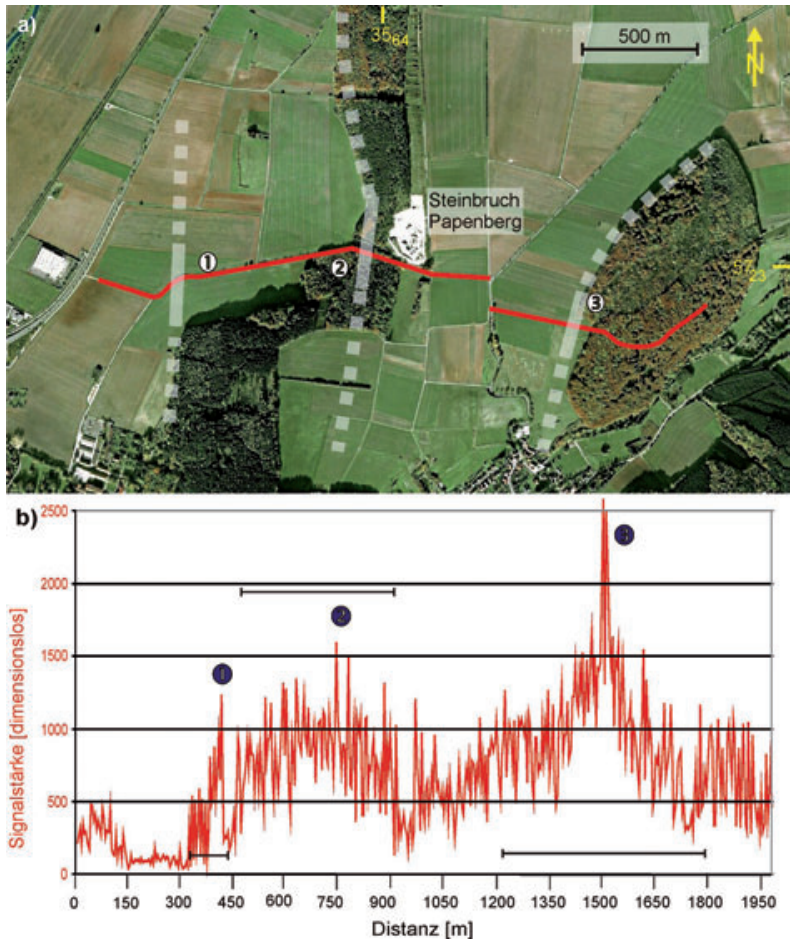

Abbildung 4. a) Messprofil (rote Linie) senkrecht zur östlichen Grabenschulter $500 \mathrm{~m}$ nördlich von Nörten Hardenberg. Das Profil überquert drei Störungen (gekennzeichnet durch weiBe Balken), die anhand von Geländebefunden nachgewiesen werden können. b) Verlauf der Signalstärke entlang des Messprofils. Punkte 1 bis 3 markieren die Lokationen der Störungen in a), wobei Störungen 1 und 3 durch asymmetrische Signalmuster charakterisiert sind. Die Breite der Peaks ist durch Balken gekennzeichnet. Weitere Erläuterungen im Text. Bildgrundlage: Google Earth.

einen Anstieg der Signalstärke von W nach E, gefolgt von einem steilen Abfall. Daraus kann gefolgert werden, dass diese Störung relativ steil nach W einfällt. Über Störung (2), die exakt an der Grabenkante liegt, zeigt der Signalverlauf auf einer Strecke von über 100 m erhöhte Intensitäten, jedoch kein ausgeprägtes Maximum, was auf keine diskrete Ausbissstelle hinweist. Vermutlich liegt hier ein komplexes System aus mehreren Störungen vor. Da in diesem Abschnitt das Muster der Intensitäten entlang des Profils keine eindeutige Asymmetrie aufweist, kann angenommen werden, dass diese Störungszone wenigsten in den obersten $100 \mathrm{~m}$ insgesamt subvertikal orientiert ist. Möglicherweise liegt aber auch ein komplexer
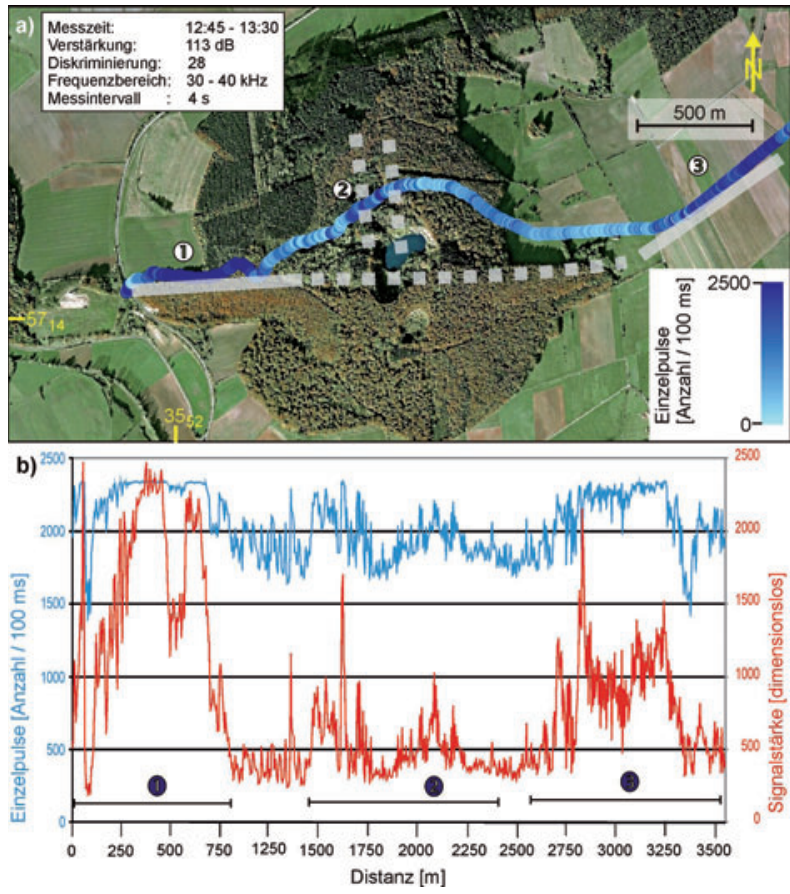

Abbildung 5. a) VLF-Messung auf der westlichen Grabenschulter des Leinetalgrabens südlich von Adelebsen. Das Profil quert die Grefenburg, einen tertiären Basaltkegel, der von einer $\mathrm{E}-\mathrm{W}$ streichenden Störung (weißer Balken) durchzogen ist. Die Signalstärken sind an den geographisch definierten Koordinaten aufgetragen. b) Verlauf der Signalstärke entlang des Messprofils. Die Breite der Peaks ist durch Balken gekennzeichnet. Bildgrundlage: Google Earth.

Überschneidungsbereich zweier Störungsscharen vor, worauf WNW-streichende Lineamente im Satellitenbild (z.B. Waldkante) hinweisen könnten. Störung (3) ist durch erhöhte Intensitäten über eine Distanz von wenigstens $400 \mathrm{~m}$ gekennzeichnet, wobei der asymmetrische Verlauf der Intensitäten hier wieder auf das zu erwartende Einfallen nach W schließen lässt.

Das Profil in Abbildung 5 (Lage siehe Abb. 1) überquert die Grefenburg, einen tertiären Basaltkegel auf der westlichen Grabenschulter des Leinetalgrabens, der von einer $\mathrm{E}-\mathrm{W}$ verlaufenden Störung durchschnitten wird (siehe GK 25, 4424 Dransfeld; v. Koenen 1900; s.a. Blockbild von Nagel et al. 1981). Die erhöhten Intensitäten in Bereich 1 lassen sich mit dem Verlauf dieser Störung korrelieren. Des Weiteren konnten in Profilabschnitt (2) zwei weitere Erhöhungen der Signalintensitäten festgestellt werden, welche auf 

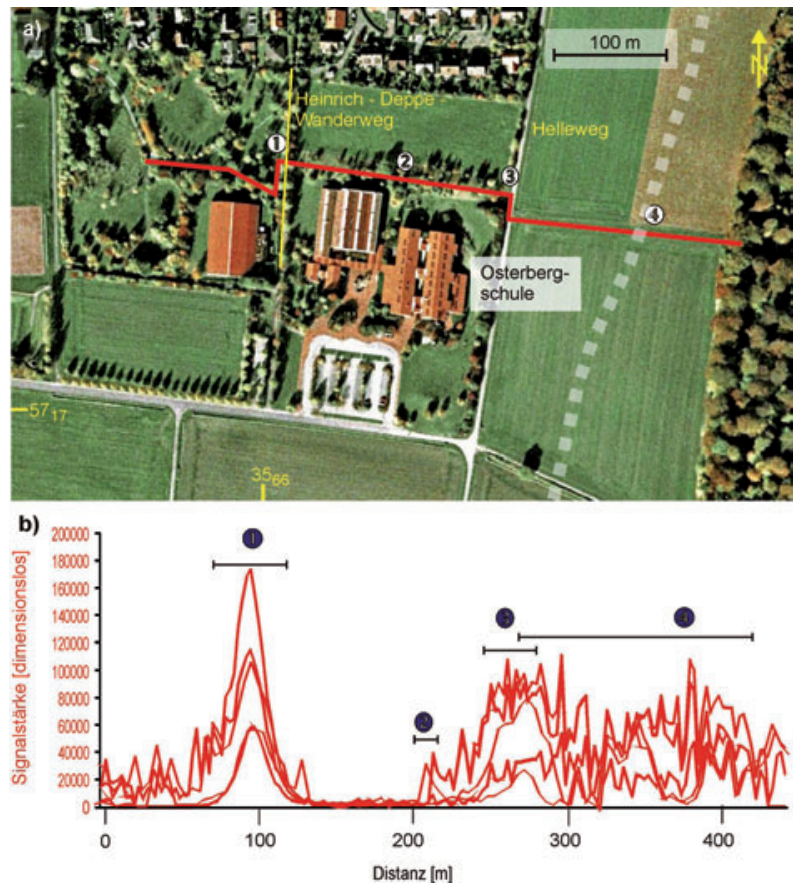

Abbildung 6. a) VLF-Messungen im Bereich der östlichen Grabenschulter des Leinetalgrabens im südlichen Bovenden (nördlich der Osterbergschule, Lage siehe Abb. 1) b) Verlauf der Signalstärke von 5 Messungen entlang des Messprofils. Die Breite der Peaks ist durch Balken gekennzeichnet. Bildgrundlage: Google Earth.

zusätzliche N-S streichende Störungen schließen lassen. Die westliche dieser beiden Störungen fällt, entsprechend dem Signalverlauf, vermutlich steil nach Osten ein. Die östliche Störung in Abschnitt (2) steht evtl. in Zusammenhang mit den erhöhten Intensitäten in Profilabschnitt (3). Dieser Bereich ist in der geologischen Karte (v. Koenen 1900) komplex strukturiert. Hier setzt sich zum einen die o.g. E-W-Störung fort, zum anderen verläuft unmittelbar nördlich davon die südliche Randstörung einer N-S streichenden Grabenstruktur.

Ziel des dritten Beispiels (Abb. 6, Lage des Profils in Abb. 1, siehe auch Großmann 2010) war es, den östlichen Leintalgraben-Hauptstörungsbereich in Bovenden zu detektieren. Fünf direkt nacheinander durchgeführte Messungen über das gleiche Profil unmittelbar nördlich der Osterbergschule zeigen, dass die Messungen relativ gut reproduzierbar sind. Von Westen beginnend zeigt sich der erste starke Intensitätsanstieg bei der Querung des Heinrich-Deppe-Wanderweges und kann auf die dort verlaufende Wasserleitung zurückgeführt werden. Im weiteren Verlauf der Messungen sind die Intensitäten sehr gering, bis sie im Bereich des Helleweges wieder stark ansteigen. Dieser breite Intensitätsanstieg könnte mit den zahlreichen dort verlaufenden Versorgungsleitungen (Wasser, Gas, Strom, Telekommunikation) im Zusammenhang stehen. Nach einem kurzen Abfall steigen die Intensitäten kontinuierlich an und fallen in Richtung Waldrand wieder deutlich ab. Diese letzteren höheren Intensitäten können klar mit der dort als vermutet verlaufenden Grabenrandhauptstörung (vgl. Geologische Karte von Niedersachsen 1:25.000, Blatt 4425 Göttingen) in Verbindung gebracht werden. Aus dem leicht asymmetrischen Verlauf der Intensitäten kann ein sehr steiles Westeinfallen der Störung abgeleitet werden. Der vermutete Störungsverlauf wäre dann allerdings weiter nach Osten zu verlegen. Der breite, unruhige Intensitätsverlauf könnte aber auch auf ein Störungsbündel (Störungsbereich) zurückgeführt werden.

Eine weitere wichtige Beobachtung wird aus diesen Profilen deutlich: Nicht nur die gestörten Bereiche sind für eine geologische Interpretation wichtig sondern auch die potentiell ungestörten Bereiche, wie sie sich zwischen den Profilmetern 120 und 210 anhand von bemerkenswert niedrigen Intensitäten ableiten lassen.

Zwei weitere VLF-Profile (Abb. 7) im Bereich der östlichen Grabenschulter befinden sich nördlich von Sudheim. Ziel des ersten Profils war es, eine in der Diplomkartierung von Rittmeyer (1979) vermutete Störung im Grabeninneren zu detektieren. Diese ließ sich allerdings nicht durch ein erhöhtes Signal bestätigen. Ein deutlich erhöhtes Signal weiter östlich im Profil (Punkt 1) lässt sich mit einer Wasserleitung korrelieren. Auffällig ist, dass die Signalstärke westlich der Wasserleitung gegenüber der Signalstärke östlich der Wasserleitung deutlich erhöht ist. Möglicherweise handelt es sich hier um eine Störungszone, die im Bereich der Wasserleitung endet. Eine anthropogen verursachte Signalerhöhung kann allerdings nicht ausgeschlossen werden.

Ziel des zweiten Profils (Abb. 7) war es, die Leinetalgraben-Hauptstörungszone zu detektieren. Abgesehen von den erhöhten Intensitäten westlich von Punkt 1 im ersten Profil, zeigt der Signalverlauf beider Profile eine recht gute Übereinstimmung. Die höhere Intensität an Punkt 1 lässt sich, wie im ersten Profil, mit Versor- 

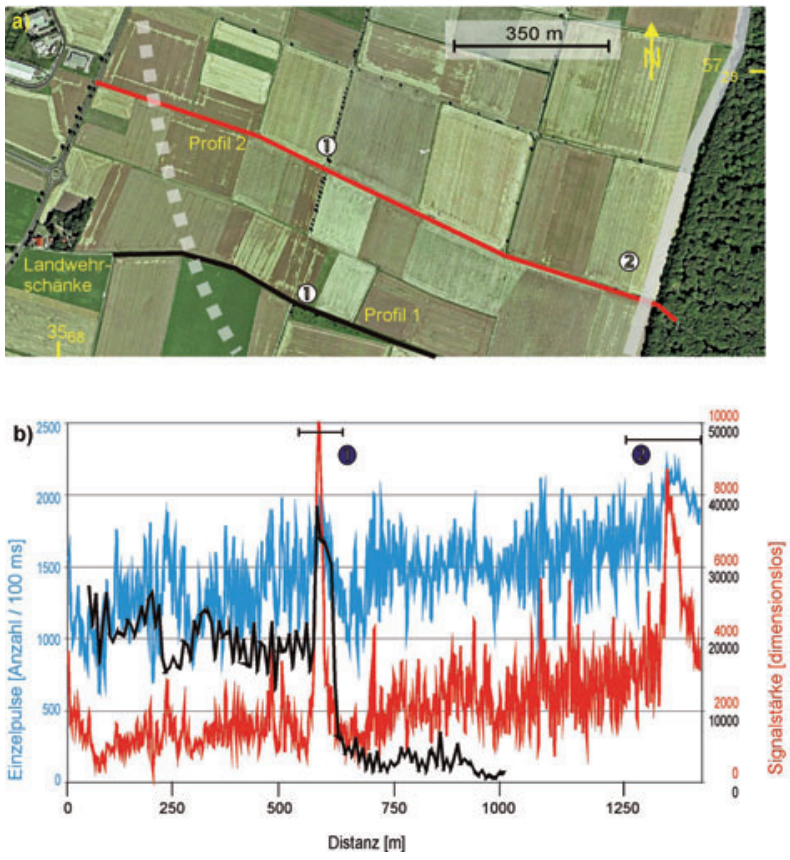

Abbildung 7. Verlauf der Signalstärke zweier Messprofile zwischen Sudheim und Northeim (Lage siehe Abb. 1). Profil 1 modifiziert nach Hachmeister (2010). Bildgrundlage: Google Earth.

gungsleitungen erklären. Der Intensitätsanstieg (Punkt 2) am östlichen Ende des Profils spricht ausgehend von seinem Verlauf für eine steil nach Osten einfallende Störung. Es könnte sich hierbei um eine antithetische Zweigstörung handeln, wie sie aus dem Bereich weiter südlich bei Sudheim bekannt sind und dort als Inversionsstrukturen interpretiert werden (Tanner et al. 2010).

Der generelle Intensitätsanstieg Richtung Osten kann auf verschiedene Weise erklärt werden: Zum einem durch eine Zunahme von kleineren Zweigstörungen in Richtung zur Hauptstörung oder aber direkt durch ein W-Einfallen der Hauptstörung.

Einen zusätzlichen Effekt könnte auch eine hangaufwärts nachlassende Mächtigkeit der Fließerden haben, verbunden mit einer entsprechend geringeren Signaldämpfung.

\section{Schlussfolgerungen}

Die Ergebnisse zeigen, dass die mit dem Cereskop ermittelten VFL-Signale zusätzliche Informationen über Störungsmuster zumindest im sedimentären Deckgebirge liefern können. So konnten bereits kartierte Störungen in ihrem Verlauf bestätigt und Hinweise auf weitere Störungen gewonnen werden. Zudem deutet sich an, dass breitere Störungszonen bzw. Überschneidungsbereiche mehrerer Störungsscharen durch entsprechend komplexe Signalmuster abgebildet werden. Umgekehrt lassen Zonen mit niedrigen Intensitäten auf einen weitgehend störungsfreien Untergrund schließen. Daraus könnten evtl. auch qualitative Aussagen über den Grad der bruchhaften Verformung abgeleitet werden.

Um die Raumlage und Form von Störungen aus entsprechenden VFL-Messungen ableiten zu können, müssten systematische Untersuchungen an mehreren Parallelprofilen durchgeführt werden. Hierzu sollten Störungen ausgewählt werden, deren Verlauf im Untergrund z.B. durch Bohrungen oder Seismik bekannt ist. Dadurch könnte evtl. in Verbindung mit anderen Parametern eine Signaleichung erzielt werden.

Als konkretes Ergebnis lässt sich aus dem Verlauf der Intensitäten und unter Berücksichtigung der erwarteten Eindringtiefen des VLF-Signals für die vorliegenden Störungen ableiten, dass diese im obersten Bereich (bis mindestens $100 \mathrm{~m}$ Tiefe) zum Grabeninneren hin steil einfallen. Eine listrische Krümmung ist demnach erst darunter zu erwarten.

\section{Danksagung}

Wir möchten uns bei allen Kollegen bedanken, die uns bei der Geländearbeit und der Diskussion der Ergebnisse unterstützt haben, besonders bei den Mitgliedern der Geothermie Gruppe Göttingen.

\section{Literatur}

Arp, G., Hoffmann, V.-E., Seppelt, S., Riegel, W. 2004. Exkursion 6: Trias und Jura von Göttingen und Umgebung. 74. Jahrestagung der Paläontologischen Gesellschaft, 02.-08. Oktober 2004, 147-192.

Großmann, J. 2010. Strukturgeologisches 3DModell des östlichen Leinetalgrabenstörungssystems bei Bovenden. Unveröffentlichte Bachelorarbeit, Universität Göttingen, $68 \mathrm{~S}$.

Hachmeister, H. 2010. Strukturgeologisches 3DModell des östlichen Leinetalgrabenstörungssystems bei Sudheim. Unveröffentlichte Bachelorarbeit, Universität Göttingen, 35 S.

Koenen, A. v. 1900. Erläuterungen zur geologischen Specialkarte von Preussen und den 
thüringischen Staaten. Lfg. 91, Blatt Dransfeld (Nr. 4424). 16 S.

Krumbholz, M. 2010. Electromagnetic radiation as a tool to determine actual crustal stresses - applications and limitations. Dissertation, Universität Göttingen, Germany. 161 S.

Nagel, U., Ritzkowski, S., Schunke, E. 1981. Geologisches und orohydrographisches Blockbild der Umgebung von Dransfeld (b. Göttingen). Schriften der Wirtschaftswissenschaftlichen Gesellschaft zum Studium Niedersachsens e.V., 79 S.

Obermeyer, H. 2001. Handbuch zur Anwendung der NPEMFE-Methode mittels des Cereskops. Ceres GmbH, Staffort $32 \mathrm{~S}$.

Rabinovitch, A., Frid, V., Bahat, D. 2007. Surface oscillations - a possible source of fracture induced electromagnetic radiation. Tectonophysics $431,15-21$.

Rittmeyer, G. 1979. Stratigraphie und Tektonik am östlichen Leinetal-Graben-Rand zwischen Northeim und Sudheim. Unveröffentlichte Diplomkartierung Universität Göttingen, $123 \mathrm{~S}$.

Tanner, D.C., Leiss, B., Vollbrecht, A., die Göttinger Geothermie Gruppe 2010. The role of strikeslip tectonics in the Leinetal Graben, Lower Saxony. Zeitschrift der deutschen Gesellschaft für Geowissenschaften 161, 369-377. 



\title{
Modellierung des Temperaturfeldes um eine tiefe Förderbohrung am östlichen Rand des Leinetalgrabens - Abschätzung des geothermischen Potentials
}

\author{
France Albero (GZG Göttingen, BGR Hannover) und David C. Tanner (GZG Göttingen, \\ LIAG Hannover)
}

\begin{abstract}
Zusammenfassung - Es wird ein stationäres 2D-Finiteelement-Konduktivwärmemodell bis $1 \mathrm{~km}$ Tiefe für die Ostschulter des Leinetalgrabens vorgestellt. Der Effekt einer $1 \mathrm{~km}$ tiefen Bohrung für geothermische Energienutzung während 40-jähriger Nutzung auf die Umgebung wird modelliert. Die Auswirkung wird mit Hilfe von Isothermen dargestellt. Der Radius der Abkühlungszone um die Bohrung kann mit $250 \mathrm{~m}$ angenommen werden, obwohl die Temperatur in der Realität in Richtung der Bohrung exponentiell abnimmt.
\end{abstract}

\begin{abstract}
We first demonstrate a 2D, finite-element, stationary $1 \mathrm{~km}$ deep conductive thermal model of a crosssection through the eastern graben shoulder of the Leinetal Graben. In this model we then visualize the effect on the temperature field after using a $1 \mathrm{~km}$ borehole to produce geothermal energy for 40 years. The effects are shown as isolines of temperature. The radius of the cooling zone around the borehole is estimated at $250 \mathrm{~m}$, although the temperature decreases exponentially towards the borehole.
\end{abstract}

Schlüsselworte—Geothermische Potential, Temperaturfeld, Bohrung, Leinetalgraben-Ostrand

\section{Einführung}

Albero (2007) bzw. Tanner et al. (2010) haben das jetzige Temperaturfeld in einem 2D Profil des Ostrandes des Leinetalgrabens vorgestellt. Das konstruierte, $1 \mathrm{~km}$ tiefe Querprofil, das zwischen den zwei tiefen Bohrungen von Sudheim und Levershausen verläuft, basiert auf Grupe (1909; Abb. 1 \& 2). Mit Hilfe eines Finitelement Software-Programms, wurde die stationäre Temperaturverteilung durch Wärmekonduktion ermittelt.

Dieser Artikel befasst sich mit einer praxisnahen Fragestellung dieser Modellierung: Was wäre der Effekt auf die Temperaturverteilung, wenn für eine Dauer von vierzig Jahren geothermische Energie aus einer $1 \mathrm{~km}$ tiefen Bohrung (in diesem Fall der Levershauser Bohrung) gewonnen würde? Diese Frage ist vor allem zur Beurteilung der Lebensdauer einer Geothermie-Bohrung und

- France Albero - GZG Göttingen, jetzt Bundesanstalt für Geowissenschaften und Rohstoffe, Stilleweg 2, D30655 Hannover. France.Albero@bgr.de

- David C. Tanner - GZG Göttingen, jetzt LeibnizInstitut für angewandte Geophysik, Stilleweg 2, D-30655 Hannover. DavidColin.Tanner@liag-hannover.de

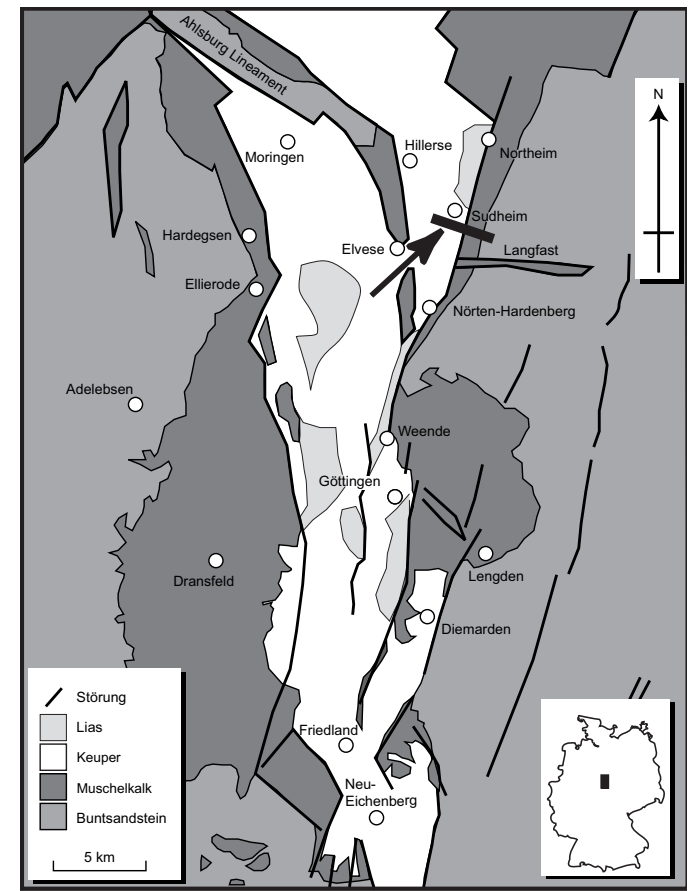

Abbildung 1. Geologische Karte mit Lage des Querprofils am Ostrand des Leinetalgrabens (Pfeil/Balken). 


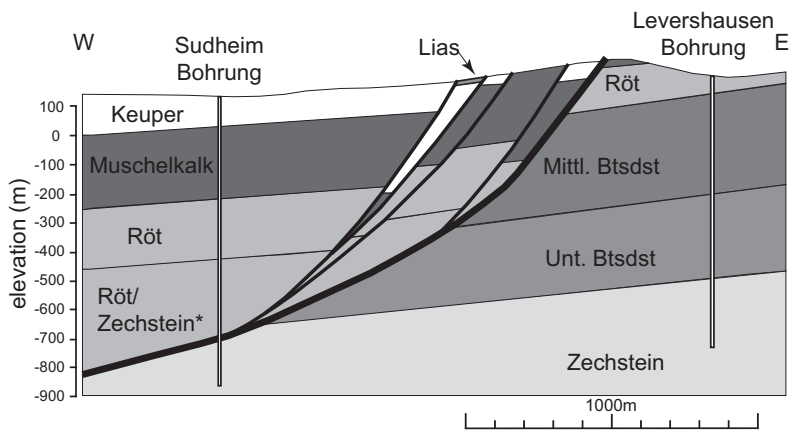

Abbildung 2. Geologisches Profil nach Grupe (1909).

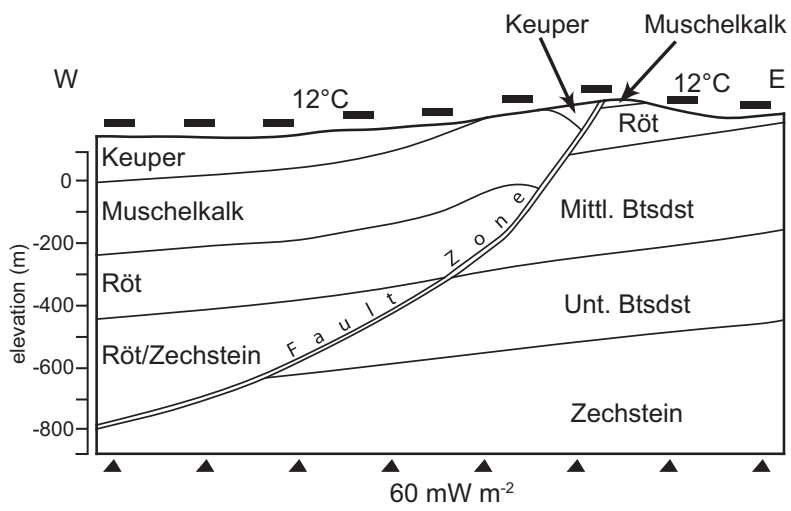

Abbildung 3. Vereinfachtes Profil, das für die vorliegende Modellierung verwendet wurde.

zur Bestimmung des Temperaturumfeldes einer Geothermiebohrung, im Hinblick auf die Positionierung weiterer benachbarter Geothermiebohrungen von Bedeutung.

\section{Methode}

Die Modellierung wurde mit dem Finitelement Software Programm COMSOL durchgeführt. Rahmenbedingungen für das Modell waren eine Oberflächentemperatur von $12^{\circ} \mathrm{C}$ und ein Basiswärmedurchfluss von $60 \mathrm{~mW} \mathrm{~m}^{-2}$ (Tanner et al. 2010). Die Lagerungsverhältnisse der Gesteine wurde dem Querprofil von Grupe (1909), leicht modifiziert, entnommen (Abb. 2); für Angaben über die in dem Modell verwendeten Parameter wie Gesteins-Wärmekapazität, thermische Leitfähigkeit und Dichte, siehe Tanner et al. (2010). Das Ergebnis ist ein Modell des geologischen Querprofils mit der stationären Temperaturverteilung durch Wärmekonduktion, die mittels Isothermen und geothermischer Gradienten

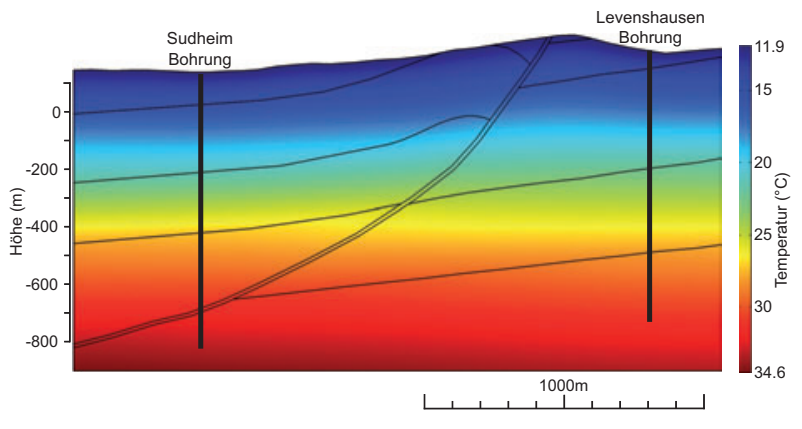

Abbildung 4. Gegenwärtiges Temperaturfeld des 1 km-tiefen Profils; Temperaturen in Grad Celsius.

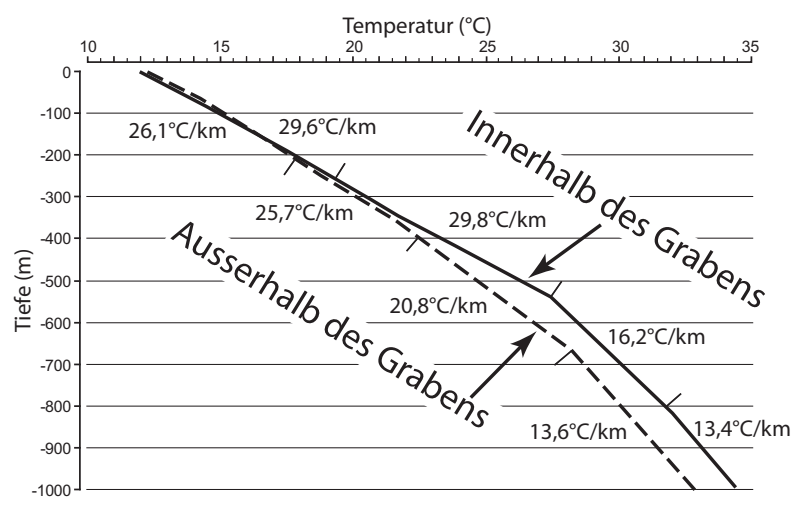

Abbildung 5. Graphische Darstellung des geothermischen Gradienten für die Levershauser(ausserhalb des Grabens) und die Sudheimer Bohrung (innerhalb des Grabens); Temperaturen in ${ }^{\circ} \mathrm{C}$.

dargestellt wird (Abb. 3, Albero 2009, Tanner et al. 2010).

Weiterhin wurde die Levershauser Bohrung als mögliche geothermische Bohrung modelliert. Dargestellt ist das Temperaturfeld nach Entzug von 120.000 $\mathrm{KWh} \mathrm{a}^{-1}$ Energie aus der Bohrung nach vierzig Jahren. Diese Menge Energie war für die Beheizung des Neubaues der Werk-statt-Schule Northeim e.V. vorgesehen (Schleevoigt 2007). In dem Gebäude sind ca. $2000 \mathrm{~m}^{2}$ Fläche zu beheizen.

\section{Ergebnisse}

Das stationäre Temperaturfeld (Abb. 4 \& 5 ) zeigt, dass mit zunehmender Tiefe die Isothermen weiter auseinanderliegen, also der geothermische Gradient sinkt (Abb. 5). Tanner et al. 2010 haben dargestellt, dass dies ein Effekt des liegenden 

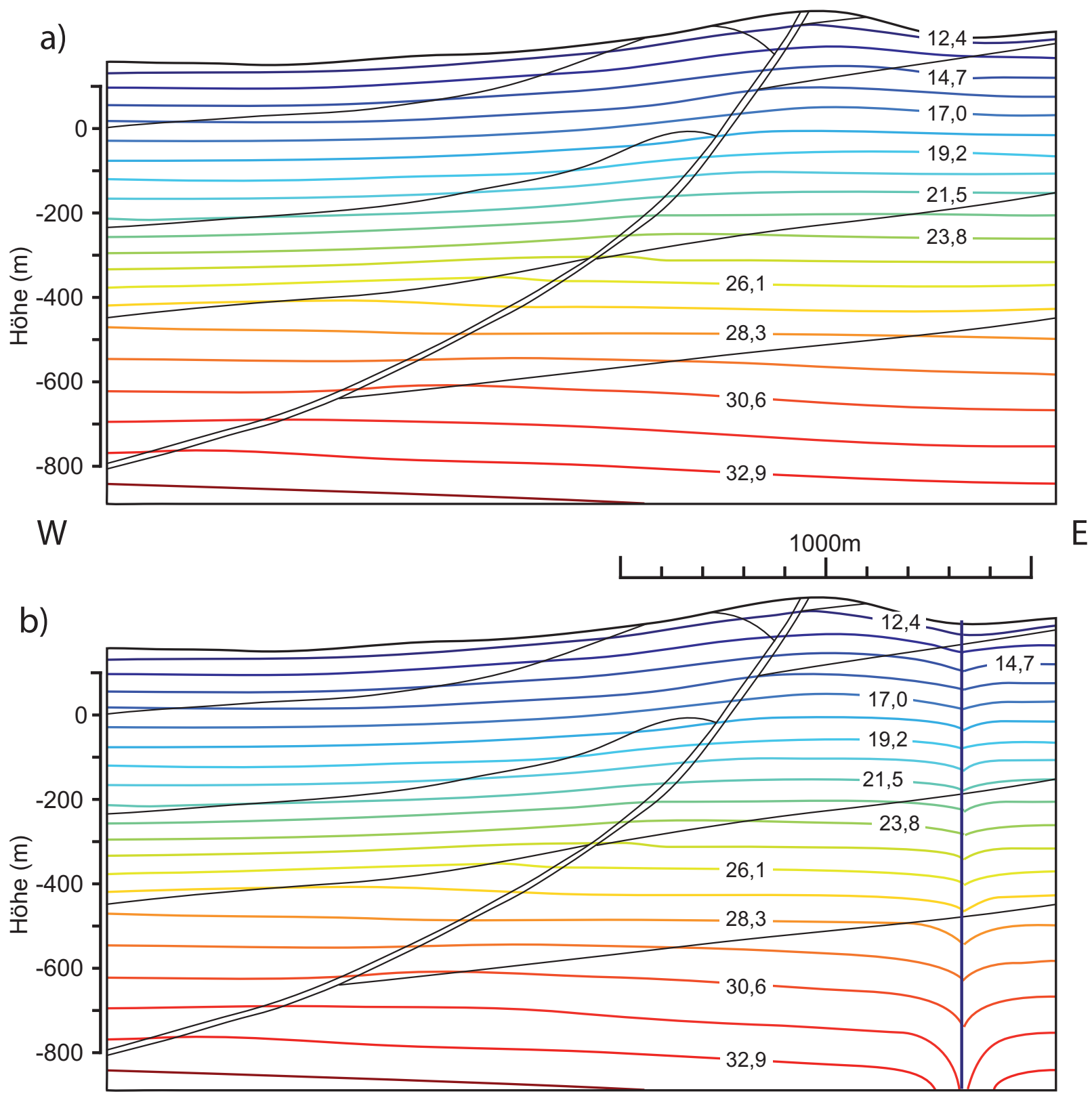

Abbildung 6. a) Gegenwärtiges stationäres Temperaturfeld des Modells des Ostleinetalgrabenrandes als Isolinien (aus Abb. 3). b) Temperaturfeld nach vierzig Jahre Nutzung der Levershauser Bohrung zur Gewinnung von geothermischer Energie. Isolinien in Grad Celsius.

Zechsteinsalzes ist, da es eine sehr hohe Wärmeleitfähigkeit besitzt. Außerhalb des Grabens, also in der Levershauser Bohrung sinkt der geothermische Gradient von $26,1^{\circ} \mathrm{C} \mathrm{km}^{-1}$ in den oberen $200 \mathrm{~m}$ auf $13,6^{\circ} \mathrm{C} \mathrm{km}^{-1}$ in den unteren $300 \mathrm{~m}$ (Abb. 5). Der geothermische Gradient innerhalb des Grabens ist höher als außerhalb (Abb. 5; Tanner et al. 2010).

Die Wärmeverteilung nach vierzig Jahren geothermischer Nutzung der Levershauser Bohrung ist in Abbildung 6b dargestellt. Die Verteilung der Isothermen zeigt, dass der Abkühlungseffekt durch den geothermischen Energiegewinn an der Basis der Bohrung, also im Bereich der höchsten Temperaturen, am stärksten ist. In Richtung Erdoberfläche wird der Effekt immer geringer. Die laterale Ausbreitung der Abkühlung besitzt durchgehend einen Radius von etwa $250 \mathrm{~m}$, wobei die Abkühlung exponentiell zur Bohrung hin zunimmt. 


\section{Schlussfolgerungen}

Mit Hilfe eines $1 \mathrm{~km}$-tiefen 2D FinitelementModells der Ostschulter des Leinetal Grabens, basierend auf dem publizierten Profil von Grupe (1909), wird das stationäre Temperaturfeld, das durch Wärmekonvektion aus dem Erdinneren verursacht wurde, dargestellt. Weiterhin wurde der Effekt auf das Temperaturfeld durch Nutzung der Levershauser Bohrung als Förderbohrung für geothermische Energie modelliert. Demzufolge ist nach 40 Jahren der Temperatur in den unteren $200 \mathrm{~m}$ der Bohrung etwa $1-2^{\circ} \mathrm{C}$ gefallen (Abb. 6 \& 7); der geothermische Gradient fällt von $13,6^{\circ} \mathrm{C} \mathrm{km}^{-1}$ auf $9^{\circ} \mathrm{C} \mathrm{km}^{-1}$ (Abb. 7).

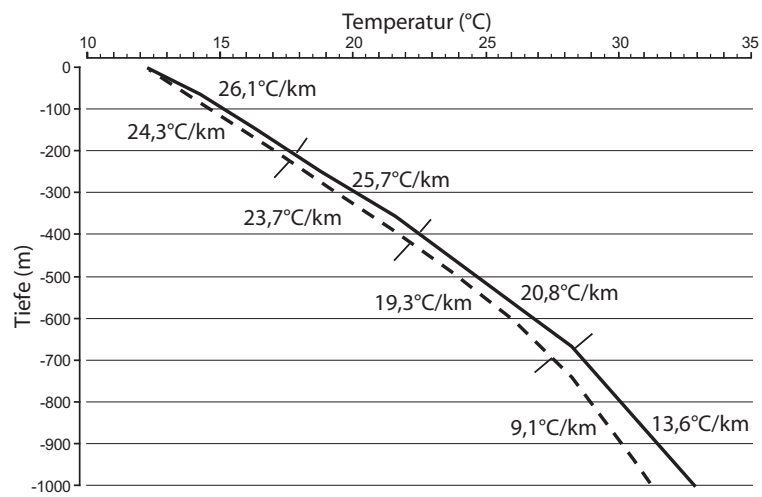

Abbildung 7. Graphische Darstellung des geothermischen Gradienten der Levershauser Bohrung. Durchgezogenen Linien - gegenwärtige, gestrichelte Linien - nach 40 Jahren Gewinn geothermischer Energie, mit jeweils geothermischen Gradienten für bestimmte Tiefenabschnitte.

Desweiteren zeigen die Ergebnisse, dass andere geothermische Bohrungen, die mehr als $250 \mathrm{~m}$ von dieser Bohrung entfernt sind, nicht vom Kühlungseffekt dieser Bohrung betroffen wären. Das zeigt das enorme Potential der möglichen Gewinnung von geothermischer Energie in dieser Gegend und vergleichbaren strukturellen Situationen, die häufig in der Erdkruste verwirklicht sind.

\section{Danksagung}

Wir danken Herrn Bührmann (Werk-statt-Schule Northeim e.V.) für nützliche Diskussionen. Bernd Leiss hat das Projekt ins Leben gerufen. Steffi Burchardt und Nadine Friese demonstrierten die Verwendung von COMSOL. Kommentare von
Axel Vollbrecht und Andreas Henk verbesserten die Arbeit.

\section{Literatur}

Albero, F. 2007. Numerical modeling in the Leinetal Graben. Unveröffentlichte M.Sc. Arbeit, Universität Göttingen/Université de Savoie, $51 \mathrm{pp}$.

Grupe, O. 1909. Über die Zechsteinformation und ihr Salzlager im Untergrunde des hannoverschen Eichsfeldes und angrenzenden Leinegebietes nach den neueren Bohrergebnissen. Zeitschrift für praktische Geologie 17, 185-205.

Schleevoigt P. 2007. Neubau einer Schule für soziale und emotionale Entwicklung in Northeim - Schätzung der voraussichtlichen Betriebkosten und Wirtschaftlichkeitsberechnung. Unveröffentlichte Studie Peter Schleevoigt Verein Deutsche Ingenieren.

Tanner, D.C., Albero, F., Leiss, B., the GGG 2010. Modelling the geothermal potential of the eastern border of the Leinetal Graben, Lower Saxony. Zeitschrift für geologische Wissenschaften $38 / 1,61-68$. 


\title{
Zur Herkunft der Sole der Saline Luisenhall (Göttingen) mittels Strontiumisotopie
}

\author{
Eike-Matthias Bultmann (GZG Göttingen), Janina Ruprecht (GZG Göttingen, LDEO \\ Columbia University), Bent T. Hansen (GZG Göttingen), Ilka Kleinhanns (GZG Göttingen, \\ IFG Tübingen)
}

\begin{abstract}
Zusammenfassung - Das geologische Alter des Steinsalzes, welches als Sole in der Saline Luisenhall gefördert wird, konnte bis dato nicht eindeutig festgelegt werden. Die ${ }^{87} \mathrm{Sr} /{ }^{86} \mathrm{Sr}$-Verhältnisse des Steinsalzes sowie der Sole sind nicht direkt mit der Strontium-Meerwasserkurve vergleichbar. Dennoch deutet die SrIsotopenzusammensetzung auf einen starken Einfluss der Salztone innerhalb der Zechstein-Evaporationsserien hin. Ferner zeigen Sauerstoff-Isotopenmessungen das die Fluide, welche das Salz lösen, meteorischen Ursprungs sind und nicht aus einem geschlossenen Reservoir stammen.
\end{abstract}

\begin{abstract}
The geological age of the rock salt, which is produced as brine in the Saline Luisenhall, is to date not unambiguously determined. ${ }^{87} \mathrm{Sr} /{ }^{86} \mathrm{Sr}$ ratios measured on rock salt and brine from the Saline Luisenhall cannot be compared directly with the seawater evolution curve of strontium. However, the isotopic compositions do point towards a strong influence by the salt shales within the Zechstein series. Furthermore it can be shown by oxygen isotopes that the fluids that dissolve the salt are of meteoric origin and do not represent a closed reservoir.
\end{abstract}

Schlüsselworte-Strontium-Isotopie, Zechstein, Saline Luisenhall

\section{Einleitung}

Dieser Beitrag gibt die Ergebnisse einer Bachelorarbeit (Bultmann 2007) wieder. Zielsetzung war es, mittels ${ }^{87} \mathrm{Sr} /{ }^{86} \mathrm{Sr}-$ Verhältnissen eine Aussage darüber zu treffen, aus welcher geologischen Einheit das in der Saline Luisenhall gewonnene Steinsalz stammt. Bisherige Arbeiten gehen von einer Herkunft aus dem Zechstein aus (Lotze, 1957). Für die relative Datierung wurden Proben direkt aus der Saline, sowie für Vergleichszwecke aus dem Göttinger Umland und aus dem Salzbergwerk $\mathrm{K}+\mathrm{S}$ Kali GmbH Neuhof Ellers (K+S Kali $\mathrm{GmbH}$ ) bei Fulda genommen. Die Proben aus dem Göttinger Umland stammen aus dem

- Eike-Matthias Bultmann - Geowissenschaftliches Zentrum der Universität Göttingen, Goldschmidtstr. 3, D37077 Göttingen. embultmann@web.de

- Janina Ruprecht - GZG, jetzt Lamont-Doherty Earth Observatory of Columbia University. New York, USA. ninarup@ldeo.columbia.edu

- Bent Hansen - Geowissenschaftliches Zentrum der Universität Göttingen, Goldschmidtstr. 3, D-37077 Göttingen. bhansen@gwdg.de

- Ilka Kleinhanns - GZG, jetzt Institut für Geowissenschaften, Universität Tübingen, Sigwartstr. 10, 72076 Tübingen. kleinhanns@ifg.uni-tuebingen.de
Röt bzw. Buntsandstein, dem Muschelkalk und dem Keuper. Als rezente Referenzen dienen eine Regenwasserprobe aus Göttingen, sowie Göttinger ${ }^{87} \mathrm{Sr} /{ }^{86} \mathrm{Sr}-$ Grundwasserdaten (Wiegand et al. 1998a, 1998b). Das Salzbergwerk Neuhof Ellers lieferte drei Proben aus dem Zechsteinsalz der Werra-Folge. Zusätzlich wurden die ${ }^{87} \mathrm{Sr} /{ }^{86} \mathrm{Sr}-$ Werte des Roten Salztons aus dem Zechstein herangezogen (Klaus 2008).

Die Sr-Isotopenanalyse der Proben wurde mittels Thermionen-Massenspektrometrie an einem TRITON TI (Thermo Electron Corporation) der Abteilung Isotopengeologie (GZG) durchgeführt. Die dabei erhaltenen ${ }^{87} \mathrm{Sr} /{ }^{86} \mathrm{Sr}$-Verhältnisse der Sole der Saline Luisenhall wurden zum einen mit denen der Festgesteinsproben und zum anderen mit denen der ${ }^{87} \mathrm{Sr} /{ }^{86} \mathrm{Sr}-$ Meerwasserkurve (Abb. 1) verglichen. Dadurch sollten Ähnlichkeiten bei der ${ }^{87} \mathrm{Sr} /{ }^{86} \mathrm{Sr}$-Isotopie erkannt und eine zeitliche Einordnung des Salinen Salzes durchgeführt werden.

\section{Strontium und Rubidium-Systematik}

Das Verhältnis von ${ }^{87} \mathrm{Sr} z u{ }^{86} \mathrm{Sr}$ wird zur Klärung verschiedenster geologischer Fragestellungen herangezogen. Strontium besitzt vier stabile Isotope: 


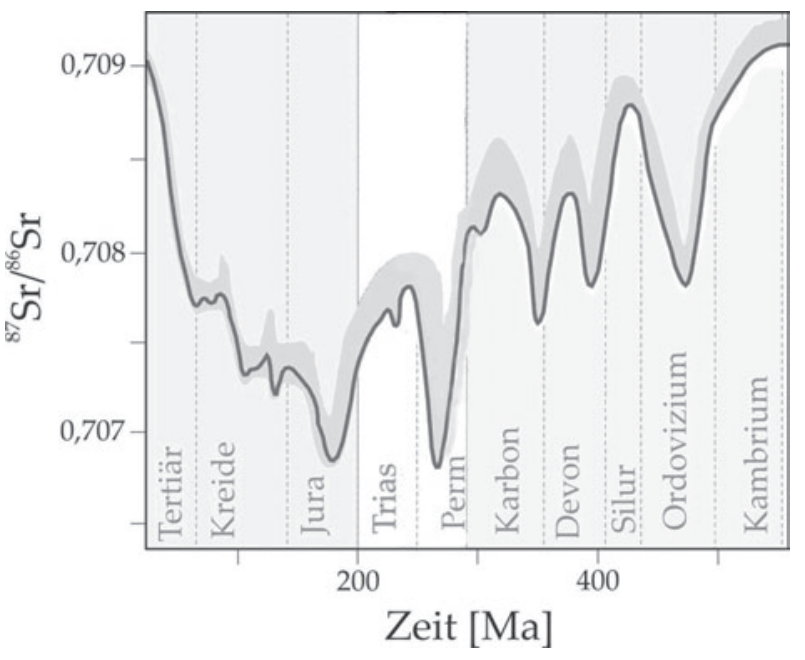

Abbildung 1. Die Variation von ${ }^{87} \mathrm{Sr} /{ }^{86} \mathrm{Sr}$ während des Phanerozoikums (Burke et al. 1982); die hier betrachtete Zeitspanne ist in Weiss hervorgehoben.

${ }^{84} \mathrm{Sr},{ }^{86} \mathrm{Sr},{ }^{87} \mathrm{Sr}$ und ${ }^{88} \mathrm{Sr}$. Die ${ }^{88} \mathrm{Sr} /{ }^{86} \mathrm{Sr}$ - und ${ }^{84} \mathrm{Sr} /{ }^{86} \mathrm{Sr}$-Verhältnisse der Erde sind stabil, das ${ }^{87} \mathrm{Sr} /{ }^{86} \mathrm{Sr}$-Verhältnis ist beeinflusst durch den Zerfall des radioaktiven Isotops ${ }^{87} \mathrm{Rb}$. Durch den fortlaufenden Zerfall des ${ }^{87} \mathrm{Rb} z u{ }^{87} \mathrm{Sr}$ variieren die ${ }^{87} \mathrm{Sr} /{ }^{86} \mathrm{Sr}$-Verhältnisse in vielen Gesteinen bzw. Mineralen und eine Datierung ist möglich (Faure \& Mensing 2005). Durch diesen Zerfall von ${ }^{87} \mathrm{Rb}$ erhöhen sich mit der Zeit die Anteile von ${ }^{87} \mathrm{Sr}$ in der ozeanischen und der kontinentalen Kruste. Durch die magmatische Fraktionierung reichert sich $\mathrm{Rb}$ in der Kruste an und somit ist der Zuwachs an radiogenen $\mathrm{Sr}$ in der Kruste höher als im Mantel.

Die ${ }^{87} \mathrm{Sr} /{ }^{86} \mathrm{Sr}-$ Schwankungen, die in Abb. 1 dargestellt sind, lassen sich damit erklären, dass zu einem bestimmten Zeitpunkt der Erdgeschichte mehr radiogenes Strontium von der kontinentalen Oberfläche oder weniger radiogenes Sr durch submarinen Vulkanismus in das Ozeanwasser gelangte.

\section{Methodik}

\subsection{Proben}

Die Proben mu1 und mm1 sind Karbonate und stammen aus dem Muschelkalk. Bei der Probe mu1 handelt es sich um Karbonat aus einer gelblichen Kalkbank des unteren Muschelkalks (242 Ma). Die Probe mm1 ist ein Lesestein, der ca. $1 \mathrm{~km}$ SSE' von Deppoldshausen einem Feld
Tabelle 1

Probenbezeichnung und deren zeitliche Einordnung nach der 6 . Stratigraphischen Tabelle von Deutschland (2002).

\begin{tabular}{llrl} 
Probe & Zeitliche Einordnung & Alter & Art \\
\hline UWS & Zechstein & 257 & Steinsalz \\
MWS & Zechstein & 257 & Steinsalz \\
OWS & Zechstein & 257 & Steinsalz \\
KBS & Buntsandstein & 244 & Karbonat \\
KBS2 & Buntsandstein & 244 & Karbonat \\
RG1 & Röt & 243 & Gips \\
RG2 & Röt & 243 & Gips \\
mu1 & unt. Muschelkalk & 242 & Karbonat \\
mm1 & mitl. Muschelkalk & 240 & Karbonat \\
GK1 & GipsKeuper & 231 & Gips \\
GK2 & GipsKeuper & 231 & Gips \\
SL1 & rezent Luisenhall & 0 & Salzkristalisat \\
SL2 & rezent Luisenhall & 0 & Sole \\
SL3 & rezent Luisenhall & 0 & Sole \\
RW & rezent Regenwasser & 0 & Regenwasser \\
\hline
\end{tabular}

\begin{tabular}{lccc}
\multicolumn{4}{c}{ Tabelle 2 } \\
Analyseergebnisse \\
Probe & ${ }^{87} \mathrm{Sr} /{ }^{86} \mathrm{Sr}^{\mathrm{a}}$ & $2 \sigma$ & $\mathrm{Sr}[\mathrm{ppm}]$ \\
\hline UWS & 0,70729 & 0,00001 & 31,8 \\
MWS & 0,70723 & 0,00002 & 18,2 \\
KBS2 & 0,70901 & 0,00002 & 13,8 \\
RG1 & 0,70864 & 0,00001 & 1332,0 \\
mu1 & 0,70842 & 0,00025 & 124,0 \\
mm1 & 0,70841 & 0,00003 & 74,7 \\
GK1 & 0,70894 & 0,00001 & 208,0 \\
SL1 & 0,70934 & 0,00001 & 50,2 \\
SL2 & 0,70932 & 0,00001 & 33,8 \\
SL3 & 0,70929 & 0,00001 & 35,2 \\
RW & 0,70832 & 0,00004 & 0,017 \\
\hline
\end{tabular}

a. ${ }^{87} \mathrm{Sr} /{ }^{86} \mathrm{Sr}$ korrigiert für Spike, Blank und Massenfraktionierung. Während des Analysenzeitraumes wurden für den NBS 987 Standard ein ${ }^{87} \mathrm{Sr} /{ }^{86} \mathrm{Sr}$-Wert von: $0,71027 \pm 0,00006(n=7)$ gemessen.

entnommen wurde und ein Alter von ca. $240 \mathrm{Ma}$ besitzt.

Etwas jünger sind die Proben GK1 und GK2 (ca. $231 \mathrm{Ma}$ ), die aus dem unteren Gipskeuper der Grabfeld-Formation entnommen wurden.

Der Saline Luisenhall wurden drei Proben entnommen. Dies sind die Probe SL1, die dem Salz entstammt, welches aus der Sole gewonnen wurde und die Proben SL2 und SL3, welche direkt aus der Sole stammen. Bei der Probe RW handelt es sich um eine Regenwasserprobe aus Göttingen. 


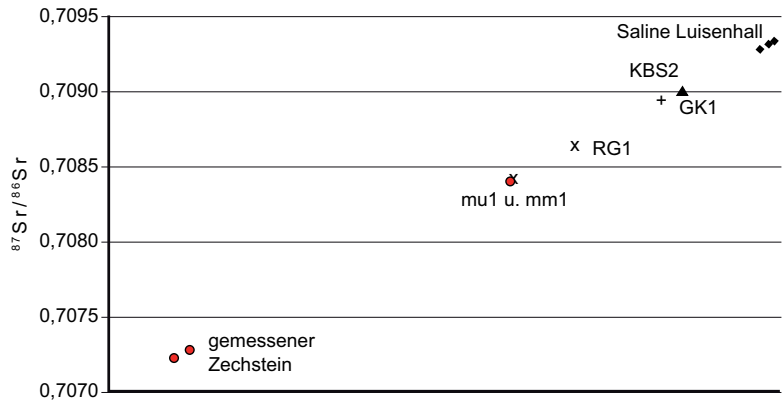

Abbildung 2. Vergleich der gemessenen ${ }^{87} \mathrm{Sr} /{ }^{86} \mathrm{Sr}$-Verhältnisse der analysierten Proben.

\subsection{Probenbearbeitung}

Das Probenmaterial wurde mit einem AchatMörser zerkleinert, bis ein feinkörniges homogenes Pulver vorlag. Davon wurde entsprechend ihres erwarteten Strontium Gehaltes mit einem an ${ }^{84} \mathrm{Sr}$ angereichertem Spike in inerte Teflon-Probenbehälter (PTFE) eingewogen. Das Probenpulver wurde dann mit wenigen Millilitern Salzsäure in Lösung gebracht. Feste Probenrückstände wurden durch Zentrifugieren von der Lösung getrennt. Das Separieren des Probenstrontiums und die Isotopenanalyse folgten den Standard-Methoden, beschrieben in Tütken et al. (2002).

\section{Disskussion/Ergebnisse}

Die Messergebnisse sind in Tabelle 2 und Abb. 2 zusammengefasst. Vier der Proben (OWS, GK2, SL2 und KBS) konnten wegen zu geringer Messgenauigkeit vermutlich wegen zu niedriger Konzentrationen bzw. silikatischer Kontamination nicht verwendet werden.

Aus Abb. 2 geht hervor, dass die ${ }^{87} \mathrm{Sr} /{ }^{86} \mathrm{Sr}-$ Werte des Luisenhaller Salzes mit keiner der Vergleichsproben übereinstimmen. Die größte Annährung besitzt der Wert der Probe KBS2, welche der Top-Solling Folge des Buntsandsteines entstammt. Generell haben alle Proben der Saline ${ }^{87} \mathrm{Sr} /{ }^{86} \mathrm{Sr}$-Isotopensignaturen, die bis zu 0,002 oberhalb der gemessenen Zechstein Salze liegen, wenngleich auch diese Werte leicht oberhalb der Meerwasserkurve liegen.

\subsection{Einordnung der Proben in die Meerwas- serkurve}

Da die ${ }^{87} \mathrm{Sr} /{ }^{86} \mathrm{Sr}$-Werte der Saline (Abb. 2) keiner Isotopensignatur der Vergleichsproben eindeutig zugeordnet werden konnten, wurden alle

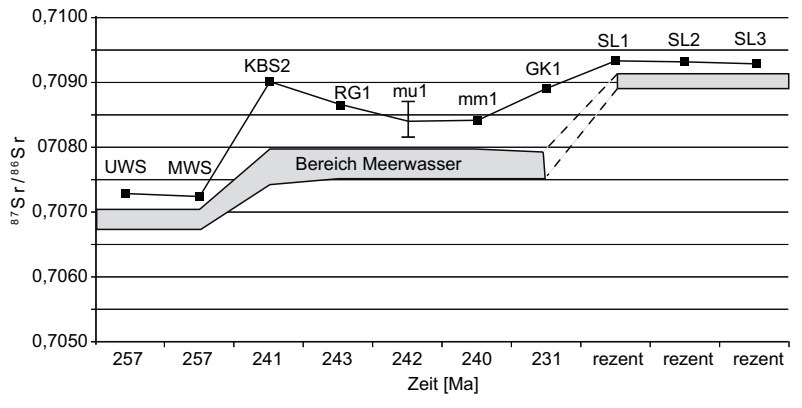

Abbildung 3. Vergleich der Meerwasserkurve (nach Burke et al. 1982) mit den Proben.

Proben mit den entsprechenden Zeitabschnitten und Daten der Meerwasserkurve (Burke et al. 1982) verglichen. In Abb. 3 sind die ${ }^{87} \mathrm{Sr} /{ }^{86} \mathrm{Sr}$ Verhältnisse über die Zeit aufgetragen. Der graumelierte Bereich stellt dabei die für den jeweiligen Zeitpunkt zu erwartenden ${ }^{87} \mathrm{Sr} /{ }^{86} \mathrm{Sr}$-Verhältnisse im Meerwasser dar. Auf der blauen Linie sind die tatsächlich gemessenen ${ }^{87} \mathrm{Sr} /{ }^{86} \mathrm{Sr}$-Werte der Proben für diese Zeitpunkte zusammengestellt. Bei der Betrachtung ist ersichtlich, dass die jeweiligen gemessenen ${ }^{87} \mathrm{Sr} /{ }^{86} \mathrm{Sr}$-Werte der Proben immer über dem Erwartungsbereich des Meerwassers liegen. Allerdings wird der allgemeine Trend der Meerwasserkurve von den Proben verfolgt.

In Abb. 3 sind außerdem die drei ${ }^{87} \mathrm{Sr} /{ }^{86} \mathrm{Sr}-$ Werte der Saline Luisenhall (SL1, SL2 \& SL3) abgebildet, welche im Mittel bei 0,7093 liegen. Dieses hohe Verhältnis überschreitet alle in Frage kommenden Werte der Meerwasserkurve. Somit kann die relative Datierung der Sole-Werte der Saline Luisenhall nicht mit dieser Methode durchgeführt werden.

\subsection{Einflussfaktoren auf die Sr-Isotopie des Luisenhaller Salzes}

Aufgrund der großen Unterschiede innerhalb der ${ }^{87} \mathrm{Sr} /{ }^{86} \mathrm{Sr}$-Isotopensignaturen der Sole zu denen der Vergleichsproben und der Meerwasserkurve, wird die geförderte Sole vermutlich von einem oder mehreren Faktoren beeinflusst worden sein. Die beiden wahrscheinlichsten Erklärungen sind entweder der Kontakt Grundwasser, welches das Salz löst, oder Fremdminerale in den analysierten Proben. Für die Klärung dieser Frage musste zunächst untersucht werden, ob das Grundwasser, welches das Salz löst, jungen oder alten Ursprungs ist. Dazu wurde eine Probe des Göttinger Regenwassers (RW) und der Sole (SL2) mittels der 


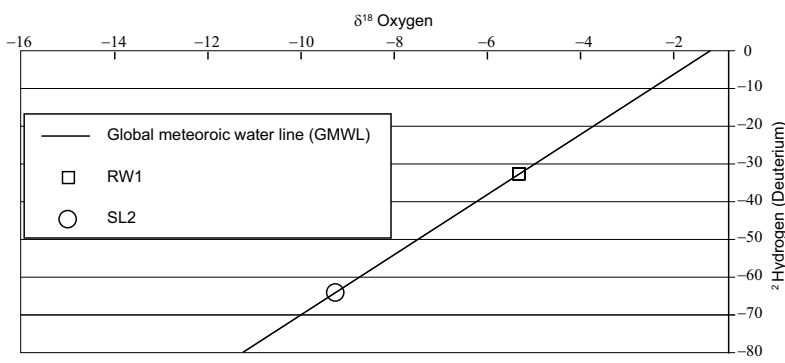

Abbildung 4. Korrelation der GMWL mit RW1 und SL2.

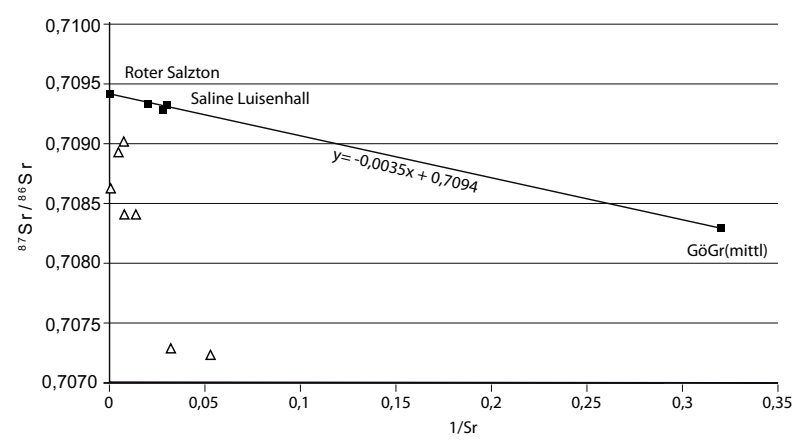

Abbildung 5. Mischungskurve vom Roten Salzton (Zechstein), dem Göttinger Grundwasser mit den Daten des Luisenhaller Salzes und den restlichen Proben (Dreiecke).

$\delta^{18} \mathrm{O}$-Methode (Klaus 2008) auf ihre Übereinstimmung zur GMWL (Global Meteoric Water Line) untersucht (Abb. 4).

Aus Abb. 4 wird ersichtlich, dass sowohl das Regenwasser als auch die Sole auf der GMWL liegen. Das bedeutet, dass das Wasser, welches das Salz löst, jungen meteorischen Ursprungs ist und nicht aus einem geschlossenen System stammt (Stosch 1999). Durch diese Tatsache kann das Göttinger Grundwasser als Lösemittel des Salzes der Saline als Näherung angenommen werden. Dieses besitzt einen Sr-Gehalt von 1,7-19 ppm und einen ${ }^{87} \mathrm{Sr} /{ }^{86} \mathrm{Sr}$-Wert von 0,7080 bis 0,7086 (Wiegand et al. 1998a, 1998b).

Als weitere Einflussfaktoren kommen Tonminerale in Frage. Um dies genauer zu untersuchen wurde eine Mischungskurve (Abb. 5) erstellt, deren Endglieder das Göttinger Grundwasser und der Rote Salzton aus der Aller Folge des Zechsteins (254 Ma) sind. Dieser Ton besitzt ein ${ }^{87} \mathrm{Sr} /{ }^{86} \mathrm{Sr}$-Verhältnis von 0,7094 und eine $\mathrm{Sr}$ Konzentration von 913 ppm (Klaus 2008).

In Abb. 5 ist das ${ }^{87} \mathrm{Sr} /{ }^{86} \mathrm{Sr}$-Verhältnis des Roten Salztons, der Saline Luisenhall, des mitt- leren Göttinger Grundwassers und der restlichen Proben gegenüber deren 1/Sr-Werten aufgetragen. Das Göttinger Grundwasser und der Rote Salzton sind die Endglieder der Mischungskurve. Die Probenwerte der Saline Luisenhall befinden sich in der Nähe des Roten Salztons fast genau auf dieser Kurve. Dies spricht dafür, dass die ${ }^{87} \mathrm{Sr} /{ }^{86} \mathrm{Sr}$-Werte der Salze aus der Saline aus dem Einfluss von feinen Tonlagen resultieren.

Der Unterschied der restlichen Proben (grüne Symbole) zur Meerwasserkurve resultiert vermutlich aus lokalen Einflüssen durch Detritus.

\section{Fazit}

Die gemessenen ${ }^{87} \mathrm{Sr} /{ }^{86} \mathrm{Sr}$-Isotopensignaturen der Proben konnten nicht mit denen der Meerwasserkurve (Burke et al. 1982) korreliert werden. Die Werte des Luisenhaller Salzes wurden durch Tonlagen ähnlich dem Roten Salzton der Aller Folge verfälscht. Die Annahme der Herkunft des Salzes aus dem Zechstein kann somit direkt weder bestätigt, noch widerlegt werden. Die hier vorgestellten Analysendaten widersprechen aber auch nicht der stratigraphischen Einstufung der Salze der Saline Luisenhall in die Zechstein-Folgen. Die erhöhten ${ }^{87} \mathrm{Sr} /{ }^{86}$-Verhältnisse der Sole scheinen durch Einlagerungen der Salztone des Zechsteins hervorgerufen zu sein. Ferner kann festgestellt werden, dass die Fluide, welche das Salz lösen, jungen meteorischen Ursprungs sind und nicht aus einem geschlossenen System stammen.

\section{Danksagung}

Für die Überlassung der Vergleichsproben möchten wir uns bei der $\mathrm{K}+\mathrm{S}$ Kali $\mathrm{GmbH}$, G. Arp, M. Reich und den Mitarbeitern der Saline Luisenhall bedanken. Ferner gilt unsere Dank N. Nolte und B. Dietrich für die Unterstützung bei der Probenaufbereitung und der Analytik. Abschließend bedanken wir uns bei K. Wemmer für Anregungen und die kritische Durchsicht des Manuskriptes.

\section{Literatur}

Bultmann, E.-M. 2007. Zur Herkunft der Sole der Saline Luisenhall (Göttingen) mittels Strontiumisotopie. Unveröff. Bachelorarbeit der Abteilung Isotopengeologie der Universität Göttingen: $37 \mathrm{~S}$.

Burke, W.H., Denison, R.E., Hetherington, R.B., Koepenick, H.F., Otto, J.B. 1982. Variation 
of Seawater ${ }^{87} \mathrm{Sr} /{ }^{86} \mathrm{Sr}$ throughout Phanerozoic time. Geology, 10, 516-519.

Faure, G., Mensing, T. M. 2005. Isotopes. Principles and Applications. John Wiley \& Sons, Inc., $897 \mathrm{pp}$.

Klaus, J. S. 2008. Evaluation of ${ }^{87} \mathrm{Sr} /{ }^{86} \mathrm{Sr}, \delta^{18} \mathrm{O}$, $\delta^{2} \mathrm{H}$, and Cation Contents as Geochemical Tracers for Provenance and Flow Paths of Saline Solutions in German Zechstein Deposits. Dissertation, University of Göttingen, 157 pp.

Lotze, F. 1957. Steinsalz und Kalisalze. Allgemeiner Geologischer Teil p. 264-265. Gebr. Borntraeger (2. Auflage).

Stratigraphische Tabelle von Deutschland 2002. Hersg. Menning, H. \& Hendrich, A. Deutsche Stratigraphische Kommission. ISBN 3-00010197-7.

Stosch, H.-G. 2004. Einführung in die Isotopengeochemie.

http://petrol.natur.cuni.cz/ janousek/ izokurz/PDF/Stosch_Isotopengeochemie.pdf

Tütken, T., Eisenhauer, A., Wiegand, B., Hansen, B. T. 2002. Glacial-interglacial cycles in $\mathrm{Sr}$ and $\mathrm{Nd}$ isotopic composition of Arctic marine sediments: changes in sediment provenance triggered by the Barents Sea ice sheet. Marine Geology, 182, 351-372.

Wiegand, B., Bielert, U., Groth, P., Eisenhauer, A., Hansen, B. 1998a. Use of ${ }^{87} \mathrm{Sr} /{ }^{86} \mathrm{Sr}$-ratios as tracer in hydrology. Terra Nostra 98/3, 388389.

Wiegand, B., Bielert, U., Eisenhauer, A Hansen, B. T. 1998b. Sr-Isotopie als Tracer zur Bestimmung von Mischungsverhältnissen zweier unterschiedlicher Wässer am Beispiel des Göttinger Trinkwassers. Tagung junger Geochemiker, Hannover 1998, S. 15. 



\title{
Gravitative Massenverlagerungen an der Röt/Muschelkalk-Schichtstufe des Göttinger Waldes GPS-gestützte Strukturkartierungen
}

\author{
Frithjof A. Bense (GZG Göttingen), Gabriele Ertl (GZG Göttingen, LBEG Hannover), \\ Axel Vollbrecht (GZG Göttingen) und Maurizio Battaglia (GZG Göttingen, USGS Menlo \\ Park)
}

\begin{abstract}
Zusammenfassung - Entlang der Röt/Muschelkalk-Schichtstufe am N-Rand des Göttinger Waldes wurden verschiedenartige Strukturen gravitativer Massenverlagerungen mit Hilfe von Zweifrequenz-GPS-Messungen kartiert. Die dadurch erzielte hoch auflösende Abgrenzung einzelner Strukturelemente ermöglicht genauere Rückschlüsse auf die stattgefundenen Bewegungsabläufe und deren zeitliche Abfolge. Die aufgenommenen Rutschmassen gehören größtenteils der Historischen Generation an. An Bewegungsarten herrschen GleitDrift- und Kriechbewegungen vor. Von einigen Bereichen abgesehen, erwies sich die einsetzte GPS-Methode als brauchbares Werkzeug. Die damit erzeugten detaillierten Karten könnten als Grundlage für ein LangzeitMonitoring zur Erfassung aktueller Verlagerungsgeschwindigkeiten und der beteiligten Bewegungsformen dienen.
\end{abstract}

\begin{abstract}
Detailed mapping of different types of landslides along the Upper Buntsandstein/Muschelkalk escarpment at the northern border of the Göttinger Wald were carried out using a dual frequency GPS tool. Highresolution delineation of individual structural units allows a characterization of the kinematics and chronology of their relocation. Most of the mapped landslides can be attributed to the so-called 'historical generation', as defined for this region. Prevailing types of relocation are sliding, drifting and creeping. In general, GPS proved to be a suitable tool for this purpose, however with certain limitations. The detailed maps may serve as a basis for long-time monitoring to detect the rates of actual relocation and the related kinematics.
\end{abstract}

Schlüsselworte-Rutschmasse, GPS-Kartierung

\section{Einführung}

Der Göttinger Wald liegt auf der östlichen Schulter des Leinetalgrabens (Abb. 1a), und wird im Wesentlichen durch Gesteine des Unteren $\mathrm{Mu}-$ schelkalks aufgebaut, die eine sehr flach nach SW geneigte Hochfläche bilden, so dass an deren

- Frithjof A. Bense - Geowissenschaftliches Zentrum der Georg-August-Universität Göttingen, Goldschmidtstr. 3, D-37077 Göttingen. fbense@gwdg.de

- Gabriele Ertl - GZG Göttingen, jetzt Landesamt für Bergbau, Energie und Geologie, Stilleweg 2, D-30655 Hannover. Gabriele.Ertl@lbeg.de

- Axel Vollbrecht - Geowissenschaftliches Zentrum der Georg-August-Universität Göttingen, Goldschmidtstr. 3, D-37077 Göttingen. avollbr@gwdg.de

- Maurizio Battaglia - U.S. Geological Survey, 345 Middlefield Road, MS 901, Menlo Park, CA 94025-3591, USA. battag@seismo.berkeley.edu nordöstlichen und östlichen Rändern der unterlagernde Obere Buntsandstein (Röt) zu Tage tritt (Abb 1a und b).

Wegen der unterschiedlichen Erosionsresistenz der Ton- und Mergelsteine des Röts und der Kalksteine des Unteren Muschelkalks (Wellenkalk) hat sich im Übergang dieser Einheiten ein Steilhang ausbildet. Ein unruhiges Relief entlang dieser Schichtstufe zeugt an vielen Stellen von unterschiedlichen Formen gravitativer Massenverlagerungen. Des Weiteren wird die Reliefbildung noch durch Auslaugung von Gipshorizonten in verschiedenen Abschnitten des Röts geprägt (z.T. auch Dolinenbildung).

Bereits v. Koenen \& Ebert (1894) beschreiben in den Erläuterungen zur geologischen Karte TK 25, Blatt 4426 Waake Rutschungserscheinungen an der Röt/Muschelkalk-Grenze und heben dabei den Hünstollen, die Mackenröder Spitze sowie die Fuchslöcher hervor. Sehr viel später 


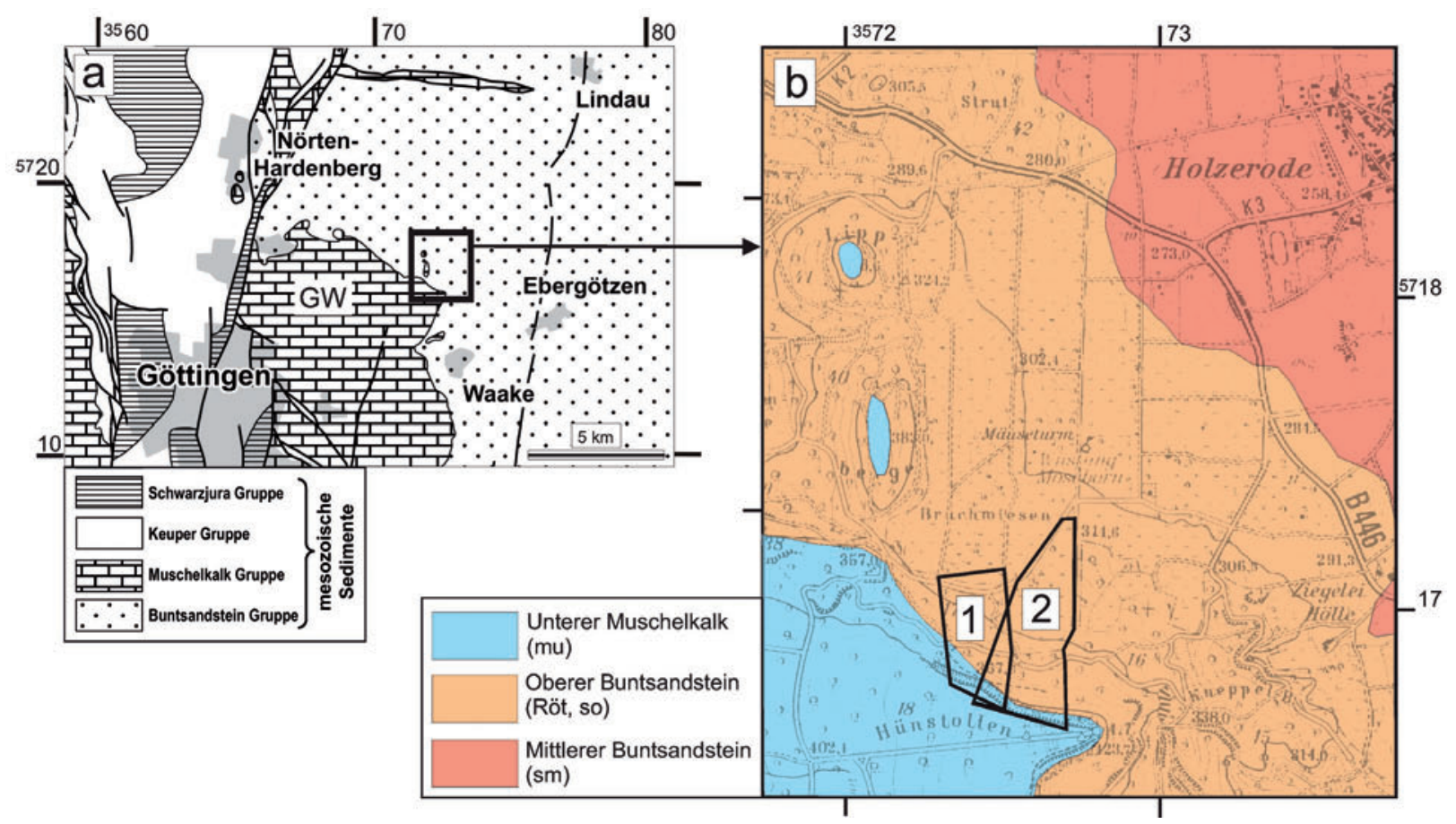

Abbildung 1. Lage der Kartiergebiete. a) Geologische Übersichtskarte für das Gebiet NE' von Göttingen; Ausschnitt einer Karte von Arp et al. (2004); GW-Göttinger Wald; Rahmen zeigt Ausschnitt von b). b) Geologische Karte mit Lage der Kartiergebiete: 1 (Bense 2005), 2 (Ertl 2005); Kartengrundlage: TK 25, 4426 Ebergötzen; geologische Grenzen nach GK 25, 4426 Waake (v. Koenen \& Ebert 1894) und Beer (1977).

führte Ackermann (z.B. 1953, 1955, 1959) erste umfassende und grundlegende Arbeiten zu den Mechanismen gravitativer Massenverlagerungen an dieser Schichtstufe durch. Dabei untergliedert er die Hangbewegungen in ,ältere holozäne und ,historische Generationen' (Ackermann 1959).

Als wesentlicher Mechanismus der Massenverlagerungen wurde ein Zusammenspiel der Bewegungsarten Stürzen, Gleiten und Fließen angenommen. Des Weiteren kommt Ackermann (1959) zu dem Ergebnis, dass Auslaugungen von Evaporiten im Untergrund nicht, wie bis dahin angenommen, eine der Hauptursachen für Rutschungen an der Röt/Muschelkalk-Schichtstufe darstellen.

Wesentliche Grundlage für die vorliegende Pilotstudie stellen zwei Bachelor-Arbeiten dar, in denen deutlich ausgeprägte Massenverlagerungen in zwei angrenzenden Gebieten durch GPS-gestützte Strukturkartierungen aufgenommen wurden (Bense 2005, Ertl 2005; Abb. 1b). Vorrangige Ziele dieser Untersuchungen waren, die Anwendbarkeit der Methode zu testen und ggf. eine möglichst genaue Kartierung der ver- schiedenen Strukturformen durchzuführen, einschließlich einer Zuordnung von Bewegungsarten. Wegen des engen Zeitrahmens für BachelorArbeiten konnte auf andere Aspekte gravitativer Massenverlagerungen wie mögliche Ursachen (lithologische Vorgaben, Klima, Hydrologie, Vegetation, Tektonik, Subrosion) sowie ihre zeitliche Einstufung nur in Form vergleichender Betrachtungen mit vorliegenden Literaturdaten eingegangen werden.

\section{Methodik}

Die strukturelle Detailkartierung der Massenbewegungen erfolgte mit einem 12-KanalZweifrequenz-GPS-Empfänger (L1 Code und Trägerphase / L2 Trägerphase) der Marke Trimble Pathfinder Pro XH mit Trimble H-Star Technologie und EVEREST-Mehrwegeunterdrückung. Zusätzlich wurde eine externe Zephyr-Antenne verwendet, wodurch Genauigkeiten von $30 \mathrm{~cm}$ und höher erreicht werden konnten (s.u.). Zur Datenaufzeichnung im Gelände diente ein Feldcomputer der Marke Trimble Ranger mit der 
Felderfassungssoftware Trimble TerraSync Professional V2.52. Als Kartengrundlage diente die Deutsche Grundkarte im Maßstab 1:5.000, Blatt Ziegelei Hölle Nr. 4426/7 mit Höhenlinien (Stand 1990). Alle Angaben zu Koordinaten und Karten beziehen sich auf das deutsche Gauss-Krüger Koordinatensystem DHDN 09 (Zone 3). Durch die Nachbearbeitung der Daten konnten die Fehler in der horizontalen Präzision der Positionsbestimmung auf bis $\mathrm{zu} 10 \mathrm{~cm}$ auf freier Fläche und bis $\mathrm{zu} 40 \mathrm{~cm}$ im Wald verbessert werden. Für eine ausführliche Beschreibung der angewendeten Methodik (Apparatur, Fehlerquellen, Korrekturverfahren etc.) sei auf Bense (2005) und Ertl (2005) verwiesen.

Um Aussagen über Rotationsprozesse (Bewegungsarten, s.u.) treffen zu können, wurden auf den verlagerten Schollen Einfallrichtungen und Einfallwinkel der Schichtflächen mit einem Gefügekompass eingemessen. Die entsprechenden Daten (Schichtflächenpole) wurden mit Hilfe des Programms StereoNett V2.46 in Form von Schmidt-Netz-Diagrammen dargestellt. Zur genaueren morphologischen Charakterisierung der Massenverlagerungen wurden mittels Inklinometer auch die Hangneigungen bestimmt.

\section{Charakterisierung gravitativer Mas- senverlagerungen}

Gravitative Massenverlagerungen zeichnen sich durch verschiedene Bewegungsarten aus, die sich im Laufe einer Entwicklung ablösen bzw. als Mischformen auftreten können. Die reinen Prozesse werden wie folgt definiert (z.B. Prinz 1997):

1) Fallen

Abstürzen von Boden- und Felsmaterial entlang einer Fläche, an der keine oder nur geringe Scherbewegung auftritt. Die bewegten Massen verlieren dabei zumindest zeitweilig den Kontakt zum Untergrund und stürzen frei fallend, rollend oder springend ab. Dabei verlieren die bewegten Massen völlig den inneren Zusammenhalt und bilden am Hangfuß Schutthalden, Berg- und Felsstürze.

2) Kippen

Vorwärtsrotation von Blöcken, Fels- oder kohäsiven Bodenmassen aus dem Hang heraus, Rotation um einen Punkt, der unterhalb des Schwerpunktes der bewegten Masse liegt.

3) Gleiten

Bewegung von Boden- oder Felsmassen auf
Gleitflächen oder Zonen intensiver Scherverformung (Rutschung im engeren Sinne). Man unterscheidet zwischen Translationsrutschungen auf ebenen Gleitflächen (z.B. Schichtflächen) und Rotationsrutschungen auf gekrümmten. Kombinationen treten bei komplex geformten Gleitfächen auf.

4) Driften

Laterale Bewegung von Fels- oder kohäsiven Bodenmassen durch Einsinken in weniger kompetente Schichten. Die Gesteinblöcke lösen sich an vorgegebenen Trennflächen ab und driften auf ihrer tonigen Unterlage hangabwärts. Dem Driften gehen meist Kippbewegungen voraus.

5) Fließen

Kontinuierliche Bewegung aufgeweichter Bodenmassen, Gesteinsschutt und Geröllen aller Größen mit sehr unterschiedlichen Geschwindigkeiten in Abhängigkeit von der Bodenart, dem Wassergehalt sowie dem Gefüge. Fließvorgänge führen zu lang gestreckten, murenartigen Formen. Die Bewegungsart des Fließens umfasst eine Vielzahl einzelner Erscheinungsformen. Schutt- oder Geröllstromrutschungen beispielsweise sind Fließungen mit einem Anteil von $>80 \%$ grobkörnigen Materials. Erdoder Schlammströme hingegen weisen einen mindestens 50\%-igen Anteil an Ton- Silt- und Sandmaterial auf. Kriechen als Sonderform des Fließens umfasst über lange Zeiträume anhaltende, langsame zumeist unstete Verformungen. Kriechen tritt sowohl in Locker- als auch in Festgesteinen auf. Solifluktion bezeichnet Kriechen oberflächennaher Bodenschichten bei Wasserübersättigung.

\section{Ergebnisse}

Folgende Strukturen wurden bei der Kartierung abgegrenzt (vergl. Abb. 2):

a) Verlauf der Abrisswand

b) Zerrspalten

c) Zusammenhängende Schollen mit ihren Kammlinien

d) Schuttmäntel

e) Erdgletscher

Nach Prozessierung der GPS-Daten in mehreren Schritten (genaue Erläuterung s. Bense 2005 und Ertl 2005) wurde für das westliche Teilgebiet eine Karte dieser Verlagerungsstrukturen erstellt und ein entsprechendes geologisches Querprofil konstruiert (Abb. 3). 

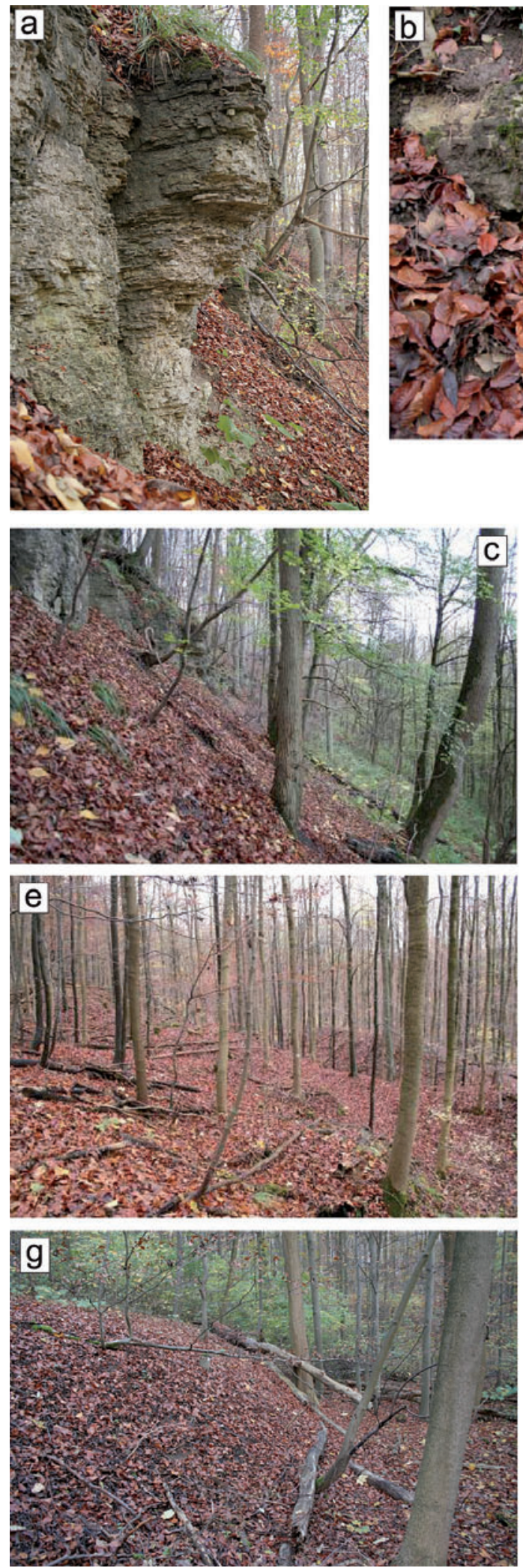
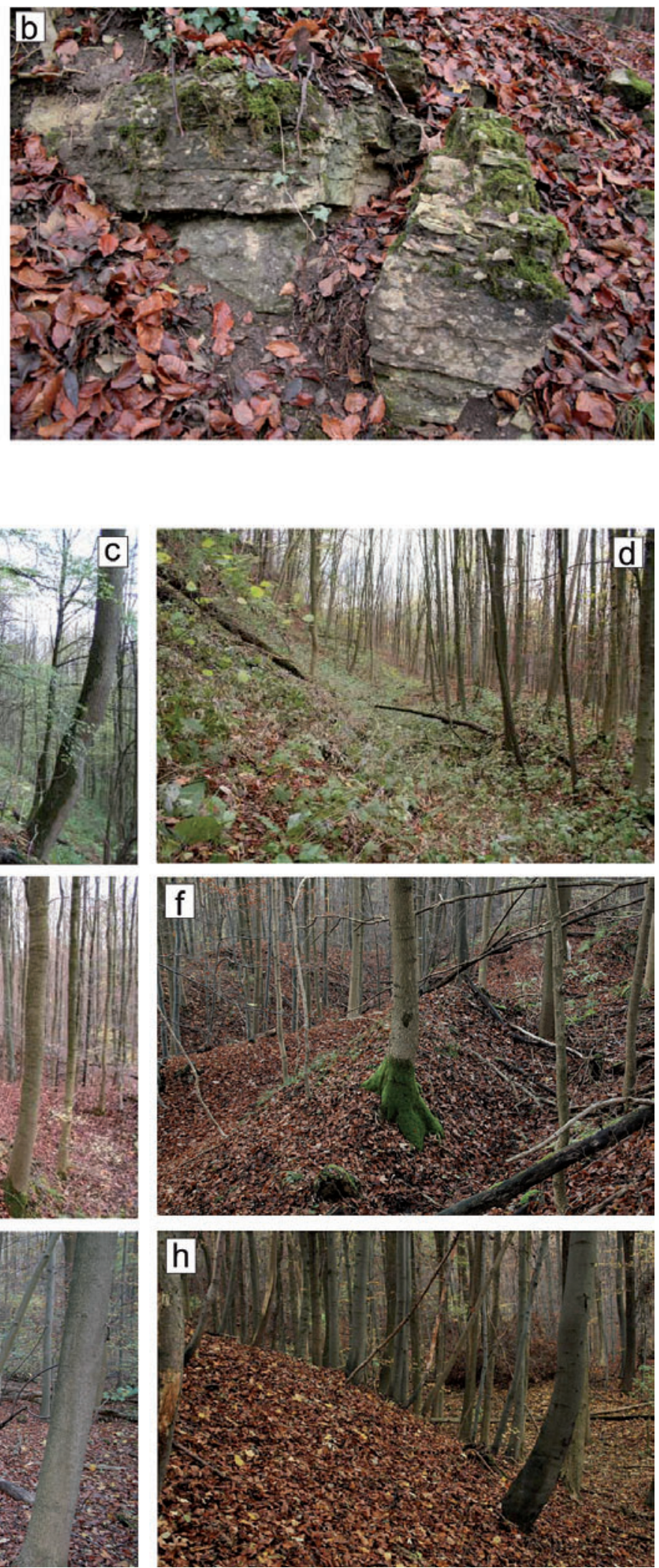

Abbildung 2. Abb. 2: Im Arbeitsgebiet beobachtete Strukturformen. a) Übersteilung an der Abrisswand sowie initiale Zerrspalte (Höhe der Abrisswand ca. $5 \mathrm{~m}$, Blickrichtung NW), b) Rezente Kipp- und Driftbewegungen einzelner Blöcke (Blickrichtung NW), c) Teilweise mit Schutt verdeckte Abrisswand (Blickrichtung W, Höhe der sichtbaren Wand ca. $4 \mathrm{~m}$ ), d) Deutliche mulden- und trogförmige Ausprägung von Zerrspalten (Blickrichtung W, links Abrisswand, rechts Oberscholle), e) Stark ausgeprägte bucklige Hangmorphologie (Blickrichtung schräg hangabwärts nach NW), f) Wulstförmige Oberscholle nach Ackermann (1959), (Blickrichtung NE), g) Steile Erdgletscherflanke (35 ${ }^{\circ}$ Hangneigung) im Nordbereich des Kartiergebietes (Blickrichtung NW), h) Front eines Erdgletschers Sichelwachstum der Bäume (Blickrichtung W). 


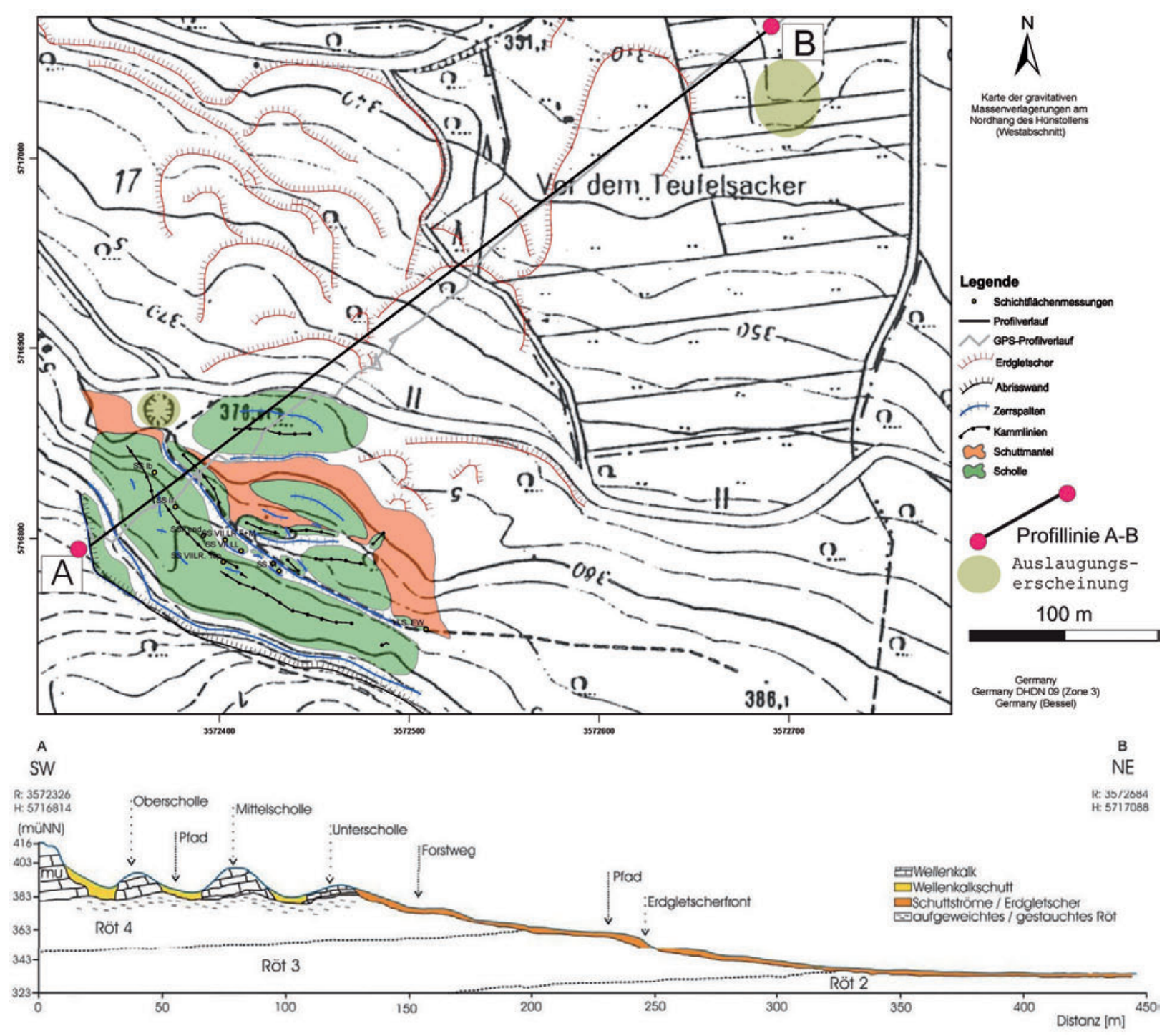

Abbildung 3. Zusammenfassende Darstellung der GPS-gestützten Kartierungen — Oben) Nach Mehrfachprozessierung der GPS-Daten erstellte Karte der gravitativ erzeugten Strukturen; Unten) Anhand der Karte und eines GPS-Profils konstruiertes geologisches Querprofil A-B (Profilverlauf siehe oben).

Anhand des Rotationssinns einzelner Schollen gegenüber der ungestörten Lagerung des $\mathrm{Mu}-$ schelkalks konnte auf die vorherrschenden Verlagerungsprozesse geschlossen werden (Abb. 4). Grundsätzlich gilt, dass Rotationen um etwa hangparallele Achsen Kipp- und Gleitkomponenten anzeigen. Abweichungen davon lassen dagegen auf Driftkomponenten schließen. Danach ergibt sich für das gesamte Arbeitsgebiet in der Summe eine Entwicklung mit Vorherrschen von Drift- und Gleitbewegungen, während Kippbewegungen nur untergeordnet stattfanden. Letztere wurden nur lokal begrenzt als dominant nachgewiesen (Ertl
2005; Kartiergebiet 2). Die größten Rotationsbeträge wurden durch Gleiten mit Rotation der Schollen gegen den Hang erreicht (Rotationsrutschen, s.o.). Inwieweit die genannten Bewegungen im vorliegenden Gebiet auch aktuell stattfinden, kann anhand der Beobachtungen nicht entschieden werden. Eindeutig (sub-) rezente Bewegungen lassen sich nur in Form von Kriechbewegungen in Erdgletscher-Zonen feststellen, dokumentiert durch Sichelwuchs (s. Abb. 2h). Auch für die offenen Zerrspalten unmittelbar an der Abrisskante (Abb. 2a) kann eine rezente Bildung angenommen werden. 


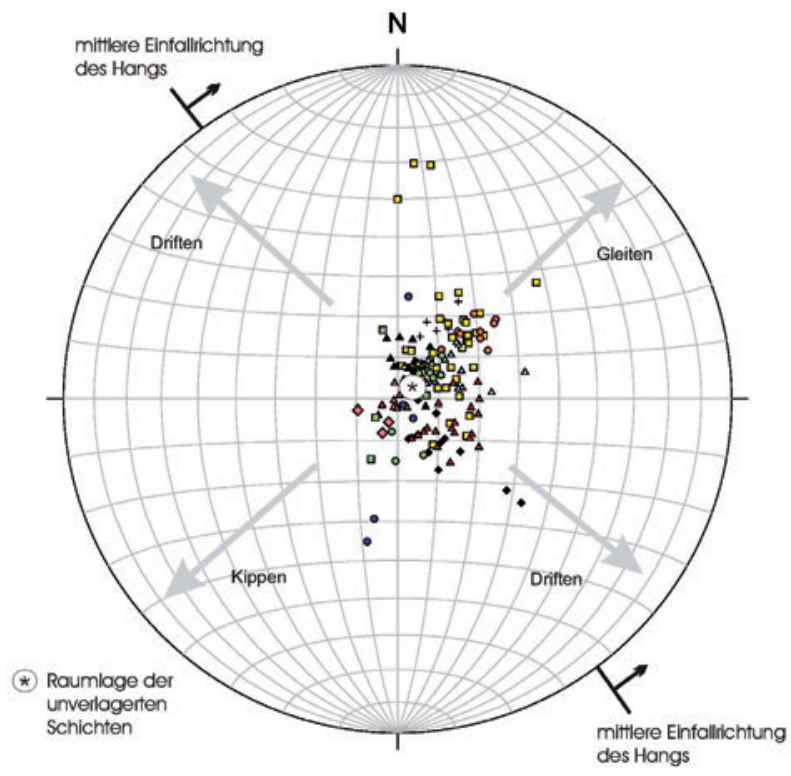

Abbildung 4. Schichtflächenpole in verlagerten Muschelkalkschollen (verschiedene Symbole/Farben beziehen sich jeweils auf einzelne Körper innerhalb von Schollen); Rotationssinn gegenüber unverlagerten Schichten (flaches Einfallen nach SW) und Beziehung zur Hangneigung (NE) erlaubt Rückschlüsse auf die vorherrschende Bewegungsart (hier: Gleiten mit Rotation gegen die Hangneigung); Schmidt-Netz, untere Halbkugel.

Eine genaue Alterseinstufung der verschiedenen Formen ist im Einzelfall problematisch (Ackermann 1959, Meyer 1985). Innerhalb des Arbeitsgebietes lässt sich jedoch anhand von morphologischen Kleinformen eine Tendenz von jüngeren Strukturen im Westen (Kartiergebiet 1) zu älteren, stärker ausgereiften Strukturen im Osten (Kartiergebiet 2) erkennen. Verschiedene Merkmale sprechen dafür, dass die markanteren Strukturen im Westteil größtenteils der sog ,Historischen Generation' zuzuordnen sind, die Ackermann (1959) in den Zeitraum zwischen 1550 bis 1850 v. Chr. stellt.

Im Nahbereich zur Hauptabrisskante hat, wahrscheinlich in Verbindung mit den gravitativen Massenverlagerungen, auch eine leichte Rotation der ungestört anstehenden Schichten stattgefunden. Dies ergibt sich aus kleinräumig stark variierenden Einfallwinkeln zwischen $1^{\circ}$ und $8^{\circ}$, z.T. mit Einfallrichtung nach NE. Dagegen wurde in größerer Entfernung zur Abrisskante ein konstantes Einfallen mit $3^{\circ}$ bis $15^{\circ}$ nach SW beobachtet.
Entsprechend gibt Beer (1977) für einen größeren Betrachtungsbereich ein gemitteltes Einfallen von etwa $3^{\circ}$ bis $6^{\circ}$ nach SW an. Eine ähnliche Schichtverstellung an der Grenze Röt-Muschelkalk führen Wilczewski \& Steinmetz (2003) auf die Auslaugung von Röt-Salinar zurück und sehen hierin eine Hauptursache für die Hanginstabilität und damit für die Entstehung der initialen Rutschkörper. Spuren von Auslaugungsprozessen im Untergrund in Form von Einsturzdolinen und Subrosionssenken finden sich auch im nördlichen Bereich des untersuchten Gebietes (Ertl 2005).

\section{Schlußfolgerungen}

Mit den erzielten Ergebnissen kann eine Anwendbarkeit von GPS in der vorliegenden Konstellation für Kartierungen/Grenzziehungen in bewaldeten Gebieten bestätigt werden. So konnten die Position und Dimension verschiedener Körper und Strukturelemente wesentlich genauer als bisher durch konventionelle geologische Kartierungen erfasst werden. Zusätzlich konnten durch Schichtflächenmessungen die Rotationsbewegungen einzelner Schollen beschrieben und damit Rückschlüsse auf vorherrschende Bewegungsarten gewonnen werden.

Rezente Bewegungen beschränken sich vor allem auf das Abstürzen, Driften und Gleiten kleinerer Gesteinspakete sowie Kriechbewegungen der Schutthänge. Für die gezielte Bearbeitung dieser Fragestellung könnten die erstellten Datensätze als Grundlage für ein Langzeitmonitoring dienen. Dabei könnten rezente Bewegungsraten (und -arten) durch langfristige Vermessungen mittels optischer Verfahren oder relativer GPS-Verfahren bestimmt werden. Hierzu sollten ergänzende Kluftrichtungsanalysen durchgeführt werden, um auch Aussagen über mögliche Rotationen um die Schichtflächen-Normale treffen zu können.

\section{Danksagung}

Wir danken K. Wemmer und T. Heinrichs für anregende Diskussionen während einer gemeinsamen Geländebegehung. 


\section{Literatur}

Ackermann, E. 1953. Der aktive Bergrutsch südlich der Mackenröder Spitze in geologischer Sicht. Nachrichten der Akademie der Wissenschaften Göttingen 5, 67-83.

Ackermann, E. 1955. Zur Unterscheidung glazialer und postglazialer Fließerden. Geologische Rundschau 43, 328-341.

Ackermann, E. 1959. Der Abtragungsmechanismus bei Massenverlagerungen an der Wellenkalk-Schichtstufe. Zeitschrift für Geomorphologie 3, 193-226 u. 283-304.

Arp, G., Hoffmann, V.-E., Seppelt, S., Riegel, W. 2004. Exkursion 6: Trias und Jura von Göttingen und Umgebung. In: Exkursionsführer zur 74. Jahrestagung der Paläontologischen Gesellschaft, 147-192.

Beer, W. 1977. Röt und Unterer Muschelkalk am Ostrand des Göttinger Waldes zwischen Södderich und Billingshausen (TK25: 4425 Göttingen, 4426 Waake, 4326 Lindau). Unveröff. Diplom-Arbeit, Georg-AugustUniversität Göttingen, $176 \mathrm{~S}$.

Bense, F. 2005. Gravitative Massenverlagerungen an der Röt/Muschelkalk-Grenze im Göttinger Wald - Eine GPS-gestützte Strukturkartierung am Nordhang des Hünstollens (WAbschnitt). Unveröff. Bacherlor-Arbeit, GeorgAugust-Universität Göttingen, $85 \mathrm{~S}$.

Ertl, G. 2005. Gravitative Massenverlagerungen an der Röt/Muschelkalk-Schichtstufe im Göttinger Wald. - Eine GPS-gestützte Strukturkartierung am Nordhang des Hünstollens (Ost-Abschnitt). Unveröff. Bacherlor-Arbeit, Georg-August-Universität Göttingen, $57 \mathrm{~S}$.

Koenen, A. v., Ebert, Th. 1894. Erläuterungen zur Geologischen Specialkarte von Preussen und den Thüringischen Staaten. 62. Lieferung - Blatt Waake [4426 Ebergötzen], Königlich Preußische Geologische Landesanstalt, Berlin, $22 \mathrm{~S}$.

Meyer, R. 1985. Die Stabilität der Hänge an der Wellenkalk-Schichtstufe des Göttinger Waldes. Unveröff. Diplomarbeit, Institut für Geologie und Dynamik der Lithosphäre, Georg-AugustUniversität Göttingen, Göttingen.

Prinz, H. 1997. Abriß der Ingenieurgeologie, 3. Auflage, ENKE-Verlag, Stuttgart, 415 S.

Wilczewski, W., Steinmetz, S. 2003. Rutschungen an der Grenze Buntsandstein/Muschelkalk. Göttinger Arbeiten zur Geologie und Paläonto- logie Sb. 5, Festschrift zum 70. Geburtstag von Professor Dr. Hans-Jürgen Behr, 121-123. 



\title{
Mittlerer Keuper im direkten Hangenden des Göttinger Sprungs, Göttingen, Georg-Dehio-Weg 5
}

\author{
Till Heinrichs (GZG Göttingen) und Bianca Wagner (GZG Göttingen)
}

\begin{abstract}
Zusammenfassung-Mittlerer Keuper unter Mittlerem Muschelkalk in quartären Gleitkörpern belegt die N-Fortsetzung des von Stille (1932) unterhalb des Rohns angegebenen Keuper-Vorkommens direkt im Hangenden der Hauptabschiebung, mit mm im Liegenden der Störung. Der Göttinger Sprung ist auf dem W-Hang des Hainberges durch mächtige Rutschkörper verdeckt. Detailbeobachtungen belegen, daß die quartären Deckschichten bis in die ältere Weichselzeit zurückreichen.
\end{abstract}

\begin{abstract}
Middle Keuper below Middle Muschelkalk in Quaternary landslides document the northern extension of the main normal fault that was predicted by Stille (1932) to be under the Keuper outcrop directly in the hangingwall of the main normal fault, with Middle Muschelkalk in the footwall. The Göttinger Sprung is covered, on the western flank of the Hainberg, by thick landslide deposits. Detailed observations prove that the Quaternary overburden reaches oldest Weichselian in age.
\end{abstract}

Schlüsselworte—Keuper, Abschiebung, Klüftung

\section{Einführung}

Im März 2007 wurde eine ca. $40 \times 20 \mathrm{~m}$ große Baugrube geologisch aufgenommen, die 4 bis $8 \mathrm{~m}$ tief in den W-Hang des Hainberges reichte, und trotzdem dort kein autochthones Anstehendes erschloss. Es wurde ein Komplex von Rutschkörpern angetroffen, dessen unruhige Oberfläche von (älterem) Löß ausgefüllt und dann von einem Mantel aus Solifluktionsschutt, Grobfraktion vorwiegend ruditische Kalksteine $\left(\mathrm{mo}_{1}\right)$, überdeckt wird (Abb. 1, 3).

\section{Aufschlussbeschreibung}

Die Karte in Verbindung mit dem Profil (Abb. 1) zeigt, daß die intern deformierten Rutschkörper vom Liegenden zum Hangenden aus den lithologischen Einheiten der Bunten Tonsteine (4 a, b, c), kalkreichen Brekzien (5a, b, c; 7) und Mergel (6, 8 ) bestehen. Die Tonsteineinheit wird aus schichtungslosen rotbraunen und grüngrauen Tonstei-

- Till Heinrichs - Abt. Angewandte Geologie, Geowissenschaftliches Zentrum der Universität Göttingen, Goldschmidtstr. 3, D-37077 Göttingen. theinri@gwdg.de

- Bianca Wagner - Abt. Angewandte Geologie, Geowissenschaftliches Zentrum der Universität Göttingen, Goldschmidtstr. 3, D-37077 Göttingen. bwagner1@gwdg.de nen gebildet, die aus Analogiegründen zum mittleren Keuper gestellt werden. Die Mergel-Einheit setzt sich aus beigen, z.T. hellgrauen KalkDolomit-Mergeln und -Mergelsteinen mit auffälligen Mn-Filmen zusammen. Ganz untergeordnet wurden darin grüngraue Siltsteine beobachtet, die wohl eingeschleppt sind. Die Einheit entstammt sicher dem Mittleren Muschelkalk. Beide Einheiten sind intern brekziös, durchsetzt im $\mathrm{cm}$ - bis dm-Maßstab von einem Netzwerk von calcitisch mineralisierten Brüchen.

Die kalkreiche Brekzie besteht aus einem ebensolchen Netzwerk, das cm- bis dm-große Mergelstein-Bruchstücke umschließt. Die mm- bis cm-dicken, harten, mineralisierten Septen bilden jedoch die Hauptmasse, so dass ein sehr festes Gestein vorliegt. Sie bestehen aus einer Masse kleinster (cm bis sub-mm) Fragmente von Mergelsteinen, z.T. dolomitisch, älterer Calcitzemente, durchsetzt von Mn-Oxiden, oft in GlaskopfHabitus, und einem Tonmineral, evtl. neugebildet, wohl ein Montmorillonit oder WechsellagerungsMineral (Abb. 2), sowie späten Calcit-Tapeten.

Die kalkreiche Brekzie tritt nach der Kartierung im Grenzbereich rotbunte Tonsteine/Mergel gehäuft auf. Da eine ähnliche Brekziierung auch in den anderen Gesteinseinheiten beobachtet wird, hängt diese Bildung möglicherweise mit dem Transportvorgang der Rutschung zusammen, in 


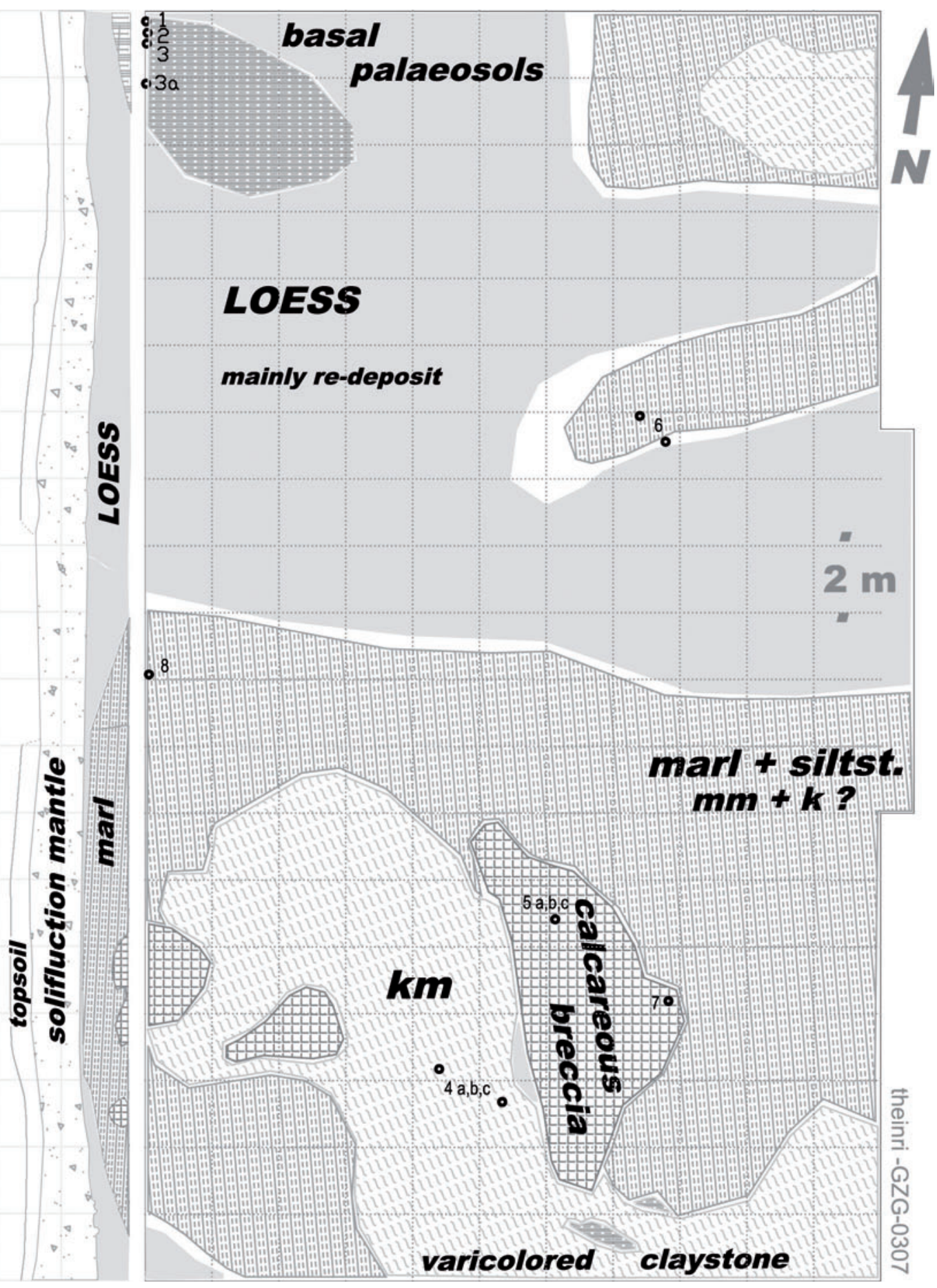

Abbildung 1. Geologie der ebenen Baugruben-Sohle und Profil des West-Stoßes, Dehio-Weg 5; ,Loess mainly redeposit" $=$ ältere Löss-Einheit mit ,Palaeosol' an der Basis = solifluidal verlagerter Ah-Horizont der Abb. 3; ältere Löss-Einheit füllt E-W verlaufende rinnenartige Vertiefungen zwischen Aufwölbungen der Rutschkörper aus; Profil (Paneel links) zwischen Meter 16 und 22 über den Zuweg interpoliert. Kreise mit No. = Probe. 


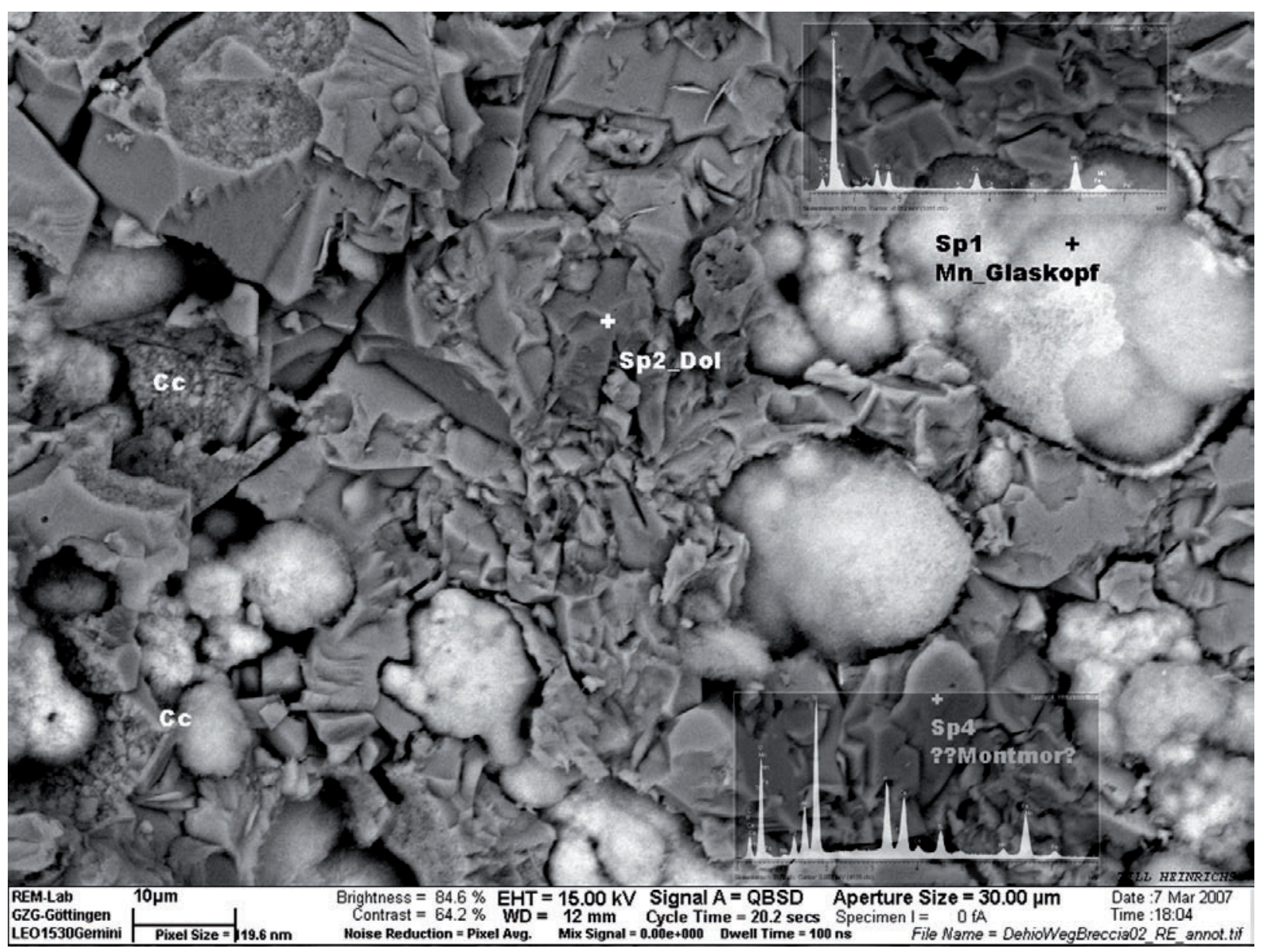

Abbildung 2. Kalkreiche Brekzie, mineralisiertes Septum; RE-Bild und qualitative EDX, blättriges Tonmineral/mixed Layer: (C), O, Na, Al, Mg, Si, K,(Ca), Ti, Mn, Fe, Glaskopf: O, Al, Si, K,(Ca), Mn, $\mathrm{Fe}$; fett = Hauptbestandteil; Probe 4b in Abb. 1.

Verbindung mit der besonderen hydrologischen Situation im Grenzbereich Mergel/ Tonsteine. Eine alternative Deutung der kalkreichen Brekzie als verfrachtete Störungsbrekzie zwischen mm aus der Liegendscholle und $\mathrm{km}$ aus dem Hangendblock der Hauptstörung ist denkbar, aber auf Grund der monomikten Zusammensetzung der enthaltenen Fragmente nicht sicher belegbar. Eine Deutung als Residualbrekzie würde die feine Fragmentierung kaum erklären, auch wurde weder Quarz noch Hellglimmer in der Brekzie beobachtet (vgl. Dünkel \& Vath 1990).

Die quartären Sedimente, die den Rutschkörpern auflagern, erreichen eine Gesamtmächtigkeit von ca. 3,50 m. Die Abfolge ist als Überblicksprofil in Abb. 3 dargestellt. Generell können ein älteres Lösspaket, eine mächtige, mehrgliedrige Solifluktionsdecke und ein jüngeres Lösspaket unterschieden werden.
Die beiden letztgenannten Einheiten treten in allen unverbauten Bereichen der Baugrube auf. Das ältere Lösspaket ist nur lokal und mit wechselnden Schichtmächtigkeiten vorhanden.

Die Basis des Profils bilden verkippte, dunkelgraue bis schwarze, stark schluffige, humusreiche Tone. Sie sind nur in der NW-Ecke der Baugrube erschlossen. Die auflagernden stark tonigen Schluffe sind heller gefärbt, enthalten jedoch noch Reste von Humusmaterial. Daneben führen sie viele Holzkohlebruchstückchen und Feinkiese. Die hangenden, kräftigbraunen, verschwemmten, schluffigen Feinsande sind deutlich verlehmt und verbraunt. Ihnen liegt eine hellgraurötliche Schlufflage mit zahlreichen Rost- und Gleyflecken auf. Einige Eiskeile, die mit hellerem, schluffreichen Material gefüllt sind, durchziehen diese Einheit. Kleine Eiskeile wurden auch dort beobachtet, wo der Löss dem $\mathrm{mm}$ aufliegt (SW- 


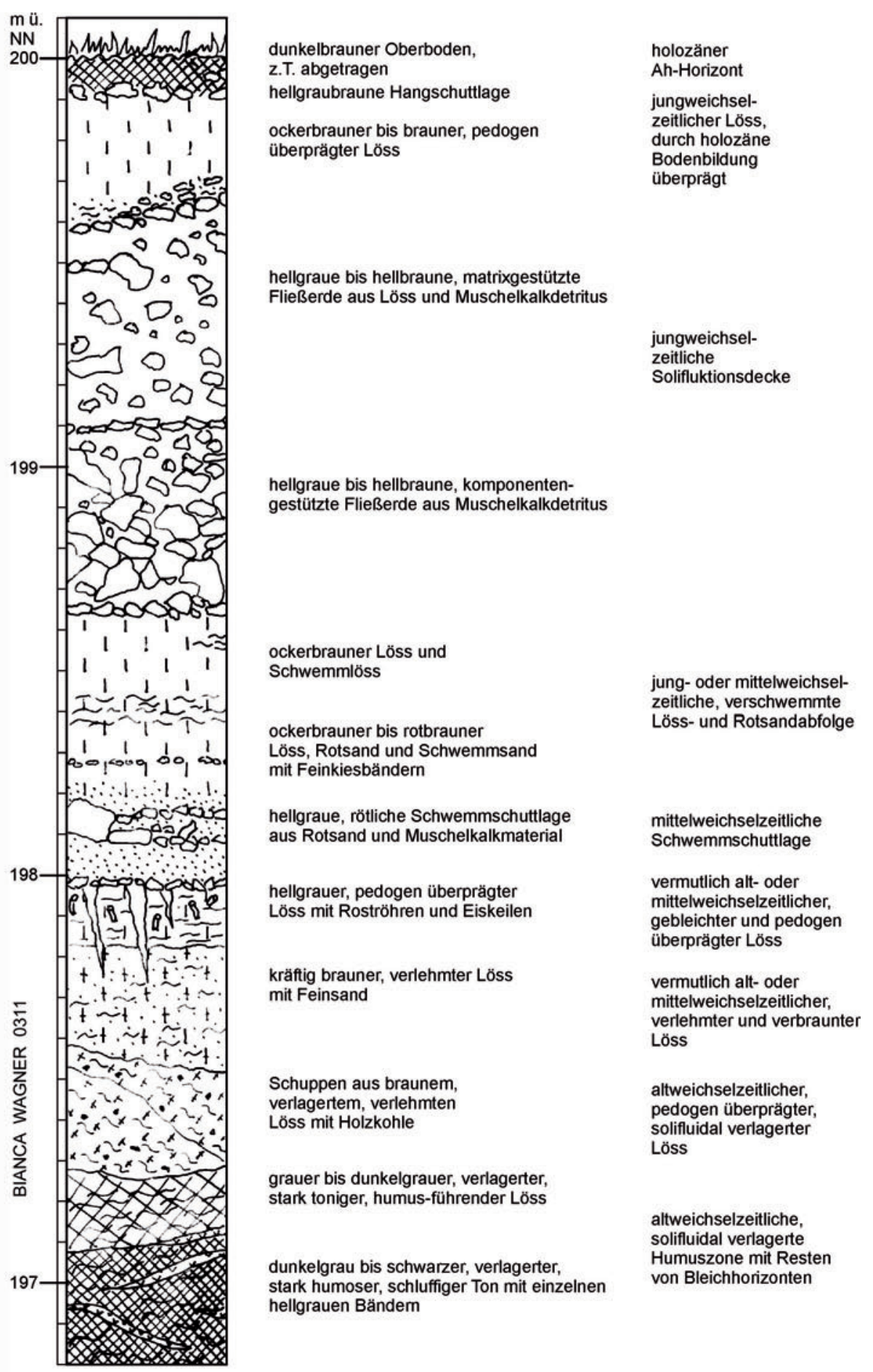

Abbildung 3. Profil der quartären Lockergesteinsabfolge oberhalb der Rutschkörper (aus 3 Teilprofilen zusammengesetzt) 
Ecke).

Darüber folgt mit markanter Grenze eine klastenreiche Schwemmschuttlage mit einem hohen Feinsandanteil. Im Hangenden geht sie allmählich in fluviatil überprägte Lösse und Feinsande (sogennante Rotsande) über. Eine mächtige Solifluktionsdecke aus Muschelkalkmaterial lagert dem unteren Lösspaket auf. Zonenweise lässt sich die Decke in einzelne Fließerdelagen untergliedern, die sich im Verhältnis von Matrix und Komponenten unterscheiden. Die überlagernden jüngere Lösse sind stark von der holozänen Bodenbildung überprägt oder wurden teilweise durch die Bauarbeiten abgeräumt. Während die oberen Schichten der Lockergesteinsabfolge nur sanft geneigt sind und nach Westen einfallen, ist der untere Abschnitt des älteren Lösspaketes deutlich verkippt und verschuppt. Die zeitliche Einstufung der Lockergesteinsabfolge, die den Rutschkörpern auflagert, ist über die Lösse möglich.

Generell kann die Abfolge als weichselzeitliche Bildung angesehen werden, da präweichselzeitliche Lösse bisher nur in Subrosionssenken oder reliktisch in Bodenbildungen des letzten Interglazials nachgewiesen wurden.

Die humusreichen Tone an der Basis der Abfolge können als Reste fossiler Ah-Horizonte (Humuszonen) angesprochen werden. Eine direkte Altersbestimmung war aufgrund der oxidativen Zersetzung der darin enthaltenen Palynomorphen nicht möglich (mdl. Mitt. W. Riegel) Da jedoch mittel- und jungweichselzeitlichen Lösse in Südniedersachsen keine Ah-Horizonte enthalten (Rohdenburg \& Meyer 1966), können sie in das Altweichsel eingestuft werden.

Weitere Hinweise auf die zeitliche Zuordnung der anstehenden Lockergesteine finden sich bei Rohdenburg (1965). Er beschreibt eine vergleichbare Abfolge aus Lössen und Fließerden, die nahe der Baugrube in einem Kanalgraben entlang des Georg-Dehio-Weges aufgeschlossen war. Rohdenburg (1965) weist eine altweichselzeitliche Humuszone nach und beschreibt den Niedervellmarer Bodenkomplex, eine Verlehmungszone des oberen Altweichsels, die möglicherweise bis ins Mittelweichsel reicht. Zudem beschreibt er eine LössFließerde und eine kräftige Muschelkalkfließerde. Letztgenannte ordnet er der kräftigen Solifluktionsphase nach dem Denekamp-Interstadial zu, also in das beginnenden Jungweichsel.

Es ist somit $\mathrm{zu}$ vermuten, dass der obere Abschnitt der älteren Lösse das Alt- bis Mittel- weichsel repräsentiert. Die Solifluktionsdecke und die jüngeren Lösse lassen sich dem Jungweichsel zuordnen.

\section{Literatur}

Dünkel, H., Vath, U. 1990. Ein vollständiges Profil des Muschelkalks von der Dransfelder Hochfläche. Geologisches Jahrbuch Hessen 118, 87-126.

Rohdenburg, H. 1965. Untersuchungen zur pleistozänen Formung am Beispiel der Westabdachung des Göttinger Waldes. Giessener Geographische Schriften, H. 7, 5-83.

Rohdenburg, H., Meyer, B. 1966. Zur Feinstratigraphie und Paläopedologie des Jungpleistozäns nach Untersuchungen an südniedersächsischen und nordhessischen Lößprofilen. Mitteilungen der Deutschen Bodenkundlichen Gesellschaft 5, 1-137.

Stille, H. 1932. Geologische Karte von Preußen und benachbarten deutschen Ländern. Erläuterungen zu Blatt Göttingen Nr. 2520. - 3 . Auflage, 40 S., 1 Kte., Berlin. 



\title{
Quartäre Akkumulation und Erosion am östlichen Rand der Leineaue bei Göttingen — Detaillierte Quartärprofile aus den Baugruben am Windausweg
}

\author{
Bianca Wagner (GZG Göttingen), Christian Nieding (IG Limburg), \\ Christina Janssen (GZG Göttingen) und Maria Herold (GZG Göttingen)
}

\begin{abstract}
Zusammenfassung - Eine quartäre Lockergesteinsabfolge am östlichen Rand der Leineaue wurde in Baugruben südlich des Göttinger Stadtzentrums detailliert aufgenommen. Die ältesten aufgeschlossenen Gesteine sind weichselzeitliche, fluviatile Schotter und Schwemmsande, auf denen sich im östlichen Teil des Baugeländes eine jungweichselzeitliche Flugsand-Löss-Decke erhalten hat. Im Westen des untersuchten Gebietes wurde eine ausgedehnte Rinne nachgewiesen, die mit spätpleistozänen bis altholozänen Kiesen, Auensanden, Schwemmsedimenten und Mudden gefüllt ist. Älterer und jüngerer Auenlehm bedecken als jüngste geologische Bildung einen großen Teil der älteren Lockergesteine. Anhand der aufgenommenen Profile wurde die weichselzeitliche bis jungholozäne Akkumulations- und Erosionsdynamik des Auenrandes rekonstruiert.
\end{abstract}

\begin{abstract}
A Quaternary sequence of unconsolidated sediments on the eastern border of floodplain of the River Leine, south of the city centre of Göttingen, is investigated in detail. The oldest exposed rocks, Weichselian in age, are fluvial gravels and alluvial sands, which are preserved in Young Weichselian layer of sand drifts and loess which is to be found to the east of the building area. To the west of the investigated area, a expansive channel has been proven that is filled with Late Pleistocene to Early Holocene gravels, flood sands, alluvial sediments and organic silts. The sections are used to reconstruct the Weichselian to Early Holocene accumulation and erosion dynamics of the flood plain.
\end{abstract}

Schlüsselworte-Quartär, Leineaue, Akkumulations- und Erosionsdynamik

\section{Einführung}

Im Jahr 2010 war der nähere Untergrund der Göttinger Leineaue in fünf $\max .3,5 \mathrm{~m}$ tiefen, temporären Baugruben aufgeschlossen. Das Baugelände befindet sich südlich der Altstadt auf der Ostseite der Leine und wird vom Windausweg, dem Parkplatz des Badeparadieses ,Eiswiese' und einer Kleingartenanlage (Abb. 1) begrenzt. Die Gebäude der ehemaligen Pathologie, die vorher

- Bianca Wagner - Geowissenschafltiches Zentrum der Universität Göttingen, Goldschmidtstr. 3, D-37077 Göttingen. bwagner1@gwdg.de

- Christian Nieding - Institut für Geotechnik in Limburg/Lahn chrisni86@gmx.de

- Christina Janssen - Geowissenschafltiches Zentrum der Universität Göttingen, Goldschmidtstr. 3, D-37077 Göttingen. tina-schaefler@gmx.de

- Maria Herold - Geowissenschafltiches Zentrum der Universität Göttingen, Goldschmidtstr. 3, D-37077 Göttingen.mherold@gwdg.de auf dem Gelände standen, waren im Vorjahr abgerissen worden. Drei der insgesamt fünf Baugruben auf dem ca. $100 \times 180 \mathrm{~m}$ großen Areal konnten geologisch aufgenommen werden. Die bisher vorliegenden Ergebnisse werden in dieser Arbeit vorgestellt.

\section{Methode}

In den drei tieferen Baugruben wurden die Wände, an denen detaillierte Profile mit wenig anthropogenen Veränderungen sichtbar waren, im Maßstab 1:50 aufgenommen. Die Aufnahme wurde mit Lot, Richtschnur und Bandmaß durchgeführt. Zahlreiche Proben für geotechnische und sedimentologische Untersuchungen sowie für OSLDatierungen wurden in allen drei Gruben entnommen. Die Probenentnahmepunkte und die Grenzen der Baugruben wurden mit einem differentiellen GPS-Gerät vermessen. Im Labor wurde die Korngrößenverteilung der Gesteine durch 


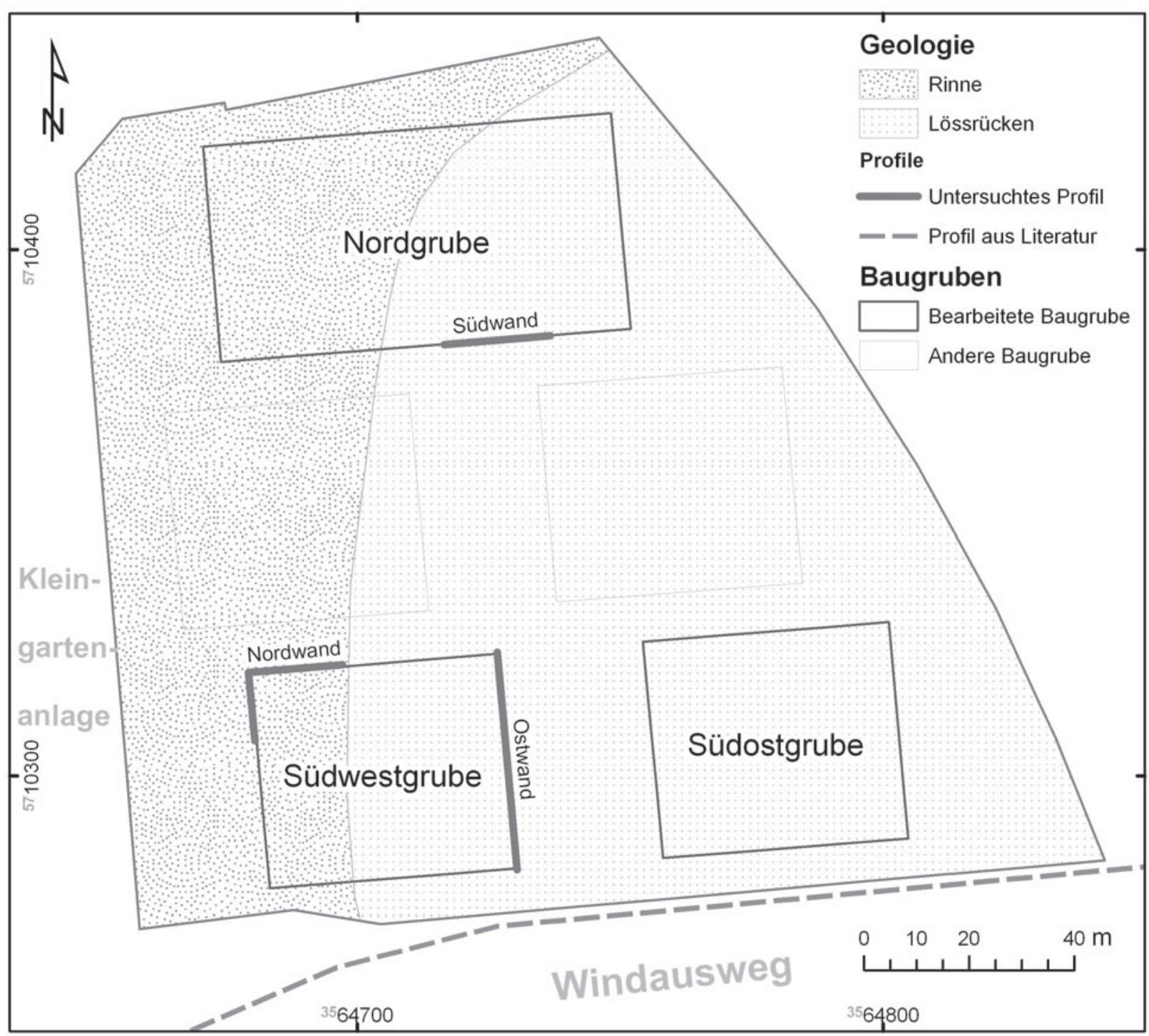

Abbildung 1. Übersichtskarte des Baugeländes mit den Umrissen der Baugruben und dem Verlauf des Lössrückens und der jüngeren Rinne.

Naßsiebung und LPS-Messung bestimmt (Nieding 2011).

\section{Ergebnisse}

Das Baugebiet befindet sich in der sanft nach Nordwesten geneigten Leineaue zwischen der Leine im Westen und dem im Osten angrenzenden Unterhang. Die Geländehöhe variiert zwischen 148 bis $150 \mathrm{~m}$ ü. NN. Die recht ebene Oberfläche ist weiträumig mit Bau- und Abrissmaterial bedeckt. Vereinzelt stecken im Untergrund noch Streifenfundamente und Rohreste. Die zahlreichen Eisen- und Manganausscheidungen in den Schottern sowie die Gleyfleckung und Bleichung der Sande und Schluffe sind auf den hohen Grundwasserstand zurückzuführen. Aus diesem Grund musste während der Bautätigkeit das Grundwasser abgepumpt werden.

Die Quartärbasis - verwitterte rotbraune Tonund Schluffsteine des mittleren Keupers - wurde bei den Erkundungsbohrungen in 128 bis 129 m ü. NN angetroffen. Die quartären Sedimente sind im Bereich des Baugebietes demzufolge ca. $20 \mathrm{~m}$ mächtig. Die in den Baugruben zugänglichen 3 bis $3,5 \mathrm{~m}$ zeigen eine starke horizontale und vertikale Variabilität. Die einzelnen Schichten, die vom Liegenden zum Hangenden in den Baugruben angetroffen wurden, werden nachfolgend näher erläutert. 


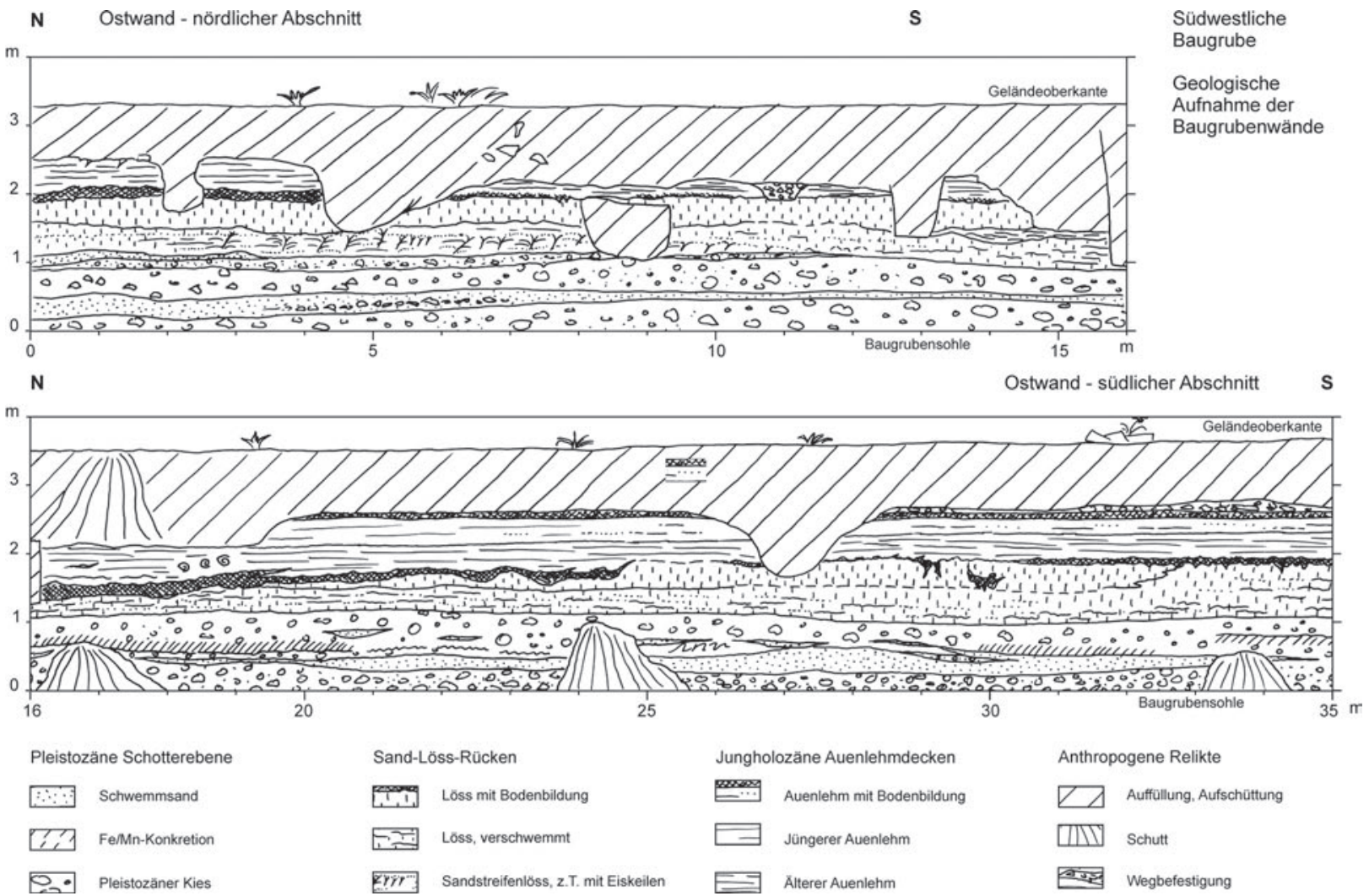

Abbildung 2. Geologische Aufnahme der Ostwand in der südwestlichen Baugrube am Windausweg.

\subsection{Fluviatile Kiese und Schwemmsande}

An der Basis der drei bearbeiteten Baugruben stehen weitflächig hellgraue bis hellbraune Mittelkiese (,Schotter $\left.{ }^{6}\right)$ an (Abb. 2, Abb. 3). Die gut gerundeten Gerölle bestehen zum größten Teil aus Kalkstein, vereinzelt aus Sandstein, Schluffstein oder Quarzit. Die Korngrößenbestimmung ergab einen schwach fein- und grobkiesigen Mittelkies mit einem teilweise hohen Anteil an Mittelsand sowie geringen Schluff- und Feinsandgehalten. Innerhalb der Kiese sind rötlich-braune, schwach fein- und grobsandige Mittelsande als $\mathrm{cm}$ - bis dm-mächtige Linsen oder diffuse sandreiche Zonen eingeschaltet. Die rötlich-braune Farbe der Sande lässt auf die nahen Buntsandsteinareale als Herkunftsgebiete schließen. Die mächtigeren Sandlagen, die sich horizontal bis über $10 \mathrm{~m}$ nachverfolgen ließen, enthalten einzelne Kieslagen und -bänder. Das Top der fluviatilen Kiese und Schwemmsande wird häufig aus einer sandreichen, nur leicht undulierenden Kiesschicht gebildet.

\section{2 Äolische Sande und Schluffe}

Im östlichen Bereich des Baugeländes stehen im Hangenden der Kiese und Schwemmsande rötli- che, rötlich-beige, beige und hellgraue Sande und Schluffe auf (Abb. 2). Nach Westen keilt diese Abfolge allmählich aus. Ihre Basis bildet ein gut sortierter Fein- bis Mittelsand mit hohem Grobschluffanteil. Darüber liegt eine Schicht aus gebänderten Feinsanden und Grobschluffen mit flaseriger Schichtung an. Lokal sind dünne Lagen aus Grobsand eingeschaltet. Im oberen Bereich der Wechselfolge sind kleine Eiskeile mit Längen von 10 bis $50 \mathrm{~cm}$ ausgebildet (,Kleinkeile'). Einzelne sehr markante Sand- bzw. Schlufflagen ziehen sich über mehrere Meter entlang der Braugrubenwände.

Im Hangenden schließt sich äolischer Löss an, der basal Merkmale fluviatiler Verspülung zeigt (gröbere Lagen, Bänderung). Die graubraune Färbung und die Gleyflecken sind auf einen Staunässeeinfluss aufgrund des zeitweise hohen Grundwasserstandes zurückzuführen. Auf den Lössen sind an mehreren Stellen Reste einer humusreichen Bodenbildung erhalten geblieben. Stellenweise wurden die Bodenreste durch spätere Bioturbation in den Löss hinein geschleppt. Vereinzelt ist dort auch der hellgraue Laacher Tuff in geringen Mengen zu finden. Auf den fluviatilen 


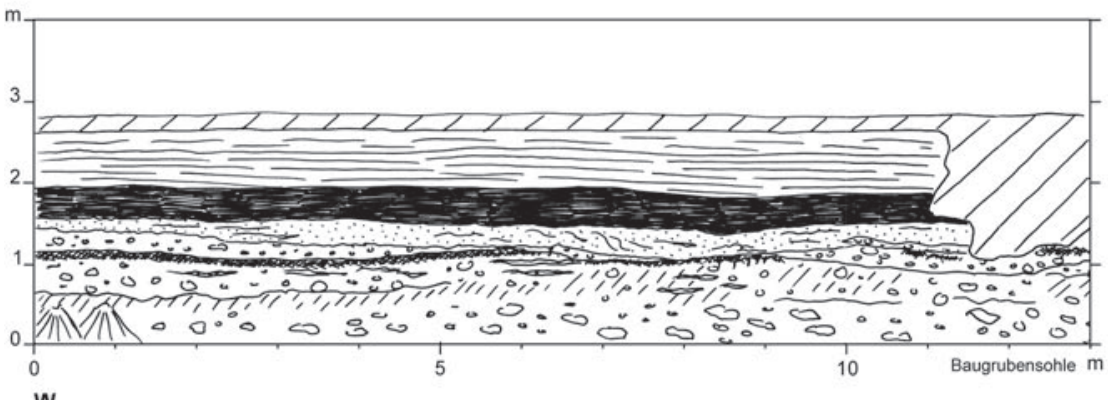

Südwestliche Baugrube

Geologische Aufnahme der Baugrubenwănde

W

Nordwand - westlicher Abschnitt

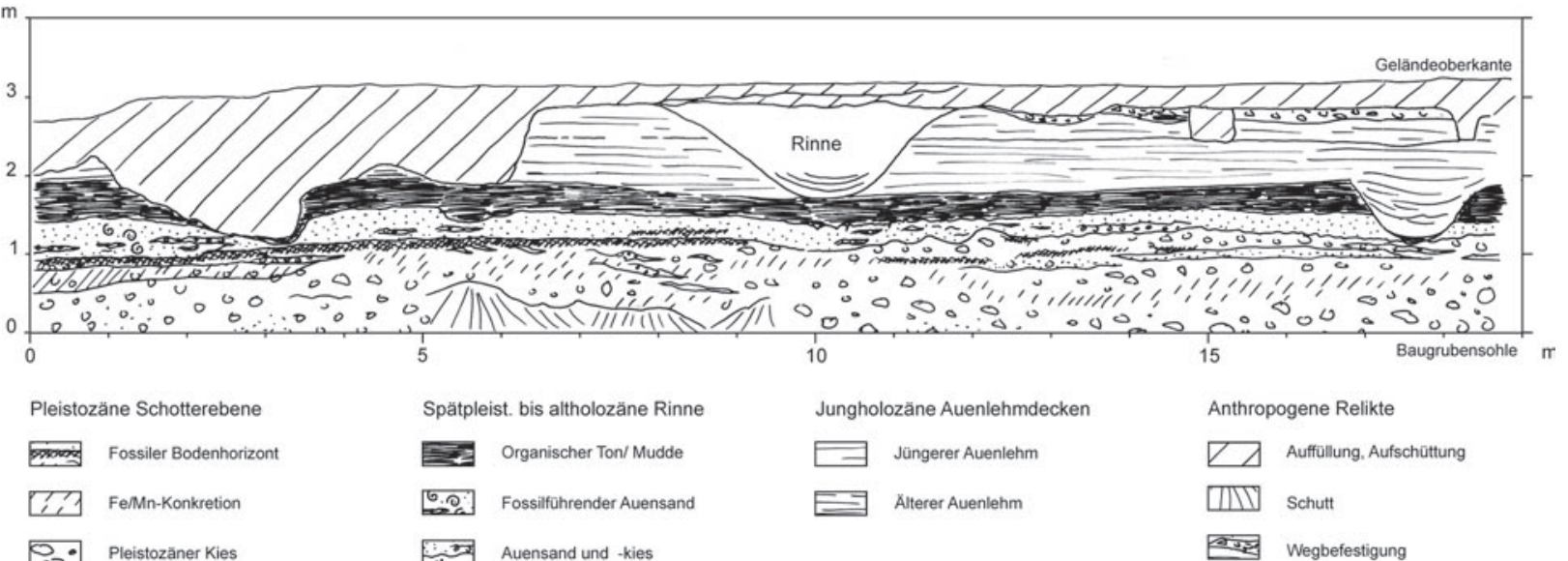

Abbildung 3. Geologische Aufnahme der Westwand (nördlicher Abschnitt) und der Nordwand (westlicher Abschnitt) in der südwestlichen Baugrube am Windausweg.

Kiesen und Schwemmsanden ohne Lössbedeckung ist eine ähnliche humusreiche, dunkelgraue bis schwarze Lage mit senkrechten Wurzelresten ausgebildet.

\subsection{Fluviatile Kiese und Auensande mit Hu- musmaterial}

Im westlichen Teil des Baugeländes bedeckt eine heterogene, hell- bis dunkelgraue Schicht aus schwach fein- und grobsandigen Mittelsanden und weitgestuften Fein- bis Mittelkiesen (sehr schwach feinsanding, mittelsandig, schwach grobsandig, schwach grobkiesig) das humusreiche Bodenrelikt (Abb. 3). Die Sande und Kiese wechseln sich in Form schmaler Bänder und Linsen ab, stellenweise gehen sie ineinander über. Kleine Rinnenstrukturen im cm- bis dm-Bereich sind ebenfalls erkennbar. Die Sande enthalten Schalenbruchstücke von Schnecken und schwarzes, humoses Material. Die Oberfläche der Schicht ist unruhig und wellig.

\subsection{Organische Tone und Mudden}

Dunkelgraue bis schwarze Tone bis Mudden mit einem hohen Anteil an organischer Substanz be- decken die Auensande und Kiese sowie einen schmalen Saum der Lössdecke. Die Korngröße umfasst schwach tonige bis tonige, fein- bis mittelsandige Fein- und Grobschluffe. Nach längerer Trockenheit zeigt die ca. 20 bis $50 \mathrm{~cm}$ mächtige, durchgängige Schicht Trockenrisse in Form eines Polyedergefüges. An mehreren Stellen wurden Holzreste gefunden. Es konnte sogar ein ca. 1,50 m langer Erlenstamm mit Rindenresten in der nördlichen Baugrube geborgen werden (jetzt im GZG).

\subsection{Auenlehme}

Eine 20 bis $40 \mathrm{~cm}$ mächtige, tonig-feinsandige, leicht humose Schluffschicht bedeckt sowohl die organischen Tone und Mudden, als auch die Lösse mit Bodenbildung im östlichen Baugebiet (Abb. 2 \& 3). Nur im äußersten Osten keilt die Schluffschicht aus.

Dieser als Auenlehm einzustufender, hellbrauner bis mittelbrauner Schluff enthält zahlreiche Schnecken und vereinzelt Knochenreste. Er füllt Unebenheiten und Rinnen der unterlagernden Sedimente aus und schließt mit einer ebenen Oberfläche ab. 
Eine vergleichbare, jedoch rötlicher gefärbte Auenlehmdecke überlagert die braunen Auenlehme. Das Erscheinungsbild beider Schichten ist sehr ähnlich, so dass nur bei starker Durchfeuchtung oder nach einer längeren Trockenphase beide aus der Entfernung unterscheidbar sind. Eine jüngere, ca. $3 \mathrm{~m}$ breite und 0,8 $\mathrm{m}$ tiefe Rinne durchschneidet beide Auenlehmdecken bis auf die Mudde. Sie ist mit dunkelgrauen bis schwarzen Tonen und Schluffen gefüllt und quert die SWBaugrube in N-S-Richtung.

\subsection{Anthropogene Relikte}

An einigen Baugrubenwänden lagern dünne Reste aus Kalksteinaufschüttungen auf den Auenlehmen. Dominiert wird der obere Abschnitt der Profile jedoch von heterogenen Auffüllungen und Aufschüttungen aus natürlichem Material (Schluffe, Kiese) und aus anthropogenen Komponenten (Bauschutt, Ziegel, Scherben, Betonfundamente)

\section{Diskussion}

Auf Grundlage der aufgenommenen Profile und der Beobachtungen im gesamten Baugelände kann die Genese und die flächenhafte Verbreitung der untersuchten quartären Gesteine rekonstruiert werden. Zusätzlich dazu ermöglichen die Untersuchungen von Rohdenburg et al. (1962) an einem am Windausweg aufgenommenen Kanalgraben (Verlauf siehe Abb. 1) mit vergleichbaren Sedimenten ihre zeitliche Einordnung.

Die Oberfläche der Kiese und Schwemmsande bildet eine flach nach Westen und Nordwesten geneigte Ebene. Die fluviatilen Ablagerungen wurden eindeutig von der Leine transportiert und abgelagert, da sie im Gegensatz zum Schwemmschutt der Nebenzuflüsse Buntsandsteingerölle führen. Der in den Baugruben aufgeschlossene Abschnitt der sicherlich noch mächtigeren Sandund Kiesfolge enthält keine warmzeitlichen Einschaltungen oder Paläoböden und zeigt kaum Anzeichen einer kryoturbaten Überprägung. Er kann somit der letzten Vereisung - der Weichseleiszeit - zugeordnet werden. Die weiträumige Akkumulation der fluviatilen Sedimente endete vermutlich an der Grenze zwischen Mittel- und Jungweichsel infolge der nachlassenden Transportkraft der Leine (Rohdenburg et al. 1962). Diese zog sich auf eine schmalere, weiter westlich gelegene Rinne zurück, so dass weite Bereiche des Flussbettes freilagen.

Während des trockenen und kalten Hochglazials im Jungweichsel wurde Sande und Schluffe aus der Schotterebene ausgeblasen und an deren Rand als Flugsand-Sandlöss-Löss-Decke oder möglicherweise auch als Düne abgelagert. Die westliche Verbreitungsgrenze des durch die Baugruben angeschnittenen ,Lössrückens' quert in SSW-NNERichtung das Baugelände (Abb. 1). Die allmähliche Klimaerwärmung im Spätglazial begünstigte die Zunahme der Vegetationsbedeckung und das Einsetzen der Bodenbildung auf den äolischen und fluviatilen Ablagerungen. Der allerödzeitliche Paläoboden blieb mit Resten des Laacher Bimses (ca. 12.5000 a) stellenweise auf dem Löss erhalten.

In der nun viel schmaleren Rinne der Leine westlich des Lössrückens (Abb. 1) wurde während dieser Phase nur wenig Material verlagert. Erst mit der Klimaverschlechterung in der Jüngeren Tundrenzeit setzte einer erneute Phase stärkerer Erosion und Verspülung ein (Rohdenburg et al. 1962, Wildhagen \& Meyer 1972a). Die Leine transportierte wieder deutlich mehr Fracht in den kleineren, jedoch sich häufig verändernden Rinnen. Die abgelagerten Sedimente sind in den Baugruben als humus- und fossilreiche Kiese und Auensande sowie Schwemmlehme erhalten. Die allmähliche Erwärmung im einsetzenden Holozän und die damit verbundene Vegetationszunahme zeigen sich an den auflagernden organischen Tonen und Mudden, die viele Wurzel- und Stammreste enthalten. Diese gleichmäßige Schicht wurde möglicherweise in einem Stillwasser- oder Altarmabschnitt der Leine mit Erlenbruchwald (Rohdenburg et al. 1962) akkumuliert. Die flächenhaft abgelagerten Auenlehme repräsentieren die jüngste Phase stärkerer fluviatiler Aktivität. Infolge der zunehmenden Besiedlung des Leinetals und den damit verbundenen Rodungen wurden weitflächig der Boden und die Lösslehme freigelegt, abgetragen und in der Leineaue sedimentiert. Wildhagen \& Meyer (1972b) korrelieren den älteren Auenlehm mit der Besiedlungswelle zwischen 600 und $900 \mathrm{n}$. Chr. Den jüngeren Auenlehm datieren sie auf die Zeit nach 1500 (schwerpunktmäßig in den Zeitraum 1500-1700).

Der holozäne Boden, der sich auf dem $\mathrm{Au}$ enlehm entwickelte, alte Wegbefestigungen und Rinnenfüllungen sind vereinzelt im oberen Bereich der Baugrubenwände erkennbar. In weiten Teilen des Baugeländes besteht jedoch die ober- 
ste Schicht aus durchschnittlich $20 \mathrm{~cm}$ bis $1 \mathrm{~m}$ mächtigen Aufschüttungen. Einzelne Fundamentreste und anthropogene Auffüllungen reichen bis zu $1,8 \mathrm{~m}$ unter GOK hinab.

In der bereits erwähnten Untersuchung von Rohdenburg et al. (1962) wird die Lage des Lössrückens (,Löss-Schwarzerde-Insel ${ }^{\circ}$ ) und der spätpleistozönen bis altholozänen Rinne südlich des Windausweges dokumentiert. Anhand der Ergebnisse der hier vorgestellten Arbeiten können diese Strukturen bis an die Nordgrenze des Baugeländes erweitert werden.

\section{Ausblick}

Die hier vorgestellten Ergebnisse zeigen den Stand der derzeitigen Auswertung. Die Analyse der geotechnischen Kennwerte, die Bestimmung weiterer sedimentologischen Parameter und die Datierung der OSL-Proben (optisch-stimulierte Lumineszenz) werden in der nächsten Phase erfolgen. Nach der Auswertung der Profilaufnahmen der beiden anderen Baugruben (Nord- und Südwestgrube) ist die Konstruktion eines 3D-Modells des Baugeländes geplant.

\section{Danksagung}

Die Autoren möchten Herrn Dannhauer und Herrn Dr. Neuhaus (onp planungs+projekt GmbH, Göttingen) für die Erlaubnis danken, die Baugruben wiederholt und auch zu ungewöhnlichen Uhrzeiten betreten zu dürfen. Dank gilt auch Dr. Witten (Witten Geotechnik, Göttingen) für die Bereitstellung der Bohrbeschreibungen und des Bohrgutes. Wir möchten ebenfalls Herrn Geisthardt (Fachdienst Umwelt, Stadt Göttingen) für den intensiven fachlichen Austausch danken.

Wir danken Dr. Leuschner für die Bestimmung des Baumfundes und Dr. Reich für die Bergung des Baumstammes. Frau Alena Ohlmeyer sei herzlich für das Einscannen der Aufschlußzeichnungen gedankt.

\section{Literatur}

Nieding, C. 2011. Geotechnische Untersuchung und Bewertung quartärer Lockergesteine in den Baugruben am Windausweg (Göttingen).Unveröffentlichte Bachelorarbeit, Geowissenschaftliches Zentrum Göttingen (GZG), Georg-August-Universität Göttingen, 42 S.

Rohdenburg, H., Meyer, B., Willerding, U., Jahnkuhn, H. 1962. Quartärgeomorphologische, bodenkundliche und archäologische Untersuchungen an einer Löß-Schwarzerde-Insel mit einer wahrscheinlich spätneolithischen Siedlung im Bereich der Göttinger Leineaue. Göttinger Jahrbuch 1962, 36-56.

Wildhagen, H., Meyer, B. 1972a. Holozäne Bodenentwicklung, Sedimentbildung und Geomorphogenese im Flussauenbereich des Göttinger Leinetalgrabens - 1. Spätglazial und Holozän bis zum Beginn der eisenzeitlichen AuenlehmAblagerung. Göttinger Bodenkundliche Berichte $21,1-75$.

Wildhagen, H., Meyer, B. 1972b. Holozäne Bodenentwicklung, Sedimentbildung und Geomorphogenese im Flussauenbereich des Göttinger Leinetalgrabens - 2. Die Auenlehm-Decken des Subatlantikums. Göttinger Bodenkundliche Berichte 21, 77-158. 


\title{
Geoelektrische Profile entlang der Leineaue im nördlichen Göttingen
}

\author{
Bianca Wagner (GZG Göttingen) und Andreas Weller (TU Clausthal)
}

\begin{abstract}
Zusammenfassung - Entlang der Leineaue im nordwestlichen Stadtgebiet von Göttingen wurden zwei geoelektrische Profile in SSE-NNW-Erstreckung gemessen. Das Ziel der Messkampagnen war die Erkundung des näheren Untergrundes anhand des unterschiedlichen spezifischen elektrischen Widerstandes von Gesteinen. Die prozessierten und visualisierten Messergebnisse zeigen eine gute Übereinstimmung mit den geologischen Daten aus mehreren Bohrbeschreibungen und der geologischen Karte. Direkt unter der Geländeoberkante konnte der bis maximal $3 \mathrm{~m}$ mächtige Auenlehm im südlichen Profil identifiziert werden. Die liegenden fluviatilen Schotter zeichneten sich in beiden Profilen anhand der hohen, nach unter wieder abnehmenden Widerstandswerte ab. Die Quartärbasis am Top der verwitterten Festgesteine wurde in 10 bis $25 \mathrm{~m}$ Tiefe nachgewiesen. Die Festgesteinsoberfläche steigt nach Norden an. Ihre wellige Oberfläche lässt auf Rinnen schließen.
\end{abstract}

\begin{abstract}
Two SSE-NNW striking geoelectric sections were measured along the River Leine in the northwestern part of the town of Göttingen. The goal of the measurement campaign was to investigate the near subsurface by using the different specific electric resistances of the strata.

The processed and visualized measurement results show a good correlation to geological information from borehole descriptions and the geological map. Directly underneath the topography, up to $3 \mathrm{~m}$ of alluvial loam could be identified in the southern section. Below this, fluvial gravels are demonstrated by resistivity values that decrease with depth. The base of the Quaternary on the top of the weathered solid rock is recognised at a depth of 10 bis $25 \mathrm{~m}$. The top of the solid rock layer rises to the north. The undulating surface suggests the presence of channels.
\end{abstract}

Schlüsselworte-Quartär, Geoelektrik

\section{Einführung}

Während der Durchführung des Projektes ,3D Göttingen" und des Teilprojektes ,Untergrund der DB-Trasse' in Kooperation mit dem LBEG Hannover und der Stadt Göttingen wurden die quartären Lockergesteine im Göttinger Stadtgebiet erkundet und räumlich konstruiert.

Da ein Großteil der verfügbaren Daten in Form von Bohrbeschreibungen und Kartenmaterial vorlag, wurden die Untersuchungen durch Geländekampagnen ergänzt. Ein Teilbereich im nördlichen Stadtgebiet wurde im Rahmen von Diplomkartierungen aufgenommen und abgebohrt

- Bianca Wagner - Geowissenschaftiches Zentrum der Universität Göttingen, Goldschmidtstr. 3, D-37077 Göttingen. bwagner1@gwdg.de

- Andreas Weller - Institut für Geophysik, TU Clausthal, Arnold-Sommerfeld-Str. 1, D-38678 ClausthalZellerfeld. Andreas.Weller@tu-clausthal.de (siehe auch Jahnke, Ohlmeyer, Rebens \& Wagner 2011, dieser Band). Da die Bohrungen nur räumlich begrenzte Aussagen zulassen, wurden geoelektrische Profile gemessen, um den Verlauf und die Teufe markanter Schichtgrenzen in einem längeren Bereich zu erkunden.

\section{Methode}

Die geoelektrischen Profile wurden entlang des Ostufers der Leine in der Leineaue gemessen (Abb. 1). Das Südprofil verläuft zwischen dem Zufluss des Leinekanals in die Leine bis an die Nordgrenze der Abwasserreinigungsanlage und hat eine Gesamtlänge von $1.400 \mathrm{~m}$. Die Messung erfolgte im Sommer 2006. Eine Fortsetzung des Profils nach Norden wurde im Sommer 2008 gemessen. Das Nordprofil verläuft vom Pfeiler des Autobahnzubringers $980 \mathrm{~m}$ entlang der Leine nach Norden und endet in der markanten Leinebiegung. Die Messung wurde mit einem GMS 150 der Firma GeoSys GmbH, Leipzig in Wenner-alpha- 


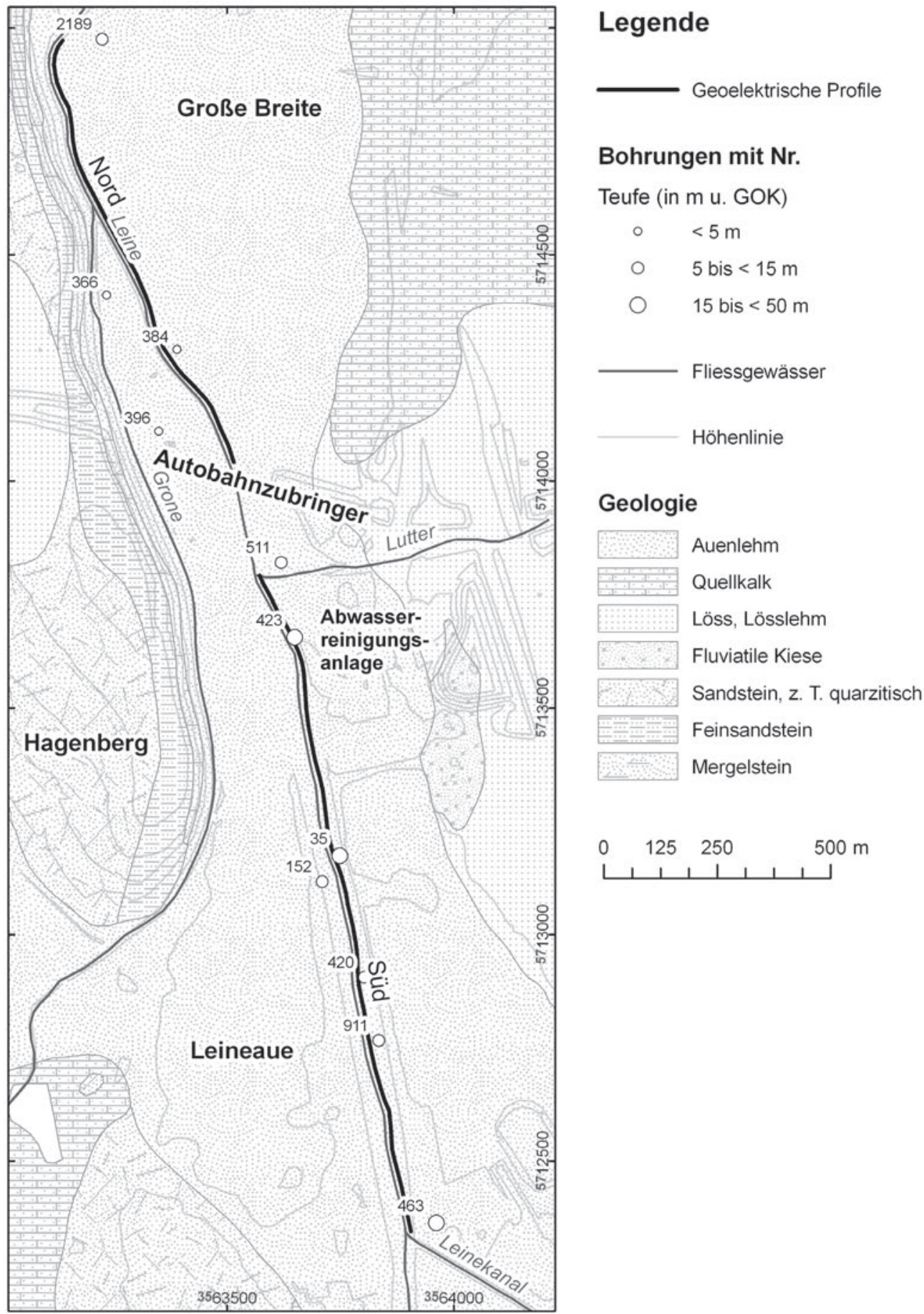

Abbildung 1. Geologische Übersichtskarte des Leineabschnitts zwischen dem Zufluss des Leinekanals und der Großen Breite (westlich Weende). 


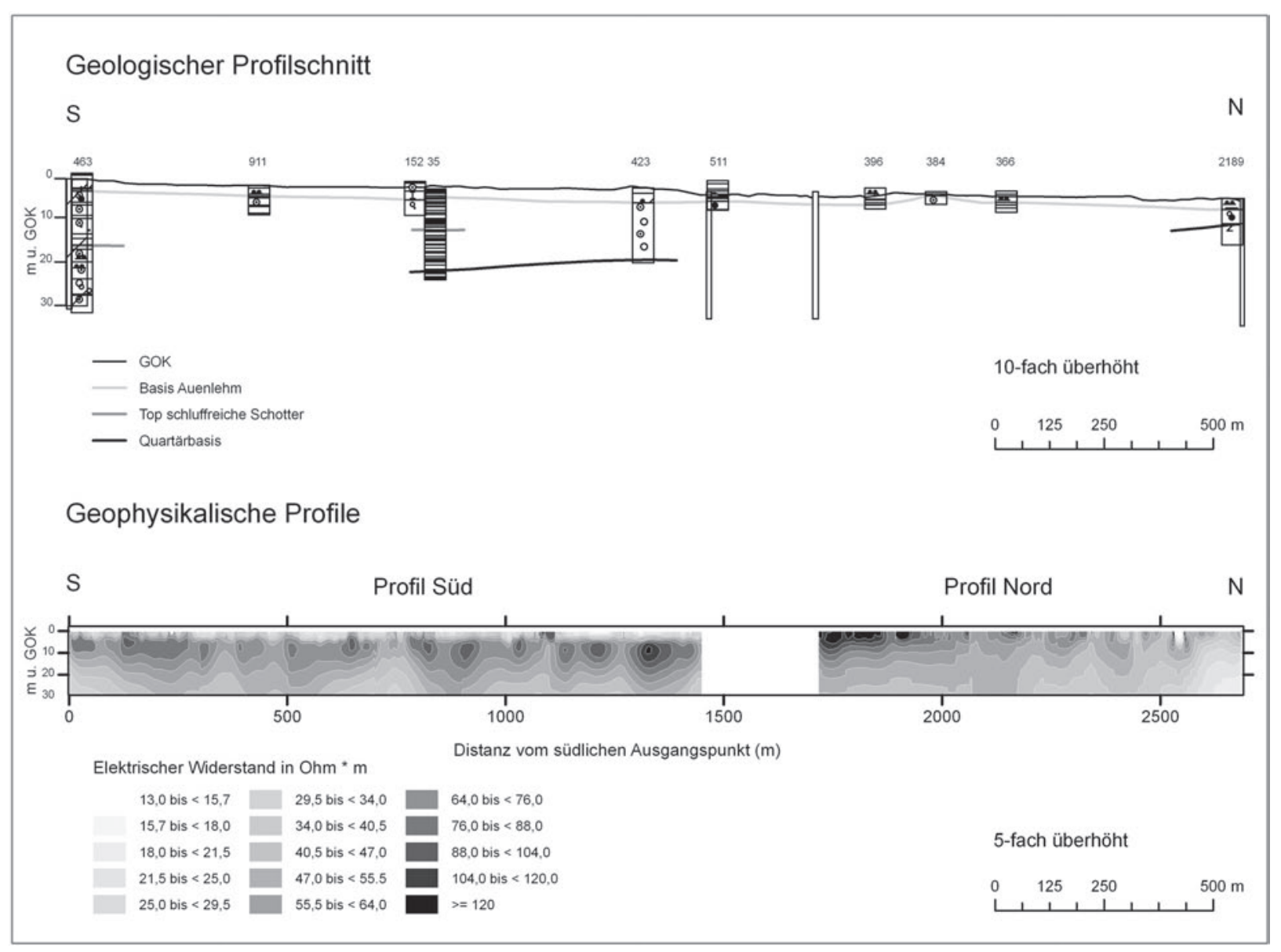

Abbildung 2. Oben: Geologischer Profilschnitt entlang der Messstrecke mit Bohrsäulen und markanten Schichtgrenzen. Die Ansatzpunkte der geoelektrischen Profile sind als schmale Rechtecke dargestellt. Das Profil wurde 10-fach überhöht, um die Schichtabfolge in den Bohrsäulen sichtbar zu machen. Kreise zeigen Kiese an, Punkte und dunkle Streifen symbolisieren Schluffe und Tone. Unten: Geoelektrisches Gesamtprofil aus Profil Süd und Profil Nord. Die Profile wurden 5-fach überhöht.

Anordnung $(A-n a-M-n a-N-n a-B)$ durchgeführt. Der Elektrodenabstand betrug $a=4 \mathrm{~m}$ mit $n=1 \ldots 15$, woraus sich eine maximale Eindringtiefe von $30 \mathrm{~m}$ ergibt. Die Inversion der Messdaten wurde mit dem Programm AC2DSirt von Axel Kampke berechnet.

Während der Messungen wurde der Profilverlauf mit Maßbändern, Messtisch und HandheldGPS der Firma Garmin dokumentiert. Die für die Auswertung verwendeten Bohrbeschreibungen wurden während zeitnaher Bohrungen aufgenommen bzw. in der Niedersächsischen Bohrdatenbank recherchiert.

\section{Ergebnisse}

Beide Profilabschnitte konnten durchgängig aufgezeichnet und prozessiert werden. Sie wurden jedoch mit einem zeitlichen Abstand von 2 Jahren und unter unterschiedlichen Witterungsbedingungen aufgenommen. Ein Zwischenraum von ca. $250 \mathrm{~m}$ musste übersprungen werden, da der Abschnitt oberflächenversiegelt ist.

Die spezifischen elektrischen Widerstandswerte liegen bei beiden Profilen in einem Bereich von 10 bis ca. $120 \Omega \mathrm{m}$ und lassen verschiedene Zonen erkennen (Abb. 2). Direkt unter der Geländeoberkante wurden sehr niedrige Widerstandswerte gemessen. Im südlichen Profil ist dieser Abschnitt durchgängig erkennbar und wird maximal $3 \mathrm{~m}$ mächtig. Im nördlichen Profil finden sich nur vereinzelte Hinweise auf eine solche niedrigohmige Schicht.

In beiden Profilen ist ein hochohmiger Bereich mit Widerstandswerten zwischen 65 und 
$100 \Omega \mathrm{m}$ vorhanden. Im südlichen Profil verläuft das Top der Zone in ca. $3 \mathrm{~m}$ Tiefe, im nördlichen Profil steigt die Zone bis zur Oberfläche an. Die Mächtigkeit dieses Bereiches liegt zwischen 5 und $17 \mathrm{~m}$. Nach unten nimmt der spezifische Widerstand allmählich bis auf Werte zwischen 50 und $65 \Omega \mathrm{m}$ ab.

Dieser mittelohmige, stärker undulierende Abschnitt tritt in beiden Profilen auf. Nach ca. 10 bis $15 \mathrm{~m}$ geht er in noch geringere Widerstandswerte von 45 bis $50 \Omega \mathrm{m}$ über. Die niedrigsten Messwerte von weniger als $46 \Omega \mathrm{m}$ wurden zur Profilbasis hin gemessen. Von Süd nach Nord wird dieser Bereich deutlicher und steigt bis auf $8 \mathrm{~m}$ unter GOK an.

\section{Diskussion}

Die geologische Karte 1:25.000 (Blatt 4425 Göttingen) und Bohrbeschreibungen wurden zur Interpretation der Ergebnisse herangezogen. 10 Bohrungen mit Tiefen zwischen 5 und $30 \mathrm{~m}$ und einer Entfernung zur Profillinie von maximal $90 \mathrm{~m}$ wurden ausgewertet.

Die oberflächenahe, niedrigohmige Zone korreliert mit der Auenlehmdecke, die in der Leineaue kartiert und erbohrt wurde. Die Auenlehme bestehen aus feinsandigen, tonigen Schluffen mit leicht humosen Beimengungen, vereinzelten Sandbändern und organischen Lagen. In Bohrung 384 gibt es Anzeichen, dass der Auenlehm lokal sehr geringmächtig ist. Das könnte eine Ursache für das Fehlen der niedrigohmigen Zone in Bereichen des Nordprofils sein. Die auffälligen hochohmigen Stellen innerhalb der obersten $3 \mathrm{~m}$ wurden durch Kabelkanäle hervorgerufen. Der sich darunter anschließende hochohmige Abschnitt korreliert mit den fluviatilen Leineschottern, die in einigen Bohrungen angetroffen bzw. auch durchteuft wurden. Die stark wasserführenden Leineschotter bestehen aus schwach schluffigen bis schwach sandigen Kiesen. Die Hauptkomponenten sind Gerölle aus Kalkstein, untergeordnet treten Sandstein-, Tonstein- und Quarzitgerölle auf. Die Abnahme des Widerstandes zum Liegenden hin lässt auf zunehmende Einschaltungen von Sanden und Schluffen (als Nebenkomponente oder als Linsen) schließen. Vermutlich zeichnet sich ein älterer Kieskörper ab.

Die in einigen Bohrungen angetroffene Oberfläche des Festgesteins deckt sich gut mit den niedrigohmigen Zonen (Werte zwischen 50 und $45 \Omega \mathrm{m}$ ). Laut Bohrbeschreibungen handelt es sich um die 1 bis $5 \mathrm{~m}$ mächtige Verwitterungszone des mittleren Keupers. Der Übergang zu noch niedrigern Widerstandswerten (unter $45 \Omega \mathrm{m}$ ) deutet den Übergang zum weniger verwitterten bis unverwitterten Festgestein an (Schluff- und Tonsteine).

Die aus den geoelektrischen Profilen und den Bohrbeschreibungen abgeleitete Quartärbasis verläuft im Süden in einer Tiefe von mehr als $30 \mathrm{~m}$ unter Geländeoberkante und steigt von nach Norden allmählich auf eine Tiefe von 8 bis $10 \mathrm{~m}$ an. Die flache Wellung kann als alte Rinnenstruktur der fluviatilen Schotter in der Festgesteinsoberfläche gedeutet werden.

\section{Ausblick}

Anhand der genannten Ergebnisse wird deutlich, dass die geoelektrischen Widerstandsmessungen in dieser Anordnung wirksam zur Erkundung des oberflächenahen Untergrundes bis in ca. $30 \mathrm{~m}$ Tiefe eingesetzt werden können. Die Methode sollte noch in weiteren, wenig bebauten und unversiegelten Gebieten Verwendung finden, um weitere Hinweise auf die Verbreitung und die Mächtigkeit der quartären Lockergesteine im Leinetal zu geben. Denkbar wäre auch die Kombination mehrerer, rechtwinklig verlaufender Profile, die eine flächenhafte Auswertung ermöglichen. Parallel dazu sollten die petrophysikalischen Eigenschaften der typischen Lockergesteine im Labor ermittelt und mit den Profilen verglichen werden.

\section{Danksagung}

Wir danken der AWIA Umwelt GmbH, dem LBEG Hannover und der Stadt Göttingen für die konstruktive Zusammenarbeit und die Bereitstellung der Bohrbeschreibungen. Weiterer Dank gilt den Clausthaler Doktoranden Marcus Möller und Sven Nordsiek, den Studierenden des ersten Durchgangs des Masterstudiengangs ,Hydrogeology and Environmental Geosciences ${ }^{6}$ und den Diplomanden Frau Alena Ohlmeyer und Sven Hellbach für die Unterstützung bei den Messkampagnen. Wir möchten Dr. Till Heinrichs für die Hilfe bei der Vermessung danken.

\section{Literatur}

Geologische Karte, 1:25.000, GK25, Blatt 4425, Göttingen. 


\title{
Geotechnische Kartierungen im Norden von Göttingen
}

\author{
Wiebke Jahnke, Alena Ohlmeyer, Marlene Rebens und Bianca Wagner (GZG Göttingen)
}

\begin{abstract}
Zusammenfassung - Im Rahmen von drei Diplomkartierungen wurde ein ca. 5,1 km² großes Gebiet in den Göttinger Stadtteilen Weende und Holtensen geotechnisch untersucht. Für die Beurteilung des Untergrundes wurden bereits vorhandene Bohrungen aus der Datenbank der Angewandten Geologie des GZGs verwendet, Bohrungen von Göttinger Ingenieurbüros zusammengetragen sowie von den Kartierern abgeteuft. Das gewonnene Probenmaterial wurde im Labor auf verschiedene bodenmechanische Parameter untersucht. Es erfolgte eine Beurteilung der Kartiereinheiten bezüglich ihrer Baugrundeigenschaften und die Erstellung einer Ingenieurgeologischen Karte, die die Verbreitung der Einheiten zwei Meter unter Geländeoberkante darstellt. Die Beurteilung der Einheiten als Baugrund hat ergeben, dass die Festgesteine und die Kiese die günstigsten Eigenschaften für Gründungszwecke besitzen.
\end{abstract}

\begin{abstract}
As part of three Diploma mapping projects, a ca. $5.1 \mathrm{~km}^{2}$ large area around the city of Göttingen quarters of Weende and Holtensen was geotechnically investigated. To determine the type of subsurfaces in this area, we used available boreholes from the database of the applied geology department of GZG, as well as boreholes from engineering firms in Göttingen and our own mapping. The representive samples were analysed in a laboratory as to their geomechanical properties. From this we divided the mapped geological units by their use as building ground and portrayed them as a engineering geological map that shows the units $2 \mathrm{~m}$ below topography. The determination of units as building ground has shown that solid rock and gravels are the most favourable for building.
\end{abstract}

Schlüsselworte-Geotechnische Kartierungen, Baugrund

\section{Einführung}

Der nördliche Bereich des Göttinger Stadtgebietes wurde in fünf geotechnischen Kartierungen im Jahr 2008/2009 erkundet. Drei dieser Kartierungen werden hier vorgestellt. Die vorwiegend quartären Ablagerungen wurden mit Hilfe von ingenieurgeologischen Parametern bodenmechanisch beschrieben. Die begrenzende Tiefe unter Geländeoberkante stellt die Quartärbasis dar. Die Auswertung erfolgte anhand von Schichtprofilen aus Fremddaten, sowie aus Bohrungen, die im Rahmen der Arbeiten aufgenommenen wurden. Die Einteilung der Schichten in Kartiereinheiten erfolgte nach DIN 18196 und DIN 18 300. Diese dienten folgend als Grundlage für die Erstellung von Nord-Süd- und West-Ost-Profilschnitten, an-

- Wiebke Jahnke — wiebkejahnke@googlemail.com

- Alena Ohlmeyer - aohlmeyer@gmx.de

- Marlene Rebens - Marlene.Rebens@gmx.de

- Bianca Wagner - bwagner1@gwdg.de Geowissenschafltiches Zentrum der Universität Göttingen, Goldschmidtstr. 3, D-37077 Göttingen. hand derer eine drei-dimensionale Bewertung erfolgte. Abschließend wurde eine Ingenieurgeologische Karte erstellt, die den Untergrund zwei Meter unter Geländeoberkante darstellt und der Bewertung des Untergrundes für baugrundliche Zwecke diente.

\section{Gebietsbeschreibung}

Die Arbeitsgebiete der Kartierungen von Ohlmeyer (2009), Jahnke (2009) und Rebens (2009) liegen im Göttinger Leinetalgraben und umfassen Bereiche der Stadtteile Weende, Holtensen und Holtenser Berg (Abb. 1). Die Gebiete nehmen insgesamt eine Fläche von ungefähr $5,1 \mathrm{~km}^{2}$ ein. Zusammengefügt ergeben die Teilgebiete ein Lförmiges Areal.

Die nördliche Grenzlinie des Gesamtgebietes verläuft von der BAB 7 im Westen Richtung Osten über den Niederangergraben, die Leine, entlang des Arbeckswegs und endet im Osten im Göttinger Wald. Die Westgrenze des Gesamtgebietes ist aufgrund der L-Form in zwei Grenzlinien gegliedert. Die Westgrenze des Teilgebietes Ohlmeyer beginnt im Norden unterhalb der BAB 7 


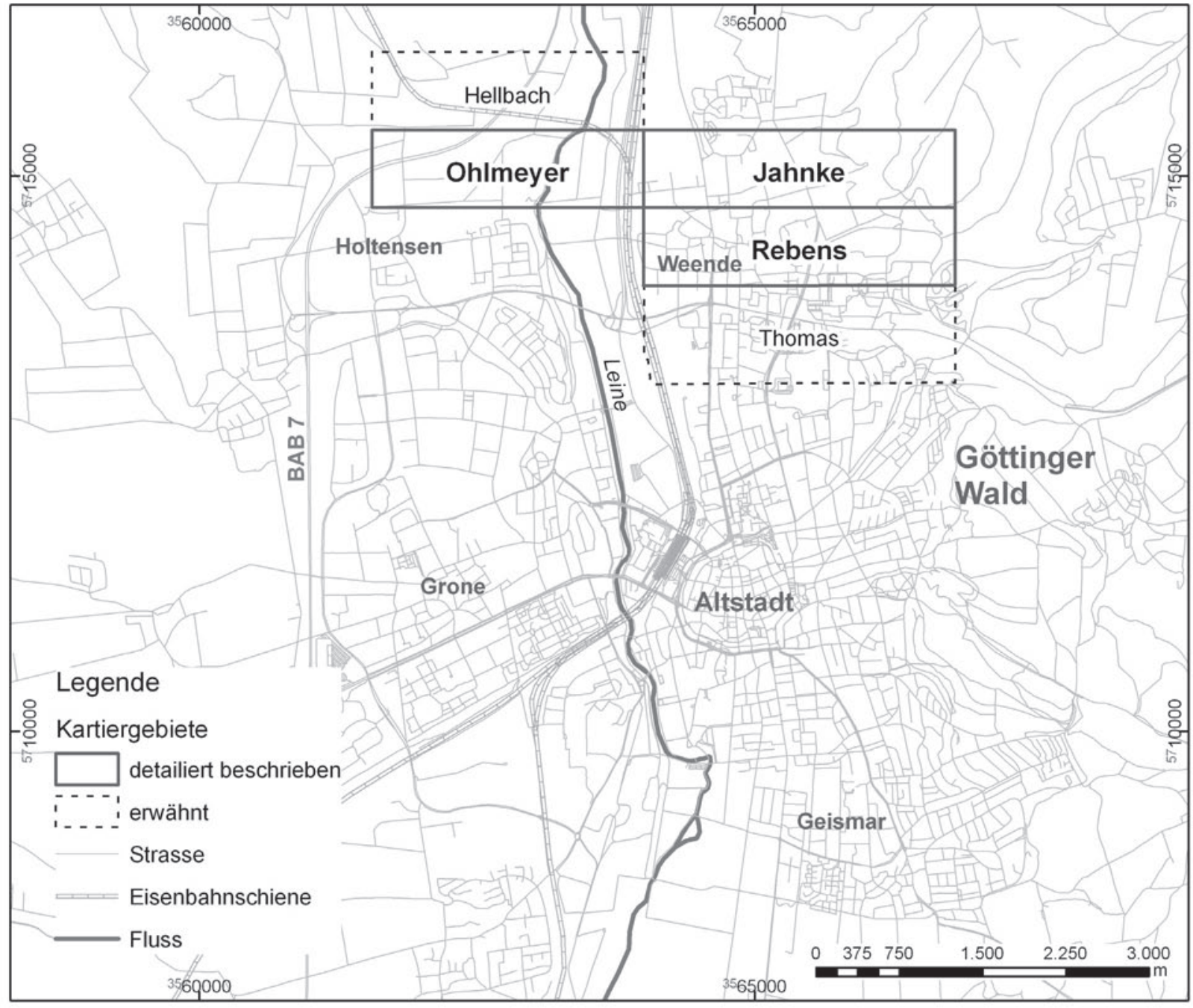

Abbildung 1. Topographische Übersichtskarte — Lage der Kartiergebiete.

und verläuft nach Süden über den Feuerteichgraben der Gartenkolonie von Holtensen. Die Westgrenze entlang des Teilgebietes Rebens verläuft entlang der Bahntrasse. Die südliche Grenzlinie ist ebenfalls untergliedert in zwei Teilstücke und erstreckt sich am Teilgebiet Ohlmeyer nördlich der Den-Haag-Straße und nördlich des Holtenser Berges. Während die Südgrenze des Teilabschnitts Rebens oberhalb des Autobahnzubringers und der B 27 verläuft. Die östliche Grenzlinie des Gesamtgebietes verläuft im Göttinger Wald.

Im Westen ist das Gebiet vorwiegend durch die Erhöhung des Holtenser Berges (Keuperrücken) geprägt. Dieser bildet in Bezug auf seine Umgebung, die bei 140-145 m ü NN liegt, eine Erhebung von ca. $172 \mathrm{~m}$ ü NN. Weiter in östlicher Richtung besitzt das Gelände eine durchschnittliche topographische Höhe von $145 \mathrm{~m}$ ü NN und steigt nach Osten auf eine Höhe von $295 \mathrm{~m}$ ü NN an, wobei der Grad der Steigung von etwa $20^{\circ} \mathrm{im}$ Westen auf etwa 40-50 zur östlichen Begrenzung zunimmt. Bedingt durch die unterschiedlichen Neigungswinkel entsteht eine Geländekante. Der höchste Punkt des Gebietes befindet sich im Nord-Osten mit einer topographischen Höhe von $298 \mathrm{~m}$ ü NN.

Die südliche Grenze ist durch das Luttertal geprägt, in dem die Lutter von Osten nach Westen fließt und auf Höhe der Abwasserreinigungsanlage in Weende in die Leine mündet. Die Weende entspringt im Nordosten des Gebietes im Geländeknick auf einer topographischen Höhe von $170 \mathrm{~m}$ ü NN und mäandriert annähernd von Ost nach West durch das Gebiet von Jahnke/Rebens. Das Einzugsgebiet der Weende befindet sich auf der Anhöhe im Nordosten des Arbeitsgebietes. 
Der Holtenser Berg bildet auf seiner Ostseite einen Steilhang, an dem die Leine entlang fließt, die 1 bis $2 \mathrm{~m}$ in die Talaue eingetieft ist (Nagel \& Schunke 1979). Durch das Kartiergebiet fließt darüber hinaus ein Bach, die Hassel. Die Hassel entspringt nördlich vom Fassberg, verläuft nahezu von Nord nach Süd durch den östlichen Teil des Kartiergebietes und mündet schließlich in die Lutter.

Im Zentrum der Kartiergebiete verläuft die Schnellfahrstrecke Würzburg-Hannover von Nord nach Süd. Westlich der Trasse wird das Gebiet vorwiegend für landwirtschaftliche Zwecke genutzt. Lediglich der Keuperrücken dient der Forstwirtschaft. Östlich der Trasse finden sich Kleingartenanlagen und Gewerbeflächen. Anschließend gibt es ein eng bebautes Wohngebiet sowie universitäre Anlagen und ein Forschungszentrum. Im nordöstlichen Bereich wird das Gebiet landwirtschaftlich genutzt und geht mit ansteigender Höhe nach Osten in einen forstwirtschaftlich genutzten Wald über. Dieser Laubwald erstreckt sich über den Nordosten des Gesamtgebietes.

\section{Planung, Datengrundlage und Methodik}

Als Datengrundlage für die Kartierungen dienen u. a. topographische Karten, geologische Karten und Luftbilder. Diese wurden mit dem Programm ArcGIS (Version 9.2) bearbeitet.

Um die räumliche Verbreitung der geologischen Einheiten möglichst detailliert erfassen zu können, wurden Profillinien netzartig über die Gebiete gelegt. Dies erfolgte unter Berücksichtigung der Geologie, Topographie und Morphologie des Untersuchungsgebietes, vorhandener Bohrungen aus dem Aktenarchiv der Abteilung Angewandte Geologie des GZGs und aus Gutachten von Firmen sowie der Lage der Profillinien der angrenzenden Kartiergebiete.

Zusammengefasst gibt es 19 West-OstProfillinien und 18 Nord-Süd-Profillinien in den Kartierungen. Für Bereiche in denen wenige bzw. keine Untergrundinformationen vorhanden waren, wurden entlang der Profillinien in einem Abstand von etwa $200 \mathrm{~m}$ vorläufige Bohrpunkte platziert. Von 89 geplanten Bohrpunkten, konnten nach der Genehmigung der Flurstückseigentümer, der Überprüfung von Leitungsplänen und der Freigabe durch den Kampfmittelräumdienst, 73 erfolgreich abgeteuft werden.
Die Bohrungen wurden vorwiegend mit dem Wackerhammer sowie dem Pürckhauer und dem Geoprobe niedergebracht. Ebenso wurden Fremdbohrungen, Baugruben und künstliche Aufschlüsse im Gelände aufgenommen. Die Erfassung und Bearbeitung der Bohrverzeichnisse erfolgte mit GeODIN (Version 6.0).

Anschließend wurde das gewonnene Probenmaterial im Labor auf bautechnische Eigenschaften untersucht. Es wurden der Wassergehalt nach DIN 18 121-1 (1998), die Korndichte nach DIN 18124 (1997), der Glühverlust nach DIN 18128 (2002), die Konsistenzgrenzen nach DIN 18 122-1 (Juli 1997), die Fließund Ausrollgrenze und die Korngrößenverteilung mittels Nasssiebung sowie LDPSA (Laser Diffraction Particle Size Analyzer) bestimmt. Zudem wurden Mikrofossilanalysen an ausgewählten Festgesteinsproben durchgeführt, um anhand der Biostratigraphie die Alter zu bestimmen.

Mit Hilfe der ermittelten Bodenkennwerte und bautechnischer Eigenschaften aus Erfahrungswerten der Literatur nach DIN 18196 in Bodengruppen und nach DIN 18300 in Bodenklassen eingeteilt. Die angefertigten Profilschnitte, Dokumentationskarten, Baugrundkarten und Quatärbasiskarten fungierten als Grundlage für die Untergrundbewertung.

\section{Kartiereinheiten}

Im Folgenden werden die Kartiereinheiten detailliert beschrieben und geotechnisch beurteilt.

\subsection{Mesozoikum}

\subsubsection{Muschelkalk}

Der östliche Hang der Kartiergebiete von Jahnke (2009) und Rebens (2009) wird von Muschelkalk aufgebaut. Es handelt sich hauptsächlich um die Schichten des Unteren Muschelkalkes und an der östlichen Grenze sowie im Norden des Kartiergebietes Jahnke (2009) um ein Vorkommen von Mittlerem Muschelkalk.

Der Untere Muschelkalk besteht aus dem Unteren, Mittleren und Oberen Wellenkalk mit drei zwischen geschalteten Werksteinhorizonten (Oolithzone, Terebratelzone, Schaumkalkzone) im Wellenkalk.

In Fremdbohrungen innerhalb der Kartiergebiete werden der Mittlere und der Obere Wellenkalk als plattiger, welliger, grauer Kalkstein beschrieben. Vom Mittleren Wellenkalk wurden 
$5 \mathrm{~m}$ erbohrt und der Obere Wellenkalk weist eine durchschnittliche Mächtigkeit von $13 \mathrm{~m}$ auf. Die Terebratelzone besteht aus einem zelligen, rötlichgrauen Kalkstein mit einer Mächtigkeit von $5 \mathrm{~m}$. Die Schaumkalkzone ist aus schaumigen, gelbbraunen Kalksteinen aufgebaut und weist als hangende Schicht vermutlich infolge von Erosion nur noch eine Mächtigkeit von $0,8 \mathrm{~m}$ auf. Nach Meyer (1987) besitzt der Untere Muschelkalk eine Gesamtmächtigkeit von ca. $100 \mathrm{~m}$.

Der Mittlere Muschelkalk besteht aus grauen bis gelblichgrauen Mergelsteinen und Gipsablagerungen, die in Form von 2-3 m mächtigen Linsen zwischen die Mergelsteine geschaltet sind (Meyer 1987). Der Mittlere Muschelkalk wurde in den Kartiergebieten nicht erbohrt.

Die Formation des Muschelkalkes kann gemäß DIN 18300 in die Bodenklasse 6-7 der leicht bis schwer lösbaren Felse eingeteilt werden. Das Einfallen der Schichten mit dem Hang in Richtung Westen (Brunotte 1985), kann vor allem bei den stärker verwitternden Wellenkalken und Mergelsteinen zu Rutschungen führen.

Mögliche Auslaugungen der Karbonat- und Sulfatgesteine im Untergrund lassen Hohlräume entstehen, die nahe der Oberfläche zu Erdfällen führen können. Eine genaue Baugrunderkundung und Erfassung solcher Hohlräume im Untergrund sollte daher durchgeführt werden. Generell besitzt die Einheit Muschelkalk jedoch eine gute Tragfähigkeit und stellt einen guten Baugrund dar. Aufgrund der teilweise sehr festen Gesteine ist der aufwendigere Aushub der Baugrube zu berücksichtigen.

\subsubsection{Keuper}

In den Kartiergebieten stehen die Gesteine des Mittleren und des Oberen Keupers an. Während der Obere Keuper im Westen des Gebietes ein flächenhaftes Auftreten aufweist und am Rand zu den Flussschottern von Mittlerem Keuper überlagert ist, tritt der Mittlere und Obere Keuper im Osten nur vereinzelt auf.

Die Schichtenfolge des Mittleren Keupers gliedert sich lithologisch in die vier Einheiten Gipskeuper, Schilfsandstein, Rote Wand und Steinmergelkeuper (Ritzkowski 1985). Der Mittlere Keuper besitzt eine Gesamtmächtigkeit von über $300 \mathrm{~m}$ (Nagel \& Wunderlich 1976), die im Gebiet nicht durch Bohrungen erschlossen werden konnten.

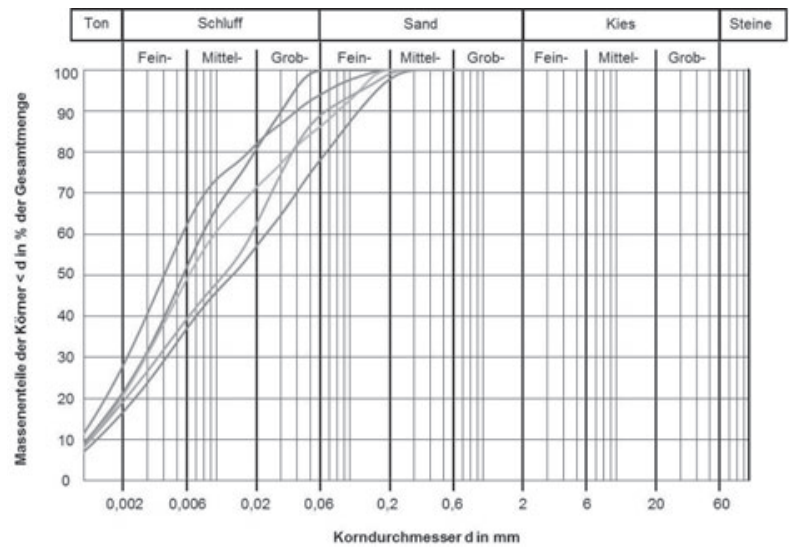

Abbildung 2. Summenkurven Keuper (5 Proben).

Die Einheiten des Mittleren Keupers werden in den Kartiergebieten wie folgt beschrieben: Der Gipskeuper besteht aus braunroten bis braunvioletten Tonsteinen, die lokal grüngrau gefärbt sind. Die nachfolgende Einheit, der Schilfsandstein, besteht aus feldspatreichen, glimmerführenden Sandsteinen, die schwach tonig bis tonig sind und eine hellolivbraune bis gelblich-olivbraune Farbe aufweisen. Die Sedimente der Roten Wand werden als schluffige, rotviolette Tonsteine beschrieben, in denen teilweise rotbraune Quarzitbänke mit Mächtigkeiten im Dezimeterbereich eingeschaltet sind. Bei der Einheit des Steinmergelkeupers bzw. dem Übergangsbereich zum Oberen Keuper handelt es sich um grüne und violette, lokal graue Siltsteine.

Der Obere Keuper (Rhät) beginnt mit fluviatildeltaischen Quarziten gefolgt von marinen Tonsteinen, welche den mittleren Teil des Rhätkeupers bilden und von den marin-deltaischen Feinsandsteinen des Oberen Rhätkeupers überlagert werden (Arp et al. 2004). Er weist Mächtigkeiten von $55 \mathrm{~m}$ auf (Nagel \& Wunderlich 1976).

In den Kartiergebieten treten gelbe bis hellgraue, kalkfreie, stark verwitterte, sandige Quarzitsandsteine auf sowie dunkle, kalkfreie Tonsteine mit rotbraunen Verwitterungsrändern in toniger bis schluffiger, hellbrauner Matrix auf. Des Weiteren wird ein sandiger, kiesiger, schwach toniger Schluff, der eine rote bis graubraune Färbung aufweist, beschrieben. Eine detaillierte Zuordnung dieser beschrieben Gesteine in die einzelnen Einheiten des oberen Keupers ist aufgrund der geringen Probenmengen nicht möglich.

Der Wassergehalt des mittleren Keupers be- 
trägt im Mittel 10,1\%, der des oberen Keupers $19,6 \%$. Beide Werte entsprechen dem natürlichen Wassergehalt von schwach bindigen Böden. Die schluffigen Ablagerungen des oberen Keupers ändern ihre Zustandsform in Abhängigkeit vom Wassergehalt. Die nur gering wasserdurchlässigen Tone des Mittleren Keupers sind quellfähig und bilden eine Basisfläche für Grundwasser. Das hohe Wasserhaltevermögen kann zur Eislinsenbildung führen und wirkt sich negativ auf die Frostsicherheit aus. Der Glühverlust von 7,5\% weist auf mittelorganische Böden hin.

Die Festgesteine bestehen überwiegend aus Schluff- bis Tonsteinen. Sie werden je nach Gefügefestigkeit gemäß DIN 18300 den Bodenklassen 6 oder 7 , leicht bis schwer lösbarer Fels, zugeordnet. Die Festigkeit von Fels ist abhängig vom Grad der Verwitterung und dem Trennflächengefüge. Ton- und Schluffsteine sind im Allgemeinen weich bis mittelhart und (ver)witterungsempfindlich (Reuter et al. 1992).

Die Gesteine des Mittleren Keupers sind gekennzeichnet durch Einschaltungen von Gipslagen und -knollen. Es besteht die Gefahr von Hohlraumbildungen durch die Auslaugung der Sulfatgesteine und daraus resultierende Erdfälle. Sind Hohlräume weitgehend durch ausführliche Erkundungen ausgeschlossen, stellen die festen Tonsteine und die Schilfsandsteine des Mittleren Keupers aufgrund ihrer Tragfähigkeit einen guten Baugrund dar.

Die stark schrumpf- und quellfähigen Schiefertone des Rhät können nachhaltige Bauwerksschäden hervorrufen. Wenn die Sedimentgesteine gegen Wasser und Frost geschützt werden, übertrifft ihre Tragfähigkeit die aller Lockergesteine beträchtlich (Reuter et al. 1992).

\subsubsection{Unterer Jura (Lias)}

Die Lias-Schichten sind in den kartierten Gebieten nur lokal erhalten geblieben. In Senken oder auf in die Tiefe versetzen Schollenbruchstücken wurden sie vor der Erosion geschützt.

Generell bildeten sich die Sedimente des Lias in einem vollmarinen Milieu (Arp et al. 2004). Im Göttinger Raum sind von der Abfolge des Unteren Jura noch mehr als 225 m Mächtigkeit erhalten (Meyer 1987).

In den Kartiergebieten treten blauschwarze oder dunkelgraue bis braune, zum Teil geschichtete Tonschiefer mit einen schwachen Kalkgehalt

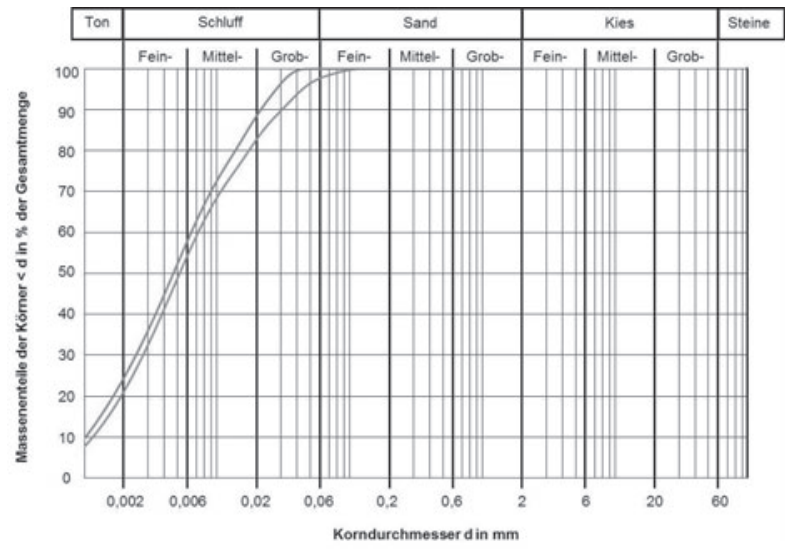

Abbildung 3. Summenkurven Jura (2 Proben).

sowie dunkelgraue oder gelbolive, kalkfreie Schluffe mit geringen Ton- und Feinsandanteilen auf.

Die Sedimente des Lias weisen im Mittel einen Glühverlust von 6,0\% auf. Die Korngrößenverteilung zweier Proben sind in Abbildung 3 dargestellt, demnach handelt es sich um eng gestufte, stark tonige Schluffe.

Den Beschreibungen nach kann eine Zuordnung gemäß DIN 18300 in die Bodenklassen 4, 6 oder 7 erfolgen. Die stark tonigen Schluffe des Unteren Juras sind sehr schrumpf- und quellfähig und können nachhaltige Bauwerksschäden (z. B. Rissbildungen) hervorrufen. Wenn die Sedimentgesteine gegen Wasser und Frost geschützt werden, übertrifft ihre Tragfähigkeit die aller Lockergesteine beträchtlich (Reuter et al. 1992).

\subsection{Quartär}

\subsubsection{Kiese}

Die fluviatilen Ablagerungen werden in den Kartiergebieten in die Einheiten Leineschotter und Lutterschwemmschutt unterteilt:

4.2.1.1 Leineschotter: Die Leineschotter erstrecken sich vom Auebereich der Leine bis in das Zentrum des Gebietes, wo sie sich mit den Schottern des Lutterschwemmfächers verzahnen. Sie werden von Auelehm oder Schwemmsand bedeckt.

Bei den Leineschottern handelt es sich, den Körnungslinien nach, um weit gestufte, schwach sandige bis sandige Fein- bis Mittelkiese, die größtenteils schwach schluffig sind. Der Anteil an Grobkiesen beträgt meistens unter 20\%, welches aber auch darauf zurückzuführen sein kann, dass das Material durch den Bohrvorgang 

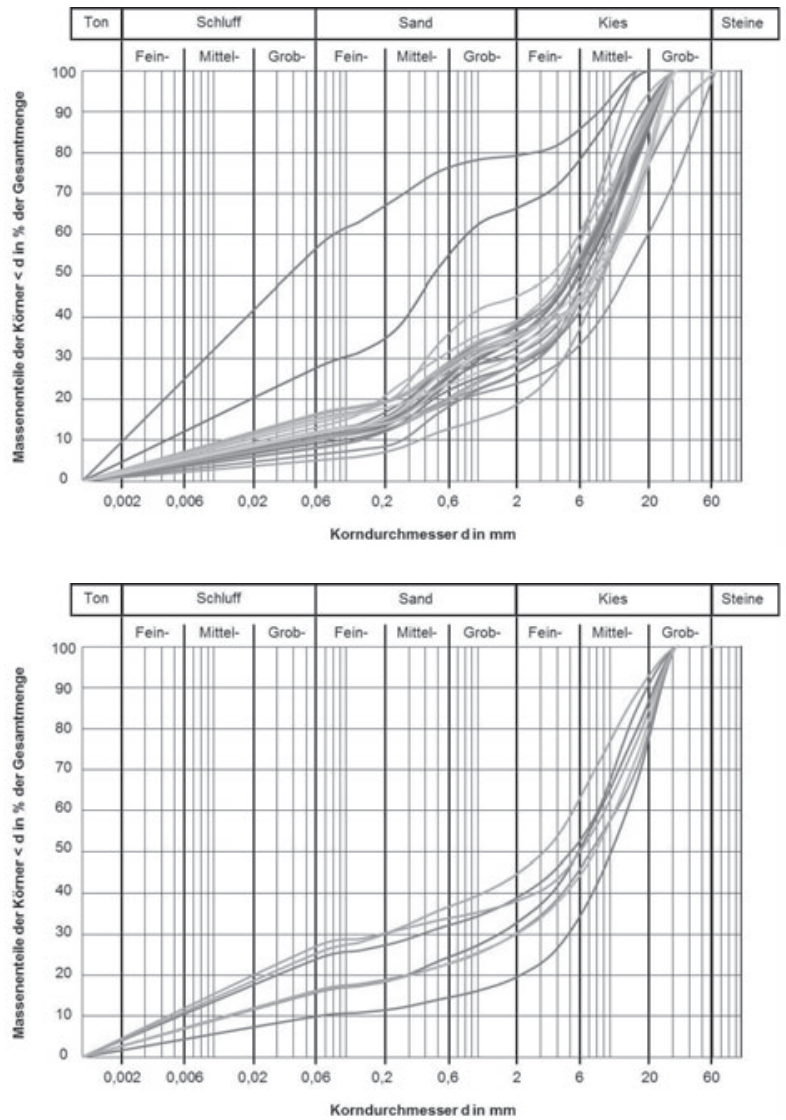

Abbildung 4. Oben a: Summenkurven Leineschotter, untern b: Summenkurven Lutterschwemmschutt.

zerstört wurde. Vermutlich besaßen die Proben ursprünglich einen größeren Gehalt an Grobkiesen als in den Körnungslinien zu sehen ist. Die Kiese sind im Allgemeinen locker bis sehr dicht gelagert, stark kalkhaltig, kantengerundet bis gut gerundet und weisen hauptsächlich hellgraue bis graue Komponenten auf. Untergeordnet sind auch rötliche, grünliche und gelbe Kiese vorhanden. Die Schotter führen Keuper-, Muschelkalk- und Buntsandsteingerölle aus der näheren Umgebung.

4.2.1.2 Lutterschwemmschutt: Die Schotter des Lutterschwemmfächers lagerten sich vor der Einmündung des Luttertals in das Tal der Leine ab. Die Ursache dafür war die verringerte Transportkraft des Flusses durch die starke Gefälleänderung beim Eintritt in die Talsohle. Die Schotterkörper der Lutter bestehen aus Muschelkalkgeröllen, die von den östlichen Grabenschulter des Leinetalgrabens abgetragen wurden und lassen sich daher von den bunt- sandsteinführenden Leineschottern unterscheiden. Der Lutterschwemmfächer wird vorwiegend von Schwemmlöss, aber auch von Auelehm bedeckt.

Die Kartiereinheit besteht, wie aus den Körnungslinien in Abbildung 4b hervorgeht, aus schwach sandigen bis sandigen, stark feinkiesigen, schwach grobkiesigen bis grobkiesigen Mittelkiesen, die oft schluffig bis stark schluffig sind. Wie bei den Leineschottern ist ebenso bei dem Lutterschwemmschutt ein ursprünglich höherer Anteil an Grobkiesen und Steinen anzunehmen. Die Kiese sind als weit gestuft zu klassifizieren.

Es treten eckige bis gut gerundete, graue bis hellgraue und gelbe Kiese auf, die im Allgemeinen mäßig locker bis dicht gelagert und stark bis sehr stark kalkhaltig sind.

Die allgemeine Senkung des Untergrundes im Zentrum des Gebietes durch die anhaltende Subrosion schuf eine Art Sedimentbecken, das vor allem mit den Flussschottern der Lutter verfüllt wurde. Diese erreichen Mächtigkeiten von bis zu $20 \mathrm{~m}$.

Die Kiese der Kartiereinheiten Leineschotter und Lutterschwemmfächer werden für die Beurteilung als Baugrund zusammengefasst, weil sie sich in ihren bodenmechanischen Eigenschaften nur wenig voneinander unterscheiden.

Bedingt durch ihren geringen Feinkornanteil besitzen die Flusskiese eine hohe Durchlässigkeit. Der Wasseranteil liegt im Mittel bei 9-12\%. Nach Nendza (1992) werden sie den nichtbindigen und schwachbindigen Böden zugeteilt. Zusätzlich besitzen sie durch die hohe Durchlässigkeit einen geringen Wassergehalt, weshalb sie unempfindlich gegenüber Frost sind.

Nach DIN 18196 handelt es sich um die Bodengruppen GU und $\mathrm{GU}^{\star}$, die gemäß DIN 18300 den Bodenklassen 3 und 4 zugeordnet werden können und nach ZTVE-StB 94 gering bis mittel frostempfindlich (F2) oder frostempfindlich (F3) sind.

Für die Schotter sind eine große Scherfestigkeit, eine mittlere bis gute Verdichtungsfähigkeit, eine sehr geringe bis vernachlässigbar kleine Zusammendrückbarkeit, eine mittlere Durchlässigkeit und ein mittleres Wasserbindevermögen zu erwarten (nach Hinweisen auf bautechnische Eigenschaften aus der DIN 18 196).

Die Setzungsempfindlichkeit der Schotter ist als sehr gering und die Grundbruchsicherheit 
als hoch einzustufen. Geringe Setzungsprozesse sind nach erster Auflast abgeschlossen. Somit gibt es keinen Langzeiteinfluss auf aufliegende Gebäude. Ebenso hat der hohe Kalkgehalt einen günstigen Einfluss auf die bautechnischen Eigenschaften dieses Lockergesteins (Prinz \& Strauß 2006). Kalkbindung bzw. -verkittung führen zu einer Erhöhung der Standfestigkeit. Die Schotter besitzen eine gute Tragfähigkeit bei dynamischer Belastung und sind für Gründungszwecke sehr gut geeignet (Reuter et al. 1992).

\subsubsection{Fließerde}

Fließerden entstehen durch Solifluktion. Ein Prozess, bei dem durch Frostsprengung zerkleinertes Material hangabwärts fließt. Der Verwitterungsschutt der Muschelkalkhänge und der auf den Hängen abgelagerte Löss haben sich während des Pleistozäns aufgrund von Bodenfließen vermengt und überwiegend an den Hangflanken und vor allem am Hangfuß abgelagert.

Fließerden sind im Gebiet weit verbreitet. Sie sind teils von Löss überdeckt und können mit den fluviatilen Kiesen verzahnt sein. Die Mächtigkeit der Fließerden im Kartiergebiet variiert von wenigen Dezimetern bis $4 \mathrm{~m}$.

Die Zusammensetzung der Fließerden ist sehr heterogen und variiert ebenso in der Korngröße. Zur Einheit der Fließerde wurden demnach alle Bodenarten mit dem Hauptbestandteil Schluff bis Sand und geringem bis starkem Kiesanteil als Nebenbestandteil gezählt.

Abbildung 5 stellt einen Auszug aus den Kornsummenkurven der Fließerden dar, demnach lassen sie sich in eng bis mittel gestufte, tonige bis sehr stark tonige, schwach feinsandige Schluffe und weit gestufte, sandige Schluff-Kies-Gemische unterteilen. Erstere sind im Wesentlichen kalkfrei, steif bis halbfest und besitzen vorwiegend Farben im Bereich zwischen gelb und braun. Die Böden können ebenso graue bis dunkelgraue Schlieren aufweisen oder gänzlich grau sein. Des Weiteren können die Fließerden schwach steinig bis steinig sein. In diesem Fall sind die Steine kantig und verwittert und liegen im Korngrößenbereich zwischen Fein- und Mittelkies.

Der Wassergehalt von 24,6\% liegt im Bereich des natürlichen Wassergehalts von stark bindigen Böden. Sie besitzen lagenweise einen hohen Anteil an quellfähigen Tonen, die je nach Wassergehalt ihr Volumen verändern. Der Glühverlust von 5,6\% steht für schwach organische Böden.

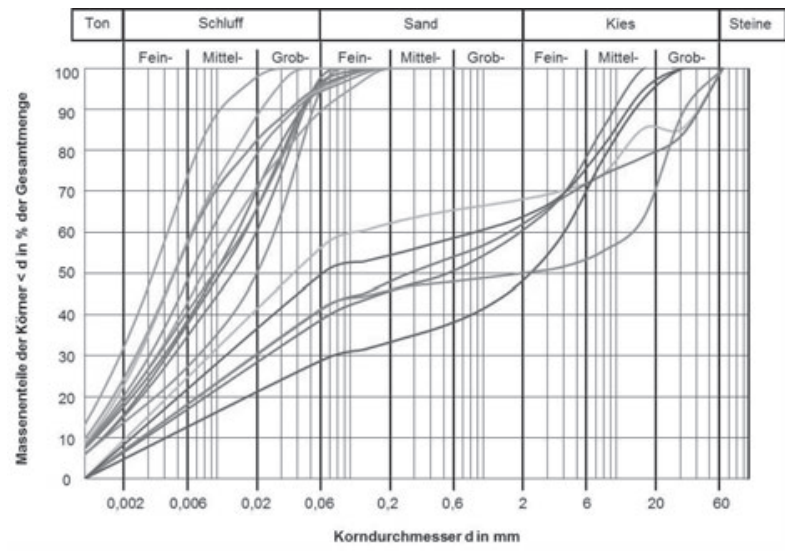

Abbildung 5. Summenkurven Fließerde (16 Proben).

Die Fließerden verhalten sich bindig und gehören gemäß DIN 18196 Bodengruppen TL sowie GU*an. Nach DIN 18300 gelten diese Gruppen als mittelschwer lösbare Bodenarten der Bodenklasse 4 und sind nach ZTVE-StB 94 als sehr frostempfindlich (F3) einzustufen.

Eine gute Durchlässigkeit ist durch den teilweise sehr hohen Feinkornanteil nur bedingt gegeben. Es kann aufgrund der Kapillarität zu Eislinsenbildung unter Frosteinwirkung führen. Ebenso können Hebungen und Frostaufbrüche des Bodens entstehen.

Durch die Heterogenität der Erscheinungsform können die bautechnischen Eigenschaften der Fließerden sehr stark variieren. Es kommen vor: mäßige bis große Scherfestigkeit, mäßige bis gute Verdichtungsfähigkeit, sehr geringe bis mittlere Zusammendrückbarkeit, sehr geringe Durchlässigkeit sowie mittlere bis große Verwitterungs- und Erosionsempfindlichkeit (nach Hinweisen auf bautechnische Eigenschaften aus der DIN 18 196).

Die Einheit ist aufgrund des Kiesanteils mitteldicht gelagert und wird als mittelguter Baugrund bewertet. Der Gehalt an Steinen hat einen positiven Einfluss auf die Eignung des Bodens für Gründungszwecke. Es ist jedoch zu beachten, dass lokal die Anteile an Schluff deutlich überwiegen können, was zu einer Verschlechterung des Baugrundes führen kann. Aufgrund ihrer Eigenschaften ist bei dieser Einheit eine lokale Untergrunduntersuchung notwendig, wenn der Bedarf einer Gründung besteht. 


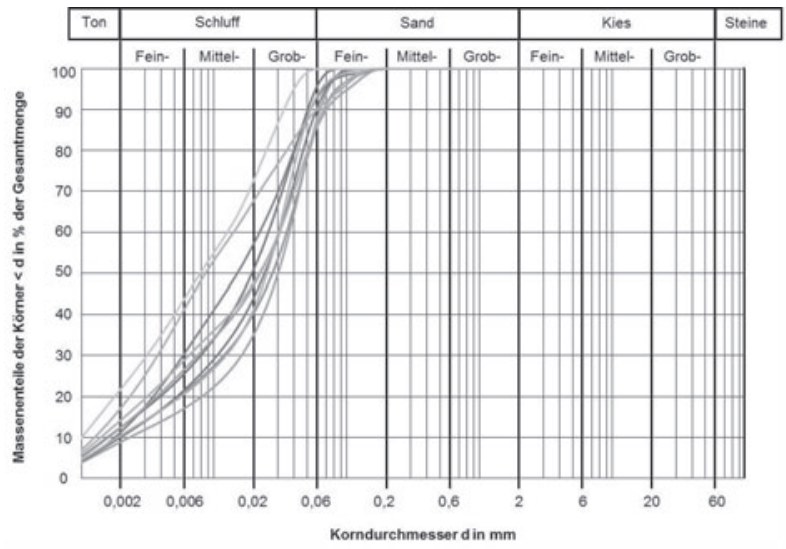

Abbildung 6. Summenkurven Löss, Lösslehm, Schwemmlöss (10 Proben).

\subsubsection{Löss, Lösslehm, Schwemmlöss}

In dieser Kartiereinheit werden Löss, Lösslehm und Schwemmlöss zusammengefasst, weil ihre Korngrößenzusammensetzungen und ihre bodenmechanischen Eigenschaften ähnlich sind. Löss ist ein äolisches Sediment und wurde im trockenen, kontinentalen Klima der Eiszeiten im Pleistozän in Gebieten mit Steppenvegetation, in Niederungen sowie an windgeschützten Hängen abgelagert (Meyer 1987). Als Lösslehm wird der durch versickertes Niederschlagswasser heute weitgehend entkalkte Löss bezeichnet. Schwemmlöss entsteht durch örtliche Hangabspülung im Holozän und ist teilweise schwierig von Auelehm zu unterscheiden.

Löss, Lösslehm und Schwemmlöss sind im gesamten Gebiet weit verbreitet und bedecken die Fließerden, die Flussschotter und die Muschelkalkhänge mit einer durchschnittlichen Mächtigkeit von $2 \mathrm{~m}$.

Bei der Mehrzahl der Böden dieser Einheit handelt es sich um schwach tonige bis tonige, schwach feinsandige Schluffe, die eng bis mittel gestuft sind. Typische Korngrößenverteilungen sind in Abbildung 6 dargestellt. Sie sind teilweise sehr schwach feinkiesig bis feinkiesig und können dunkle Schlieren und Rostflecken aufweisen. Dies lässt auf eine Umlagerung der Sedimente schlieBen, was ein Indiz für Schwemmlöss ist.

In der Regel weisen die Schichten dieser Kartiereinheit Farben von braun über gelbbraun bis hellbraungelb (ocker) auf. Außerdem werden sie mit zunehmender Tiefe oft grau oder besitzen lokal graue Lagen, die Reduktionshorizonte darstellen. Die Sedimente besitzen sehr unterschiedliche Konsistenzen. Die Zustandsform im Gelände ist vorwiegend weich und steif, aber auch halbfest.

Der Glühverlust beträgt durchschnittlich 3\%. Somit sind die Böden dieser Einheit schwach organisch. Untergeordnet kommen auch mittelorganische Horizonte vor. Der mittlere Wassergehalt von $22,2 \%$ lässt auf überwiegend stark bindige Böden schließen.

Die Böden der Kartiereinheit werden gemäß DIN 18196 in die Bodengruppen UL und TL eingeteilt. Diese bindigen Bodenarten gehören nach DIN 18300 bei flüssiger bis breiiger Konsistenz zur Bodenklasse 2, bei weicher bis halbfester Konsistenz zur Bodenklasse 4. Sie sind nach ZTVEStB 94 sehr frostempfindlich (F3).

Die Durchlässigkeit ist als gering einzustufen. Außerdem sind eine mäßige Scherfestigkeit, mäßige Verdichtungsfähigkeit sowie mittlere Zusammendrückbarkeit zu erwarten. Die Böden zeigen eine sehr große Verwitterungs- und Erosionsempfindlichkeit (nach Hinweisen auf bautechnische Eigenschaften aus der DIN 18 196).

Es handelt sich um veränderlich feste Bodenarten, die bei starker Durchfeuchtung zum Fließen neigen. Die Tragfähigkeit ist abhängig vom Grad der Bindigkeit und dem Wassergehalt. Mit zunehmendem Wassergehalt nimmt die Haftfestigkeit von bindigen Lockergesteinen ab und die Rutschungs- und Setzungsempfindlichkeit steigt an. Deshalb sollte gesichert sein, dass es zu keinem Grundwasseranstieg kommt, der die Standfestigkeit der Bauwerke gefährdet (nach Nagel \& Wunderlich 1976; Henningsen 1992; Reuter et al. 1992; Prinz \& Strauß 2006).

\subsubsection{Schwemmsand}

Schwemmsand ist ein holozänes, fluviatiles Sediment, welches sich über den Kiesen ablagelagert hat. Der Sand ist ein Anzeiger für die stark verringerte Transportkraft der Flüsse. Er ist maximal $3 \mathrm{~m}$ mächtig und wird größtenteils von Auelehm bedeckt und ist wie der Auelehm im Bereich der Leineaue vorzufinden.

Diese Kartiereinheit besteht aus feinkörnigen und gemischtkörnigen, locker gelagerten Sedimenten, die je nach Korngrößenzusammensetzung eng bis weit gestuft sind (Abb. 7). Es handelt sich um schwach schluffige bis stark schluffige Mittelsande, welche in der Regel geringe Anteile an Fein- und Grobsand sowie Fein- und Mittelkies enthalten. Des Weiteren treten schwach schluffige bis schluffige Sand-Kies-Gemische auf oder Böden mit unterschiedlich starken Anteilen an Schluff 


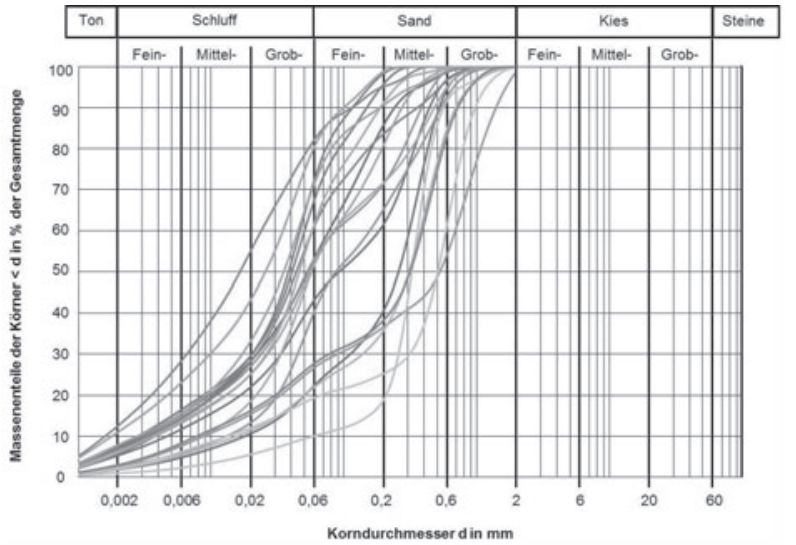

Abbildung 7. Summenkurven Schwemmsand (19 Proben).

und Sand. Untergeordnet können organische und organogene Beimengungen enthalten sein. Das Farbspektrum der Schwemmsande erstreckt sich von grau, gelb, braun und seltener rot sowie Mischfarbtöne dieser Farben. Die Böden der Kartiereinheit sind kalkhaltig bis sehr stark kalkhaltig und weich bzw. locker gelagert. Zum Teil sind helle Schalenbruchstücke und organische Beimengungen, vor allem dunkle Holzreste, enthalten.

Die Böden sind mit einem Glühverlust von 2,3\% schwach organisch. Die Wassergehalte liegen zwischen 12,1 und 57,4\%, was auf schwach bis stark bindige Böden hindeutet. Nach DIN 18196 handelt es sich um die Bodenarten SU-SU* sowie GU-GU*. Sie können gemäß DIN 18300 den Bodenklassen 2, 3 oder 4 zugeordnet werden. Die unterschiedlichen Bodengruppen sind nach ZTVE-StB 94 gering bis mittel (F2) oder sehr frostempfindlich (F3).

Aufgrund des teilweise hohen Schluffgehaltes können die bodenmechanischen Eigenschaften des Schwemmsandes lokal schwanken. Es sind $\mathrm{zu}$ erwarten: große bis sehr große Scherfestigkeit, mittlere bis gute Verdichtungsfähigkeit, mittlere bis vernachlässigbar kleine Zusammendrückbarkeit sowie sehr geringe bis mittlere Durchlässigkeit (nach Hinweisen auf bautechnische Eigenschaften aus der DIN 18 196).

In Bereichen mit hohem Schluffanteil oder organischen und organogenen Beimengungen sind größere Setzungsempfindlichkeiten zu erwarten, die zum Beispiel über ein Streifenfundament ausgeglichen werden können. Von Vorteil wäre auch ein Teilbodenaustausch, die Einbringung von Schotterpfählen (Stopfverdichtung) oder, wenn möglich, das Einrütteln von Steinen (Steinskelettgründung). Außerdem kann eine Verdichtung der Böden in Betracht gezogen werden, wenn gesichert ist, dass keine oder nur wenige und geringmächtige organische und organogene Lagen vorhanden sind.

\subsubsection{Wiesenkalk}

Der Wiesenkalk bildete sich im Holozän an kalkreichen Quellen an den Muschelkalkhängen. Im Arbeitsgebiet ist dies vor allem im Bereich des Weendesprings der Fall. Durch den abnehmenden $\mathrm{CO}_{2}$-Partialdruck fällt an der Quelle Kalk aus, der Organismen und Pflanzenreste umlagert. Die darauf folgende Vermoderung und Lösung der organischen Anteile führt zur Entstehung der hohen Porosität des Gesteins (Meyer 1987). Die Wiesenkalke wurden teilweise umgelagert und mit Bachlehm vermischt abgelagert.

Der Wiesenkalk besteht aus weichem hochporösem Mergel mit beigem bis hellgrauem Farbton. Das Material setzt sich aus weißen bis grauen Kalkkonkretionen zusammen, oft umgeben von einer schluffigen Matrix. Die Konkretionen liegen in Größen von Sand bis Feinkies vor. Charakteristisch ist der sehr hohe Ca-Gehalt.

Die Ablagerungen beschränken sich auf den zentralen Bereich östlich der Bundesautobahn 7 . Der Wiesenkalk steht in geringen Tiefen von 1$4 \mathrm{~m}$ unter Geländeoberkante an und ist in der Regel von Auelehmen überdeckt.

Nach Nendza (1992) wird Wiesenkalk mit einem Wassergehalt von 33\% in die stark bindigen Böden eingeteilt. Der Organikanteil von 1\% ist vernachlässigbar klein. Korngrößen können nicht ermittelt werden, da das Material aus weichen Kalkkonkretionen zusammengesetzt ist. Aufgrund des hohen Wassergehaltes ist Wiesenkalk als Baugrund stark frostgefährdet. Hebungen und Frostaufbrüche des Bodens durch Eislinsenbildung im Winter wirken sich negativ auf die Standsicherheit des Bodens aus. Aufgrund der lockeren Lagerung ist die Einheit des Wiesenkalkes stark setzungsempfindlich. Das Material ist infolge seines hohen Ca-Gehaltes leicht wasserlöslich. Die Einheit Wiesenkalk gehört zu den bindigen/organischen Lockergesteinen und den organischen und organogenen Bodenarten.

Nach DIN 18196 entspricht diese Einheit der Bodengruppe OK. In die DIN 18300 wird der Wiesenkalk der Bodenklasse 2 zugeordnet und 


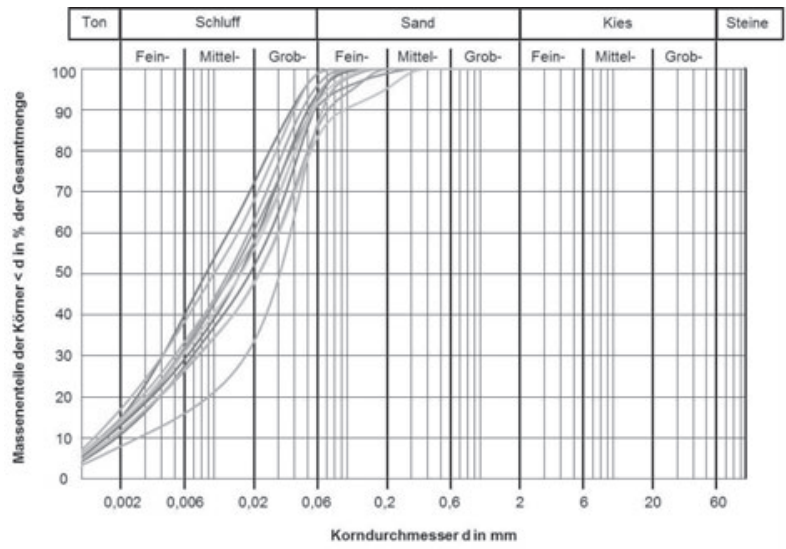

Abbildung 8. Summenkurven Auelehm (11 Proben).

ist gemäß ZTVE-StB 94 als sehr frostempfindlich (F3) einzustufen.

Im Allgemeinen ist die Tragfähigkeit dieser Einheit sehr schlecht. Infolge der starken Rutschungsund Fließgefährdung dieses Untergrundes ist eine Gründung bis zu den tragfähigen Flussschottern im Untergrund empfehlenswert.

\subsubsection{Auelehm}

Bei Auelehm handelt es sich um umgelagerten Oberboden, Löss bzw. Lösslehm und Schwemmlöss, der im Überschwemmungsbereich der Leine sedimentierte. Die Entstehung ist überwiegend anthropogen bedingt. Durch die Besiedlung und Landnutzung entstanden ungeschützte Flächen, die leicht erodiert und abgetragen werden konnten. Der Auelehm ist im Bereich der Leineniederung weit verbreitet und 2 bis maximal $4 \mathrm{~m}$ mächtig.

Ein feinkörniges, vorwiegend weiches, aber auch steif bis halbfestes, mittel gestuftes Lockermaterial aus Schluff mit unterschiedlichen Anteilen an Ton und Sand dargestellt. Bei den Bodenproben kommen die Farben braun, gelb und grau sowie Übergänge zwischen diesen vor. Der Auelehm ist kalkfrei bis sehr stark kalkhaltig und enthält untergeordnet organische Beimengungen (Holzreste, Kohle) und helle Schalenbruchstücke. In das Sediment sind Lagen von Feinsand und Ton sowie organische und organogene Böden (OU, OT, OH, HN-HZ) eingeschaltet. Weit gestufte Sand-KiesLagen sind seltener enthalten.

Mit einem mittleren Glühverlust bei 4,1\% ist der Boden schwach organisch. Der mittlere Wassergehalt von $24,4 \%$ weist auf einen stark bindigen
Boden hin, zumal der höchste Wert bei 48,3\% liegt. Deshalb ist ein gutes Wasserbindevermögen zu erwarten.

Der Auelehm kann gemäß DIN 18196 den Bodengruppen OU, UL, TL und TM zugeordnet werden. Die Bodengruppen entsprechen nach DIN 18300 den Bodenklassen 2 bis 4 und sind gemäß ZTVE-StB 94 als sehr frostempfindlich (F3) einzustufen.

Für Auelehm ist eine geringe Durchlässigkeit anzunehmen. Das feinkörnige Lockersediment weist eine mäßige bis schlechte Verdichtungsfähigkeit sowie eine mittlere bis große $\mathrm{Zu}$ sammendrückbarkeit auf. Dies lässt auf eine hohe Setzungsempfindlichkeit schließen. Die Scherfestigkeit ist als gering einzustufen (nach Hinweisen auf bautechnische Eigenschaften aus der DIN 18 196).

Die zwischengeschalteten organischen und organogenen Böden haben einen mittleren Wassergehalt von $64,2 \%$. Der Höchste liegt bei knapp über $90 \%$. Bei organischen Böden kann der Wassergehalt sogar weit über $100 \%$ betragen. Der Glühverlust beträgt im Mittel 12,8\% (mit einem Maximum bei 24,7\%). Die Böden sind somit mittel bis stark organisch. Die Tragfähigkeit der organischen und organogenen Horizonte ist meist so gering, dass eine Bebauung ohne Sicherheitsmaßnahmen nicht möglich ist (Reuter et al. 1992). Dadurch werden die bautechnischen Eigenschaften des Auelehms verschlechtert.

Aufgrund der mäßigen bis schlechten Verdichtbarkeit, kann von einer Verdichtung des Baugrundes abgesehen werden. Es ist denkbar, einen Bodenaustausch durch tragfähiges Material, wie zum Beispiel ein Schottergemisch, durchzuführen. Auelehm kann als Dichtungsbaustoff für z. B. Erd- und Staudämme verwendet werden (nach DIN 18 196).

\subsubsection{Künstliche Auffüllungen}

Die Auffüllungen gehören zu den aufgeschütteten Bodenarten und bestehen aus natürlichen Böden (Ton, Schluff, Sand, Kies und Steine) und/oder Fremdstoffen (Ziegelreste, Schlacke, Straßenschotter). Neben Auffüllungen im Bereich von Straßenbauten und vereinzelte Auffüllungen zur Geländeanpassung in den Wohngebieten treten vor allem im Bereich der Gleisanlagen im Zentrum des Gebietes größere Mengen an Auffüllungen auf. Je nach Lagerungsdichte sowie Art und Zusammensetzung der unterschiedlichen Bestandteile ist eine 
Zuordnung in Bodenklassen 3 bis 6 möglich.

\section{Schlussfolgerung und Ingenieurgeo- logische Karte}

Für die Auswertung in Bezug auf baugrundtechnische Eigenschaften wurde eine ingenieurgeologische Karte erstellt. Abbildung 9 zeigt eine Übersicht der räumlichen Verteilung der Baugrundtypen in $2 \mathrm{~m}$ Tiefe. Diese dient der Bewertung des Untergrundes für Bauzwecke jeglichen Ausmaßes. Die Schichten von 0-2 m spielen nur eine untergeordnete Rolle. Zudem ist hier die Frostsicherheit vernachlässbar, da die Frosttiefe bei $0,8 \mathrm{~m}$ liegt und während der Gründung berücksichtigt werden muss.

Der Osten des Arbeitsgebietes ist durch $\mathrm{Mu}-$ schelkalk geprägt. Dieser setzt sich aus Mittlerem und Oberem Muschelkalk zusammen und wird als eine Einheit zusammengefasst. Aufgrund der guten Tragfähigkeit stellt diese Einheit einen guten Baugrund dar. Mögliche Auslaugungsprozesse der Karbonat- und Sulfatgesteine sowie der aufwendigere Aushub sind jedoch zu berücksichtigen.

Die Einheit Löss, Lösslehm und Schwemmlöss ist flächenhaft im Osten sowie im Nordwesten des Gebietes vertreten. Die Tragfähigkeit ist bei dieser Einheit stark abhängig vom Wassergehalt und damit nur bedingt gegeben. Demzufolge stellt die Einheit einen eher ungünstigen Baugrund dar. Eine Gründung bis zu einer tragfähigeren Schicht und eine besondere Sicherung der Baugrube ist empfehlenswert.

Fließerden bedecken die Hänge des Muschelkalks im Osten sowie die Hänge des Keuperrückens im Westen und verzahnen sich mit der Einheit Löss. Aufgrund des Kiesanteils besitzt diese Einheit eine gute Tragfähigkeit und stellt einen mittelguten Baugrund dar. Es ist jedoch $\mathrm{zu}$ beachten, dass lokal die Anteile an Schluff (Schwemmlöss) deutlich überwiegen können, was zu einer Verschlechterung des Baugrundes führen kann.

Wiesenkalk findet geringe Verbreitung im zentralen Bereich des Gebietes. Die schlechten Baugrundeigenschaften basieren auf der hohen Empfindlichkeit gegenüber Rutschungs- und Fließprozessen zudem ist ein Auslaugungsprozess nicht auszuschließen. Eine Gründung zu den unterlagernden tragfähigeren Flussschottern ist empfehlenswert.

Die Einheit der Kiese setzt sich aus dem Lutterschwemmschutt und den Leineschottern zu- sammen. Der Lutterschwemmschutt erstreckt sich vom Südosten bis in den zentralen Bereich des Gebietes. Die Leineschotter befinden sich im südlichen Bereich der Leineaue. Die Kiese weisen eine gute Tragfähigkeit und eine geringe Setzungsempfindlichkeit auf und sind für Gründungszwecke gut geeignet.

Ebenfalls im Bereich der Leineaue ist die Einheit Schwemmsand abgelagert. Diese verfügt über gute Baugrundeigenschaften. In Bereichen mit hohem Schluffanteil oder organischen/organogenen Beimengungen sind jedoch größere Setzungsempfindlichkeiten zu erwarten.

Der Auelehm ist im Bereich der Leineniederungen weit verbreitet. Aufgrund der hohen Setzungsempfindlichkeit weist er eine geringe Tragfähigkeit auf. Ein Bodenaustausch oder eine Gründung bis auf die unterlagernden Schotter ist von Nöten.

Keupervorkommen finden sich flächenhaft im Südwesten sowie lokal im östlichen Gebiet. Diese Einheit setzt sich aus Mittlerem und Oberem Keuper zusammen. Die festen Tone und der Schilfsandstein eignen sich gut als Baugrund. Hohlräume aufgrund von Sulfatvorkommen im Untergrund sind durch weitere Erkundungen auszuschließen. Die Schiefertone des Rhät neigen zu Rutschungen und sind quellfähig. Sie stellen daher einen schlechten Baugrund dar.

Im Allgemeinen liefert die Karte eine gute Übersicht der Baugrundeinheiten. Weitere Erkundungen können anhand von Profilschnitten durchgeführt werden. Ebenso sind die ersten Meter unter GOK auf kritischen Schichten, wie z.B. Torf zu prüfen. Dieser wurde innerhalb der Arbeiten nur lokal und geringmächtig angetroffen, sollte bei einer möglichen Gründung dennoch nicht vernachlässigt werden. Insgesamt stellen die Kiese und Festgesteine in dem Gebiet den besten Baugrund dar. 

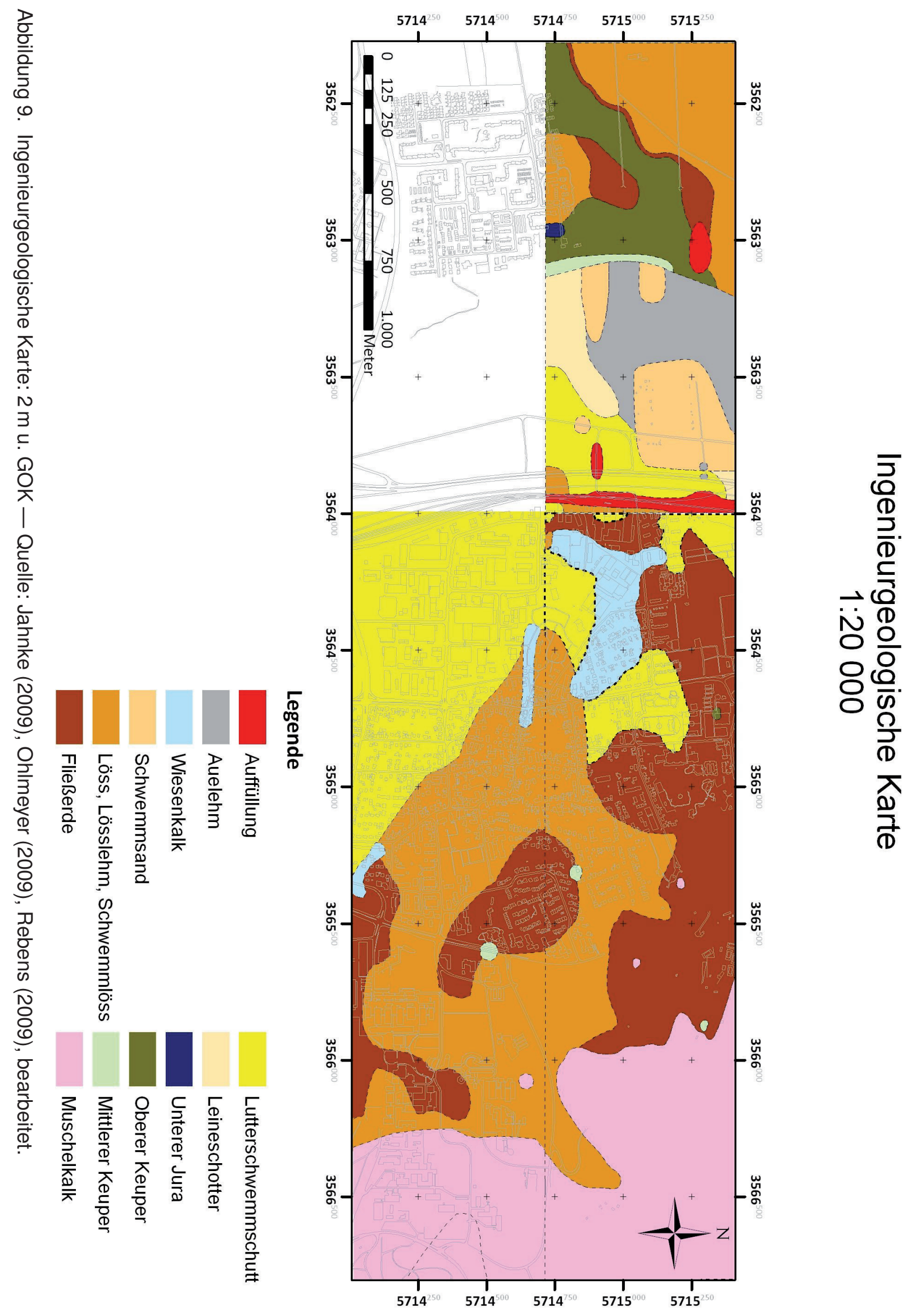


\section{Danksagung}

Bedanken möchten wir uns besonders bei Herrn Prof. Dr. T. Ptak-Fix für die hervorragende und hilfsbereite Betreuung. Bei Herrn Dr. V. Karius bedanken wir uns für die Einführung in die Bohrung mit dem Wackerhammer und die Messungen am LPS. Des Weiteren gilt unser Dank den Mitarbeitern der Werkstatt des GZGs für die schnellen Reparaturen und Einsätze im Gelände. Den Eigentümern und Pächtern der Flurstücke gilt ein besonderer Dank für die Genehmigung der Bohrarbeiten. Wir bedanken uns ebenfalls bei der Stadt Göttingen für die Auskunft über die Besitzer der Liegenschaften. Ein weiteres Dankeschön an die Ingenieurbüros und Ämtern, für die Informationen und die Bereitstellung von Daten. Ein großes Dankeschön gilt zum Schluss den Bohrhelfer und Mitkartierern für die großartige Hilfe und die anregenden Diskussionen.

\section{Literatur}

Arp, G., Hoffmann, V.-E., Seppelt, S., Riegel, W. 2004. Trias und Jura von Göttingen und Umgebung. - 74. Jahrestagung der Paläontologischen Gesellschaft, 02.-08. Oktober 2004, Exkursion 6, 147-192, Göttingen (Universitätsdruck).

Brunotte, E. 1985. Wandern in Göttingen, C. Geomorphologische Erläuterungen (Oberflächenformen). 57-69, Göttingen (Göttinger Tageblatt).

DIN 18 121-1 1998-04. Baugrund, Untersuchung von Bodenproben, Wassergehalt, Teil 1: Bestimmung durch Ofentrocknung. Normenausschuss Bauwesen im DIN Deutsches Institut für Normung e. V.

DIN 18 122-1 1997-07. Baugrund, Untersuchung von Bodenproben, Zustandsgrenzen (Konsistenzgrenzen), Teil 1: Bestimmung der Fließund Ausrollgrenze. Normenausschuss Bauwesen im DIN Deutsches Institut für Normung e. V.

DIN 18124 1997-07. Baugrund, Untersuchung von Bodenproben, Bestimmung der Korndichte, Kapillarpyknometer - Weithalspyknometer. Normenausschuss Bauwesen im DIN Deutsches Institut für Normung e. V.

DIN 18128 2002-12. Baugrund, Untersuchung von Bodenproben, Bestimmung des Glühverlustes. Normenausschuss Bauwesen im DIN Deutsches Institut für Normung e. V.
DIN 18196 1988-10. Erd- und Grundbau, Bodenklassifikation für Bautechnische Zwecke. Normenausschuss Bauwesen im DIN Deutsches Institut für Normung e. V.

DIN 18300 2006-10. VOB Vergabe- und Vertragsordnung für Bauleistungen - Teil C: Allgemeine Technische Vertragsbedingungen für Bauleistungen (ATV) - Erdarbeiten. Normenausschuss Bauwesen im DIN Deutsches Institut für Normung e. V.

Henningsen, D. 1992. Geologie für Bauingenieure, Eine Einführung. 131pp, Springer- Verlag $\mathrm{GmbH}$.

Jahnke, W. 2009. Geotechnische Kartierung in Göttingen - Weende-Nord. Unveröffentlichte Diplomkartierung, Georg-August-Universität Göttingen, 41pp.

Meyer, R.H. 1987. Streifzüge durch die Erd- und Landschaftsgeschichte des Flecken Bovenden. 100pp, Göttingen (Verlag Erich Goltze GMBH u. CO. KG).

Nagel, U., Schunke, E. 1979. Geomorphologische Erläuterungen zum Orohydrographischen Blockbild der Umgebung von Göttingen. Neues Archiv für Niedersachsen 28, 200-222.

Nagel, U., Wunderlich, H.-G. 1976. Geologisches Blockbild der Umgebung von Göttingen. 50pp, Göttingen (Göttinger Tageblatt).

Nendza, H. 1992. Bodenmechanisches Praktikum, Einführung und Leitfaden. 168pp, Essen (Verlag Glückauf $\mathrm{GmbH}$ ).

Ohlmeyer, A. 2009. Geotechnische Kartierung im Leinetal bei Göttingen/Holtensen. Unveröffentlichte Diplomkartierung, GeorgAugust-Universität Göttingen, 62pp.

Prinz, H., Strauß, R. 2006. Abriss der Ingenieurgeologie. 671pp, München (Elsevier $\mathrm{GmbH}$ ).

Rebens, M. 2009. Geotechnische Kartierung in Göttingen - Weende-Süd. Unveröffentlichte Diplomkartierung, Georg-August-Universität Göttingen, 48pp.

Reuter, F., Klengel, J., Pašek, J. 1992. Ingenieurgeologie. 603pp, Leipzig, Stuttgart (Deutscher Verlag für Grundstoffindustrie $\mathrm{GmbH}$ ).

Ritzkowski, S. 1985. Wandern in Göttingen, B. Erläuterungen zur Geologie, 48-56, Göttingen (Göttinger Tageblatt).

ZTVE-StB 94/Fassung 97. Zusätzliche Technische Vertragsbedingungen und Richtlinien für Erdarbeiten im Straßenbau, Klassifikation der Frostempfindlichkeit von Bodengruppen. 



\title{
Tiefengeothermisches Potential in der Region Göttingen - geologische Rahmenbedingungen
}

\author{
Bernd Leiss (GZG Göttingen), David Tanner (GZG Göttingen, LIAG Hannover), \\ Axel Vollbrecht (GZG Göttingen) und Klaus Wemmer (GZG Göttingen)
}

\begin{abstract}
Zusammenfassung - Um die ehrgeizigen energiepolitischen Ziele der Region zu erreichen, wird die geothermische Energie künftig wesentlich stärker als wichtiger Bestandteil des regenerativen Energiemixes berücksichtigt werden müssen. Die geologischen Rahmenbedingungen für die tiefengeothermische Energienutzung in 3.500 bis $5.000 \mathrm{~m}$ Tiefe sind für die regional prägende Struktur des Leinetalgrabens noch weitgehend unbekannt und zwar in Hinsicht auf Lithologie, Struktur und geothermischen Gradienten. Vor einer richtungsentscheidenden Konzeption eines tiefengeothermischen Systems sind zunächst seismische Erkundungsmaßnahmen sowie eine Erkundungsbohrung Voraussetzung.

Abstract-In order to reach the ambitious political energy goals of the region, geothermal energy must play a more important role in the regenerative energy mix. The required geological framework to extract deep thermal energy from depths of 3,500 to 5,000 m within the regional structure of the Leinetal graben, in terms of lithology, structure and geothermal gradient, is widely unknown. A landmark decision concerning the conception of a deep geothermal system firstly prerequisites seismic investigation as well as a deep research borehole.
\end{abstract}

Schlüsselworte-Tiefengeothermische Nutzungssysteme, Leinetalgrabenstruktur, Stockwerkstektonik, Zechsteinsalinar

\section{Einführung}

\subsection{Ziele}

Im Dezember 2010 hat der Kreistag des Landkreises Göttingen beschlossen, dass bis zum Jahr 2025 über $50 \%$ der im Landkreis verbrauchten Energie an Strom und Wärme aus erneuerbaren Energienquellen stammen soll. Bis zum Jahr 2040 soll dieser Anteil auf 100 Prozent steigen und damit Energieautarkie erreicht werden.

Die Ziele der Stadt Göttingen definieren sich etwas anders: Reduktion der $\mathrm{CO}_{2}-$ bzw. Treibhausgasemissionen um $40 \%$ bis zum Jahr 2020, um $50 \%$ bis zum Jahr 2030 und um mindesten $95 \%$ bis zum Jahr 2050, jeweils bezogen

- Bernd Leiss - Geowissenschaftliches Zentrum der Universität Göttingen, Goldschmidtstr. 3, D-37077 Göttingen. bleiss1@gwdg.de

- David Tanner - GZG, jetzt Leibniz-Institut für angewandte Geophysik, Stilleweg 2, D-30655 Hannover. DavidColin.Tanner@liag-hannover.de

- Axel Vollbrecht - Geowissenschaftliches Zentrum der Universität Göttingen, Goldschmidtstr. 3, D-37077 Göttingen. avollbr@gwdg.de

- Klaus Wemmer - Geowissenschaftliches Zentrum der Universität Göttingen, Goldschmidtstr. 3, D-37077 Göttingen. kwemmer@gwdg.de auf das Jahr 1990. Es ist dabei nicht explizit festgelegt, wie dieses Ziel erreicht werden kann - inwieweit durch Einsatz erneuerbarer Energien oder durch Steigerung der Energieeffizienz. Gleichzeitig will die Georg-August Universität Göttingen bis zum Jahr 2020 eine Verminderung der klimarelevanten Emissionen um 40\% bezogen auf das Jahr 1990 erreichen und die Rahmenbedingungen des ,Erneuerbare-Energien-Wärmegesetzes (EEWärmeG) ${ }^{\natural}$ einhalten. Im Mai 2011 hat sich die ,Metropolregion Hannover Braunschweig Göttingen Wolfsburg، das ehrgeizige Ziel gesetzt, bis 2050 den Energiebedarf für Strom, Wärme und Verkehr vollständig aus erneuerbaren Energiequellen zu decken (http://www. metropolregion.de).

Die grundlastfähige, d.h. saisonal und vom Tages- und Jahresverlauf unabhängige tiefengeothermische Energienutzung spielt dabei in den bisherigen Diskussionen über einen effizienten Energiemix aus erneuerbaren Energien nur eine untergeordnete Rolle. Dies liegt unter anderem daran, dass die Erkundung der geologischen Rahmenbedingungen und die anschließende Erschließung in Tiefen von 3.500 bis $5.000 \mathrm{~m}$ sehr aufwendig ist und bislang erst wenige tiefengeo- 
thermische Konzepte inklusive Kraftwerkstechnik realisiert worden sind.

Die damit verbundenen, vergleichsweise hohen Investitionskosten sind mit einem relativ hohen Fündigkeitsrisiko verbunden, das aber durch eine möglichst fundierte Kenntnis der geologischen Rahmenbedingungen deutlich reduziert werden kann.

Ziel des vorliegenden Beitrags ist es, einen kurzen Status Quo der aktuellen Kenntnisse der regionalgeologischen Rahmenbedingungen für die verschiedenen tiefengeothermischen Energiekonzepte in allgemein verständlicher Form zusammenzufassen, um sie Entscheidungsträgern in Politik und Wirtschaft, öffentlichen und privaten Investoren, aber auch der interessierten Öffentlichkeit zugänglich zu machen. Unter Region Göttingen wird hier im Wesentlichen die Stadt und der Landkreis Göttingen sowie der südliche Landkreis von Northeim verstanden. Die Region Göttingen ist hier somit weniger durch politische Grenzen, als vielmehr durch die geologischen Gegebenheiten definiert.

\subsection{Tiefengeothermische Nutzungssysteme}

Zur Gewinnung geothermischer Energie für die Wärmeversorgung über Nah- und Fernwärmenetze sollten je nach Nutzungssystem Gesteinstemperaturen von mindestens $80^{\circ} \mathrm{C}$ und zur Stromerzeugung von mindestens $120^{\circ} \mathrm{C}$ zur Verfügung stehen. Diese Temperaturen können in Deutschland im allgemeinen ab ca. $2.500 \mathrm{~m}$ Tiefe angetroffen werden, man strebt aber in Abhängigkeit vom Nutzen-Kosten-Verhältnis Teufenbereiche von bis zu $5.000 \mathrm{~m}$ an, um möglichst hohe Temperaturen zu erreichen. Bei solchen Nutzungskonzepten wird von Tiefengeothermie gesprochen, die im Gegensatz zur oberflächennahen Erdwärmegewinnung steht, bei der die Wärmeenergie in der Regel dem ca. $8-12^{\circ} \mathrm{C}$ warmen Gestein aus 80 bis $150 \mathrm{~m}$ Tiefe mit Hilfe von Wärmepumpen entzogen wird. Auf die Region bezogene Informationen zur oberflächennahen Erdwärmegewinnung (z.B. Anzahl, Standort und Effizienz bislang installierter Systeme) finden sich bei Großmann (2010) und auf dem Kartenserver des Niedersächsischen Bodeninformationssystems (NIBIS 2011).

Für die geothermische Energiegewinnung aus großen Tiefen können hydro- und petrothermale Systeme unterschieden werden (z.B. Stober et al. 2010). Beim hydrothermalen System wird warmes Wasser aus einem tiefen Grundwasserleiter
(Aquifer) über eine Förderbohrung gewonnen und das nach Entzug der Wärmeenergie abgekühlte Wasser über eine Injektionsbohrung wieder verpresst (Dublettensystem). Um die notwendigen Förderraten zu realisieren, sind entsprechende Gesteinspermeabilitäten notwendig. Eine wichtige, aber bisher kaum verwirklichte Variante dieses Systems ist die Nutzung von Störungszonen. Störungszonen sind i.d.R. permeabler als die ,intakten' Nebengesteine und bieten dadurch eine große Wärmetauscherfläche für ein hydrothermales System an. Bei den petrothermalen Systemen gibt es zum Einen die tiefe Erdwärmesonde, bei der in einem geschlossenen System in der Bohrung ein Wärmeträgermedium zirkuliert. Zum Anderen gib es das so genannte ,Hot-Dry-Rock'-Verfahren (HDR) oder heute zunehmend auch als ,Enhanced Geothermal Systems (EGS)' bezeichnet, bei dem das trockene heiße Gestein zunächst (bei Bedarf) durch ein unter hohem Druck eingepresstes Fluid hydraulisch aufgebrochen wird (Stimulierung) und die dabei entstandenen Risssysteme (neben den natürlich vorhandenen) als Wärmetauscherflächen für das später zirkulierende Fluid über ein Dublettensystem genutzt werden können.

Welches Nutzungssystem realisierbar ist, hängt in erster Linie von den geologischen Rahmenbedingungen und von den vorhandenen oder zu installierenden Verbraucherstrukturen an der Oberfläche ab. Dies definiert dann auch das theoretische und technische Potential einer Region.

\section{Strukturgeologische Rahmenkennt- nisse}

Die Region Göttingen wird durch die N-S streichende, ca. 5 bis $12 \mathrm{~km}$ breite Leinetalgrabenstruktur geprägt (Abb. 1; siehe u.a. Arp et al. 2004; Tanner, Leiss et al. 2010; Arp et al. 2011, dieser Band; Vollbrecht und Tanner 2011, dieser Band und jeweils Referenzen darin). Das Grabenrandstörungsystem weist im mesozoischen Deckgebirge einen vertikalen Versatz von bis zu $800 \mathrm{~m}$ auf. An der Oberfläche werden die Grabenschultern vor allem durch Gesteine des Muschelkalks, das Grabeninnere durch Gesteine des Keupers aufgebaut. In ca. 800 bis $1500 \mathrm{~m}$ Tiefe folgt das Zechsteinsalinar (Abb. 2). Die durch Extensionsund die später überprägende Inversionstektonik entstandene Grabenstruktur (Tanner, Leiss et al. 2010; Vollbrecht und Tanner 2011, dieser Band) kann durch das leicht lösliche und plastisch leicht 


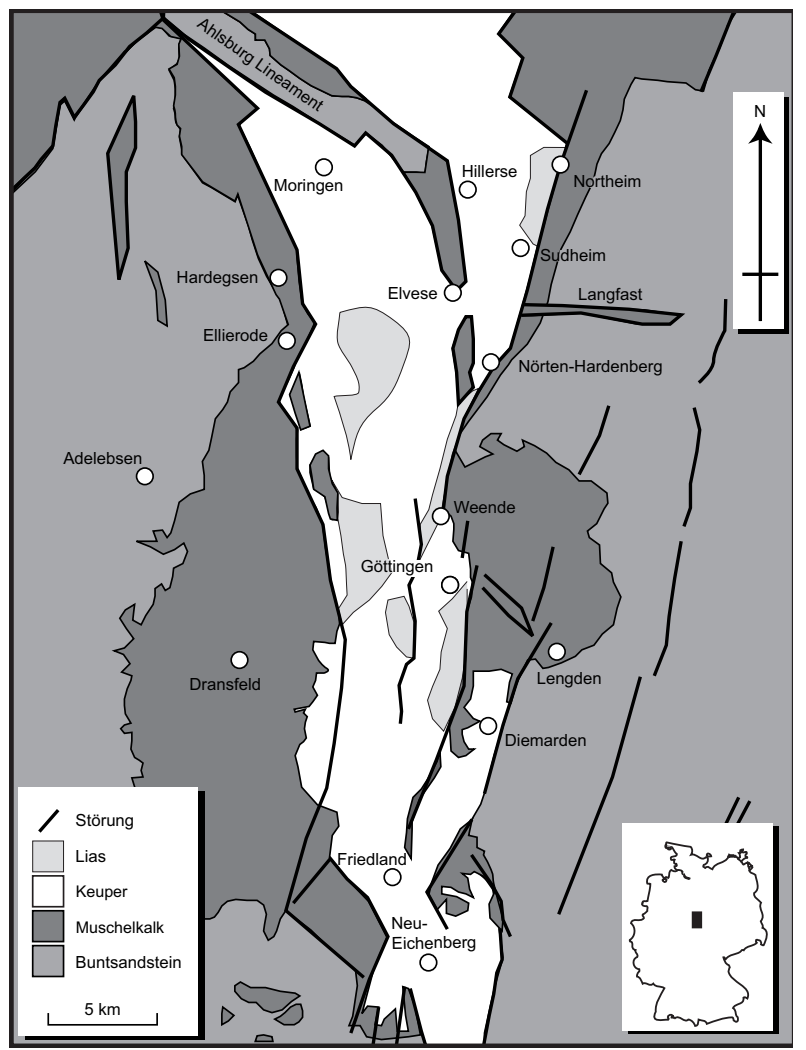

Abbildung 1. Geologische Übersichtskarte der $\mathrm{N}-\mathrm{S}$ streichenden Leinetalgrabenstruktur.

verformbare Kali- und Steinsalz lokal modifiziert sein (siehe Abb. 2). So können die Salinargesteine an Störungen aufsteigen und u.a. lakkolithartige Aufwölbungen verursachen oder es kann oberflächennah gelöst werden und kleinräumig Erdfälle oder großräumige Depressionen verursachen. Entsprechende Strukturen innerhalb der Schichtenfolge des Deckgebirges sind vor allem aus geologischen Oberflächenkartierungen abgeleitet worden. Nur aus dem ehemaligen KalisalzBergbau bei Reyershausen gibt es bedingt Kenntnisse zur Stratigraphie und Struktur des Zechsteinsalinars im Bereich der östlichen Grabenschulter für Tiefen zwischen 800 und $900 \mathrm{~m}$ (Oelrich et al. 2008). Zusätzliche Kenntnisse aus tieferen Bohrungen mit Endteufen zwischen knapp 800 und $1500 \mathrm{~m}$ gibt es in der Region nur von vier Standorten außerhalb des Grabens (Dransfeld, Sudheim/Levershausen, Reyershausen, Northeim, siehe NIBIS 2011, GeotIS 2011 und Fabian 1957, Müller et al. 1961, Pöhlig 1986, Grupe 1909). Im Jahr 2010 erfolgten über den Forschungsverbund ,Geothermie und Hochleistungsbohrtechnik (gebo, siehe http://www.gebo-nds.de)' erste seis- mische und geoelektrische Untersuchungen, die in einem E-W Profil über die östliche GrabenrandStörungszone bis in eine Teufe von ca. $1000 \mathrm{~m}$ reichen und bei denen zunächst methodische Aspekte im Vordergrund stehen (Musmann et al. 2011).

Das unter dem Zechstein liegende Grundgebirge (Präsalinar) gehört mit seinen Metasedimenten und möglichen Intrusiva des mittleren bis höheren Paläozoikums zum externen Falten- und Überschiebungsgürtel des variszischen Rhenoherzynikums, wie es im Harz und Rheinischen Schiefergebirge zu Tage tritt. In den Bohrungen Northeim 1 und Dransfeld 1 wurden in knapp $1.500 \mathrm{~m}$ Teufe oberkarbonische Tonsteine und Grauwacken angetroffen. Darüber folgen in der Bohrung Dransfeld 1 Zechsteinsedimente und in der Bohrung Northeim 1 sind noch ca. $70 \mathrm{~m}$ Rotliegend zwischengeschaltet. Aufgrund dieser geringmächtigen und offensichtlich nur lokal vorkommenden Rotliegendgesteine, spielen die entsprechenden Sandsteine und Vulkanite, die anderenorts als Zielgesteine in Betracht gezogen werden, im Raum Göttingen keine wesentliche Rolle.

Informationen über den Teufenbereich $>1.500$ $\mathrm{m}$ gibt es bislang nur in Form eines westlich der Grabenstruktur von der Solling-Aufwölbung bis in die Hessische Senke verlaufenden seismischen Profils aus dem DEKORP-Projekt (Deutsches Kontinentales Reflexionsseismisches Programm) von 1990 (DEKORP Research Group 1994). Diese Daten können allerdings nur sehr bedingt zur vorliegenden lokalen Fragestellung beitragen, da dort die Krustenstruktur bis in eine Tiefe von $60 \mathrm{~km}$ Ziel der Untersuchungen war und damit die notwendige hohe strukturelle Auflösung des tiefengeothermischen Zielhorizontes in 3 bis $5 \mathrm{~km}$ Tiefe nicht gegeben ist.

Die in der Region Göttingen in entsprechend tiefen Zielhorizonten zu erwartenden devonischen und unterkarbonischen Metasedimente und mögliche Intrusiva weisen durch Falten- und Überschiebungstektonik eine deutliche Verdickung ihrer primären Mächtigkeit von weit über $5.000 \mathrm{~m}$ auf. Es dürfte daher auszuschließen sein, dass eine Tiefengeothermie-Bohrung noch bis in das prävariszische Basement reicht. Der strukturelle Aufbau im Tiefenbereich eines möglichen Zielhorizonts (z.B. Tanner, Brandes et al. 2010), könnte demnach dem heutigen oder einem bereits erodierten Aufschlußniveau im Harz oder im Rheinischen Schiefergebirge ähneln (z.B. Franke 


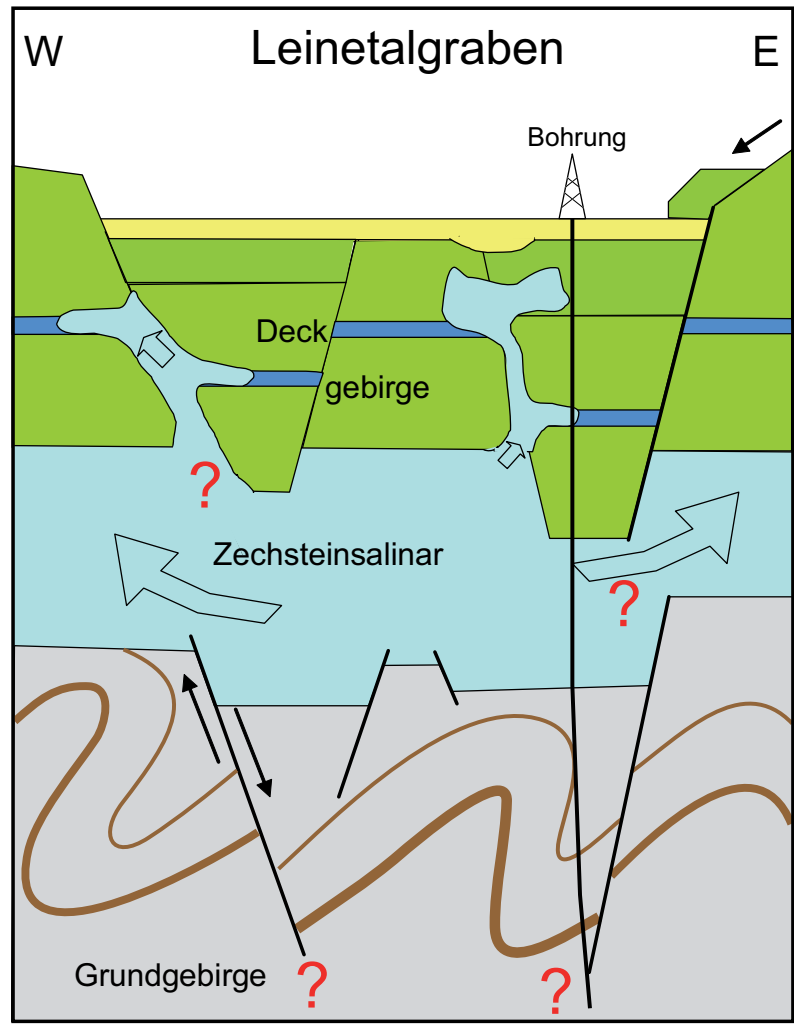

Abbildung 2. Stark schematisiertes, nicht skaliertes W-E Profil der Leinetalgrabenstruktur, das die ausgeprägte Stockwerkstektonik zeigt.

\section{0).}

Wie in dem stark vereinfachten Schema der Leinetalgrabenstruktur in Abb. 2 dargestellt, ist davon auszugehen, dass das Zechsteinlager als tektonischer Entkoppelungshorizont bzw. Abscherhorizont zwischen Deckgebirge und Grundgebirge fungieren kann. Die von der Oberflächenkartierung her bekannten, als steil einfallend interpretierten Störungssysteme können daher im Zechsteinsalz enden oder listrisch auslaufen, wie dies z.B. bereits von Grupe (1909) aufgrund von Bohrungen abgeleitet wurde (siehe auch Abb. 2 in Arp et al. 2011, dieser Band). Ob, wo und wie sich diese Störungssysteme im Zechsteinlager und Grundgebirge fortsetzen, ist aufgrund der mangelnden Datenbasis bislang weitgehend unbekannt.

\section{Regionaler geothermischer Gradient und hydrogeothermales Potential}

Temperaturdaten aus der Bohrung Dransfeld und aus dem ehemaligen Kalisalzberbergwerk bei Reyershausen weisen auf einen durchschnittlichen geothermischen Gradienten von ca. $30^{\circ} \mathrm{km}^{-1} \mathrm{im}$
Deckgebirge hin. Weitere direkte Informationen über den geothermischen Gradienten im Zechsteinsalinar und den tieferen Einheiten liegen nicht vor. Auch über mögliche Modifikationen des geothermischen Gradienten (auch im Sinne von Anomalien) durch erhöhte Wärmeströme in den stärker leitenden Salzen des Zechstein gibt es keine für die Region verwertbaren Angaben. Hinsichtlich dieser Fragestellung wurden erste spezielle Szenarien im Bereich der Grabenostrandstörung von Tanner, Albero et al. (2010), und Albero und Tanner (2011, dieser Band) modelliert. Die Temperaturangaben des tieferen Untergrundes in den vom Leibniz-Institut für Angewandte Geophysik (LIAG) in Hannover erstellten Temperaturgleichen-Karten beruhen auf Interpolationen von Bohrlochtemperaturmessungen (Abb. 3a, b). Aus Abb. 3a wird deutlich, dass die hohe Dichte an Bohrungen, wie sie im norddeutschen Becken vorliegen, ein lokal sehr differenziertes Bild des geothermischen Gradienten ergeben. Aus den vergleichsweise wenigen Bohrungen, die bis $500 \mathrm{~m}$ Tiefe reichen, ergibt sich für die Region Göttingen ein normaler geothermischer Gradient. Für den Teufenbereich von $3.000 \mathrm{~m}$ beruht der Verlauf der Isothermen nur noch auf der Basis einer einzigen Bohrung im weiteren Umfeld (Solling-Devon-1: $4.406 \mathrm{~m}$, NIBIS 2011; Abb. 3b). Betrachtet man die Region nördlich von Göttingen, so wird deutlich, dass sich kein genereller Trend für lokale Temperaturgradienten aus den $500 \mathrm{~m}$ und $3.000 \mathrm{~m}$ Isothermenkarten ableiten lässt. Dies wird auch am Beispiel des Wärmehochs bei Hannover sichtbar, das auf der $500 \mathrm{~m}$-Isothermenkarte noch nicht auffällt und erst auf der $3.000 \mathrm{~m}$-Isothermenkarte deutlich erscheint.

Hinweise auf ein mögliches paläogeothermisches Wärmehoch nordwestlich des Untersuchungsgebietes ergeben sich z.B. aus erhöhten Illitkristallinitäten und diagenetischen Mineralneubildungen (Vollbrecht und Tanner 2011, Abb. 2a-d, dieser Band). Die 500 m-Isothermenkarte (Abb. 3a) z.B. gibt einen Hinweis darauf, dass diese Wärmeanomalie möglicherweise auch heute noch von Bedeutung ist. Aber auch hier kann die $3.000 \mathrm{~m}$ Isothermenkarte aufgrund von mangelnden Bohrdaten keine Bestätigung liefern.

Abb. 4 zeigt die hydrogeothermalen Energieressourcen Deutschlands. Göttingen liegt dort in einem Bereich, der als ,Becken ohne nachgewiesene bzw. mit geringen hydrothermalen Energie- 

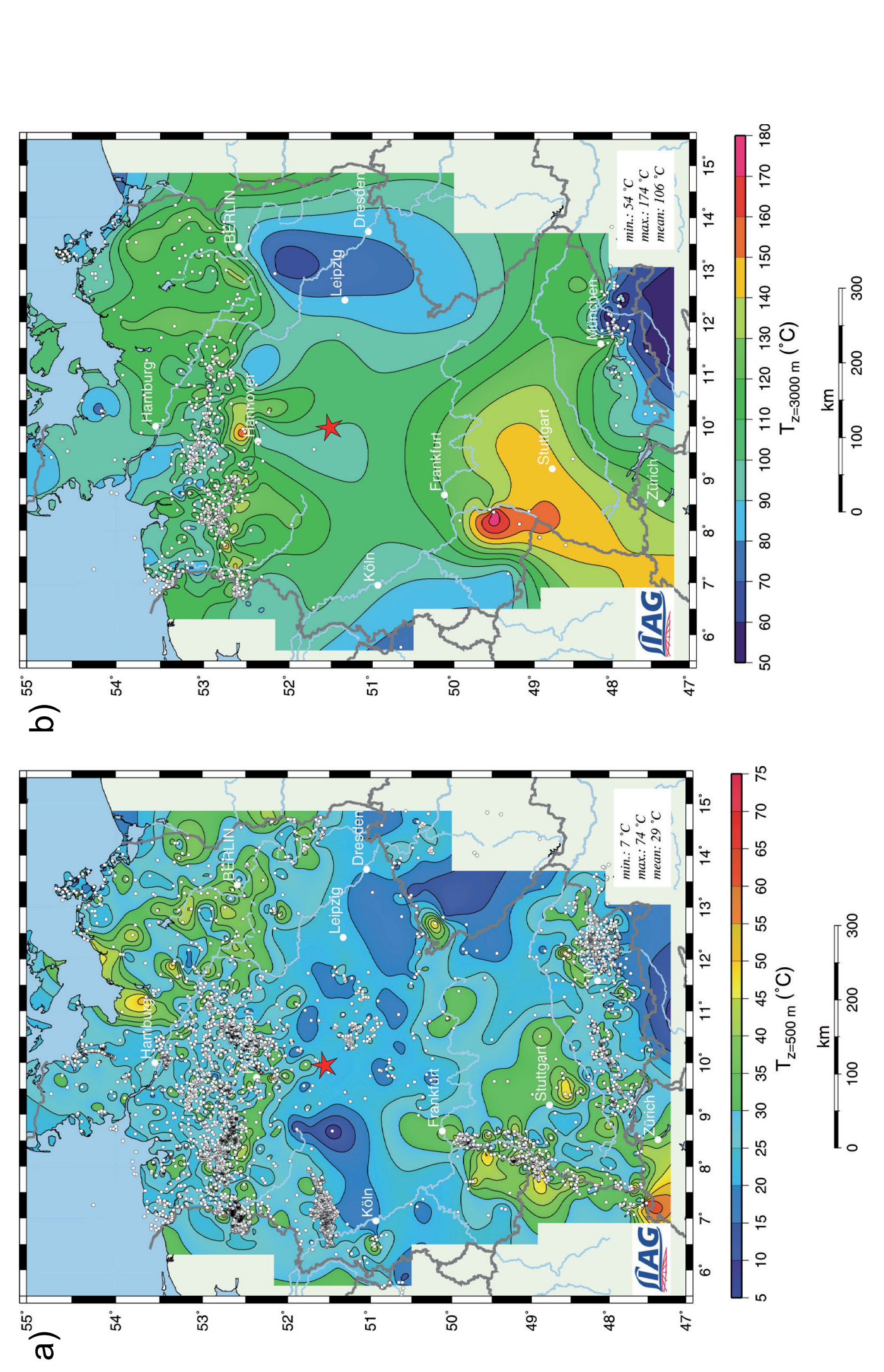

है

๑ำ

点 竞

๓

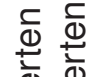

产

可 焉

으으 흘



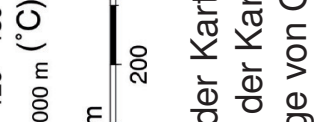

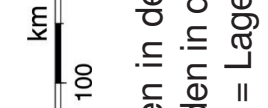

क्ष

I. $\begin{aligned} & 0 \\ & 0\end{aligned}$

ब离 㐫

ब。

次

त

등 오

员

"II

NN

ऽ

(1)

뜬 응 중

๘

的

$\cdots \bar{\Phi}$

응 땅

든 $\frac{\pi}{5}$

등

志这

동

ऽ

Q 논

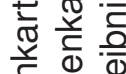

ब ह

힝

ब

으을

ชิ ह่

๓ 8

이

든 1 ㄷํㅇ

응 $\frac{1}{\frac{1}{2}}$ 


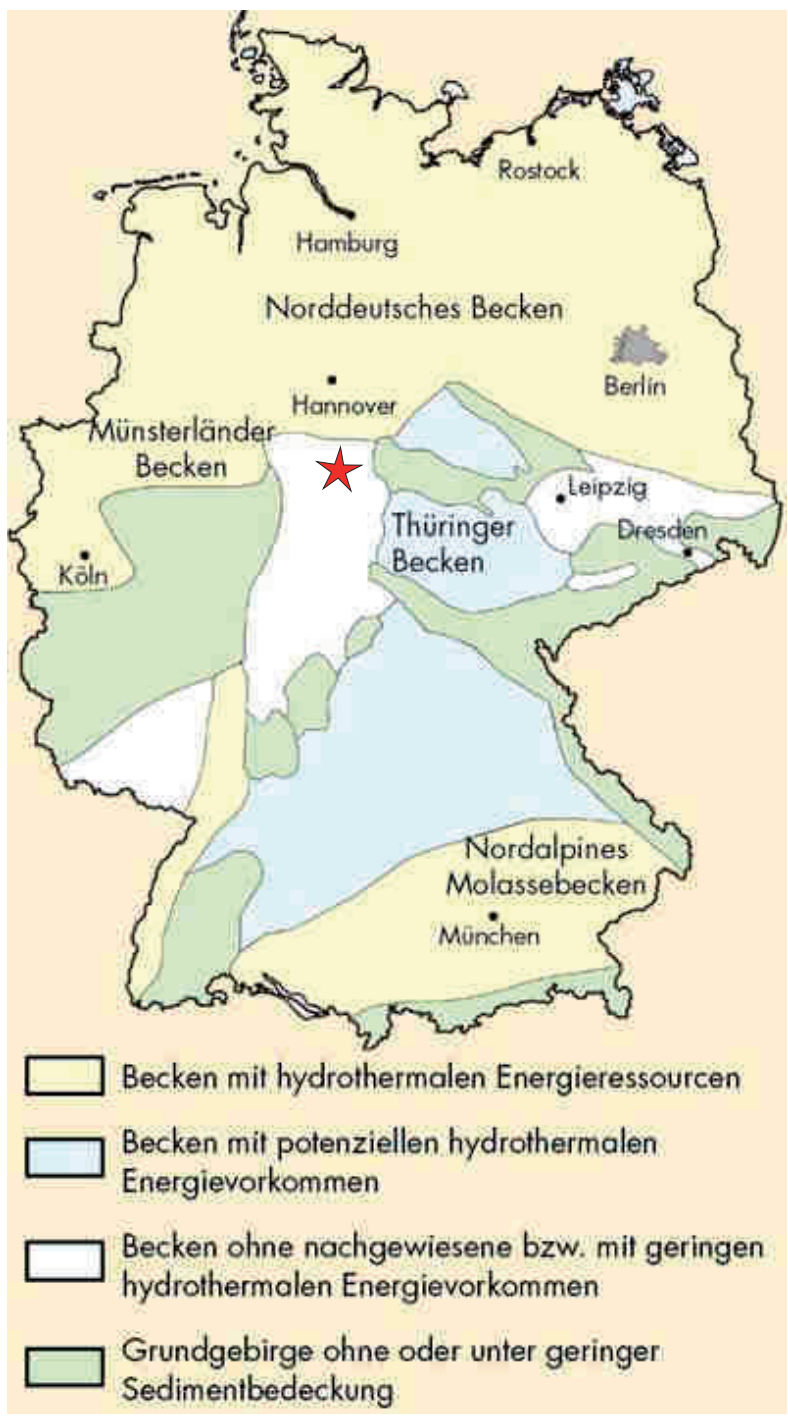

Abbildung 4. Hydrothermale Energievorkommen Deutschlands (Quelle: http://www.g-o.de, @ GFZ Potsdam). Stern = Lage von Göttingen .

vorkommen' ausgewiesen ist. Bezogen auf das mesozoische Deckgebirge liegt Göttingen am Westabfall der NE-SW verlaufenden Eichsfeldschwelle und damit sowohl in der lokalen Graben- als auch in einer überregionalen Beckensituation. Aus den teilweise für geothermale Ressourcen sehr gut geeigneten Gesteinen und Störungssystemen sind aus den bisherigen tieferen Bohrungen hydrothermale Energievorkommen nur aus der Saline Luisenhall bekannt (z.B. Bultmann et al. 2011, dieser Band), in der Sole aus $460 \mathrm{~m}$ mit einer in situ-Temperatur von $26^{\circ} \mathrm{C}$ gefördert wird. Hydrothermale Vorkommen sind aber auch aus dem ehemaligen Kalisalzbergwerk Königshall-Hindenburg bei Reyershausen bekannt. Der Zufluss von Ober- flächenwasser, möglicherweise vom Südwestrand des Harzes durch die nach SW einfallenden Schichten, führte während des Bergwerkbetriebes immer wieder zu Flutungen und schließlich zur Schließung des Bergwerkes. Die Frage, ob sich diese Situation auch im Grundgebirge wieder findet und dort Thermalwässer in permeablen Gesteinen oder an Störungszonen gebunden vorkommen, ist ungeklärt.

\section{Schlussfolgerungen und Ausblick}

Insgesamt ist für die Region Göttingen festzustellen, dass für eine quantitative Abschätzung des theoretischen geothermischen Potentials noch erhebliche Kenntnislücken vorliegen und damit risikominimierte Erschließungsstrategien und Nutzungssysteme noch nicht konzipiert werden können. Es sind daher zunächst zielgerichtete Erkundungsmaßnahmen erforderlich, die zu folgenden grundlegenden Fragestellungen beitragen müssen:

1) Inwieweit lassen sich kartierte Bruchstrukturen des oberflächennahen Deckgebirges in die potentiellen Zielhorizonte in 3-5 km Tiefe projizieren? Zentrale Problematik ist dabei die wahrscheinliche strukturelle Entkopplung von Deck- und Grundgebirge durch das zwischengelagerte, duktile Zechsteinsalinar. Zur Klärung dieser Fragestellung ist eine tiefenseismische Erkundung am effektivsten. Dadurch könnten auch die Mächtigkeiten der Stockwerkseinheiten, die nur punktuell aus Bohrungen bekannt sind, großräumiger modelliert werden.

2) Lassen sich die Strukturen wie sie im heutigen Erosionsniveau von Harz und Rheinischen Schiefergebirge aufgeschlossen sind auf subsalinare Zielhorizonte übertragen? Diese Frage betrifft sowohl die variszisch geprägten als auch die dort entwickelten jüngeren Bruchstrukturen. Derart komplex überlagerte Strukturen können nur mit einer hochauflösenden 3D-Seismik erfasst werden, um sie in störungsgebundene petro- und hydrothermale Modelle einzubringen.

3) Mit welchen in situ-Gesteinspermeabilitäten ist im Zielbereich geothermischer Nutzung zu rechnen? Hierbei sind alle aus dem Rhenoherzynikum bekannten Lithologien in Betracht zu ziehen (z.B. Ton- und Siltsteine, Grauwacken, Riffkalke, Intrusiva). Diese Daten 
können nur über eine entsprechend tiefe Erkundungsbohrung erbracht werden. Gleichzeitig können die erbohrten Lithologien in die geophysikalische und geologische Interpretation der seismischen Daten einfließen.

4) Mit welcher in situ-Spannungskonstellation ist im Zielgebiet zu rechnen? Wie aus der Worldstress-Map (WSM 2011) hervorgeht, liegen keine Messdaten für den betrachteten Großraum vor. Nördlich angrenzend ist die maximale horizontale Normalspannung etwa WNW-ESE, im Süden dagegen NNW-SSE gerichtet. In einer Bohrung könnte durch in situ-Messungen geklärt werden, welchem dieser Regime die Region Göttingen zuzuordnen ist. Die Kenntnis der Spannungssituation ist von großer Bedeutung für die hydrodynamische Modellierung von Geothermalsystemen.

5) Gibt es an Strukturstockwerke gebundene Hydrothermalsysteme? Auch diese, für das später zu konzipierende geothermische Nutzungssystem richtungsentscheidende Frage, kann nur mit einer tiefen Erkundungsbohrung beantwortet werden.

6) Sind potentiell geothermisch nutzbare Störungszonen möglicherweise mineralisiert, durch vorhandene Tonsteine oder durch neugebildete Tonletten verschmiert, d.h. hinsichtlich ihrer Permeabilität reduziert oder vollständig versiegelt? Auch dieser Aspekt kann nur durch eine Bohrung verfolgt werden, da oberflächennahe Beobachtungen nicht auf die Zielhorizonte übertragbar sind. So hängen Mineralisationen u.a. vom Chemismus des Ausgangsgesteins und vom Fluidangebot ab, sowohl hinsichtlich primärer Ausfällung, als auch möglicher sekundärer Lösung.

7) Können die Bereiche um potentiell geothermisch nutzbare Störungszonen durch die Zirkulation meteorischer Wässer ausgekühlt sein und inwieweit bzw. bis in welche Tiefe modifiziert das gut wärmeleitende Salzlager den geothermischen Gradienten? Eine Zirkulation meteorischer Wässer und eine damit verbundene Auskühlung bis in die Tiefen des Zielhorizontes kann als unwahrscheinlich angenommen werden. Auch hierzu und zur Frage, ob es nach einer unter dem Salzlager möglicherweise ausgekühlten Zone zu einem steileren Anstieg des geothemischen Gradienten kommt, kann ebenfalls nur durch eine
Bohrung beantwortet werden. Grundsätzlich und insbesondere hinsichtlich des geologischen Rahmens in der Region Göttingen, kann von einem oberflächennahen geothermischen Gradienten nicht auf einen tiefen geothermischen Gradienten geschlossen werden (vgl. Abb. 3a und b).

8) Könnte aus den durch das Salinar getrennten Deckgebirgs- und Grundgebirgsstockwerken durch kombinierte Nutzung ein spezifisches Geothermiesystem entwickelt werden?

Eine in jüngster Zeit häufig gestellte Frage, ist die nach einem erhöhten Erdbebenrisiko durch die hydraulische Stimulierung geothermischer Zielhorizonte (induzierte Seismizität), insbesondere im Zusammenhang mit den Störungssystemen der Leinetalgrabenstruktur. Im Vergleich zu anderen Regionen (z.B. Basel, http://www.wsu.bs.ch/ geothermie) ist das Risiko einer Aktivierung von Störungssystemen für den Raum Göttingen als gering einzustufen, da die natürliche Seismizität extrem niedrig ist (z.B. Leydecker 2005). Dies weist darauf hin, dass in historischen Zeiträumen keine hohen krustalen Spannungen aufgebaut werden und die lokalen Störungszonen quasi inaktiv sind. Eine quantitative Aussage der Stärke induzierter Seismizität kann im Vorfeld jedoch nicht getroffen werden, das Erdbebenrisiko durch eine projektbegleitende seismische und hydraulische Überwachung jedoch minimiert werden (z.B. Baisch 2009).

\section{Danksagung}

Wir danken zahlreichen Kolleginnen und Kollegen für viele anregende Diskussionen.

\section{Literatur}

Albero, F., Tanner, D. 2011. Modellierung des Temperaturfeldes um eine tiefe Förderbohrung am östlichen Leinetalgrabenrand zur Abschätzung des geothermischen Potentials, dieser Band.

Arp, G., Hoffmann V.-E., Seppelt S., Riegel W. 2004. Trias und Jura von Göttingen und Umgebung, 74. Jahrestagung der Paläontologischen Gesellschaft, 2.-8.10.2004, Exkursion 6, 147192, Universitätsdrucke.

Arp, G. Vollbrecht, A., Tanner, D., Leiss, B. 2011. Zur Geologie des Leinetalgrabens - ein kurzer Überblick, dieser Band. 
Baisch, S. 2009. Induzierte Seismizität in Geothermischen Reservoiren: Warum, Wo und Wie stark? Beitrag ,Der Geothermiekongress 2009' Bochum (www.geothermie.de/fileadmin/ useruploads/aktuelles/Geothermiekongress/ vortraege/EV_Baisch.pdf).

Bultmann, E.-M.', Ruprecht, J., Hansen, B.T., Kleinhanns, I. 2011. Zur Herkunft der Sole der Saline Luisenhall (Göttingen) mittels Strontiumisotopie, dieser Band.

DEKORP Research Group 1994. The deep reflection seismic profiles DEKORP 3/MVE-90. Zeitschrift für Geologische Wissenschaften 22, 627-824.

Fabian, H. J. 1957. Die Bohrung 'Northeim 1'. Ergebnisse eines regionalgeologisch interessanten Aufschlusses am Leinetalgraben. Neues Jahrbuch Geologie Paläontologie Abhandlungen 105, 113-122.

Franke, W., 2000. The mid-European segment of the Variscides: Tectonostratigraphic units, terrane boundaries and plate tectonic evolution, in W. Franke, V. Haak, O. Oncken, and D. Tanner, eds., Orogenic processes: Quantification and modeling in the Variscan belt: Geological Society (London) Special Publication 179, 1-3.

GeotIS 2011. Geothermisches Informationssystem für Deutschland. www.geotis.de

Großmann, S. 2010. Die Nutzung oberflächennaher Geothermie durch Privathaushalte Gründe für und gegen die Nutzung bei Neubauten und Sanierungsvorhaben. Unveröffentlichte Diplomarbeit Georg-August-Universität Göttingen, $120 \mathrm{~S}$.

Grupe, O. 1909. Über die Zechsteinformation und ihr Salzlager im Untergrunde des hannoverschen Eichsfeldes und angrenzenden Leinegebietes nach den neueren Bohrergebnissen. Zeitschrift Praktische Geologie 17, 185-205.

Leydecker, G. 2005. Erdbebenkatalog für die Bundesrepublik Deutschland mit Randgebieten für die Jahre 800-2004. Online-Datenfile, Bundesanstalt für Geowissenschaften und Rohstoffe, Hannover (http://www.bgr.bund.de).

Musmann, P., Thomas, R., Buness, H. 2011. Seismische Erkundung von geologischen Störungszonen am Beispiel des Leinetalgrabens: Erste Ergebnisse, in '71. Jahrestagung der Deutschen Geophysikalischen Gesellschaft, Köln, 21.-24. Februar 2011'.

Müller, M., Boigk, H., Füchtbauer, H., Malzahn, E., Eckardt, F.J., Mattiat, B. 1961. Schichten- verzeichnis und geologischer Bericht über die Aufschlussbohrung Dransfeld 1, Niedersächsisches Landesamt für Bodenforschung, Bohrakte 33198, Hannover.

NIBIS 2011: Niedersächsisches Bodeninformationssystem. NIBIS@ KARTENSERVER: http://http://www.lbeg.niedersachsen.de/ live/live.php?navigation_id $=608 \&$ article_id $=$ 841\&_psmand $=4$

Oelrich, A.R.I., Leiss, B., Tanner, D., Hansen, B.T. 2008. Geologisches 3D-Modell des ehemaligen Kalibergwerkes Reyershausen (Südniedersachsen). TSK 12 Karlsruhe, Geotectonic Research 95/01, 130-131.

Pöhlig, Ch. 1986. Sedimentologie des Zechsteinkalks und des Werra-Anhydrits (Zechstein 1) in Südost-Niedersachsen. Göttinger Arbeiten zur Geologie und Paläontologie, 99 S.

Stober, I. Fritzer, T., Obst, K., Schulz, R. 2010. Nutzungsmöglichkeiten in Deutschland. Bundesministerium für Umwelt, Naturschutz und Reaktorsicherheit (Hrsg.), Bonn, 75 S.

Tanner, D.C., Leiss, B., Vollbrecht, A., the GGG 2010. The Role of Strike-Slip Tectonics in the Leinetal Graben, Lower Saxony. Zeitschrift der deutschen Gesellschaft für Geowissenschaften 161, 369-377.

Tanner, D.C., Albero, F., Leiss, B., the GGG 2010. Modelling the Geothermal Potential of the Eastern Border of the Leinetal Graben, Lower Saxony. Zeitschrift für Geologische Wissenschaften 38, 61-68.

Tanner, D. C., Brandes, C., Leiss, B. 2010. Structure and kinematics of an outcrop-scale, foldcored triangle zone. AAPG Bulletin 94, 1799 1809.

Vollbrecht, A., Tanner, D.C. 2011. Der Leinetalgraben als Teil einer regionalen Pull-ApartStruktur, dieser Band.

WSM (2011): World Stress Map Project (Internetbasiert). Helmholtz Zentrum Potsdam, GFZ - Deutsches GeoForschungszentrum. http://www.world-stress-map.org. 
Der vorliegende Band enthält eine Sammlung von Arbeiten, die sich mit unterschiedlichen Aspekten der geologischen Entwicklung des Leinetalgrabens und seines strukturellen Rahmens befassen. Grundlage stellen neue und ältere, bislang nicht veröffentlichte Ergebnisse dar, die teilweise im Rahmen von geowissenschaftlichen Examensarbeiten erbracht wurden. Diese häufig in Archiven „versteckten“ Daten sollen hiermit interessierten Fachleuten aber auch geologisch interessierten Laien zugänglich gemacht werden. Herausgeber ist die $\mathrm{Ge}-$ othermieGruppeGöttingen (GCG), in der GeowissenschaftlerInnen aus verschiedenen Abteilungen des Geowissenschaftlichen Zentrums der Georg-August-Universität Göttingen (GZG) tätig sind. Aktuelles Forschungsziel der GGG ist, ein dreidimensionales Strukturmodell für die Region Göttingen mit dem Leinetalgraben als zentrale Struktur zu erstellen, das als Grundlage für eine anwendungsbezogene Bewertung geothermischer Potentiale dienen soll. 